\title{
Design, Synthesis, and Structure-activity Relationships for Chimeric Inhibitors of Hsp90
}

\author{
Gang Shen, Mingwen Wang, Timothy R. Welch, and Brian S. J. Blagg* \\ Supporting Information
}

Department of Medicinal Chemistry, The University of Kansas, 1251 Wescoe Hall Dr.,

Malott Hall 4070, Lawrence, KS 66045-7563

bblagg@ku.edu 
Table of Contents

${ }^{1} \mathrm{H}$ NMR spectrum of 2

${ }^{13} \mathrm{C}$ NMR spectrum of $\mathbf{2}$ S9

${ }^{1} \mathrm{H}$ NMR spectrum of $4 \quad \mathrm{~S} 10$

${ }^{13} \mathrm{C}$ NMR spectrum of $\mathbf{4} \quad \mathrm{S} 11$

${ }^{1} \mathrm{H}$ NMR spectrum of $\mathbf{5} \quad \mathrm{S} 12$

${ }^{13} \mathrm{C}$ NMR spectrum of $\mathbf{5} \quad \mathrm{S} 13$

${ }^{1} \mathrm{H}$ NMR spectrum of $\mathbf{5 . 1} \quad \mathrm{S} 14$

${ }^{13} \mathrm{C}$ NMR spectrum of $\mathbf{5 . 1} \quad \mathrm{S} 15$

${ }^{1} \mathrm{H}$ NMR spectrum of $6 \quad \mathrm{~S} 16$

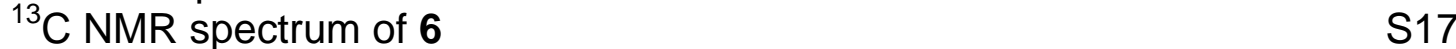

${ }^{1} \mathrm{H}$ NMR spectrum of $\mathbf{3} \quad \mathrm{S} 18$

${ }^{13} \mathrm{C}$ NMR spectrum of $3 \quad \mathrm{~S} 19$

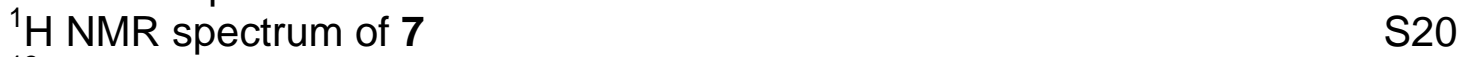

${ }^{13} \mathrm{C}$ NMR spectrum of $7 \quad$ S21

${ }^{1} \mathrm{H}$ NMR spectrum of $8 \quad$ S22

${ }^{13} \mathrm{C}$ NMR spectrum of 8

${ }^{1} \mathrm{H}$ NMR spectrum of $10 \quad$ S24

${ }^{13} \mathrm{C}$ NMR spectrum of $10 \quad \mathrm{~S} 25$

${ }^{1} \mathrm{H}$ NMR spectrum of $\mathbf{9} \quad$ S26

${ }^{13} \mathrm{C}$ NMR spectrum of $\mathbf{9} \quad \mathrm{S} 27$

${ }^{1} \mathrm{H}$ NMR spectrum of 11

${ }^{13} \mathrm{C}$ NMR spectrum of $11 \quad$ S29

${ }^{1} \mathrm{H}$ NMR spectrum of 12

${ }^{13} \mathrm{C}$ NMR spectrum of 12

${ }^{1} \mathrm{H}$ NMR spectrum of 13

${ }^{13} \mathrm{C}$ NMR spectrum of 13

${ }^{1} \mathrm{H}$ NMR spectrum of 14

${ }^{13} \mathrm{C}$ NMR spectrum of $\mathbf{1 4}$

${ }^{1} \mathrm{H}$ NMR spectrum of 15

${ }^{13} \mathrm{C}$ NMR spectrum of $15 \quad$ S37

${ }^{1} \mathrm{H}$ NMR spectrum of $16 \quad$ S38

${ }^{13} \mathrm{C}$ NMR spectrum of $16 \quad$ S39

${ }^{1} \mathrm{H}$ NMR spectrum of $\mathbf{1 7} \quad \mathrm{S} 40$

${ }^{13} \mathrm{C}$ NMR spectrum of $17 \quad$ S41

${ }^{1} \mathrm{H}$ NMR spectrum of $18 \quad \mathrm{~S} 42$

${ }^{13} \mathrm{C}$ NMR spectrum of 18

${ }^{1} \mathrm{H}$ NMR spectrum of 19

${ }^{13} \mathrm{C}$ NMR spectrum of 19

${ }^{1} \mathrm{H}$ NMR spectrum of $\mathbf{2 0} \quad \mathrm{S} 46$

${ }^{13} \mathrm{C}$ NMR spectrum of $20 \quad \mathrm{~S} 47$

${ }^{1} \mathrm{H}$ NMR spectrum of $\mathbf{2 1} \quad \mathrm{S} 48$

${ }^{13} \mathrm{C}$ NMR spectrum of $\mathbf{2 1} \quad$ S49

${ }^{1} \mathrm{H}$ NMR spectrum of $\mathbf{2 2} \quad$ S50

${ }^{13} \mathrm{C}$ NMR spectrum of 22 
${ }^{1} \mathrm{H}$ NMR spectrum of 25

${ }^{13} \mathrm{C}$ NMR spectrum of 25

${ }^{1} \mathrm{H}$ NMR spectrum of $\mathbf{2 6} \quad$ S54

${ }^{13} \mathrm{C}$ NMR spectrum of $\mathbf{2 6} \quad$ S55

${ }^{1} \mathrm{H}$ NMR spectrum of $27 \quad$ S56

${ }^{13} \mathrm{C}$ NMR spectrum of $27 \quad$ S57

${ }^{1} \mathrm{H}$ NMR spectrum of $\mathbf{2 5 . 1}$

${ }^{13} \mathrm{C}$ NMR spectrum of $25.1 \quad$ S59

${ }^{1} \mathrm{H}$ NMR spectrum of $29 \quad$ S60

${ }^{13} \mathrm{C}$ NMR spectrum of $29 \quad$ S61

${ }^{1} \mathrm{H}$ NMR spectrum of $\mathbf{3 0} \quad$ S62

${ }^{13} \mathrm{C}$ NMR spectrum of $\mathbf{3 0} \quad$ S63

${ }^{1} \mathrm{H}$ NMR spectrum of $\mathbf{3 1} \quad \mathrm{S64}$

${ }^{13} \mathrm{C}$ NMR spectrum of $\mathbf{3 1} \quad \mathrm{S65}$

${ }^{1} \mathrm{H}$ NMR spectrum of $\mathbf{3 2} \quad$ S66

${ }^{13} \mathrm{C}$ NMR spectrum of $\mathbf{3 2} \quad \mathrm{S67}$

${ }^{1} \mathrm{H}$ NMR spectrum of $\mathbf{3 3} \quad$ S68

${ }^{13} \mathrm{C}$ NMR spectrum of $33 \quad 569$

${ }^{1} \mathrm{H}$ NMR spectrum of $35 \quad S 70$

${ }^{13} \mathrm{C}$ NMR spectrum of $\mathbf{3 5} \quad \mathrm{S71}$

${ }^{1} \mathrm{H}$ NMR spectrum of $\mathbf{3 6} \quad \mathrm{S} 72$

${ }^{13} \mathrm{C}$ NMR spectrum of $\mathbf{3 6} \quad \mathrm{S} 73$

${ }^{1} \mathrm{H}$ NMR spectrum of $\mathbf{3 7} \quad \mathrm{S74}$

${ }^{13} \mathrm{C}$ NMR spectrum of $37 \quad \mathrm{S75}$

${ }^{1} \mathrm{H}$ NMR spectrum of $\mathbf{3 8} \quad \mathrm{S} 76$

${ }^{13} \mathrm{C}$ NMR spectrum of $\mathbf{3 8} \quad \mathrm{S77}$

${ }^{1} \mathrm{H}$ NMR spectrum of $\mathbf{4 0} \quad \mathrm{S78}$

${ }^{13} \mathrm{C}$ NMR spectrum of $40 \quad \mathrm{S79}$

${ }^{1} \mathrm{H}$ NMR spectrum of $\mathbf{4 1} \quad S 80$

${ }^{13} \mathrm{C}$ NMR spectrum of $\mathbf{4 1} \quad$ S81

${ }^{1} \mathrm{H}$ NMR spectrum of $42 \quad$ S82

${ }^{13} \mathrm{C}$ NMR spectrum of $\mathbf{4 2} \quad$ S83

${ }^{1} \mathrm{H}$ NMR spectrum of $\mathbf{4 3} \quad \mathrm{S84}$

${ }^{13} \mathrm{C}$ NMR spectrum of $\mathbf{4 3} \quad S 85$

${ }^{1} \mathrm{H}$ NMR spectrum of $\mathbf{4 5} \quad$ S86

${ }^{13} \mathrm{C}$ NMR spectrum of $\mathbf{4 5} \quad \mathrm{S} 87$

${ }^{1} \mathrm{H}$ NMR spectrum of $46 \quad$ S88

${ }^{13} \mathrm{C}$ NMR spectrum of $46 \quad$ S89

${ }^{1} \mathrm{H}$ NMR spectrum of $\mathbf{4 7} \quad S 90$

${ }^{13} \mathrm{C}$ NMR spectrum of $\mathbf{4 7} \quad \mathrm{S} 91$

${ }^{1} \mathrm{H}$ NMR spectrum of $\mathbf{4 8} \quad$ S92

${ }^{13} \mathrm{C}$ NMR spectrum of $\mathbf{4 8} \quad$ S93

${ }^{1} \mathrm{H}$ NMR spectrum of $\mathbf{5 0} \quad \mathrm{S94}$

${ }^{13} \mathrm{C}$ NMR spectrum of $\mathbf{5 0} \quad \mathrm{S95}$

${ }^{1} \mathrm{H}$ NMR spectrum of $\mathbf{5 1} \quad$ S96

${ }^{13} \mathrm{C}$ NMR spectrum of $\mathbf{5 1} \quad \mathrm{S} 97$ 
${ }^{1} \mathrm{H}$ NMR spectrum of $\mathbf{5 2}$

${ }^{13} \mathrm{C}$ NMR spectrum of $\mathbf{5 2}$

${ }^{1} \mathrm{H}$ NMR spectrum of $\mathbf{5 3}$

$\mathrm{S} 100$

${ }^{13} \mathrm{C}$ NMR spectrum of 53

S101

${ }^{1} \mathrm{H}$ NMR spectrum of $\mathbf{5 5}$

S102

${ }^{13} \mathrm{C}$ NMR spectrum of $\mathbf{5 5}$

$\mathrm{S} 103$

${ }^{1} \mathrm{H}$ NMR spectrum of $\mathbf{5 6}$

S104

${ }^{13} \mathrm{C}$ NMR spectrum of $\mathbf{5 6}$

S105

${ }^{1} \mathrm{H}$ NMR spectrum of $\mathbf{5 8}$

S106

${ }^{13} \mathrm{C}$ NMR spectrum of $\mathbf{5 8}$

S107

${ }^{1} \mathrm{H}$ NMR spectrum of $\mathbf{5 9 . 1}$

$\mathrm{S} 108$

${ }^{13} \mathrm{C}$ NMR spectrum of $\mathbf{5 9 . 1}$

S109

${ }^{1} \mathrm{H}$ NMR spectrum of 59

S110

${ }^{13} \mathrm{C}$ NMR spectrum of $\mathbf{5 9}$

S111

${ }^{1} \mathrm{H}$ NMR spectrum of 60

$\mathrm{S} 112$

${ }^{13} \mathrm{C}$ NMR spectrum of $\mathbf{6 0}$

S113

${ }^{1} \mathrm{H}$ NMR spectrum of $\mathbf{6 2}$

S114

${ }^{13} \mathrm{C}$ NMR spectrum of $\mathbf{6 2}$

S115

${ }^{1} \mathrm{H}$ NMR spectrum of 63

S116

${ }^{13} \mathrm{C}$ NMR spectrum of 63

${ }^{1} \mathrm{H}$ NMR spectrum of 64

S118

${ }^{13} \mathrm{C}$ NMR spectrum of 64

S119

${ }^{1} \mathrm{H}$ NMR spectrum of 65

S120

${ }^{13} \mathrm{C}$ NMR spectrum of 65

S121

${ }^{1} \mathrm{H}$ NMR spectrum of $\mathbf{6 6}$

S122

${ }^{13} \mathrm{C}$ NMR spectrum of $\mathbf{6 6}$

S123

${ }^{1} \mathrm{H}$ NMR spectrum of $\mathbf{6 7}$

S124

${ }^{13} \mathrm{C}$ NMR spectrum of 67

S125

${ }^{1} \mathrm{H}$ NMR spectrum of $\mathbf{6 8}$

S126

${ }^{13} \mathrm{C}$ NMR spectrum of $\mathbf{6 8}$

S127

${ }^{1} \mathrm{H}$ NMR spectrum of 69

S128

${ }^{13} \mathrm{C}$ NMR spectrum of 69

S129

${ }^{1} \mathrm{H}$ NMR spectrum of $\mathbf{7 0}$

$\mathrm{S} 130$

${ }^{13} \mathrm{C}$ NMR spectrum of 70

S131

${ }^{1} \mathrm{H}$ NMR spectrum of $\mathbf{7 1}$

$\mathrm{S} 132$

${ }^{13} \mathrm{C}$ NMR spectrum of $\mathbf{7 1}$

$\mathrm{S} 133$

${ }^{1} \mathrm{H}$ NMR spectrum of 72

S134

${ }^{13} \mathrm{C}$ NMR spectrum of 72

$\mathrm{S} 135$

${ }^{1} \mathrm{H}$ NMR spectrum of 73

S136

${ }^{13} \mathrm{C}$ NMR spectrum of 73

S137

${ }^{1} \mathrm{H}$ NMR spectrum of $\mathbf{7 4}$

S138

${ }^{13} \mathrm{C}$ NMR spectrum of $\mathbf{7 4}$

S139

${ }^{1} \mathrm{H}$ NMR spectrum of 75

S140

${ }^{13} \mathrm{C}$ NMR spectrum of 75

S141

${ }^{1} \mathrm{H}$ NMR spectrum of 76

S142

${ }^{13} \mathrm{C}$ NMR spectrum of 76

S143 
${ }^{1} \mathrm{H}$ NMR spectrum of $\mathbf{7 8}$

${ }^{13} \mathrm{C}$ NMR spectrum of $\mathbf{7 8}$

${ }^{1} \mathrm{H}$ NMR spectrum of 79

S146

${ }^{13} \mathrm{C}$ NMR spectrum of 79

${ }^{1} \mathrm{H}$ NMR spectrum of $\mathbf{8 0}$

${ }^{13} \mathrm{C}$ NMR spectrum of $\mathbf{8 0}$

${ }^{1} \mathrm{H}$ NMR spectrum of $\mathbf{8 1}$

S150

${ }^{13} \mathrm{C}$ NMR spectrum of $\mathbf{8 1}$

S151

${ }^{1} \mathrm{H}$ NMR spectrum of $\mathbf{8 2}$

S152

${ }^{13} \mathrm{C}$ NMR spectrum of $\mathbf{8 2}$

S153

${ }^{1} \mathrm{H}$ NMR spectrum of $\mathbf{8 3}$

S154

${ }^{13} \mathrm{C}$ NMR spectrum of 83

S155

${ }^{1} \mathrm{H}$ NMR spectrum of $\mathbf{8 4}$

S156

${ }^{13} \mathrm{C}$ NMR spectrum of $\mathbf{8 4}$

S157

${ }^{1} \mathrm{H}$ NMR spectrum of $\mathbf{8 5}$

S158

${ }^{13} \mathrm{C}$ NMR spectrum of $\mathbf{8 5}$

S159

${ }^{1} \mathrm{H}$ NMR spectrum of $\mathbf{8 8}$

S160

${ }^{13} \mathrm{C}$ NMR spectrum of $\mathbf{8 8}$

S161

${ }^{1} \mathrm{H}$ NMR spectrum of $\mathbf{8 9}$

S162

${ }^{13} \mathrm{C}$ NMR spectrum of $\mathbf{8 9}$

S163

${ }^{1} \mathrm{H}$ NMR spectrum of $\mathbf{9 0}$

S164

${ }^{13} \mathrm{C}$ NMR spectrum of $\mathbf{9 0}$

S165

${ }^{1} \mathrm{H}$ NMR spectrum of 91

S166

${ }^{13} \mathrm{C}$ NMR spectrum of 91

${ }^{1} \mathrm{H}$ NMR spectrum of 93

S168

${ }^{13} \mathrm{C}$ NMR spectrum of 93

S169

${ }^{1} \mathrm{H}$ NMR spectrum of 96

S170

${ }^{13} \mathrm{C}$ NMR spectrum of $\mathbf{9 6}$

S171

${ }^{1} \mathrm{H}$ NMR spectrum of 97

S172

${ }^{13} \mathrm{C}$ NMR spectrum of 97

S173

${ }^{1} \mathrm{H}$ NMR spectrum of $\mathbf{9 8}$

S174

${ }^{13} \mathrm{C}$ NMR spectrum of $\mathbf{9 8}$

S175

${ }^{1} \mathrm{H}$ NMR spectrum of 95

S176

${ }^{13} \mathrm{C}$ NMR spectrum of 95

S177

${ }^{1} \mathrm{H}$ NMR spectrum of $\mathbf{9 2}$

S178

${ }^{13} \mathrm{C}$ NMR spectrum of 92

S179

${ }^{1} \mathrm{H}$ NMR spectrum of 94

S180

${ }^{13} \mathrm{C}$ NMR spectrum of 94

S181

${ }^{1} \mathrm{H}$ NMR spectrum of 99

S182

${ }^{13} \mathrm{C}$ NMR spectrum of 99

S183

${ }^{1} \mathrm{H}$ NMR spectrum of $\mathbf{1 0 0}$

$\mathrm{S} 184$

${ }^{13} \mathrm{C}$ NMR spectrum of 100

S185

${ }^{1} \mathrm{H}$ NMR spectrum of 101

S186

${ }^{13} \mathrm{C}$ NMR spectrum of 101

${ }^{1} \mathrm{H}$ NMR spectrum of $\mathbf{1 1 2}$

S188

${ }^{13} \mathrm{C}$ NMR spectrum of $\mathbf{1 1 2}$

S189 
${ }^{1} \mathrm{H}$ NMR spectrum of $\mathbf{1 1 6 a}$

${ }^{13} \mathrm{C}$ NMR spectrum of $\mathbf{1 1 6} \mathbf{a}$

${ }^{1} \mathrm{H}$ NMR spectrum of $117 \mathrm{a}$

S192

${ }^{13} \mathrm{C}$ NMR spectrum of $117 \mathrm{a}$

S193

${ }^{1} \mathrm{H}$ NMR spectrum of $\mathbf{1 1 8 a}$

S194

${ }^{13} \mathrm{C}$ NMR spectrum of $\mathbf{1 1 8 a}$

${ }^{1} \mathrm{H}$ NMR spectrum of $119 \mathrm{a}$

S196

${ }^{13} \mathrm{C}$ NMR spectrum of $119 a$

S197

${ }^{1} \mathrm{H}$ NMR spectrum of $120 \mathrm{a}$

S198

${ }^{13} \mathrm{C}$ NMR spectrum of $\mathbf{1 2 0 a}$

S199

${ }^{1} \mathrm{H}$ NMR spectrum of $116 \mathrm{c}$

$\mathrm{S} 200$

${ }^{13} \mathrm{C}$ NMR spectrum of $116 \mathrm{c}$

$\mathrm{S} 201$

${ }^{1} \mathrm{H}$ NMR spectrum of $\mathbf{1 1 8 \mathrm { c }}$

S202

${ }^{13} \mathrm{C}$ NMR spectrum of $118 \mathrm{c}$

$\mathrm{S} 203$

${ }^{1} \mathrm{H}$ NMR spectrum of $119 \mathrm{c}$

$\mathrm{S} 204$

${ }^{13} \mathrm{C}$ NMR spectrum of $119 \mathrm{c}$

S205

${ }^{1} \mathrm{H}$ NMR spectrum of $120 \mathrm{c}$

S206

${ }^{13} \mathrm{C}$ NMR spectrum of $120 \mathrm{c}$

S207

${ }^{1} \mathrm{H}$ NMR spectrum of $121 \mathrm{c}$

S208

${ }^{13} \mathrm{C}$ NMR spectrum of 121c

S209

${ }^{1} \mathrm{H}$ NMR spectrum of $122 \mathrm{c}$

$\mathrm{S} 210$

${ }^{13} \mathrm{C}$ NMR spectrum of $122 \mathrm{c}$

$\mathrm{S} 211$

${ }^{1} \mathrm{H}$ NMR spectrum of $110 \mathrm{a}$

$\mathrm{S} 212$

${ }^{13} \mathrm{C}$ NMR spectrum of 110a

$\mathrm{S} 213$

${ }^{1} \mathrm{H}$ NMR spectrum of 111a

$\mathrm{S} 214$

${ }^{13} \mathrm{C}$ NMR spectrum of $\mathbf{1 1 1 a}$

S215

${ }^{1} \mathrm{H}$ NMR spectrum of $\mathbf{1 1 6 e}$

$\mathrm{S} 216$

${ }^{13} \mathrm{C}$ NMR spectrum of $\mathbf{1 1 6 e}$

$\mathrm{S} 217$

${ }^{1} \mathrm{H}$ NMR spectrum of $118 \mathrm{e}$

S218

${ }^{13} \mathrm{C}$ NMR spectrum of $\mathbf{1 1 8 e}$

S219

${ }^{1} \mathrm{H}$ NMR spectrum of $120 \mathrm{e}$

S220

${ }^{13} \mathrm{C}$ NMR spectrum of $120 \mathrm{e}$

$\mathrm{S} 221$

${ }^{1} \mathrm{H}$ NMR spectrum of $121 \mathrm{e}$

$\mathrm{S} 222$

${ }^{13} \mathrm{C}$ NMR spectrum of $121 \mathrm{e}$

$\mathrm{S} 223$

${ }^{1} \mathrm{H}$ NMR spectrum of $110 \mathrm{~b}$

$\mathrm{S} 224$

${ }^{13} \mathrm{C}$ NMR spectrum of $\mathbf{1 1 0 b}$

$\mathrm{S} 225$

${ }^{1} \mathrm{H}$ NMR spectrum of $\mathbf{1 1 1 b}$

$\mathrm{S} 226$

${ }^{13} \mathrm{C}$ NMR spectrum of $\mathbf{1 1 1 b}$

$\mathrm{S} 227$

${ }^{1} \mathrm{H}$ NMR spectrum of $\mathbf{1 1 6 f}$

$\mathrm{S} 228$

${ }^{13} \mathrm{C}$ NMR spectrum of $\mathbf{1 1 6 f}$

$\mathrm{S} 229$

${ }^{1} \mathrm{H}$ NMR spectrum of $\mathbf{1 1 8 f}$

$\mathrm{S} 230$

${ }^{13} \mathrm{C}$ NMR spectrum of $\mathbf{1 1 8 f}$

$\mathrm{S} 231$

${ }^{1} \mathrm{H}$ NMR spectrum of $120 \mathrm{f}$

$\mathrm{S} 232$

${ }^{13} \mathrm{C}$ NMR spectrum of $120 \mathrm{f}$

$\mathrm{S} 233$

${ }^{1} \mathrm{H}$ NMR spectrum of $\mathbf{1 2 1 f}$

$\mathrm{S} 234$

${ }^{13} \mathrm{C}$ NMR spectrum of $\mathbf{1 2 1 f}$

$\mathrm{S} 235$ 
${ }^{1} \mathrm{H}$ NMR spectrum of $\mathbf{1 2 2 f}$

$\mathrm{S} 236$

${ }^{13} \mathrm{C}$ NMR spectrum of $\mathbf{1 2 2 f}$

$\mathrm{S} 237$

${ }^{1} \mathrm{H}$ NMR spectrum of $\mathbf{1 1 3}$

$\mathrm{S} 238$

${ }^{13} \mathrm{C}$ NMR spectrum of $\mathbf{1 1 3}$

$\mathrm{S} 239$

${ }^{1} \mathrm{H}$ NMR spectrum of $\mathbf{1 1 4}$

$\mathrm{S} 240$

${ }^{13} \mathrm{C}$ NMR spectrum of $\mathbf{1 1 4}$

$\mathrm{S} 241$

${ }^{1} \mathrm{H}$ NMR spectrum of $\mathbf{1 1 6} \mathrm{g}$

S242

${ }^{13} \mathrm{C}$ NMR spectrum of $\mathbf{1 1 6} \mathbf{g}$

$\mathrm{S} 243$

${ }^{1} \mathrm{H}$ NMR spectrum of $\mathbf{1 1 8} \mathbf{g}$

$\mathrm{S} 244$

${ }^{13} \mathrm{C}$ NMR spectrum of $\mathbf{1 1 8 g}$

$\mathrm{S} 245$

${ }^{1} \mathrm{H}$ NMR spectrum of $120 \mathrm{~g}$

S246

${ }^{13} \mathrm{C}$ NMR spectrum of $120 \mathrm{~g}$

$\mathrm{S} 247$

${ }^{1} \mathrm{H}$ NMR spectrum of $\mathbf{1 2 1 \mathrm { g }}$

$\mathrm{S} 248$

${ }^{13} \mathrm{C}$ NMR spectrum of $\mathbf{1 2 1} \mathbf{g}$

S249

${ }^{1} \mathrm{H}$ NMR spectrum of $\mathbf{1 1 6 i}$

$\mathrm{S} 250$

${ }^{13} \mathrm{C}$ NMR spectrum of $116 \mathrm{i}$

$\mathrm{S} 251$

${ }^{1} \mathrm{H}$ NMR spectrum of $\mathbf{1 1 8 i}$

$\mathrm{S} 252$

${ }^{13} \mathrm{C}$ NMR spectrum of $\mathbf{1 1 8} \mathrm{i}$

$\mathrm{S} 253$

${ }^{1} \mathrm{H}$ NMR spectrum of $\mathbf{1 2 0 i}$

S254

S255

${ }^{1} \mathrm{H}$ NMR spectrum of $121 \mathrm{i}$

S256

${ }^{13} \mathrm{C}$ NMR spectrum of $121 \mathrm{i}$

$\mathrm{S} 257$

${ }^{1} \mathrm{H}$ NMR spectrum of $122 \mathrm{i}$

${ }^{13} \mathrm{C}$ NMR spectrum of $\mathbf{1 2 2 i}$

S258

S259

${ }^{1} \mathrm{H}$ NMR spectrum of $\mathbf{1 1 6 h}$

$\mathrm{S} 260$

${ }^{13} \mathrm{C}$ NMR spectrum of $\mathbf{1 1 6 h}$

S261

${ }^{1} \mathrm{H}$ NMR spectrum of $\mathbf{1 1 8 h}$

S262

${ }^{13} \mathrm{C}$ NMR spectrum of $\mathbf{1 1 8 h}$

S263

${ }^{1} \mathrm{H}$ NMR spectrum of $120 \mathrm{~h}$

S264

${ }^{13} \mathrm{C}$ NMR spectrum of $\mathbf{1 2 0 h}$

S265

${ }^{1} \mathrm{H}$ NMR spectrum of $121 \mathrm{~h}$

S266

${ }^{13} \mathrm{C}$ NMR spectrum of $\mathbf{1 2 1 h}$

S267 


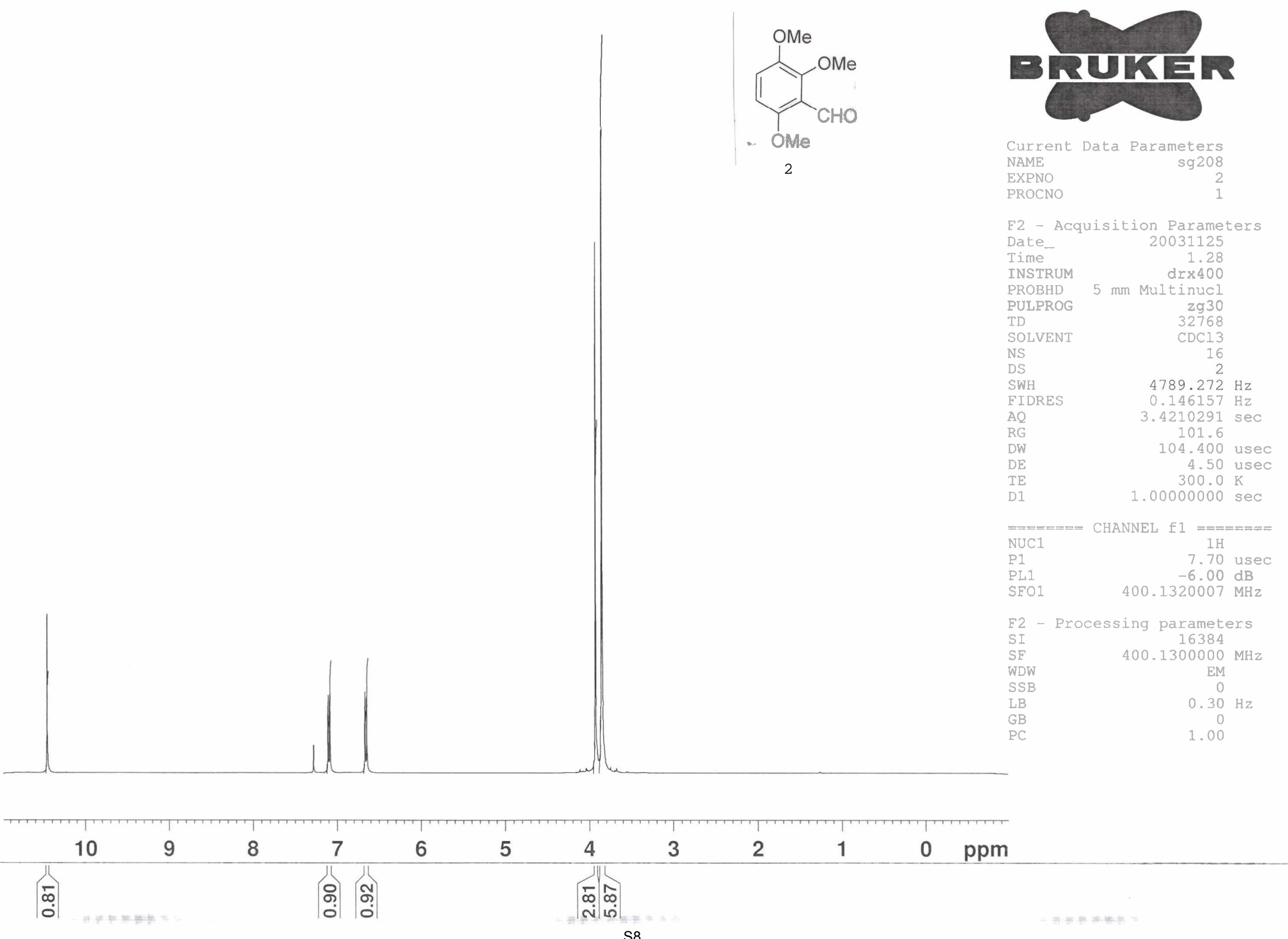




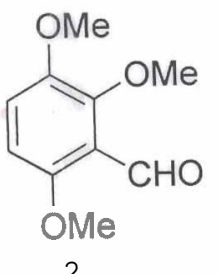

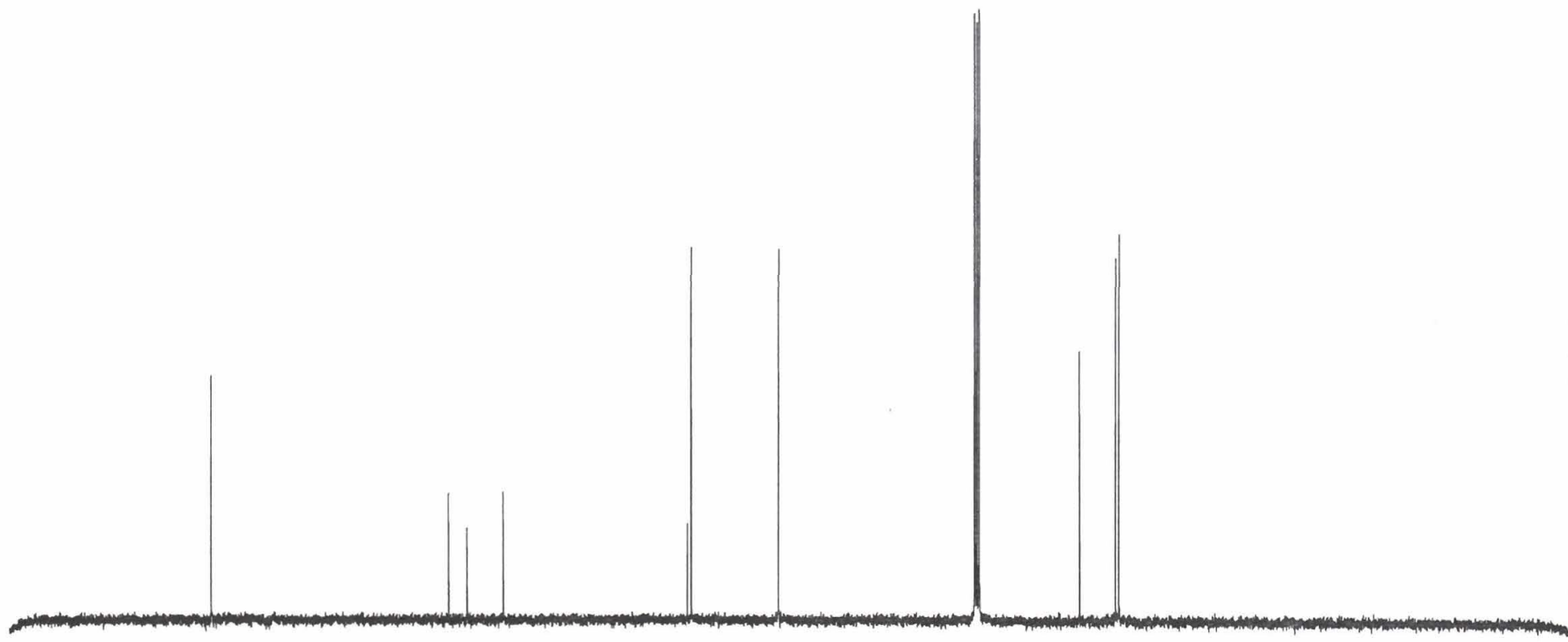

- RUKER

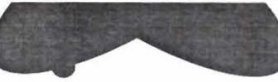

Current Data Parameters

NAME

EXPNO

PROCNO

sg209

F2 - Acquisition Parameters

Date_ 20031125

Time $\quad 1.57$

INSTRUM

drx 400

PROBHD $5 \mathrm{~mm}$ Multinucl

PULPROG

zgpg 30

TD

65536

SOLVENT

NS

DS

FIDRES

$A Q$

RG

DW

DE

d11

d12

$\mathrm{CDCl3}$
500
2

$23148.148 \mathrm{~Hz}$

$0.353213 \mathrm{~Hz}$

$1.4156276 \mathrm{sec}$

1290.2

21.600 usec

4.50 usec

$300.0 \mathrm{~K}$

$2.00000000 \mathrm{sec}$

$0.03000000 \mathrm{sec}$

$0.00002000 \mathrm{sec}$

\section{CHANNEL $f$}

NUC 1

P1

$\mathrm{f} 1==\mathrm{x}=\mathrm{x}=\mathrm{m}=\mathrm{=}$

$13 \mathrm{C}$

12.30 usec

2.00 dB

SFO1

$100.6232933 \mathrm{MHz}$

$========$ CHANNEL $f$

CPDPRG2

f2

NUC2

PCPD2

PL2

PL12

PL13

$\mathrm{SFO} 2$

waltz 16

100.00 usec

$0.00 \mathrm{~dB}$

$18.00 \mathrm{~dB}$

$18.00 \mathrm{~dB}$

$400.1316005 \mathrm{MHz}$

2 - Processing parameters 32768

$100.6127290 \mathrm{MHz}$

60

40

20
ppm WDW 


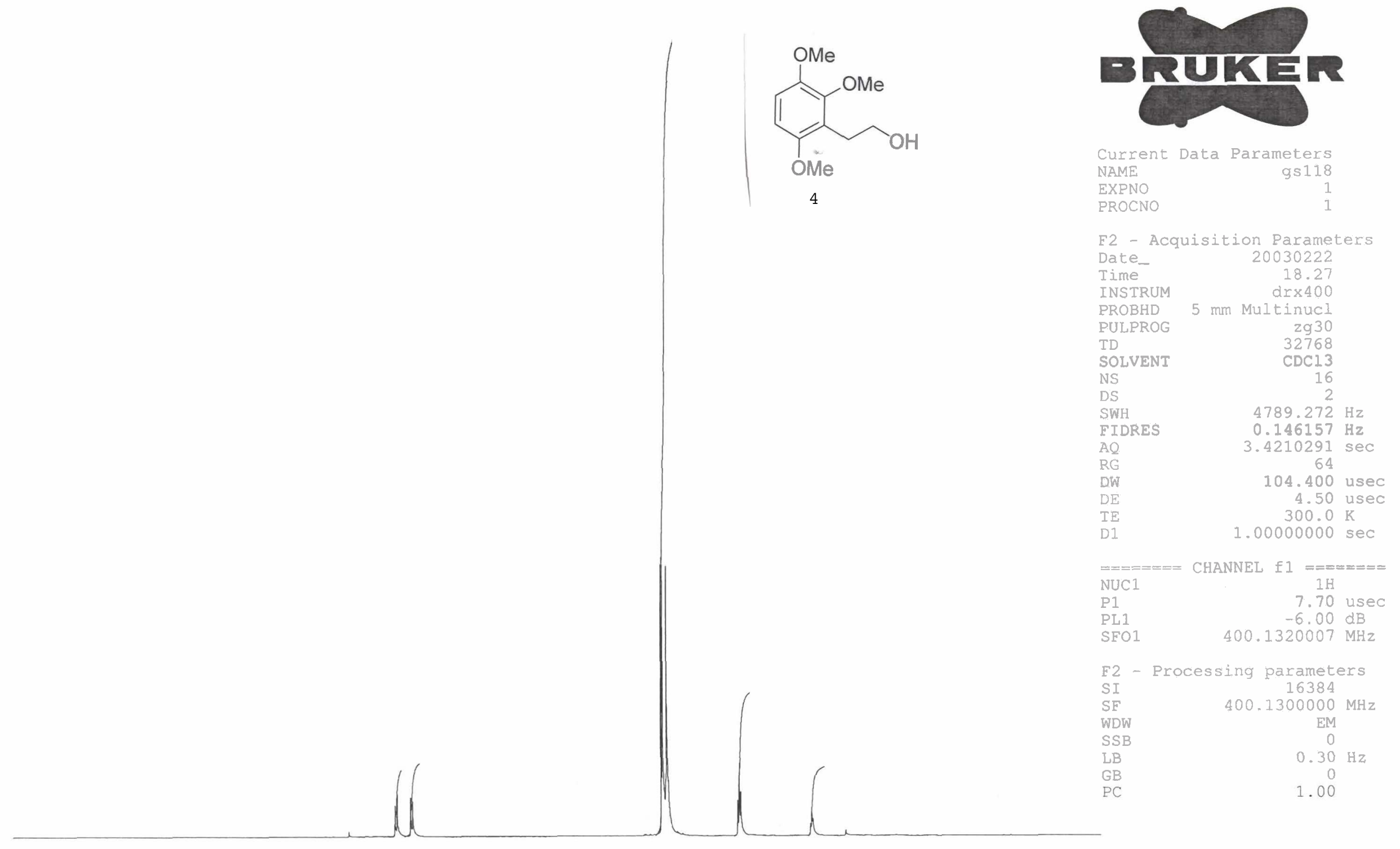

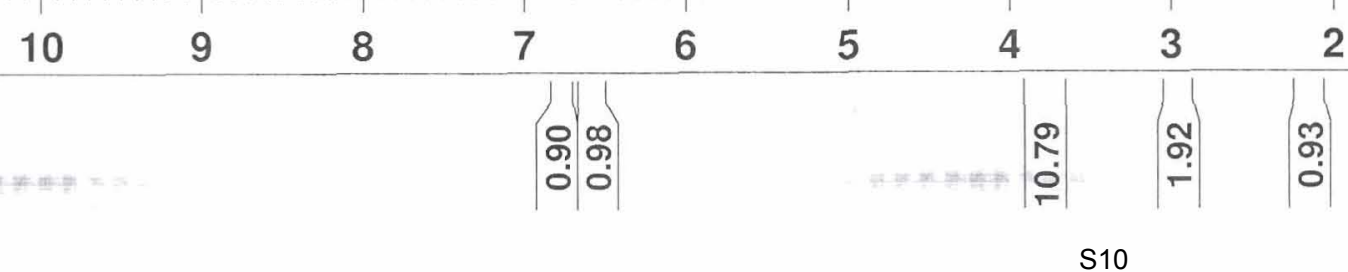




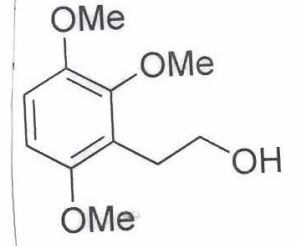

4

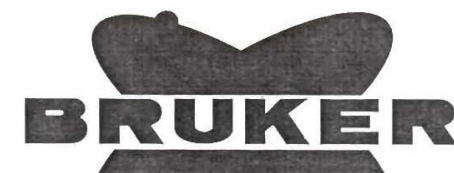
$\longrightarrow$

Current Data Parameters NAME

EXPNO

PROCNO

F2 - Acquisition Parameters Date_ 20030222
Time
18.42

INSTRUM

PROBHD

PULPROG

TD

drx400

$5 \mathrm{~mm}$ Multinucl

zgpg 30

$\mathrm{CDCl} 3$

403

NS

DS

SWH

FIDRES

$\mathrm{AQ}$

RG

DW

DE

D1

d11

d12

$==1$
NUC1

PL1

SFO1

$23148.148 \mathrm{~Hz}$ $0.353213 \mathrm{~Hz}$

$1.4156276 \mathrm{sec}$ 512

21.600 usec 4.50 usec $300.0 \mathrm{~K}$

0.05000000 sec $0.03000000 \mathrm{sec}$ 0.00002000 sec

\section{CHANNEI}

CPDPRG 2

$\mathrm{NUC2} 2$

PCPD2

PL2

PL12

PL13

$5 F O 2$

- Processing parameters 32768

$100.6127290 \mathrm{MHz}$

180

160

140

120

100

80

60

40

20

ppm ${ }_{S S}^{\text {WD }}$

$\begin{array}{lc}\mathrm{SSB} & \mathrm{EM} \\ \mathrm{LB} & 1.00 \mathrm{~Hz} \\ \mathrm{~GB} & 0\end{array}$




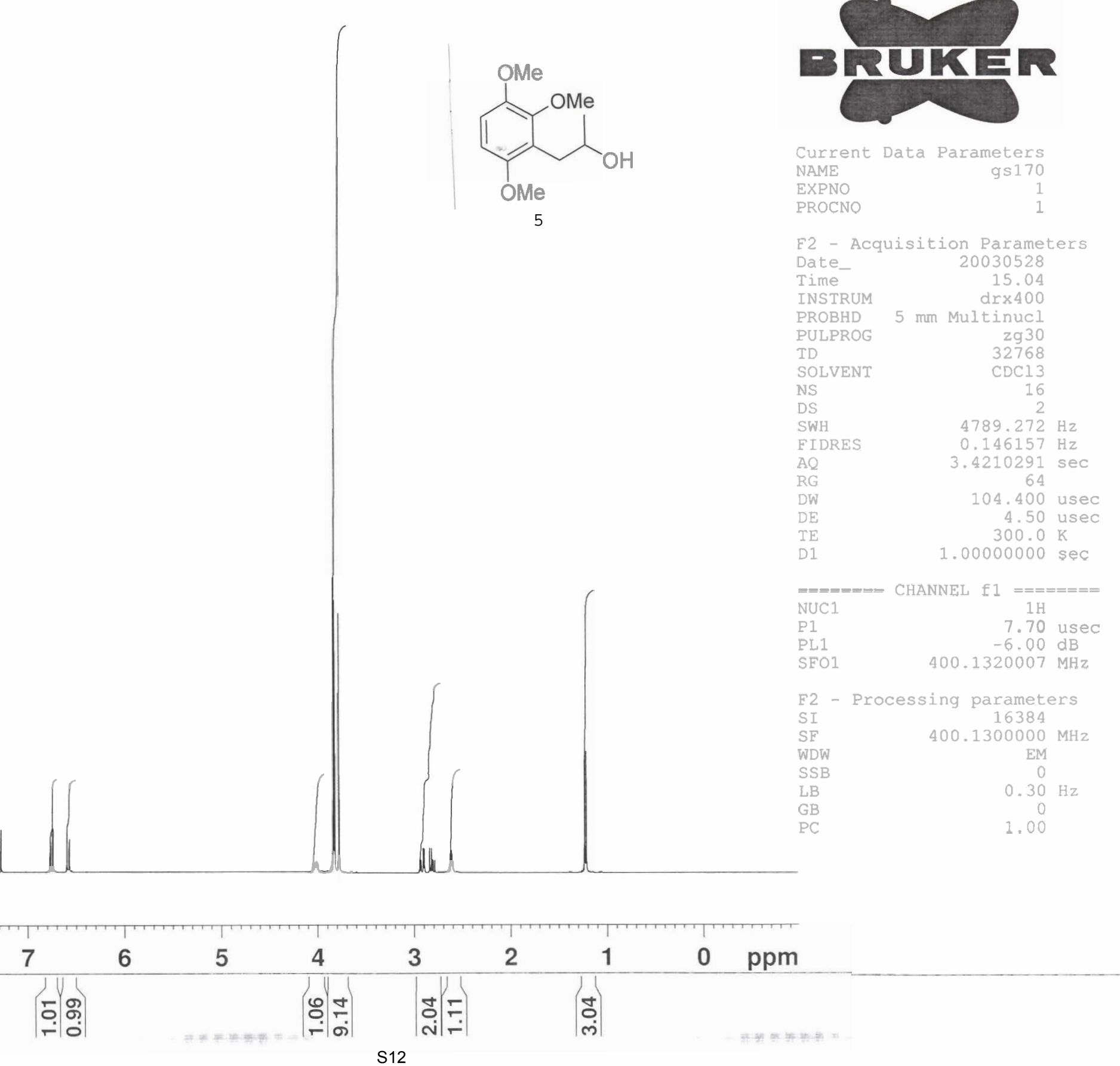




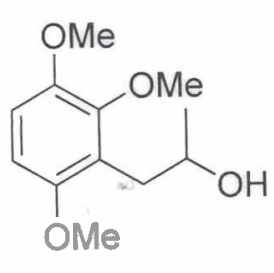

5
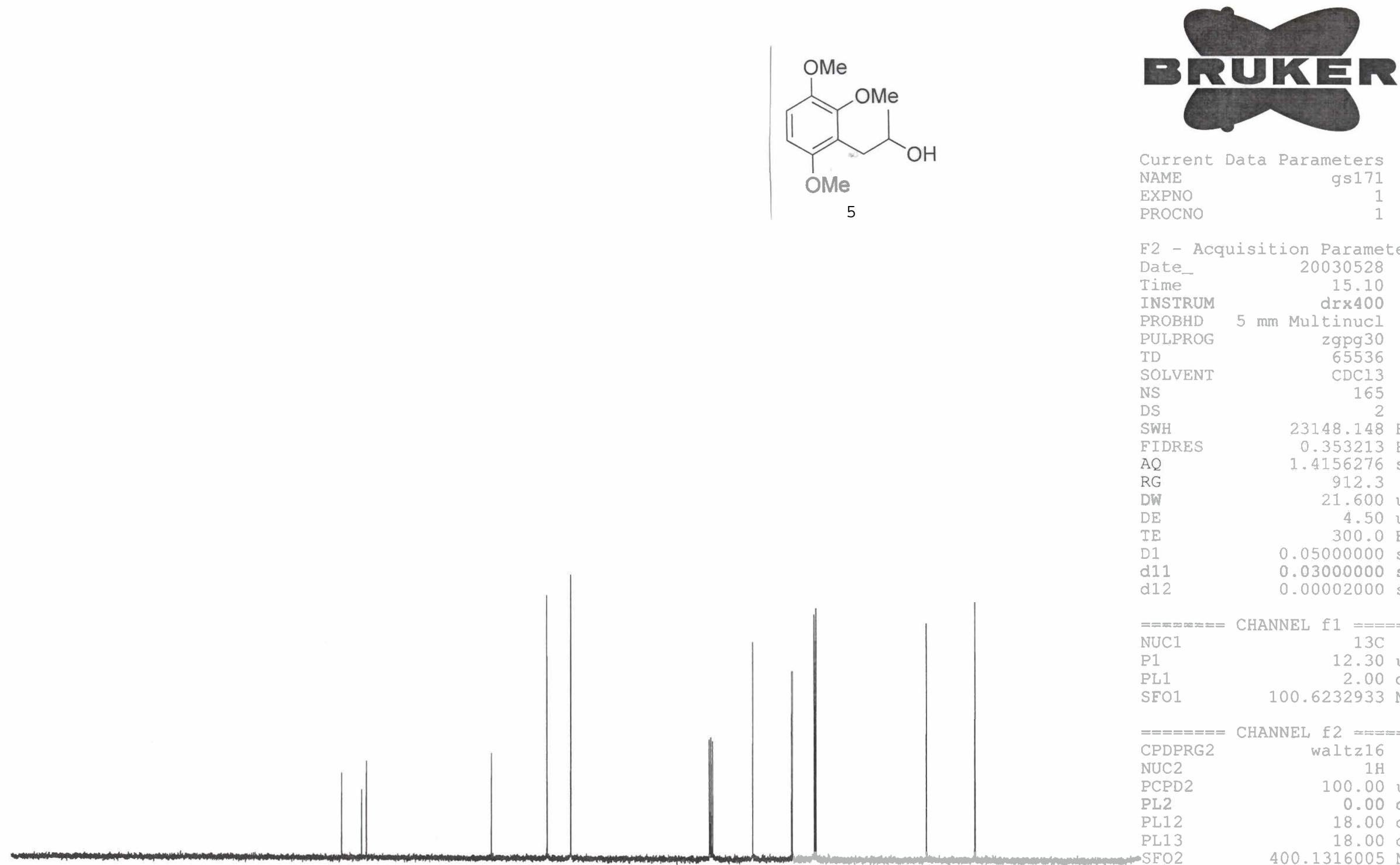

Current Data Parameters

NAME

EXPNO

PROCNO

gs 171

F2 - Acquisition Parameters

Date_ 20030528

Time

15.10

INSTRUM

PROBHD $5 \mathrm{~mm}$ Multinucl

drx 400

PULPROG zgpg 30

TD

SOLVENT

NS

DS

SWH

FIDRES

AQ

RG

DW

DE

IE

d11

$$
\text { d12 }
$$

65536

CDC13

$23148.148^{2} \mathrm{~Hz}$

$0.353213 \mathrm{~Hz}$

$1.4156276 \mathrm{sec}$ 912.3

21.600 usec

4.50 usec $300.0 \mathrm{~K}$

$0.05000000 \mathrm{sec}$

$0.03000000 \mathrm{sec}$

d12 $0.00002000 \mathrm{sec}$

$== \pm=$

NUC 1
P1

PL1

CHANNEL
$1=$
12.30 use

SEO1

$100.6232933 \mathrm{MHz}$

$========$ CHANNEL $f 2$

CPDPRG2

壬2 $=\div= \pm====$

CPDPRG

$\mathrm{PCPD} 2$

PL2

PL12

PL13

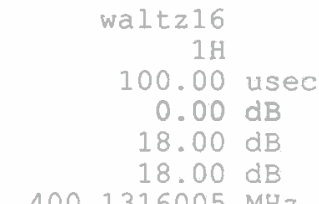

2 - Processing parameters SI $\quad 32768$

60

40

20

ppm ${ }_{S S P}^{W D}$

$\mathrm{GB}$
$\mathrm{PB}$ 


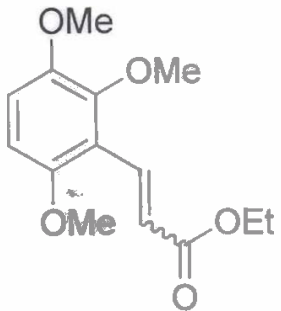

5.1

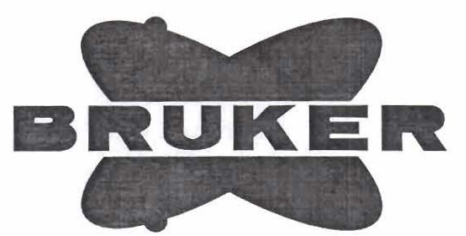

Current Data Parameters NAME

EXPNO

PROCNO

F2 - Acquisition Parameters

Date 20031125

Time

2.57

drx 400

INSTRUM

PROBHD

mu Multinucl

PUIPROG zgpg 30

TD 65536

SOLVENT

NS

DS

SWH

FIDRES

AQ

RG

DW

TE

D1

d 11

65536
CDC13

966

$23148.148 \mathrm{~Hz}$

$0.353213 \mathrm{~Hz}$

$1.4156276 \mathrm{sec}$ 2048

21.600 usec 4.50 used $300.0 \mathrm{~K}$

$2.00000000 \mathrm{sec}$

$0.03000000 \mathrm{sec}$

$0.00002000 \mathrm{sec}$

$====== \pm=$ CHANNEL $\mathrm{f}$

NUC1

P1

$1==$

12.30 usec

$2.00 \mathrm{~dB}$

SFO1

$100.6232933 \mathrm{MHz}$

$======$ CHANNEL

CPDPRG2

NUC2

PCPD2

PL2

PL12

PL13

SFO2

- Processing parameters

$400,1316005 \mathrm{MHz}$ 32768

$100.6127290 \mathrm{MHz}$

waltzi6

100.00 usec $0.00 \mathrm{~dB}$

EM 


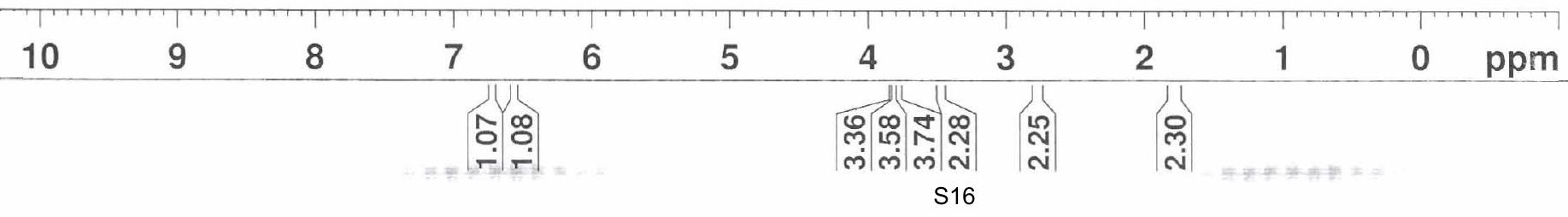




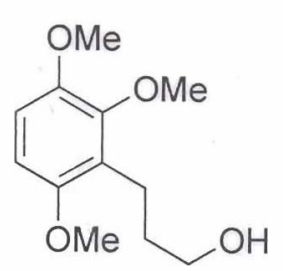

6
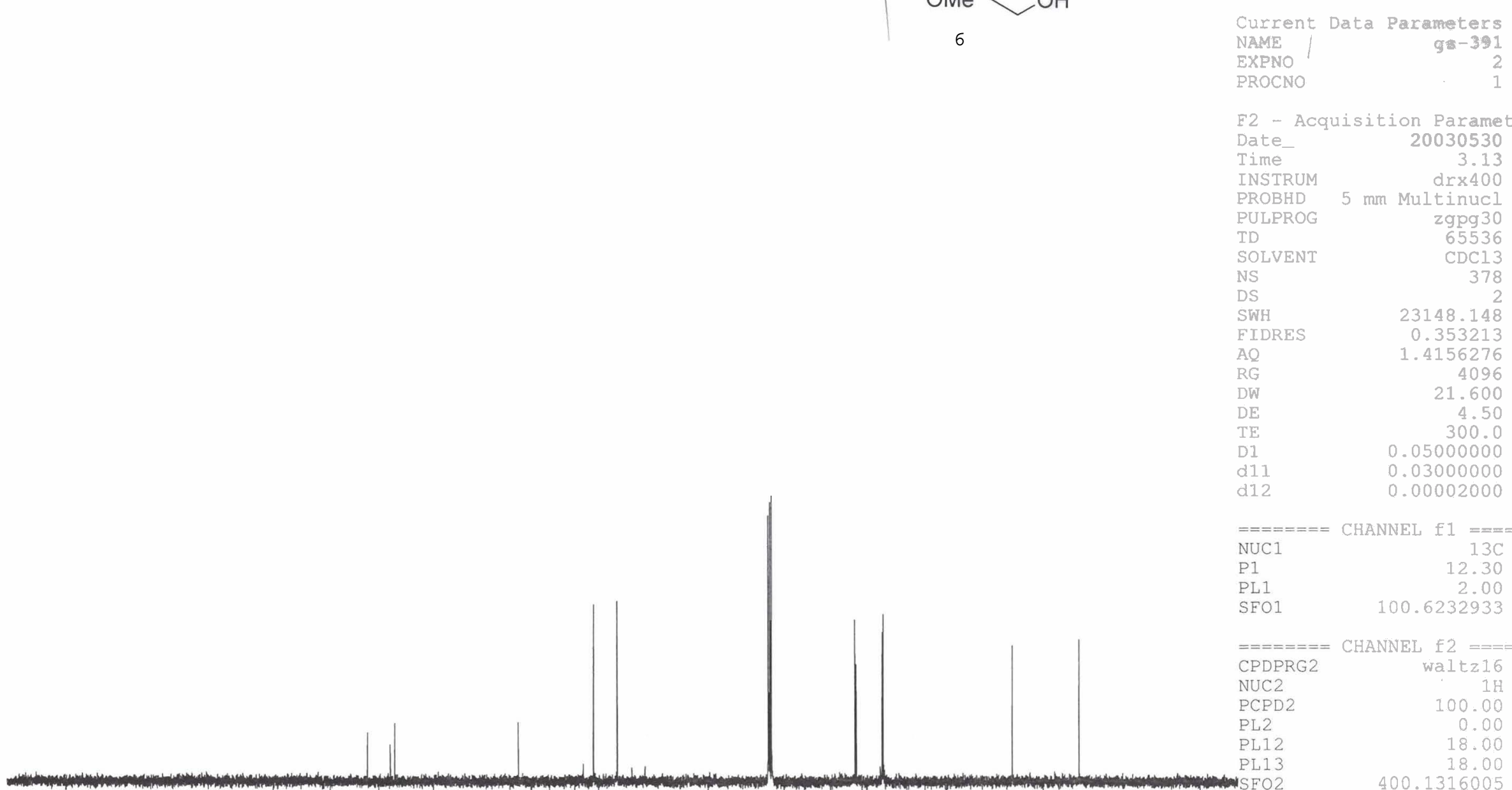

F2 - Acquisition Parameters

Date_ 20030530

Time

3.13

INSTRUM

PROBHD drx400

PULPROG

5 mrn Multinucl

TD

zgpg 30

SOLVENT

NS

SW

FIDRES

AQ

RG

DW

DE

TE

D1

d12

CDCl3

378

$23148,148 \mathrm{~Hz}$

$0.353213 \mathrm{~Hz}$

$1.4156276 \mathrm{sec}$ 4096

21.600 usec 4.50 used $300.0 \mathrm{~K}$

$0.05000000 \mathrm{sec}$

$0.03000000 \mathrm{sec}$

$0.00002000 \mathrm{sec}$

- Processing parameters $13 \mathrm{C}$

12.30 used $2.00 \mathrm{~dB}$

$100.6232933 \mathrm{MHz}$

180

160

140

120

100

80

60

40

20

$\begin{array}{ll}\mathrm{LB} & 1.00 \mathrm{~Hz} \\ \mathrm{~GB} & 0 \\ \mathrm{PC}=1.40 & 1.4\end{array}$




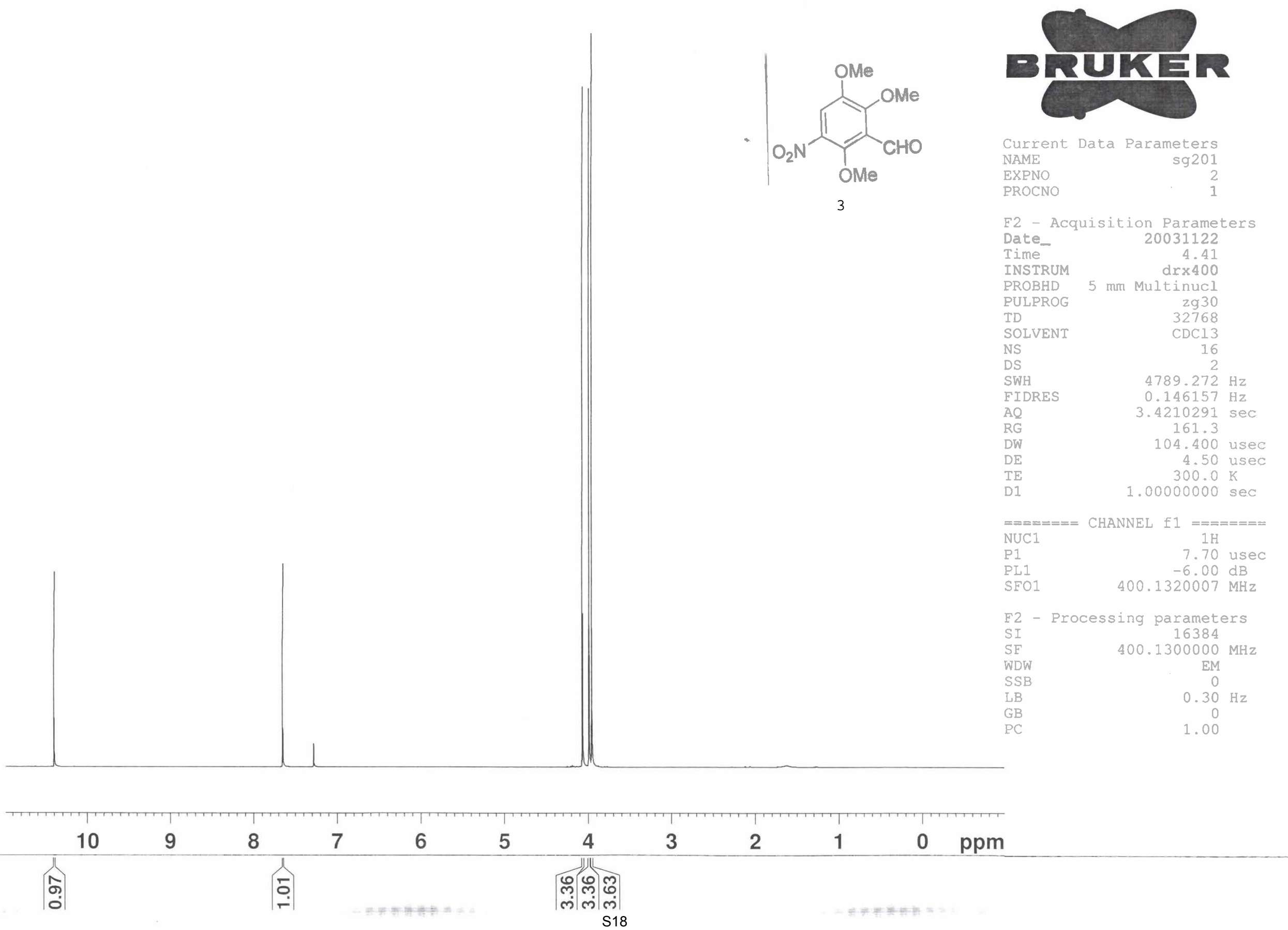




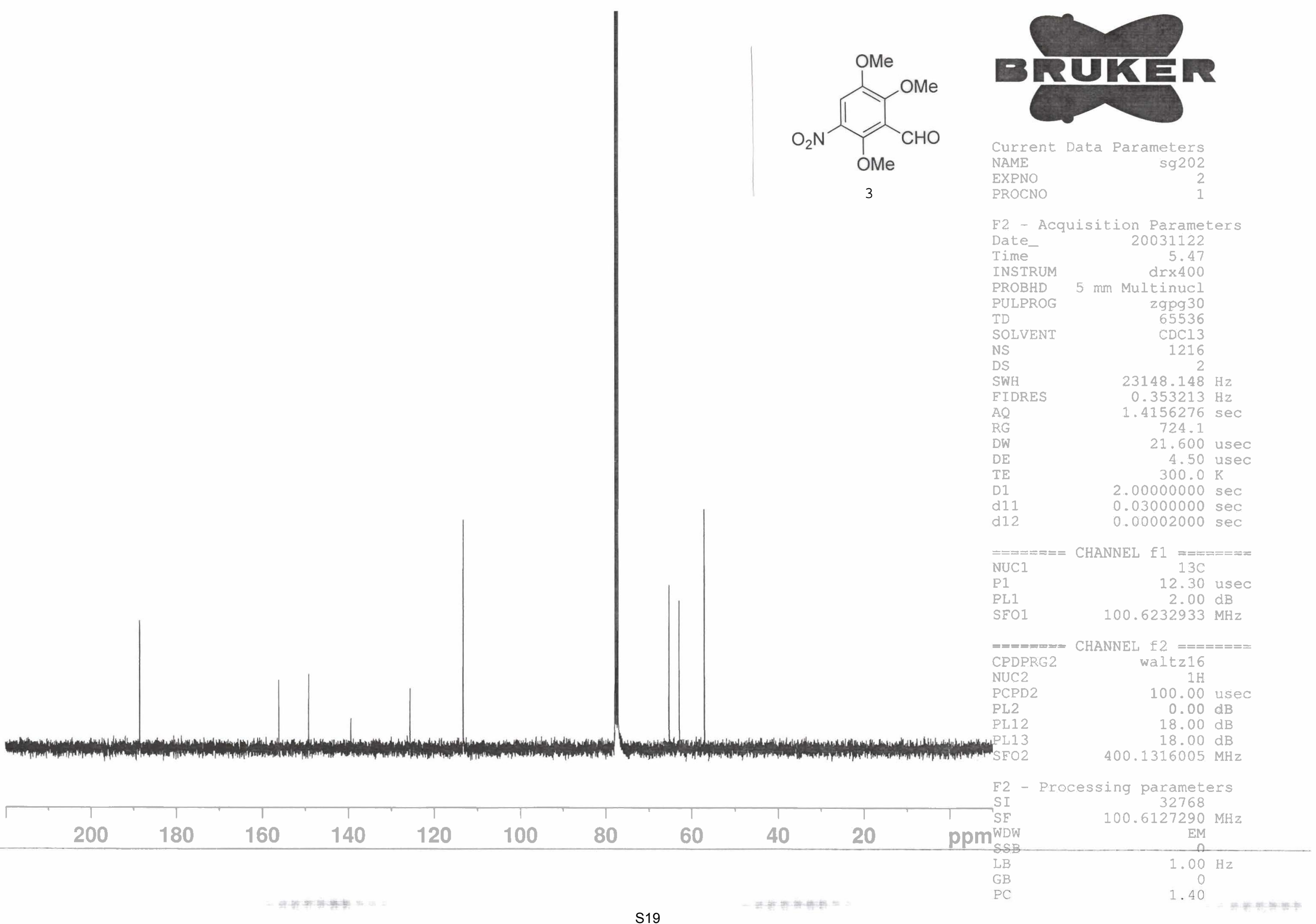




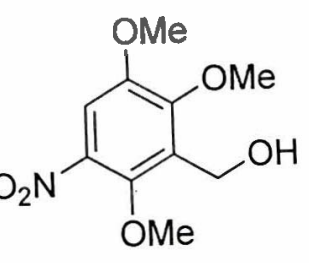

E. EUKE

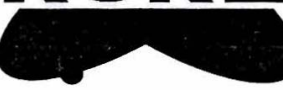

Current Data Parameters NAME

EXPNO

PROCNO

F2 - Acquisition Parameters

Date 20030308

20030308
23.43

dr $\times 400$

PROBHD

PULPROG

mn Multinucl

SOLVENI

NS

DS

SWH

FIDRES

AQ

RG

DW

DE 32768 CC13
2
4789.272
0.146157
. 146157
143.7
104.400 usec 4.50 usec $300.0 \mathrm{~K}$
$1.00000000 \mathrm{sèc}$

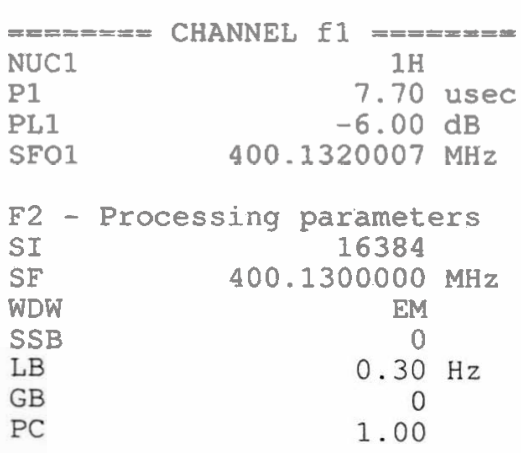

$\begin{array}{llllllllllll}10 & 9 & 8 & 7 & 6 & 5 & 4 & 3 & 2 & 1 & 0 & \mathrm{ppm}\end{array}$




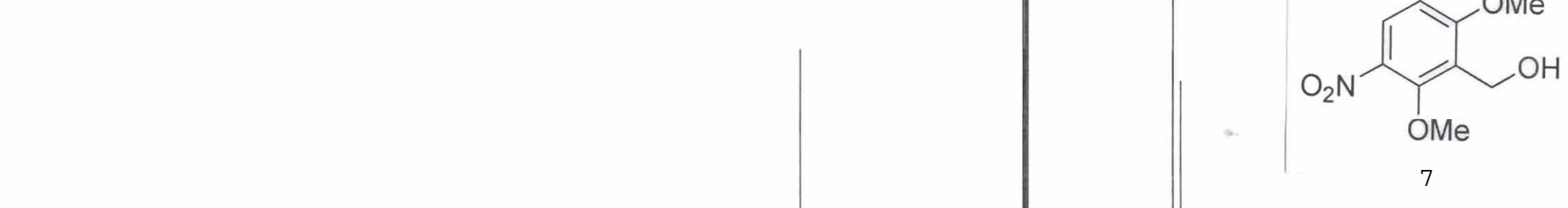

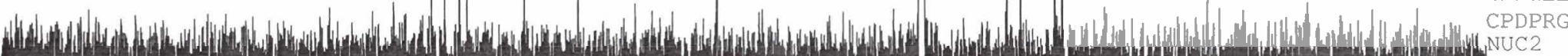

2 - In

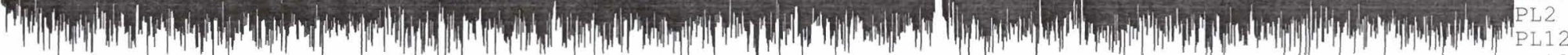

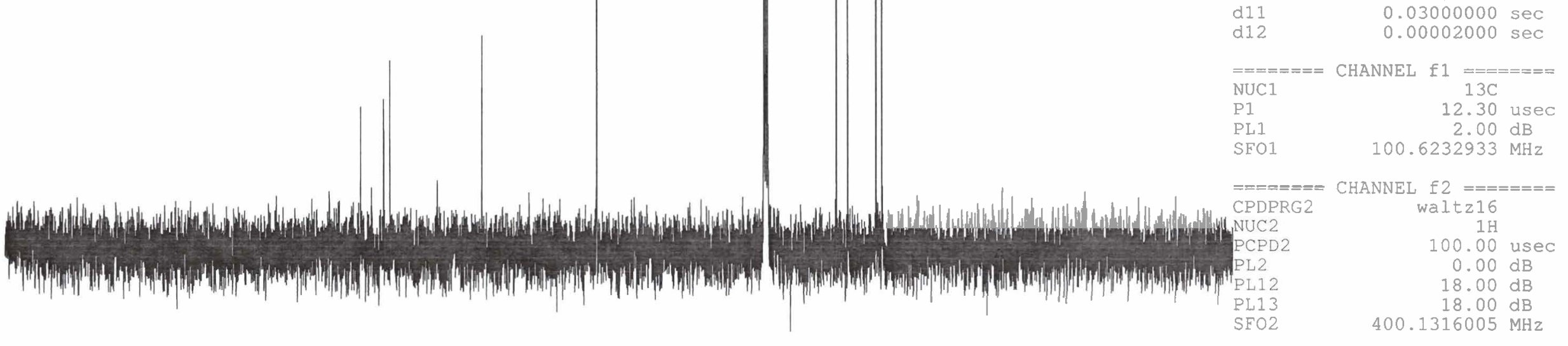

$\begin{array}{lr}\text { Current Data Parameters } \\ \text { NAME } & \text { gs } 124 \\ \text { EXPNO } & 1 \\ \text { PROCNO } & 1\end{array}$

F2 - Acquisition Parameters

Date_ 20030308

Time $\quad 23.49$

INSTRUM

PROBHD

$\operatorname{dr} \times 400$

PULPROG

$5 \mathrm{~mm}$ Multinucl

SOLVENT

NS

DS

FIDRES

AQ

RG

DE

TE

D1

d11

zgpg 30

CDCl3

452
2

$23148.148 \mathrm{~Hz}$

$0.353213 \mathrm{~Hz}$

$1.4156276 \mathrm{sec}$ 2580.3

21.600 usec 4.50 used $300.0 \mathrm{~K}$

$0.05000000 \mathrm{sec}$

0.00002000 sec

$\mathrm{SFO} 2$

$400.1316005 \mathrm{MHz}$

$\begin{array}{r}\hline \\ 200 \\ \hline\end{array}$

180

160

140

120

100

80

60

40

20

F2 - Processing parameters

$\begin{array}{lc}\text { SSB } & 0 \\ \mathrm{LB} & 1.00 \mathrm{~Hz} \\ \mathrm{~GB} & 0 \\ \mathrm{PC} & 1.40\end{array}$



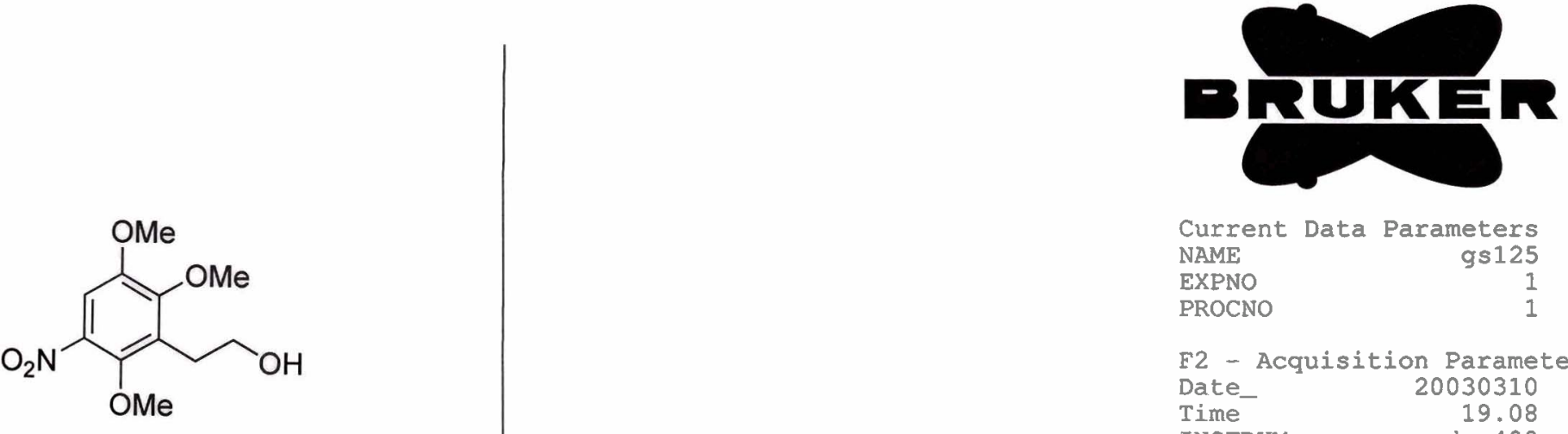

Current Data Parameters NAME

EXPNO gs 125

PROCNO

F2 - Acquisition Parameters

Date_ 20030310

Time 19.08

INSTRUM

drx 400

$5 \mathrm{~mm}$ Multinucl

PULPROG

TD

SOLVENT

NS

DS

SWH

IDRES

AQ

RG

DE

TE

zg30
32768

(1)

CDC13

16

$4789.272 \mathrm{~Hz}$

$0.146157 \mathrm{~Hz}$

$3.4210291 \mathrm{sec}$

64

104.400 usec 4.50 usec $300.0 \mathrm{~K}$

$1.00000000 \mathrm{sec}$

\begin{tabular}{|c|c|c|}
\hline $\begin{array}{l}== \pm=-1 \\
\mathrm{NUC1}\end{array}$ & $\begin{array}{r}m==\text { CHANNEL fl }=\begin{aligned} &== \\
& 1 \mathrm{H}\end{aligned}\end{array}$ & $=====$ \\
\hline P1 & 7.70 & usec \\
\hline PL1 & -6.00 & $\mathrm{~dB}$ \\
\hline SFO1 & 400.1320007 & $\mathrm{MHz}$ \\
\hline F2 - & Processing paramet & ers \\
\hline SI & 16384 & \\
\hline $\mathrm{SF}$ & 400.1300000 & $\mathrm{MHz}$ \\
\hline WDW & EM & \\
\hline $\mathrm{SSB}$ & 0 & \\
\hline LB & 0.30 & $\mathrm{~Hz}$ \\
\hline GB & 0 & \\
\hline PC & 1.00 & \\
\hline
\end{tabular}

3 :

$\begin{array}{lllll}2 & 1 & 0 & \mathrm{ppm} \\ \left.\mid \begin{array}{c}1 \\ 0\end{array}\right) & & & & \end{array}$




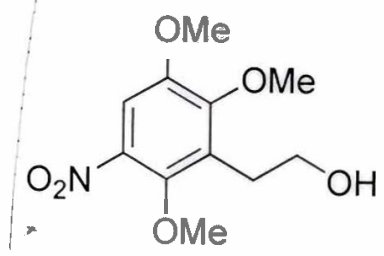

8

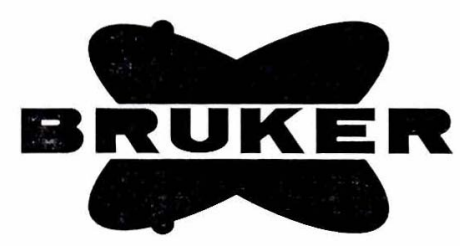

Current Data Parameters NAME EXPNO

PROCNO

F2 - Acquisition Parameters Date $\quad 20030310$

Time

INSTRUM

PROBHD

PULPROG

TD

SOLVENT

NS

DWH

FIDRES

$A Q$

RG

DW

DE

dII

d12

\section{$== \pm t====$}

NUC1

P1

PL1

SFO1

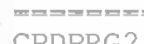

NUC2

PCPD2

PL2

PL12

PL13 $\begin{array}{r}19.16 \\ \hline\end{array}$

dr 4000

$5 \mathrm{~mm}$ Multinucl

zgpg 30

65536

$\mathrm{CDC13}$

200

$0.353213 \mathrm{~Hz}$

$1.4156276 \mathrm{sec}$

$$
812.7
$$

21.600 usec

4.50. usec $300.0 \mathrm{~K}$

$0.05000000 \mathrm{sec}$

$0.03000000 \mathrm{sec}$

$0.00002000 \mathrm{sec}$

CHANNEL

$1==$
$13 \mathrm{C}$

12.30 usec

$2.00 \mathrm{~dB}$

$100.6232933 \mathrm{MHz}$

CHANNEL

walt $16=$

waltz16

100.00 usec

$0.00 \mathrm{~dB}$

$18.00 \mathrm{~dB}$

$18.00 \mathrm{~dB}$

$400,1316005 \mathrm{MHz}$
$23148.148 \mathrm{~Hz}$

60

40

20

- Processing parameters 32768

$\begin{array}{lc}W D W & E M \\ \text { LB } & 1.00 \mathrm{~Hz} \\ \mathrm{~GB} & 0 \\ \mathrm{PC} & 1.40\end{array}$


<smiles>COc1cc([N+](=O)[O-])c(OC)c(CCCO)c1OC</smiles>

10

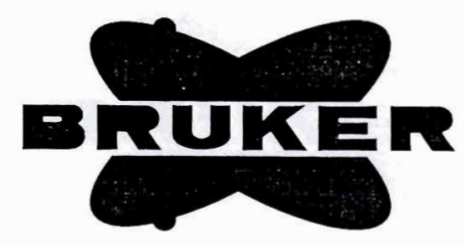

Current Data Parameters NAME

EXPNO

PROCNO

gs 129

1

F2 - Acquisition Parameters

Date_ 20030312

Time

INSTRUM

PROBHD

2.22
$r \times 400$

PUIPROG

TD

SOLVENT

NS

DS

WH

FIDRES

AQ

RG

$\mathrm{DE}$

TE

D1

d11 12

$5 \mathrm{~mm}$ Multinucl

zgpg 30

65536

CDCl 13

237

$23148.148 \mathrm{~Hz}$

$0.353213 \mathrm{~Hz}$

$1.4156276 \mathrm{sec}$ 1625.5

21.600 usec

4.50 usec $300.0 \mathrm{~K}$

$0.05000000 \mathrm{sec}$

$0.03000000 \mathrm{sec}$

$0.00002000 \mathrm{sec}$

$==x=====$ CHANNEL $\mathrm{f}$

NUC1

P1

PL1

SFO1

$===== \pm=$

NUC2

NUC2
PCPD2

PL2

PL12

P

2 - Processing parameters

SI

180

160

140

120

100

80

60

40

20

ppm $_{\text {LS }}^{\text {WD }}$

$\mathrm{LB}$
$\mathrm{GB}$

$\mathrm{PC}$ 


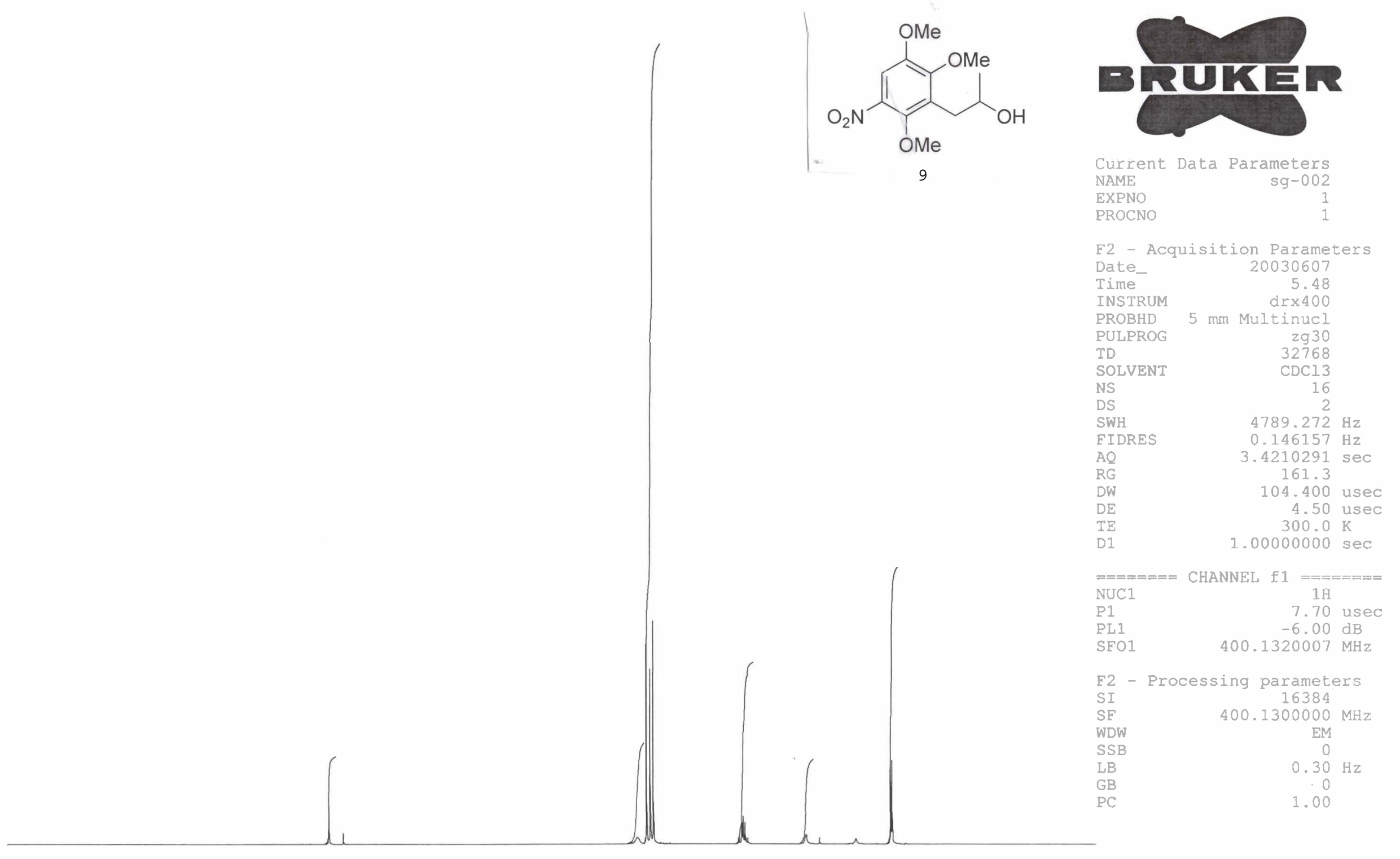

9

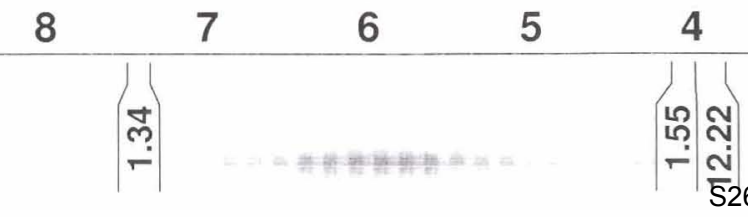

$\begin{array}{lllll}3 & 2 & 1 & 0 & \mathrm{ppm}\end{array}$

\begin{tabular}{|c|c|}
\hline $\begin{array}{c}\hat{N} \\
\mathrm{~N}\end{array}$ & 帝 \\
\hline
\end{tabular}




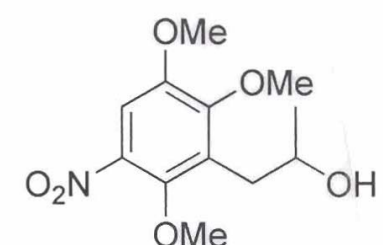

9

(2): (a)

Current Data Parameters NAME

EXPNO

PROCNO

F2 - Acquisition Parameters

Date

Time

INSTRUM

PROBHD

PULPROG

SOLVENT

NS

DS

FIDRES

AQ

RG

DW

$\mathrm{DE}$

TE

d11

d12

$-===$
NUCI

PL1

SFO1

$\simeq=====$
CPDPRG2

NUC?

PCPD2

PL2

PL12

PL13 13

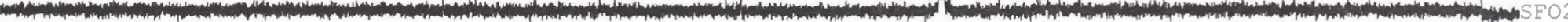

SI

$1.4156276 \mathrm{sec}$ 1290.2

1.600 ustec

4.50 usec

$0.05000000 \mathrm{sec}$

$0.03000000 \mathrm{sec}$

$0.00002000 \mathrm{sec}$

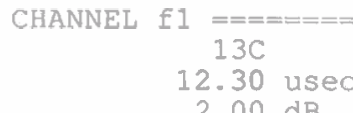
EM 


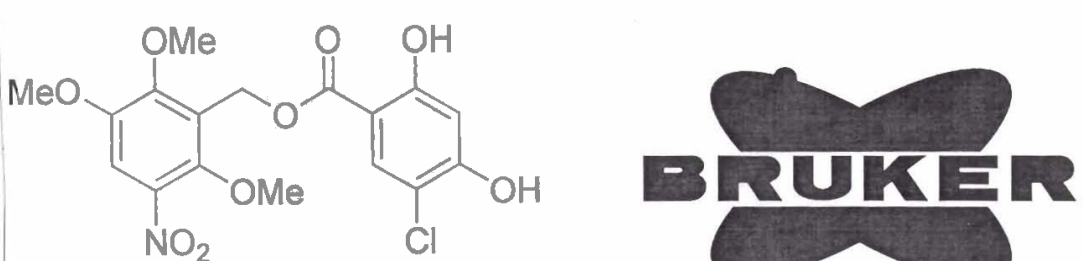

11

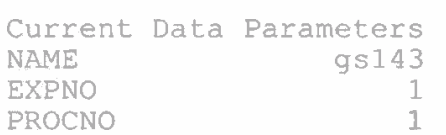

PROCNO

F2 - Acquisition Parameters Date_ 20030325

Time 16.20

INSTRUM $\quad \operatorname{dr} \times 400$

PROBHD $5 \mathrm{~mm}$ Multinucl

PULPROG $\quad$ zg30

SOLVENT

NS

DS

SWH

FIDR
AQ

RG

DW

DE

2930
32768

CDC13

2
$4789.272 \mathrm{~Hz}$

$3.4210291 \mathrm{sec}$

143.7

104.400 usec

4.50 usec

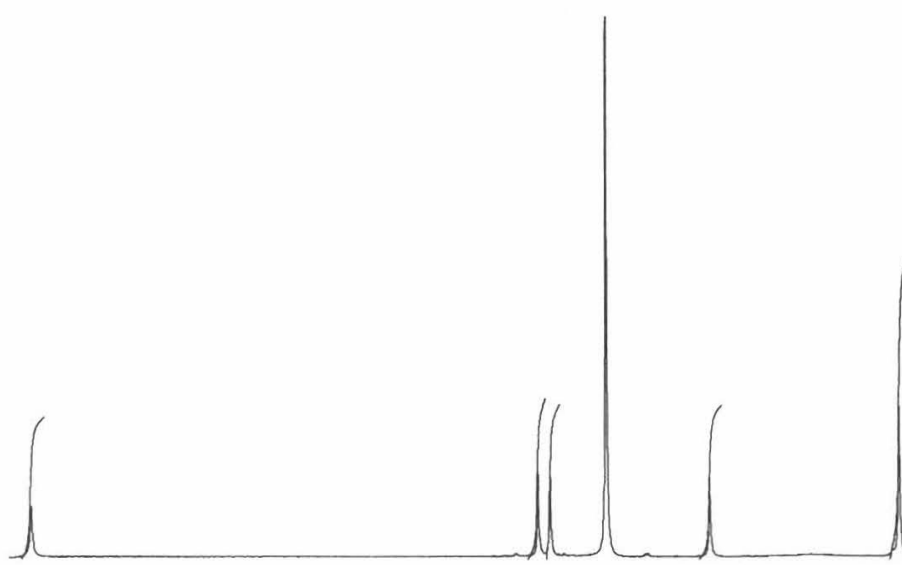

$1.00000000 \mathrm{sec}$

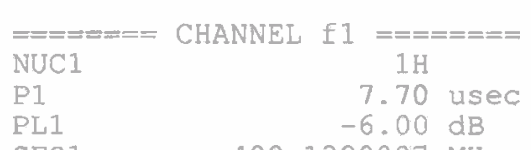

PL1

$-6.00 \mathrm{~dB}$

$400.1320007 \mathrm{MHz}$

F2 - Processing parameters

SI $\quad 16384$

SF $\quad 400.1300000 \mathrm{MHz}$

WDW

SSB

LB

EM

EM
0
$0.30 \mathrm{~Hz}$
0
1.00

\section{0}

9

8

7

6

5

4

3

2

1

0

ppm

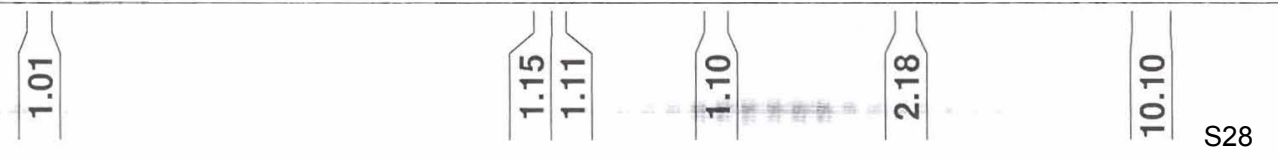




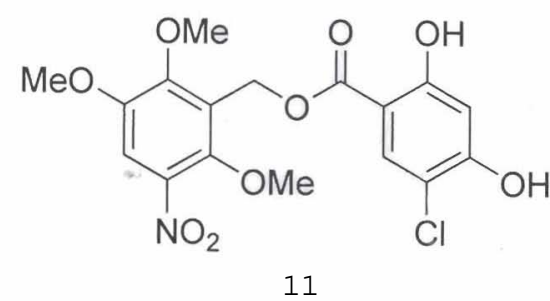

BRUKER

$\begin{array}{lr}\text { Current Data } & \text { Parameters } \\ \text { NAME } & 9 \$ 159 \\ \text { EXPNO } & 1 \\ \text { PROCNO } & 1\end{array}$

F2 - Acquisition Parameters

Date 20030517

$\begin{array}{lr}20030517 \\ \text { Time } & 21.59\end{array}$

Time

drx 400

PROBHD $5 \mathrm{~mm}$ Multinucl

PULPROG

zgpg 30

TD

SOLVENT

NS

DS

SWH

FIDRES

$\mathrm{CDC} 13$

FIDR

$\mathrm{AQ}$
$\mathrm{RG}$

DW

DE

d1 1

$$
\text { d12 }
$$

$23148.148 \mathrm{~Hz}$

$0.353213 \mathrm{~Hz}$

$1.4156276 \mathrm{sec}$

574.7

21.500 usec 4.50 usec $300.0 \mathrm{~K}$

0.05000000 sec

$0.03000000 \mathrm{sec}$

$0.00002000 \mathrm{sec}$

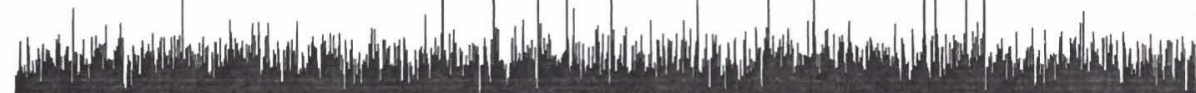

CPDPRG2

PCPD2

PL 2

PL12

$\mathrm{FO} 2$

CHANNEL

f $2========$ waltz 16

100.00 usec $0.00 \mathrm{~dB}$ $18.00 \mathrm{~dB}$ $18.00 \mathrm{~dB}$ $400.1316005 \mathrm{MHz}$

\begin{tabular}{|c|c|c|c|c|c|c|c|c|c|c|}
\hline 1 & & 1 & 1 & 1 & 1 & 1 & 1 & $T$ & $T$ & 1 \\
\hline 200 & 80 & 160 & 140 & 120 & 100 & 80 & 60 & 40 & 20 & ppm \\
\hline
\end{tabular}

- P pcessing parameters

SI 32768

GB

$\begin{array}{lc}\text { WD } & \text { EM } \\ \text { LB } & 0 \\ \text { GB } & 1.00 \mathrm{~Hz} \\ \text { PC } & 0 \\ \text { PC } & 1.40\end{array}$




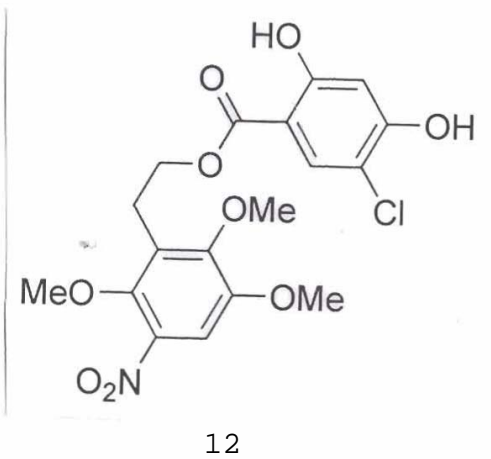

B UNRER

Curcent Data Parameters NAME

EXPNO

PROCNO

22 - Acquisition Parameters Date 20030326

Time

INSTRUM

PROBHD

PULPROG

TD

SOLVENT

NS

DS

SWH

FIDRES

AQ

$\mathrm{RG}$

DW

DE

D1

19.44
$\operatorname{dr} 8400$

$5 \mathrm{~mm}$ Multinucl

zg30

32768

$\mathrm{CDC} 13$

$4789.272 \mathrm{~Hz}$

0. $146157 \mathrm{~Hz}$

$3.4210291 \mathrm{sec}$ 90.5

104.400 usec 4.50 usec $300.0 \mathrm{~K}$

$1.00000000 \mathrm{sec}$

$\begin{array}{lc}=======\text { CHANNEL } f 1===== \pm= & 1 \mathrm{H} \\ \text { NUC1 } & 7.70 \mathrm{useC} \\ \text { P1 } & -6.00 \mathrm{~dB} \\ \text { PL1 } & 400.1320007 \mathrm{MHz} \\ \text { SFO1 } & \\ & \\ \text { F2 - Processing parameters } \\ \text { SI } & 16384 \\ \text { SF } & 400.1300000 \mathrm{MHz} \\ \text { WDW } & \text { EM } \\ \text { SSB } & 0 \\ \text { LB } & 0.30 \mathrm{~Hz} \\ \text { GB } & 0 \\ \text { PC } & 1.00\end{array}$

\begin{abstract}
9
\end{abstract}

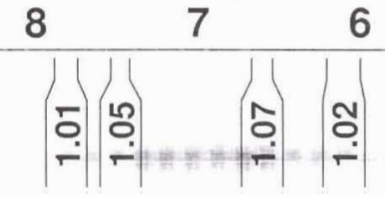

5

4

3

2

1

o ppm

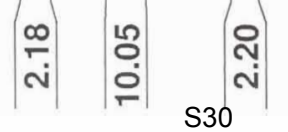



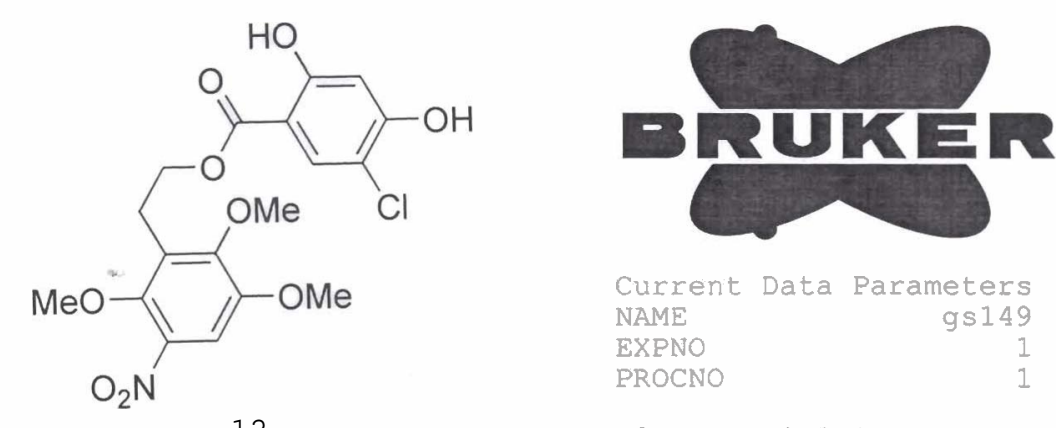

\section{Current Data Parameters NAME EXPNO \\ PROCNO gs 149}

F2 - Acquisition Parameter Date_ 20030326 Time $\quad 19.48$

INSTRUM

PROBHD

PULPROG

TD

SOLVENT

NS

DS

SWH

FIDRES

AO

RG

DW

DE

TE

di1

d 112

5 mm Multinuco

arx 400

zgpg 30
65536

$\mathrm{CDC} 13$

564

$23148.148 \mathrm{~Hz}$

$0.353213 \mathrm{~Hz}$

$1.4156276 \mathrm{sec}$

$$
812.7
$$

21.600 usec 4.50 usec $300.0 \mathrm{~K}$

$0.05000000 \mathrm{sec}$

$0.03000000 \mathrm{sec}$

$0.00002000 \mathrm{sec}$

$======$
NUC1
P1
PL1
SFO1
$======$
CPDPRG2
NUC2
PCPD2
PL2
PL12
PL13

CHANNEL f

f $1===$

12.30 usec

$2.00 \mathrm{~dB}$

$100.6232933 \mathrm{MHz}$

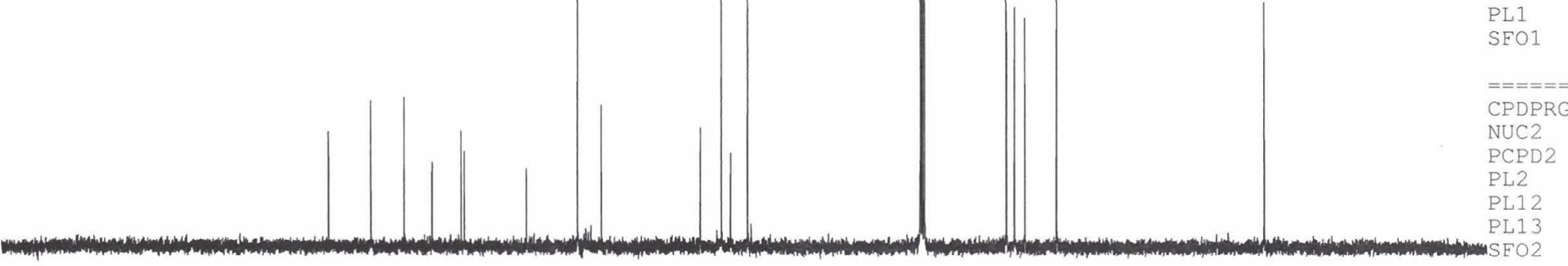

CHANNEL

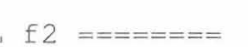

100.00 usec

$0.00 \mathrm{~dB}$

$18.00 \mathrm{~dB}$

$18.00 \mathrm{~dB}$

$400.1316005 \mathrm{MHz}$

200

180

160

140

120

100

80

60

40

20

SE

32768

$100.6127290 \mathrm{MHz}$ LB

GB

PC 


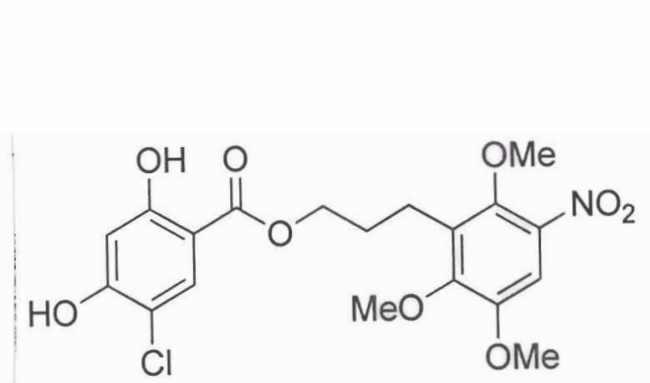

13
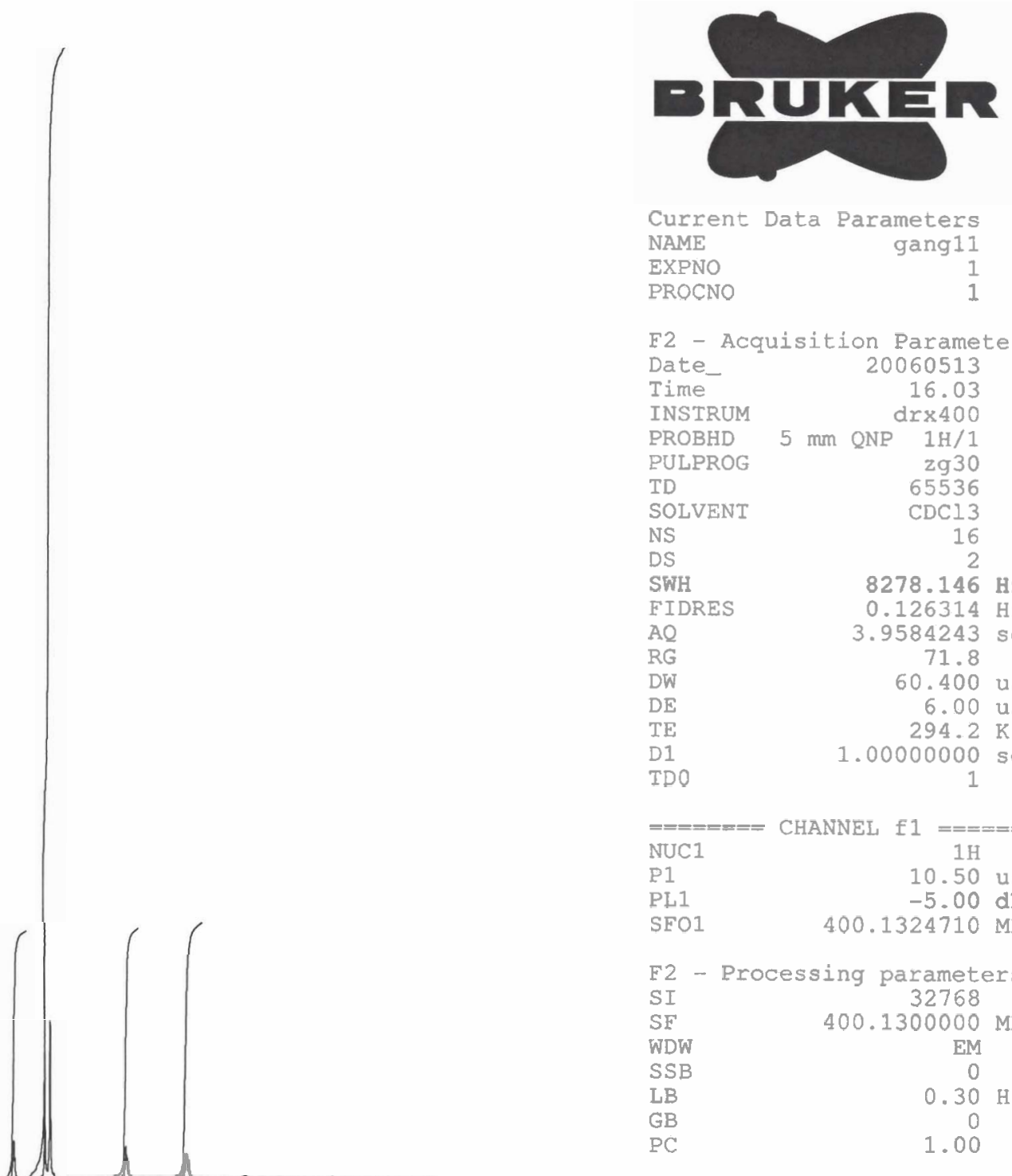

Current Data Parameters NAME

EXPNO

PROCNO

gang 11
1
1

F2 - Acquisition Parameters

Date 20060513

Time $\quad 16.03$

INSTRUM dr $\times 400$

PROBHD

$1 \mathrm{H} / 1$

TD

SOLVENI

NS

DS

FIDRES

AQ

RG

DW

$\mathrm{DE}$

D1

$\mathrm{zg} 30$
65536

65536

$\mathrm{CDC} 13$

16
2

$8278.146 \mathrm{~Hz}$

$0.126314 \mathrm{~Hz}$

$3.9584243 \mathrm{sec}$ 71.8

60.400 usec 6.00 usec $294.2 \mathrm{~K}$

$1.00000000 \mathrm{sec}$

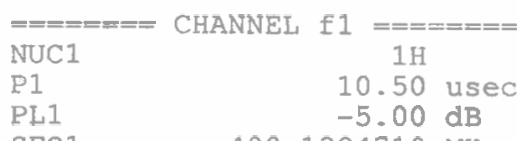

SFO 1

$400.1324710 \mathrm{MH}$

F2 - Processing parameters

$\begin{array}{cc}\text { SI } & 32768 \\ \text { SF } & 400.1300000 \mathrm{MHz}\end{array}$

WDW

SSB

LB

$\mathrm{GB}$
$\mathrm{PC}$
$\begin{array}{lllll}16 & 15 & 14 & 13 & 12\end{array}$

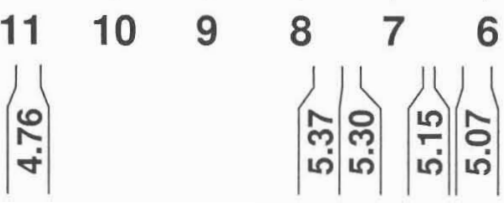

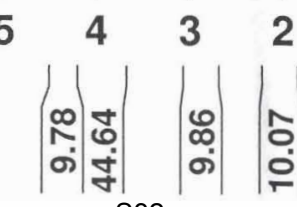
ppm 

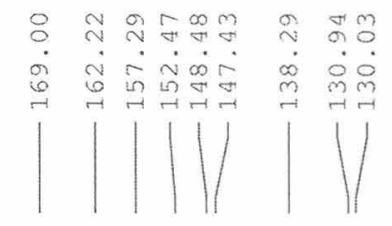

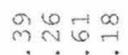

$\rightarrow$
$\Rightarrow 0$
7

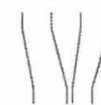

Cl<smiles>COC(=O)OCCCc1c(OC)c(OC)cc([N+](=O)[O-])c1OC</smiles>

13

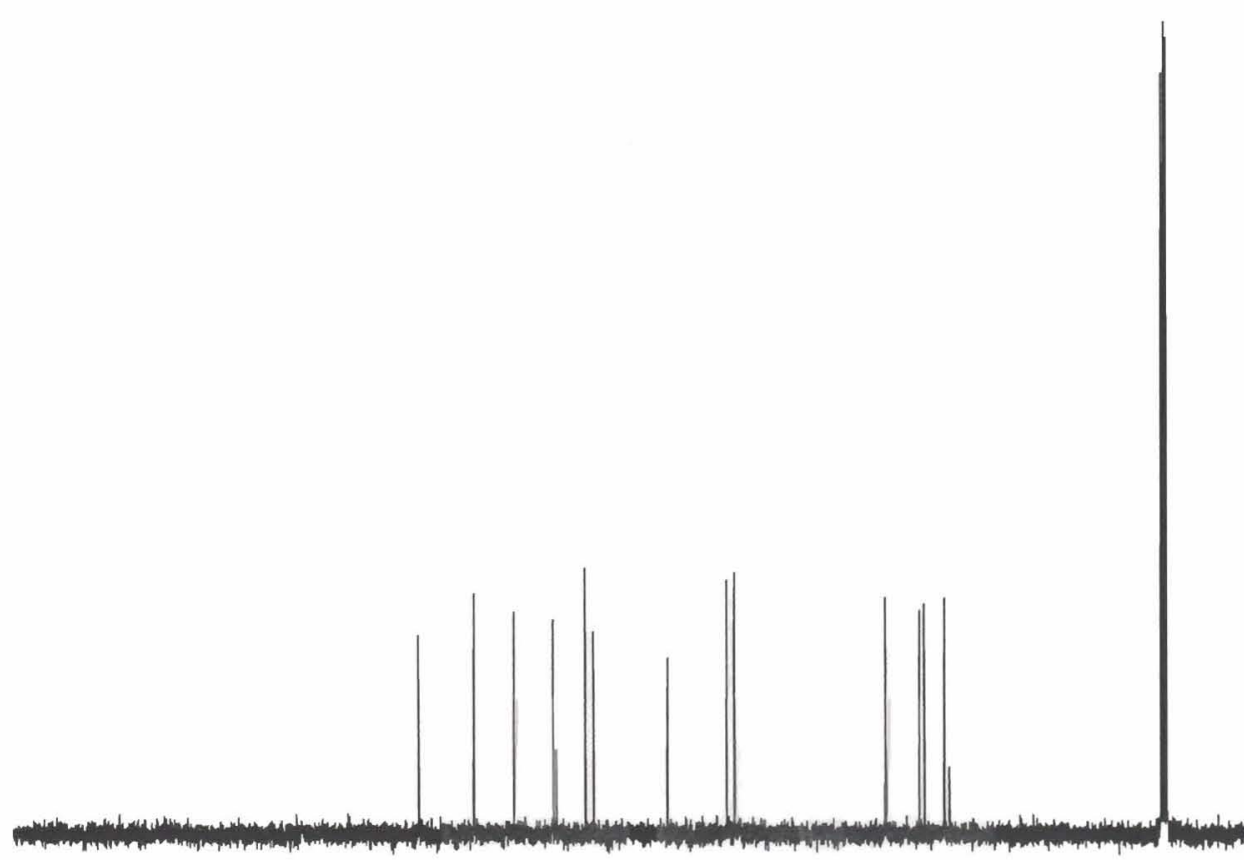

또금ำ

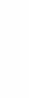



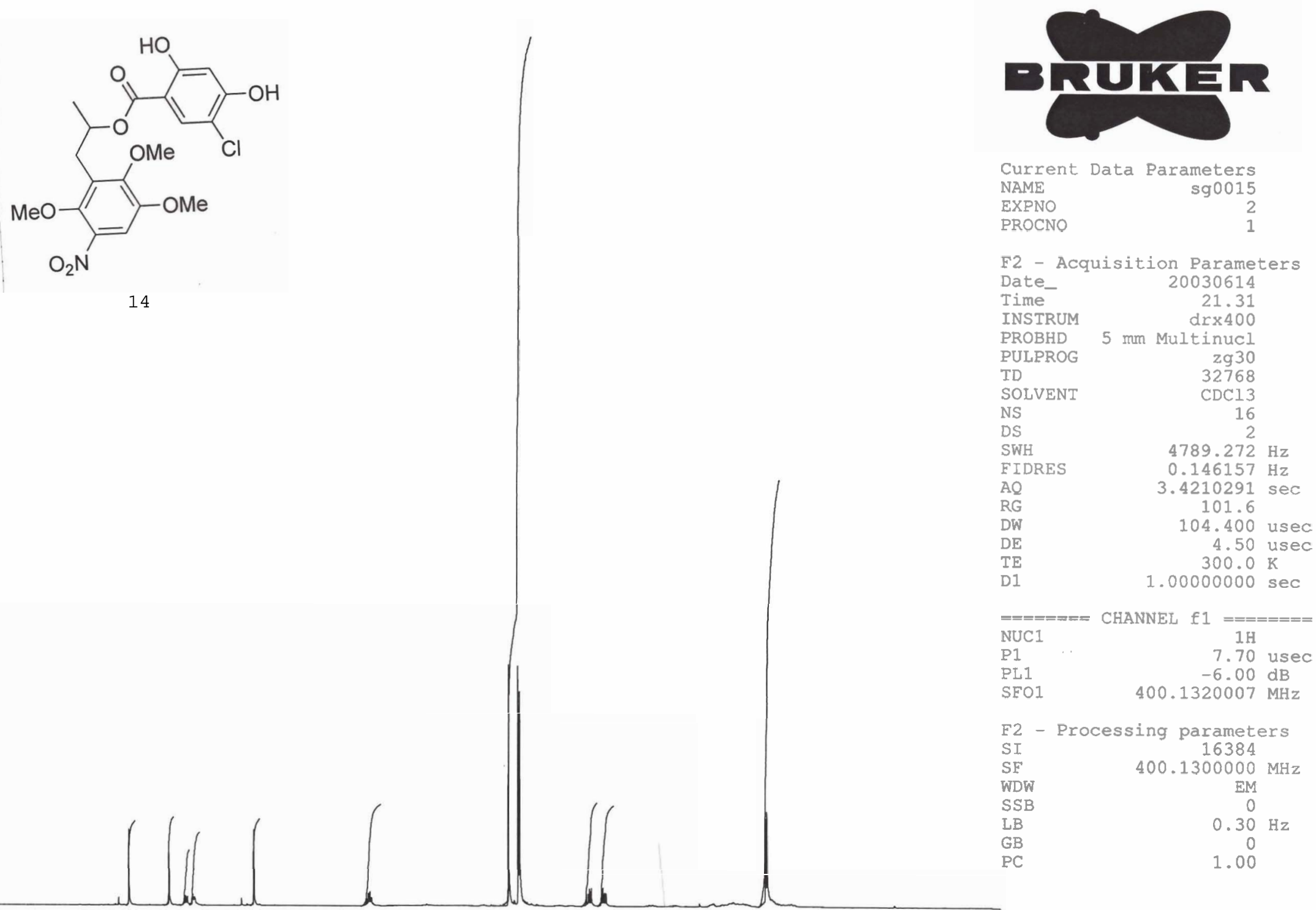

$===== \pm==$ CHANNEL $f 1$

NUC1

P1

PL1

$0000 \mathrm{sec}$

SEO1

7.70 usec $-6.00 \mathrm{~dB}$

$400.1320007 \mathrm{MHz}$

F2 - Processing parameters

SI 16384

SF $\quad 400.1300000 \mathrm{MHz}$

WDW

SSB

$\angle B$

EM

$\mathrm{EM}$
0
$30 \mathrm{~Hz}$
0
0

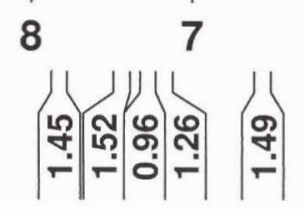

6

5

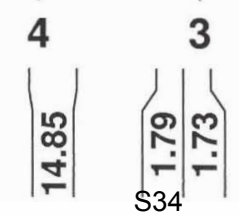

2

100 ppm

$$
\text { 일 }
$$




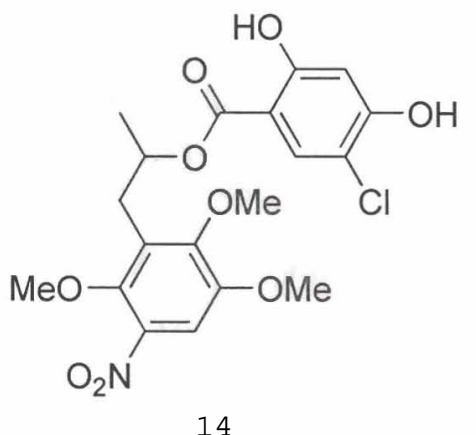

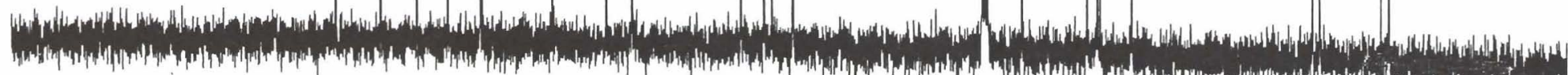

\begin{tabular}{|c|c|c|c|c|c|c|c|c|c|}
\hline ppm & 200 & $\begin{array}{c}1 \\
175\end{array}$ & $\begin{array}{c}1 \\
150\end{array}$ & 125 & 100 & $\begin{array}{l}1 \\
75\end{array}$ & $\begin{array}{l}1 \\
50\end{array}$ & 25 & 0 \\
\hline
\end{tabular}

Current Data Parameters

F2 - Acquisit ion Parameters

Date_ 20021114

Time $\quad 23.05$

23.05

drx400

$\begin{array}{lr}\text { PROBHD } & 5 \text { mal Multinucl } \\ \text { PULPROG } & \text { zgog30 }\end{array}$

$\begin{array}{lr}\text { PULPROG } & \text { zgpg30 } \\ \text { TD } & 65536\end{array}$

SOLVENT CDCI3

124

NS

DS

SWH $\quad 23148.148 \mathrm{~Hz}$

FIDRES $\quad 0.353213 \mathrm{~Hz}$

$\mathrm{AQ} \quad 1.4156276 \mathrm{sec}$

RG

DW

$\mathrm{DE}$

D1

$\mathrm{d} 11$
$\mathrm{~d} 12$

21.600 usec 4.50 usec $300.0 \mathrm{~K}$

$2.00000000 \mathrm{sec}$

$2.00000000 \mathrm{sec}$

$0.00002000 \mathrm{sec}$

$=======$ CHANNEL

PL1

12.30 usec

SF01

$1006232.0033 \mathrm{~dB}$

$== \pm==$ + CHANNEL f2 $== \pm====+$

CPDPR waltz16

$\begin{array}{lc}\text { NUC2 } & 1 \mathrm{H} \\ \text { PCPD2 } & 100.00 \text { us }\end{array}$

PL2

PL12

PL13

100.00 usec

$18.00 \mathrm{~dB}$

$18.00 \mathrm{~dB}$

$400.1316005 \mathrm{MHz}$

F2 - Processing parameters

SI 32768

SF $\quad 100.6127290 \mathrm{MHz}$

WDW

$\begin{array}{lll}\mathrm{LB} & 1.00 \mathrm{~Hz}\end{array}$

$\mathrm{GB}$

GB

$1.00 \mathrm{~Hz}$

1.10

1D NMR plot parameters

CX $\quad 20.00 \mathrm{~cm}$

CY $\quad 12.50 \mathrm{~cm}$

FIP $215.000 \mathrm{ppm}$

F1 21631.74 Hz

F2P $\quad-5.000 \mathrm{ppm}$

F2 $\quad-503.06 \mathrm{~Hz}$

PPMCM $11.00000 \mathrm{ppm} / \mathrm{cm}$

$\mathrm{HOCM}$

$1106.73999 \mathrm{~Hz} / \mathrm{cm}$ 


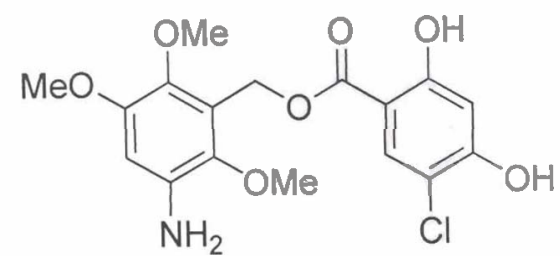

15

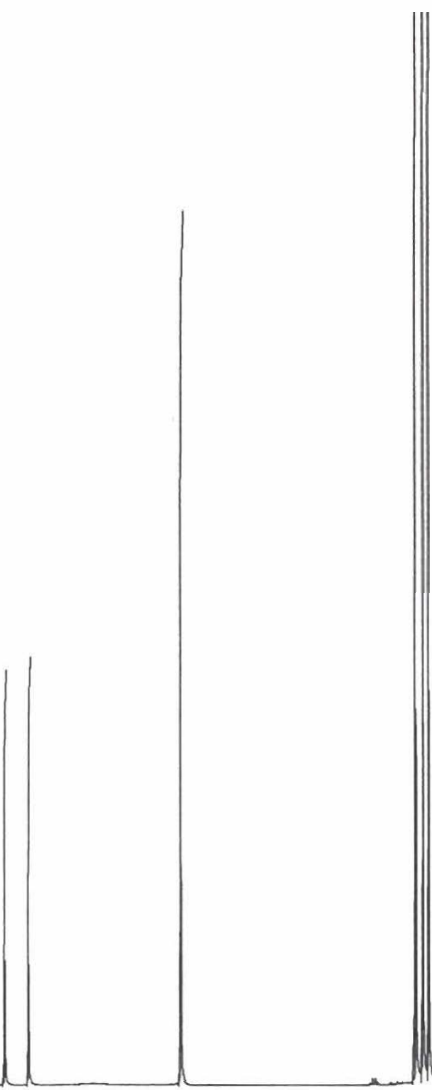

- mank

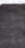

(1)

Current Data Parameters NAME

EXPNO

PROCNO

F2 - Acquisition Parameters

Date_ 20031122

Time

6.50

INSTRUM

PROBHD

$5 \mathrm{~mm}$ Multinucl

PULPROG

TD

SOLVENT

NS

SWH

FIDRES

AQ

$\mathrm{RG}$

DE

TE

D1

$\mathrm{zg} 30$
32768

32768

CDC13

2

$5208.333 \mathrm{~Hz}$

$0.158946 \mathrm{~Hz}$

$3.1457779 \mathrm{sec}$

$$
181
$$

96.000 usec

4. 50 usec

$300.0 \mathrm{~K}$

$1.00000000 \mathrm{sec}$

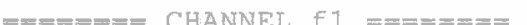
NUC1

P1

PL1

$1 \mathrm{H}$

SFO1

70 usec

$400.1326008 \mathrm{MHz}$

F2 - Processing parameters

SI $\quad 16384$

SF $\quad 400.1300000 \mathrm{MHz}$

WDW

EM

$0.30 \mathrm{~Hz}$

0

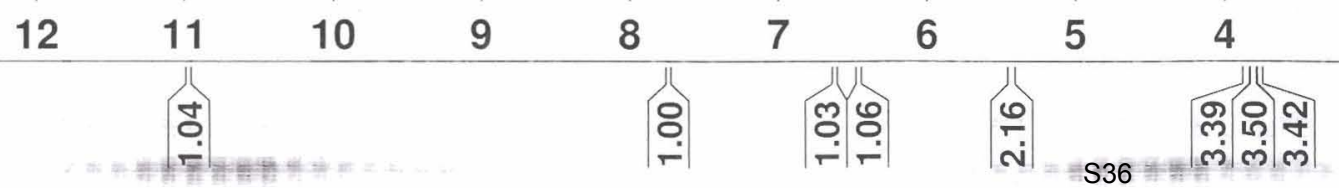

$\begin{array}{llll}3 & 2 & 1 & \mathrm{ppm}\end{array}$ 

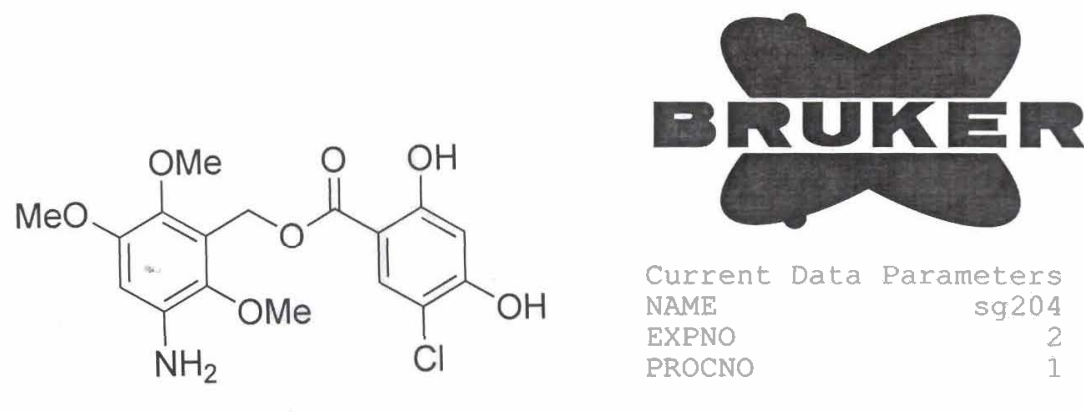

$\begin{array}{lr}\text { Current Data Parameters } \\ \text { NAME } & \text { sg204 } \\ \text { EXPNO } & 2 \\ \text { PROCNO } & 1\end{array}$

F2 - Acquisition Parameters

Date_ 20031122

Time

20031122
6.43

INSTRUM

PROBHD

PULPROG

TD

arx 400

$5 \mathrm{~mm}$ Multinucl

zgpg 30
65536

SOLVENT

NS

$\mathrm{CDCl} 3$

DS

SWH

FIDRES

AQ

RG

DW

DE

DI

$$
\text { d11 } 12
$$

d. 12

$23148.148 \mathrm{~Hz}$

$0.353213 \mathrm{~Hz}$

$1.4156276 \mathrm{sec}$ 812.7

21.600 usec

4.50 usec

$300.0 \mathrm{~K}$

$2.00000000 \mathrm{sec}$

$0.03000000 \mathrm{sec}$
$0.00002000 \mathrm{sec}$

$====$
NUC1

P1

PL1

SFO1

$1==$
$13 \mathrm{C}$

12.30 usec

$2.00 \mathrm{~dB}$

$100.6232933 \mathrm{MHz}$ NUC2

$===== \pm 2$

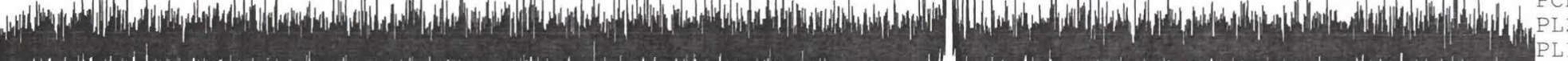

100

80

60

40

20

opmWDW

32768

$100.6127290 \mathrm{MHz}$ 

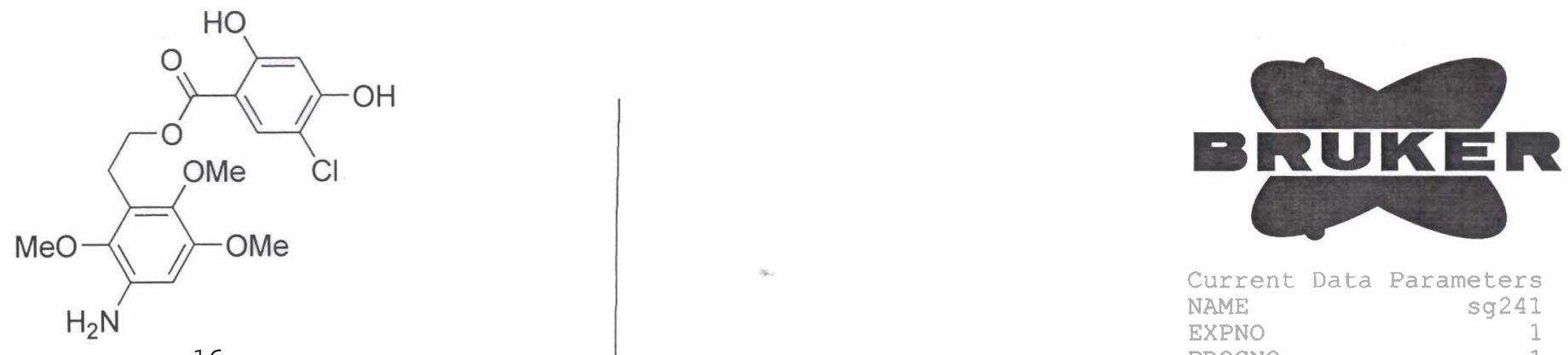

Current Data Parameters

$$
\text { NAME }
$$

EXPNO

PROCNO

F2 - Acquisition Parameters

Date_ 20031211

Time

INSTRUM

PROBHD

PULPROG

TD

SOLVENT

NS

DS

SWH

FIDRES

$A Q$

RG

DW

DE

TE

5.27
drxio0

$z 930$
32768

32768

CDC13

$4789.272 \mathrm{~Hz}$

$0.146157 \mathrm{~Hz}$

$3.4210291 \mathrm{sec}$ 228.1

104.400 usec 4.50 usec $300.0 \mathrm{~K}$

$1.00000000 \mathrm{sec}$

$========$ CHANNEL f $1==== \pm== \pm=$ NUC1

P1

PL1 1 $1 \mathrm{H}$

7.70 usec

SFO1

$-6.00 \mathrm{~dB}$

F2 - Processing parameters

SI 16384

SE $\quad 400.1300000 \mathrm{MHz}$

WDW

SSB

$G B$

$\mathrm{EM}$
0

$0.30 \mathrm{~Hz}$

0
1.00

10

9

8

7

6

5

4

3

2

1

ppm

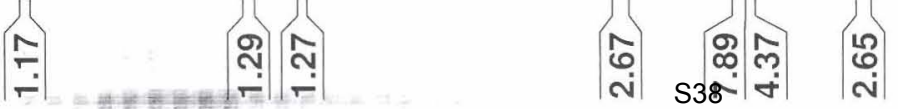




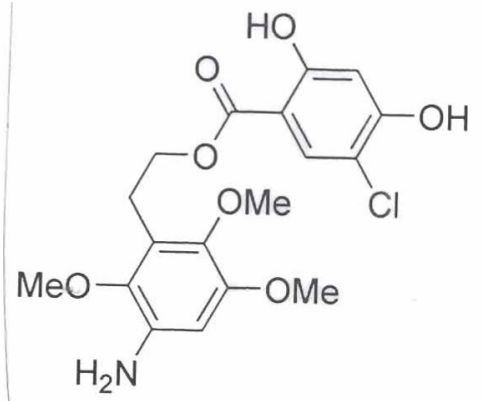

16
- RUKER Cris

Current Data Parameters NAME

EXPNO

PROCNO

F2 - Acquisition Parameters

Time

INSTRUM

PROBHD

PULPROG

TD

SOLVENI

NS

EIDRES

AQ

RG

DW

DE

TE

D1

d 112

$===$
NUC1

P1

PL1

$\mathrm{SFO} 1$

$=======$

CPDPRG2

NUC2

$\mathrm{PCPD} 2$

\section{Wh}

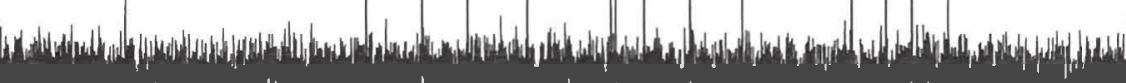

20031211 14.43
$\operatorname{dr} \times 400$

$5 \mathrm{~mm}$ Multinucl

zgpg 30

65536
CDC13

6534

$23148.148 \mathrm{~Hz}$

$0.353213 \mathrm{~Hz}$

$1.4156276 \mathrm{sec}$ 32768

21.600 usec

4.50 usec

$300.0 \mathrm{~K}$

$2.00000000 \mathrm{sec}$

$0.03000000 \mathrm{sec}$

$0.00002000 \mathrm{sec}$

CHANNEL E

1. $13 \mathrm{C}$

12.30 usec

$2.00 \mathrm{~dB}$

$100.6232933 \mathrm{MHz}$

CHANNEL $f 2$

waltz 16

100.00 usec

$0.00 \mathrm{~dB}$

$18.00 \mathrm{~dB}$

$18.00 \mathrm{~dB}$

$400.1316005 \mathrm{MHz}$

- Processing parameters 32768 $100.6127290 \mathrm{MHz}$ 200 180 160 140

120

100

80

60

40

20

ppm

EM




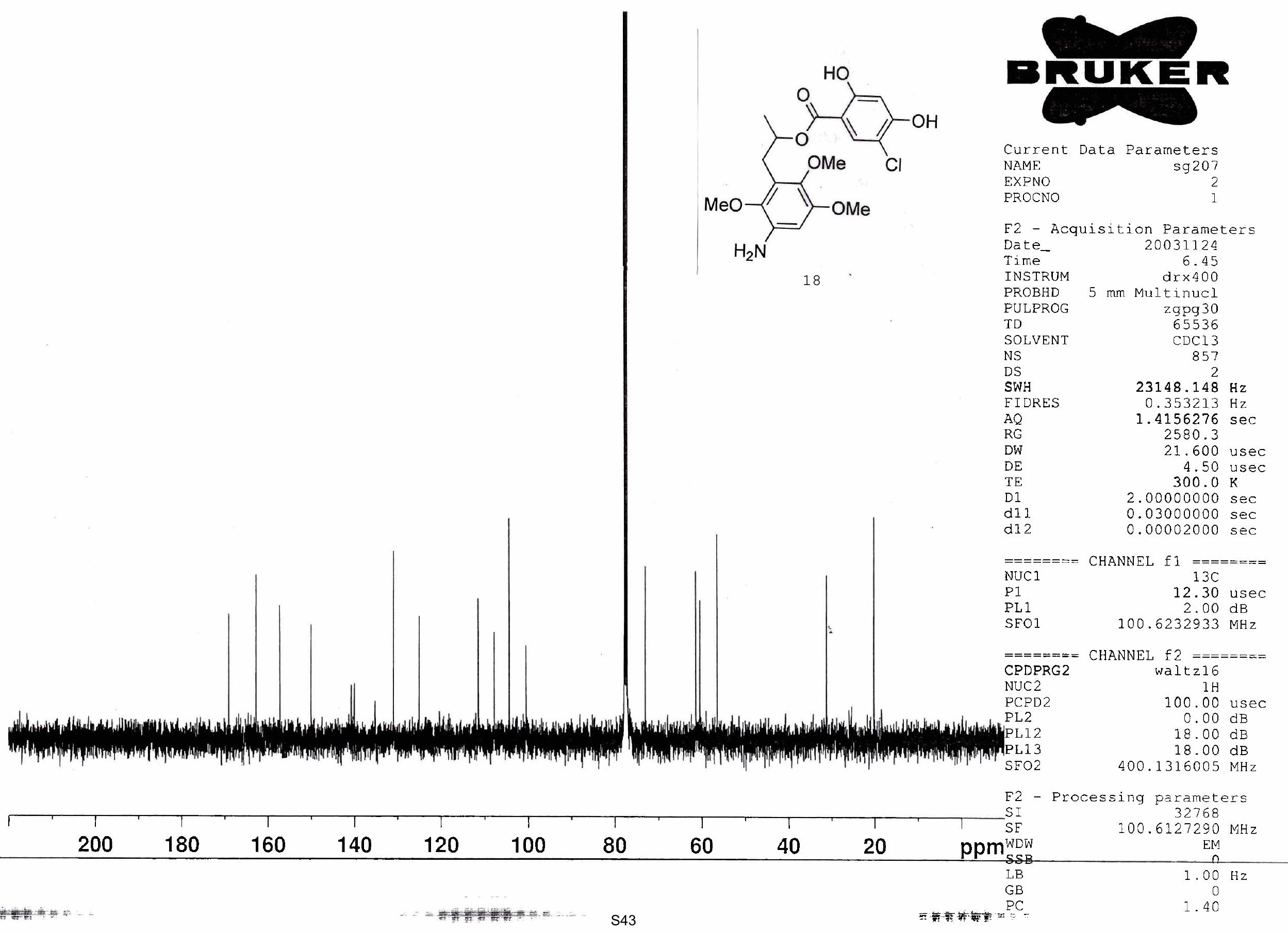




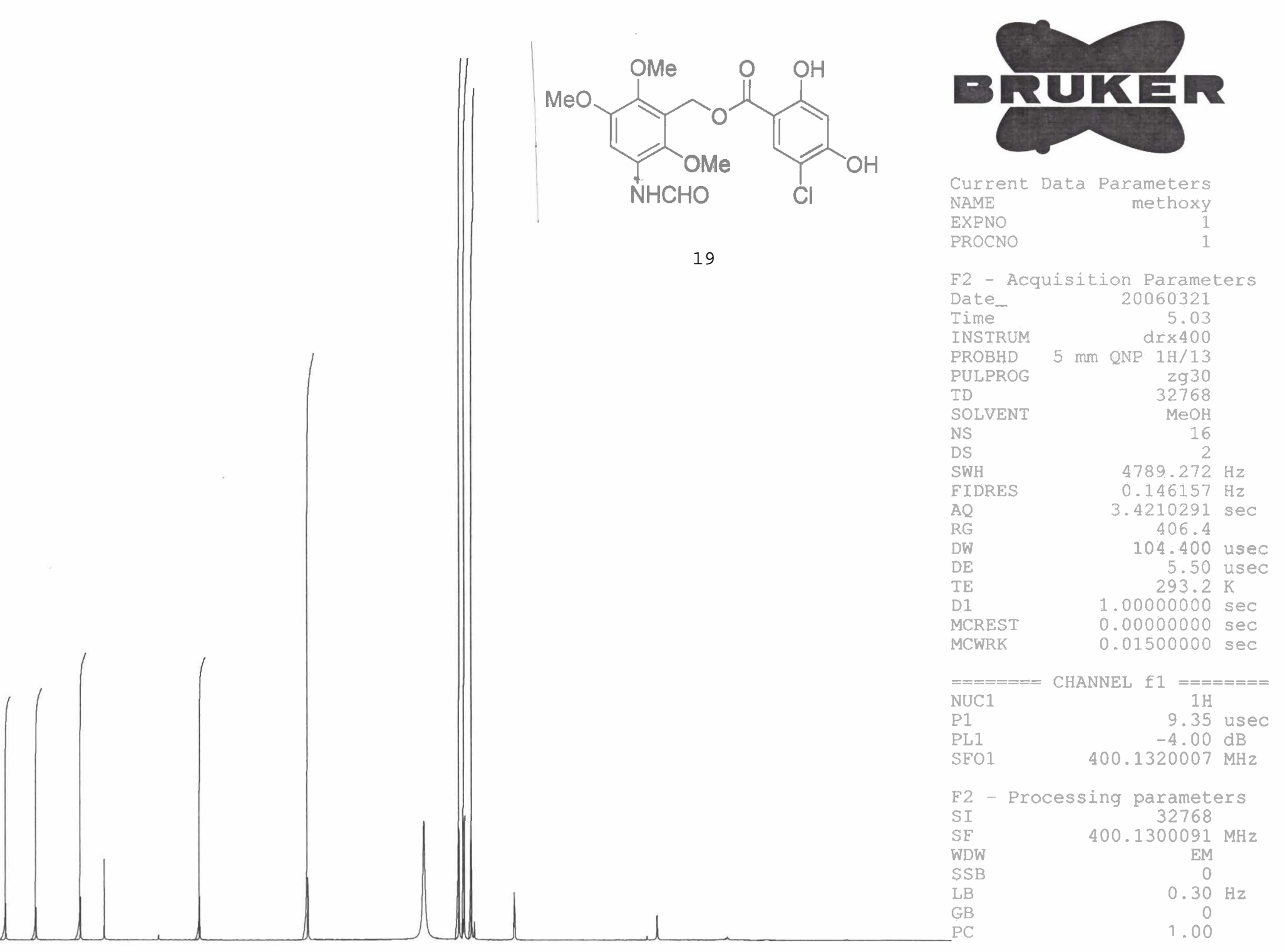

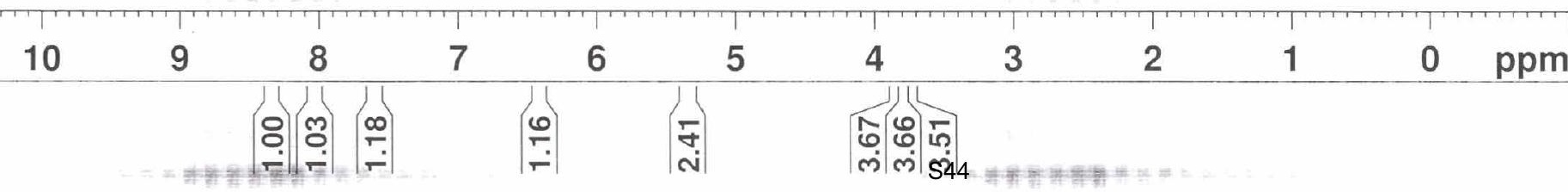




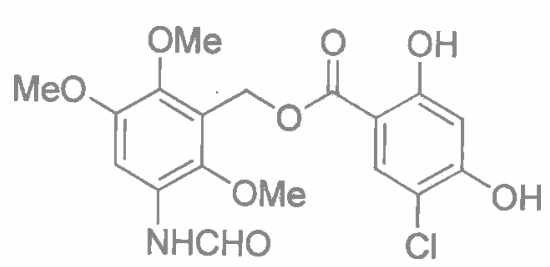

19

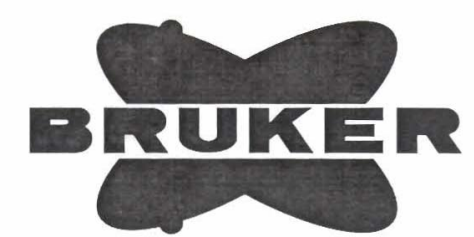

Current Data Parameters NAME

EXPNO

PROCNO

F2 - Acquisition Parameters

Time 13.37

13.37
INSTRUM drx400

PROBHD $5 \mathrm{~mm}$ QNP $1 \mathrm{H} / 13$

TULPROG

SOLVENT

NS

EIDRES

EIDRES
AQ

AQ

DW

DE

IE

D1

d11

MELTA

MCWRK

zgpg 30
65536

65536

18432

$23148.148 \mathrm{~Hz}$

$23148.148 \mathrm{~Hz}$
$0.353213 \mathrm{~Hz}$

$1.4156276 \mathrm{sec}$ 32768

21.600 usec 5.50 usec $293.2 \mathrm{~K}$

$0.15000001 \mathrm{sec}$

$0.03000000 \mathrm{sec}$

$0.05000000 \mathrm{sec}$

$0.00000000 \mathrm{sec}$

$0.01500000 \mathrm{sec}$ $==\because=====$ CHANNEL

NUC1

PI

PL1

SFO1

$$
13 \mathrm{C}
$$

11.25 usec

$2.00 \mathrm{~dB}$

$100.6232933 \mathrm{MHz}$

$========$ CHANNEL $f 2$

CPDPRG2

NUC2

PCPD2

PL2

PL12

PL13

S

waltzl

$1 \mathrm{H}$

100.00 usec

$-4.00 \mathrm{~dB}$

$16.58 \mathrm{~dB}$

$17.00 \mathrm{~dB}$

$400.1316005 \mathrm{MHz}$

\begin{tabular}{|c|c|c|c|c|c|c|c|c|c|}
\hline 200 & $\begin{array}{c}1 \\
180\end{array}$ & 160 & 140 & $\begin{array}{c}1 \\
120\end{array}$ & 100 & 80 & 60 & $\begin{array}{l}1 \\
40\end{array}$ & 20 \\
\hline
\end{tabular}

Processing parameters $\begin{array}{lr}\text { SI } & 65536 \\ \text { SF } & 100.6127290 \mathrm{MHz}\end{array}$ SSB

EM
$1.00 \mathrm{~Hz}$




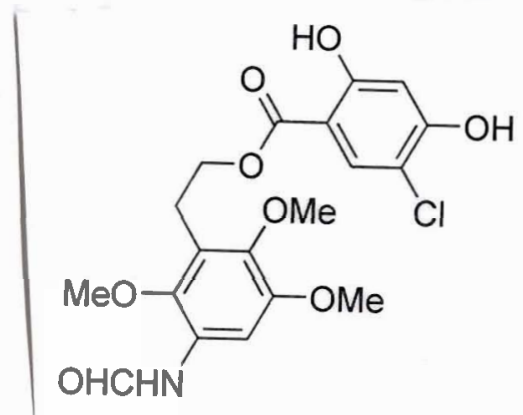

20 |이

7

6

5

4

3

2

1

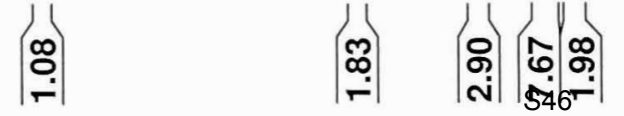

GRUKE

Current Data Parameters

NAME $\quad$ sg0037

EXPNO

PROCNO

F2 - Acquisition Parameters

Date__ 20030629

Time

INSTRUM

PROBHD

PULPROG

TD

SOLVENT

NS

DS

SWH

AQ

DW

DE

D1

$===$
NUC1

P1

SFO1

20030629

dr 4400

$5 \mathrm{~mm}$ Multinucl

zg30

32768

$\mathrm{MeOH}$

16
2

$4789.272 \mathrm{~Hz}$

$0.146157 \mathrm{~Hz}$

$3.4210291 \mathrm{sec}$ 181

104.400 usec 4.50 usec $300.0 \mathrm{~K}$

$1.00000000 \mathrm{sec}$ CHANNEL $\mathrm{f} 1$

$$
\begin{gathered}
1======= \\
1 \mathrm{H} \\
7.70 \text { usec }
\end{gathered}
$$
$400.1320007 \mathrm{MHz}$

F2 - Processing parameters

SI 16384

$\begin{array}{lr}\text { SI } & 16384 \\ \text { SF } & 400.1300000 \mathrm{MHz} \\ \text { WDW } & \text { EM }\end{array}$

WDW

SSB

LB

GB

0
$0.30 \mathrm{~Hz}$
0
1.00 


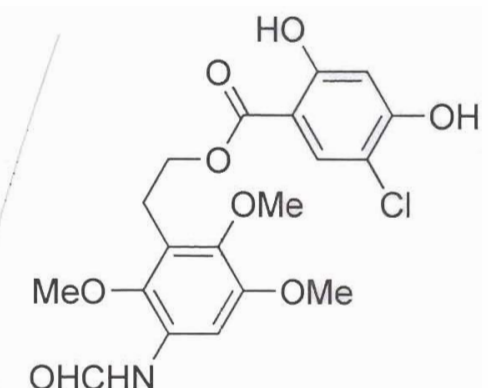

20

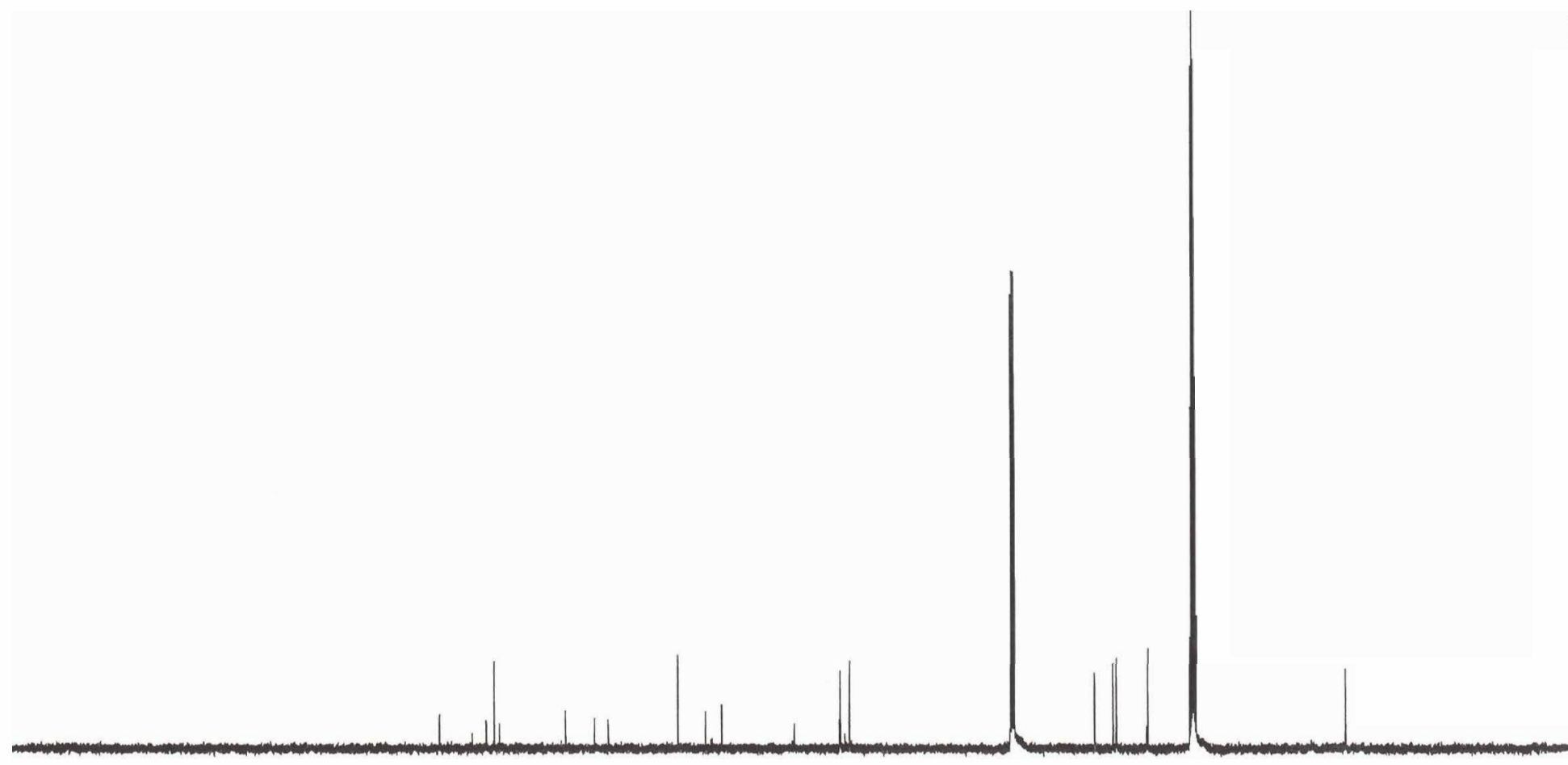

220

200

180

160

140

120
40
20

\begin{abstract}
100
\end{abstract}

80

Current Data Farameters NAME

EXPNO

82 - Acquisition Parameters

Date-

INSTRUM

PROBHD

PULPROO

TD

NS

DS

FIDRES

AQ

$R G$
$D W$
$D E$

IE

TE
d11
d12

d12

20031224
5.15

$5 \mathrm{~mm}$ BBO $\begin{array}{r}\text { spect } \\ \mathrm{BB}-1 \mathrm{H}\end{array}$

2gpg 30
65536

$\mathrm{CDC} 13$
3732

$31446.541 \mathrm{~Hz}$

$0.479836 \mathrm{~Hz}$

2048

15.900 usec

$\begin{aligned} 6.00 \mathrm{usec} & \\ 300.0 \mathrm{~K} & \end{aligned}$

$1.00000000 \mathrm{sec}$

$0.03000000 \mathrm{sec}$

$=======$ CHANNEL $\mathrm{fl}========$

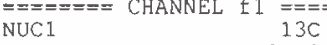

12.50 useo

SFO

$125.7719472 \mathrm{MHz}$

$==m= \pm= \pm=$ CHANNEL $\mathrm{f} 2 \mathrm{2}======$ CPDPRG2 waltz16

NUC2

PL2

PL13

95.00 use

$-4.00 \mathrm{~dB}$

$19.00 \mathrm{~dB}$

$500.1325000 \mathrm{MHz}$

F1 - Acquisition parameters NDO

TD

SFO1

SN

256
$500.1325 \mathrm{MHz}$ $23.475060 \mathrm{~Hz}$ $12.016 \mathrm{ppm}$
undefined

EnMODE undefined

E2 - Processing parameters 65536

WDW

SSB

125.7578008 MHz

$E M$
0
$1.00 \mathrm{~Hz}$
0
1.40

- Processing parameters 1024

$500.1300000 \mathrm{MHz}$

$\begin{array}{lr}\text { SF } & 500.1300000 \\ \text { WDW } & \text { no }\end{array}$

$S S B$
$L B$

$0.30 \mathrm{~Hz}$ 


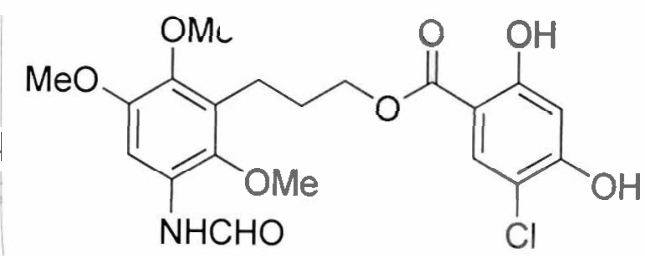

21

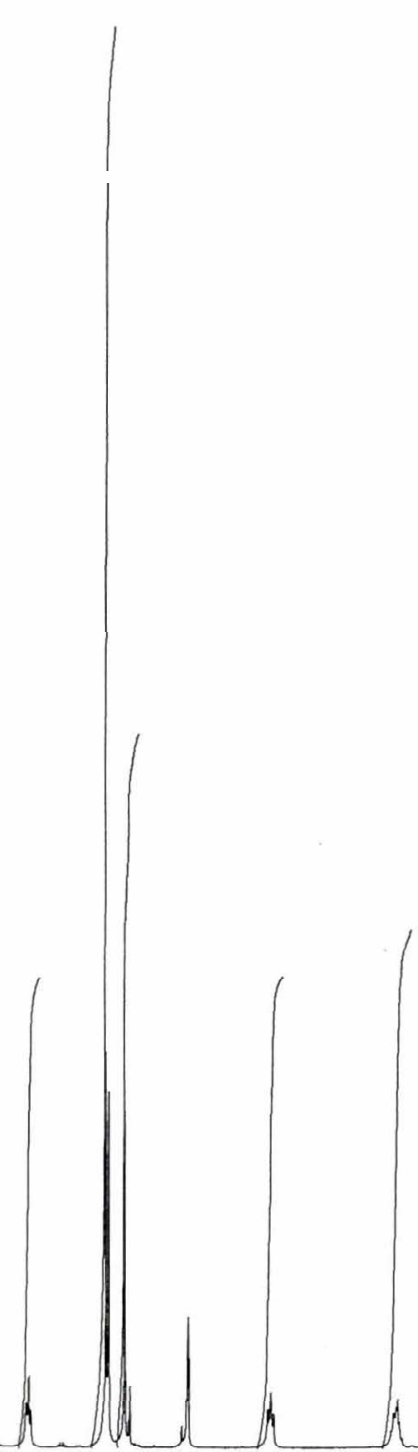

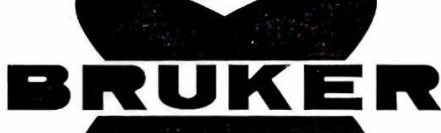 .}

Current Data Parameters

NAME

EXPNO

PROCNO

F2 - Acquisition Parameters

Date_ 20030702

Time

INSTRUM

PROBHD

PULPROG

SOLVENT

NS

DSH

FIDRES

AQ

DW

DE

D1

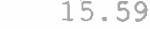

dr 8400

$5 \mathrm{~mm}$ Multinucl

zg30
32768

$4789.272 \mathrm{~Hz}$

$0.146157 \mathrm{~Hz}$

$3.4210291 \mathrm{sec}$$$
181
$$

104.400 usec 4.50 usec $300.0 \mathrm{~K}$

$1.00000000 \mathrm{sec}$

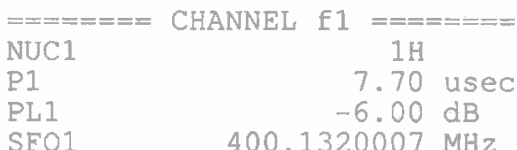

SFO1

$400.1320007 \mathrm{MHz}$

F2 - Processing parameters

SI 16384

SF $\quad 400.1300000 \mathrm{MHz}$

WDW

SSB

LB

GB
PC
EM

$0.30 \mathrm{~Hz}$

0 


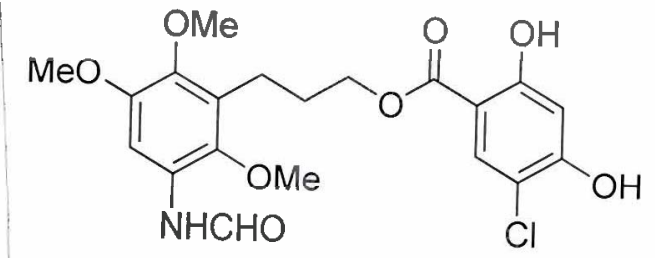

21
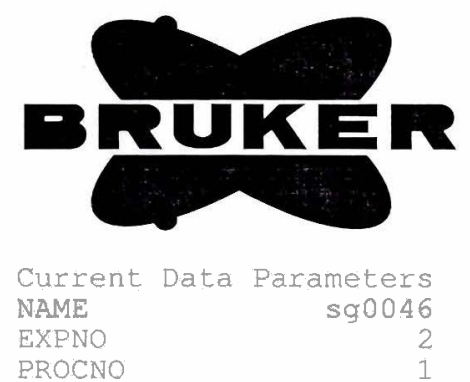

F2 - Acquisition Parameters

ision Pasame

$\begin{array}{lr}\text { Time } & 19.44\end{array}$

INSTRUM

PROBHD

dr $\times 400$

PUITRROG

$5 \mathrm{~mm}$ Multinucl

TD

SOLVENT

NS

DS

SWH

FIDRES

$\mathrm{AQ}$

RG

$\mathrm{DE}$

TE

D1

d11

$== \pm== \pm=$
NUCl

$\mathrm{P} 1$

PL1
SFO1

zgpg 30

65536

$\mathrm{MeOH}$

1109

$23148.148 \mathrm{~Hz}$

. 156276 sec

1824.6

21.600 usec

4.50 usec

$300.0 \mathrm{~K}$

0.05000000 sec

$0.03000000 \mathrm{sec}$

$0.03000000 \mathrm{sec}$
$0.00002000 \mathrm{sec}$

CPDPRG2

NUC2

PCPD?

PL2

PL12

PL13

$\mathrm{SFO} 2$

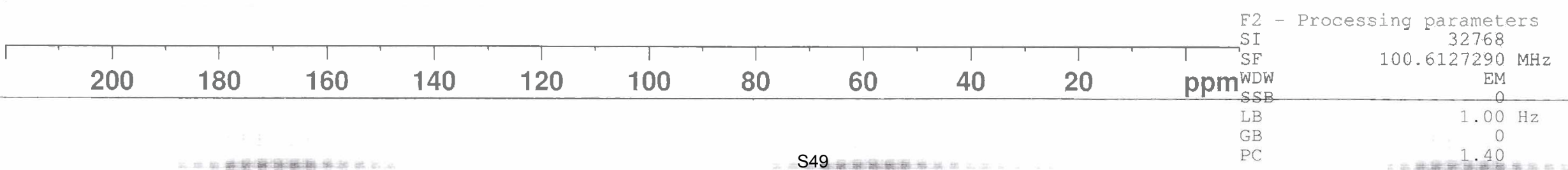




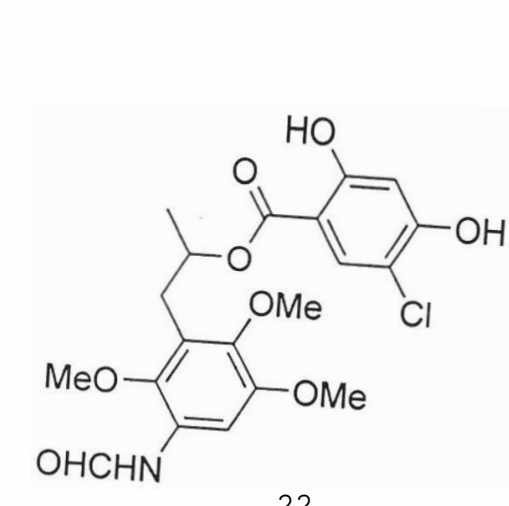

R. UURER

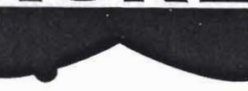

Current Data Parameters

NAME

EXPNO

PROCNO

sg-112

F2 -

Iime

INSTRUM

PROBHD

PULPROG

TD

SOLVENT

NS

DS

SWH

FIDRES

$A Q$

RG

DW

DE

TE

NUC1

P1

PL1

SFO1

F2

SI

WDW

WDW

$\mathrm{GB}$ cquisition Parameter 20030822 6.28

mm Mult:

$\mathrm{zg} 30$

32768

$\mathrm{CDCl} 3$

32

4789.272 $0.146157 \mathrm{~Hz}$ $3.4210291 \mathrm{se}$ 64 104.400 us 4.50 us $300.0 \mathrm{~K}$ $1.00000000 \mathrm{se}$ .70 us $6.00 \mathrm{~dB}$ $400.1320007 \mathrm{MH}$ Processing parameters 16384 400.1300000 EM $0.30 \mathrm{~Hz}$ 0 1.00

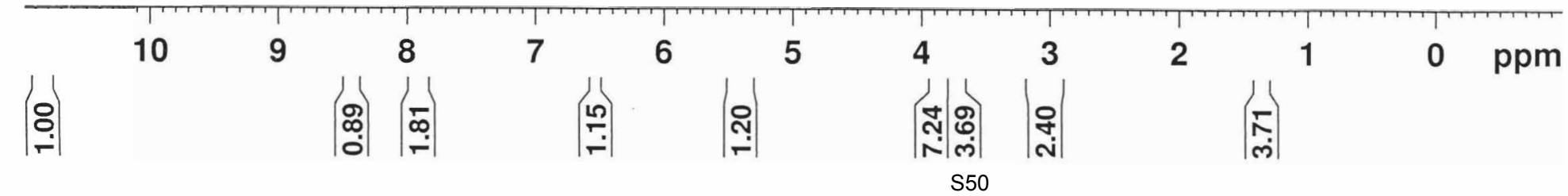


22

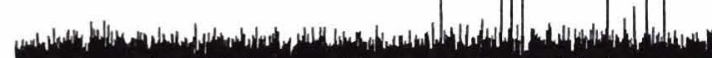

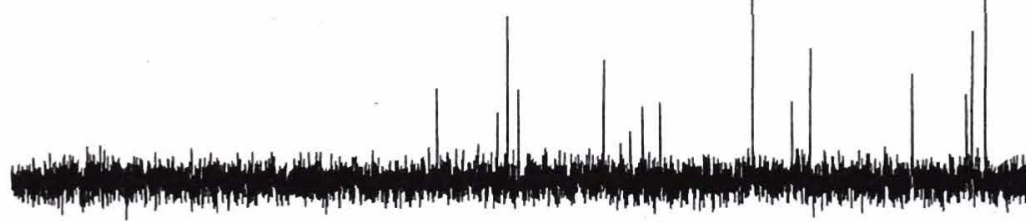

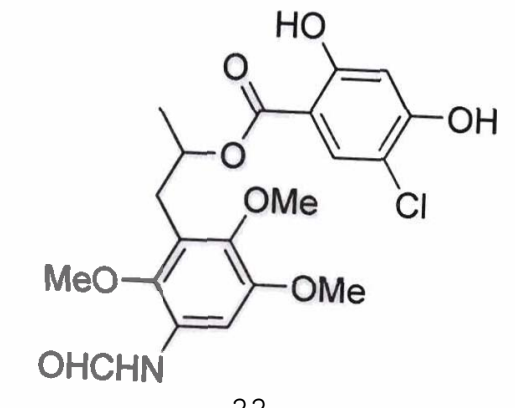
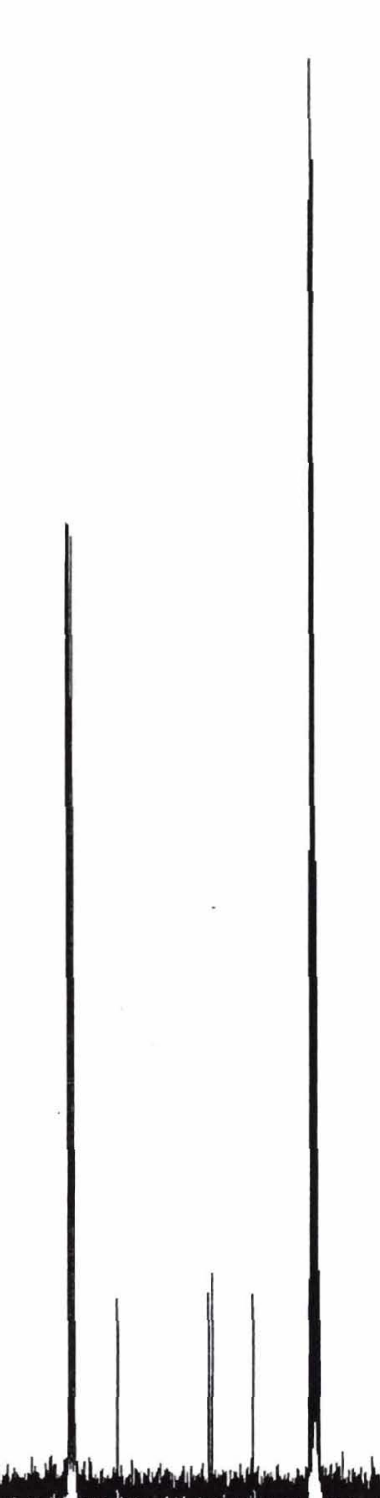

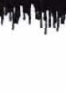

, thin

\section{DEUKE .}

Current Data Parameters NAME

EXPNO

PROCNO

sg0035

F2 - Acquisition Parameters Date_ $\quad 20030627$

Time

INSTRUM

PROBHD

PULPROG

ID

SOLVENT

NS

DS

SWH

FIDRES

AQ

RG

DW

IE

D1

d11

$=======0$ CHANNEL

NUC1

P1

PLI

SFO1

$5 \mathrm{~mm}$ Multinucl

zgpg 30

65536

$\mathrm{MeOH}$

137

$0.353213 \mathrm{~Hz}$

322.5

21.600 usec 4.50 usec

$0.05000000 \mathrm{sec}$

$0.03000000 \mathrm{sec}$

$0.00002000 \mathrm{sec}$

$f 1$

$13 \mathrm{C}$

12.30 usec $2.00 \mathrm{~dB}$ $100.6232933 \mathrm{MHz}$ CPDPRG2 waltz16 NUC2

PCPD2

PL2

PL12

PL13

$\mathrm{SFO} 2$
$23148.148 \mathrm{~Hz}$

$1.4156276 \mathrm{sec}$

$$
+
$$




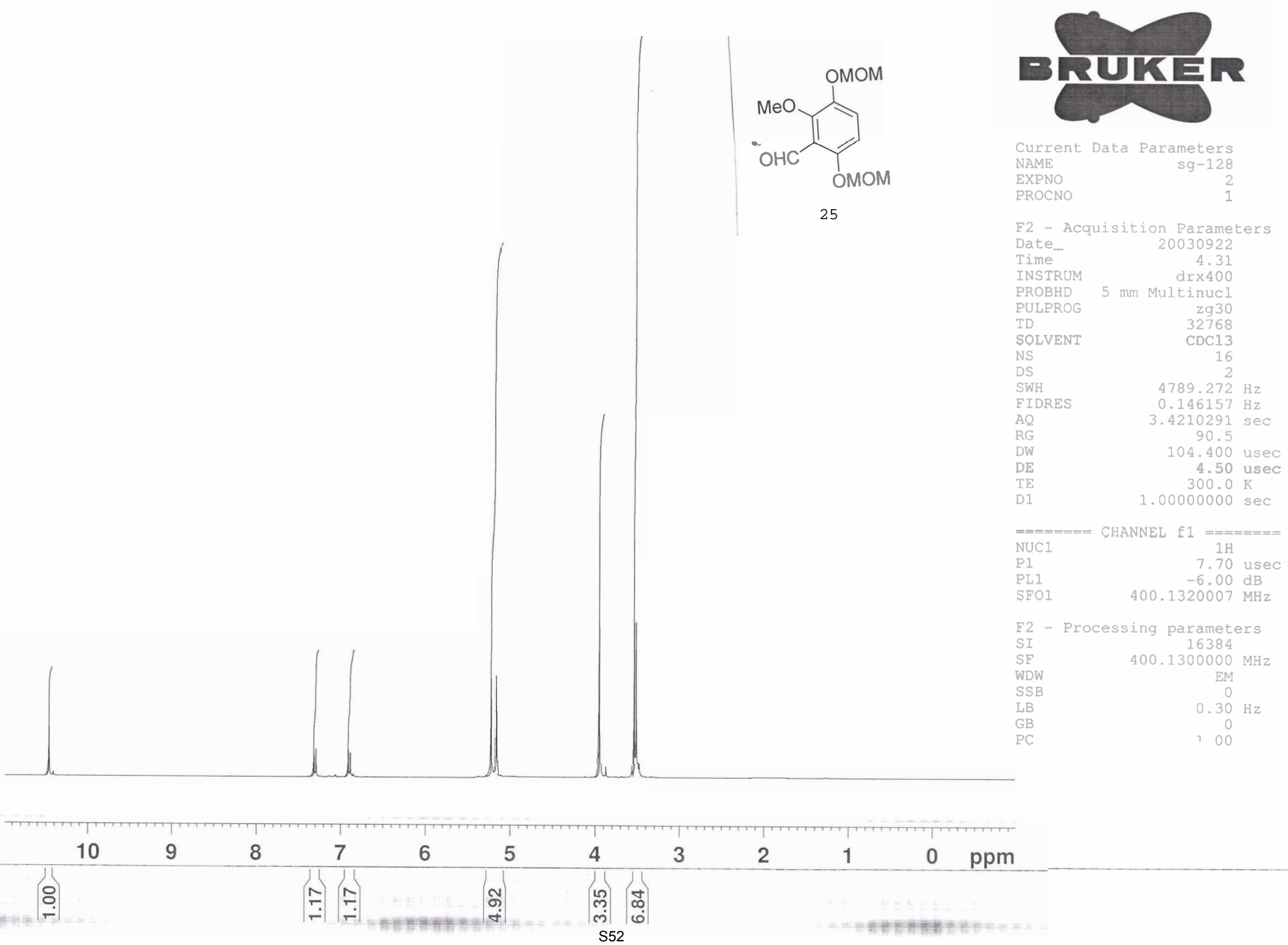




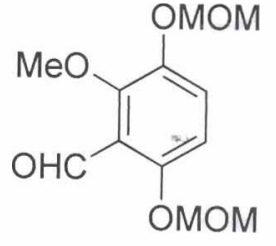

25

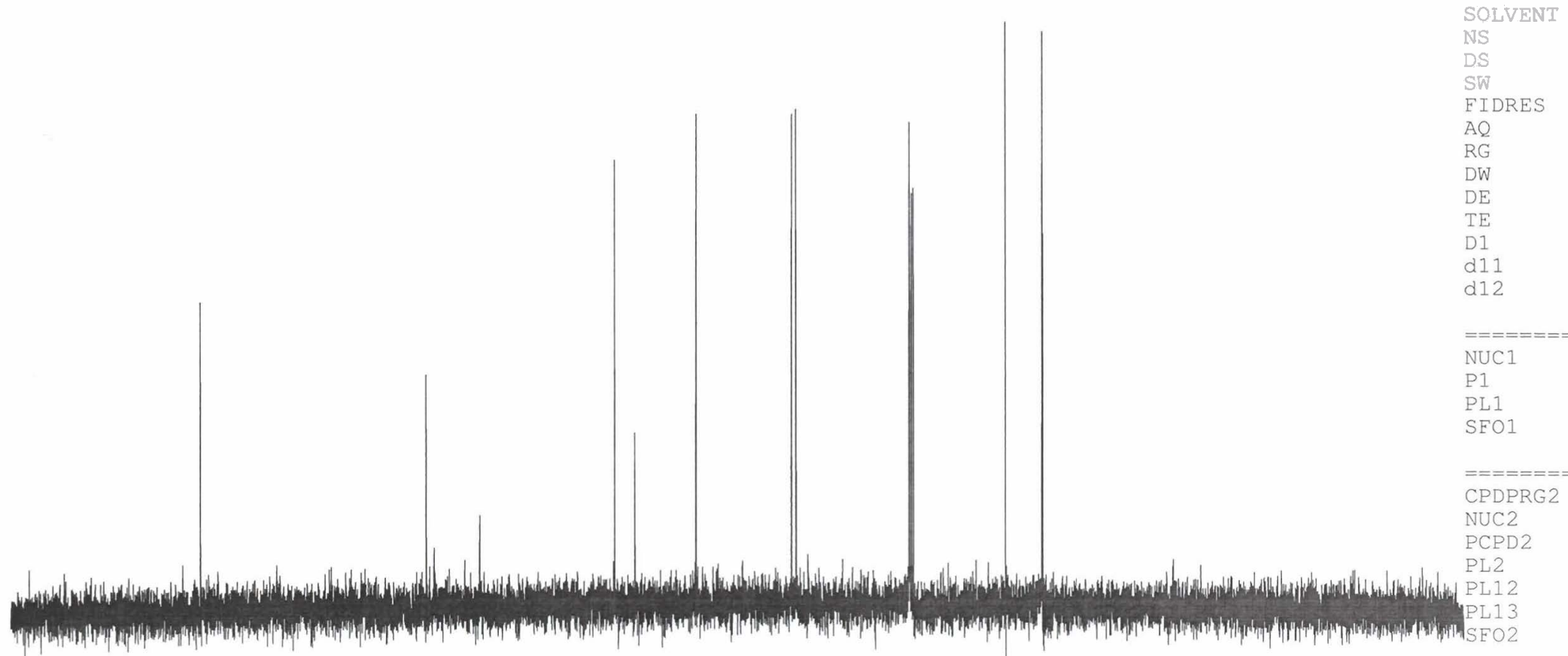

Current Data Parameters

NAME

sg-129

EXPNO

1

F2 - Acquisition Parameters

Date__ 20030922

Iime

4.35
drx 400

INSIRUM

PROBHD

$5 \mathrm{~mm}$ Multinucl

65536

CDC 13

$23148.148 \mathrm{~Hz}$

$0.353213 \mathrm{~Hz}$

1.4156276 sed 1290.2

21.600 usec 4.50 usec $300.0 \mathrm{~K}$

0.05000000 sec

$0.03000000 \mathrm{sec}$

$0.00002000 \mathrm{sec}$

CHANNEL

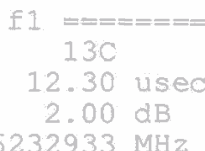

$100 \quad 6232933 \mathrm{MHz}$

CHANNEI :

waltz16

$1 \mathrm{H}$

100.00 usec

$0.00 \mathrm{~dB}$

$18.00 \mathrm{~dB}$

$18.00 \mathrm{~dB}$

$400.1316005 \mathrm{MHz}$ 

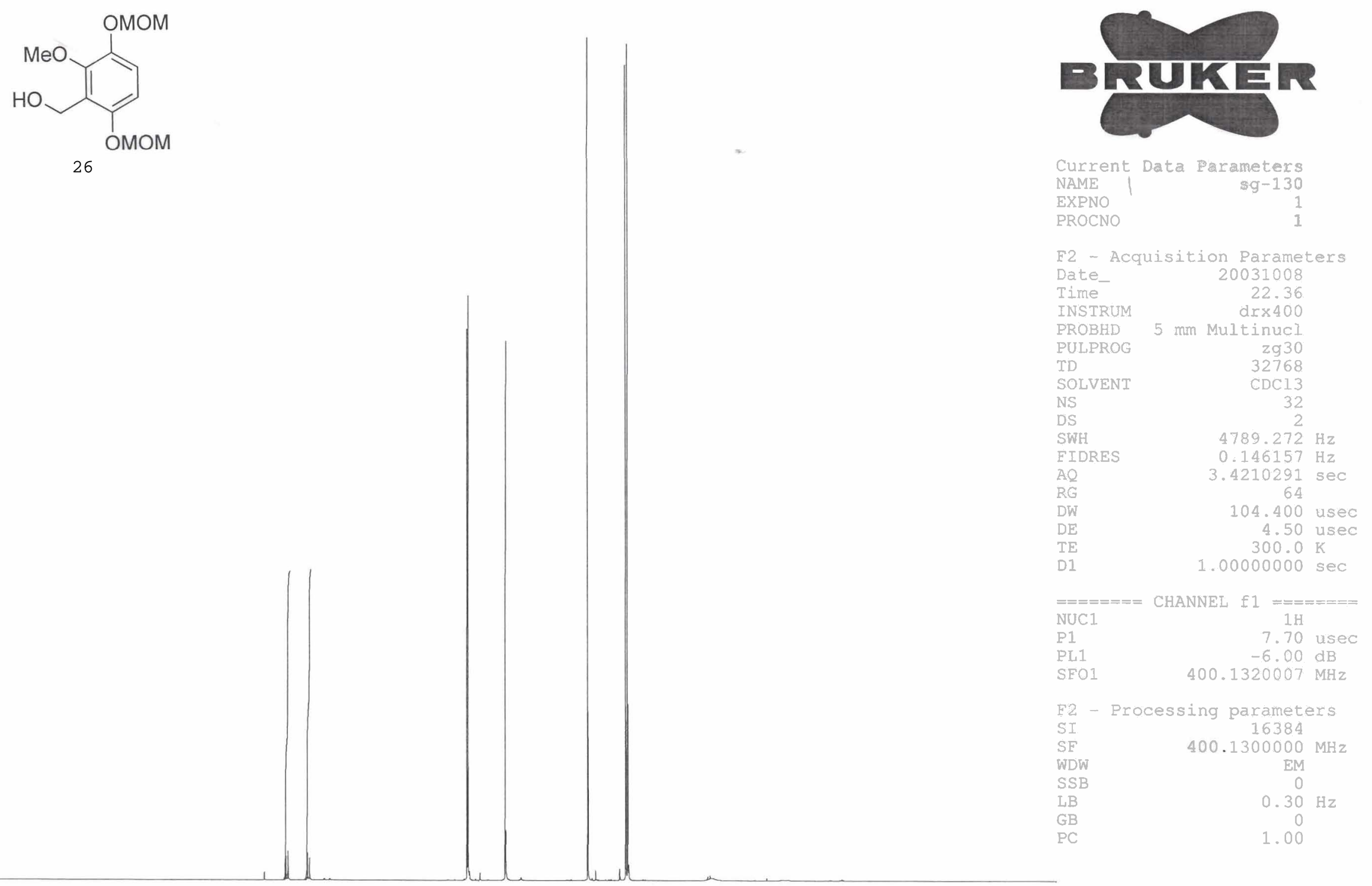

10

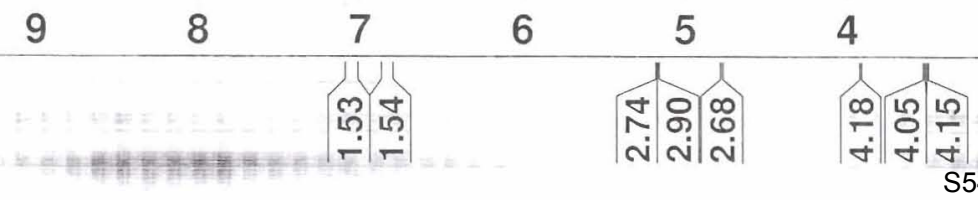




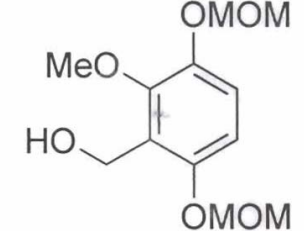

26

\section{BRUKER}

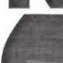

\begin{tabular}{l|r} 
Current & Data Parameters \\
NAME & $\$-131$ \\
EXPNO & 2 \\
PROCNO & 1
\end{tabular}

2 - Acquisition Parameters

Time

20031008

INSTRUM

PROBHD

dir 400

PULPROG

SOLVENT

NS

is

FIDRES

AO

DW

DE

TE

d11

d12

$5 \mathrm{~mm}$ Multinucl zgpg 30
65536 $\mathrm{CDC} 13$

82

$23148.148 \mathrm{~Hz}$

$0.353213 \mathrm{~Hz}$

$1.4156276 \mathrm{sec}$ 32768

21.600 usec

4.50 usec $300.0 \mathrm{~K}$

$0.05000000 \mathrm{sec}$

$0.03000000 \mathrm{sec}$

$0.00002000 \mathrm{sec}$

$====== \pm=$ CHANNEL f

NUC1

P1

PL1

SEO1

$13 \mathrm{C}$

12.30 usec

$2.00 \mathrm{~dB}$

$100.6232933 \mathrm{MHz}$

CHANNEL

CPDPRG2

NUTC2

PCPD2

PL2

PL12

PL13

waltzI 6

100.00 usec

$0.00 \mathrm{~dB}$

$18.00 \mathrm{~dB}$

$18.00 \mathrm{~dB}$

$400.1316005 \mathrm{MHz}$

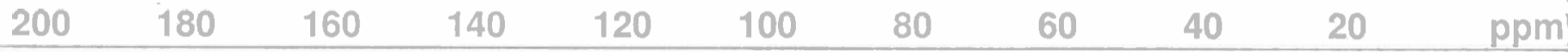



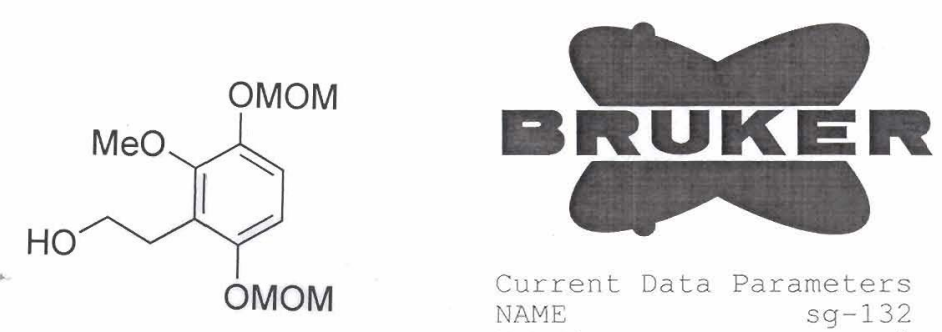

27

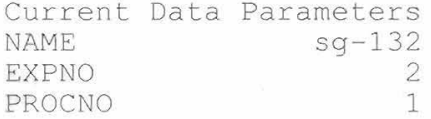

F2 - Acquisition Parameters

Date_ 20030923

Time- 5.47

INSTRUM drx400

PROBHD $5 \mathrm{~mm}$ Multinucl

PULPROG zg30

TD 32768

SOLVENT CDCl3

NS

DS

SWH

FIDRES

$\mathrm{AQ}$

$R G$

DW

DE

TE
DI

$\mathrm{CDC} 13$
16

$4789.272 \mathrm{~Hz}$

$0.146157 \mathrm{~Hz}$

$3.4210291 \mathrm{sec}$

64
104.400

4.50 usec $300.0 \mathrm{~K}$

$1.00000000 \mathrm{sec}$

$====$
NUC 1

$\mathrm{P} 1$

CHANNEL $\mathrm{f}$

$$
\text { . }
$$$$
1 \mathrm{H}
$$

7. 70 usec

$-6.00 \mathrm{~dB}$

PL1
SFO1

$400.1320007 \mathrm{MHz}$

F2 - Processing parameters

SI 16384

SE $\quad 400.1300000 \mathrm{MHz}$

WDW EM

SSB

$E M$
0

$0.30 \mathrm{~Hz}$

$\begin{array}{lr}\mathrm{GB} & 0 \\ \mathrm{PC} & 1.00\end{array}$

\section{0}

9

8

6

5

4

3

2

1 


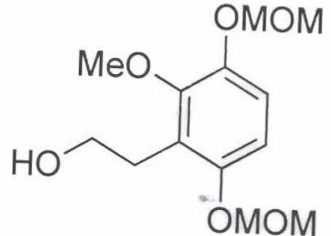

27

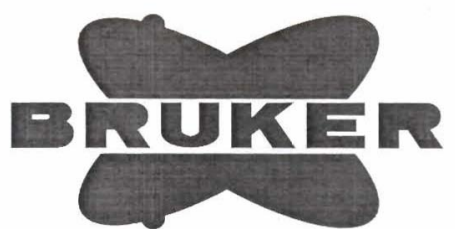

Current Data Parameters

NAME

EXPNO

PROCNO

F2 - Acquisition Parameters

Date_ 20030923

Time $\quad 5.52$

INSTRUM drX400

PROBHD $5 \mathrm{~mm}$ Multinucl

PULPROG $\quad$ zopog 30

$\begin{array}{lr}\text { TD } & 65536\end{array}$

SOLVENT CDC13

NS 111

DS

SWH 23.148.148 Hz

EIDRES $\quad 0.353213 \mathrm{~Hz}$

AQ $\quad 1.4156276 \mathrm{sec}$

RG

DW

1448.2

21.600 used

4.50 used

$300.0 \mathrm{~K}$

$0.05000000 \mathrm{sec}$

$\begin{array}{ll}\mathrm{d} 11 & 0.03000000 \mathrm{sec} \\ \mathrm{d} 12 & 0.00002000 \mathrm{sec}\end{array}$

d12

NUC1

P1

PL1

SFO1

CHANNEL

$$
\begin{aligned}
& 1======== \\
& 13 \mathrm{C} \\
& 12.30 \text { used } \\
& 2.00 \mathrm{~dB}
\end{aligned}
$$

$100.6232933 \mathrm{MHz}$

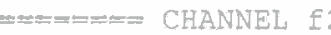

CPDPRG2 waltz16

NUC?

PCPD2

PL2

PL12

PL13

$\mathrm{SFO} 2$

100.00 usec

$0.00 \mathrm{~dB}$

$18.00 \mathrm{~dB}$

$18.00 \mathrm{~dB}$

$400.1316005 \mathrm{MHz}$

- Processing parameters 32768

200

180

160

140

120

100

80

60

40

20

PpmWDW

$100.6127290 \mathrm{MHz}$

LB

$G B$

EM



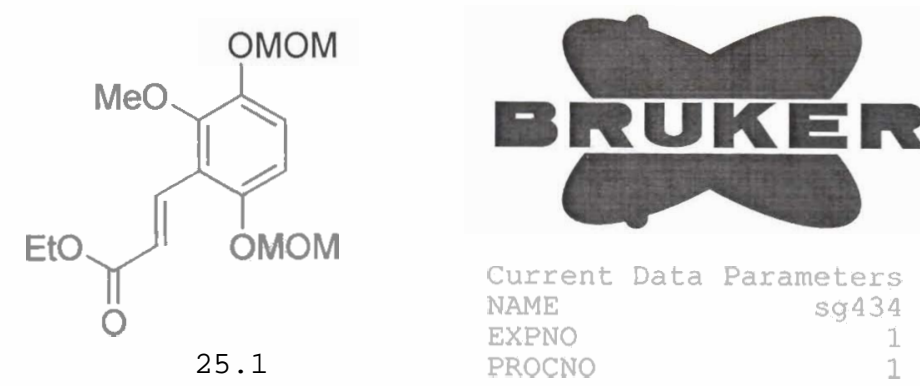

F2 - Acquisition Parameters Date 20050406

$\begin{array}{lr}\text { Dime } & 20050406 \\ \text { Time } & 2.53\end{array}$

INSTRUM drX400

PROBHD $5 \mathrm{~mm}$ QNP $1 \mathrm{H} / 13$

PULPROG $\quad \mathrm{zg} 30$

TD

SOLVENT

NS

DS

EIDRES

AQ

RG
DW

$\mathrm{DE}$

TE

MCREST

MCWRK

CDC13

$4789.272 \mathrm{~Hz}$

$0.146157 \mathrm{~Hz}$

$3.4210291 \mathrm{sec}$ 64

104.400 usec

5.50 usec $294.2 \mathrm{~K}$

1. $00000000 \mathrm{sec}$

$0.00000000 \mathrm{sec}$

$0.01500000 \mathrm{sec}$

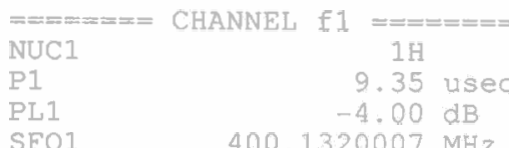

$400.1320007 \mathrm{MHz}$

F2 - Processing parameters

SI $\quad 32768$

SF $\quad 400.1300000 \mathrm{MHz}$

WDW

SSB

EM
0
0.30
0
1.02

\begin{tabular}{|c|c|c|c|c|c|}
\hline 10 & 8 & 7 & 6 & 5 & 4 \\
\hline & & & 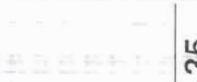 & & ำ \\
\hline
\end{tabular}

$\begin{array}{lllll}3 & 2 & 1 & 0 & \mathrm{ppm}\end{array}$

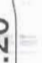




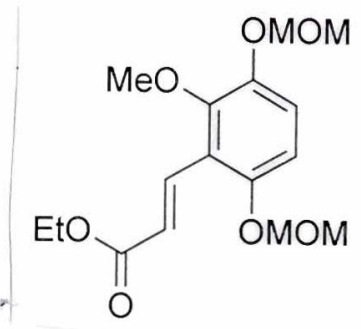

25.1

\section{0

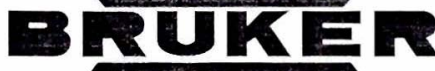 ind}

$\begin{array}{lr}\text { Current Data Parameters } \\ \text { NAME } & \$ 9434 \\ \text { EXPNO } & 2 \\ \text { PROCNO } & 1\end{array}$

E2 - Acquisition Parameters

Date 20050406

Time

INSTRUM

PROBHD

PULPROG

TD

SOLVENT

NS

DS

EIDRES

AQ

DW

DE

D1

Q11

DELTA

MCREST

MCREST

$===$
NUC1

PI

SFO1

2.56

dr $\times 400$

$5 \mathrm{~mm}$ QNP $1 \mathrm{H} / 13$

zgpg 30

$\mathrm{CDCl} 3$

83

$23148.148 \mathrm{~Hz}$

$0.353213 \mathrm{~Hz}$

$1.4156276 \mathrm{sec}$

32768

21.600 usec

5.50 usec

294.2 K

$0.15000001 \mathrm{sec}$

$0.03000000 \mathrm{sec}$

$0.05000000 \mathrm{sec}$

$0.00000000 \mathrm{sec}$

$0.01500000 \mathrm{sec}$

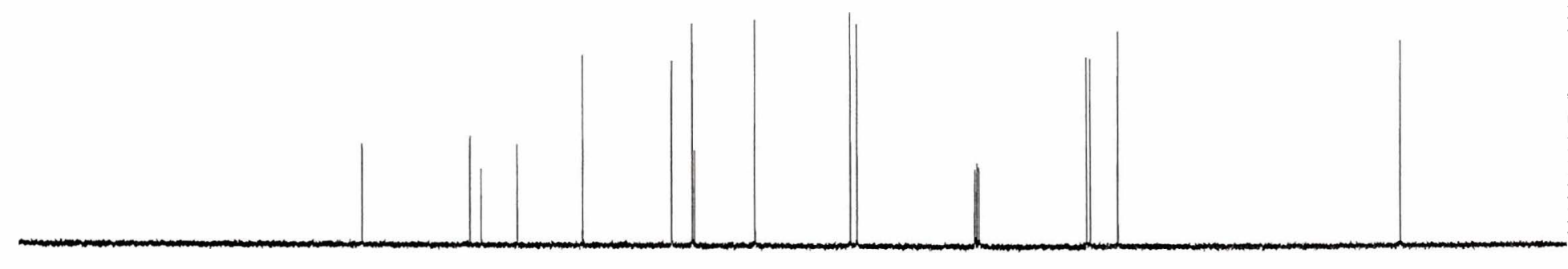

$=====$

CPDPRG

NUC2

PCPD2

PL2

PL12

$\mathrm{SEO} 2$

22

HANNEL $f 2=======$
waltz 16
$1 \mathrm{H}$
$100.00 \mathrm{usec}$
$-4.00 \mathrm{~dB}$
$16.58 \mathrm{~dB}$
$17.00 \mathrm{~dB}$
$400.1316005 \mathrm{MHz}$

$400.1316005 \mathrm{MHz}$ 


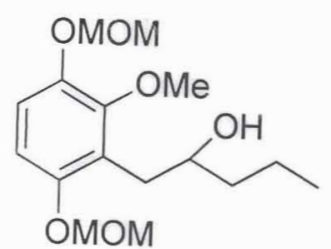

29

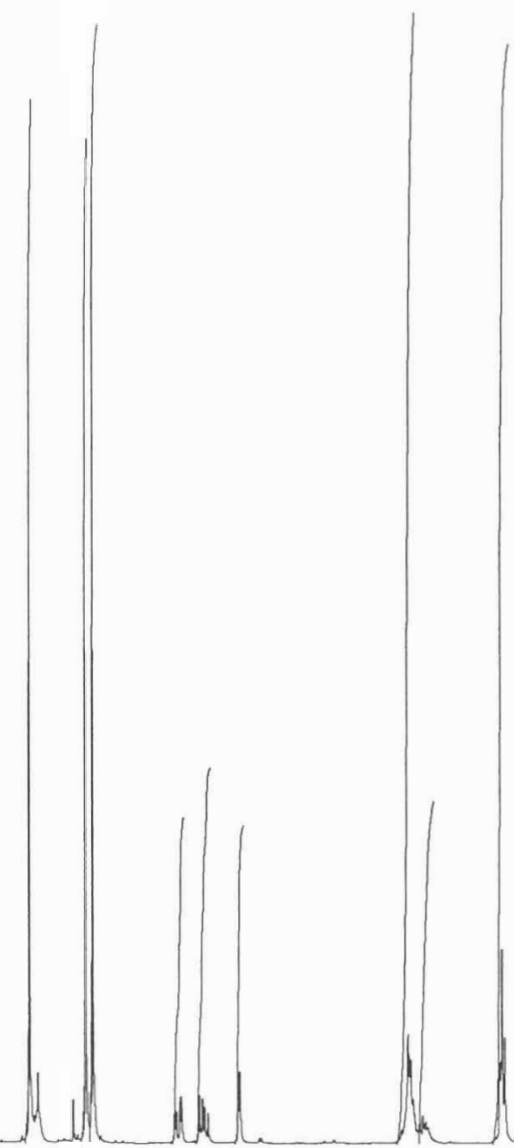

Bत्सKE C.

Current Data Parameters NAME sg424epoxypetane EXENO PROCNO

F2 - Acquisition Parameters

Date_ 20050318

Time

17.21

INSTRUM

PROBHD

PULPROG

TD

SOLVENT

NS

DS

FIDRES

AO

RG

$D W$

$D \Xi$

DI

MCREST

MCWRK

$5 \mathrm{~mm}$ ONP $1 \mathrm{H} / 13$

2930

32768

$\mathrm{CDC13}$

$4789.272 \mathrm{~Hz}$

$0.146157 \mathrm{~Hz}$

3.4210291 sec

64

104.400 usec

5.50 usec $293.2 K$

$1.00000000 \mathrm{sec}$

0.00000000 sec

$0.01500000 \mathrm{sec}$

프를

PI

PL1
SEO1.

El $1 \mathrm{H}$
9.35 usec
$-4.00 \mathrm{~dB}$

2 - Processing parameters

32768

$4001300000 \mathrm{MH}$

SE

WDW

SSB

LB

$G B$
EM

$0.30 \mathrm{~Hz}$

1.00

98

$8 \frac{7}{8+0}$

6

5

43

2

1

0

ppm

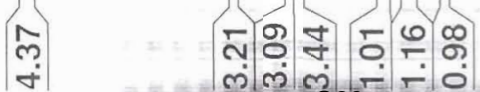

क 8 \% 

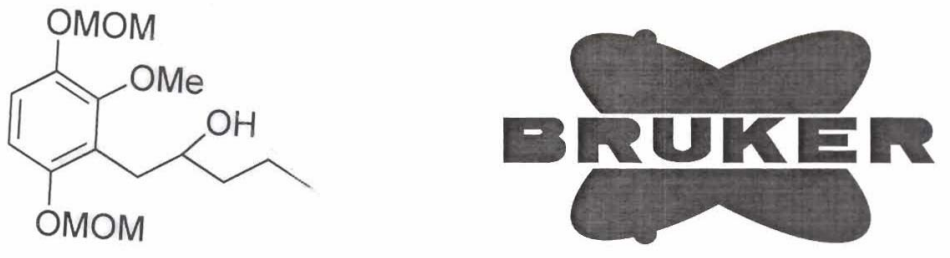

Current Data Parameters NAME Sg424epoxypetane EXPNO PROCNO

F2 - Acquisition Parameters Date 20050318

Time-

$\begin{array}{lr}\text { Time } & 17.26 \\ \text { INSTRUM } & \text { drx } 400\end{array}$

INSTRUM 5 mm QNP $1 H / 13$
PROBHD 5 m

PUILPROG

TD

SOLVENT

NS

DS

FIDRES

$A Q$

RG

DW

DE

dII

DELTA

MCREST

zgpg 30

65536

$\mathrm{CDCl}$
205
4

MCWRK

$23148.148 \mathrm{~Hz}$

$0.353213 \mathrm{~Hz}$

$1.4156276 \mathrm{sec}$ 32768

21.600 usec 5.50 usec 293.2 K

$0.15000001 \mathrm{sec}$

$0.03000000 \mathrm{sec}$

$0.05000000 \mathrm{sec}$

$0.05000000 \mathrm{sec}$

$0.01500000 \mathrm{sec}$

$== \pm== \pm==$ CHANNEL NUC1

PI

PL1 $13 \mathrm{C}$ $==$ 11.25 usec SEO1 $2.00 \mathrm{~dB}$

$=======$ CHANNEI

CPDPRG2

NUC2 2

PCPD2

PL2

PL12

PL13.

SFO2

400.1316005 MHz waltz 16

100.00 usec

$-4.00 \mathrm{~dB}$

$16.58 \mathrm{~dB}$

$17.00 \mathrm{~dB}$ 


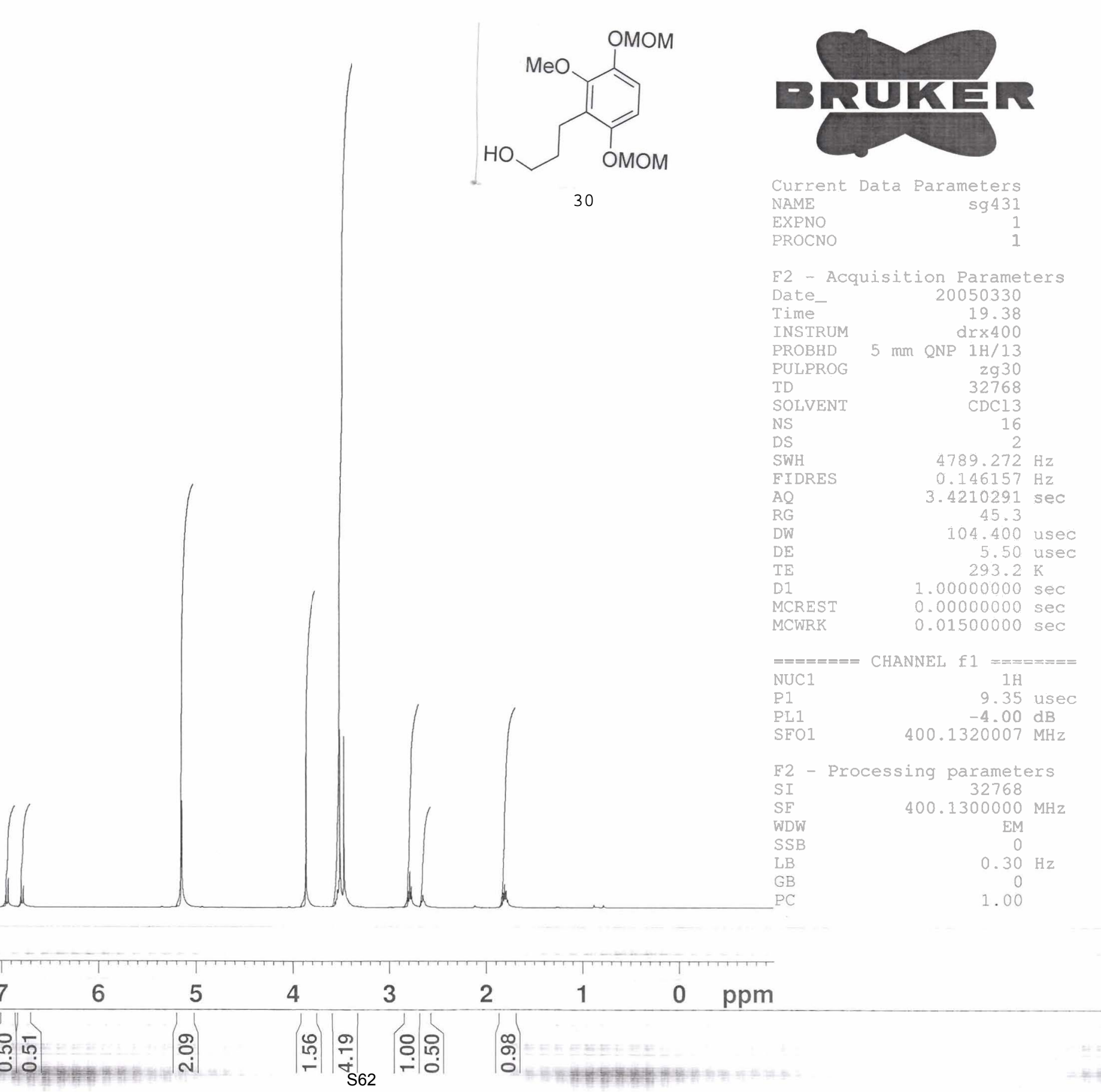



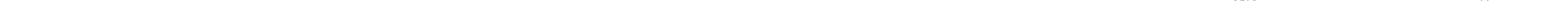


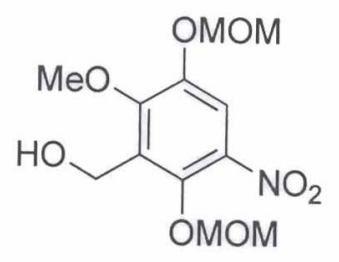

31

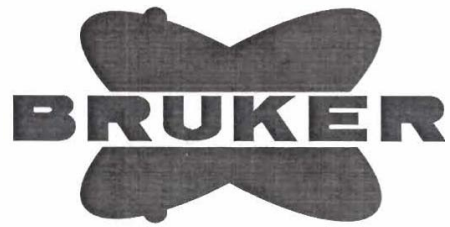

Current Data Parameters NAME Icmomitration EXPNO PROCNO

$\begin{array}{lr}\text { E2 - Acquisition Parameters } \\ \text { Date_ } \\ \text { Time } & 500324 \\ \text { INSTRUM } & 5.02 \\ \text { PROBHD } & \text { drx } 400 \\ \text { PULPROG } & \text { QNP } / 13 \\ \text { TD } & z 930 \\ \text { SOLVENT } & 32768 \\ \text { NS } & \text { CDC13 } \\ \text { DS } & 16 \\ \text { SWH } & 2 \\ \text { EIDRES } & 4789.272 \mathrm{~Hz} \\ \text { AQ } & 0.146157 \mathrm{~Hz} \\ \text { RG } & 3.4210291 \mathrm{sec} \\ \text { DW } & 256 \\ \text { DE } & 104.400 \mathrm{usec} \\ \text { TE } & 5.50 \mathrm{usec} \\ \text { D1 } & 293.2 \mathrm{~K} \\ \text { MCREST } & 0.00000000 \mathrm{sec} \\ \text { MCWRK } & 0.01500000 \mathrm{sec}\end{array}$

$0.01500000 \mathrm{sec}$

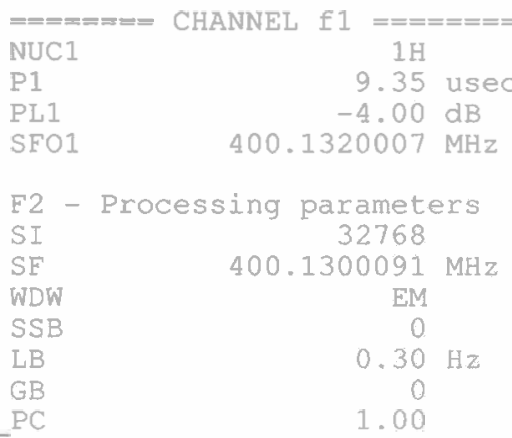

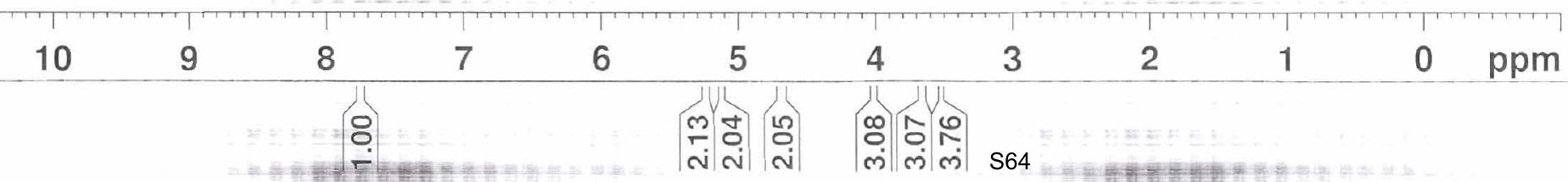



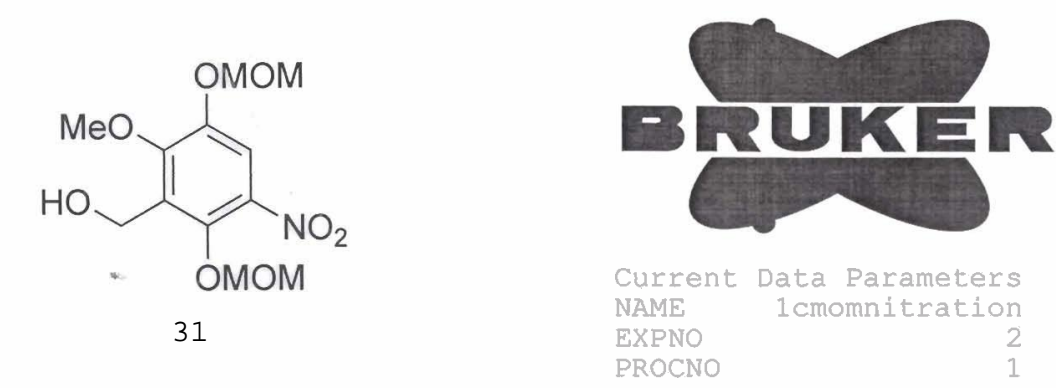

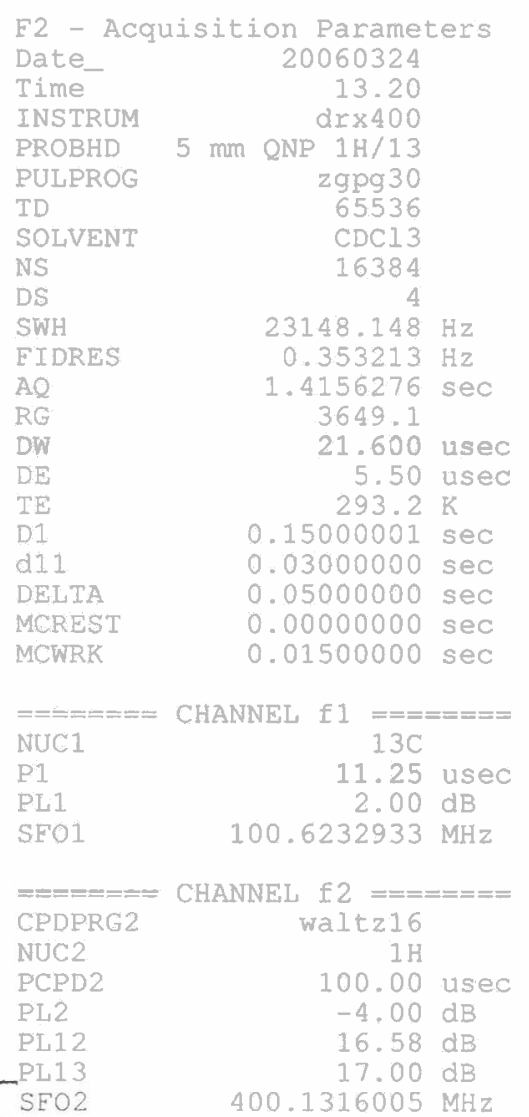

2 - processing parametersSI 65536 


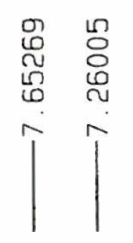

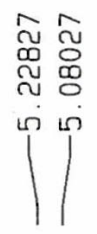

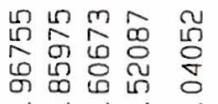

mंm $m$ m

1111
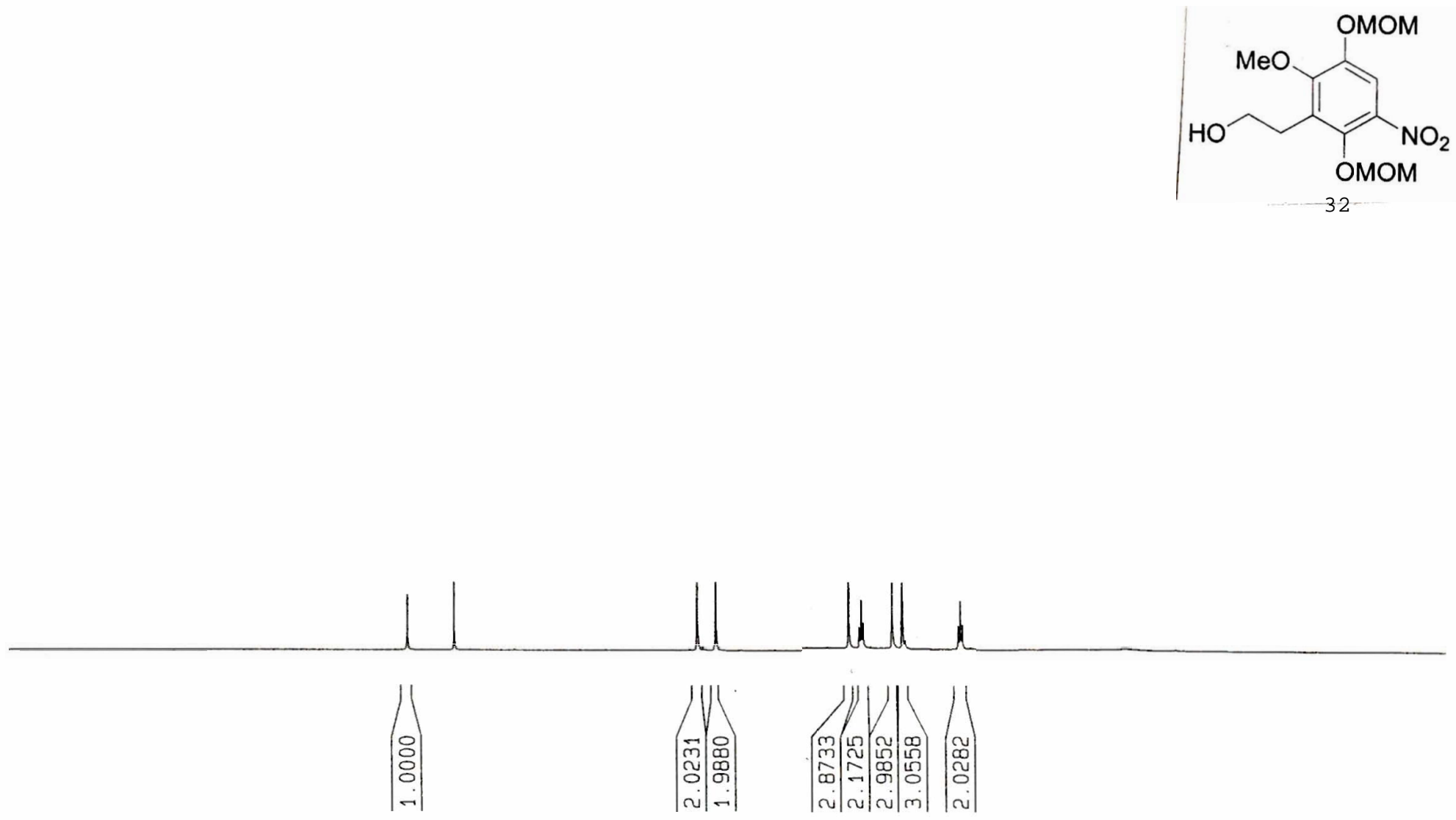

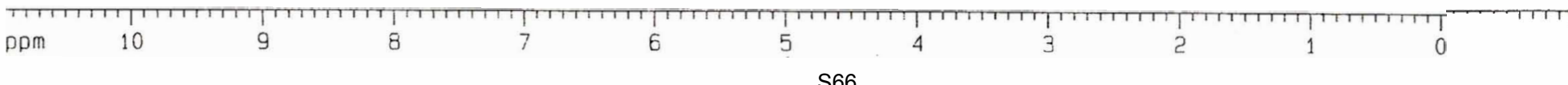



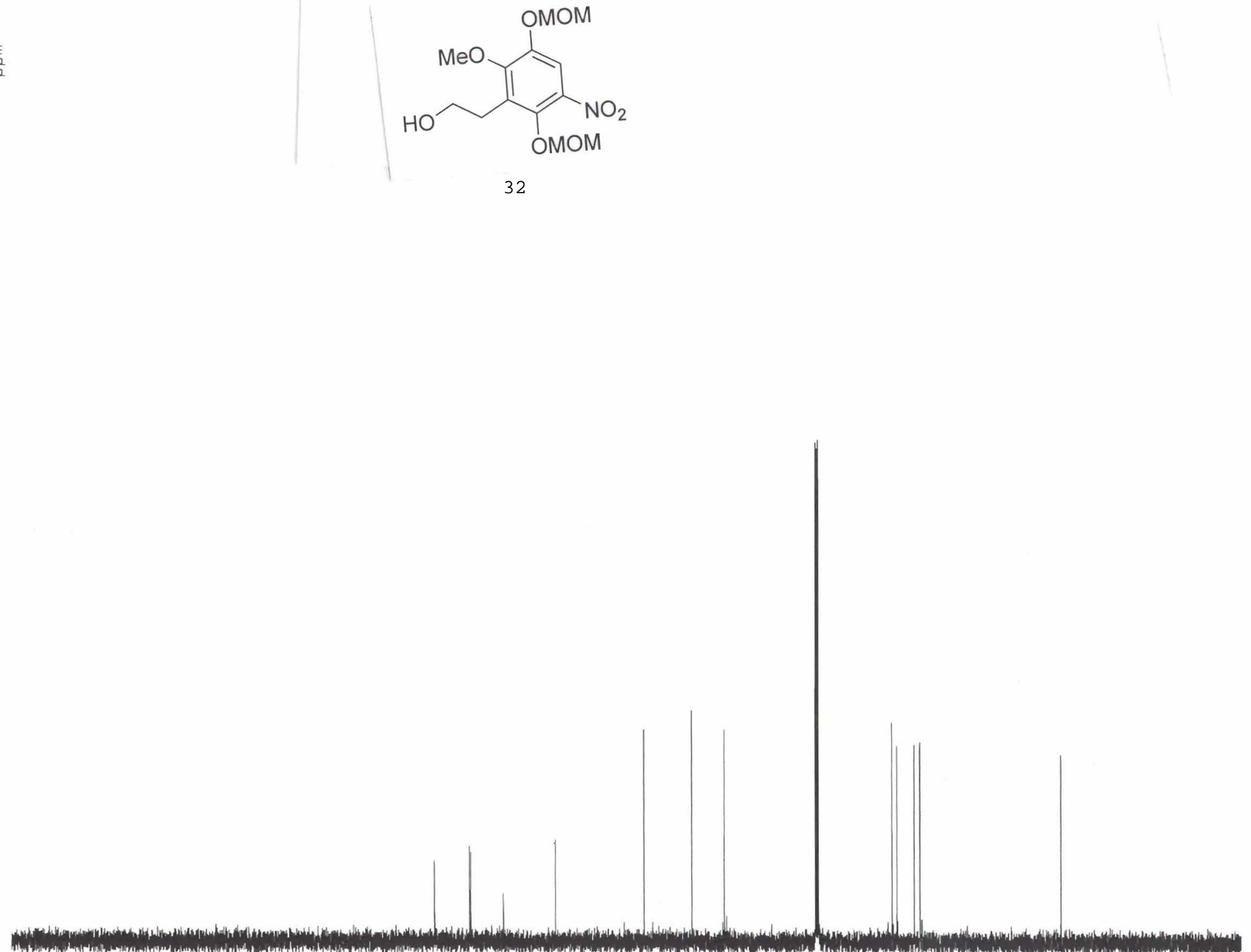


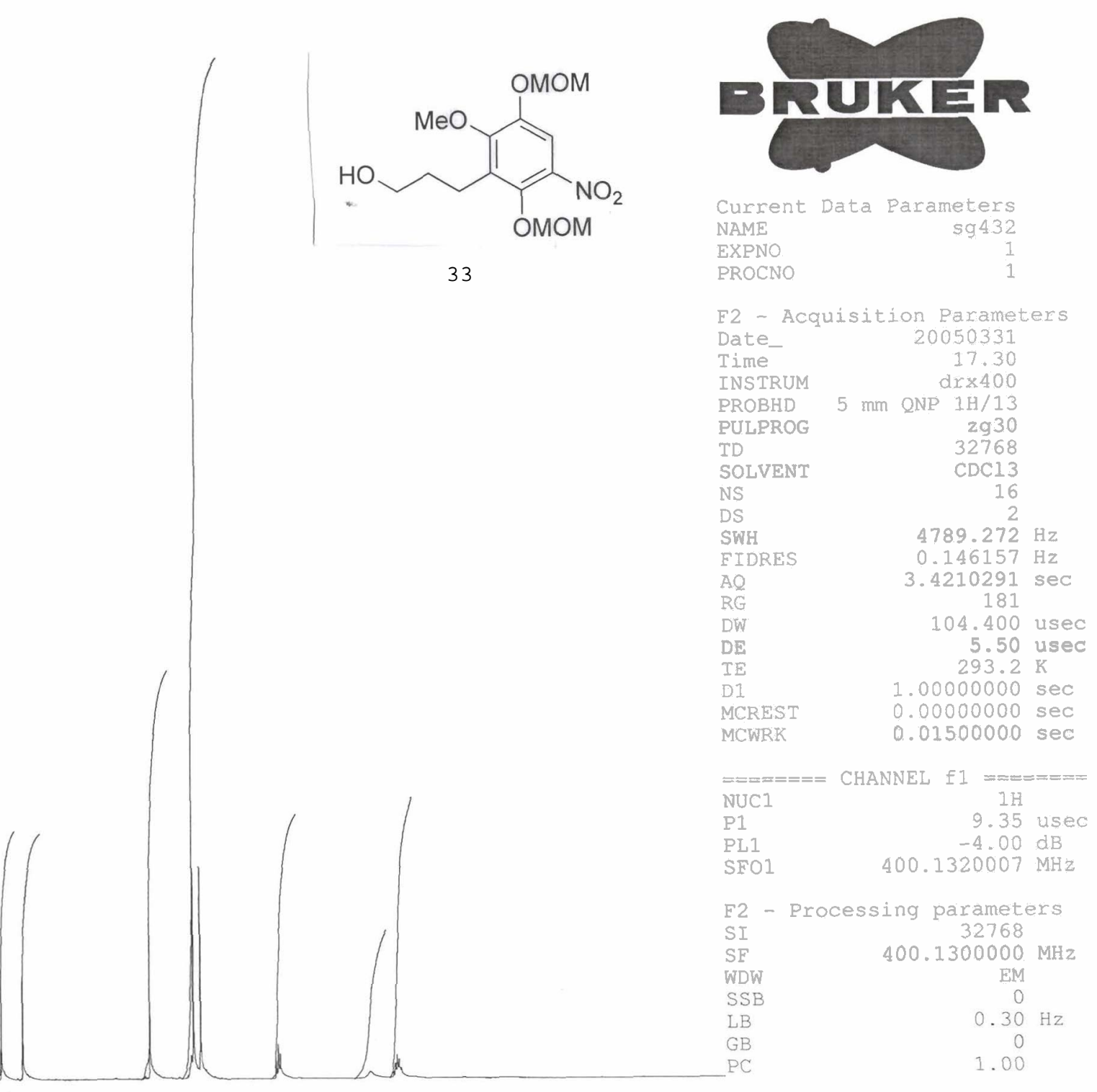

$\begin{array}{ll}9 & 8\end{array}$

$8 \quad 7$

6

$\frac{5}{4}$

$\left|\begin{array}{c}4 \\ \multirow{2}{*}{} \\ \text { ก่ }\end{array}\right|$




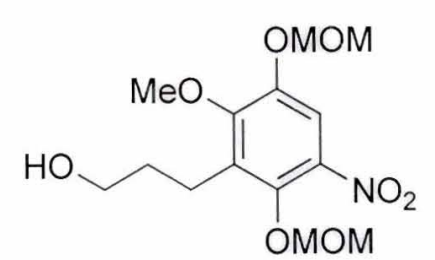

33

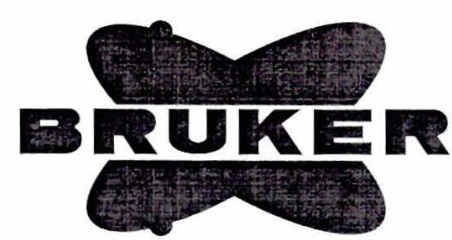

Curnent Data Parameters NAME

EXPNO

PROCNO

F2 - Acquisition Parameters Date

Date_

Time

INSTRUM

PROBHD
PULPROG

$T D$

SOLVENT

NS

DS

SWH

AQ

RG

$R G$
$D W$
$D E$

DE

D1 1

DELTA

MCRESI

MCWRK

$== \pm=$
NUC1

P1

PL1
SFO1

$== \pm====$

CPDPRG

NUC2 2

PCPD2

PL2

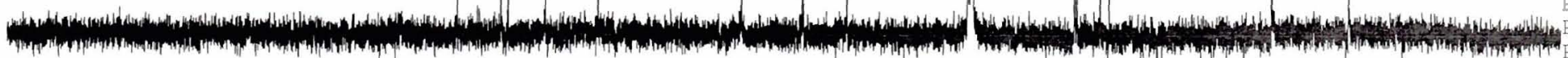

$5 \min$ QNP $1 \mathrm{H} / 13$ $1 \mathrm{H} / 13$
$\mathrm{zgpg} 30$

65536

$\mathrm{CDCl} 3$

794

$23148.148 \mathrm{~Hz}$

$0.353213 \mathrm{~Hz}$

$1.4156276 \mathrm{sec}$ 32768

21.600 usec

5.50 usec 5.50 K
$293.2 \mathrm{~K}$

$0.15000001 \mathrm{sec}$

0.15000001 sec

$0.03000000 \mathrm{sec}$

$0.05000000 \mathrm{sec}$

$0.00000000 \mathrm{sec}$

$0.01500000 \mathrm{sec}$

$=$ CHANNEL $\mathrm{f}$

$$
\begin{array}{r}
13 \mathrm{C} \\
11.25 \mathrm{usec} \\
2.00 \mathrm{~dB}
\end{array}
$$

$100.6232933 \mathrm{MHz}$

CHANNEL $f 2$ $12========$ waltz 16

$1 \mathrm{H}$

100.00 used

$-4.00 \mathrm{~dB}$

$16.58 \mathrm{~dB}$
$17.00 \mathrm{~dB}$

$400.1316005 \mathrm{MHz}$

F2 - Processing parameters 


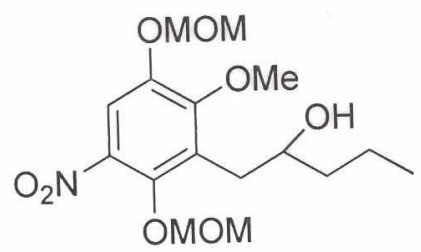

35

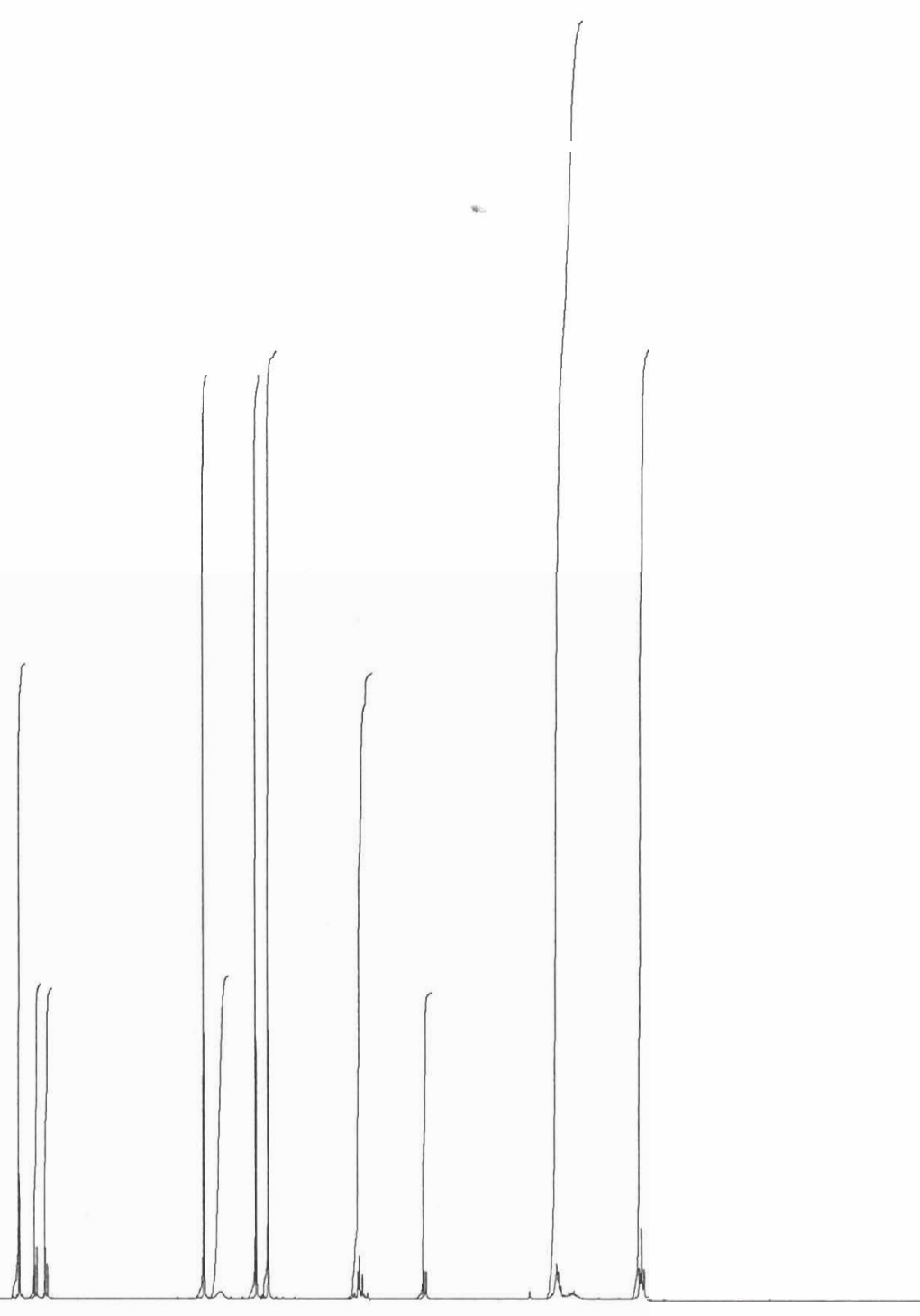

ERUKस: .

Current Data Parameters

NAME

EXPNO

PROCNO

F2 - Acquisition Parameters

Date 20050408

Iime

INSTRUM

PROBHD

PUILPROG

16.48

$\mathrm{TD}$

SOLVENT

NS

DS

EIDRES

AQ

RG

DE

TE

MCREST"

MCWRK

$5 \mathrm{~mm}$ QNP $1 \mathrm{H} / 13$

$1 \mathrm{H} / 13$
$2 \mathrm{~g} 30$

32768

CDCl3

16
2

$4789.272 \mathrm{~Hz}$

$0.146157 \mathrm{~Hz}$

$3.4210291 \mathrm{sec}$ 101.6

104.400 used 5.50 used $293.2 \mathrm{~K}$

1. $00000000 \mathrm{sec}$ $0.00000000 \mathrm{sec}$ $0.01500000 \mathrm{sec}$

$==:=$
NUC1

PI

PL1

SFO1

CHANNEL E1

$$
\begin{aligned}
& 1 \mathrm{H} \\
& 9.35 \text { usec } \\
& -4.00 \mathrm{~dB}
\end{aligned}
$$

$400.1320007 \mathrm{MHz}$

2 - Processing parameters 32768

$400,1300000 \mathrm{MHz}$

EM

WDW

SSB

LB

$\mathrm{PC}$

2

1

0

ppm 


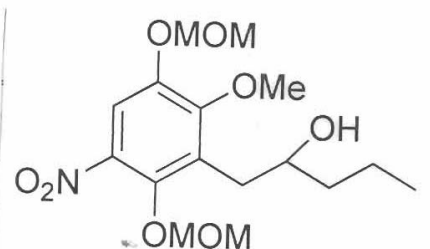

35
BRURER

Current Data Parameters NAME

XPNO

PROCNO

22 - Acquisition Parameters

Date_ $\quad 20050408$

INSTRUM

PROBHD

PULPROG

TD

SOLVENT

S

SWH

FIDRES

AQ

DW

$\mathrm{DE}$

d11

DELTA

MCREST

MCWRK

NUC

PL1

SFO1

CPDPRG2

NUC2

PCPD2

PL2

PL12

PL13

$\mathrm{SFO} 2$

16.57

$5 \mathrm{~mm}$ QNP $1 \mathrm{H} / 13$

zgpg 30

$\mathrm{CDCl} 3$

338

$23148.148 \mathrm{~Hz}$

$0.353213 \mathrm{~Hz}$

1. $4.156276 \mathrm{sec}$ 32768 $293.2 \mathrm{~K}$

$0.15000001 \mathrm{sec}$

$0.03000000 \mathrm{sec}$

$0.05000000 \mathrm{sec}$

$0.00000000 \mathrm{sec}$

$=$ CHANNEL

130

$2.00 \mathrm{~dB}$
21. 600 usec

5.50 usec

$0.01500000 \mathrm{sec}$

11.25 usec

$100.6232933 \mathrm{MHz}$
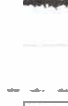

Processing parameters

180

160

140

120

100

80

60

40

$20 \quad \operatorname{ppm}_{\mathrm{WDW}}^{\mathrm{SE}}$

$$
\begin{aligned}
& \mathrm{f} 2======= \\
& \text { waltz } 16 \\
& 1 \mathrm{H} \\
& 100.00 \mathrm{usec} \\
& -4.00 \mathrm{~dB} \\
& 16.58 \mathrm{~dB} \\
& 17.00 \mathrm{~dB}
\end{aligned}
$$

$400.1316005 \mathrm{MHz}$ 65536 $100.6127290 \mathrm{MHz}$ EM 


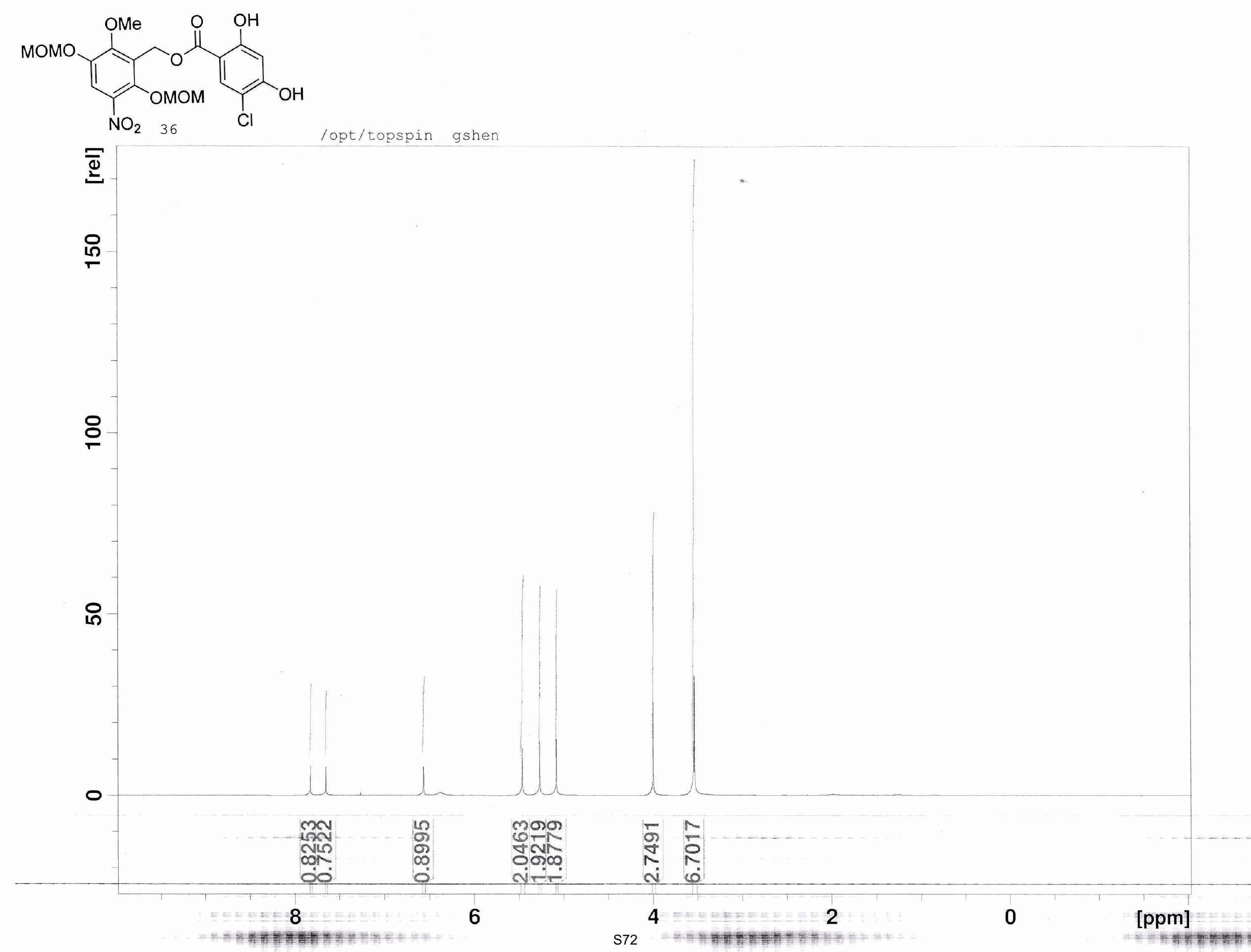



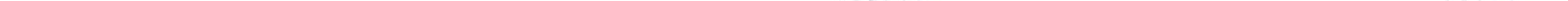

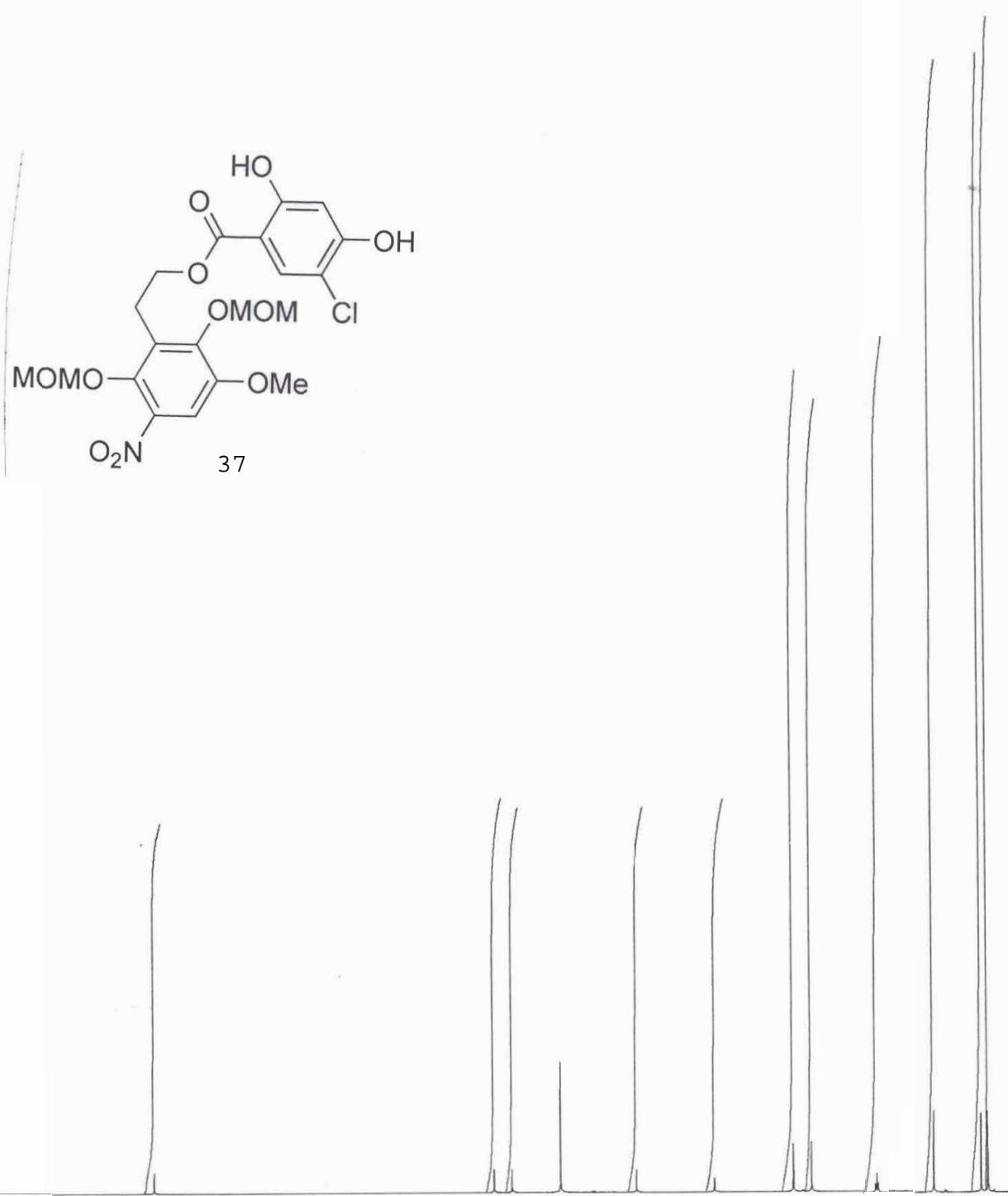

BRUKER

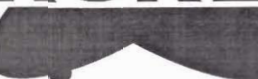

Current Data Parameters

NAME

EXPNO

PROCNO

E2 - Acquisition Parameters

Date_ 20031208

$\begin{array}{ll}\text { Time } & 15.46 \\ \text { spect }\end{array}$

PROBHD $5 \mathrm{~mm}$ BBO BB-1H

PULPROG

SOLVENT

NS

DWH

SWH

AQ

RG

DW

$\mathrm{DE}$

D1

$$
\begin{array}{r}
2930 \\
32768 \\
\mathrm{CDCl}
\end{array}
$$

$7002.801 \mathrm{~Hz}$

$0.213709 \mathrm{~Hz}$

$2.3397565 \mathrm{sec}$

71.400 usec 6.00 usec $300.0 \mathrm{~K}$

$===z====$ CHANNEL $\mathrm{f} 1===$

NUC1

$\mathrm{P} 1$

$\mathrm{PL} 1$
$\mathrm{SFO1}$

$1 \mathrm{H}$
7.70 usec
$4.00 \mathrm{~dB}$

E1 - Acquisition parameters NDO

TD

SEO1

EIDRES

$\mathrm{SW}$

EnMODE

256

$500.1325 \mathrm{MH}$

$23.475060 \mathrm{~Hz}$

undefined

E2 - Processing parameters

SI 32768

SE $\quad 500.1300000 \mathrm{MH}$

WDW

$\stackrel{L B}{G B}$

EM

0
0.30

0
1.00

F1 - Prccessing parameters 13 12

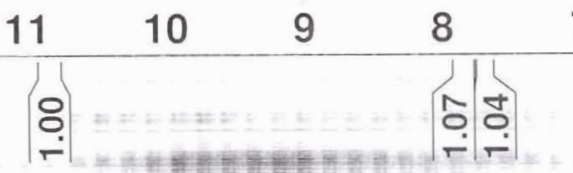
7 6 5 4 3 $\left|\begin{array}{l}\text { 苟 } \\ 0\end{array}\right|$ 2 1 ppm ${ }^{\text {MI }}$ 1024 S74 

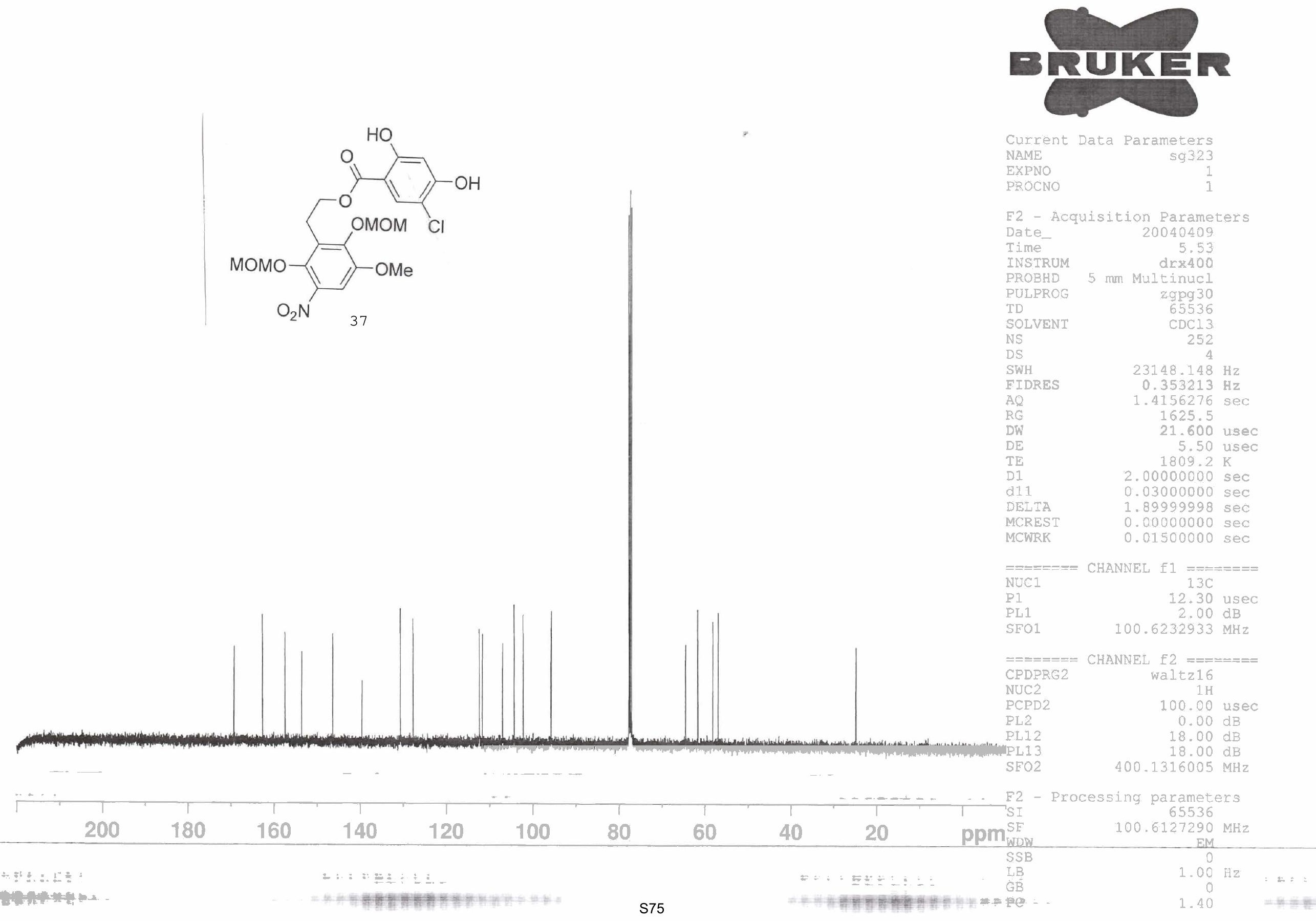


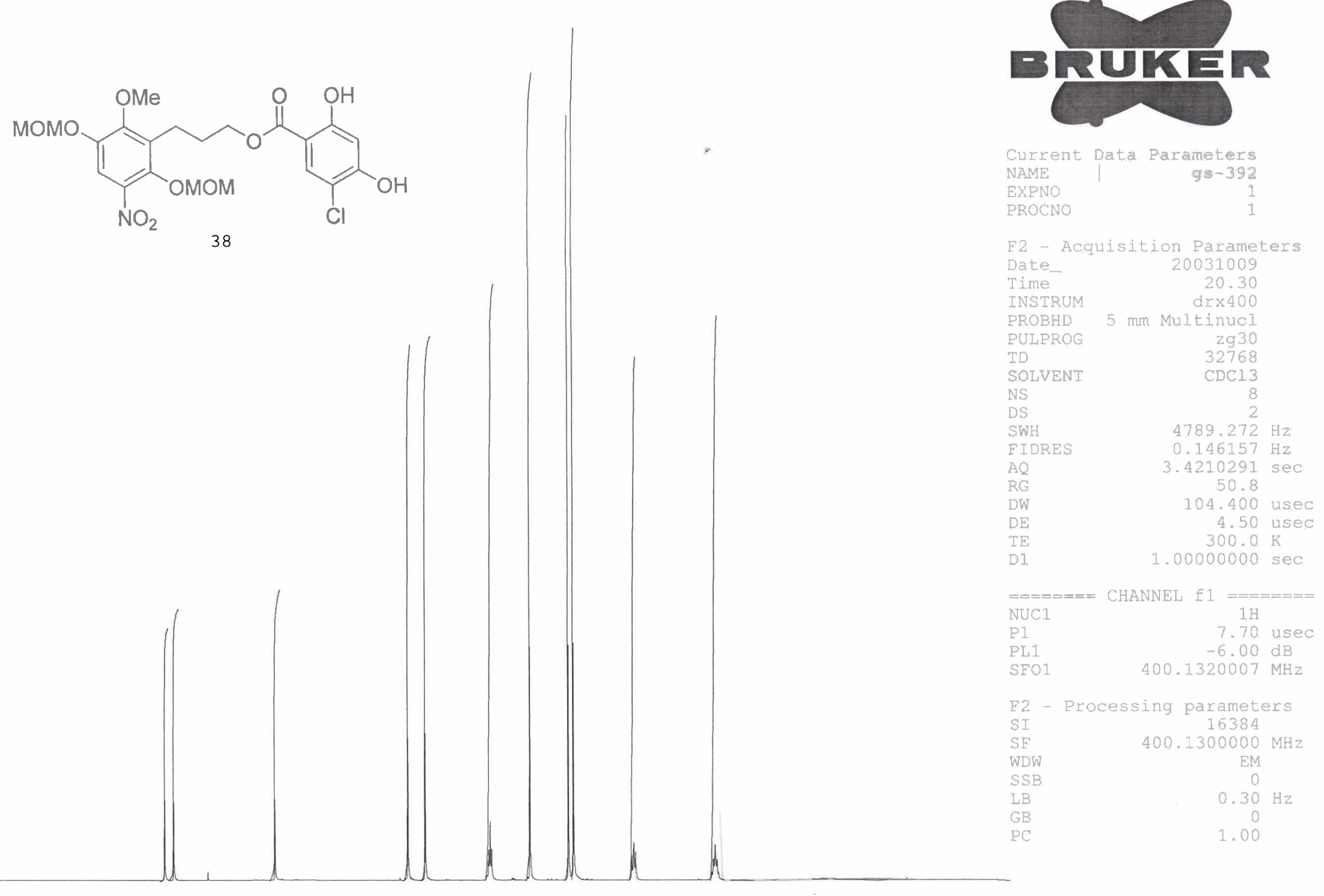

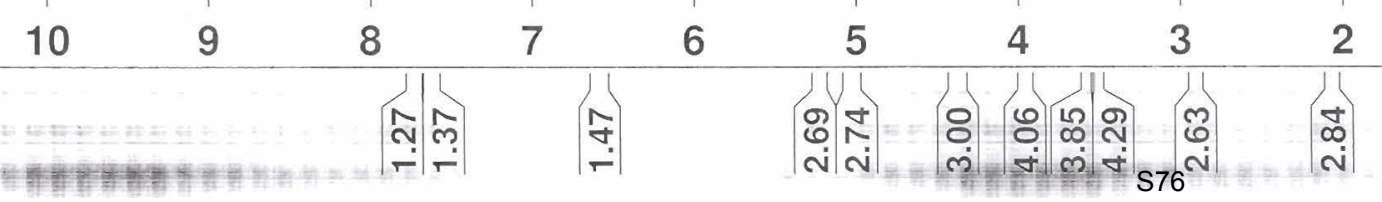




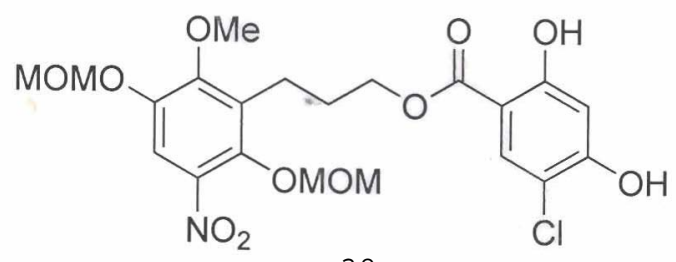

GRUKER

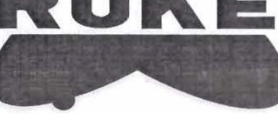

Current Data Barmeters NAME

EXPNO

PROCNO s $9-95$

22 - Acquisition Parameters Date $\quad 20031009$

$\begin{array}{lr}\text { Time } & 20.34\end{array}$

INSTRUM drx400

PROBHD 5 fum Multinucl

PULPROG

zgpg 30

TD

SOLVENT

nis

DS

SWH

EIDRES

AQ

RG

DE

IE

D1

$\mathrm{CDCl} 3$

367

$23148.148 \mathrm{~Hz}$

$0.353213 \mathrm{~Hz}$

$1.4156276 \mathrm{sec}$ 645.1

21.600 usec

4.50 usec $300.0 \mathrm{~K}$

$0.05000000 \mathrm{sec}$

$0.03000000 \mathrm{sec}$

$0.00002000 \mathrm{sec}$

NUC?

CHANNEL $\mathrm{f}$

130

12.30 .

$2.00 \mathrm{~dB}$

PL1

$100.6232933 \mathrm{MHz}$

$=====$

CHANNEL $f 2========$

CPDPRG

PCPD 2

PL2

PL12

PI 13

SEO2

waltz 16

10.0 .00 usec

$0.00 \mathrm{~dB}$

$18.00 \mathrm{~dB}$

$18.00 \mathrm{~dB}$

$400.1316005 \mathrm{MHz}$

22 - Processing parameters F.... 32768 
<smiles>CCC(Cc1c(OC)c(OC)cc([N+](=O)[O-])c1OC)OC(=O)c1cc(Cl)c(O)cc1O</smiles>

40

10 9 8 7 6 5 4 3

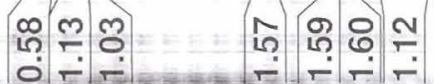

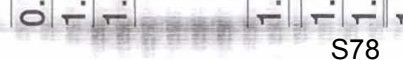

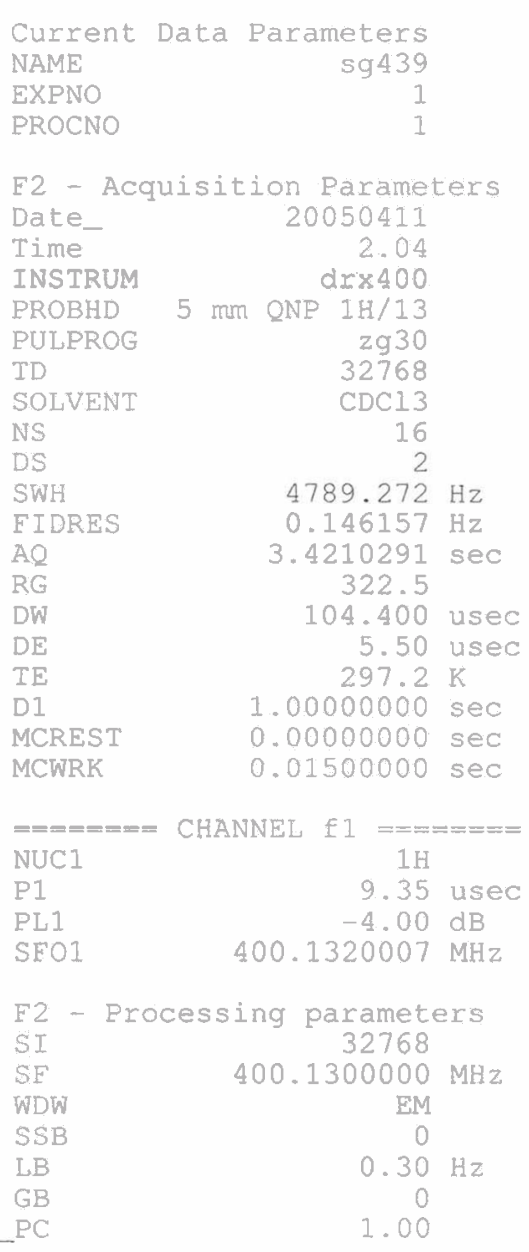

-3 Qure (a)

rura $+\sqrt{2+2}$ 


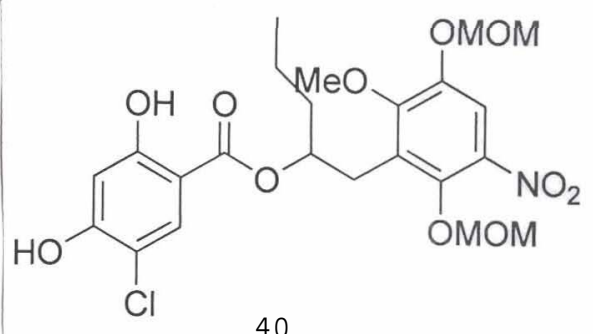

40

$$
\mid
$$

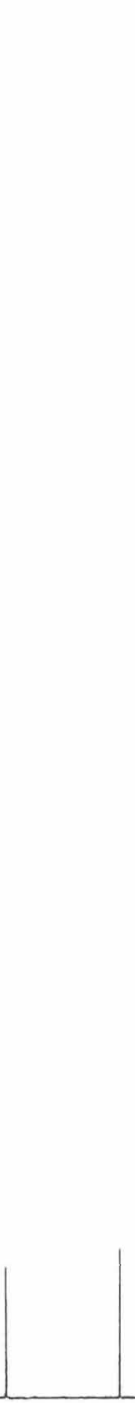

- RUKER C

Current Data Parameters

NAME

EXPNO

PROCNO

F2 - Acquisition Parameters

Date_ 20050413

Time

1.41

INSIRUM

PROBHD

5 inm CPDUL 13C

PULPROG

zgpg 30

TD

SOLVENT

NS

DS

SWH

FIDRES

AQ

RG

DW

TE

D1.

d11

DELTA.

MCREST

MCWRK

$=== \pm$
NUCI

$\mathrm{Pl}$

PL1

SFO1

65536

$\mathrm{CDCl} 3$

3072
4

$31446.541 \mathrm{~Hz}$

$0.479836 \mathrm{~Hz}$

$1.0420883 \mathrm{sec}$

$$
8192
$$

15.900 usec

6.00 usec $300.0 \mathrm{~K}$

$0.15000001 \mathrm{sec}$

$0.03000000 \mathrm{sec}$

$0.05000000 \mathrm{sec}$

$0.00000000 \mathrm{sec}$

$0.01500000 \mathrm{sec}$
CHANNEL fl $=======$ $13 \mathrm{C}$
10.00 usec $-4.90 \mathrm{~dB}$
$125.7697360 \mathrm{MHz}$

CHANNEL f2 $=========0$

CPDPRG2

NUC2

PCPD2

PL2

PL12

PL13

$\mathrm{SEO} 2$

wa1

altz16

80.00 usec

$-5.00 \mathrm{~dB}$

9. $54 \mathrm{~dB}$

500.1325000 MHz

- Processing parameters 65536 $\frac{S E}{S S B}$ $125.7577890 \mathrm{MHz}$ 


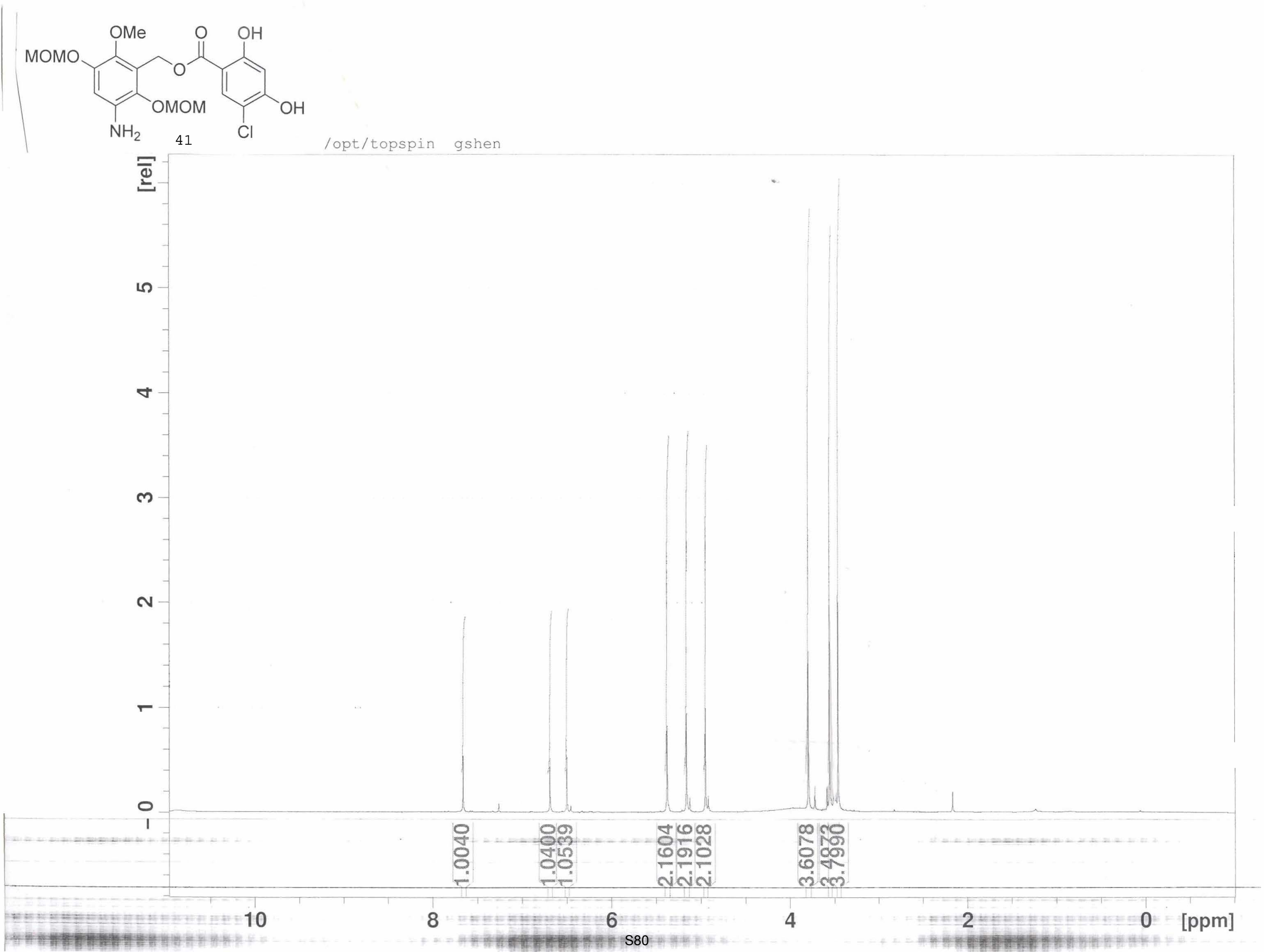



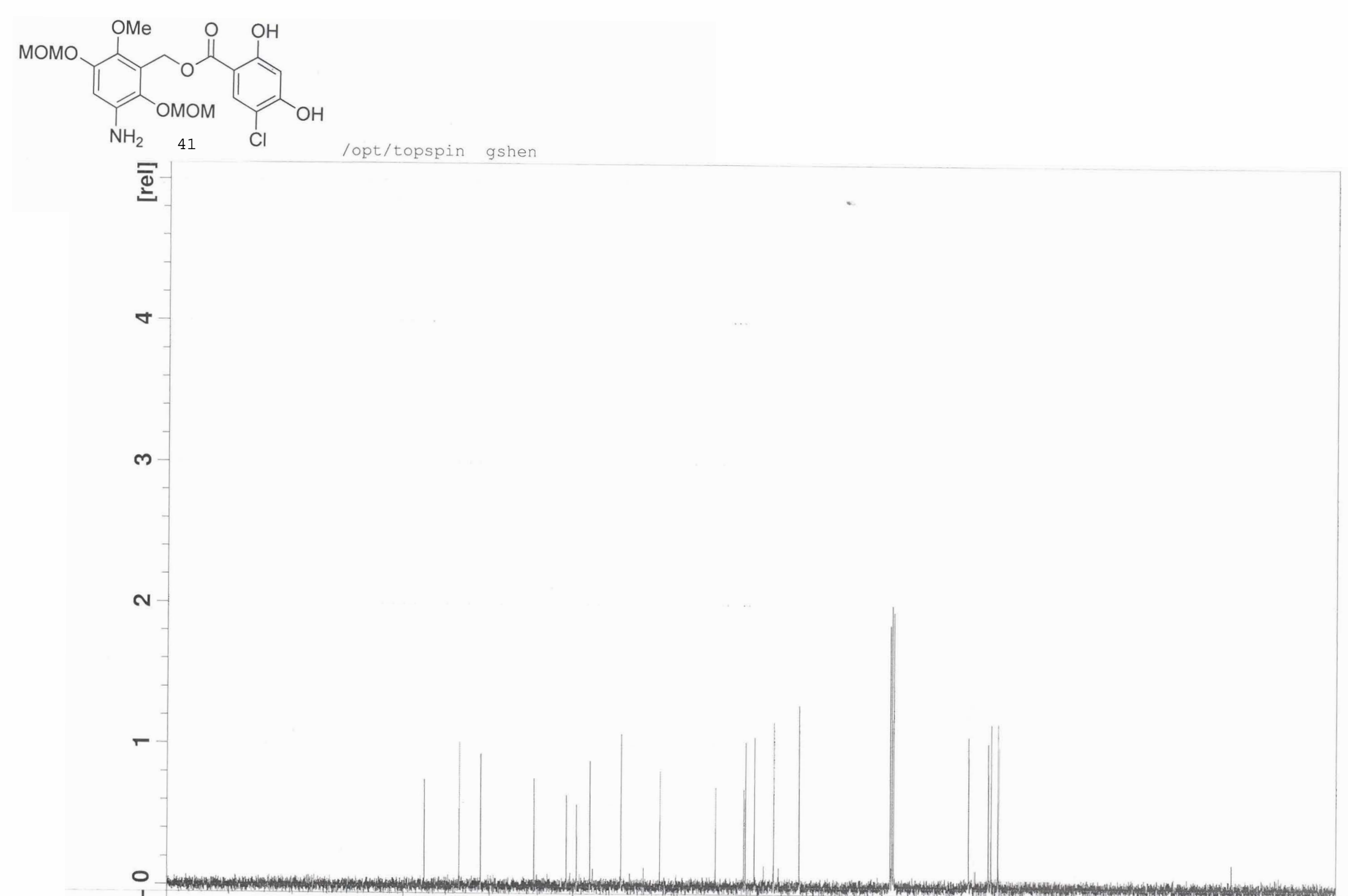

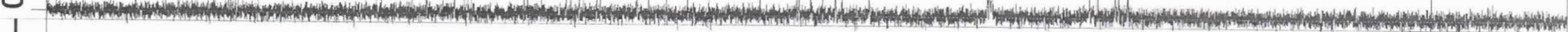



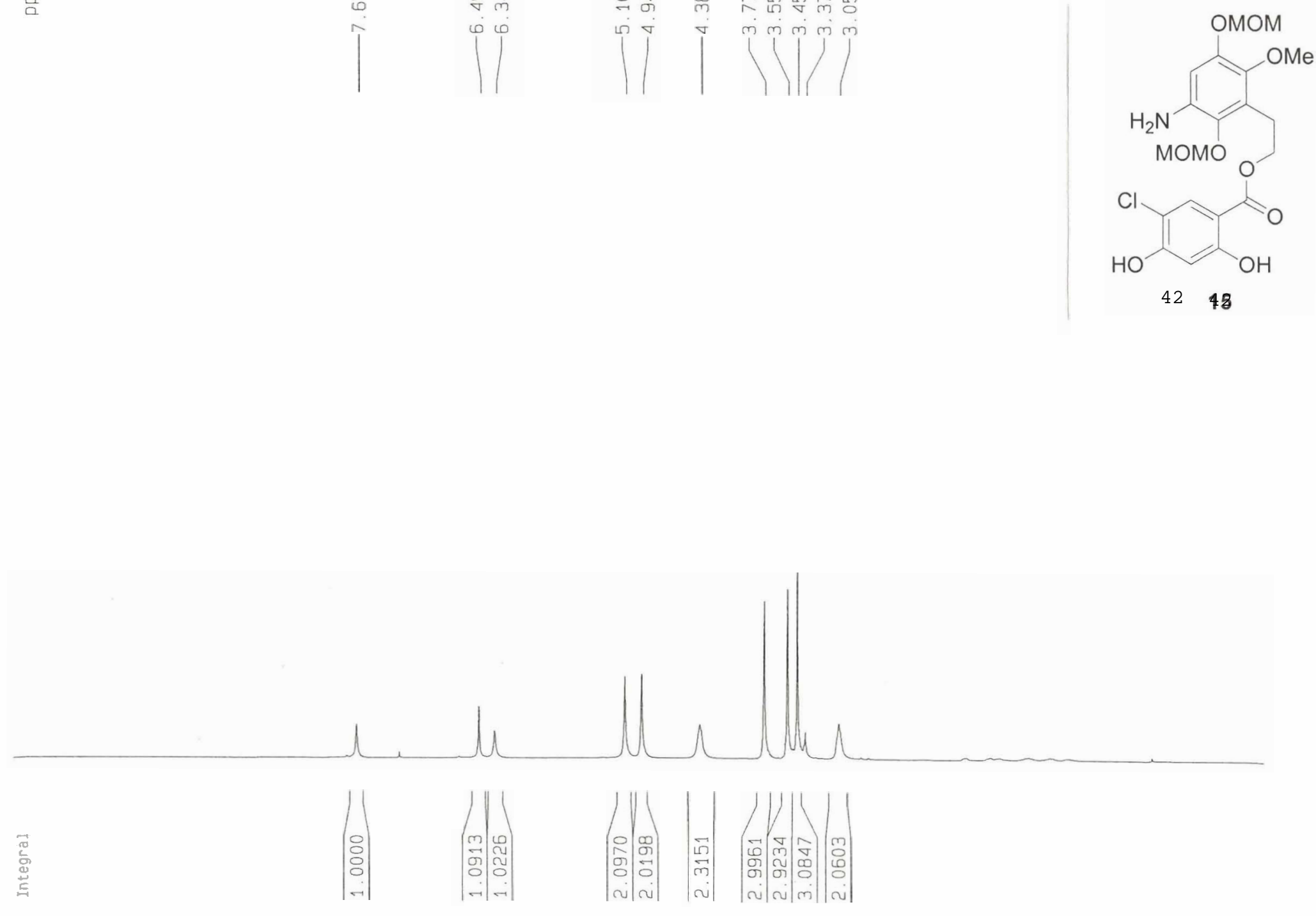

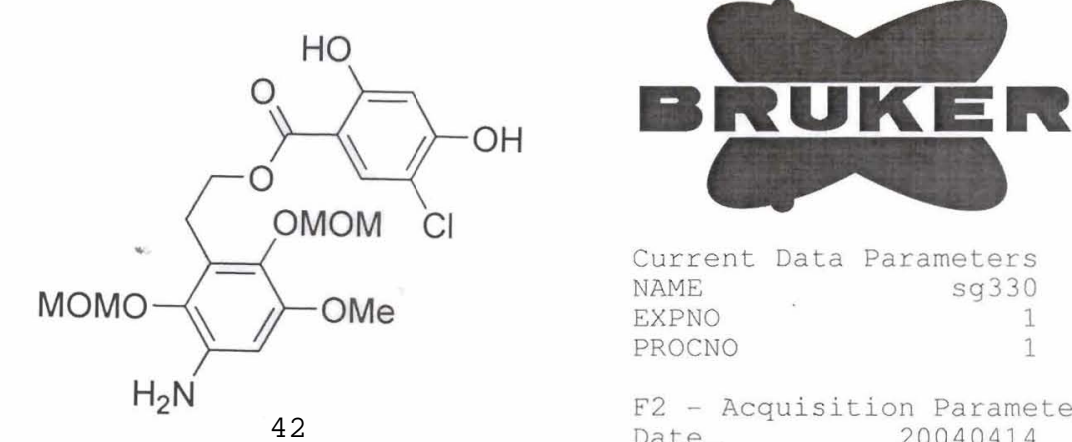

Current Data Parameters NAME

EXPNO

PROCNO

F2 - Acquisition Parameters Date_ 20040414
Time
6.08

INSTRUM

PROBHD $5 \mathrm{~mm}$ Multinucl

dr $\times 400$

PULPROG

TD

SOLVENT

NS

zgpg 30

65536

DS

FIDRES

$A Q$

RG

DW

DE

TE

d11

DELTA

MCREST

MCWRK

$$
2517
$$

$23148.148 \mathrm{~Hz}$

$0.353213 \mathrm{~Hz}$

$1.4156276 \mathrm{sec}$

512

21.600 usec

5.50 usec

$1809.2 \mathrm{~K}$

$2.00000000 \mathrm{sec}$

$0.03000000 \mathrm{sec}$

$1.89999998 \mathrm{sec}$

$0.00000000 \mathrm{sec}$

$0.01500000 \mathrm{sec}$

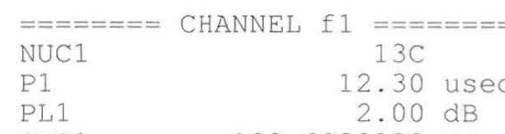

$100.6232933 \mathrm{MHz}$

$========$ CHANNEL f $2========$

CPDPRG2 waltz16

NUC2

PCPD2

PL2

PL12

$\mathrm{SFO} 2$

100.00 usec

$0.00 \mathrm{~dB}$

$18.00 \mathrm{~dB}$

$18.00 \mathrm{~dB}$

$400.1316005 \mathrm{MHz}$ 


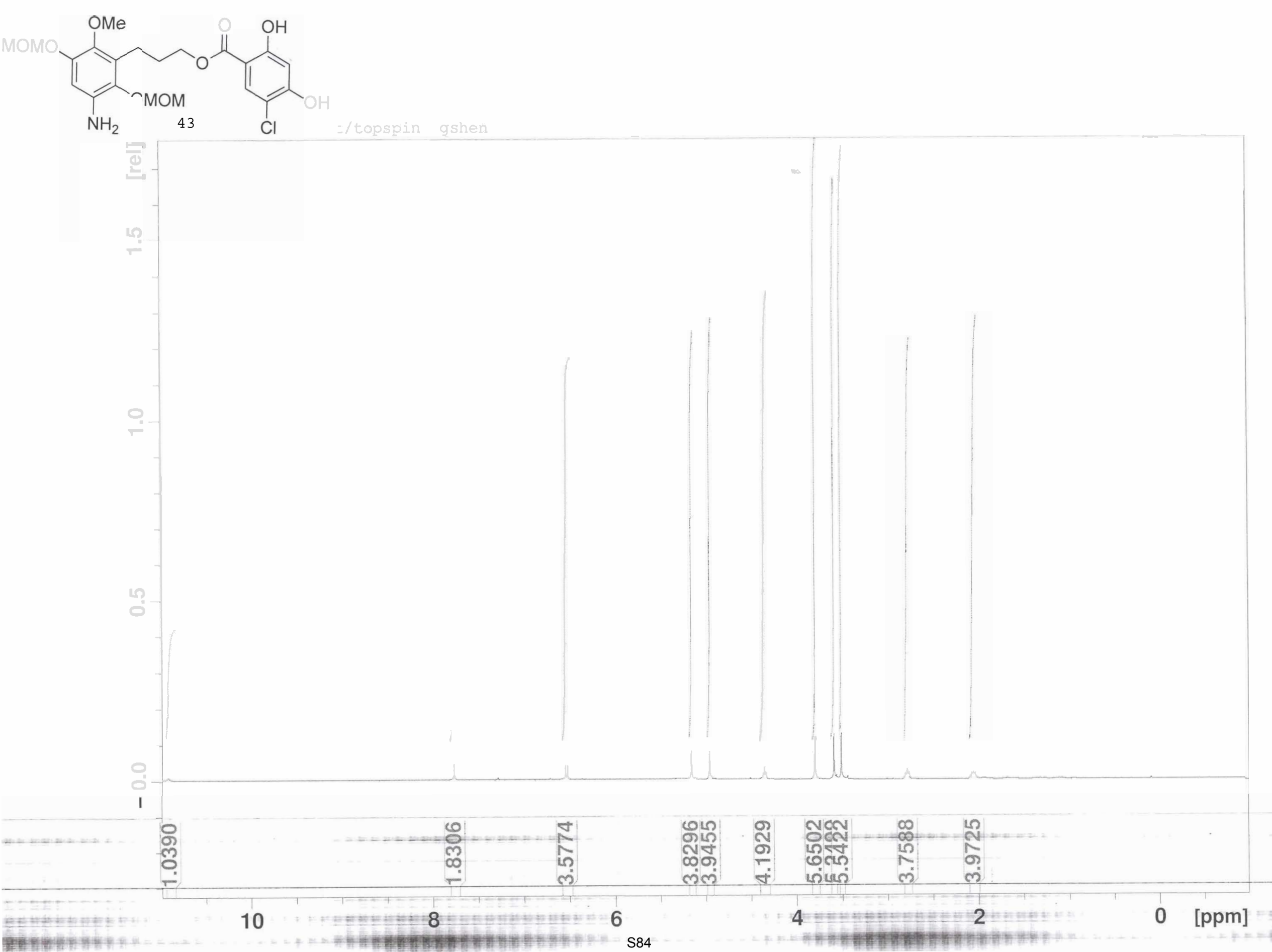


MOMO

可

요

음

으

0

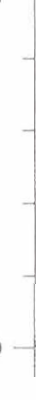



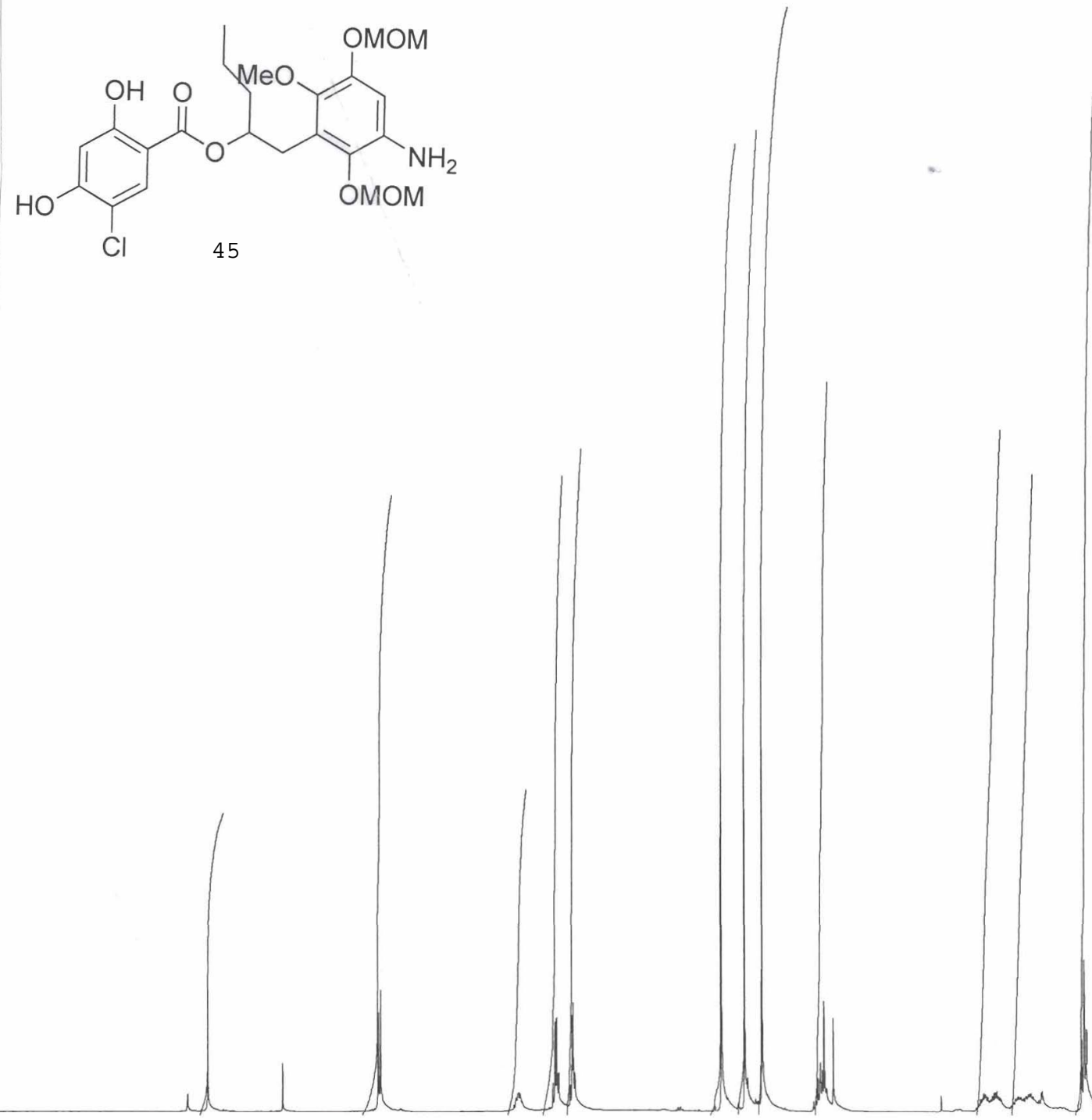

BRUKER

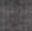

Current Data Parameters NAME

EXPNO

PROCNO

F2 - Acquisition Parameters

Date_ 20050412

Time

23.26

INSTRUM

PROBHD

$1+13$

PULPROG

SOLVENT

2930

EIDRES

RG

DW

DE

D1

MCREST

MCWRK

CDC13

16

$4789.272 \mathrm{~Hz}$

$0.146157 \mathrm{~Hz}$

$3.4210291 \mathrm{sec}$

128

104.400 usec

5.50 usec

$293.2 \mathrm{~K}$

$1.00000000 \mathrm{sec}$

$0.00000000 \mathrm{sec}$

$0.01500000 \mathrm{sec}$

$=======$ CHANNEL $f 1$

NUC1

PL1

SFO1

E1 $==$

9.35 usec

$-4.00 \mathrm{~dB}$

F2 - Processing parameters

32768

SF $\quad 400.1300000 \mathrm{MHz}$

WDW

SSB

LB

EM

EM
0
$30 \mathrm{~Hz}$

PC

$\begin{array}{lll}10 & 9 & 8\end{array}$

$8 \quad 7$

7

6

5

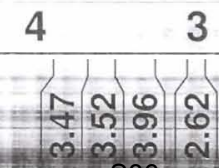

$3 \quad 2$

1

0 ppm 


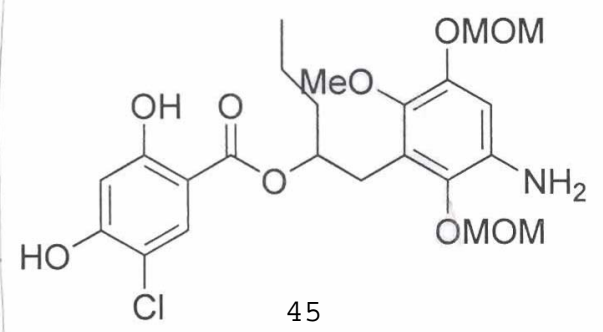
Current Data Parameters
NAME
EXPNO
PROCNO
$\operatorname{sg} 440$

F2 - Acquisition Parameters

Date 20050412

$\begin{array}{lr}\text { Time } & 23.28\end{array}$

INSTRUM $\mathrm{dr} \times 400$

PROBHD $5 \mathrm{~mm}$ QNP $1 \mathrm{H} / 13$

PULPROG

zgpg 30

TD

SOLVENT

NS

CDC13

DS

SWH

FIDRES

$A Q$

RG

DW

IE

D1

d11

DELTA

MCWRK

183
4

$23148.148 \mathrm{~Hz}$

$0.353213 \mathrm{~Hz}$

$1.4156276 \mathrm{sec}$ 32768

21.600 usec 5.50 usec $293.2 \mathrm{~K}$

$0.15000001 \mathrm{sec}$

$0.03000000 \mathrm{sec}$

$0.05000000 \mathrm{sec}$

$0.00000000 \mathrm{sec}$

$0.01500000 \mathrm{sec}$

NUC1

P1

PL1

SEO1

CPDPRG

NUC2

PCPD2

PL2

PL12

$\mathrm{SEO} 2$
$13 \mathrm{C}$
$13 \mathrm{C}$
$2.00 \mathrm{~dB}$

$100.6232933 \mathrm{MHz}$

$200 \quad 180 \quad 160$
$=:$

140

120

100

80

60

40

20

ppm

$2-\operatorname{Proc}$

SF $\quad 100.6127290$

$\begin{array}{lc}\text { WDW } & E M \\ \text { SSB } & 0 \\ \mathrm{LB}: \quad 1.00 \mathrm{~Hz} \\ \mathrm{~GB} & 0 \\ \mathrm{PC}=2 & 1.2\end{array}$



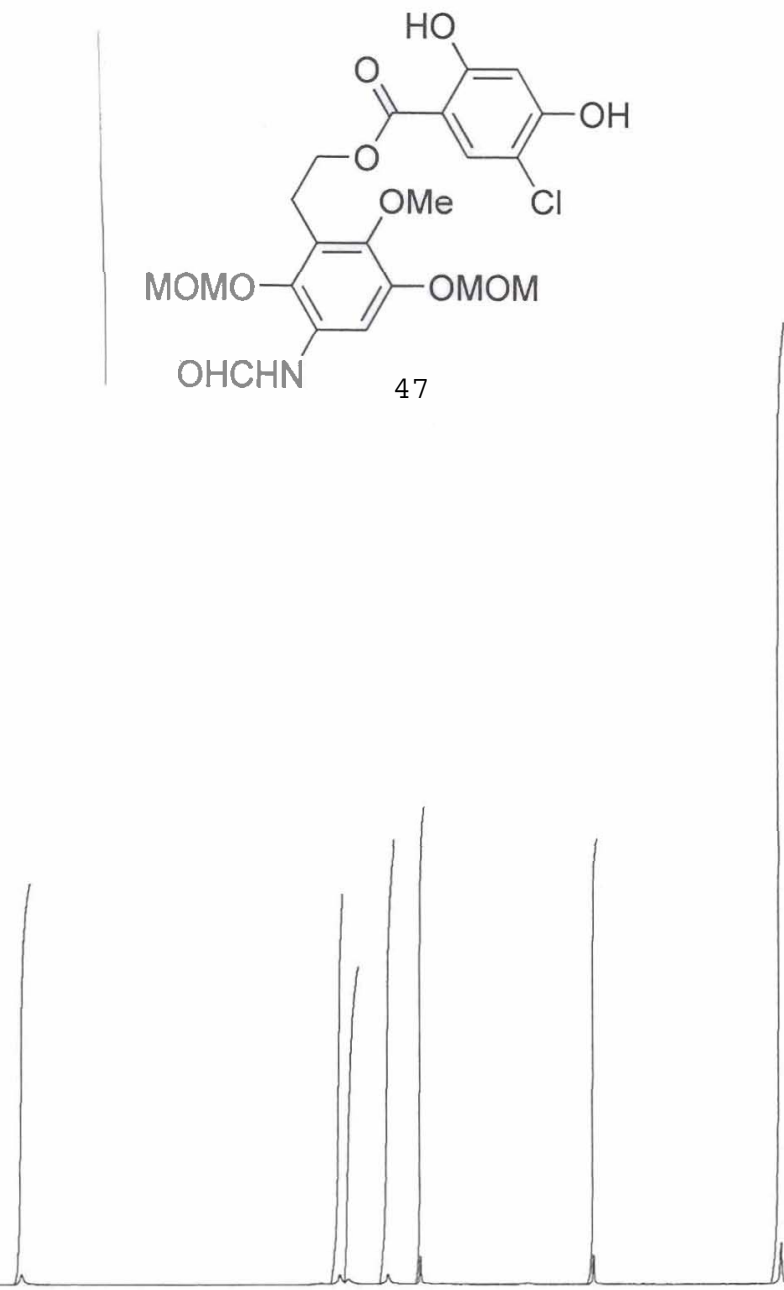

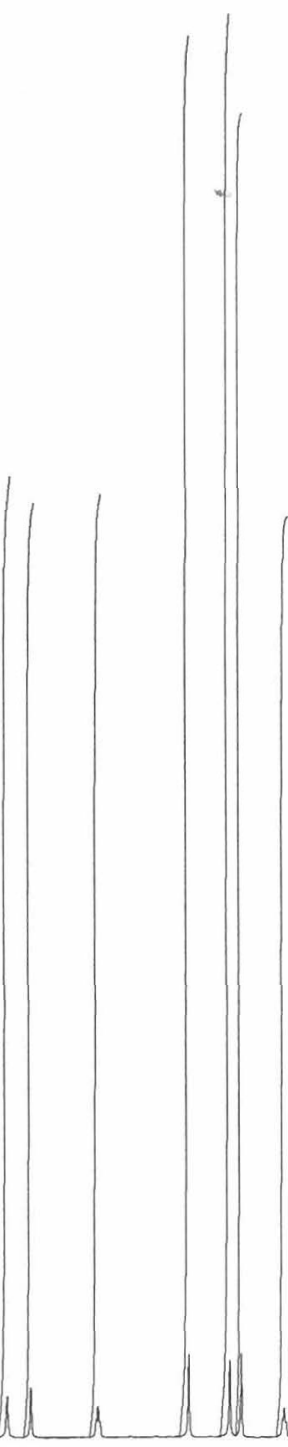

BRUKER

(

Current Data Parameters

NAME

EXPNO

PROCNO

F2 - Acquisition Parameters

Date__ 20031210

Time

8.00

$5 \mathrm{~mm} \mathrm{BBO} \mathrm{BB}-1 \mathrm{H}$

PULPROG

$\mathrm{BB}-1 \mathrm{H}$
2030
32768

TD

SOLVENT

NS

DS

SWH

AO

RG

DW

$\mathrm{DE}$

D1

$\mathrm{CD} 3 \mathrm{CN}$

$7002.801^{2} \mathrm{~Hz}$

$0.213709 \mathrm{~Hz}$

$2.3397565 \mathrm{sec}$ 574.7

71.400 usec

6.00 usec

$0.03000000 \mathrm{sec}$

$=======$ CHANNEL $f 1$

NUC1

P1

PL1

SEO1

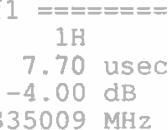

F1 - Acquisition parameters NDO

ID

SEO1

EIDRES

SW

EnMODE

$500,1325 \mathrm{MHz}$

$23.475060 \mathrm{~Hz}$

$12.016 \mathrm{ppm}$

undefined

2 - Processing parameter

SI 32768

SF $\quad 500.1300000 \mathrm{MHz}$

WDW

SSB

$B C$

$$
\begin{gathered}
E M \\
0 \\
0.30 \mathrm{~Hz} \\
0 \\
1.00
\end{gathered}
$$

8

7

6

5

4

3

2

$1 \operatorname{ppm}^{\mathrm{SI}}$

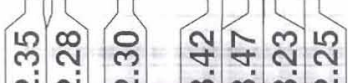
ง|ง ล 


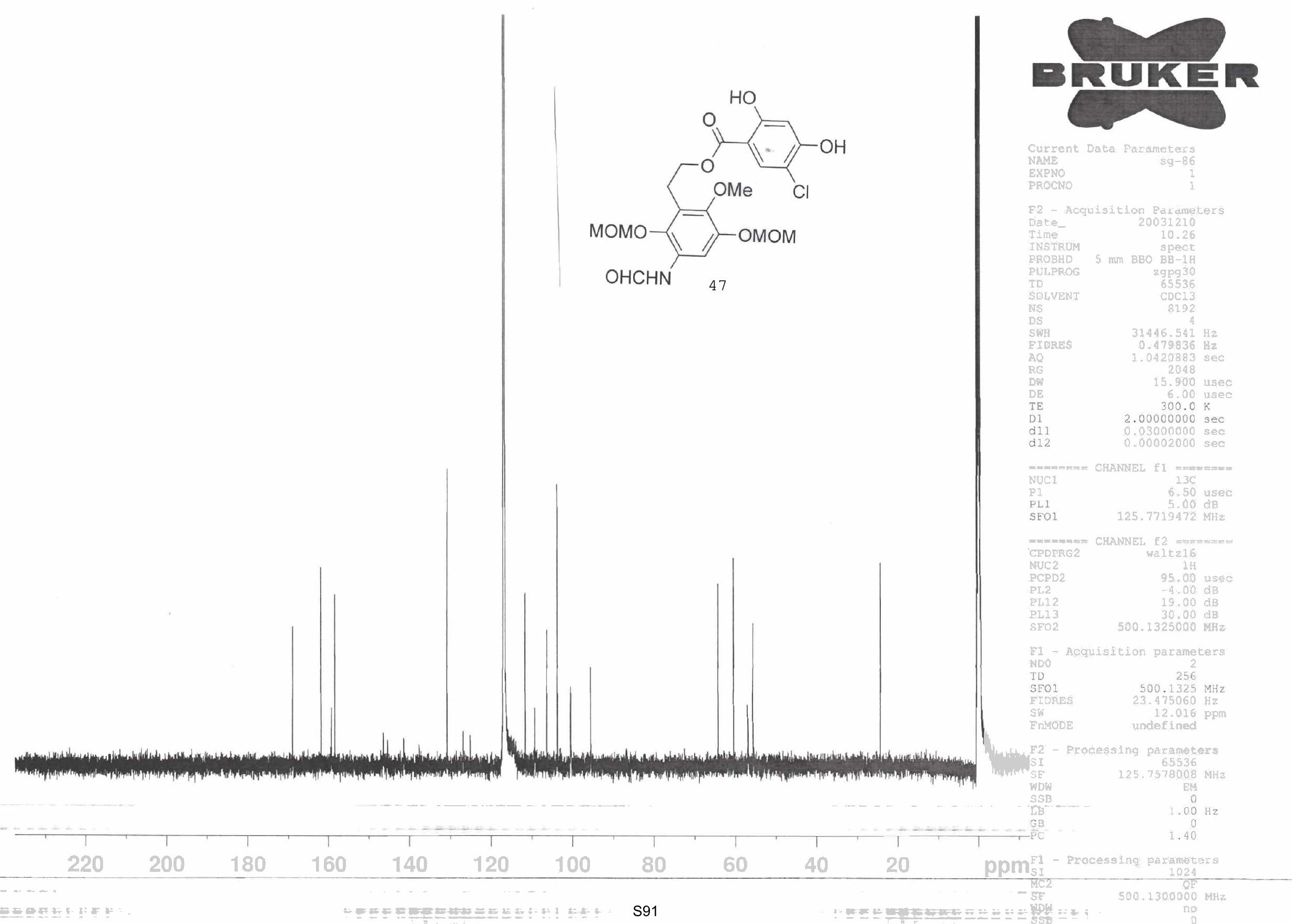




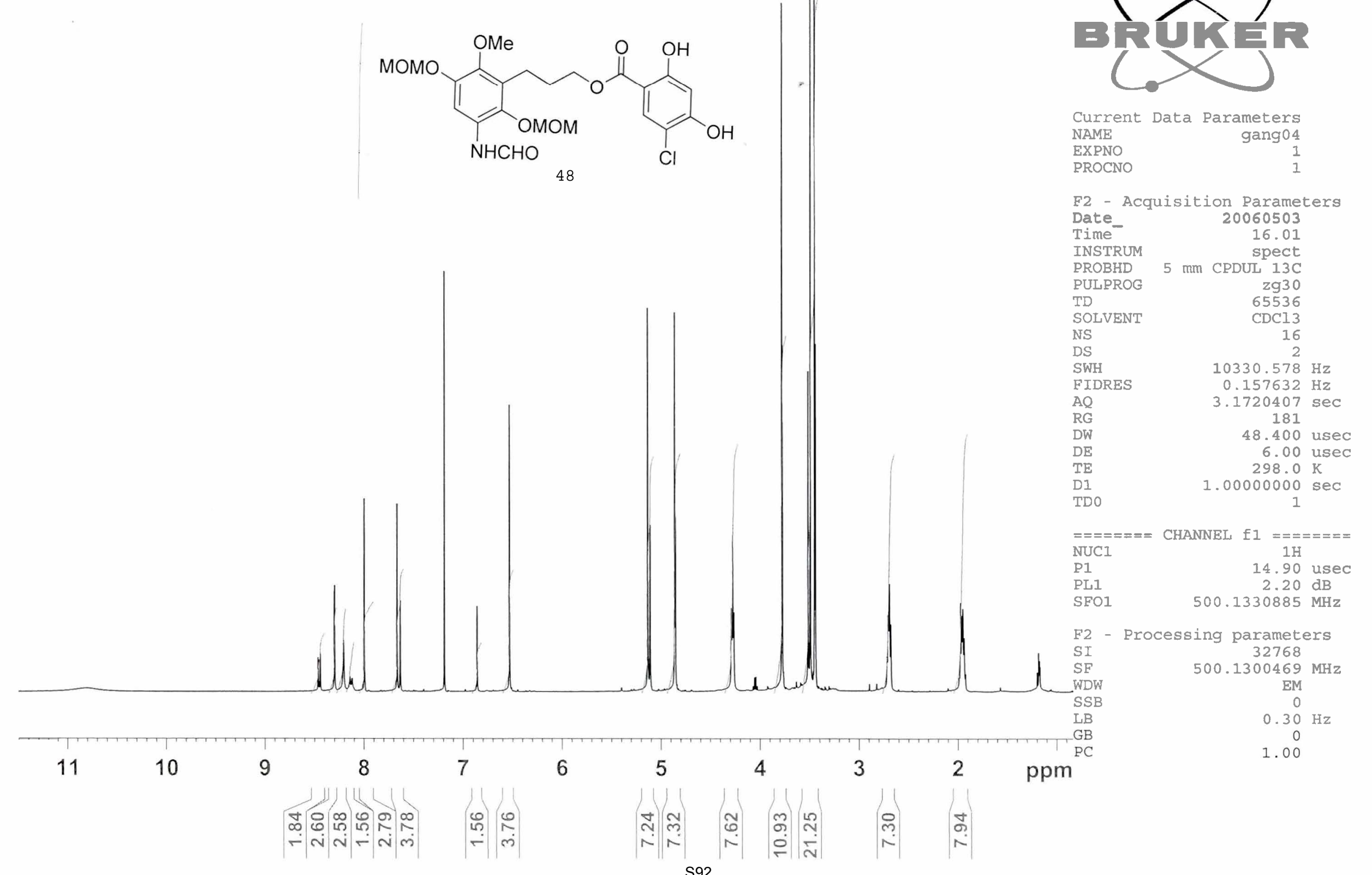




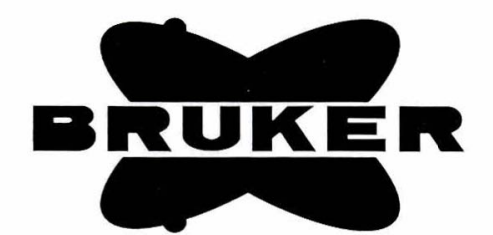

Current Data Parameters NAME

EXPNO

PROCNO

E2 - Acquisition Parameters

Date_ 20060503

$\begin{array}{lr}\text { Time } & 23.25\end{array}$

Time spect

PROBHD

PULPROG

TD

SOLVENT

NS

DS

SWH

AQ

DE

TE

D1

d11

DELTA

TDO

NUC

P1

P1

SFO1

$5 \mathrm{~mm}$ CPDUL $13 \mathrm{C}$

zgpg 30
65536

6513

1024

$30030.029 \mathrm{~Hz}$

$0.458222 \mathrm{~Hz}$

$1.0912410 \mathrm{sec}$

18390.4

16.650 usec

6.00 usec $298.0 \mathrm{~K}$

$0.15000001 \mathrm{sec}$

$0.03000000 \mathrm{sec}$

$0.05000000 \mathrm{sec}$

$$
1
$$
CHANNEL
$13 \mathrm{C}$
9.95 usec $3.80 \mathrm{~dB}$

$125.7703643 \mathrm{MHz}$

$===2===$ CHANNEI f2 $2=0$

CPDPRG2 waltz16

NUC2

PCPD2

PL 2

PL13

F2 - Processing parameters 


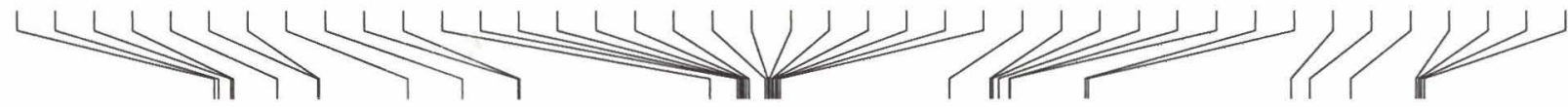<smiles>CCC(Cc1c(OC)c(NC(=O)O)cc(OC)c1OC)OC(=O)c1cc(Cl)c(O)cc1O</smiles>

50
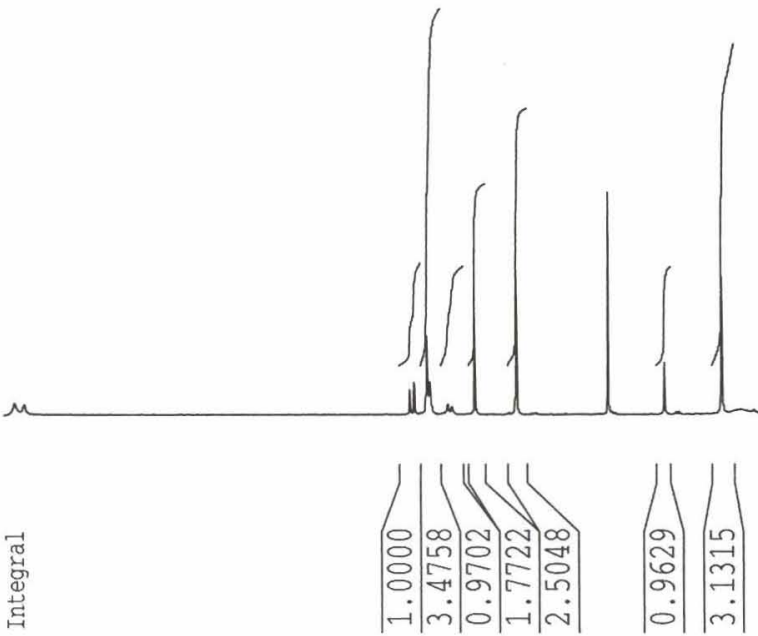
11.
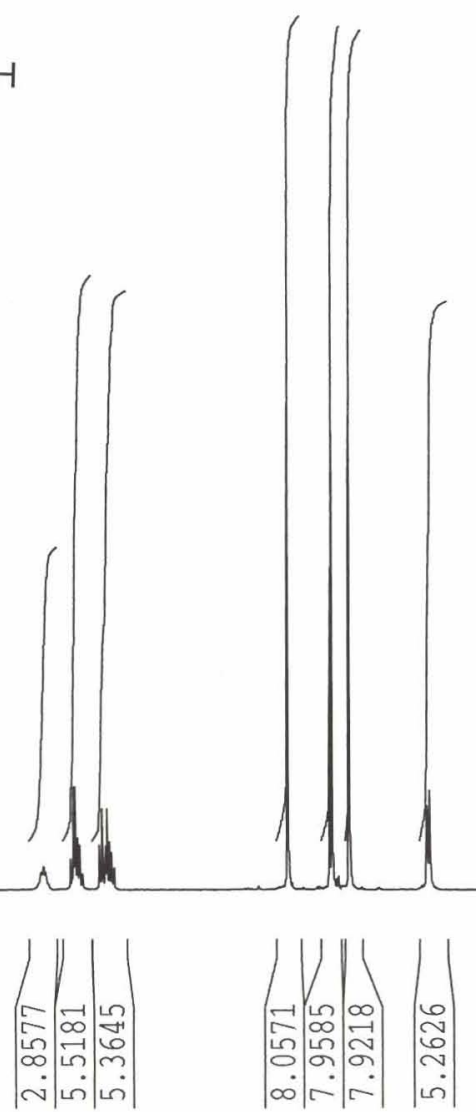

Current Data Parameters

EXENO

PROCNO

F2 - Acquisition Parameters

Date

Time $\quad 10.26$

INSTRUM drx400

PROBHD 5 m QNP 1H/13

PULPROG $\quad \mathrm{zg} 30$

32768

SOLVENT CDC13

NS

STDRS

AQ $\quad 3.4210291 \mathrm{sec}$

RG 228.1

DW $\quad 104.400$ usec

DE $\quad 5.50$ usec

TE $293.2 \mathrm{~K}$

D1 $\quad 1.00000000 \mathrm{sec}$

MCREST $\quad 0.00000000 \mathrm{sec}$

MCWRK $\quad 0.01500000 \mathrm{sec}$

$== \pm===$ CHANNEL f1 $== \pm====$ NUC1

P1 9.35 usec

PL1 $-4.00 \mathrm{~dB}$

SF01 $400.1320007 \mathrm{MHz}$

F2 - Processing parameters

SI $\quad 32768$

SF $\quad 400.1300000 \mathrm{MHz}$

WDW TM

SSB $\quad 0.30 \mathrm{~Hz}$

$\begin{array}{lr}\mathrm{LB} & 0 \\ \mathrm{~GB} & 0 \\ \mathrm{PC} & 1.00\end{array}$

1D MMR plot parameters

1D NMR plot parameters $\begin{array}{lr}\text { CX } & 20.00 \mathrm{~cm} \\ \text { CY } & 5.00 \mathrm{~cm}\end{array}$ F1P $\quad 11.000 \mathrm{ppm}$ F1 $4401.43 \mathrm{~Hz}$ F2P -1.000 pom F2 $240.07800 \mathrm{~Hz} / \mathrm{cm}$ 


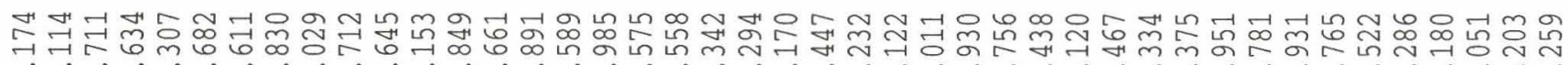

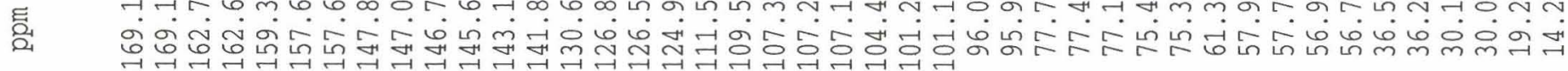

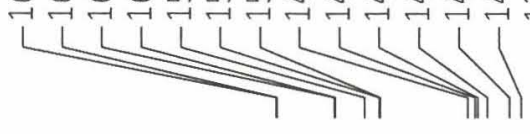<smiles>CCC(Cc1c(OC)c(NC(=O)O)cc(OC)c1OC)OC(=O)c1cc(Cl)c(O)cc1O</smiles>

50

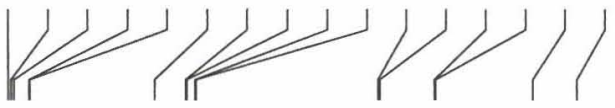

F2 - Accuisition Parameters
Date_ 20050414

$\begin{array}{lr}\text { Time } & 16.01 \\ \text { INSTRUM } & \text { drx400 }\end{array}$

PROBKDD $5 \mathrm{~mm}$ ONP $1 \mathrm{H} / 13$

PULPROG $\quad$ zgpg30

TD

SOLVENI

NS

$\begin{array}{ll}\text { DS } & 4 \\ \text { SWH } & 23148.148 \mathrm{~Hz}\end{array}$

FIDRRS $\quad \begin{aligned} & 23148.148 \mathrm{~Hz} \\ & 0.353213 \mathrm{~Hz}\end{aligned}$

$\mathrm{AQ} \quad 1.4156276 \mathrm{sec}$

DW

$\mathrm{DE}$
$\mathrm{TE}$
$\mathrm{D} 1$

DI

DELTA

\section{MELTA}

MCWRK 

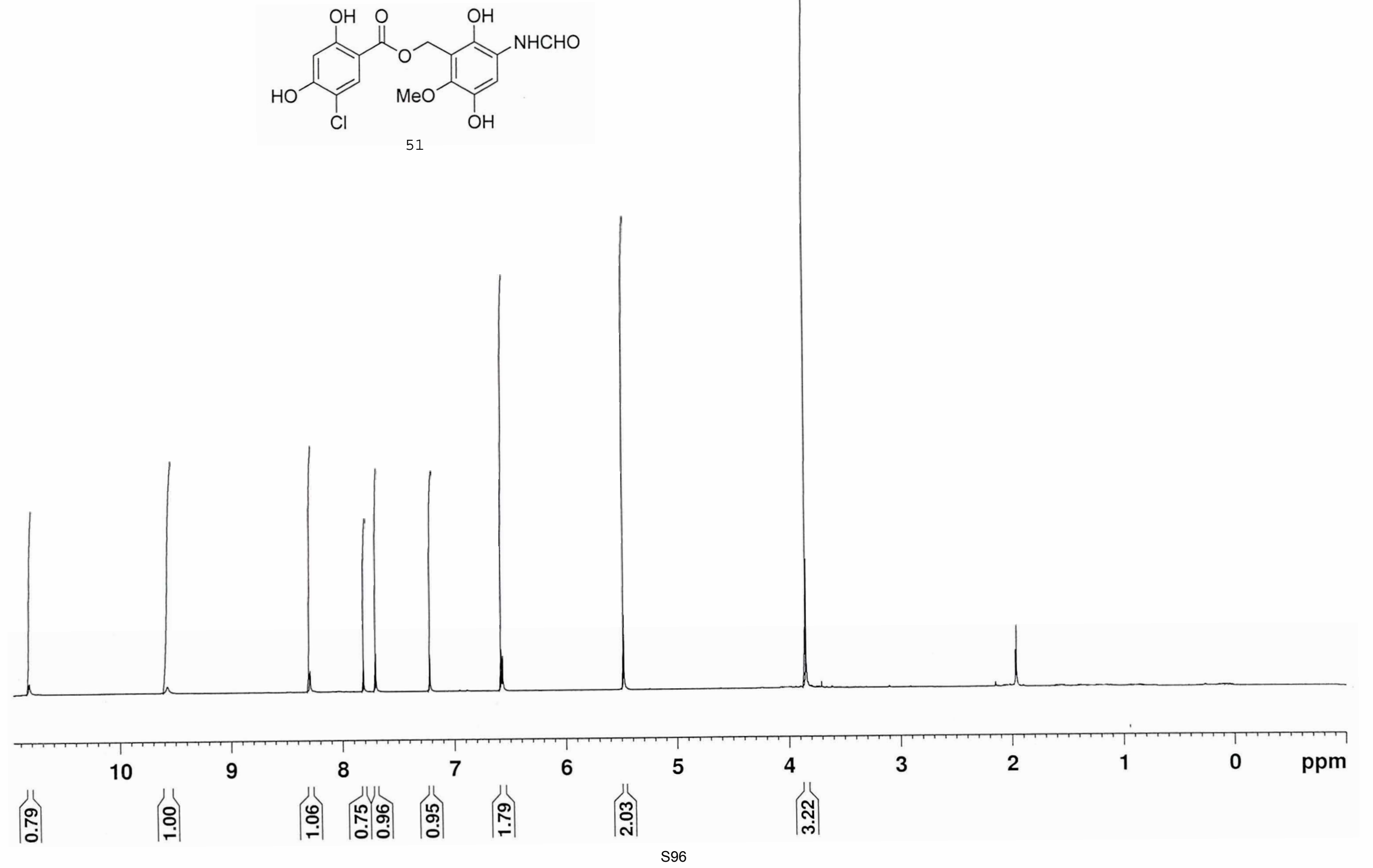

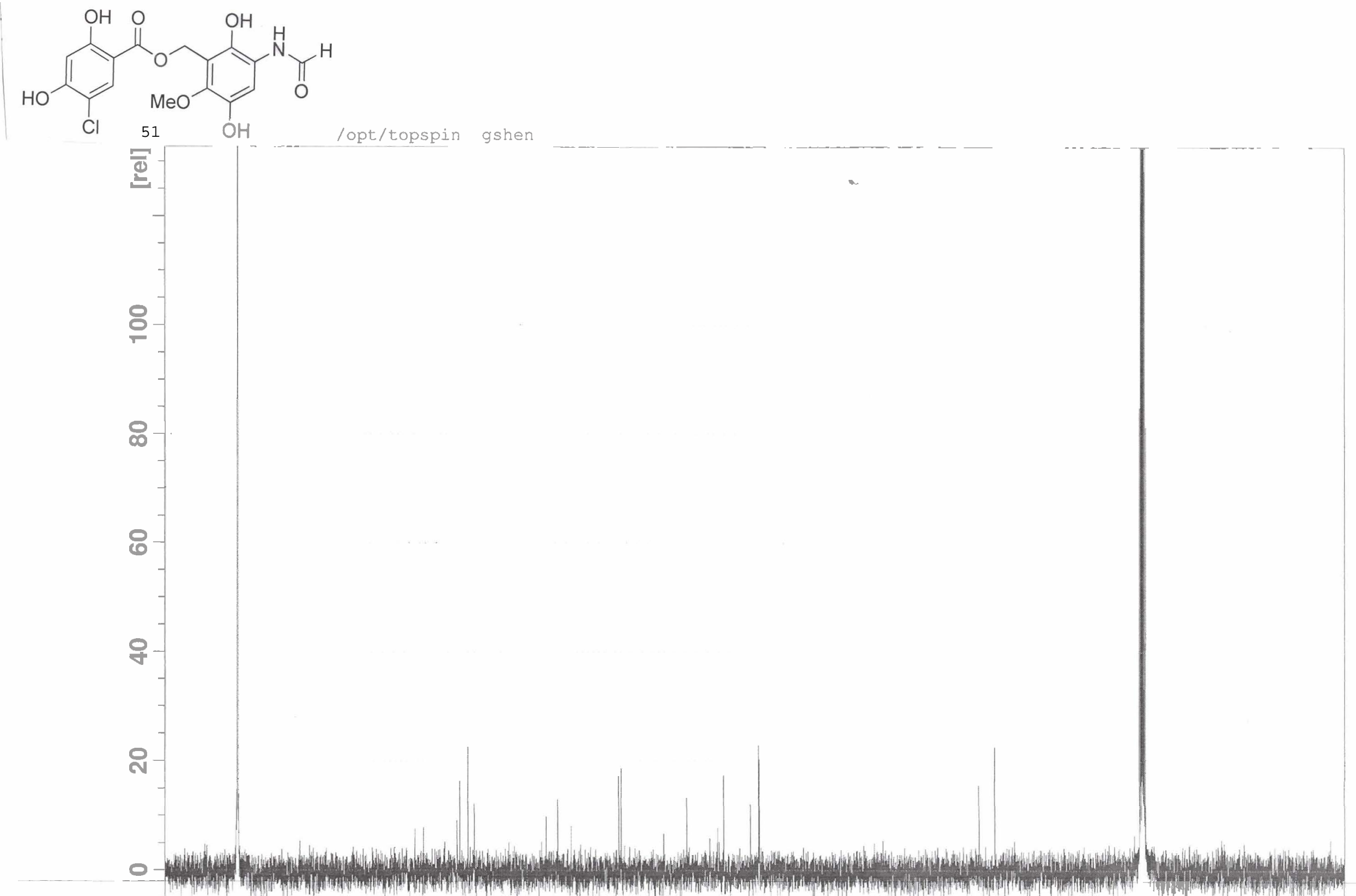

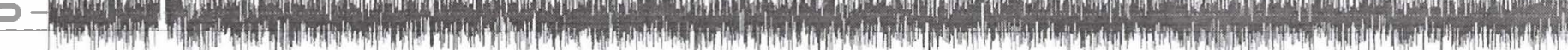



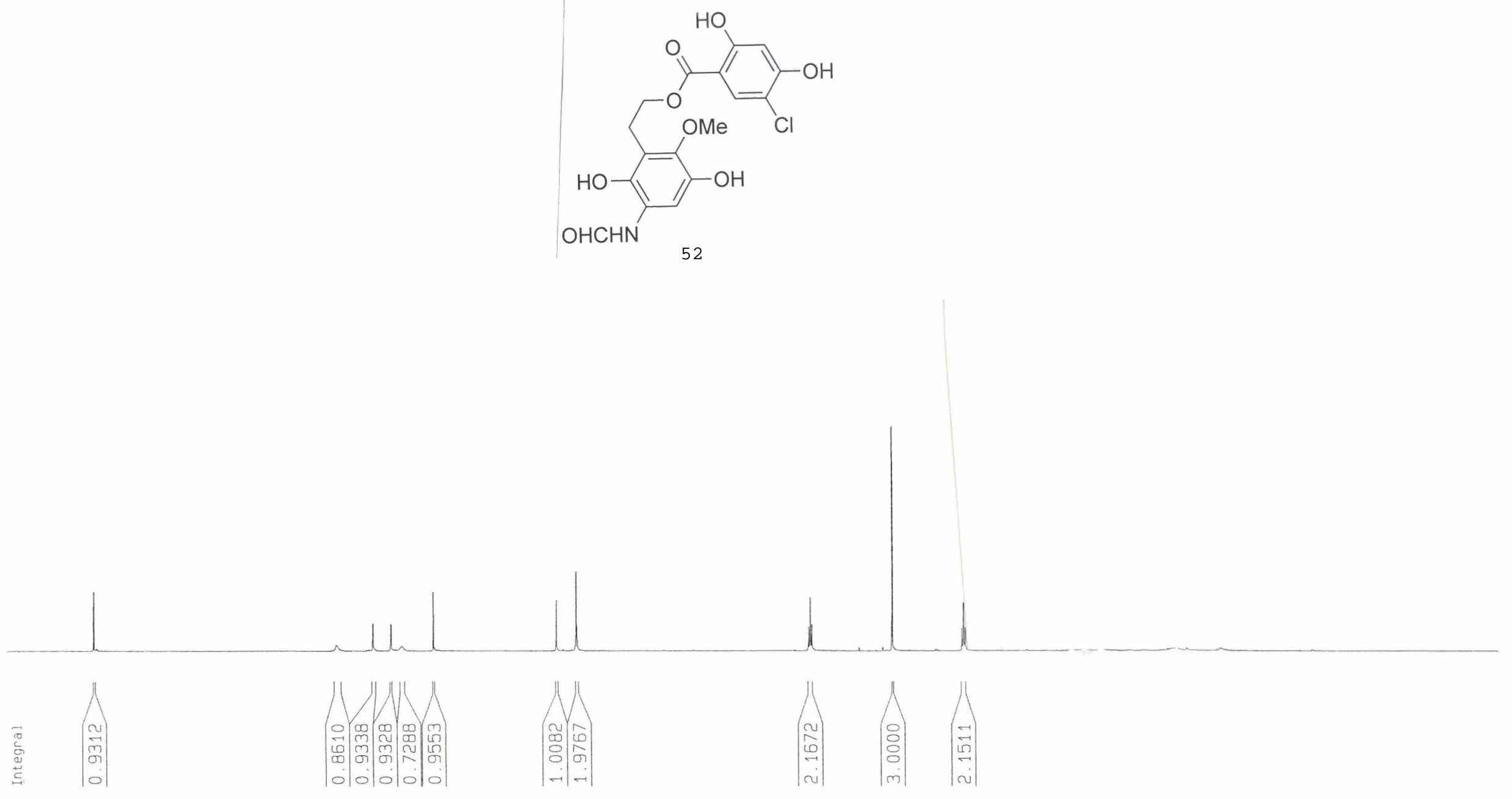


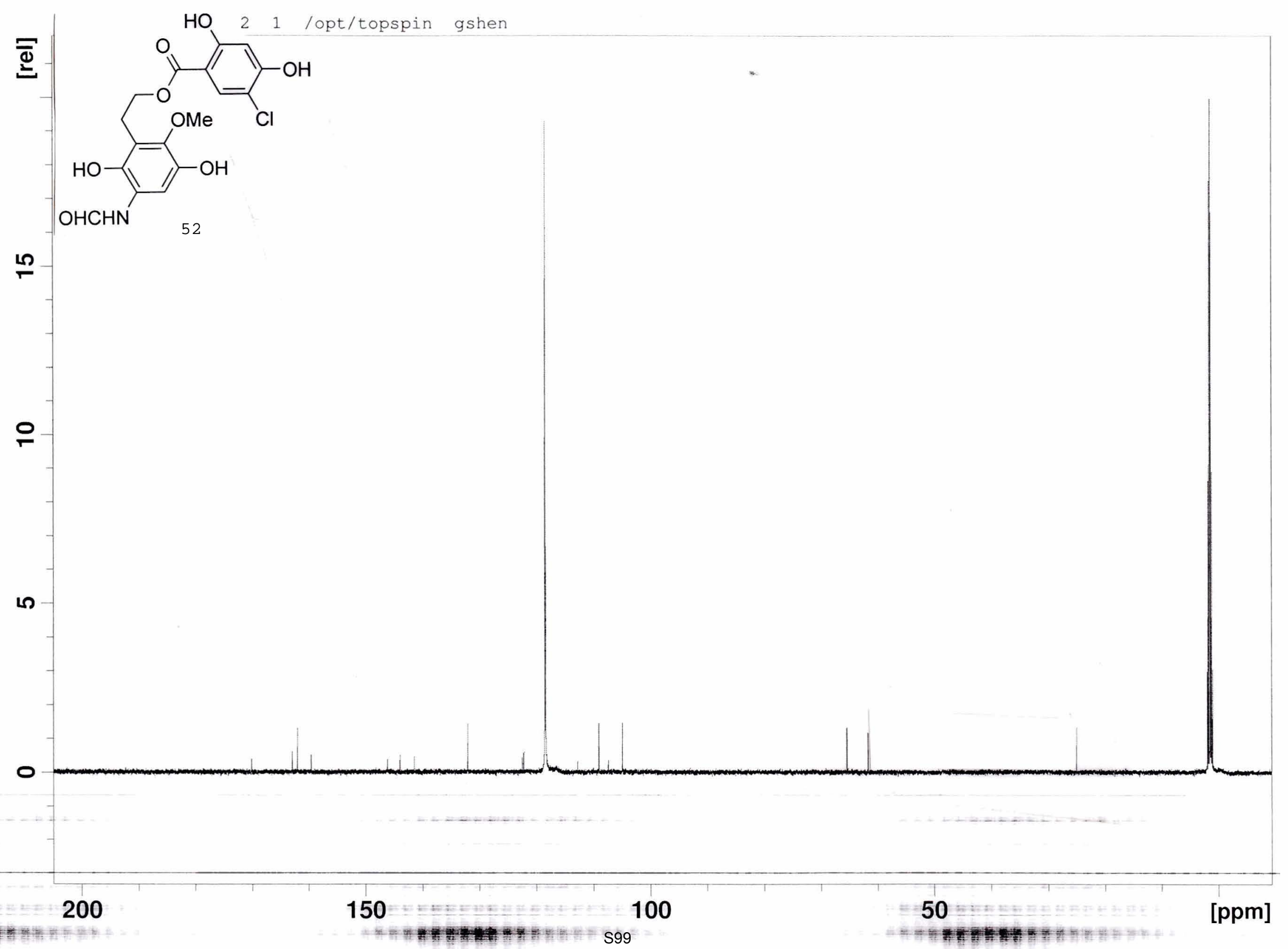


PROTON Acetone opt/topspin gshen 17<smiles>COc1c(O)cc(NC=O)c(O)c1CCCOC(=O)c1cc(Cl)c(O)cc1O</smiles>

53

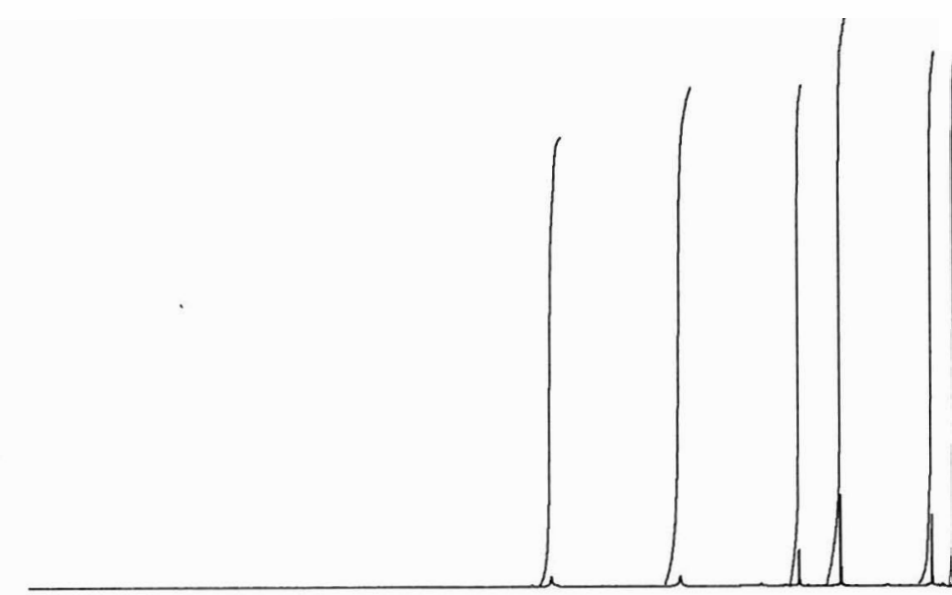

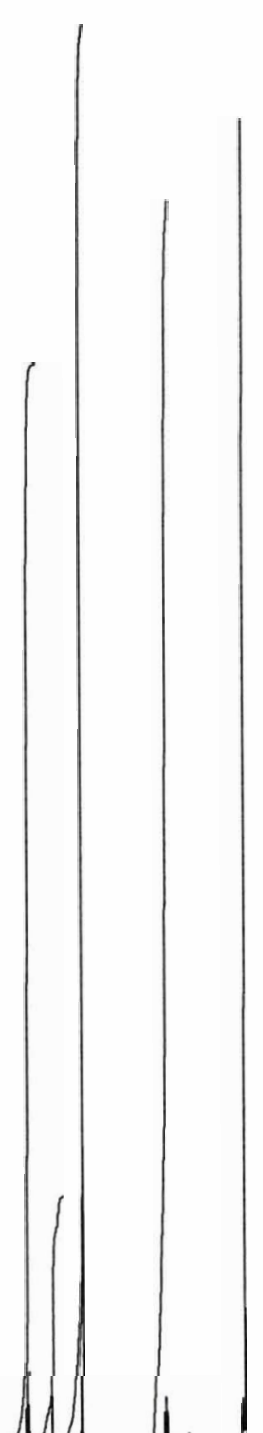

GTUKER

Current Data Parameters NAME

EXPNO

PROCNO

F2 - Acquisition Parameters Date_ 20060407

Time

INSTRUM

PROBHD

PULPROG

TD

SOLVENT

NS

DS

SWH

FIDRES

$\mathrm{AQ}$

RG

$\mathrm{DW}$

TE

D1

TDO

$====$
NUC1

P1

PL1

$\mathrm{SFO} 1$

F2 - Processing parameters

SI

SF

WDW

SSB

LB

GB
PC

1

22.50

spect

$5 \mathrm{~mm}$ CPDUL $13 \mathrm{C}$

$\mathrm{zg} 30$
65536

Acetone

16

$0.157632 \mathrm{~Hz}$

3.1720407 sec

$$
114
$$

48.400 usec 6.00 usec $298.0 \mathrm{~K}$

$1.00000000 \mathrm{sec}$ CHANNEL 1

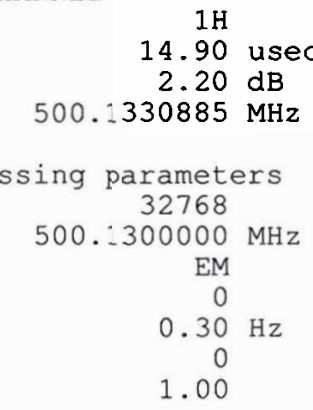

$10330.578 \mathrm{~Hz}$

\begin{tabular}{|c|c|c|c|c|c|c|c|c|c|c|c|c|c|c|c|c|c|c|c|}
\hline 16 & 15 & 14 & 13 & 12 & & & 9 & 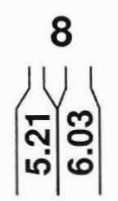 & 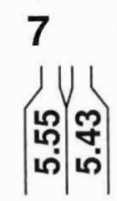 & & & 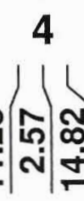 & 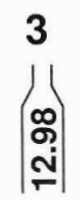 & 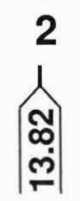 & 1 & 0 & -1 & -2 & ppm \\
\hline
\end{tabular}



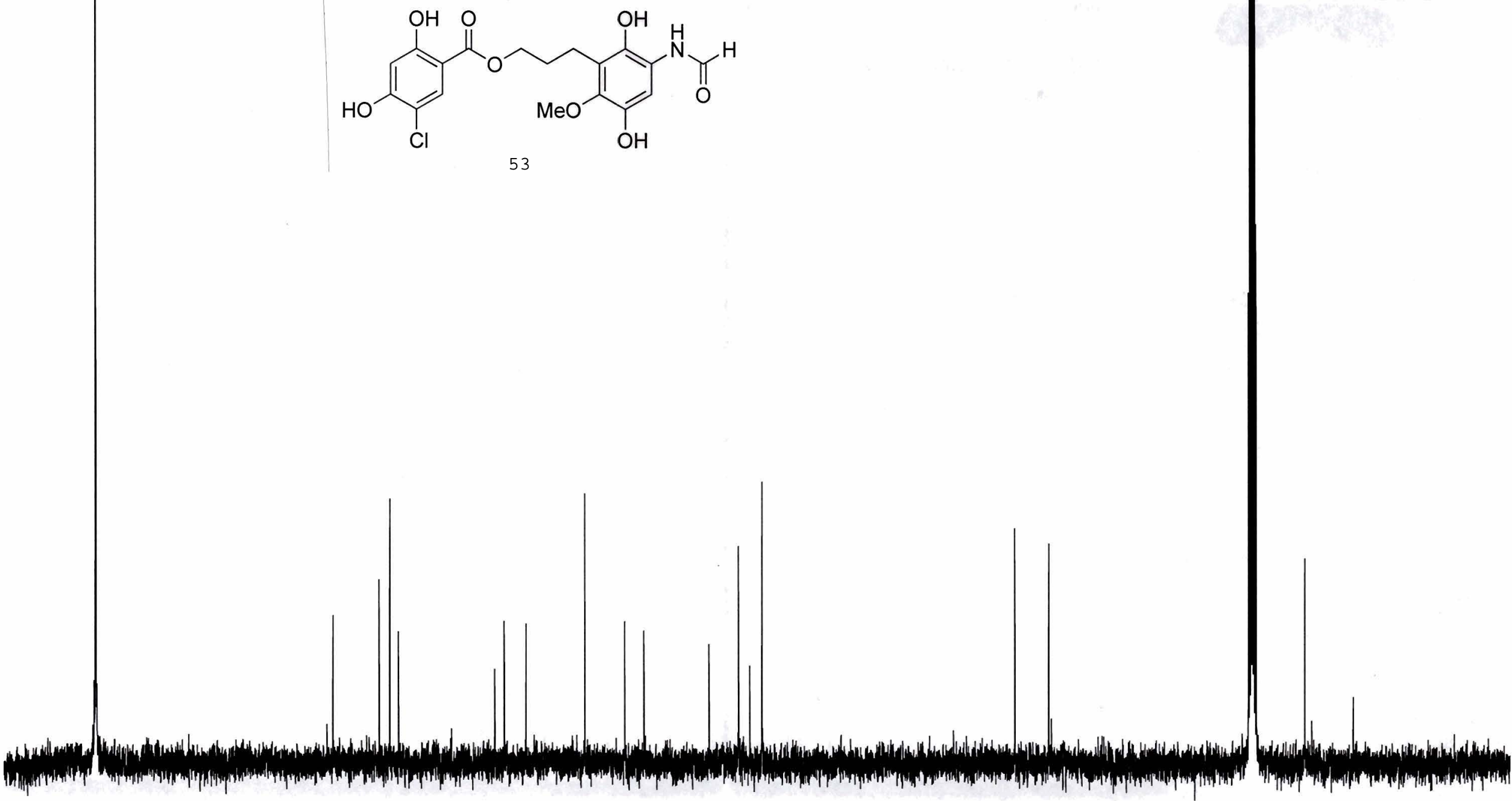
Wh 


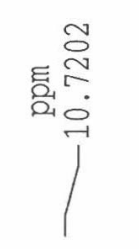

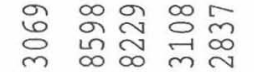

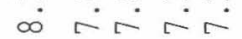

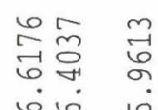

111

66 ก

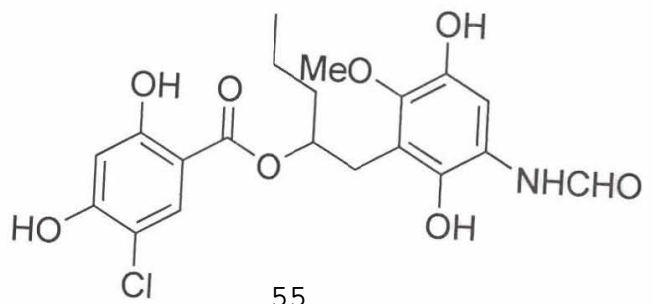

55
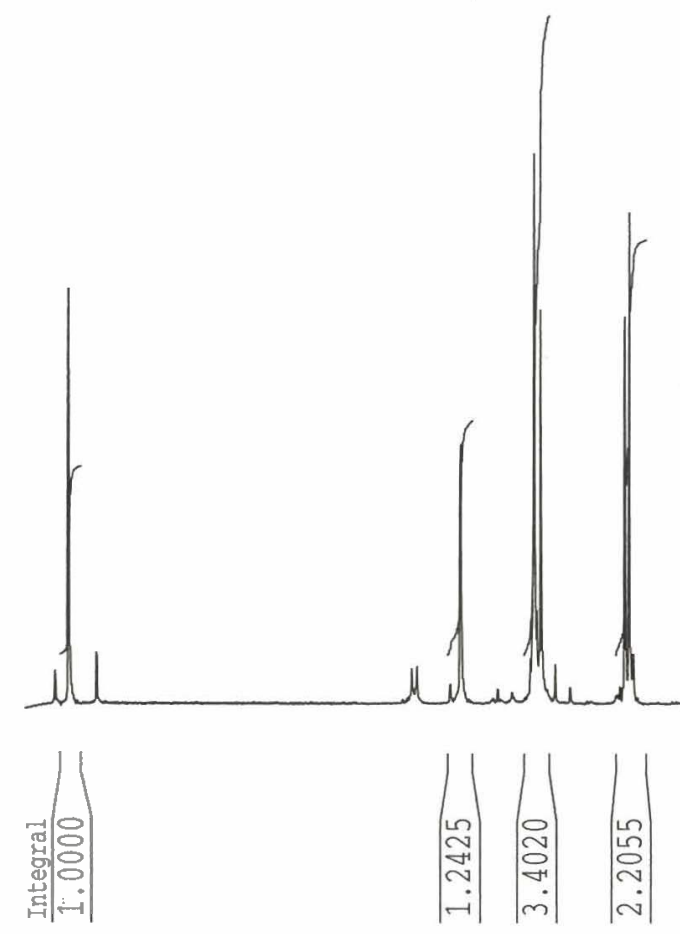

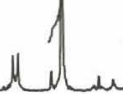
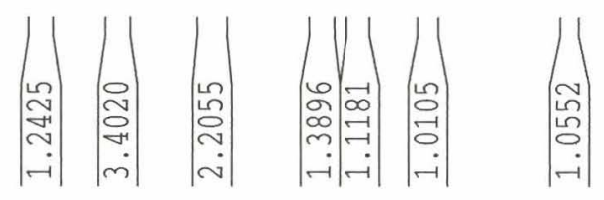

6
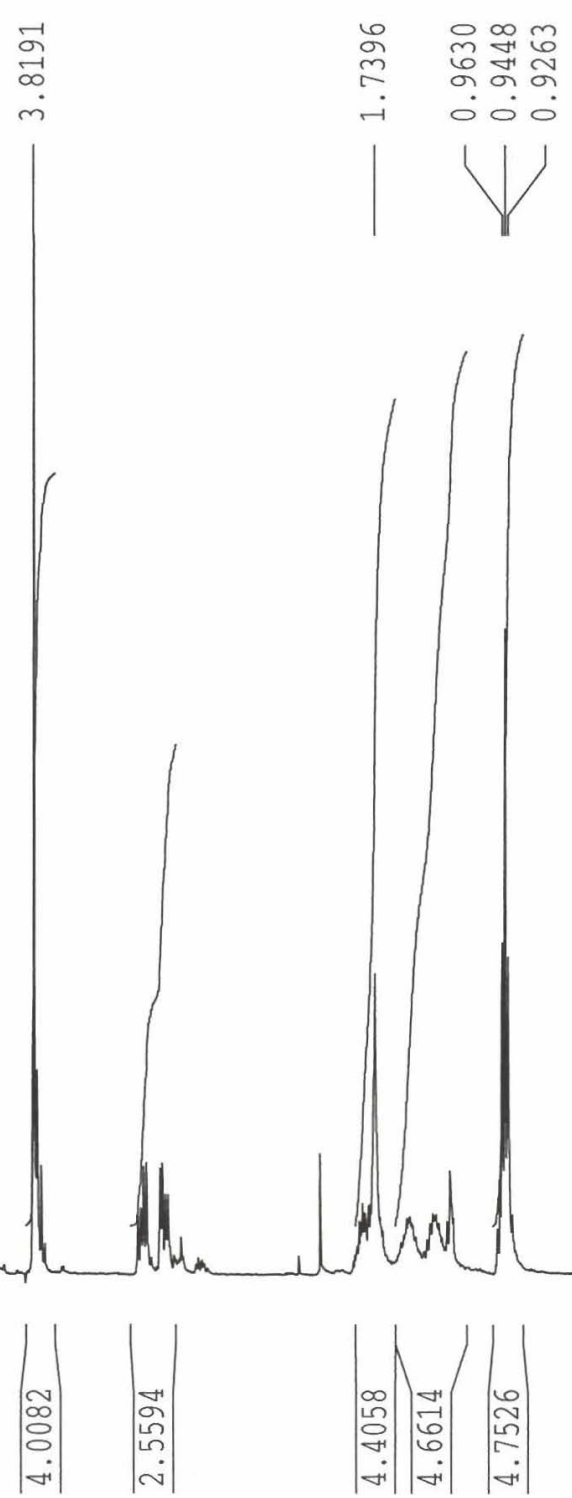

Current Data Parameters

NAME

EXPNO

PROCNO

$\operatorname{sg} 447$

F2 - Acouisition Parameters

Date_ 20050421

$\begin{array}{lr}\text { Time } & 10.19\end{array}$

INSTRUM $\quad \operatorname{drx} 400$

PULPROG $\quad \mathrm{gg} 30$

$\begin{array}{lr}\text { PULPROG } & 2930 \\ \text { TD } & 32768\end{array}$

SOLVENT $\quad$ CDC13

NS 16

SWH $\quad 4789.272 \mathrm{~Hz}$

FIDRES $\quad 0.146157 \mathrm{~Hz}$

AQ $\quad 3.4210291 \mathrm{sec}$

RG 256

$\begin{array}{lr}\text { DW } & 104.400 \text { usec } \\ D E & 5.50 \text { usec }\end{array}$

TE $294.2 \mathrm{~K}$

D1 $1.00000000 \mathrm{sec}$

MCREST $\quad 0.00000000 \mathrm{sec}$

MCWRK $\quad 0.01500000 \mathrm{sec}$

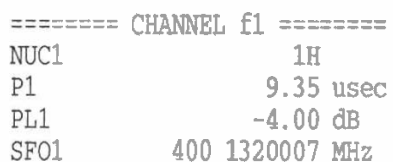

F2 - Processing parameters

SI 32768

SF $\quad 4001300000 \mathrm{MHz}$

WDW

SSB

LB

GB

EM

$0.30 \mathrm{~Hz}$

0

1.00

1D NMR plot paraneters

CX $\quad 20.00 \mathrm{~cm}$

CY $\quad 12.50 \mathrm{~cm}$

F1P $\quad 11.000 \mathrm{ppm}$

$4401.43 \mathrm{~Hz}$

F2P $-1.000 \mathrm{ppm}$

$22-400.13 \mathrm{~Hz}$

$\begin{array}{lr}\text { PPMCM } & 0.60000 \mathrm{ppm} / \mathrm{cm} \\ \text { HZCM } & 240.07800 \mathrm{~Hz} / \mathrm{cm}\end{array}$ $240.07800 \mathrm{~Hz} / \mathrm{cm}$ 


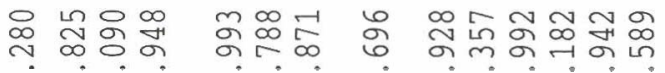

엉요

अं

걱

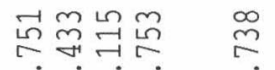

도든

i.
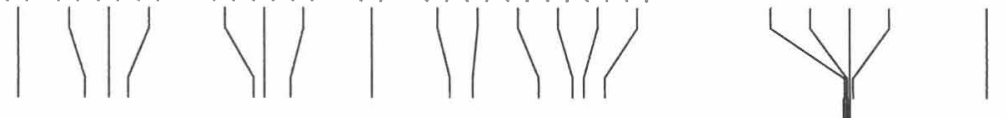

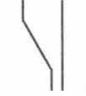

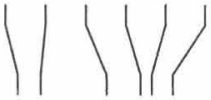<smiles>CCC(Cc1c(O)c(N=C=O)cc(O)c1OC)OC(=O)c1cc(Cl)c(O)cc1O</smiles>

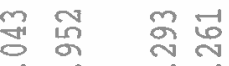

이 워

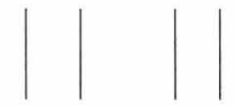

55

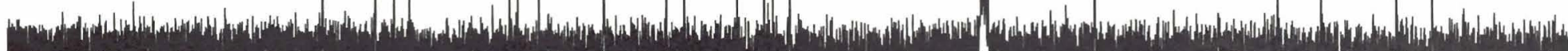

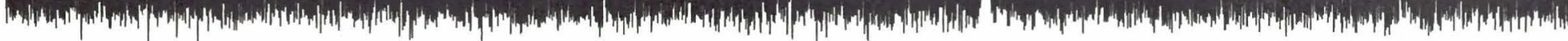

ppm $200 \quad 200 \quad 2,5$

150

125

75

50

Current Data Parameters

PROCNO

F2 - Acquisition Parameters

Date 20050421

Time $\quad 10.34$

PROBHD 5 man QNP $1 \mathrm{H} / 13$

PULPROG zgpg30

TD

SOLVENT

NS
DS
SWH

SWH $\quad 23148.148 \mathrm{~Hz}$

FIDRES $\quad 0.353213 \mathrm{~Hz}$

AQ $\quad 1 . \$ 156276 \mathrm{sec}$

$D W$

$\mathrm{DE}$

D1

d11

$0.150000012 \mathrm{~K}$

$0.03000000 \mathrm{sec}$

$0.00000000 \mathrm{sec}$

$0.01500000 \mathrm{sec}$

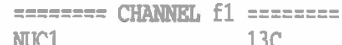

NUC1

P1

SFO1

11.25 usec

$2.00 \mathrm{~dB}$

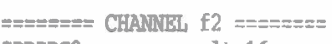

CPDPRG2

NUC2 $1 \mathrm{H}$

PCPD2 100.00 usec

$\begin{array}{ll}\text { PL2 } & -4.00 \mathrm{~dB} \\ \text { PL12 } & 16.58 \mathrm{~dB}\end{array}$

PL13 $17.00 \mathrm{~dB}$

SFO2 $400.1316005 \mathrm{MHz}$

F2 - Processing parameters

$\begin{array}{lc}\text { SI } & 65536 \\ \text { SF } & 100.6127290 \mathrm{MHz}\end{array}$

WDW 100.6127290

$\begin{array}{ll}\text { SSB } & 1.00 \mathrm{~Hz}\end{array}$

$\begin{array}{lr}L B & 1.00 \\ G B & 0\end{array}$

$9 \mathrm{C}$

1.40

1D MR plot parameters

CX $20.00 \mathrm{~cm}$

CY $\quad 20.00 \mathrm{~cm}$

F1P 215.000 ppm

F1 $21631.74 \mathrm{~Hz}$

$\begin{array}{ll}\text { 82P } & -5.000 \mathrm{ppm} \\ \mathrm{B2} & -503.06 \mathrm{gz}\end{array}$

PPMCY $\quad 11.00000 \mathrm{pDm} / \mathrm{C}$

HZCM $\quad 1106.73999 \mathrm{~Hz} / \mathrm{cm}$ 


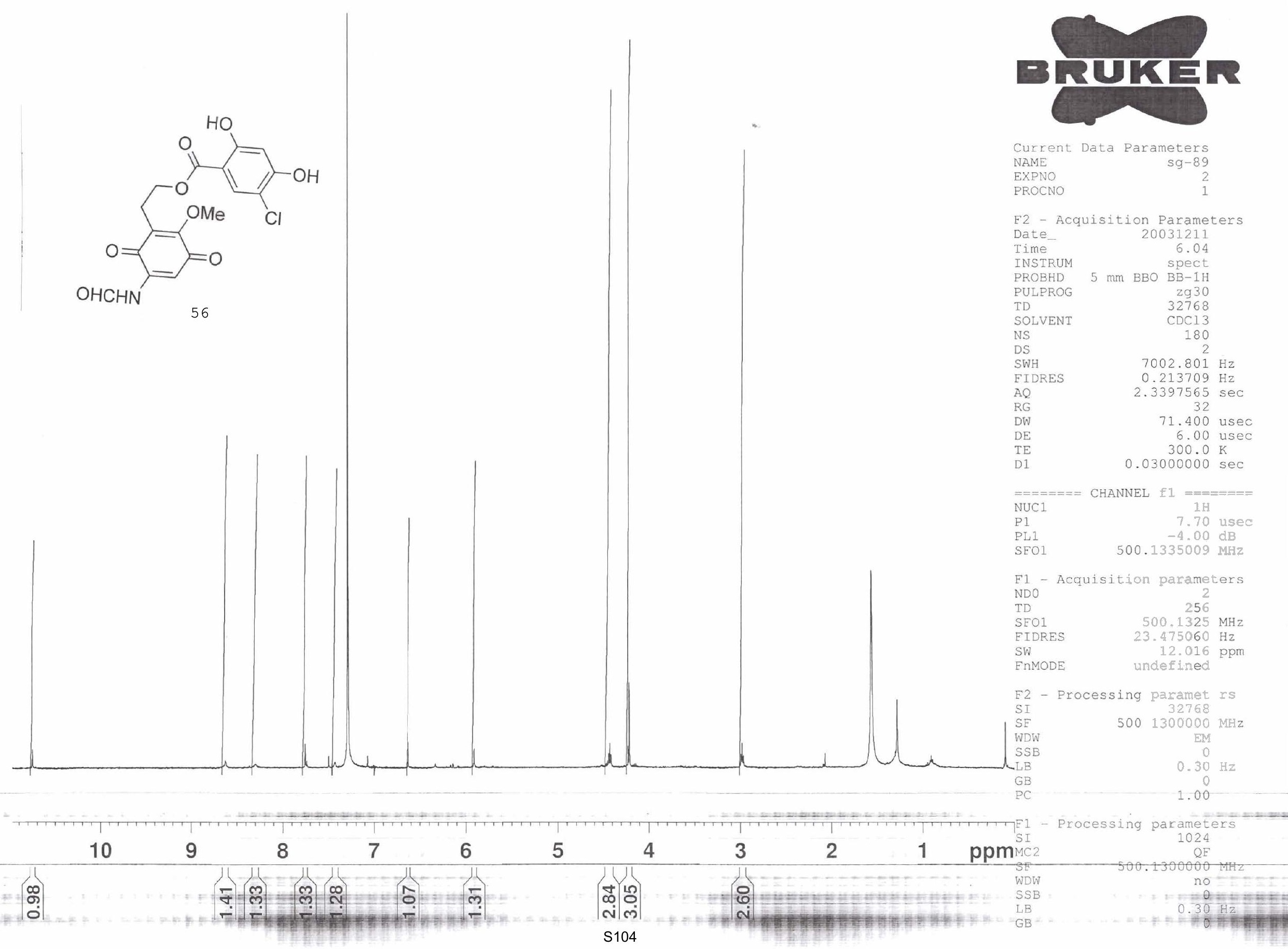



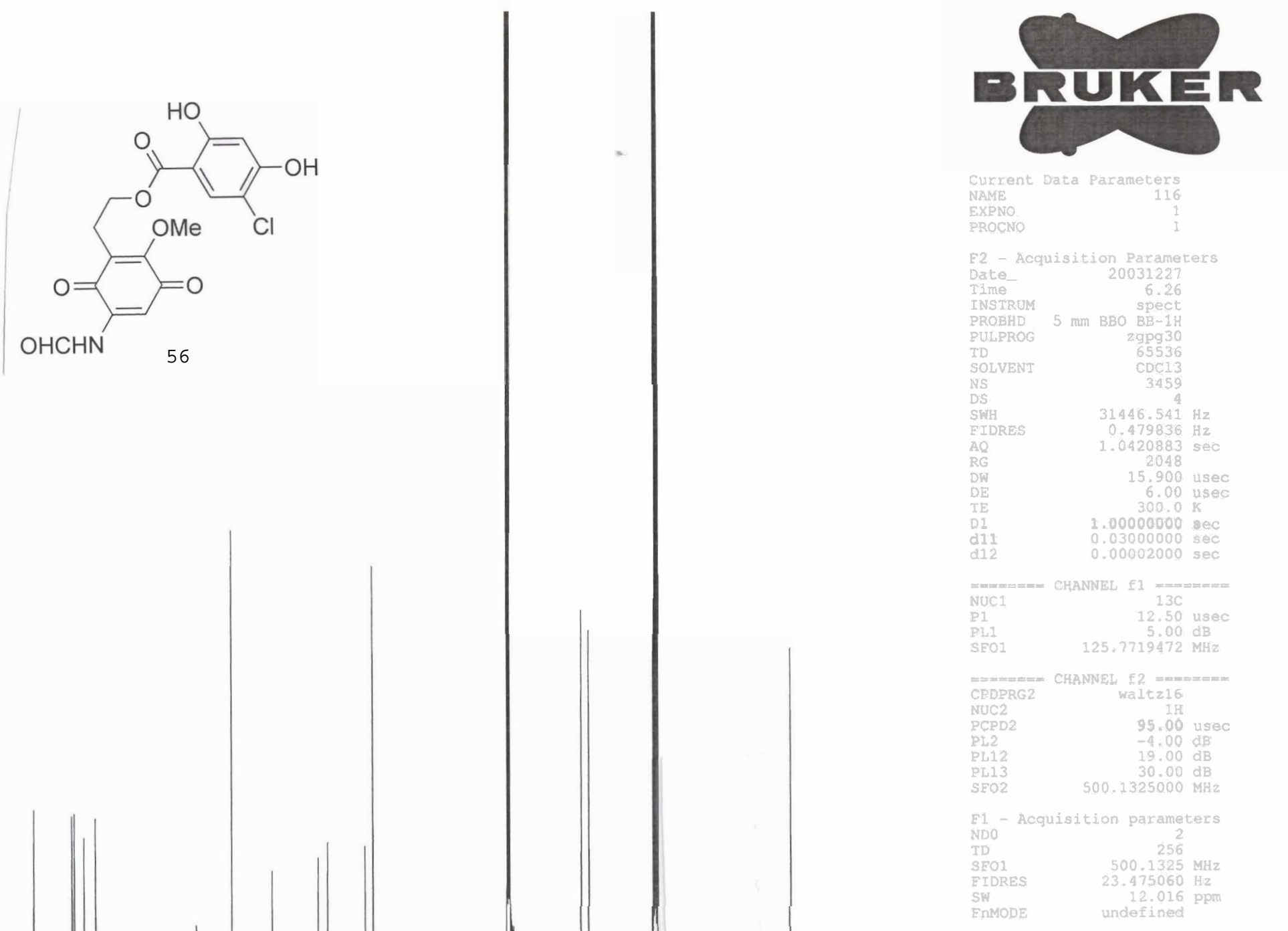

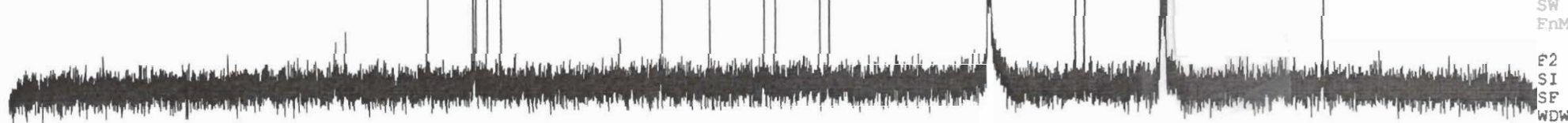

E2 - Processing parameters

65536
$125.7578008 \mathrm{MHz}$ $5 B$ EM

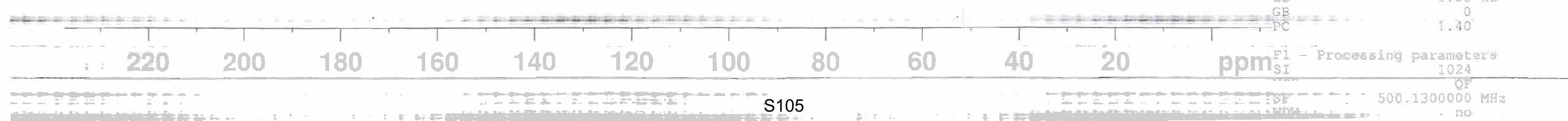


Cl

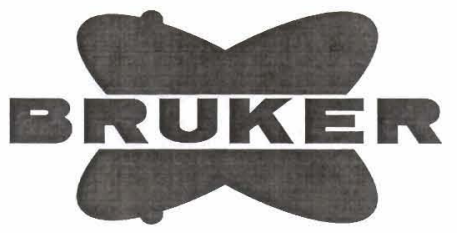

Current Data Parameters NAME

EXPNO

$\operatorname{sg} 448$

PROCNO

F2 - Acquisition Parameters

Date

20050422

Time

18.05

INSTRUM

PROBHD

L $13 \mathrm{C}$

PULPROG

zgpg 30

SOLVENT

65536

$\mathrm{CDCl} 3$

2048

NS

DS

FIDRES

$\mathrm{AQ}$

RG

DW

DE

TE

d11

DELIA

MCREST

MCWRK

$31446.541 \mathrm{~Hz}$

$0.479836 \mathrm{~Hz}$

$1.0420883 \mathrm{sec}$ 8192

15.900 usec

6.00 usec $300.0 \mathrm{~K}$

$0.15000001 \mathrm{sec}$

$0.03000000 \mathrm{sec}$

$0.03000000 \mathrm{sec}$

$0.05000000 \mathrm{sec}$

$0.00000000 \mathrm{sec}$

$0.01500000 \mathrm{sec}$

$========$ CHANNEL $f$

$\mathrm{NUCl}$

P1

PL1

$13 \mathrm{C}$

$13 \mathrm{C}$

10.00 usec

$-4.90 \mathrm{~dB}$

$125.7697360 \mathrm{MHz}$

CHANNeL 12 - =====

CPDPRG2 waltz16

NUC2

$\mathrm{PCPD}$

PL12

$\mathrm{SFO}$ 65536 $125.7577890 \mathrm{MHz}$ 


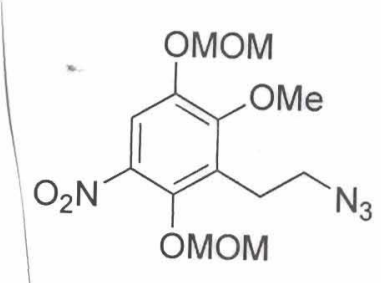

59.1

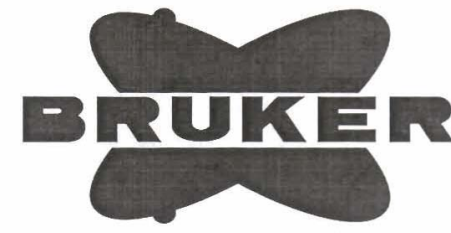

Current Data Parameters NAME

EXPNO

PROCNO

F2 Acouisition Parameter:

Date_ 20040819

Time

INSTRUM

PROBHD

PUIPROG

TD

SOLVEN

NS

SWH

FIDRES

A?
RG

DW

DE

TE

D1

MCREST

$==\pi== \pm=$ CHANNEL $\mathrm{f}$

NUC1

P1

SLO1

F2 - Processing parameters

$S I$

$S E$
WDW

SSB

LB

$G B$

4.59
$\operatorname{ar} \times 400$

mm Multinucl

2930

CDCl 13

16

$4789.272 \mathrm{~Hz}$

$0.145157 \mathrm{~Hz}$

$3.4210291 \mathrm{sec}$

64

04.400 usec 5.50 usec $293.2 \mathrm{~K}$

$1.00000000 \mathrm{sec}$

$0.00000000 \mathrm{sec}$

$0.01500000 \mathrm{sec}$

32768
400.130000 $400.1300000 \mathrm{MHz}$

EM

$0.30 \mathrm{H} \%$

0

3

2

1

0

ppm

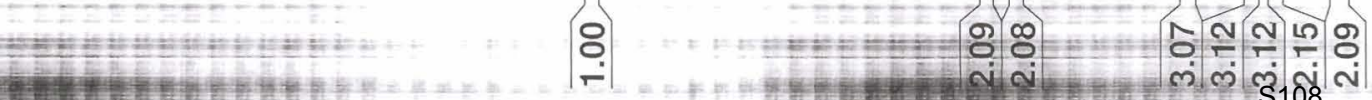




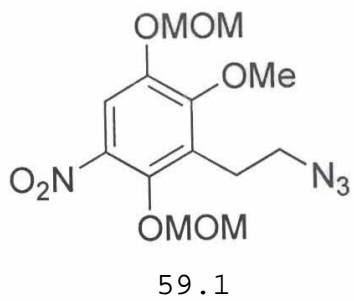

$\begin{array}{lr}\text { Current Data Parameters } \\ \text { NAME } & s g-472 \\ \text { EXPNO } & 1 \\ \text { PROCNO } & 1\end{array}$

F2 - Acquisition Parameters

Date_ 20040819

Time

5.01
$\operatorname{ar} \times 400$

INSTRUM $5 \mathrm{~mm}$ MUIt

PROBHD

PULPROG $\quad$ zgpg30

TD

65536
$C D C 13$

SOLVENT

NS

DS

SWH

FIDRES

$A Q$

RG

DW

DE

D1

d11

DELTA

MCREST

MCWRK

4
23148,148

$0.353213 \mathrm{~Hz}$

$1.4156276 \mathrm{sec}$ 32768

21.600 usec 5.50 usec $293.2 \mathrm{~K}$

$2.00000000 \mathrm{sec}$

$0.03000000 \mathrm{sec}$

$1.89999998 \mathrm{sec}$

0.00000000 sec

0.01500000 sec

$======0$ CHANNEL

NUC1

P1 $13 \mathrm{C}$

12.30 usec

PL1 $2.00 \mathrm{~dB}$

SFO1

$100.6232933 \mathrm{MHz}$

$==$ CHANNEL, $\mathrm{f} 2$

CPDPRG2 waltz16
NUC2

NUC2

PCPD2

PL2

100.00 usec

$0.00 \mathrm{~dB}$

$18.00 \mathrm{~dB}$

$18.00 \mathrm{~dB}$

$400.1316005 \mathrm{MHz}$

SEO2

$-42=$ processing parameters 


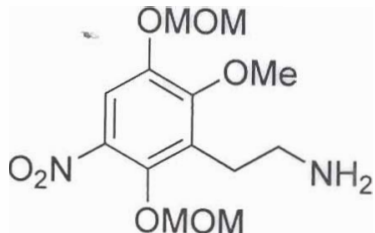

59

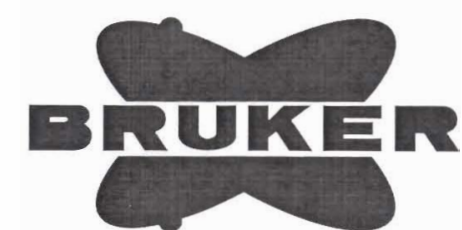

Current Data Parameters NAME:

EXPNO

PROCNO

$\mathrm{sg}-509$

1

F2 - Acquisition Parameters

Date_ $\quad 20040902$

Time

INSTRUM

PROBHD

$\operatorname{drx} 400$

$5 \mathrm{~mm}$ Multinucl

PULPROG

$z 930$
32768

SOLVENT

NS

$\mathrm{CDCI} 3$

SWH

FIDRES

AQ

RG

$D W$

$\mathrm{DE}$

IE

MCREST

MCWRK

$===$
NUC1
P1

PL1

SFO1

2

$4789.272 \mathrm{~Hz}$

$0.146157 \mathrm{~Hz}$

$3.4210291 \mathrm{sec}$ 228.1

104.400 usec 5.50 usec $293.2 \mathrm{~K}$

$1.00000000 \mathrm{sec}$

$0.00000000 \mathrm{sec}$

$0.01500000 \mathrm{sec}$

GB
CHANNEL $f$

$=======$

$1 \mathrm{H}$

7.70 usec $-6.00 \mathrm{~dB}$ $400.1320007 \mathrm{MHz}$
Processing parameters

32768

$400.1300000 \mathrm{MHz}$

EM

30

$0.30 \mathrm{~Hz}$

0

98




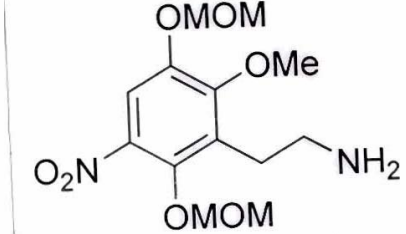

59

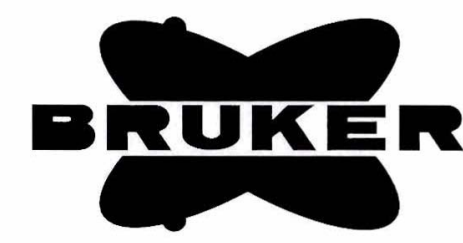

Current Data Parameters NAME EXPNO

PROCNO

F2 - Acquisition Parameters Date_ 20040907

Time

INSTRUM

PROBHD

PULPROG

TD

SOLVENT

NS

DS

SWH

FIDRES

$A Q$

RG

DW

DE

TE

D1

DELTA

MCREST

MCWRK

$==-$
NUC1

P1

PL1

SFO1

$======$

CPDPRG2

NUC2

PCPD

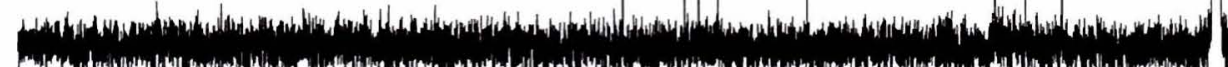

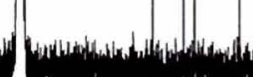

4.17
$\operatorname{dr} \times 400$

$5 \mathrm{~mm} \mathrm{Multinucl}$

zgpg 30

65536
$\mathrm{CDC} 13$

2124

$23148.148 \mathrm{~Hz}$

$0.353213 \mathrm{~Hz}$

$1.4156276 \mathrm{sec}$ 2298.8

21.600 usec

5.50 usec $293.2 \mathrm{~K}$

$2.00000000 \mathrm{sec}$

$0.03000000 \mathrm{sec}$

$1.89999998 \mathrm{sec}$

$0.00000000 \mathrm{sec}$

$0.01500000 \mathrm{sec}$

CHANNEL f

$13 \mathrm{C}$

12.30 usec

$100.6232933 \mathrm{MHz}$

CHANNEL $\mathrm{f} 2========$

waltz 16

100.00 usec

$0.00 \mathrm{~dB}$

$18.00 \mathrm{~dB}$

$18.00 \mathrm{~dB}$

$400.1316005 \mathrm{MHz}$
PL2

PL12

$\mathrm{SFO} 2$

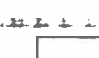

- =-2x

160

80


<smiles>COc1cc([N+](=O)[O-])c(OC)c(OC)c1CCNC(=O)c1cc(Cl)c(OCc2ccccc2)cc1OCc1ccccc1</smiles>

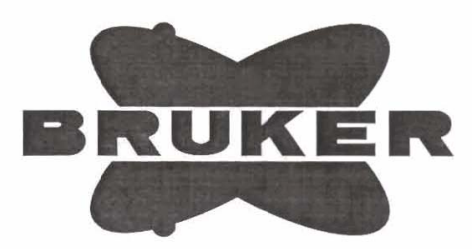

Current Data Parameters NAME

EXPNO

PROCNO

F2 - Acquisition Parameters

Date_ 20040912

Time

6.50

INSTRUM

PROBHD

irxucl

PULPROG

TD

SOLVENT

NS

DS

SWH

FIDRES

$\mathrm{AQ}$

RG

DW

$\mathrm{DE}$

MCREST

MCWRK

$5 \mathrm{~mm}$ Multinucl

$\mathrm{zg} 30$
32768

$\mathrm{CDCl} 3$

$4789.272 \mathrm{~Hz}$

(0) $146157 \mathrm{~Hz}$

$3.4210291 \mathrm{sec}$

.4210291
104.400

104.400 usec 5.50 usec $293.2 \mathrm{~K}$

$100000000 \mathrm{sec}$

$1.00000000 \mathrm{sec}$

$0.00000000 \mathrm{sec}$

$========$ CHANNEL $\mathrm{f}$

NUC1

P1

PL1

$\mathrm{SEO} 1$
$1=$
$1 \mathrm{H}$
7.70 usec
$-6.00 \mathrm{~dB}$

$400.1320007 \mathrm{MHz}$

F2 - Processing parameters

ST 32768

SE $\quad 400.1300000 \mathrm{MHz}$

WDW

SSB

LB

$G B$

$\mathrm{PC}$
$1 \mathrm{M}$
0

0.30 .4

2,00

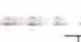

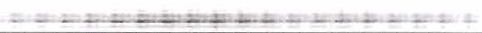

$$
1.00
$$

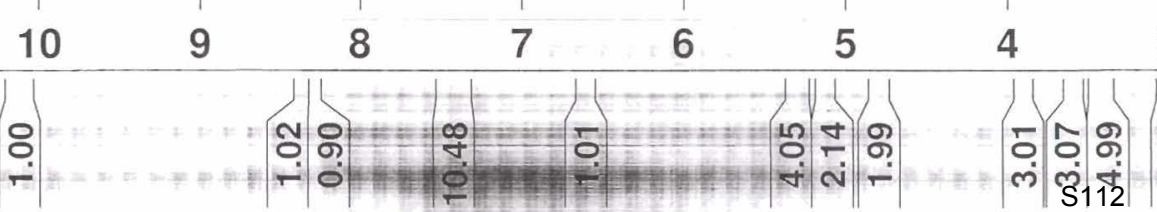

3




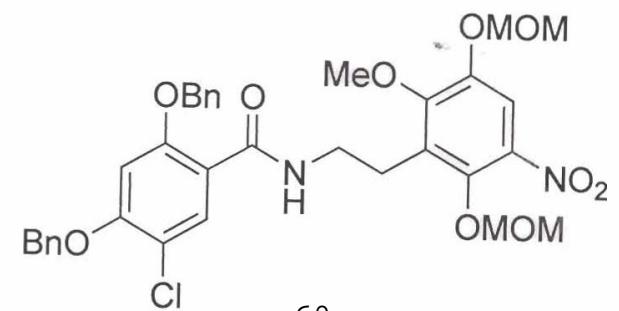

60

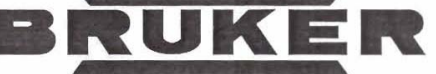
.

urrent Data Parameters NAME sg -522

EXPNO

PROCNO

F2 - Acquisition Parameters

Date_ 20040912

Time 15.46

INSTRUM

PROBHD $5 \mathrm{~mm}$ Multinucl

PULPROG zgpg30

SOLVENT

NS

DS

SWH

FIDRES

AQ

RG

DW

DE

TE

dI 1

DELTA

MCREST

MCWRK

$29 p g 30$
65536

CDCl 3

9216

$23148.148 \mathrm{~Hz}$

$0.353213 \mathrm{~Hz}$

$1.4156276 \mathrm{sec}$ 645.1

21.600 usec 5.50 usec $293.2 \mathrm{~K}$

$2.00000000 \mathrm{sec}$

$0.03000000 \mathrm{sec}$

1.0300000

$1.89999998 \mathrm{sec}$

$0.00000000 \mathrm{sec}$

$0.01500000 \mathrm{sec}$

$========$ CHANNEL $\mathrm{f}$

NUC 1

$1==$

12.30 usec

$2.00 \mathrm{~dB}$

$\begin{array}{ll}\text { PL1 } & 2.00 \mathrm{~dB} \\ \text { SFO1 } & 100.6232933 \mathrm{MHz}\end{array}$

$========$ CHANNEL f $2========$

CPDPRG2 waltz16

NUC2

nce 2

PL2

PL12

PL13

$\mathrm{SFO} 2$

100.00 usec

$0.00 \mathrm{~dB}$

$18.00 \mathrm{~dB}$

$18.00 \mathrm{~dB}$

$400.1316005 \mathrm{MHz}$

\begin{tabular}{|c|c|c|c|c|c|c|c|c|c|c|c|}
\hline \multirow[t]{2}{*}{$\Gamma$} & 7 & $T$ & $T$ & 1 & 1 & $T$ & 1 & $T$ & $T$ & $T$ & $T$ \\
\hline & 200 & 180 & 160 & 140 & 120 & 100 & 80 & 60 & 40 & 20 & ppm \\
\hline
\end{tabular}

F2 65536 $100.6127290 \mathrm{MHz}$ 
<smiles>COc1cc(N=C=O)c(OC)c(OC)c1CCNC(=O)c1cc(Cl)c(O)cc1O</smiles>

62

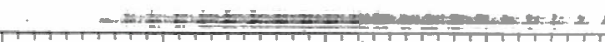

9

5

4
$-1+\frac{1}{1+1}$

$\begin{array}{lr}\text { Current Data Parameters } \\ \text { NAME } & \text { sg-564 } \\ \text { EXPNO } & 1 \\ \text { PROCNO } & 1\end{array}$

F2 - Acquisition Parameters

Date 20041019

Time $\quad 17.40$

INSTRUM dr 400

PROBHD $5 \mathrm{~mm}$ Multinucl

PULPROG $\quad \mathrm{zg} 30$

TD

SOLVENT

NS

DS

SWH

FIDRES

$A Q$

RG

$D E$

TE

D1

MCRESI

$\mathrm{MeOH}$

$\mathrm{MeOH}$
16

$4789.272 \mathrm{~Hz}$

$0.146157 \mathrm{~Hz}$

$3.4210291 \mathrm{sec}$ 181

104.400 usec 5.50 usec $292.2 \mathrm{~K}$

$1.00000000 \mathrm{sec}$

$0.00000000 \mathrm{sec}$

$0.01500000 \mathrm{sec}$ $========$ CHANNEL $f 1$ NUC1

P1

PL1

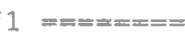
$1 \mathrm{H}$

7.70 usec

$-6.00 \mathrm{~dB}$

SFO1 $400.1320007 \mathrm{MHz}$

F2 - Processing parameters

SI 32768

SE $\quad 400.1300000 \mathrm{MHz}$

SSB

GB

400.1300000
EM

$\mathrm{EM}$
0

$0.30 \mathrm{~Hz}$

1.00 


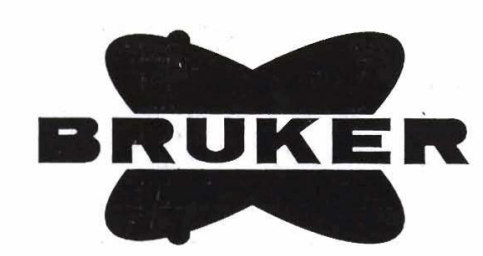<smiles>COc1cc(NC=O)c(OC)c(OC)c1CCNC(=O)c1cc(Cl)c(O)cc1O</smiles>

62 $\begin{array}{lr}\text { Current Data Parameters } \\ \text { NAME } & \text { gs_3_211 } \\ \text { EXPNO } & 2 \\ \text { PROCNO } & 1\end{array}$

E2 - Acquisition Parameters

Date_20041019

$\begin{array}{lr}\text { Time } & 23.08\end{array}$

INSTRUM spect

PROBHD $5 \mathrm{~mm}$ CPDUL $13 \mathrm{C}$

PULPROG 2gpg 30

TD 65536

SOLVENT CDC13

NS

DS

SWH

FIDRES

$\mathrm{AQ}$

RG

$\mathrm{DW}$

DE

TE

D1

d11

DELTA

MCREST

MCWRK

NUC1

P1

PL1

SFO1

$=======$

CPDPRG2

NUC2

PCPD2

PL2

PL12

PLI 3

SFO2
2888

$31446.541 \mathrm{~Hz}$

$0.479836 \mathrm{~Hz}$

$1.0420883 \mathrm{sec}$ 8192

15.900 usec 6.00 usec $300.0 \mathrm{~K}$

$0.15000001 \mathrm{sec}$

0.1500001 sec

$0.03000000 \mathrm{sec}$

$0.05000000 \mathrm{sec}$

$0.00000000 \mathrm{sec}$

$0.01500000 \mathrm{sec}$

CHANNEL $f$

$1=$

10.00 usec

$-5.20 \mathrm{~dB}$

$125.7697360 \mathrm{MHz}$

CHANNEL $f 2==== \pm===$ waltzi6

80.00 usec

$-5.10 \mathrm{~dB}$

$-5.10 \mathrm{~dB}$
$10.00 \mathrm{~dB}$

$10.00 \mathrm{~dB}$

$500.1325000 \mathrm{MHz}$

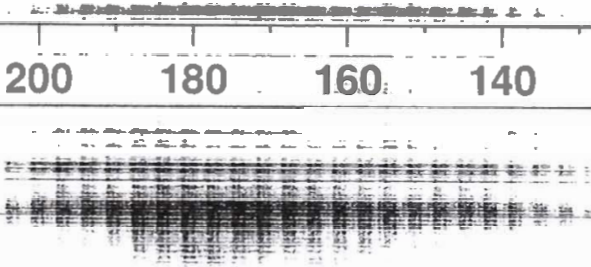

120
$-1+1$

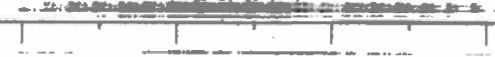

F2 SI $p p m_{W D W}^{S E}$ SSB

LB

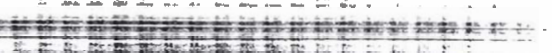

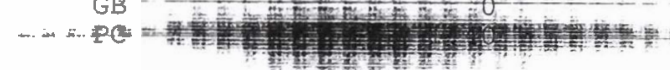



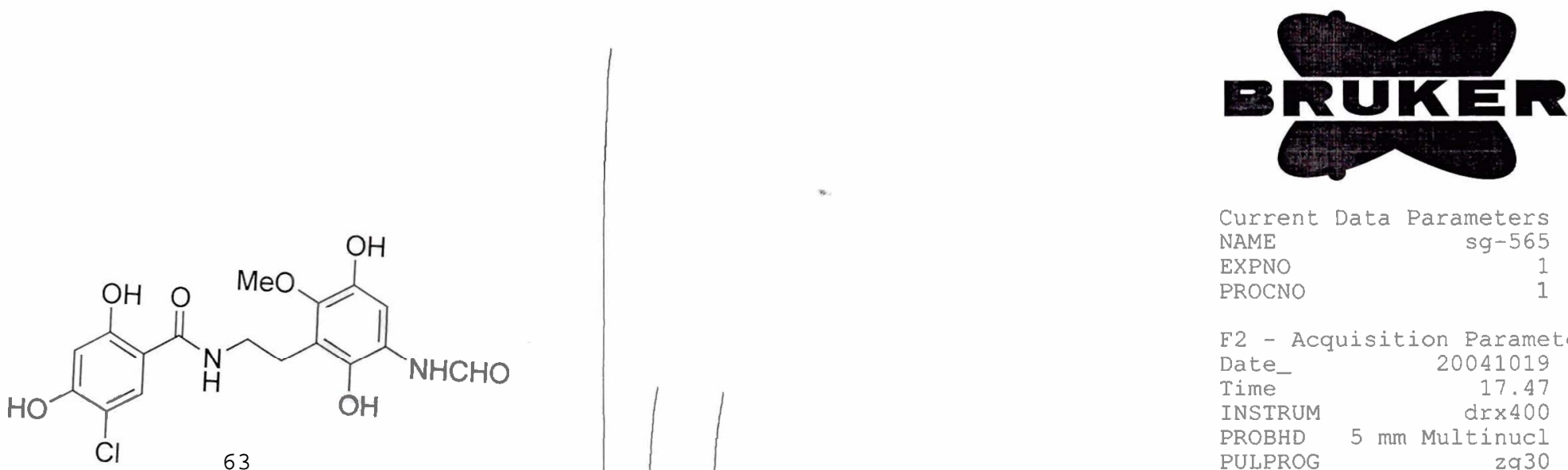

EXPNO

F2 - Acquisition Parameters

Date_ 20041019

Time

17.47

INSTRUM

PROBHD

PULPROG

drx 400

TD

$5 \mathrm{~mm}$ Multinucl

SOLVENT

NS

DS

SWH

FIDRES

AQ

RG

DW

DE

TE

MCREST

MCWRK

zg 30
32768

$\mathrm{MeOH}$

16

$4789.272 \mathrm{~Hz}$

$0.146157 \mathrm{~Hz}$

$3.4210291 \mathrm{sec}$ 181

104.400 usec 5.50 usec $292.2 \mathrm{~K}$

$1.00000000 \mathrm{sec}$

$0.00000000 \mathrm{sec}$

$0.01500000 \mathrm{sec}$

$===+===-$ CHANNEL $\mathrm{f}$

NUC1

P1

PL1

SFO1

7. 70 usec

$-6.00 \mathrm{~dB}$

$400.1320007 \mathrm{MHz}$

22 - Processing parameters

SI 32768

SF $\quad 400.1300000 \mathrm{MHz}$

WDW

SSB

$\mathrm{EM}$

$0.30 \mathrm{~Hz}$

$L B$
$G B$

0

1. 00

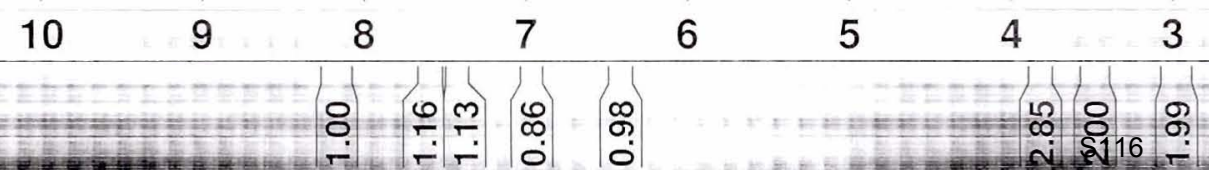



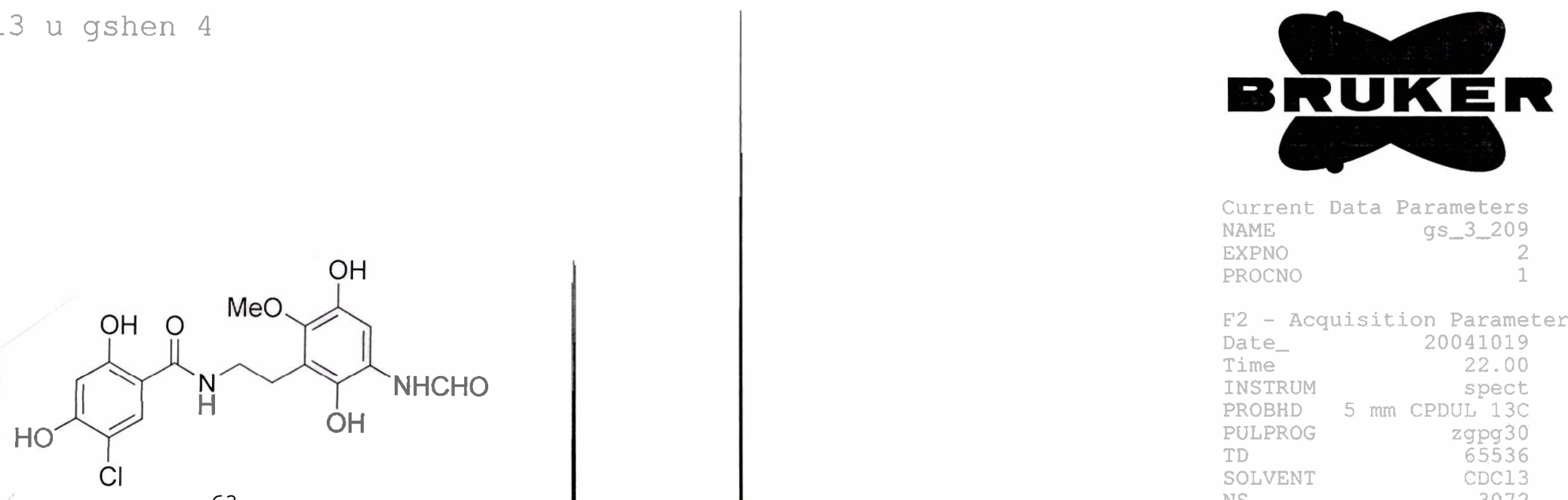

63

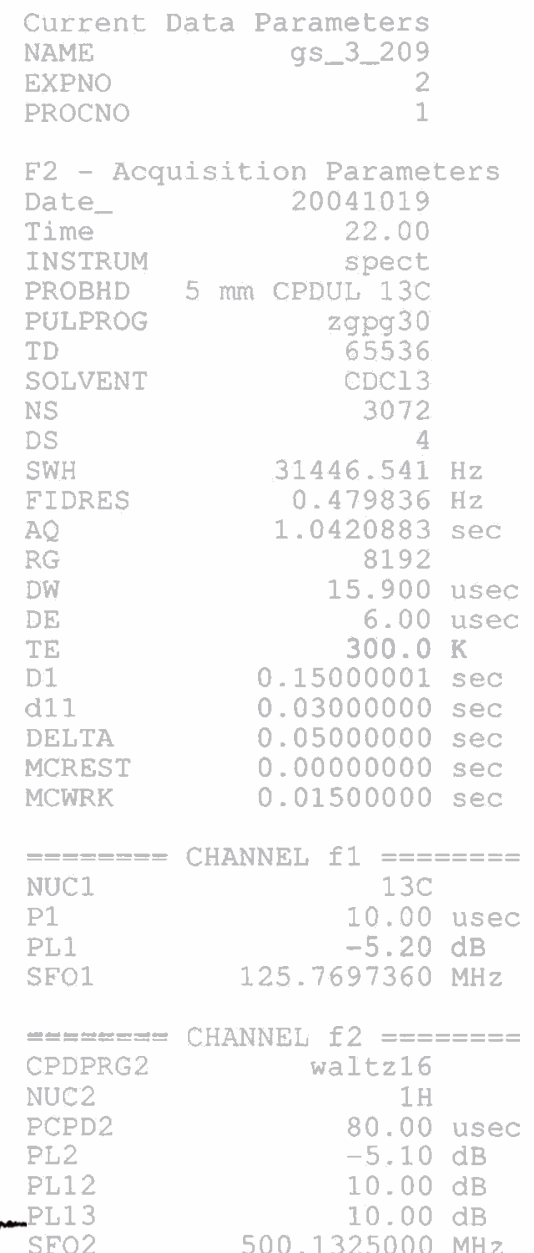


<smiles>COC1=C(CCNC(=O)c2cc(Cl)c(O)cc2O)C(=O)C(NC=O)=CC1=O</smiles>

\title{
DTUKER .
}

Current Data Parameters NAME sg-566 amide-quinone EXPNO PROCNO

F2 - Acquisition Parameters

Date 20041022

$\begin{array}{lr}\text { Date_- } & 20041022 \\ \text { Time } & 16.51\end{array}$

$\begin{array}{lr}\text { Time } & 16.51 \\ \text { INSTRUM } & \text { drx400 }\end{array}$

PULPROG

$5 \mathrm{~mm}$ Multinucl

ID

32768

$\mathrm{MeOH}$

NS

DS

FID

AQ

RG

DW

DE

TE

MCREST

MCREST

$M C W R$

$4789.272 \mathrm{~Hz}$

$0.146157 \mathrm{~Hz}$

$3.4210291 \mathrm{sec}$

256

104.400 usec 5.50 usec 293.2

1.00000000 sec

1.0000000000 secto

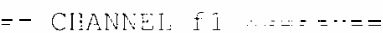
NUC]

$\mathrm{PL}$
$\mathrm{PL} 1$
$\mathrm{SFO}$

.70 use

$6.00 \mathrm{~dB}$

F2 - Processing parameters

SI 32768

SE $\quad 400.1300000 \mathrm{MHz}$

WDW

SSB

GB

EM
0
$0.30 \mathrm{~Hz}$
0
1.00

6

5

\begin{abstract}
4
\end{abstract}
3

2 


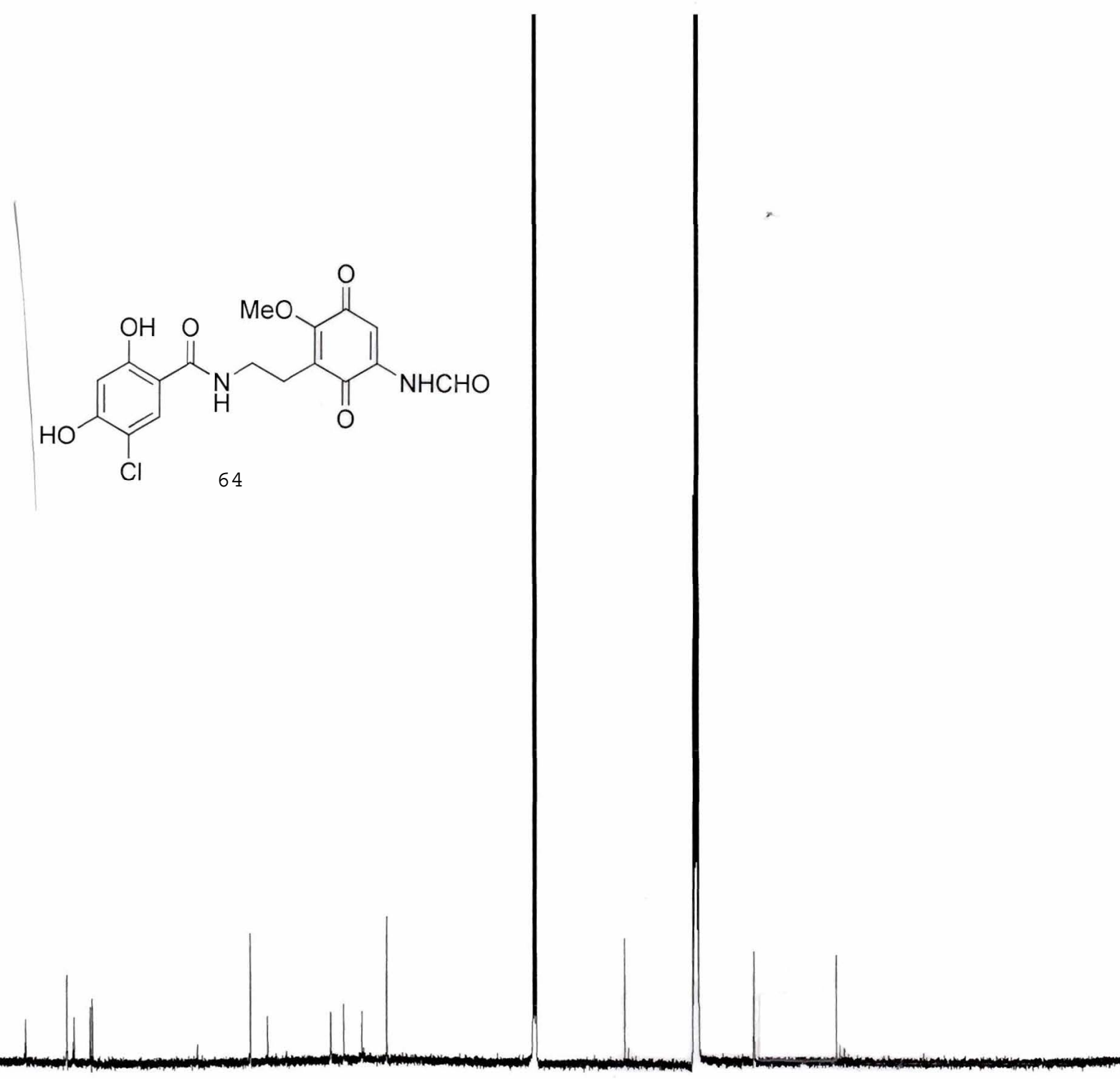

GRUKER

Current Data Parameters

NAME

EXPNO

F2 - Acquisition Parameters

Acquisition

20041029

INSTRUM

PROBHD $5 \mathrm{~mm}$ CPDUL $13 \mathrm{C}$

PULPROG

SOLYENT

DS

$\begin{array}{lr}\text { SWH } & 31446.541 \mathrm{hz} \\ \text { EIDRES } & 0.479836 \mathrm{~Hz}\end{array}$

RG

DW

TE

D11

MEREST

$=======$ CHANNEL f $1=======$
NUC1 $13 \mathrm{C}$

-5.20 dB

SFO1

125. $7697360 \mathrm{MHz}$

$=======$ CHANNEL $\mathrm{f} 2== \pm==== \pm$
Waltz16

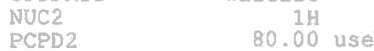

PL2

PL13

$10 \mathrm{~dB}$

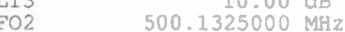

F1 - Acquisition parameters

NDO Acquisition parameters

TD $\quad 500.1325 \mathrm{MHz}$

$23.475060 \mathrm{~Hz}$

E2 - Processing parameters
SI 


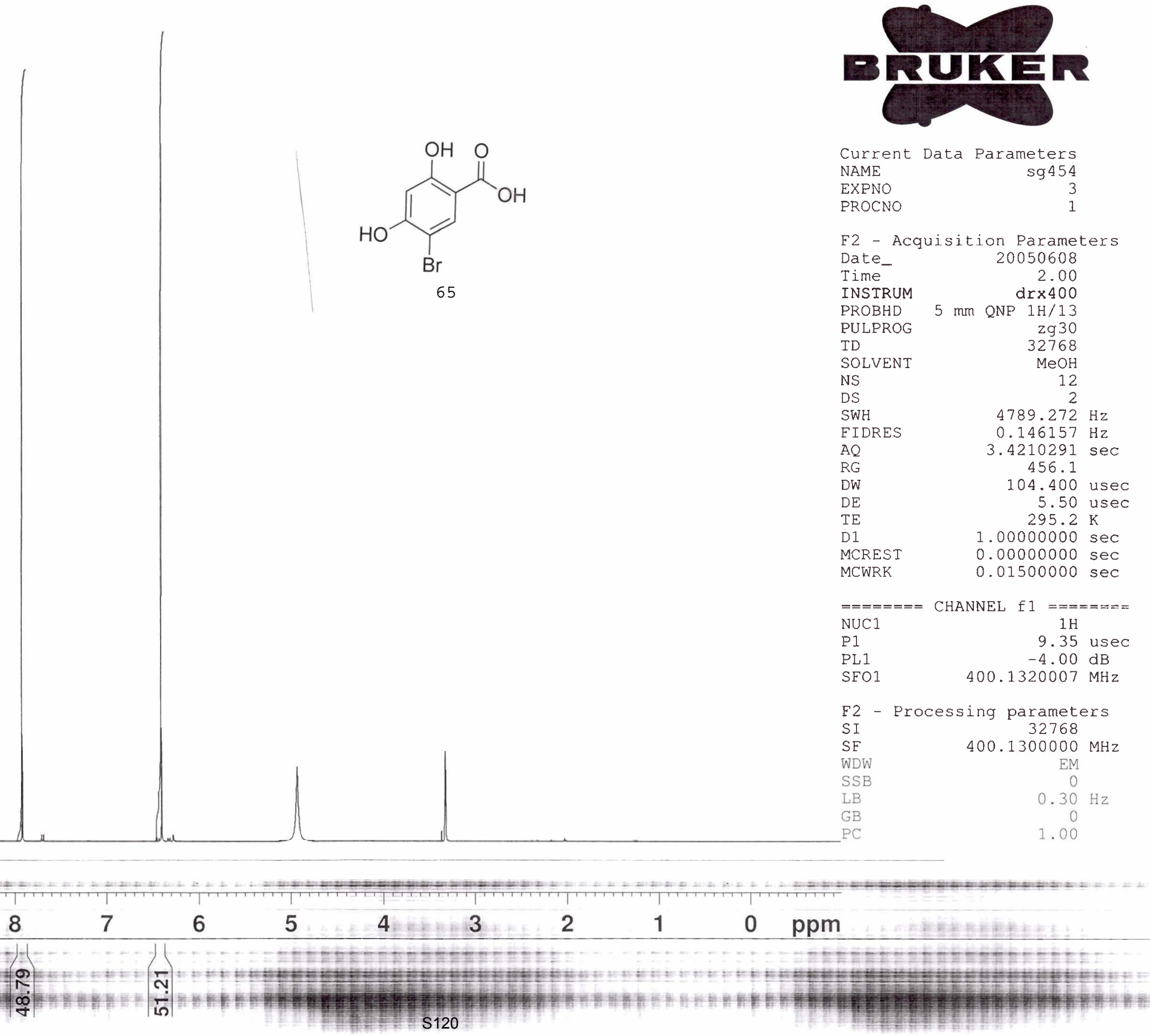




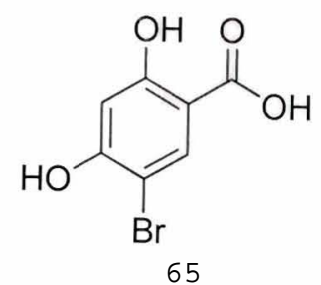

NAME

EXPNO

PROCNO

F2 - Acquisition Parameters

Date_ 20060415

Date_

INSTRUM

PROBHD

PULPROG

TD

SOLVENT

NS

DS
SWH

SWH
EIDRES

$\mathrm{AQ}$
$\mathrm{RG}$

DW

DE

D1

d11

DELTA

TD0

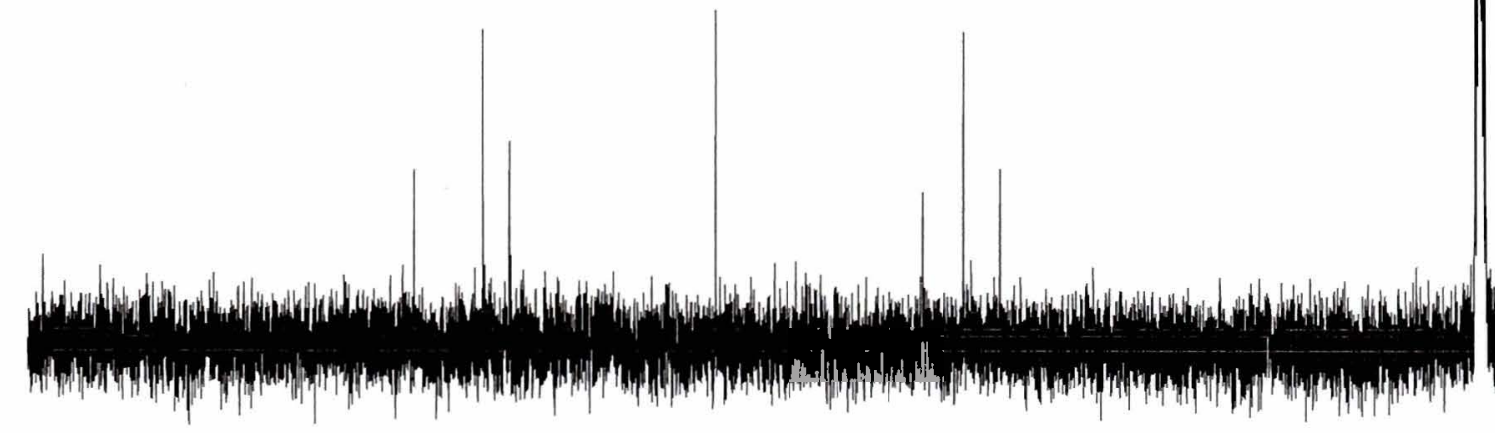

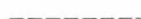

NUC

P1

PL1

SEO1

CPDPRG2

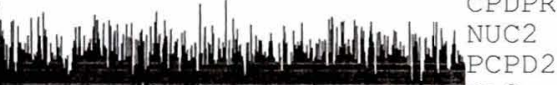

(T)

PCPD
PL2
PL12
PL13

SEO2 gang 04

2

0.24
$\operatorname{dr} \times 400$

$5 \mathrm{~mm}$ QNP $1 \mathrm{H} / 1$

zgpg 30

DMSO

253

$23980.814 \mathrm{~Hz}$

$0.365918 \mathrm{~Hz}$

$1.3664756 \mathrm{sec}$ 32768

20.850 usec

6.00 usec

$296.2 \mathrm{~K}$

$2.00000000 \mathrm{sec}$

$0.03000000 \mathrm{sec}$

$1.89999998 \mathrm{sec}$

CHANNEL $f 1========$

$13 \mathrm{C}$

9.85 usec

$100.6228298 \mathrm{MHz}$

CHANNEL $f$

waltz 16

100.00 usec

$-5.00 \mathrm{~dB}$

$14.58 \mathrm{~dB}$

$14.58 \mathrm{~dB}$

$400.2316005 \mathrm{MHz}$

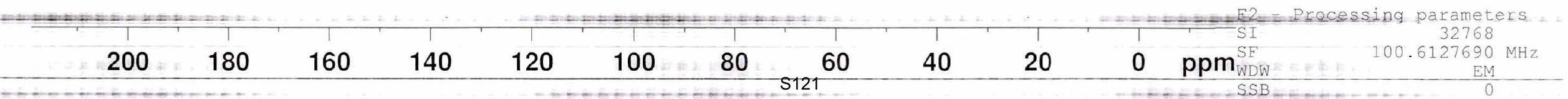




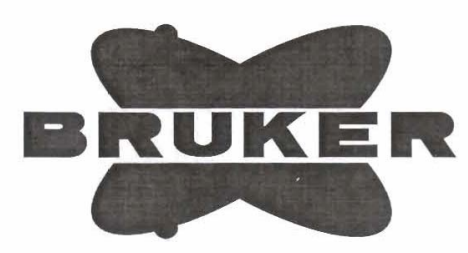

Current Data Parameters NAME

EXPNO

PROCNO

E2 - Acquisition Parameters

Date_ 20060415

Time

1.03

INSTRUM

PROBHD

PULPROG

TD

SOLVENT

NS

DS

FIDRES

AQ

AQ

DW

$\mathrm{DE}$

TE

D1

TD0

$===$
NUC 1

P1

PL1

SFO1

F2 - Processing parameters

SI 32768

SF $\quad 400.1300000 \mathrm{MHz}$

WDW

SSB

LB

$G B$

PC

EM
0
$0.30 \mathrm{~Hz}$
0
1.00


<smiles>COc1cc([N+](=O)[O-])c(OC)c(CC(C)OC(=O)c2cc(Br)c(O)cc2O)c1OC</smiles>

67
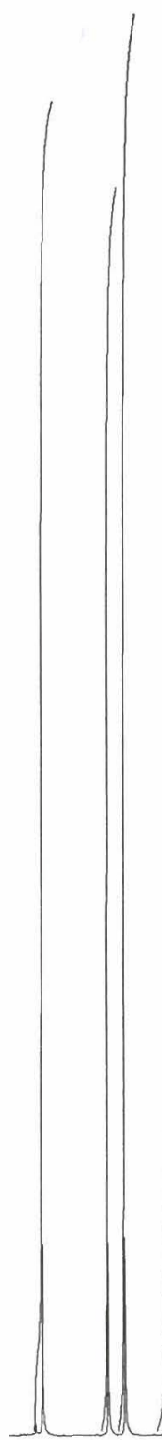

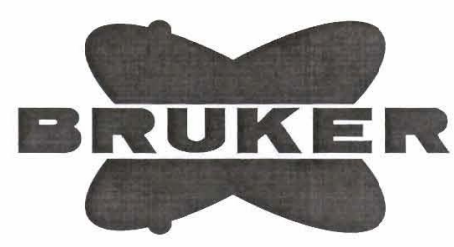

Current Data Parameters NAME

EXPNO

PROCNO

F2 - Acquisition Parameters

Date_ 20050709

Time

5.5

INSTRUM

PROBHD

PULPROG

TD

SOLVENT

NS

DS

FIDRES

$A Q$

$A Q$
$R G$

RG

$\mathrm{DE}$

TE

D1

MCWRK

$5 \mathrm{~mm}$ ONP $1 \mathrm{H} / 13$

$\mathrm{zg} 30$

2930
32768

CDCl3

16
2

$4789.272 \mathrm{~Hz}$

$0.146157 \mathrm{~Hz}$

$3.4210291 \mathrm{sec}$ 287.4

$10 \leqslant .400$ usec 5.50 usec $295.2 K$

$1.00000000 \mathrm{sec}$

$0.00000000 \mathrm{sec}$

$0.01500000 \mathrm{sec}$

$=======$ CHANNEL $\mathrm{f}$ NUC1

$\mathrm{P} 1$

$1===$

9.35 usec

$-4.00 \mathrm{~dB}$

SFO1 $400.1320007 \mathrm{MHz}$

F2 - Processing parameters

SI 32768

SF $\quad 400.1300000 \mathrm{MHz}$

WDW

SSB

LB

$\mathrm{PC}$
$E M$
0
0.30
0
0
1.0

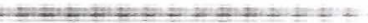

$x+10$

9

8

7

6

$5=4$

3

2

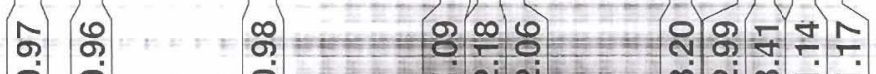

ó| 

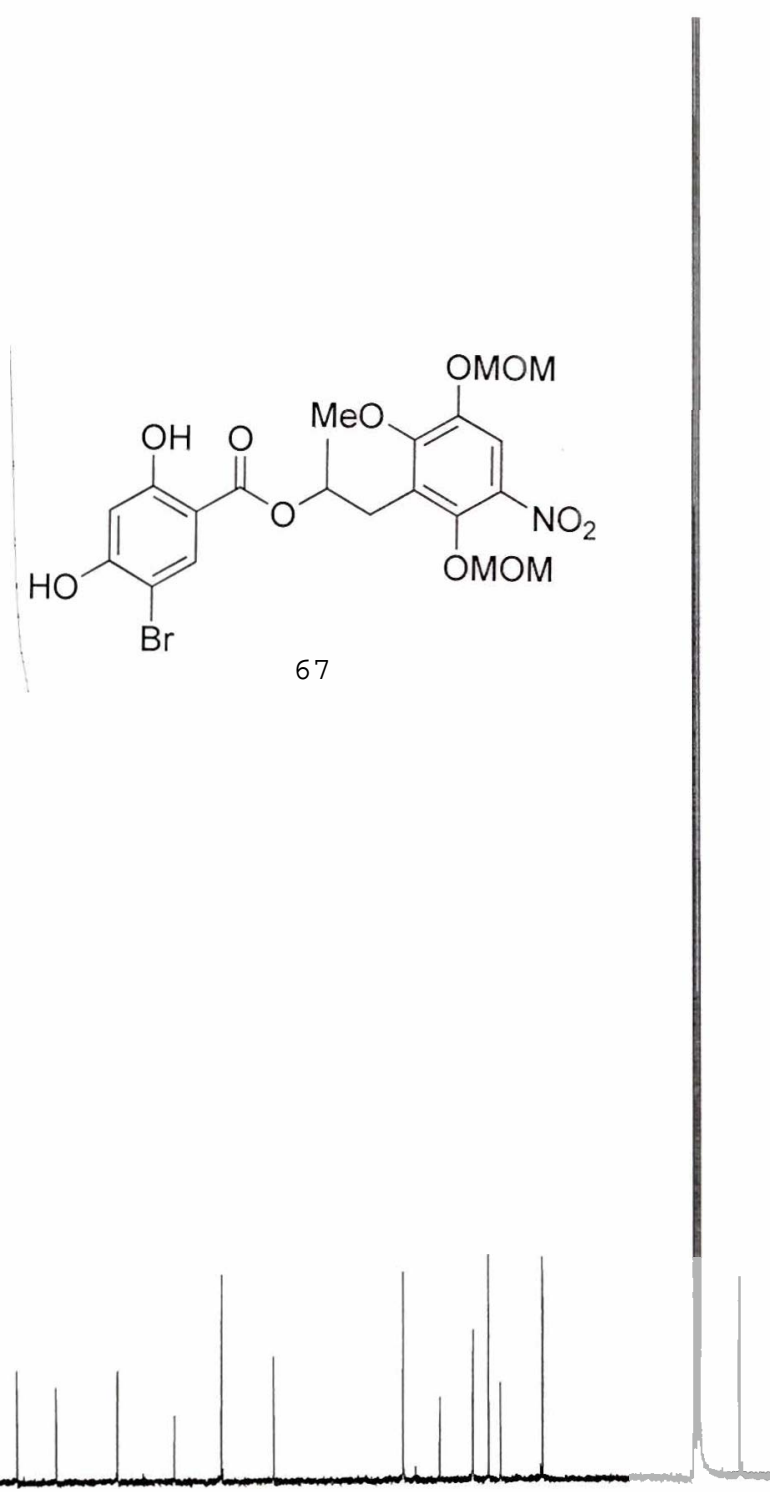

- TUKER

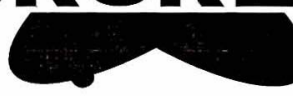

Current Data Paramec

NAME

EXPNO

PROCNO

F2 - Acquisition Parameters

Date__ 20050709

Time

INSTRUM

PROBHD

PULPROG

TD

SOLVENT

NS

DS

SWH

FIDRES

AQ

$\mathrm{RG}$
$\mathrm{DW}$
$\mathrm{DE}$

DE

D1

d.11

DELTA

MCWRK

15.37

drx 400

$5 \mathrm{~mm}$ ONP $1 \mathrm{H} / 13$

zgpg 30

$\mathrm{CDC} 13$

21504

$23148.148 \mathrm{~Hz}$

$1.4156276 \mathrm{sec}$

32768

21.600 usec

5.50 usec $295.2 \mathrm{~K}$

$0.15000001 \mathrm{sec}$

$0.03000000 \mathrm{sec}$

0.05000000 sec

$0.00000000 \mathrm{sec}$

$0.01500000 \mathrm{sec}$

$=== \pm== \pm=$ CHANNEL I 1

NUC1

P1

f $1==$

25 usec

11.25 usec

PL1 1 $2933 \mathrm{MHz}$

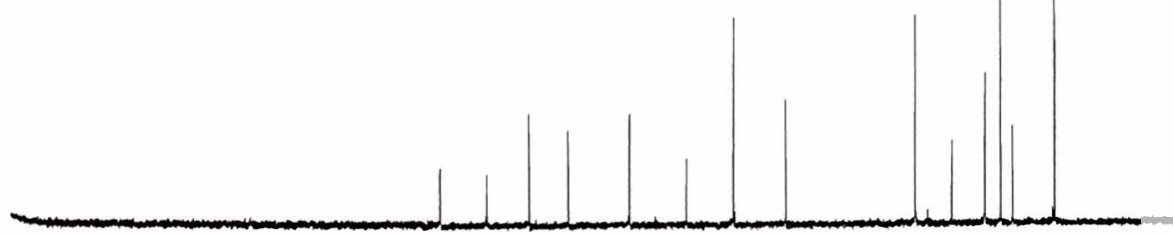

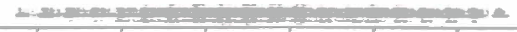

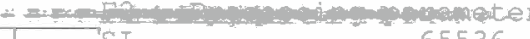

.$\overline{80}$


<smiles>COc1cc([N+](=O)[O-])c(OC)c(CC(C)OC(=O)c2cc(I)c(O)cc2O)c1OC</smiles>

68

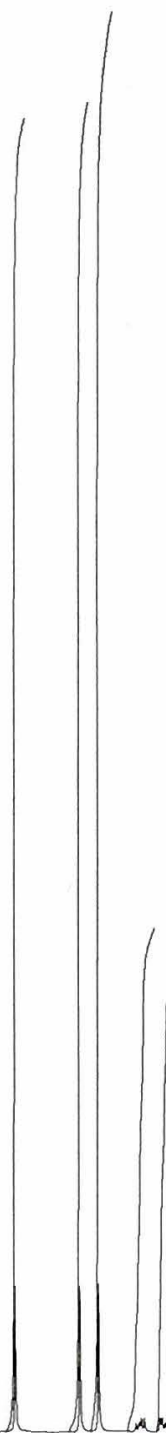

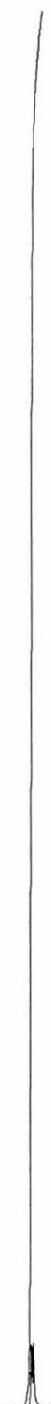

BRUKER $C$ NAME EXPNO

PROCNO

F2 - Acquisition Parameters Date_ 20050616

Time

21.22

INSTRUM dr 400

PROBHD $5 \mathrm{~mm}$ ONP 1H/13

PUTPROG ID

SOLVENT

NS

DS

SWH

EIDRES

$A Q$

RG

DW

TE

D1

MCREST

MCWRK

2930
32768

CDC13

16
2

$4789.272 \mathrm{~Hz}$

$0.146157 \mathrm{~Hz}$

$3.4210291 \mathrm{sec}$

287.4

104.400 used 5.50 usec $298.2 \mathrm{k}$

$1.00000000 \mathrm{sec}$

0.00000000 sec

NUC1

$\mathrm{P} 1$

$0.01500000 \mathrm{sec}$

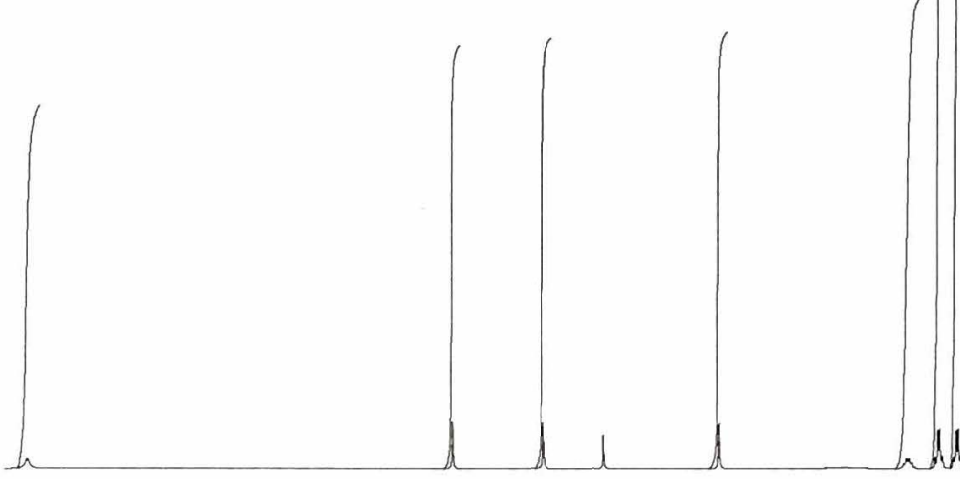

u

1

0 ppm

$1 \mathrm{H}$

9.35 used

$4.00 \mathrm{~dB}$

$400.1320007 \mathrm{MHz}$

- Processing parameters

$4001300000 \mathrm{MH}$

EM

0
0.30

1.00

$\begin{array}{llll}6 & 5 & 4 & 3\end{array}$

3

2

8

7

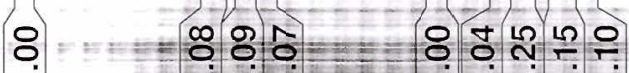

-

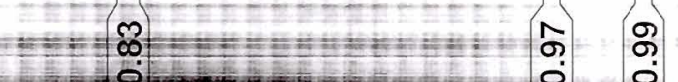
A. W a

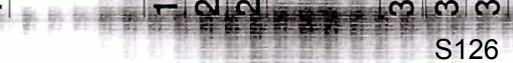

จิ

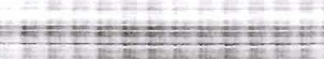

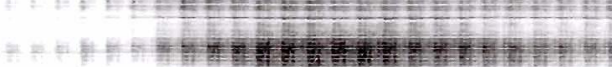

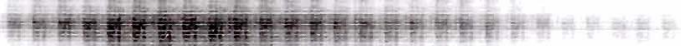




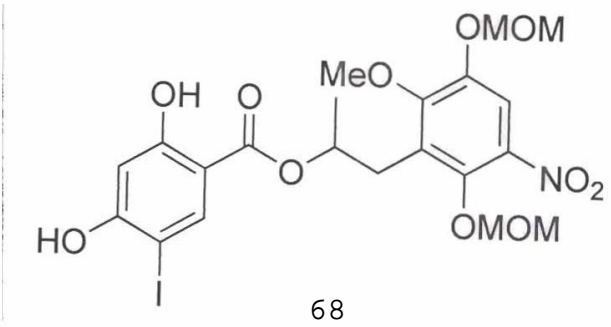

68

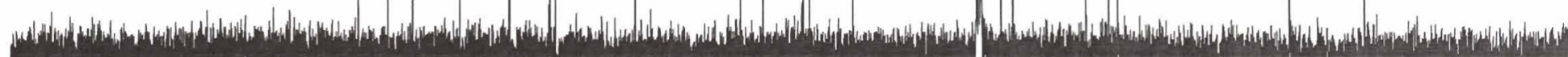

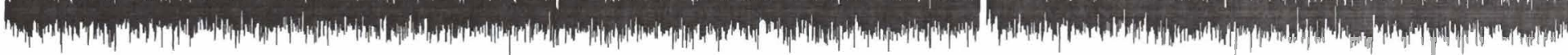

$-3 \mathrm{ROP}=\mathrm{E}$ C

current Data Parameters NAME

EXPNO

PROCNO

s.g 45

Date

Parameters

Time

20050616

INSTRUM Or\&400

PROBHD $5 \mathrm{~mm}$ ONP $1 \mathrm{H} / 13$

PULPROG 2gpg 30

SOLVENI

NS

DS

SWH

E'IDRES

$\mathrm{AO}$

RG

DW

DE

d11

DELTA

MCREST

MCWRK

645
4

$23148.148 \mathrm{~Hz}$

$0.353213 \mathrm{~Hz}$

$1.4156276 \mathrm{sec}$ 32768

21.600 usec

5.50 usec $298.2 \mathrm{~K}$

$0.15000001 \mathrm{sec}$

$0.03000000 \mathrm{sec}$

$0.05000000 \mathrm{sec}$

$0.00000000 \mathrm{sec}$

$0.01500000 \mathrm{sec}$

NUC1

P1

PL1

SEO1

$$
\begin{aligned}
& 1====== \\
& 13 \mathrm{C} \\
& 11.25 \mathrm{usec} \\
& 2.00 \mathrm{~dB}
\end{aligned}
$$

$==$ CHANNEL $f$

CPDPRG2

NUC2

PCPD

PLI
PL12
PL13

SEO2
200

180

160

140

120

100

80

S127 
<smiles>COc1cc(N)c(OC)c(CC(C)OC(=O)c2cc(Br)c(O)cc2O)c1OC</smiles>

69

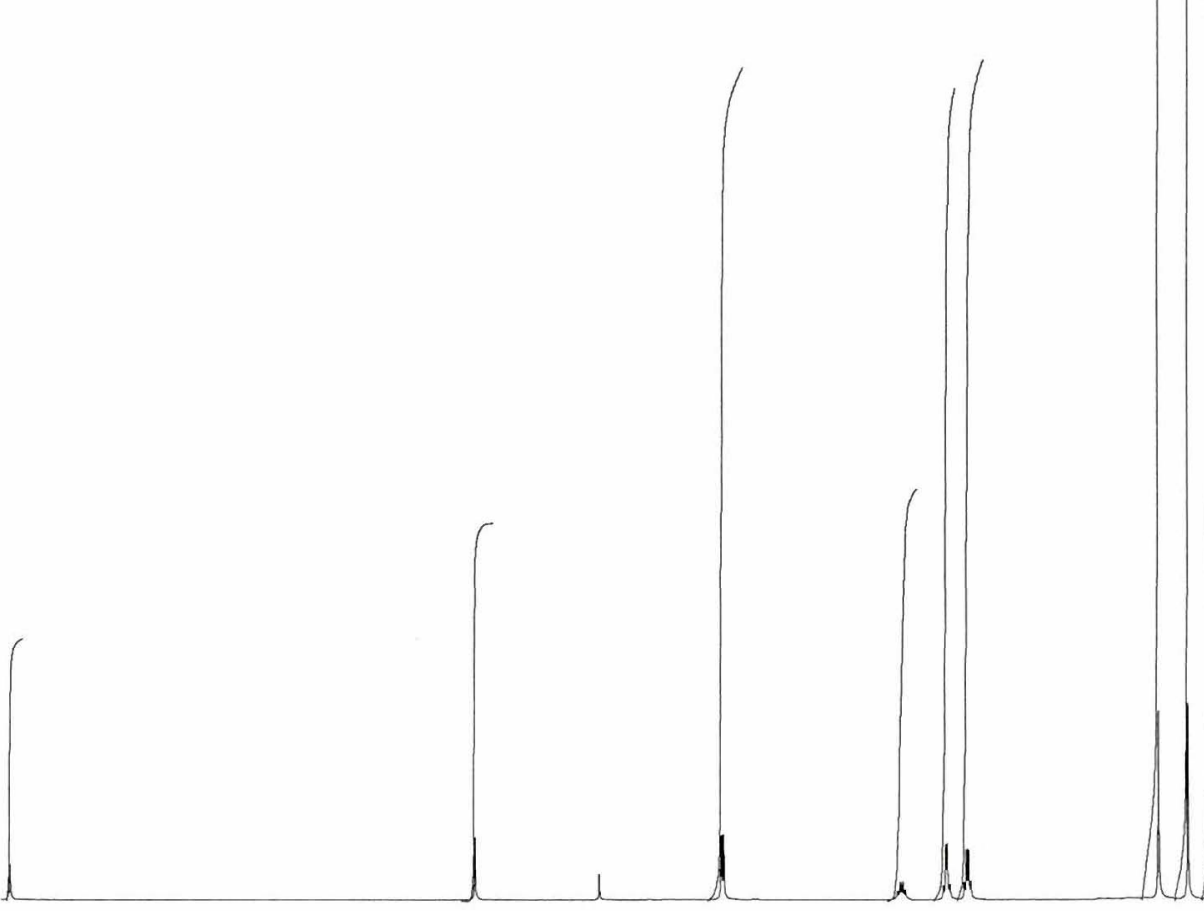

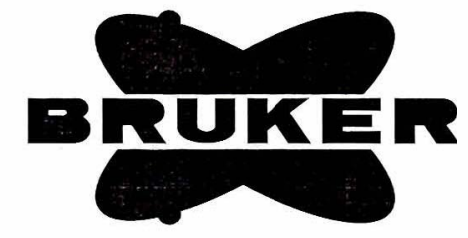

Current Data Parameters NAME

EXPNO sg458brnh2

PROCNO

$\mathrm{F} 2$ - Acquisjion Parameters Date__ 20050621

Time

2.05

INSTRUM

PROBHD

a 5400

PULPROG

TD

$5 \mathrm{~mm}$ ONP $3 \mathrm{H} / 13$

SOLVENT

NS

DS

FIDRES

FIDR
AQ
RG

DW

DE

TE

MCREST

MCWRK

$\mathrm{zg} 30$

32768

$\mathrm{CDC13}$

16
2

$4789.272 \mathrm{~Hz}$

$0.146157 \mathrm{~Hz}$

$3.4210291 \mathrm{sec}$ 203.2

104.400 usec $295.2 \mathrm{~K}$

I. $00000000 \mathrm{sec}$

0.00000000 sec

$0.01500000 \mathrm{sec}$

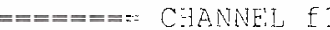

NUC1

P]

PL1

$1 \mathrm{H}$

9.35 used

$-4.00 \mathrm{~dB}$

$400.1320007 \mathrm{MHz}$

F2 - Processing parameters 32768 $400.1300000 \mathrm{MHz}$

EM

$0.30 \mathrm{~Hz}$

1.00

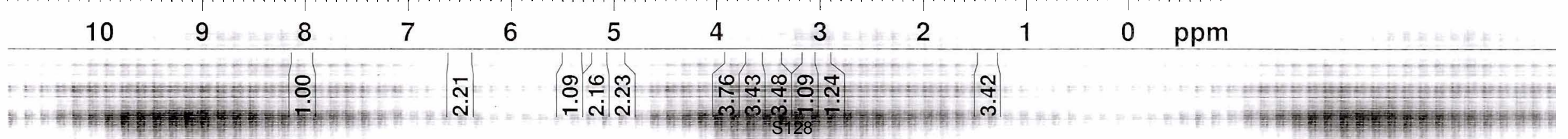


<smiles>COc1cc(N)c(OC)c(CC(C)OC(=O)c2cc(Br)c(O)cc2O)c1OC</smiles>

69
BRUKER C
NAME

EXPNO

F2 - Acquisition Parameters Date__ 20050618

Time

INSTRUM

PROBHD

PULPROG

TD

SOLVENT

NS

DS

FIDRES

AQ

RG

$\mathrm{DW}$
$\mathrm{DE}$

$\mathrm{DE}$

D1

d11

DELTA

MCREST

MCWRK

$====$

NUC1

P1

$\mathrm{SFO} 1$

$====$
CPDPRG

NUC2

NUC2
PCPD2

$\mathrm{PL} 2$
22.03

$\operatorname{dr} \times 400$

$5 \mathrm{~mm}$ QNP $1 \mathrm{H} / 13$

zgpg 30

65536

$\mathrm{CDCl} 3$

648

$23148.148 \mathrm{~Hz}$

$0.353213 \mathrm{~Hz}$

1. $4156276 \mathrm{sec}$ 32768

21.600 usec

5.50 usec 295.2 K

$0.15000001 \mathrm{sec}$

$0.03000000 \mathrm{sec}$

$0.05000000 \mathrm{sec}$

$0.00000000 \mathrm{sec}$

$0.01500000 \mathrm{sec}$

$$
\begin{gathered}
\mathrm{f} 1======= \\
13 \mathrm{C} \\
11.25 \text { usec } \\
2.00 \mathrm{~dB}
\end{gathered}
$$

$100.6232933 \mathrm{MHz}$

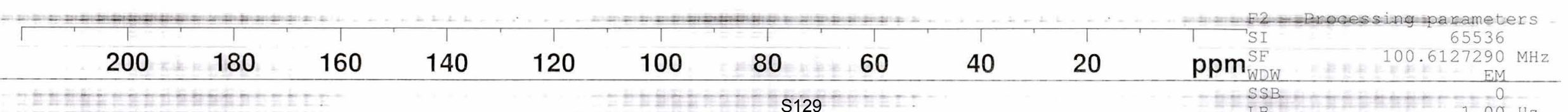

f 2

waltz16

100.00 use $-4.00 \mathrm{~dB}$

$16.58 \mathrm{~dB}$

$400.1316005 \mathrm{MHz}$ 


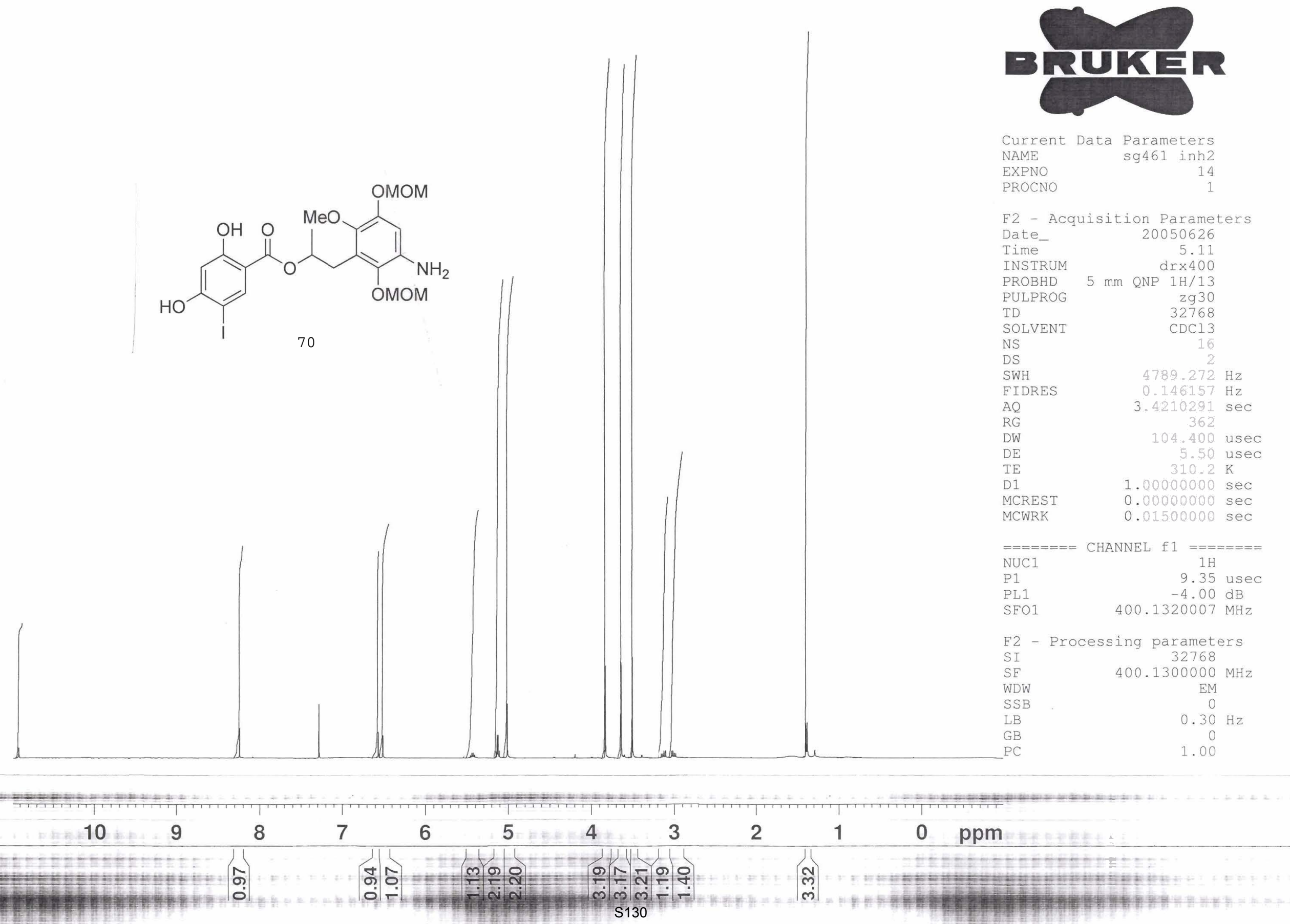



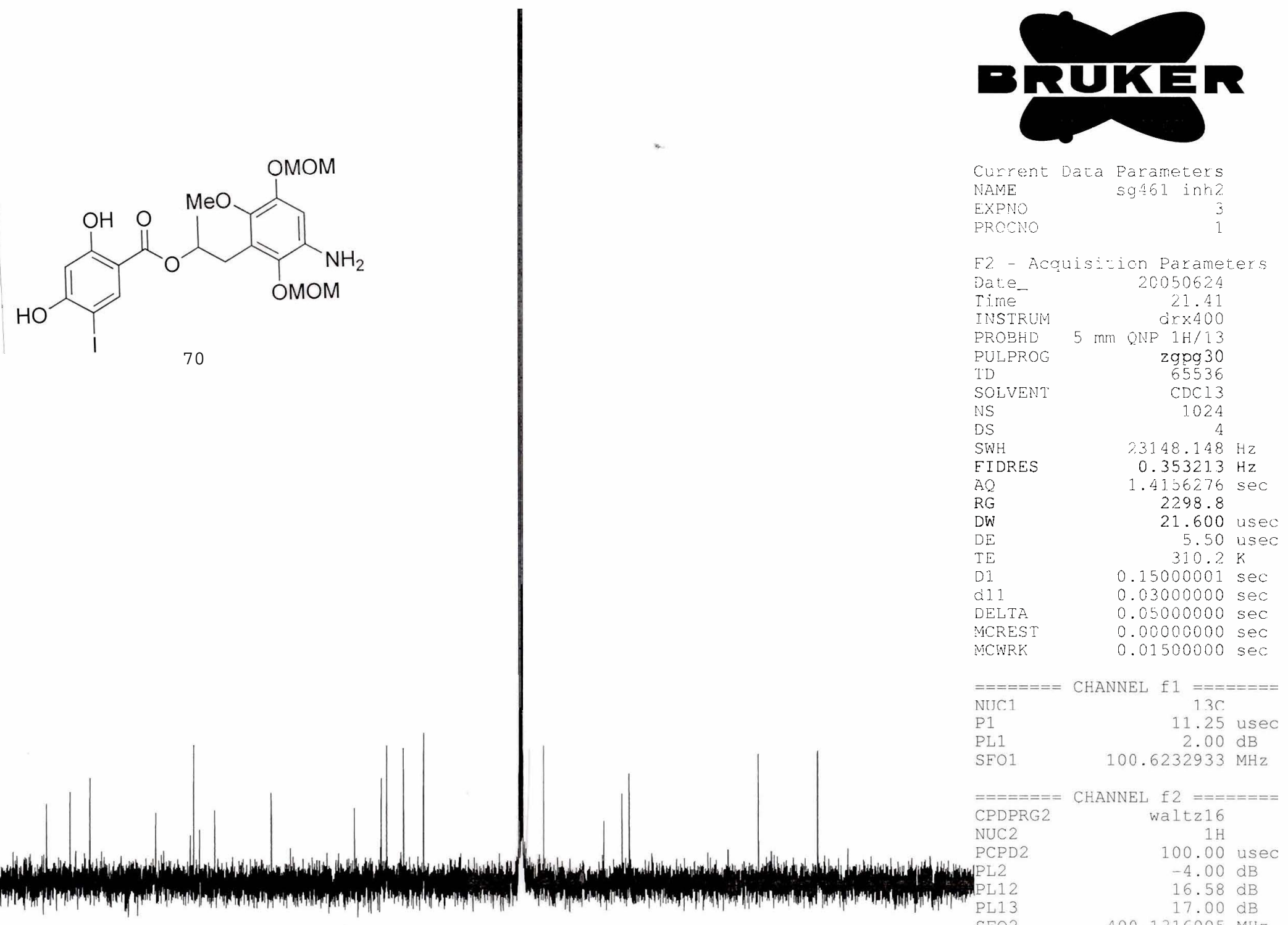

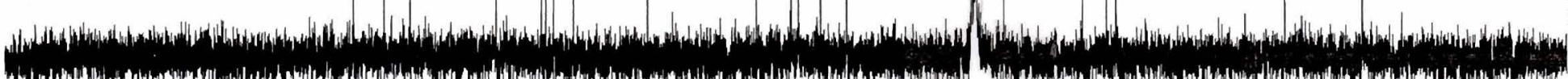

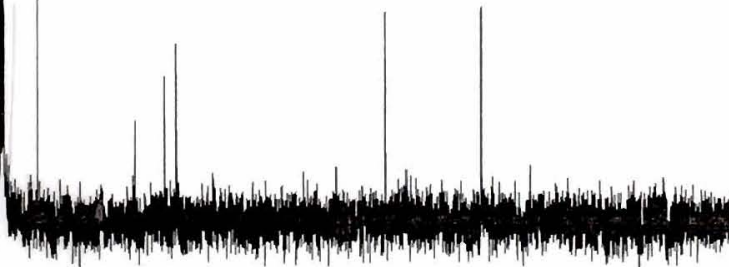

$\mathrm{SEO} 2$

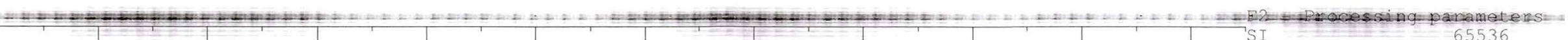




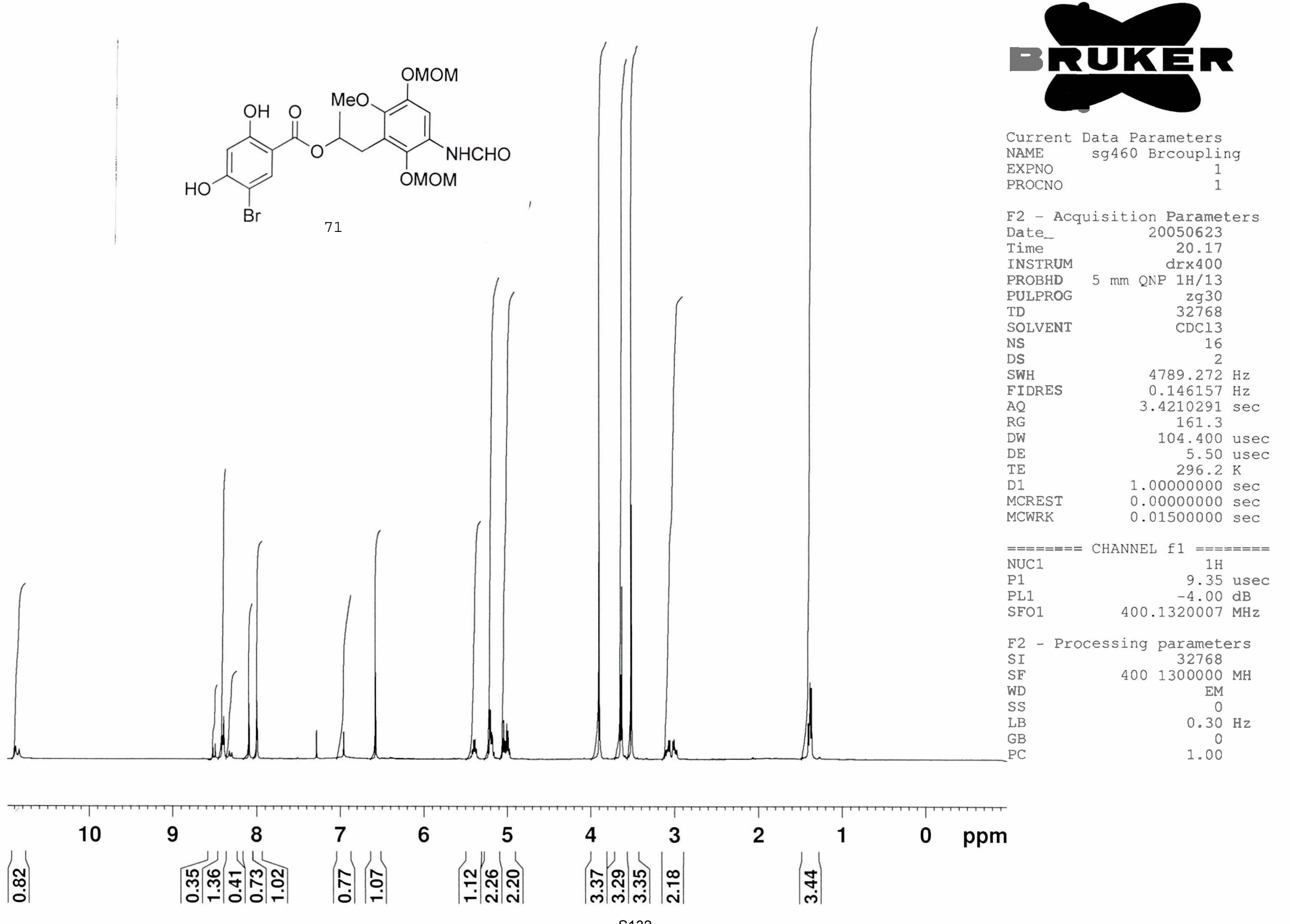



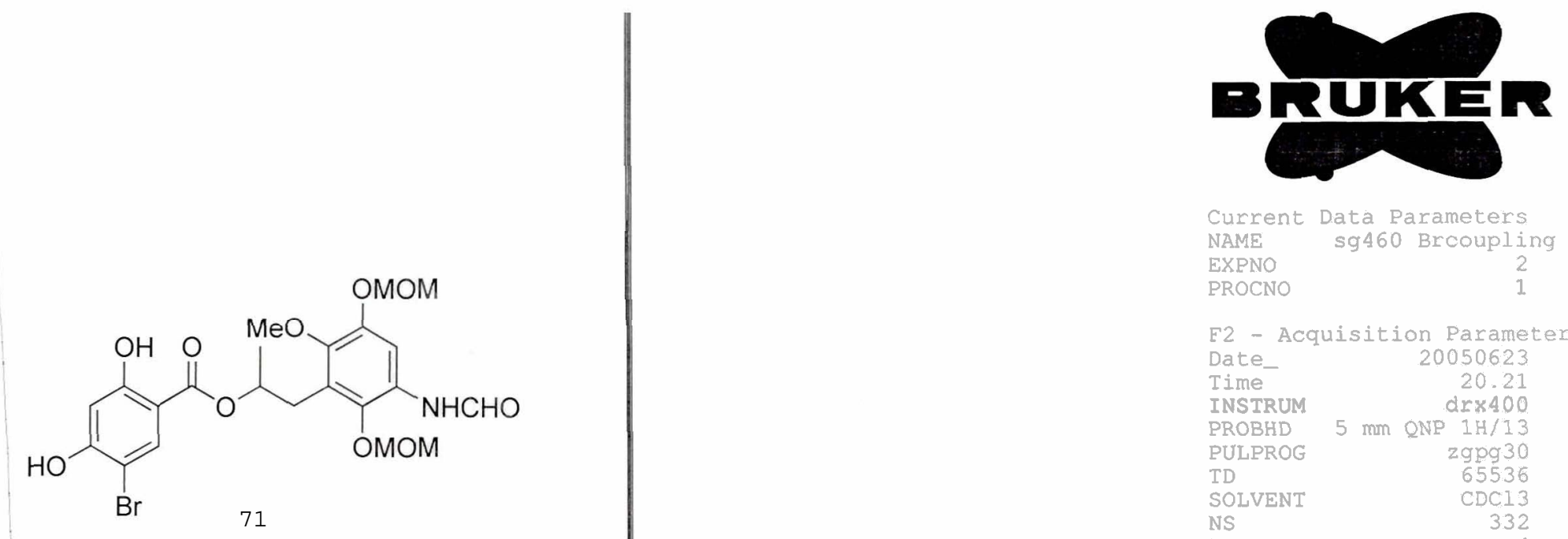

EXPNO

F2 - Acquisition Parameters

Date_ 20050623

Time

INSTRUM

PROBHD

PULPROG

TD

SOLVENT

NS

DS

SWH

FIDRES

$\mathrm{AQ}$

RG

$\mathrm{DW}$
$\mathrm{DE}$

DE

dII

DEITA

MCREST

MCWRK
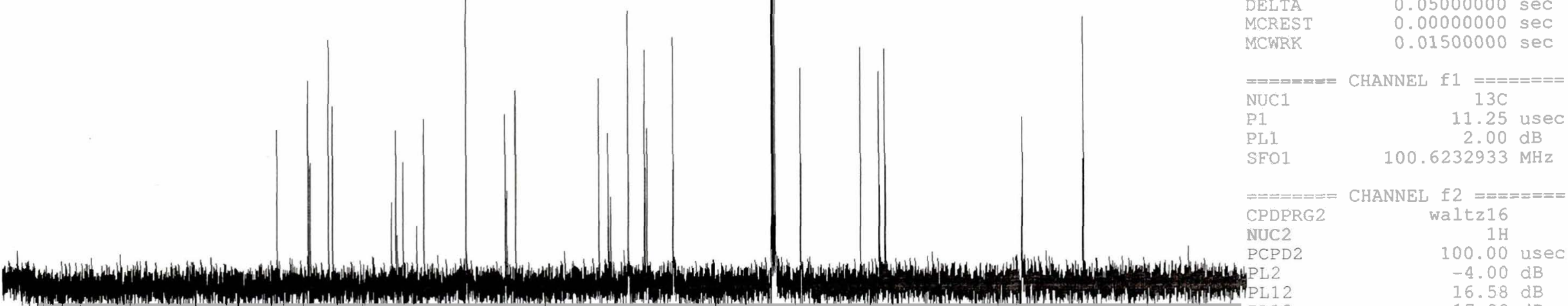

PL1

$100,6232933 \mathrm{MHz}$

CPDPRG2

NUC2

PCPD2

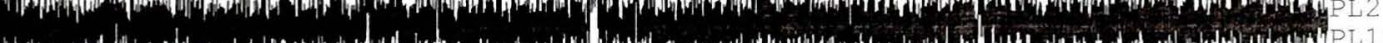

PLI3

CHANNEL f2

20.21
$0 \times 400$

mm ONP $14 / 13$

zgpg 30

$\mathrm{CDCl} 3$

332

$23148.148 \mathrm{~Hz}$

$0.353213 \mathrm{~Hz}$

$1.4156276 \mathrm{sec}$

32768

21.600 usec

5.50 usec

$296.2 \mathrm{~K}$

$0.15000001 \mathrm{sec}$

$0.03000000 \mathrm{sec}$

0.05000000 sec

$0.00000000 \mathrm{sec}$

01500000 sec 

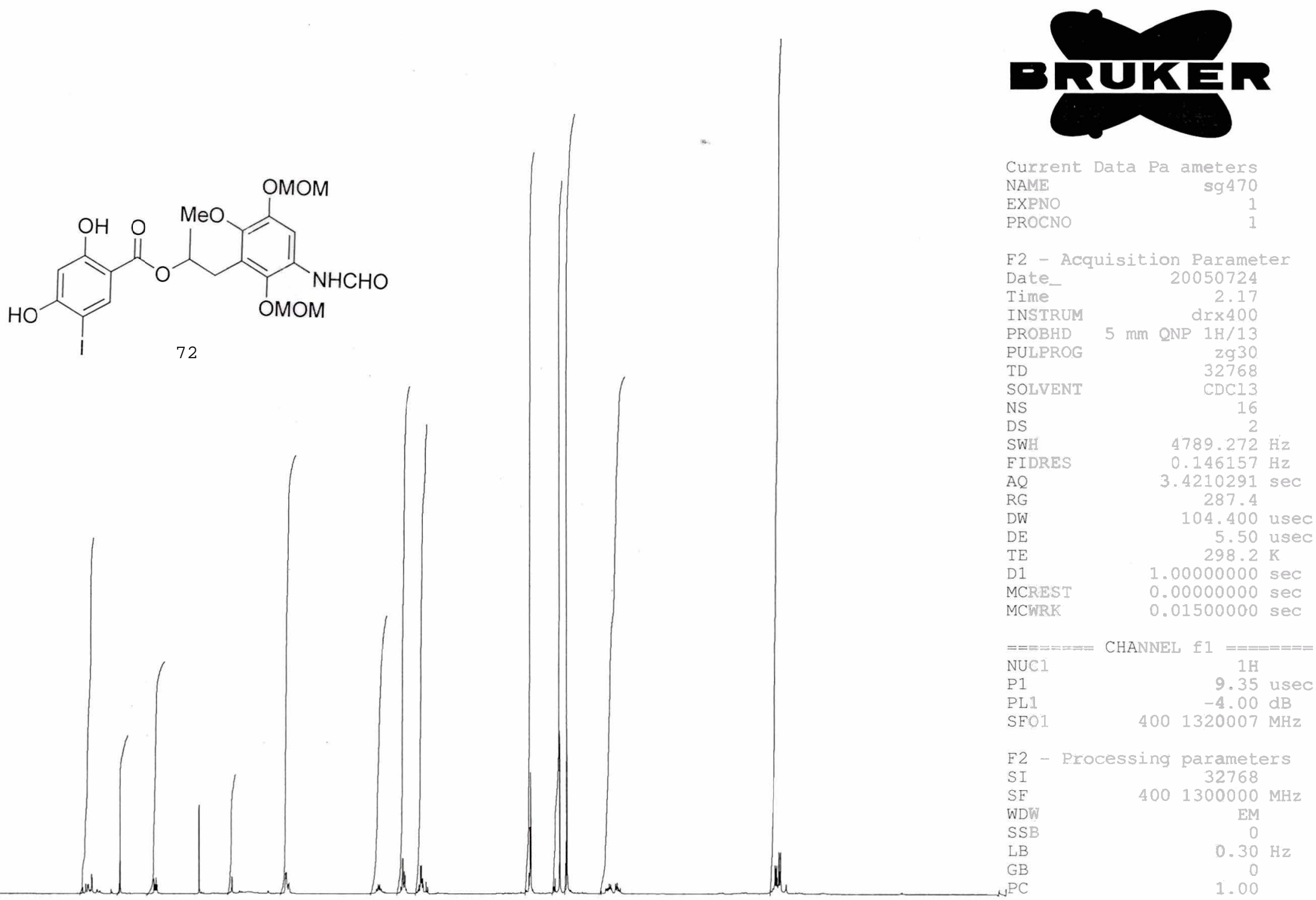

h h

$\begin{array}{lll}7 & 6 & 5\end{array}$

4

3

2

$1 \quad 0 \quad \mathrm{ppm}$

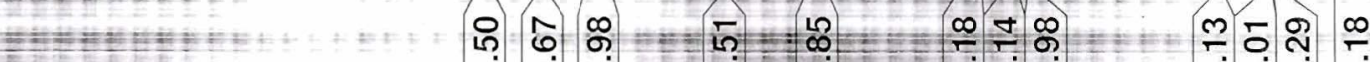

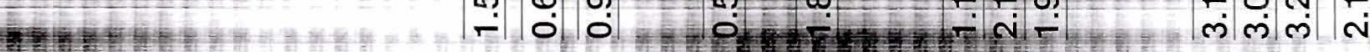




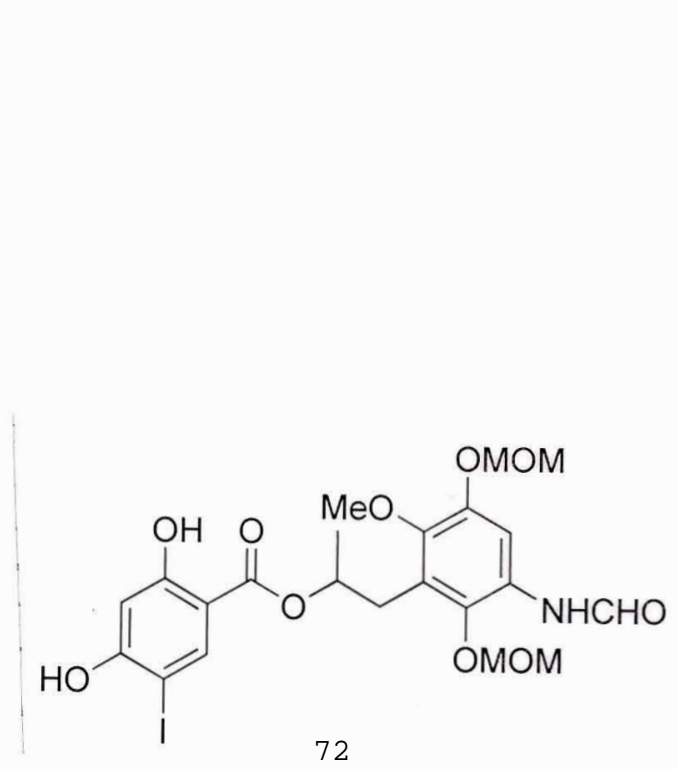

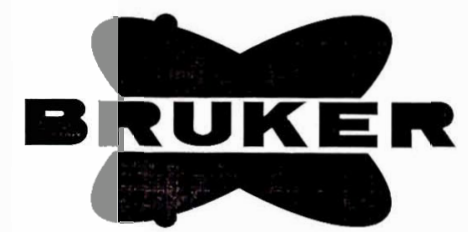

Current Data Parameters NAME $\operatorname{sg} 471$

EXPNO

PROCNO

F2 - Acquisition Parameters

Date

Time

INSTRUM

PROBHD

PULPROG

TD

SOLVENT

NS

DS

SWH

EIDRES

AQ

RG

DW

$\mathrm{DE}$

TE

d1 1

DELTA

MCREST

MCWRK

20050730

$5 \mathrm{drx} 400$

5 Im QNP 1H/13

zgpg 30

CDC13

293

$23148.148 \mathrm{~Hz}$

$0.353213 \mathrm{~Hz}$

$1.4156276 \mathrm{sec}$

4096

21.600 usec

5.50 usec $296.2 \mathrm{~K}$

$0.15000001 \mathrm{sec}$

$0.03000000 \mathrm{sec}$

$0.05000000 \mathrm{sec}$

$0.00000000 \mathrm{sec}$

$0.01500000 \mathrm{sec}$

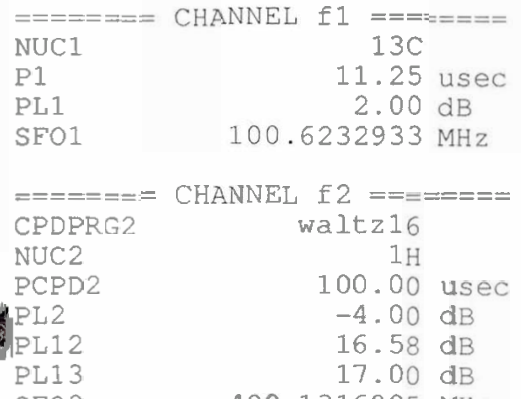

$400.1316005 \mathrm{MH}$

SSB


<smiles>COc1c(O)cc(NC=O)c(O)c1CC(C)OC(=O)c1cc(Br)c(O)cc1O</smiles>

73

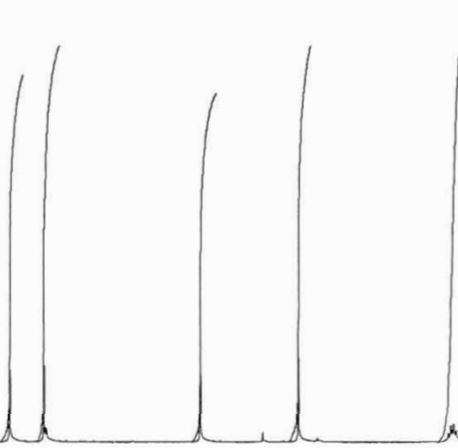

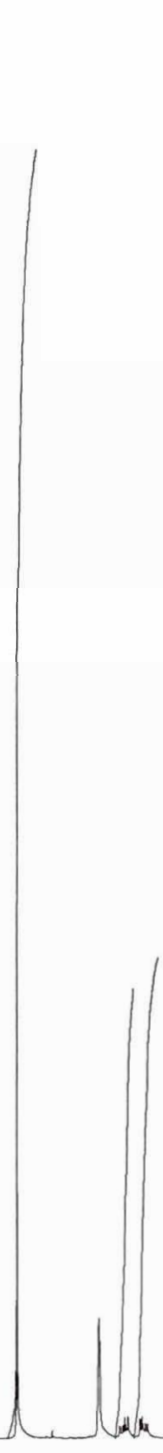

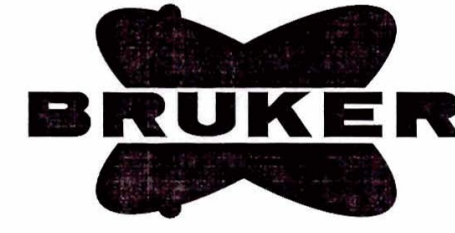

Current Data Parameters NAME

EXPNO

PROCNO

$\operatorname{sg} 465$

- Acquisition Parameters

Date_

Time

INSTRUM

PROBHD

PULPROG

TD

SOLVENT

NS

DS

SWH

FIDRES

AQ

DW

DE

TE

MCREST

MCWRK

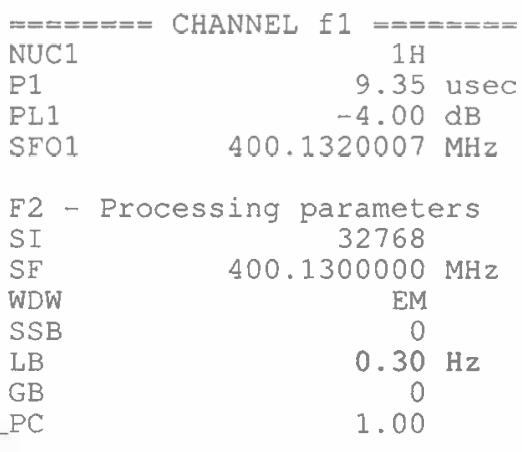



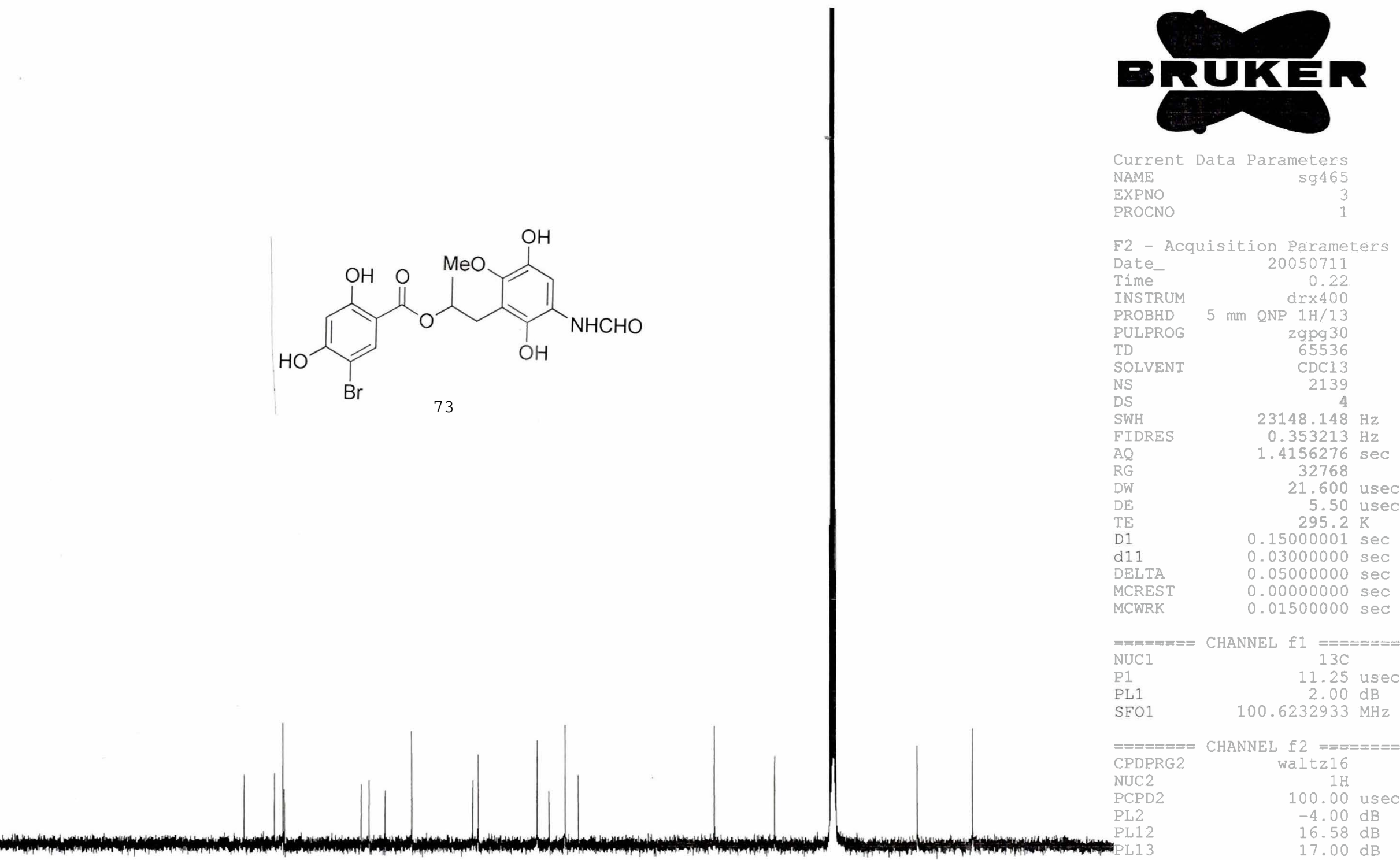

SFO

$100.6232933 \mathrm{MH}$

$\begin{array}{lr}\text { CPDPRG2 } & 1 \mathrm{H} \\ \text { NUC2 } & \text { waltz } 16 \\ \text { PCPD2 } & 100.00 \mathrm{usec} \\ \text { PL2 } & -4.00 \mathrm{~dB} \\ \text { PL12 } & 16.58 \mathrm{~dB} \\ \text { PL13 } & 17.00 \mathrm{~dB} \\ \text { SFO2 } & 400.1316005 \mathrm{MHz}\end{array}$

$400.1316005 \mathrm{MHz}$ 


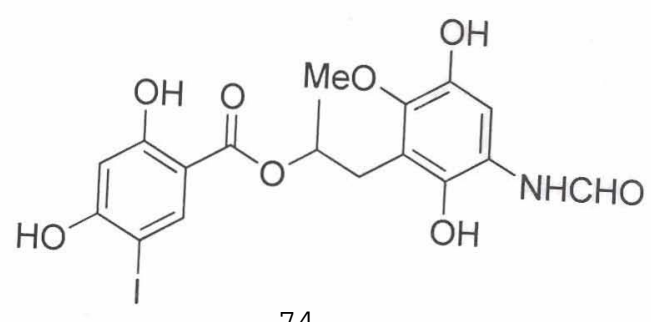

74

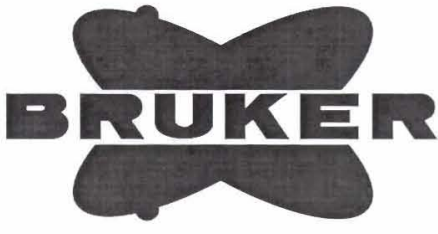

Current Data Parameters NAME

EXPNO

PROCNO

F2 - Acquisition Parameters

Date

Iime

INSTRUM

PROBHD

PULPROG

TD

SOLVENT

NS

SW

SWH

$\mathrm{AQ}$

RG

DW

PE

D1

dII

DELTA

MCREST

NUC

PL1

CPDPRG2

NUC2

PCPD2

PL1 12

PL 13

$\mathrm{SEO} 2$ so 471

1

20050729

5.24

$5 \mathrm{~mm}$ ONP $1 \mathrm{H} / 13$

zgpg 30

65536

CDC13

$23148,148 \mathrm{~Hz}$

$0.353213 \mathrm{~Hz}$

$1.4156276 \mathrm{sec}$

4156276
32768

21.600 used

5.50 used

$295.2 \mathrm{~K}$

$2.00000000 \mathrm{sec}$

$0.03000000 \mathrm{sec}$

$1.89999998 \mathrm{sec}$

0.00000000 sec

0.00000000 sec

$0.01500000 \mathrm{sec}$

CHANNEL f

$13 \mathrm{C}$

11.25 usec

$2.00 \mathrm{~dB}$

$100.6232933 \mathrm{MHz}$

CHANNEL $f$

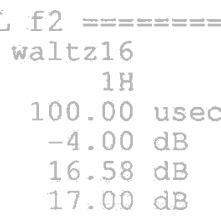

\begin{tabular}{|c|c|c|c|c|c|c|c|c|c|c|}
\hline$F=1$ & 1 & 1 & 1 & 1 & $y=1$ & 1 & 1 & 1 & & 65536 \\
\hline 200 & 180 & 160 & 140 & 120 & $100-80$ & 60 & 40 & 20 & $\mathrm{ppm}_{W D W}^{\mathrm{SE}}$ & $\begin{array}{c}100.6127290 \\
\mathrm{FM}\end{array}$ \\
\hline
\end{tabular}


<smiles>COC(CC1C(=O)C=C(NC=O)C(=O)C1=O)OC(=O)c1cc(Br)c(O)cc1O</smiles>

75
= =

\begin{tabular}{|c|c|c|c|}
\hline $\begin{array}{l}\text { Current } \\
\text { NAME } \\
\text { EXPNO } \\
\text { PROCNO }\end{array}$ & Data & $\begin{array}{r}\text { Parameters } \\
\text { sg } 466 \\
1 \\
1\end{array}$ & \\
\hline $2-A C D$ & quisi & ion Parame & ters \\
\hline Date_ & & 20050712 & \\
\hline ime & & 2.10 & \\
\hline NSTRUM & & $\operatorname{dr} \times 400$ & \\
\hline ROBHD & $5 \mathrm{~mm}$ & QNP $1 \mathrm{H} / 13$ & \\
\hline PULPROG & & $\mathrm{zg} 30$ & \\
\hline & & 32768 & \\
\hline SOLVENT & & CDCI3 & \\
\hline NS & & 16 & \\
\hline DS & & 2 & \\
\hline SWH & & 4789.272 & $\mathrm{~Hz}$ \\
\hline FIDRES & & 0.146157 & $\mathrm{~Hz}$ \\
\hline & & $\begin{array}{r}3.4210291 \\
574.7\end{array}$ & sec \\
\hline DW & & 104.400 & usec \\
\hline DE & & 5.50 & usec \\
\hline Th & & 296.2 & $\mathrm{~K}$ \\
\hline D1 & & .00000000 & sec \\
\hline & & .00000000 & sec \\
\hline NRK & & .01500000 & $\mathrm{sec}$ \\
\hline
\end{tabular}

CHANNEL $\mathrm{f}$

$\begin{array}{lr}\text { NUC1 } & 1 \mathrm{H} \\ \text { P1 } & 9.35 \mathrm{usec} \\ \text { PL1 } & -4.00 \mathrm{~dB}\end{array}$

SEO1 $400.1320007 \mathrm{MHz}$

E2 - Processing parameters

SI 32768

SF $\quad 400 \quad 1300000 \mathrm{MHz}$

WDW

SSB

LB
$E M$
0
0.30
0
0
1.0

$\begin{array}{llllllllllll}10 & 9 & 8 & 7 & 6 & 5 & 4 & 3 & 2 & 1 & 0 & \mathrm{ppm}\end{array}$

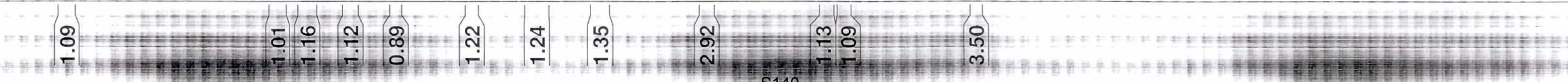



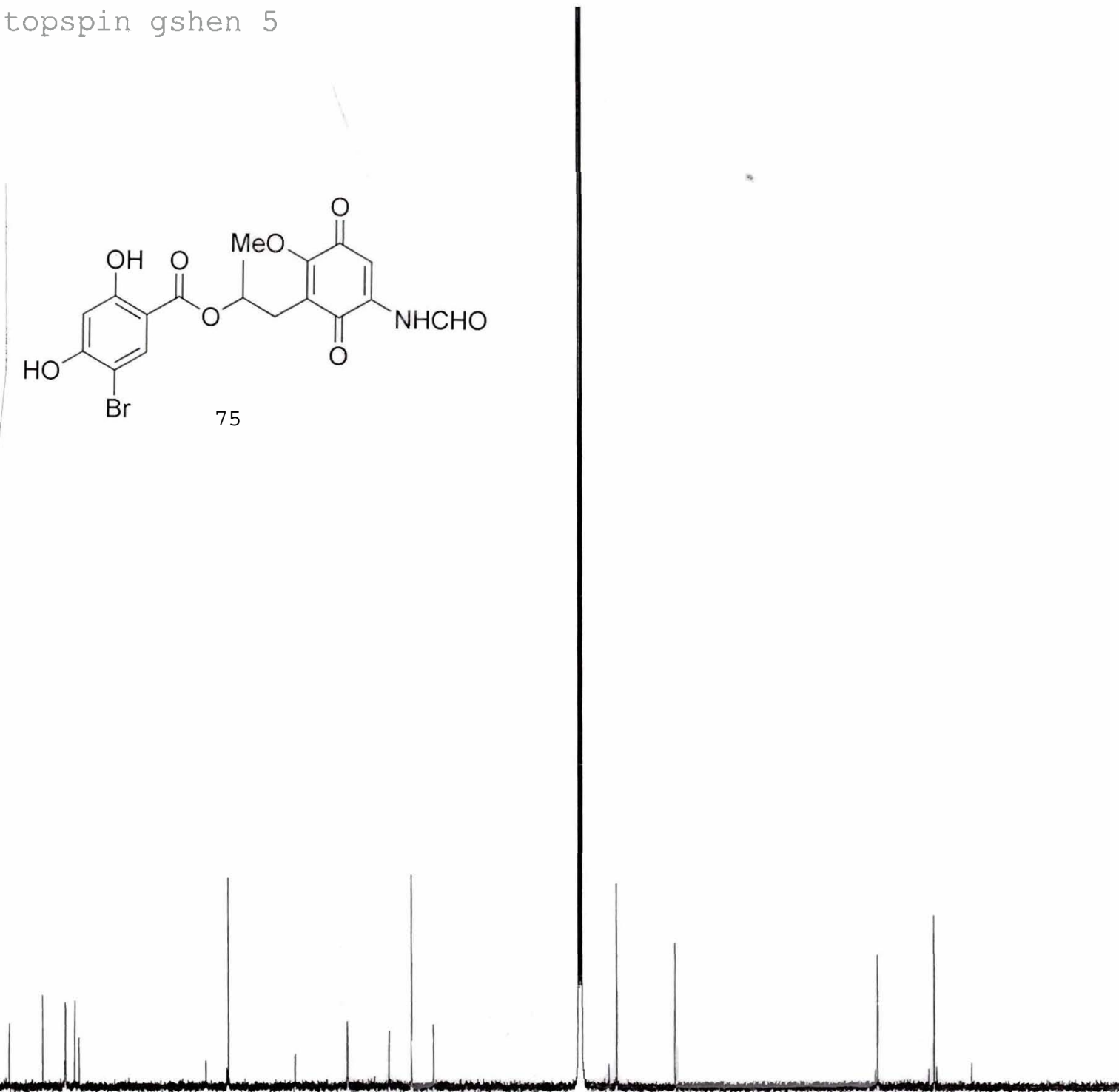

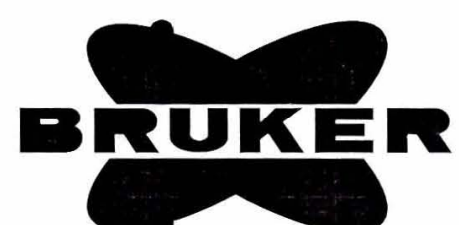

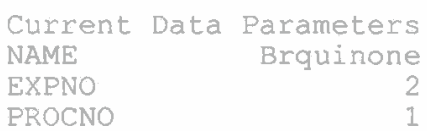

PROCNO

F2 - Acquisition Parameters

Date 20060414

Time

INSTRUM

PROBHD

PULPROG

SOLVENI

NS

SWH

FIDRES

AO

$\mathrm{RG}$

DW

TE

dI1

DELTA

TDO

20060414

spect

$5 \mathrm{~mm}$ CPDUL $13 \mathrm{C}$ zgpg 30

65536

CDC13

4

$30030.029 \mathrm{~Hz}$

$0.458222 \mathrm{~Hz}$

$1.0912410 \mathrm{sec}$

14596.5

16.650 usec

6.00 usec

$298.0 \mathrm{~K}$

$0.15000001 \mathrm{sec}$

$0.03000000 \mathrm{sec}$

$0.05000000 \mathrm{sec}$
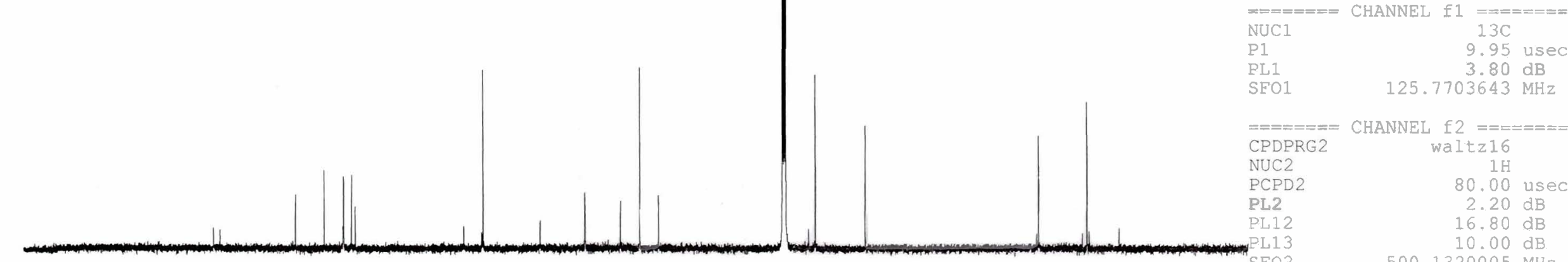

CPDPRG2 waltz16

NUC2

PCPD2

PL2

PLI2

PL 13

$1 \mathrm{H}$

80.00 usec

$2.20 \mathrm{~dB}$

$16.80 \mathrm{~dB}$

$10.00 \mathrm{~dB}$

$500.1320005 \mathrm{MHz}$ 


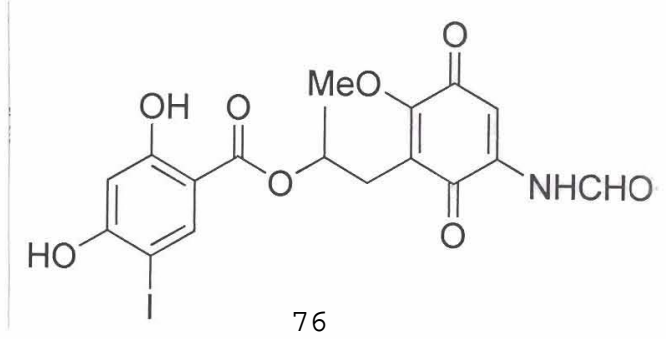

\section{a tunen}

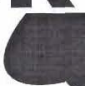

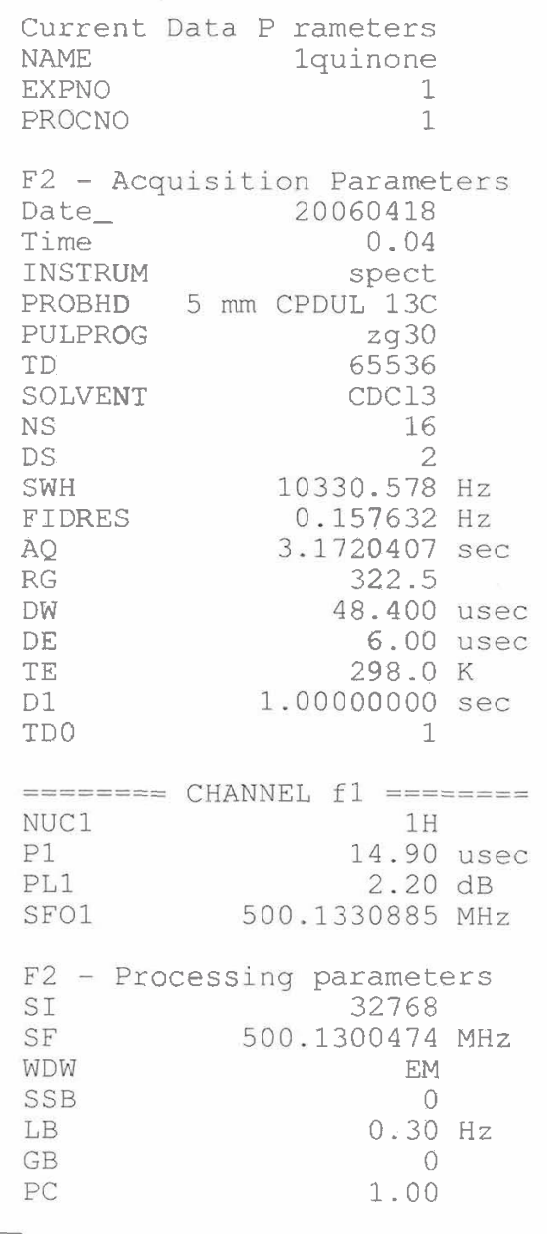



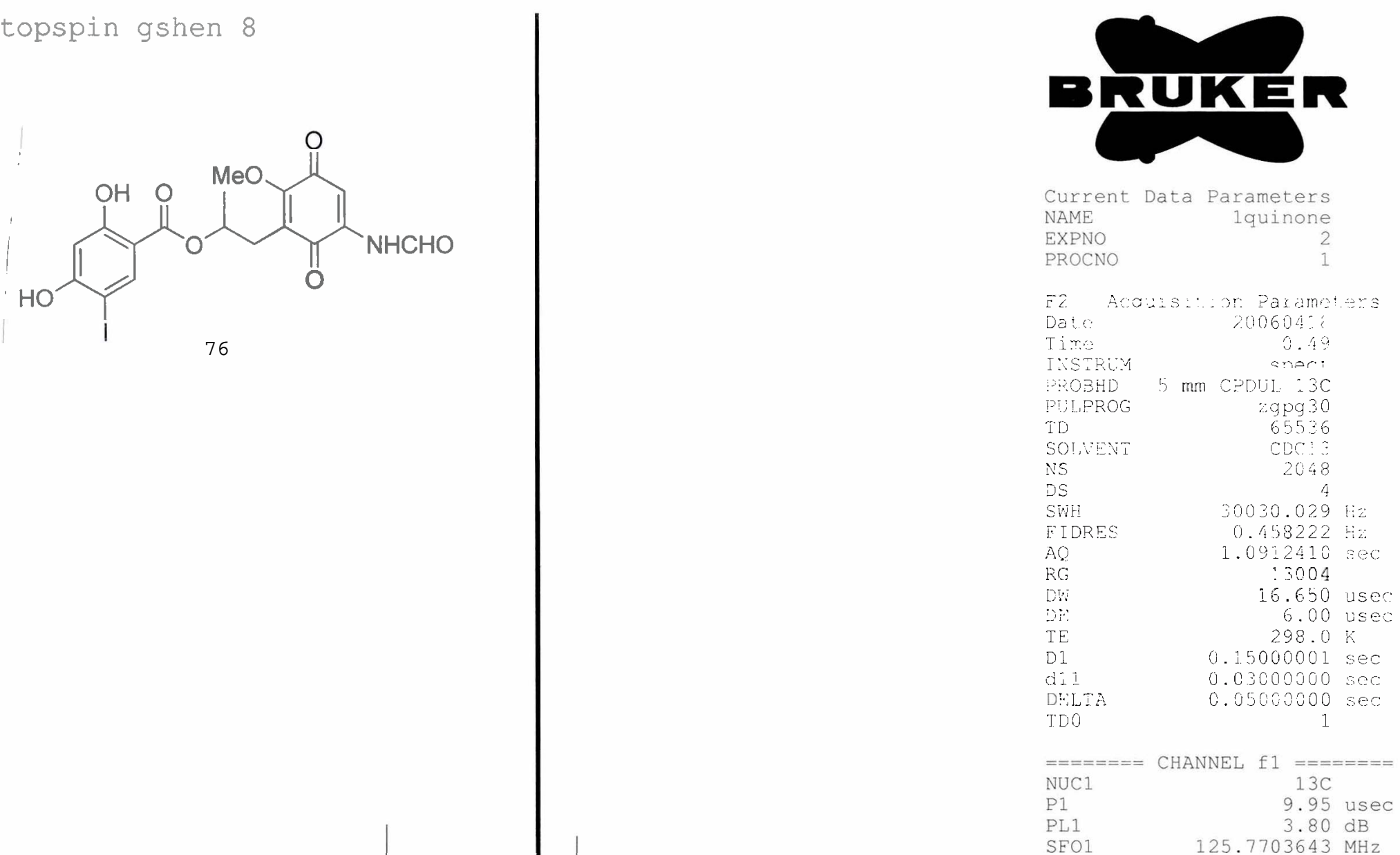

SEO1 $125.7703643 \mathrm{MH}$

$========$ CHANNEL f $2=======$

CPDPRG2 waltz16

NUC2 2

PCPD2

PL2

PL12

$\mathrm{PLO} 13$

waltz16
$1 \mathrm{H}$
80.00 usec
$2.20 \mathrm{~dB}$
$16.80 \mathrm{~dB}$
$10.00 \mathrm{~dB}$
$1320005 \mathrm{MHz}$



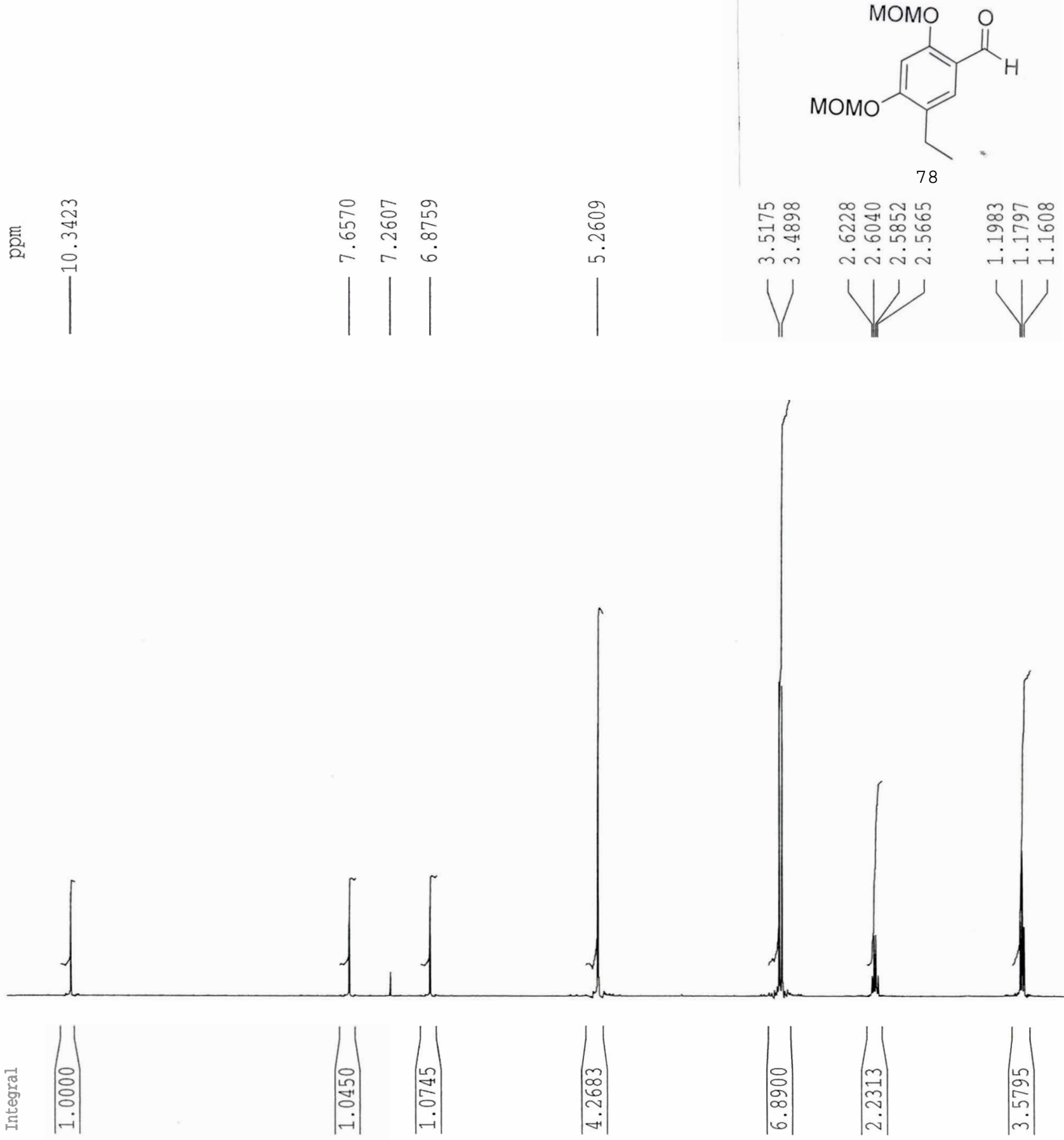

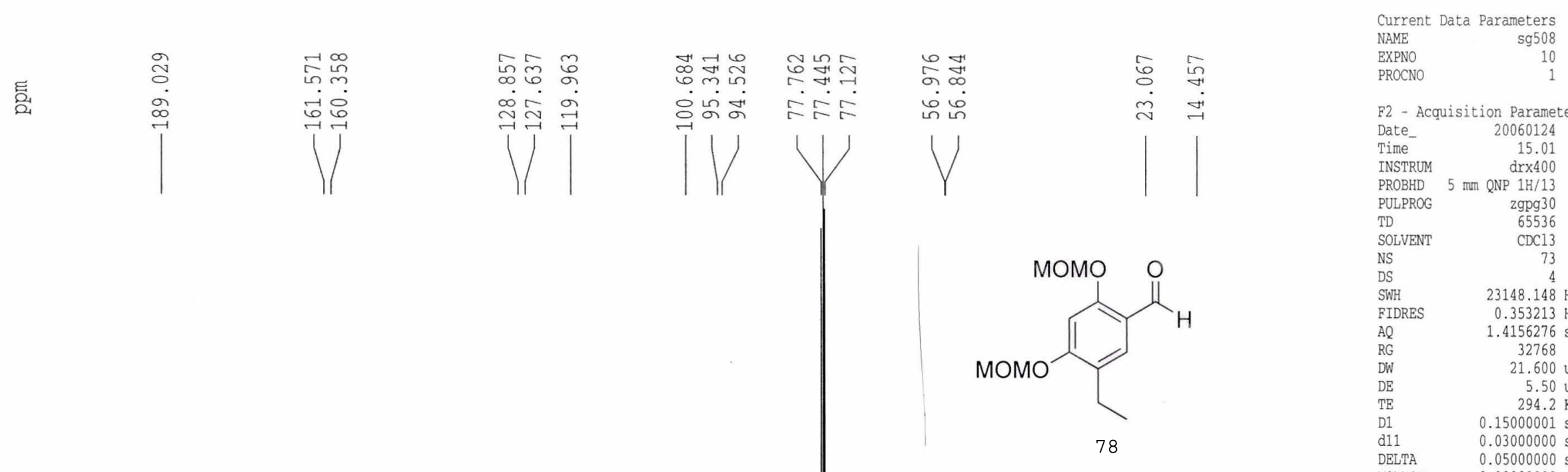

F2 - Acquisition Parameters

Date_ 20060124

$\begin{array}{lr}\text { Time } & 15.01 \\ \text { INSTRIMM } & \text { drx400 }\end{array}$

PROBHD $5 \mathrm{~mm}$ QNP $1 \mathrm{H} / 13$

PULPROG zgpg30

$\begin{array}{ll}\text { TD } & 65536 \\ \text { SOLVENT } & \mathrm{CDCl}\end{array}$

NS

DS

SWH

FIDRES $\quad 23148.148 \mathrm{~Hz}$

$0.353213 \mathrm{~Hz}$

$\begin{array}{lr}\text { AQ } & 1.4156276 \mathrm{sec} \\ \text { RG } & 32768\end{array}$

$\begin{array}{lr}\mathrm{RG} & 32768 \\ \mathrm{DW} & 21.600 \mathrm{usec}\end{array}$

DE $\quad 5.50$ usec

DI

$0.1500001 \mathrm{sec}$

DELTA $0.05000000 \mathrm{sec}$

$\begin{array}{ll}\text { DELTA } & 0.05000000 \mathrm{sec} \\ \text { MCREST } & 0.00000000 \mathrm{sec}\end{array}$

$\begin{array}{ll}\text { MCREST } & 0.00000000 \mathrm{sec} \\ \text { MCWRK } & 0.01500000 \mathrm{sec}\end{array}$

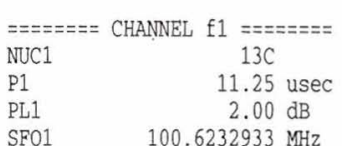

$======-$ CHANNEL f2 $=======$

CPDPRG2 waltz16

NUC2 $1 \mathrm{H}$

$\begin{array}{ll}\text { NUC2 } & 1 \mathrm{H} \\ \text { PCPD2 } & 100.00 \text { usec }\end{array}$

$\begin{array}{lr}\mathrm{PCPD2} & 100.00 \mathrm{ds} \\ \mathrm{PL2} & -4.00 \mathrm{~dB}\end{array}$

$\begin{array}{ll}\text { PL2 } & -4.00 \mathrm{~dB} \\ \text { PL12 } & 16.58 \mathrm{~dB}\end{array}$

$\begin{array}{ll}\text { PL12 } & 16.58 \mathrm{~dB} \\ \text { PL13 } & 17.00 \mathrm{~dB}\end{array}$
$\mathrm{SFO} 2$
$400.1316005 \mathrm{MHz}$

Jand
F2 - Processing parameters
SI $\quad 65536$
SF $\quad 100.6127290 \mathrm{MH}$
WDW
LB $\quad 1.00 \mathrm{~Hz}$
GB $\quad 0$
1.40

1D NMR plot parameters 
气
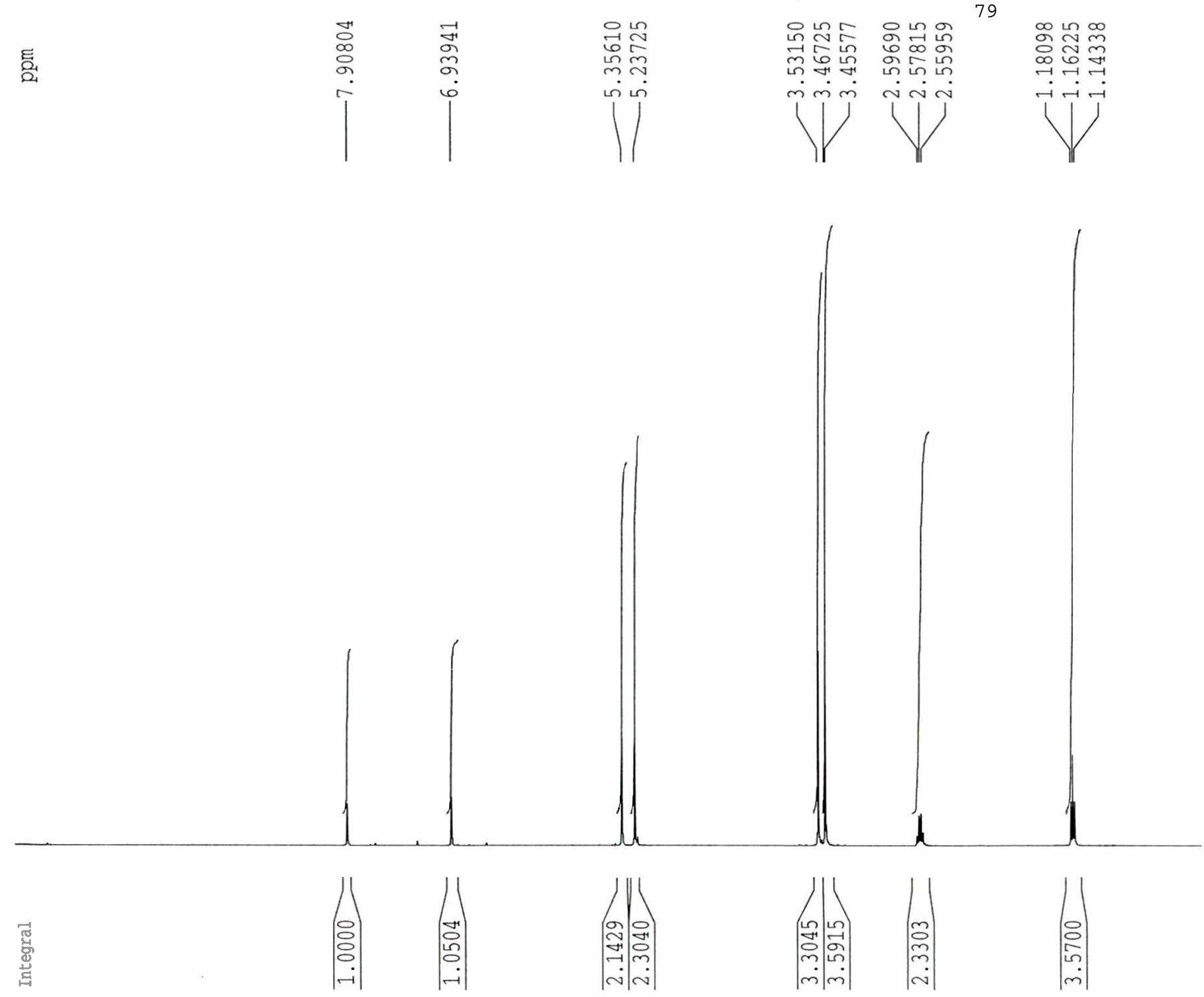
이큑ํㄱ

र्वित

당ㅎํ
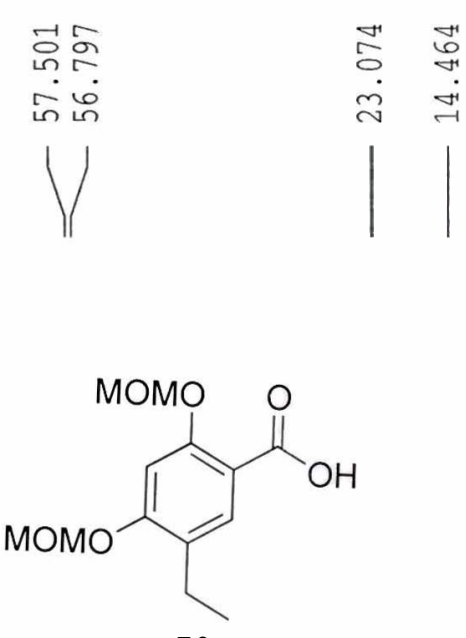

79
Current Data Parameters

NAME

EXPNO

PROCNO

F2 - Acquisition Parameters

Date_ 20060125

ime

INSTRUM

drx 400

PROBHD $5 \mathrm{~mm}$ ONP 1H/13

PULPROG zgpg30

TD $\quad 65536$

SOLVENT

NS

DS
SWH

SWH $\quad \begin{aligned} & 4 \\ & 23148.148 \mathrm{~Hz}\end{aligned}$

FIDRES $\quad 0.353213 \mathrm{~Hz}$

$\mathrm{AQ} \quad 1.4156276 \mathrm{sec}$

RG

DW

DE

$\mathrm{DE}$

D1

dil

DELTA

MCREST

32768

21.600 usec 5.50 usec $0.03000000 \mathrm{sec}$ $0.05000000 \mathrm{sec}$

$0.00000000 \mathrm{sec}$ $0.00000000 \mathrm{sec}$

$== \pm=== \pm=$ CHANNEL f1 ==s=z=s

$\begin{array}{lr}\text { NUC1 } & 13 \mathrm{C} \\ \text { P1 } & 11.25 \mathrm{usec} \\ \text { PL1 } & 2.00 \mathrm{~dB} \\ \text { SF01 } & 100.6232933 \mathrm{MHz}\end{array}$

SF01 $\quad 100.6232933 \mathrm{~dB}$

$== \pm= \pm==-$ CHANNEL f2 $== \pm= \pm===$

CPDPRG2 waltz16

$\begin{array}{lc}\text { NUC2 } & 1 \mathrm{H} \\ \text { PCPD2 } & 100.00 \text { usec }\end{array}$

100.00 us

PL2 $\quad-4.00 \mathrm{~d}$

PL12 $16.58 \mathrm{~dB}$

$\begin{array}{lr}\text { PL13 } & 17.00 \mathrm{~dB} \\ \text { SFO2 } & 400.1316005 \mathrm{MHz}\end{array}$

F2 - Processing parameters

SI 65536

SF $\quad 100.6127290 \mathrm{MHz}$

WDW

SSB

GB

PC

0

$1.00 \mathrm{~Hz}$

1.40

1D NMR plot parameter

$\begin{array}{lr}\mathrm{CX} & 20.00 \mathrm{~cm} \\ \mathrm{CY} & 2.00 \mathrm{~cm}\end{array}$

$\begin{array}{lr}\text { CY } & 2.00 \mathrm{~cm} \\ \text { F1P } & 215.000 \mathrm{ppm}\end{array}$ $21631.74 \mathrm{~Hz}$ $-5.000 \mathrm{ppm}$ $-503.06 \mathrm{~Hz}$ 


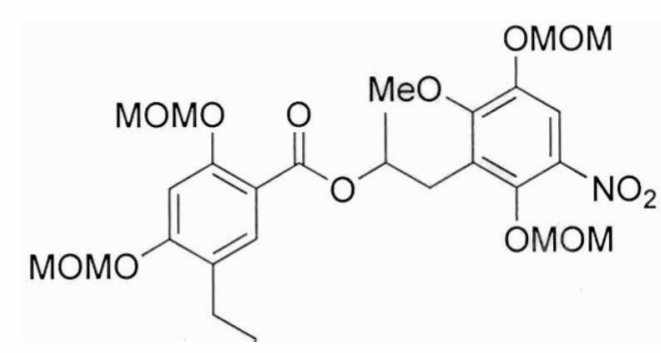

80

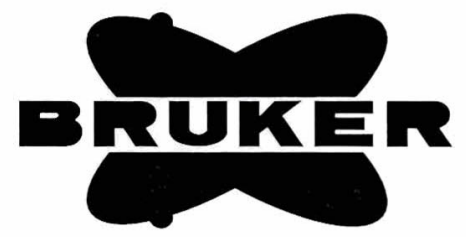

Current Data Parameters NAME

EXPNO

PROCNO

F2 - Acquisition Parameters

Date

Time

INSTRUM

PROBHD

PULPROG

TD

SOLVENT

NS

DS

SWH
FIDRES

FIDR
AQ

AQ

DW

DE

TE

MCREST

MCREST

$===$
NUC1

P1

PL1

SFOI

F2 - Processing parameters

SI 32768

SF $\quad 400.1300091 \mathrm{MHz}$

WDW

SSB

LB
20060126

18.31

$1 \mathrm{r} \times 400$
$1 \mathrm{H} / 13$

2930
32768

$\mathrm{CDCl} 3$

$4789.272 \mathrm{~Hz}$

$0.146157 \mathrm{~Hz}$

$3.4210291 \mathrm{sec}$

161.3

5.400 usec $293.2 \mathrm{~K}$

$1.00000000 \mathrm{sec}$

0.00000000 sec

0.01500000 sec

CHANNEL

$1 \mathrm{H}$

9.35 usec

$4.00 \mathrm{~dB}$

400.1300091
EM

0
30

1.00 


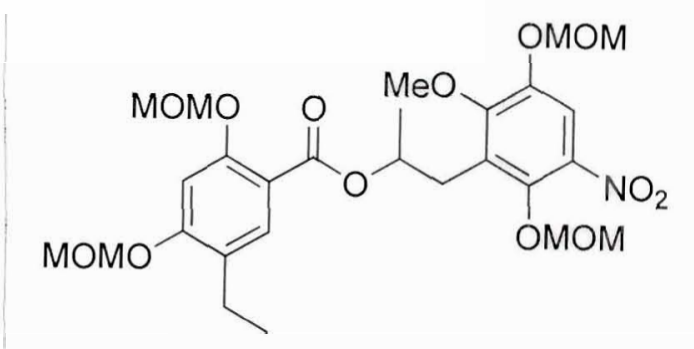

80

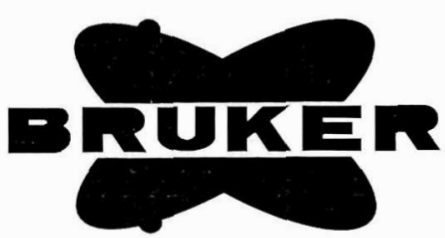

Current Data Parameters NAME

EXPNO

EXPNO

F2 - Acquisition Parameter

Date

Date_

INSTRUM

PROBHD

PULPROG

TD

SOLVENT

NS

DS

SWH

EIDRES

AQ

DW

$\mathrm{DE}$

TE

d11

DELTA

MCREST

MCWRK

P1

SFO1

$=== \pm== \pm=$

CPDPRG2

NUC2

PL2

$\mathrm{PL} 2$

PI12

$\mathrm{SFO} 2$
20060126

19.24

mm ONP $1 \mathrm{H} / 13$

zgpg 30

65536

$\mathrm{CDCl} 3$

1143

$23148.148 \mathrm{~Hz}$

$0.353213 \mathrm{~Hz}$

$1.4156276 \mathrm{sec}$

$$
32768
$$

21.600 usec

5.50 usec $293.2 \mathrm{~K}$

$0.15000001 \mathrm{sec}$

$0.03000000 \mathrm{sec}$

$0.05000000 \mathrm{sec}$

$0.00000000 \mathrm{sec}$

$0.01500000 \mathrm{sec}$
CHANNEL I $2=======$

L $2===$
waltz 16

100.00 usec

$-4.00 \mathrm{~dB}$

$16.58 \mathrm{~dB}$

$400.1316005 \mathrm{MHz}$ 


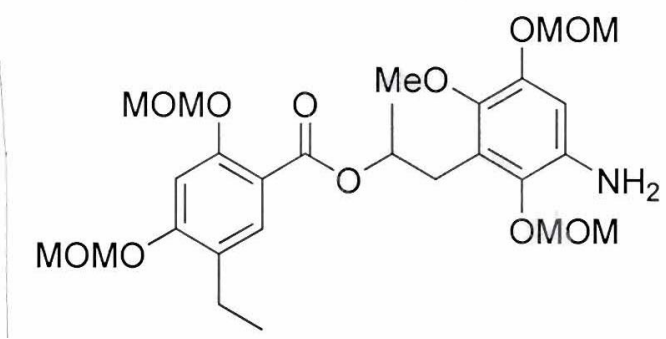

81

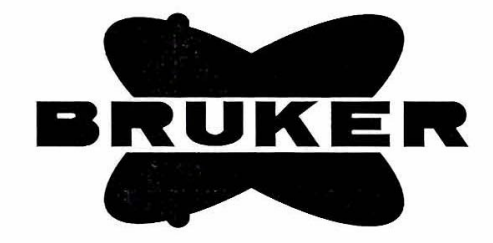

Current Data Parameters NAME

EXPNO

PROCNO

F2 - Acquisition Parameters

Date_ 20060131

Time

INSTRUM

PROBHD

PULPROG

ID

SOLVENT

NS

DS

FIDRES

$A Q$

RG

DW

TE

D1

MCREST

MCWRK

$===$

$\mathrm{NUC1}$

P1

PL1

19.43

$\operatorname{dr} \times 400$

$5 \mathrm{~mm}$ QNP $1 \mathrm{H} / 13$

$z 930$
32768

32768
$\operatorname{CDC} 13$
16

16
2

$4789.272 \mathrm{~Hz}$

$0.146157 \mathrm{~Hz}$

$3.4210291 \mathrm{sec}$ 287.4

104.400 usec 5.50 usec $293.2 \mathrm{~K}$

$1.00000000 \mathrm{sec}$

$0.00000000 \mathrm{sec}$

$0.01500000 \mathrm{sec}$

CHANNEL EI

1 = $=======$

$1 \mathrm{H}$

$-4.00 \mathrm{~dB}$

$400.1320007 \mathrm{MHz}$

2 - Processing parameters

SI 32768

SF $\quad 400.1300091 \mathrm{MHz}$

WDW

SSB

LB

$P C$ 

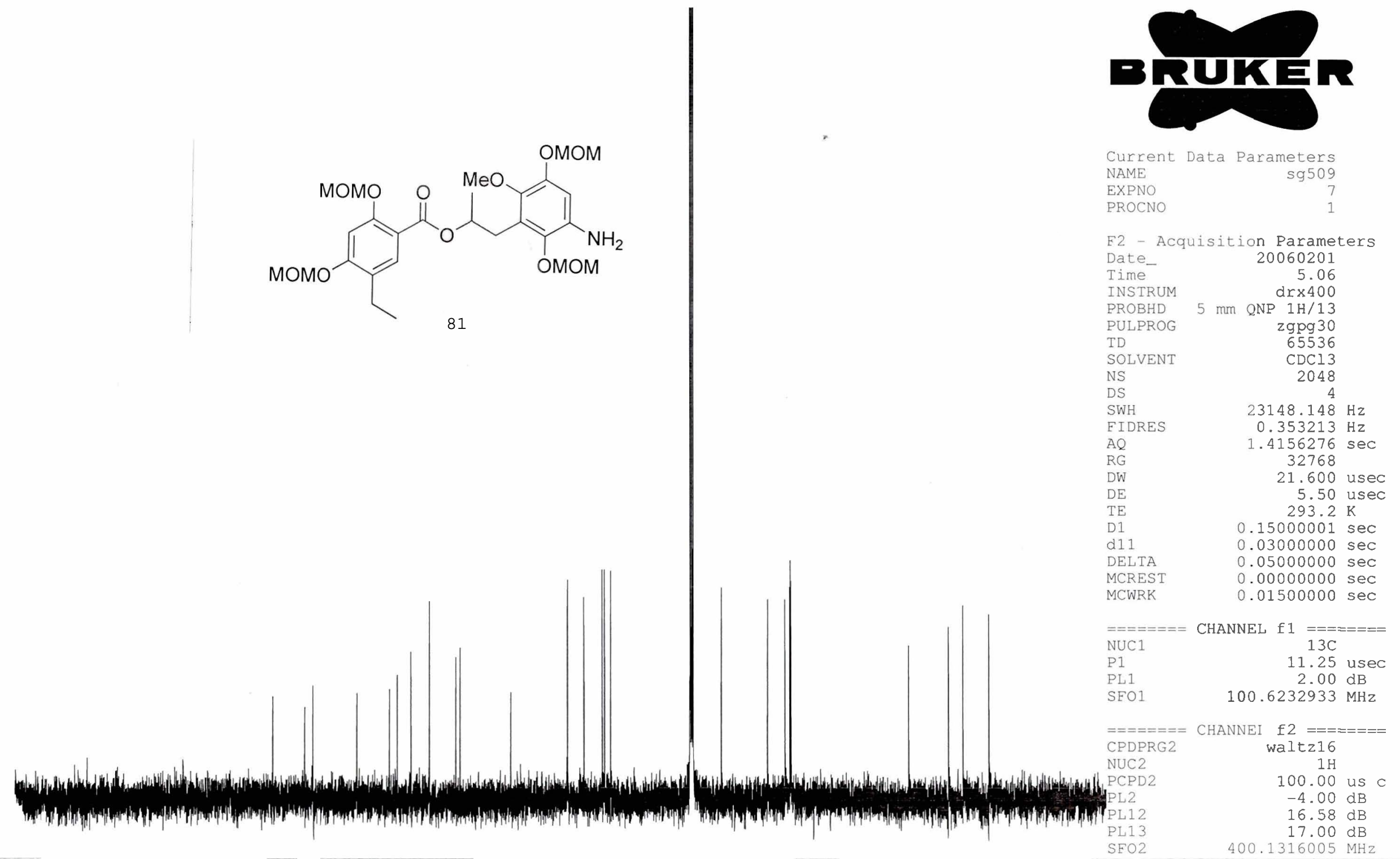

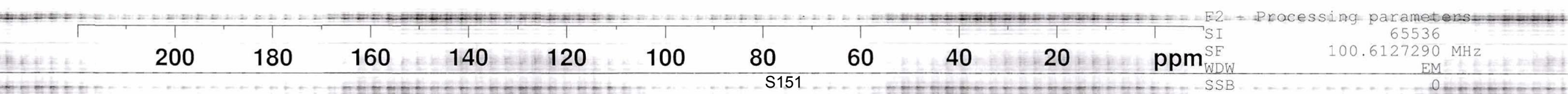



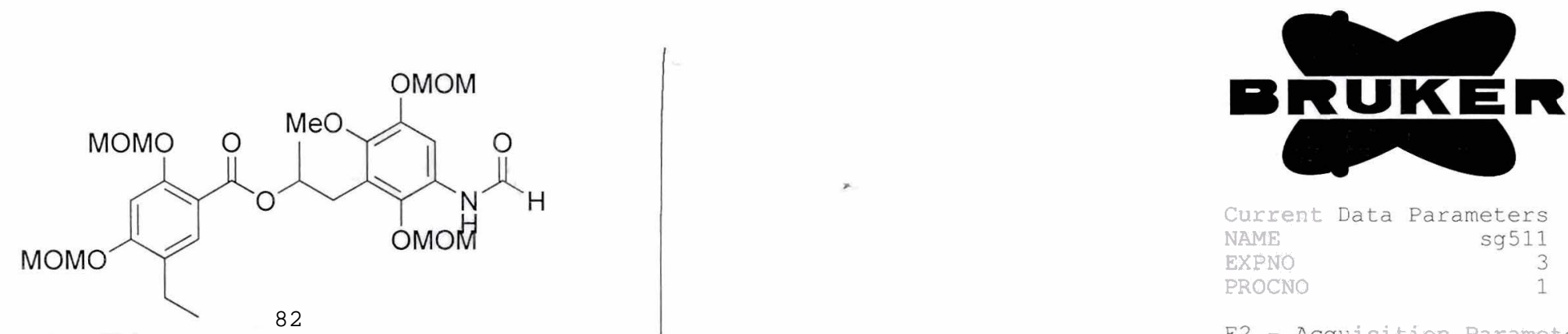

Current Data Parameters NAME EXPNO PROCNO

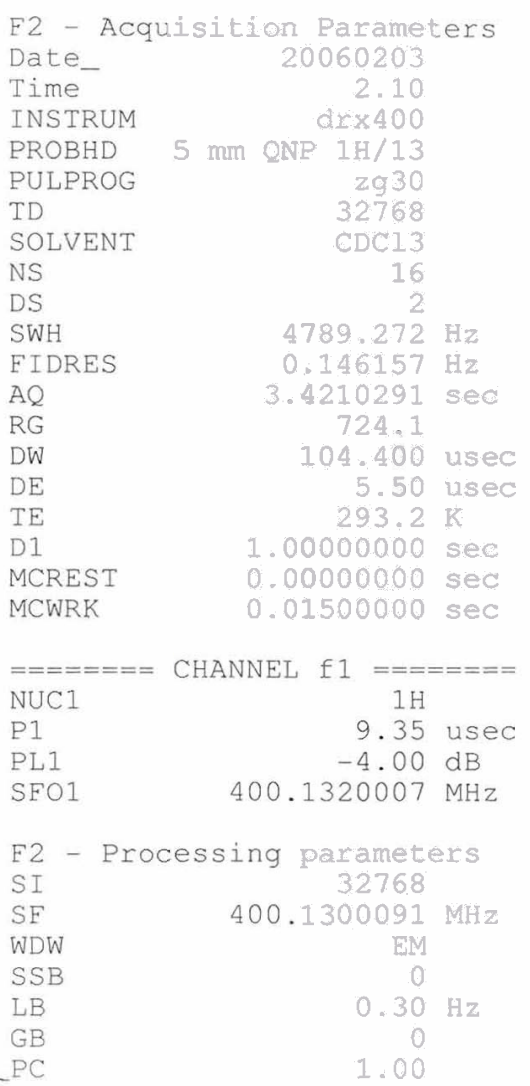




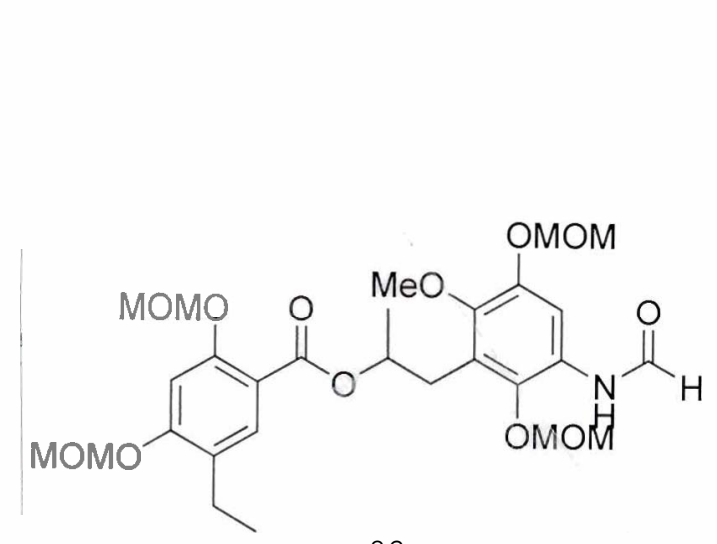

82

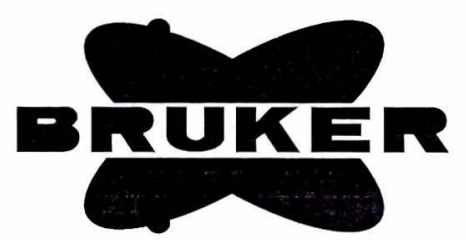

Current Data Parameters NAME

EXPNO

PROCNO

F2 - Acquisition Parameters

Time

INSTRUM

PROBHD

TD

SOLVENT

NS

DS

FIDRES

$\mathrm{AQ}$

$\mathrm{RG}$
$\mathrm{DW}$
$\mathrm{DE}$
$\mathrm{TE}$

TE

D1 11

DELTA

MCREST

$====$

NUC1

$\mathrm{P} 1$

PL1

CPDPRG2

NUC2

PCPD2

PL2

PL2

PL12

PLI3

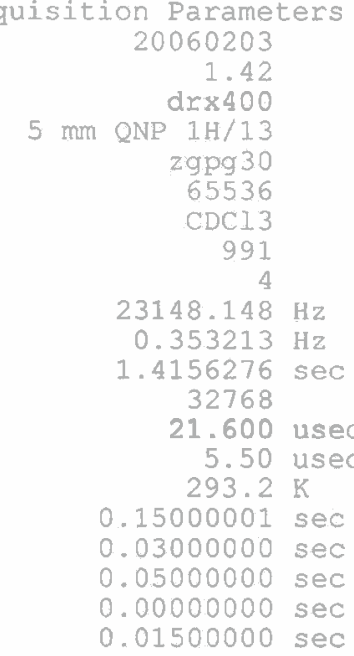

11.25 usec

$2.00 \mathrm{~dB}$

CHANNEL $\mathrm{f} 2$ waltz 16

100.00 usec

$-4.00 \mathrm{~dB}$

$17.00 \mathrm{~dB}$
$100.6232933 \mathrm{MHz}$ 


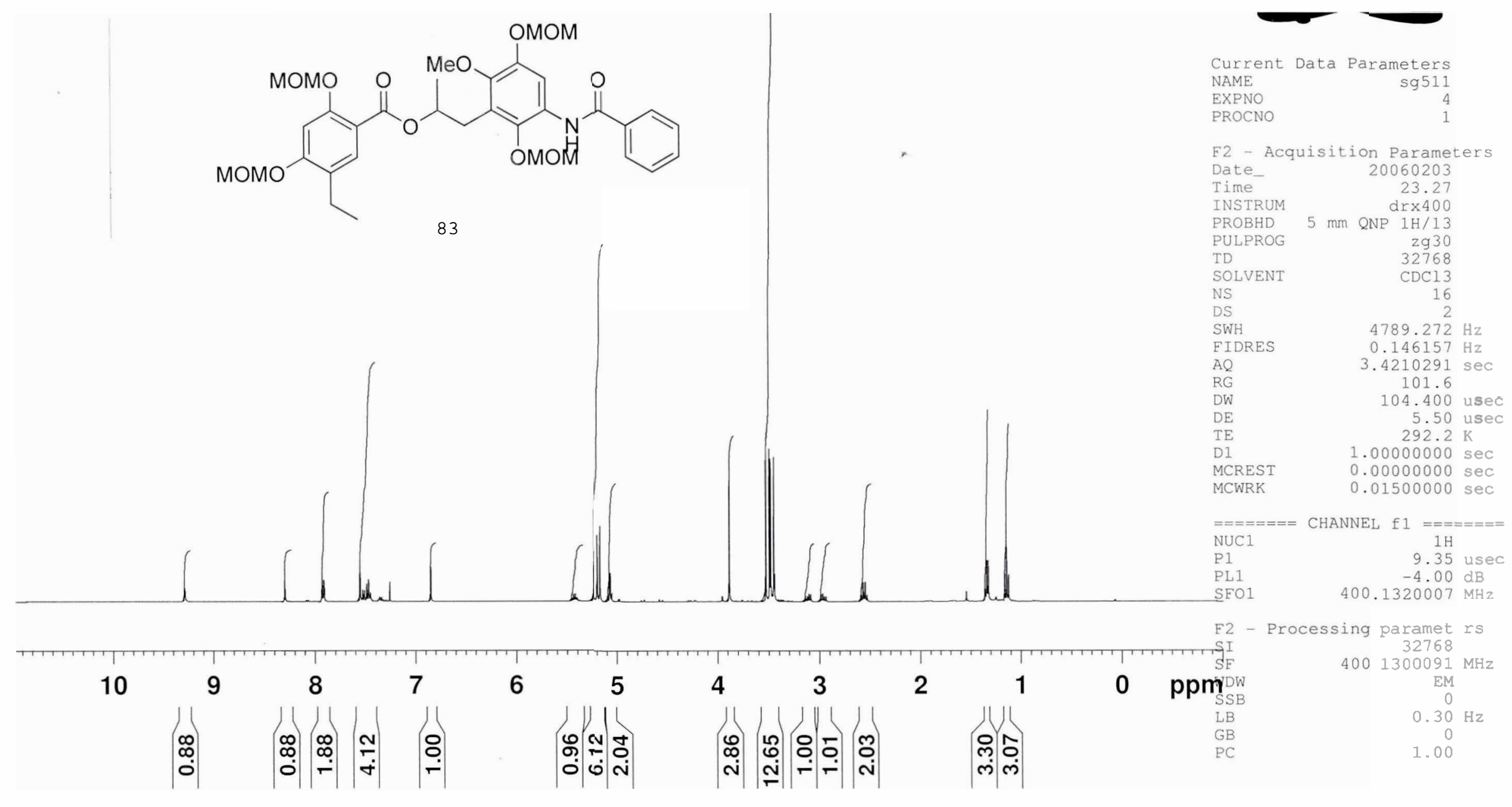




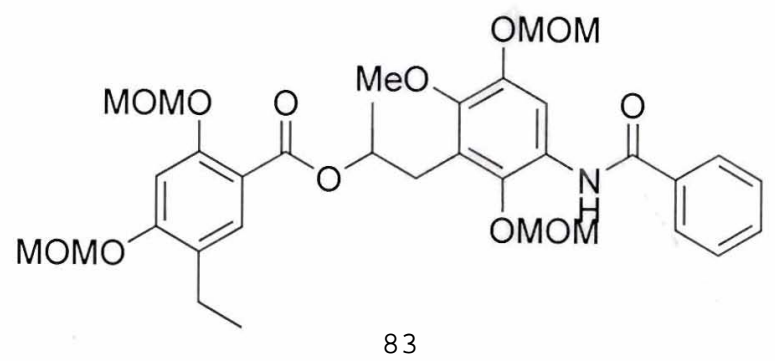

TUKER

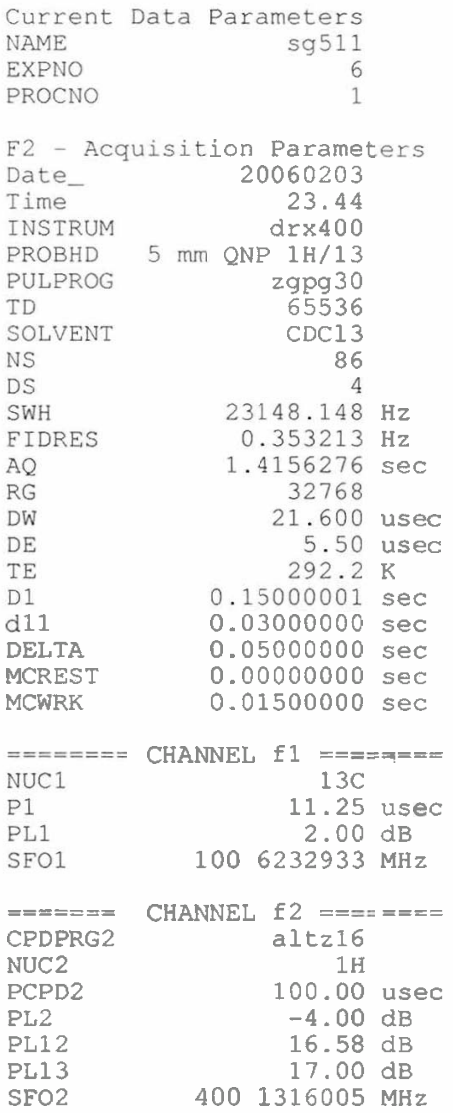

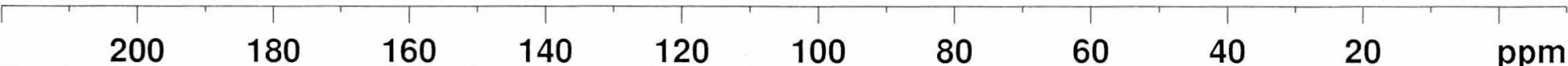




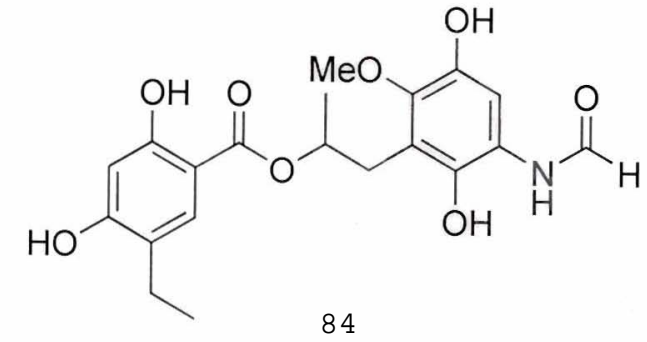

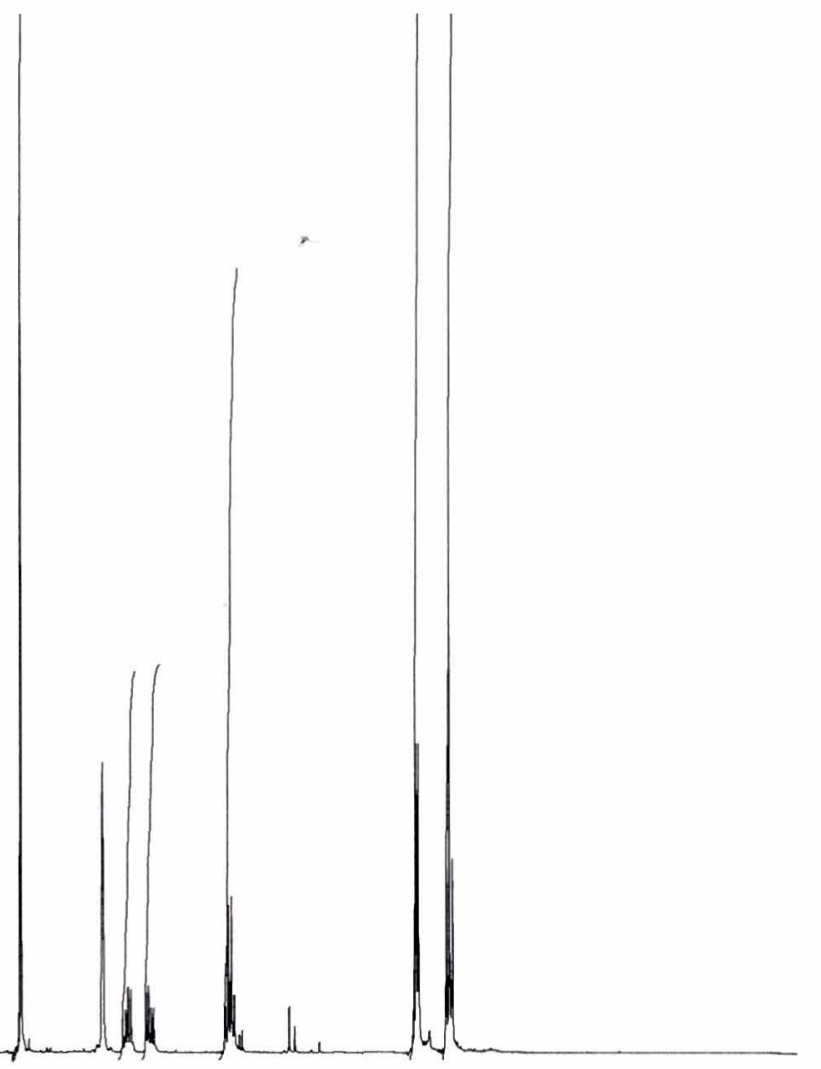

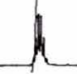

10

\begin{abstract}
9
\end{abstract}

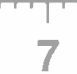

7

突
F2 - Acquisition Parameters

Date

Time

INSTRUM

PROBHD

PULPROG

TD

SOLVENT

NS

DS

FIDRES

AQ

RG

DE

D1

MCREST

MCWRK

20060205

drx400

$5 \mathrm{~mm}$ ONP $1 \mathrm{H} / 13$

$1 \mathrm{H} / 13$
$\mathrm{zg} 30$

32768

16

$4789.272 \mathrm{~Hz}$

$0.146157 \mathrm{~Hz}$

3.4210291 sec 256

104.400 usec 5.50 usec $291.2 \mathrm{~K}$

$1.00000000 \mathrm{sec}$

$0.00000000 \mathrm{sec}$

$0.01500000 \mathrm{sec}$

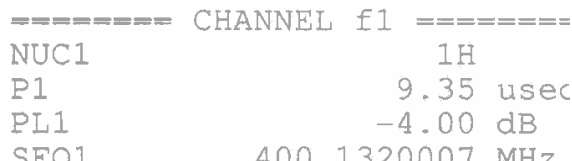

SEO1

$400.1320007 \mathrm{MHz}$

2 - Processing parameters SI $\quad 32768$ SF $\quad 400.1300091 \mathrm{MHz}$

WDW EM

$0.30 \mathrm{~Hz}$

LB

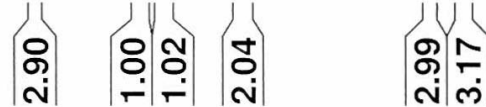

1.00 


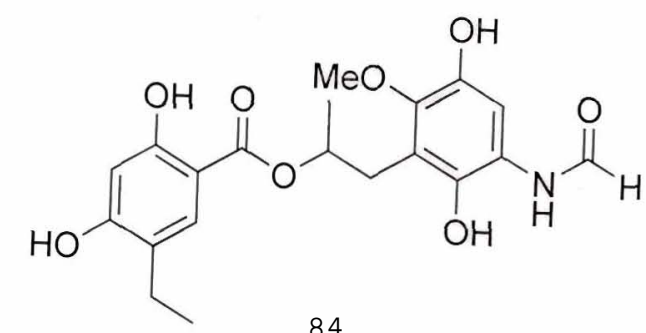

22 - Acquisition Parameter

20060205

Time 14.28

INSTRUM

drx400

PROBHD $5 \mathrm{~mm}$ QNP $1 \mathrm{H} / 13$

PULPROC

TD

NS

DS

SWH

AQQ

RG

DE

$$
\begin{aligned}
& x=== \\
& \text { NUC1 } \\
& \text { P1 } \\
& \text { PL1 }
\end{aligned}
$$$$
\begin{aligned}
& \text { PL1 } \\
& \text { SFO1 }
\end{aligned}
$$

$0.15000001 \mathrm{sec}$

0.03000000 sec

$0.00000000 \mathrm{sec}$

$0.01500000 \mathrm{sec}$

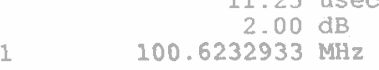

$=======$ CHANNTL

CPDPRG

NUC2

PL2

PL13 NNEL f2 216
waltzi6

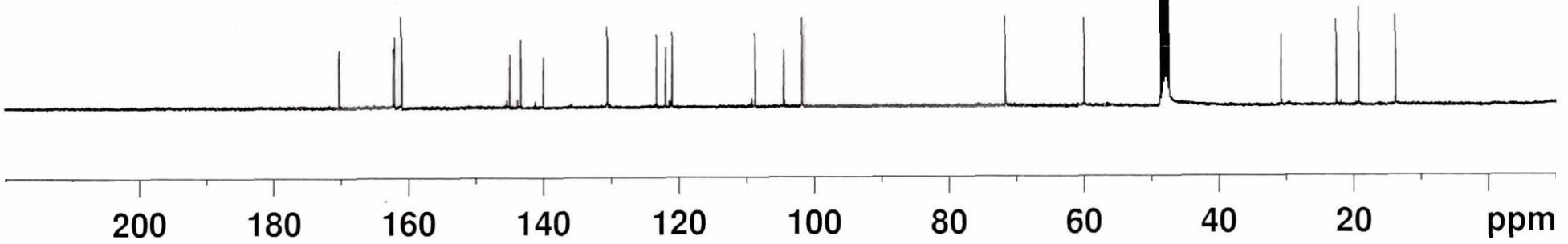

F2 - Processing pacameters

SI $\quad 65536$

SE

$\begin{array}{lc}\text { WDW } & 0 \\ \text { LB } & 1.00 \mathrm{~Hz}\end{array}$

$\begin{array}{lr}\mathrm{LB} & 1.00 \\ \mathrm{~GB} & 1.40\end{array}$ 


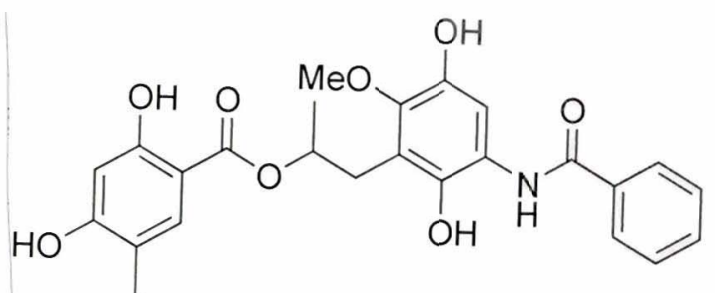

85

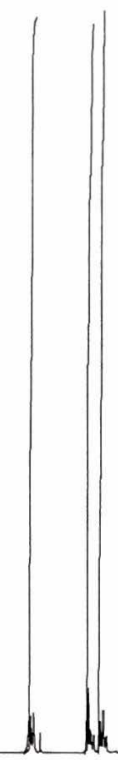

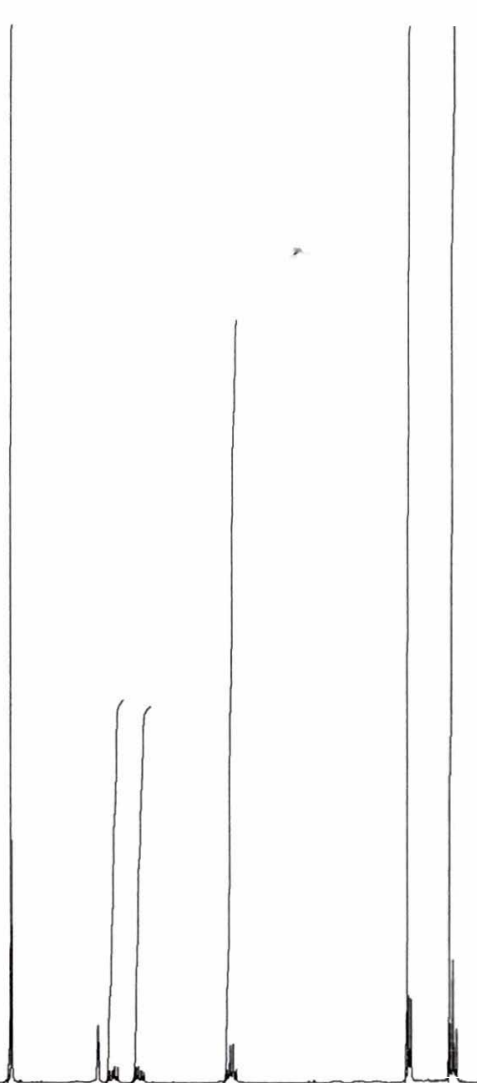

10

9

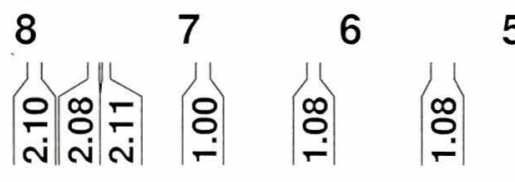

54

\section{4}

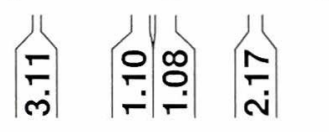

Current Data Parameters

NAME

EXPNO

sg 512 pheny

ROCNO

F2 - Acquisition Parameters

(2)

Time

20060205
18.03

INSTRUM drx400

PROBHD $5 \mathrm{~mm}$ QNP $1 \mathrm{H} / 13$

PULPROG

TD

SOLVENT

NS

DS

EIDRES

AQ

RG

DW

TE

D1

MCREST

MCWRK

2930
32768

$\mathrm{MeOH}$

16

$4789.272 \mathrm{~Hz}$

$0.146157 \mathrm{~Hz}$

$3.4210291 \mathrm{sec}$ 161.3

104.400 usec 5.50 usec $291.2 \mathrm{~K}$

$1.00000000 \mathrm{sec}$

$0.00000000 \mathrm{sec}$

$0.01500000 \mathrm{sec}$

$=======$ CHANNEL

NUC

P1

PL1

$$
===
$$

9.35 usec

FO

$400.1320007 \mathrm{MHz}$

F2 - Processing parameters

SI 32768

SF $\quad 400.1300091 \mathrm{MHz}$

SSB

$\mathrm{EM}$
0

$0.30 \mathrm{~Hz}$

1.00

GB

$\begin{array}{lllll}2 & 1 & 0 & \text { ppm } & { }_{\mathrm{B}} \mathrm{B}\end{array}$



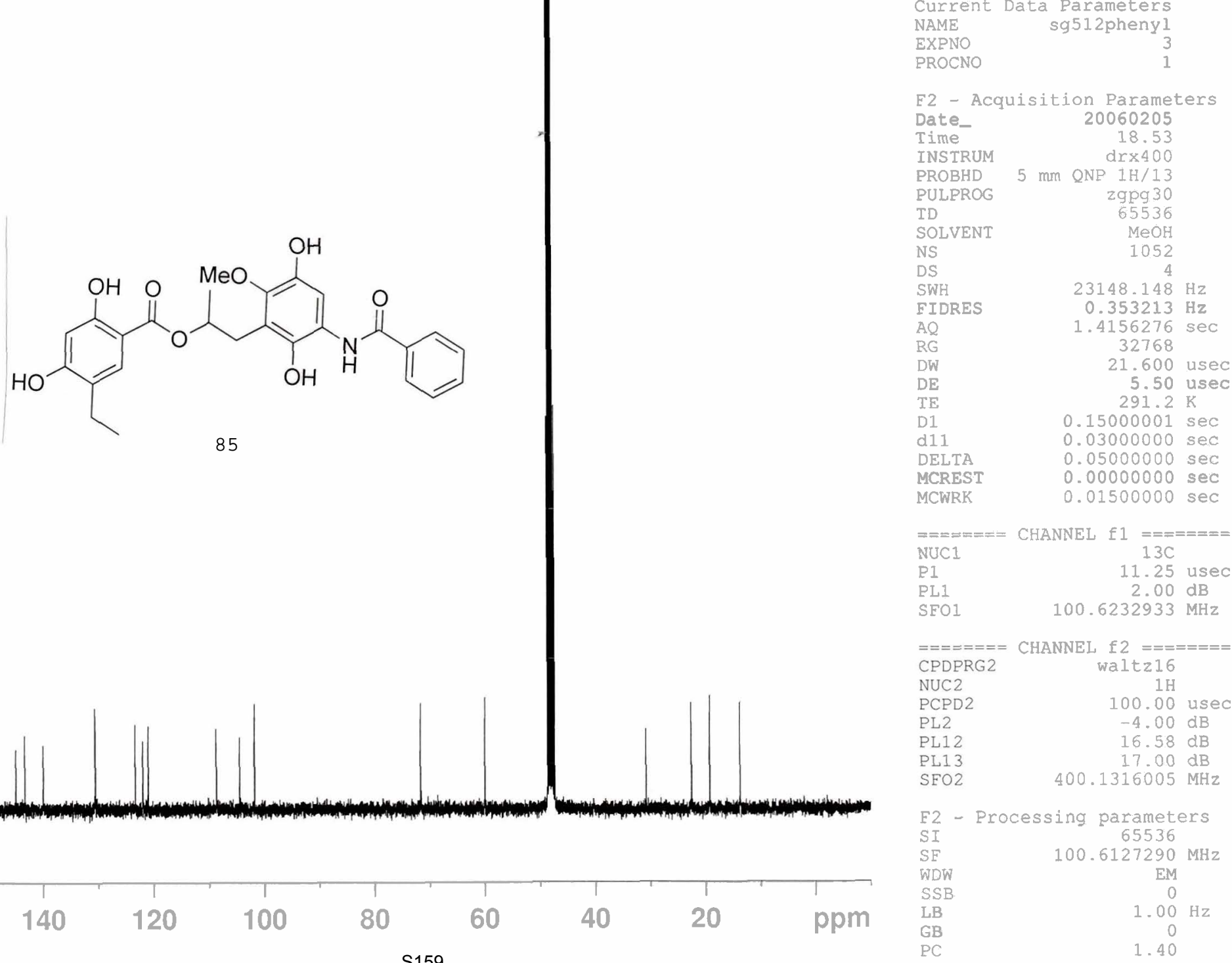

CHANNEL

NUC1

PI

SFO1

130

11.25 usec

$100.6232933 \mathrm{MHz}$

$=======$ CHANNEL

CPDPRG2

NUC2

PL2

PL1 2

$\mathrm{SFO} 2$

ANEL $f 2=======$
waltz 16
$1 \mathrm{H}$
100.00 usec
$-4.00 \mathrm{~dB}$
$16.58 \mathrm{~dB}$
$17.00 \mathrm{~dB}$
$400.1316005 \mathrm{MHz}$


F2 - Acquisition Parameters

Date_ 20051107

Time $\quad 18.21$

INSTRUM dr $\quad$ dr 400

PROBHD 5 mm ONP 1H/13

PULPROG $\quad \mathrm{zg} 30$

TD 32768

SOLVENT $\quad \mathrm{CDC} 13$

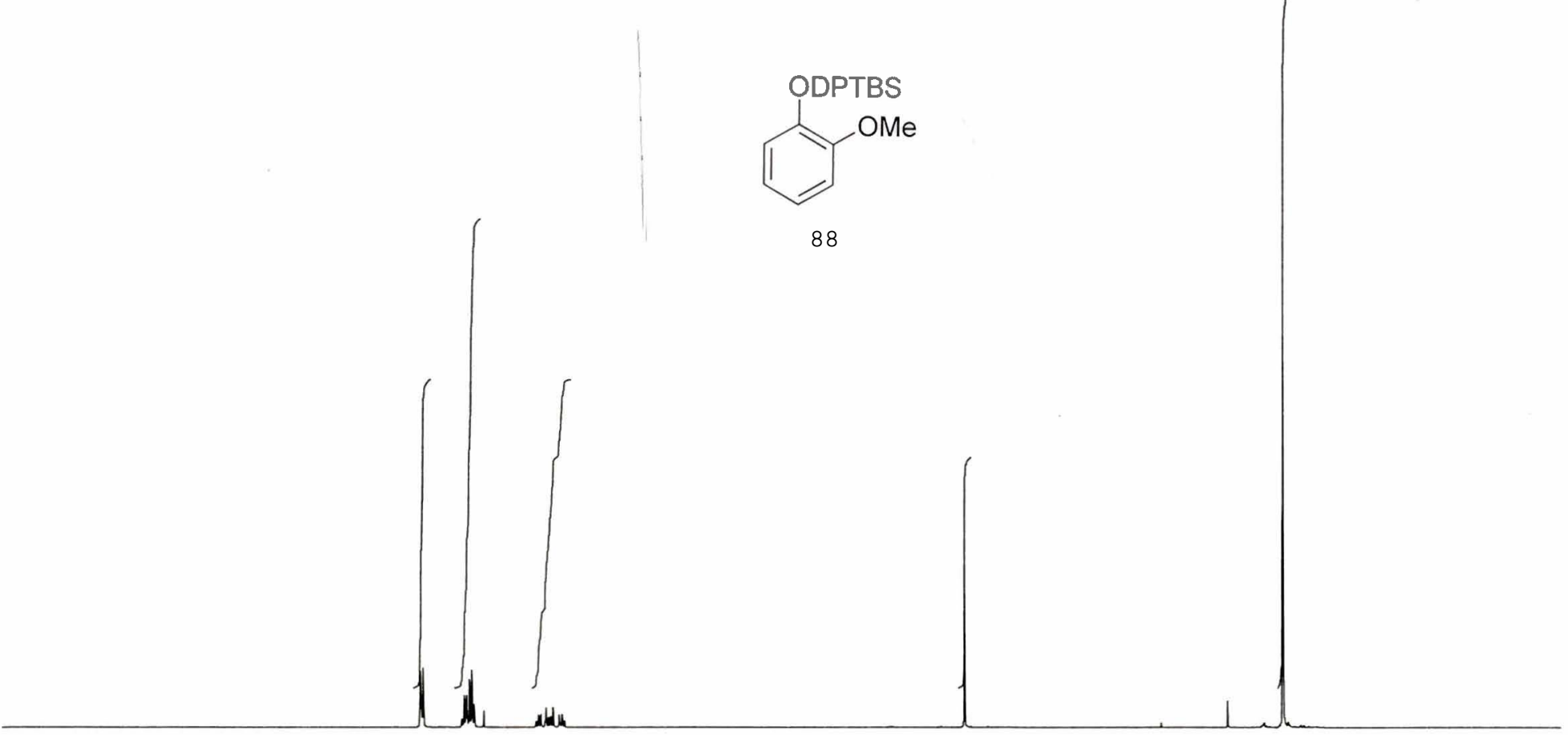

NS

DS

$\quad 0.146157 \mathrm{~Hz}$

$\mathrm{AQ} \quad 3.4210291 \mathrm{sec}$

RG $\quad 128$

DW $\quad 104.400$ usec

DE $\quad 5.50$ usec

TE $294.2 \mathrm{~K}$

D1 $1.00000000 \mathrm{sec}$

MCREST $\quad 0.00000000 \mathrm{sec}$

MCWRK $\quad 0.01500000 \mathrm{sec}$

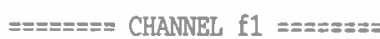

NUC1

P1 9.35 usec

$13.00 \mathrm{~dB}$

F2 - Processing parameters

SI $\quad 32768$

SF $\quad 400.1300091 \mathrm{MHz}$

WDW EM

$\begin{array}{lc}\text { SSB } & 0 \\ \text { LB } & 0.30 \mathrm{~Hz}\end{array}$

GB 0

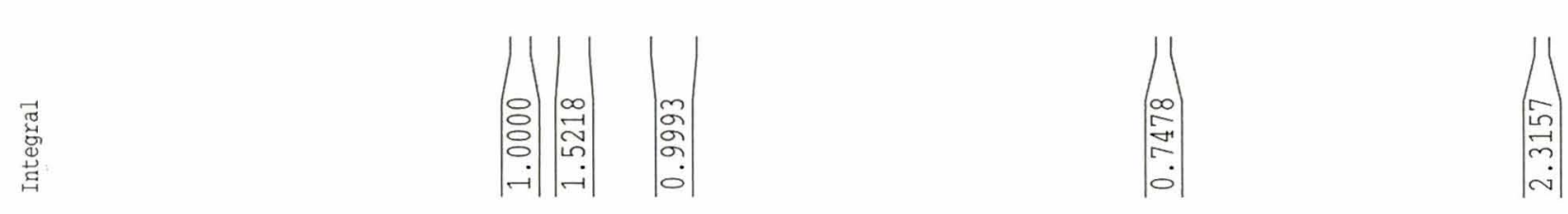

ID MNR plot parameters

$\begin{array}{lr}\text { CX } & 20.00 \mathrm{~cm} \\ \text { CY } & 5.00 \mathrm{~cm} \\ \text { F1P } & 11.000 \mathrm{ppm} \\ \text { F1 } & 4401.43 \mathrm{~Hz} \\ \text { F2P } & -1.000 \mathrm{ppm} \\ \text { F2 } & -400.13 \mathrm{~Hz} \\ \text { PPMCM } & 0.60000 \mathrm{ppm} / \mathrm{cm} \\ \text { HZCM } & 240.07800 \mathrm{~Hz} / \mathrm{cm}\end{array}$




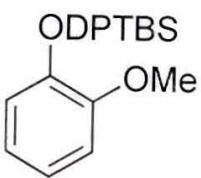

88

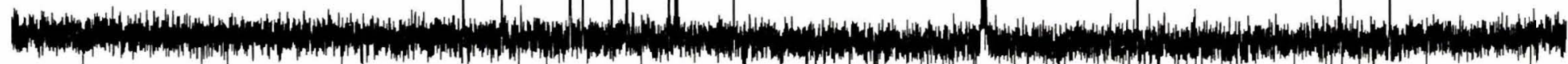

F2 - Acquisition Parameters

Date_ 20051107

Time $\quad 18.25$

PROBHD $5 \mathrm{~mm}$ ONP $1 \mathrm{H} / 13$

PULPROG 5 an

zgpg30

SOLVENT

NTS

DS

SWH

FIDRES

$A Q$
$R G$
RW

DW

TE

D1

d11

DELTA

MCRES

4

$23148.148 \mathrm{~Hz}$

$.4156276 \mathrm{sec}$

21.600 usec

5.50 usec $294.2 \mathrm{~K}$

$0.15000001 \mathrm{sec}$

$0.03000000 \mathrm{sec}$

$0.05000000 \mathrm{sec}$

$0.00000000 \mathrm{sec}$

$===$ CHANNEL f1 $==:===$

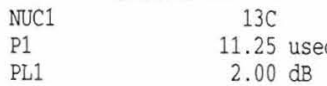

$\begin{array}{lr}\text { PL1 } & 2.00 \mathrm{~dB} \\ \text { SF01 } & 100.6232933 \mathrm{MHz}\end{array}$

$===-==-=$ CHANNEL f2 $== \pm====$ CPDPRG2 waltz16

NUC2 $1 \mathrm{H}$

PCPD2 100.00 usec

PL2

$-4.00 \mathrm{~dB}$
$16.58 \mathrm{~dB}$

$16.58 \mathrm{~dB}$

PL13 $\quad 17.00 \mathrm{~dB}$

$400.1316005 \mathrm{MHz}$

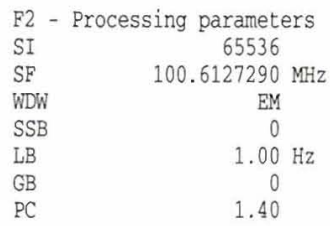

1D NMR plot parameters 
吕

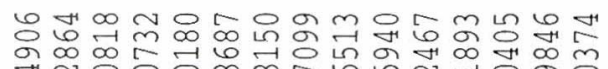

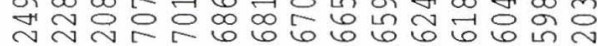
- م

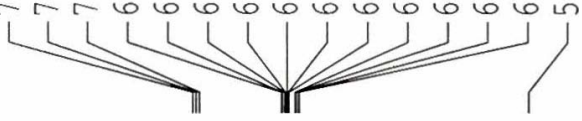

ஸे

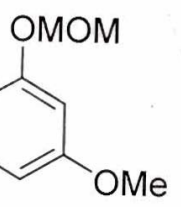

89
Current Data Parameters NAME

PROCN

F2 - Acquisition Parameters

Date_ 20050916

Time 20.50

INSTRUM drx400

PROBHD $5 \mathrm{~mm}$ ONP $1 \mathrm{H} / 13$

PULPROG $\quad \mathrm{zg} 30$

TD 32768

$\mathrm{CDCl}$

NS

SWH $\quad 4789.272 \mathrm{~Hz}$

FIDRES $\quad 0.146157 \mathrm{~Hz}$

AQ $\quad 3.4210291 \mathrm{sec}$

RG 32

DW $\quad 104.400$ usec

DB $\quad 5.50$ usec

TB $293.2 \mathrm{~K}$

D1 $\quad 1.00000000 \mathrm{sec}$

MCREST

MCWRK $\quad 0.01500000 \mathrm{sec}$

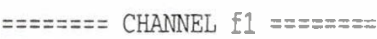
NUC1

P1 9.35 usec

PL1 $-4.00 \mathrm{~dB}$

SF01 400.1320007 MHz

F2 - Processing parameters

SI - 32768

SF $\quad 400.1300000 \mathrm{MHz}$

WDW EM

$\begin{array}{lc}\text { SSB } & 0 \\ \text { LB } & 0.30 \mathrm{~Hz}\end{array}$

GB $\quad 0$

$\mathrm{PC}$

1.00

1D NMR plot parameters

$\begin{array}{lr}\text { CX } & 20.00 \mathrm{~cm} \\ \text { CY } & 12.50 \mathrm{~cm} \\ \text { F1P } & 11.000 \mathrm{ppm} \\ \text { F1 } & 4401.43 \mathrm{~Hz} \\ \text { F2P } & -1.000 \mathrm{ppm} \\ \text { F2 } & -400.13 \mathrm{~Hz} \\ \text { PPMCM } & 0.60000 \mathrm{ppm} / \mathrm{cm} \\ \text { HZCM } & 240.07800 \mathrm{~Hz} / \mathrm{cm}\end{array}$

$240.07800 \mathrm{~Hz} / \mathrm{cm}$

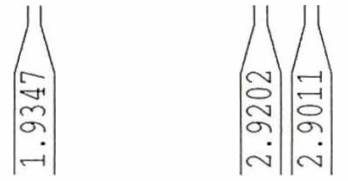




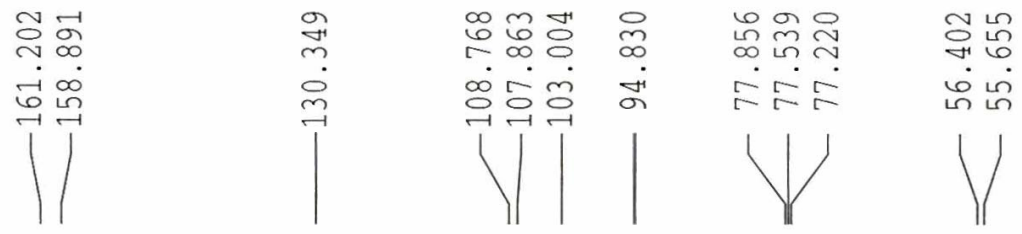

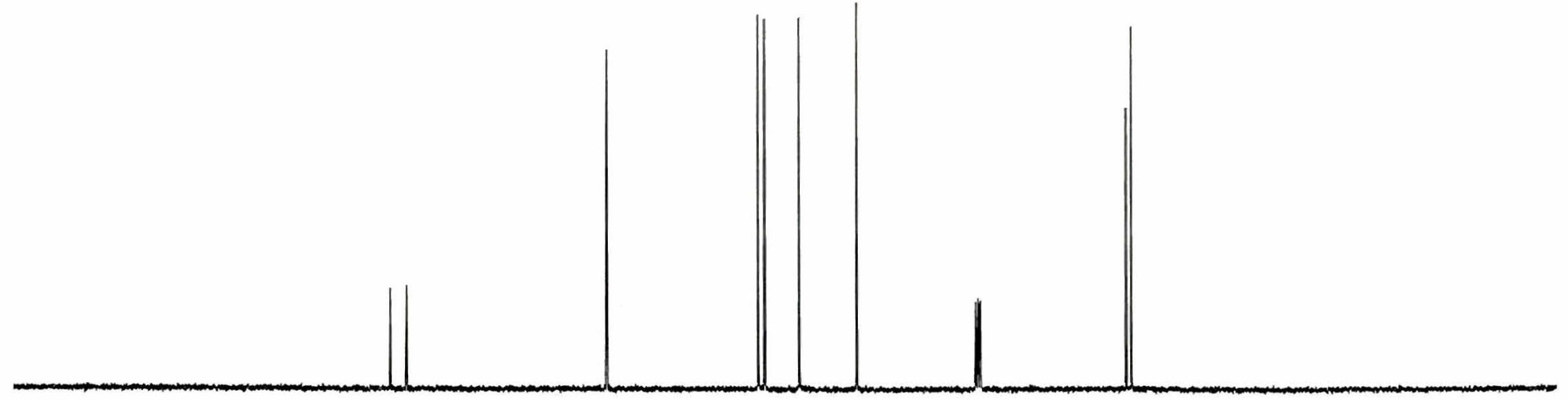

OMOM

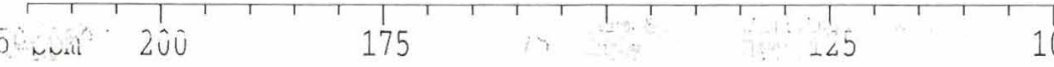

100

F2 - Acquisition Paranet rs

Date. 20050916

Time 20.54

IMSTRUNA drat00

PROBHD 5 ONP $1 \mathrm{H} / 13$

PUTPROG $\quad$ zgpg 30

PUTPROG

SOLVENT

NS

DS

$23148.148 \mathrm{~Hz}$

FIDRES $\quad 0.353213 \mathrm{~Hz}$

$\begin{array}{cc}\text { AQ } & 1.4156276 \mathrm{sec} \\ \text { RG } & 32768\end{array}$

32768

21.600 usec

DE $\quad 5.50$ usec

$01.15000001 \mathrm{sec}$

d11 $\quad 0.15000001 \mathrm{sec}$

d11 $\quad 0.03000000 \mathrm{sec}$

DELIIA $\quad 0.05000000 \mathrm{sec}$

MCREST $\quad 0.00000000 \mathrm{sec}$

MCWRR

$0.01500000 \mathrm{sec}$

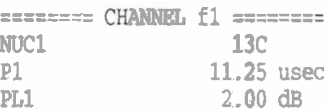

SLi $\quad 100.6232933 \mathrm{MHz}$

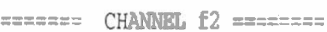

$\begin{array}{lr}\text { CPDPRG2 } & \text { waltz16 } \\ \text { NUC2 } & 1 \mathrm{H}\end{array}$

$\begin{array}{ll}\text { NUC2 } & \text { 1H } \\ \text { PCPD2 } & 100.00 \text { usec }\end{array}$

$\begin{array}{ll}\text { PL2 } & -4.00 \mathrm{~dB}\end{array}$

$\begin{array}{ll}\text { PL2 } & -4.00 \mathrm{~dB} \\ \text { PL12 } & 16.58 \mathrm{~dB}\end{array}$

PLI3 $17.00 \mathrm{~dB}$
$\mathrm{SFO} 2$
$00.1316005 \mathrm{MHz}$

F2 - Processing parameters

$\begin{array}{cc}\text { SI } & 65536 \\ \text { SP } & 100.6127290 \mathrm{kHz}\end{array}$

WDW

$\begin{array}{lc}\text { SSB } & 0 \\ \text { LB } & 1.00 \mathrm{~Hz}\end{array}$

$1.00 \mathrm{H}$
0
1.40

ID MMR pIot parameters

$\mathrm{CX} \quad 20.00 \mathrm{~cm}$

$\begin{array}{lr}\text { CY } & 5.00 \mathrm{~cm} \\ \text { F1P } & 215.000 \mathrm{ppm}\end{array}$

$\begin{array}{ll}\text { F1P } & 215.000 \mathrm{ppm} \\ \text { F1 } & 21631.74 \mathrm{~Hz}\end{array}$

$\begin{array}{ll}\text { R2P } & -5.000 \mathrm{ppm} \\ \text { F2 } & -503.06 \mathrm{~Hz}\end{array}$

F2 $\quad-503.06 \mathrm{~Hz}$

in $3999 \mathrm{~Hz} / \mathrm{cm}$ 

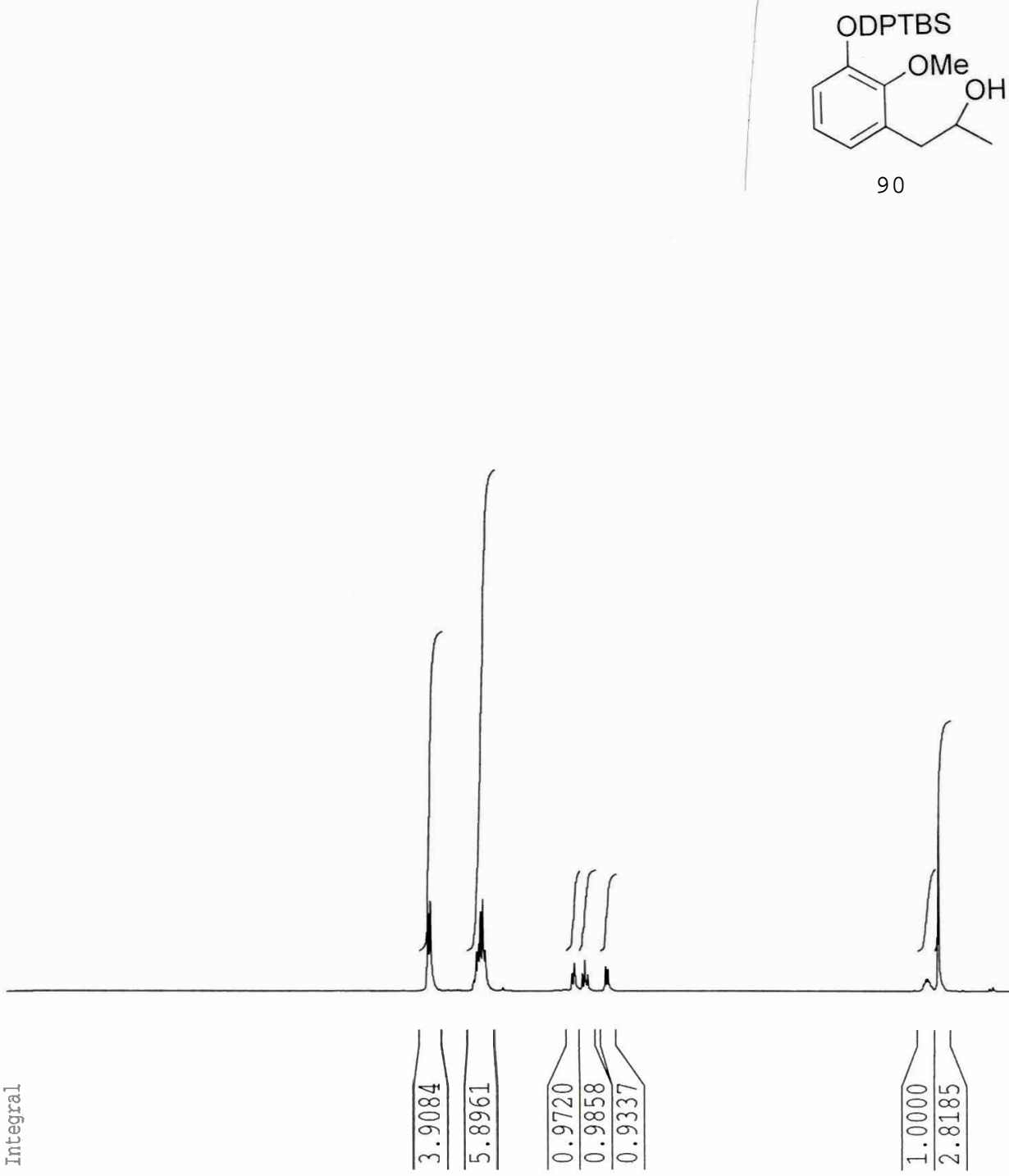

Current Data Parameters

PROCNO

$\mathrm{sg}=96$

F2 - Acquisition Parameters

Date $\quad 20051116$

Time $\quad 16.58$

TNSTRUM drx400

PROBHD $5 \mathrm{~mm}$ QNP $1 \mathrm{H} / 13$

PULPROG

$\mathrm{zg} 30$

TD

SOLVENT

NS

DS

SWH

FIDRES

AQ

$\mathrm{RG}$

$\mathrm{DW}$

$\mathrm{DE}$

DE

MCRES

MCWRK

$========$ CHANNEL
NUC1

P1

SL1

$400.1320007 \mathrm{MHz}$

F2 - Processing parameters

SI 32768

SF $\quad 400.1300091 \mathrm{MHz}$

WDW

SSB

LB

$G B$

$\mathrm{PC}$

$E M$
0

$0.30 \mathrm{~Hz}$

0

1D NMR plot parameters

CX $\quad 20.00 \mathrm{~cm}$

CY $\quad 5.00 \mathrm{~cm}$

F1P

F1

F2P

PPMCM

$\mathrm{HZCM}$

$11.000 \mathrm{pom}$ $4401.43 \mathrm{~Hz}$

-1.000 ppon $-400.13 \mathrm{~Hz}$

$0.60000 \mathrm{ppro} / \mathrm{cm}$ $240.07800 \mathrm{~Hz} / \mathrm{cm}$ 


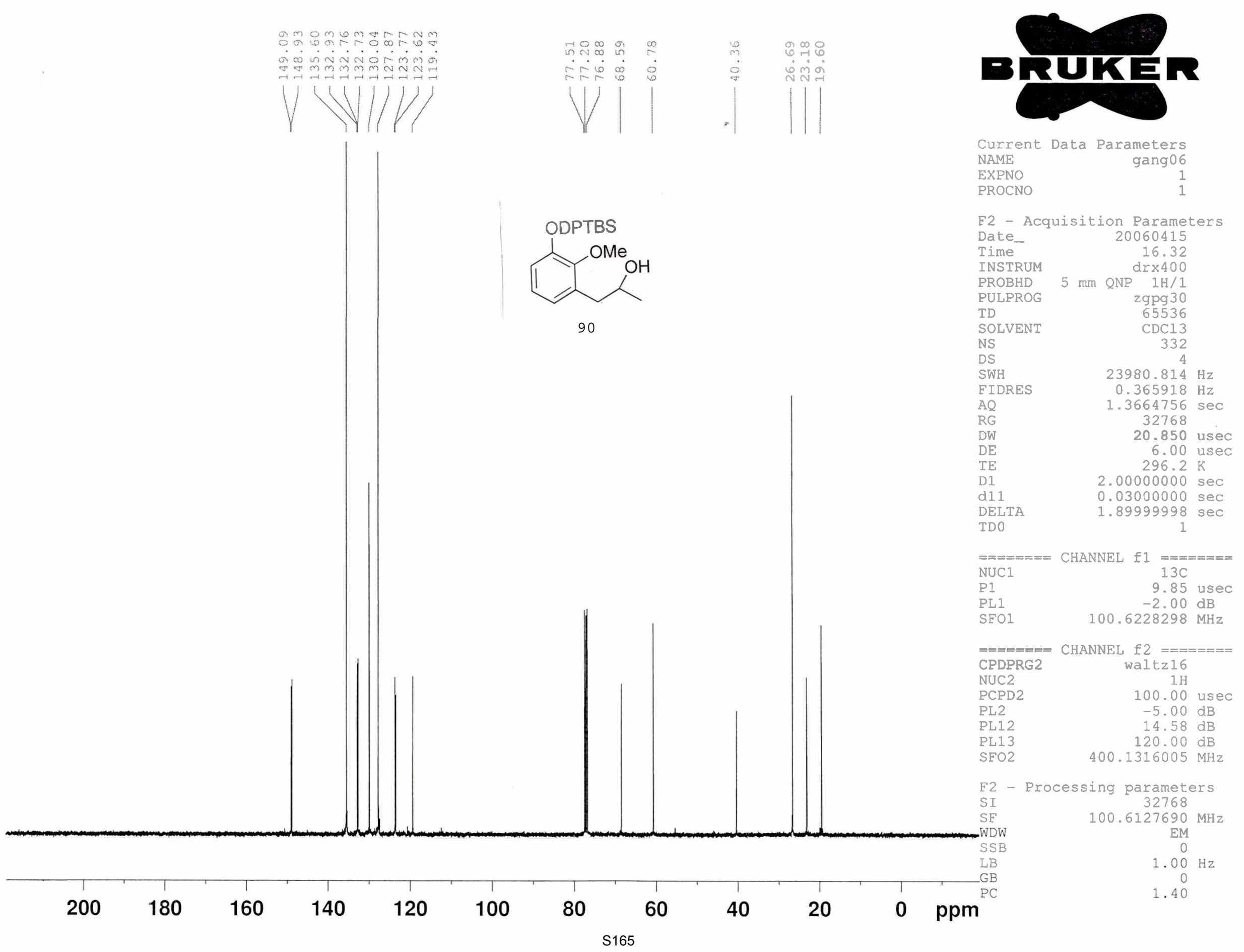




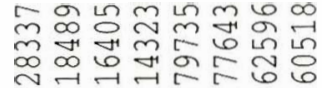

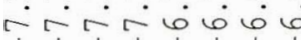
i)

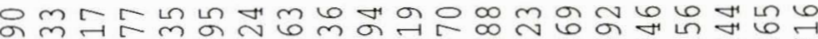

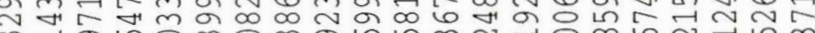
तु ने ம்
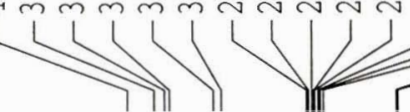

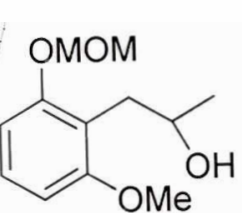

91
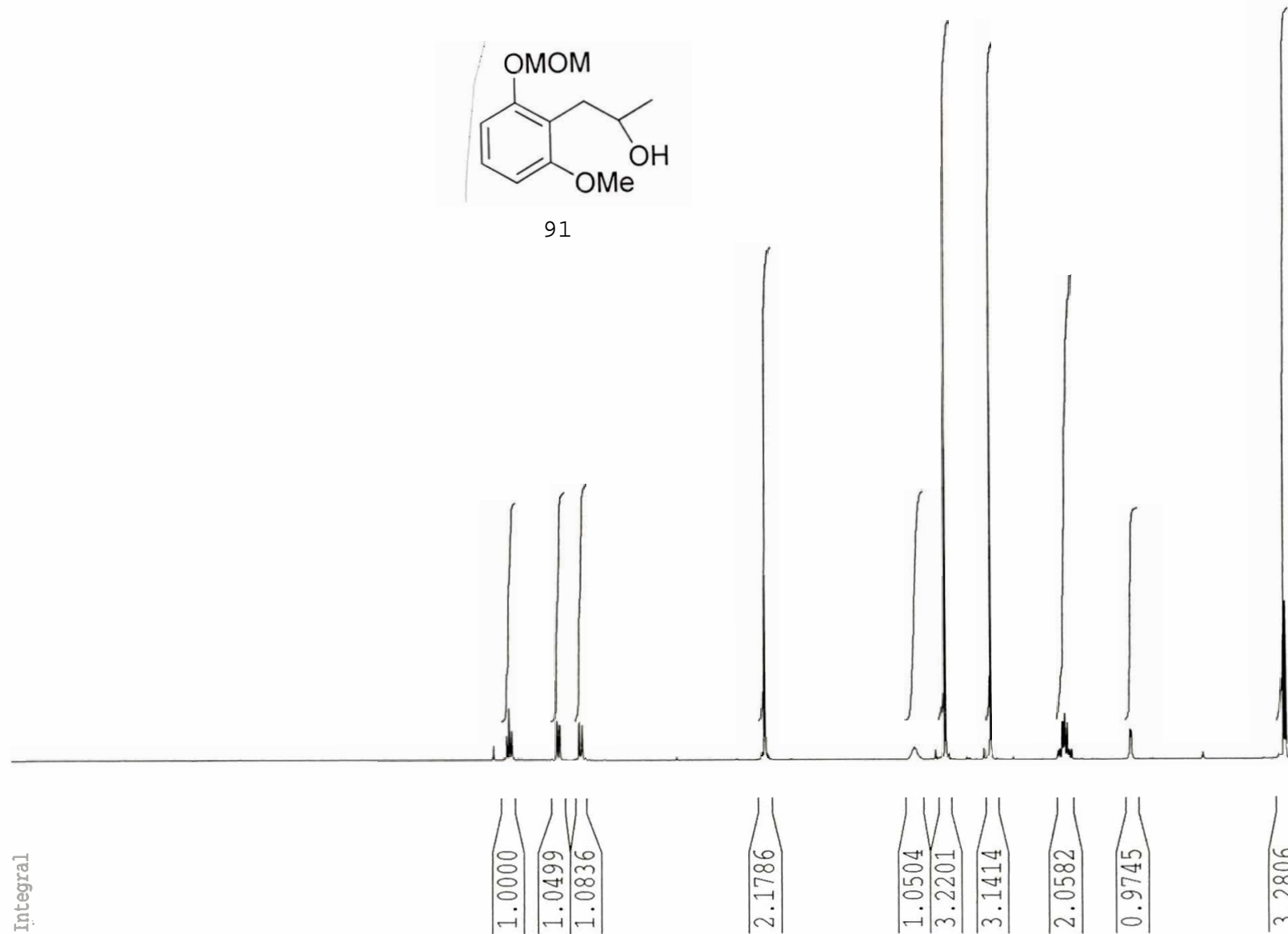

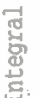
pom
10

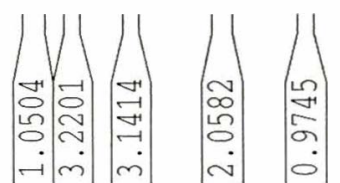

Current Data Parameters

EXPNO

PROCNO

F2 - Acquisition Parameters

Date 20050920

Time $\quad 18.42$

INSTRIMM drx400

PROBHD 5 mII ONP 1H/13

PULPRDG $\quad 2930$

32768

SOLVENT $\quad$ CDC13

NS

DS

SWH $\quad 4789.272 \mathrm{~Hz}$

FIDRES $\quad 0.146157 \mathrm{~Hz}$

AQ $\quad 3.4210291 \mathrm{sec}$

DW

DE

TE

MCRES

MCREST 64

104.400 usec 5.50 usec $295.2 \mathrm{~K}$

1.00000000 sec

$0.00000000 \mathrm{sec}$

$0.01500000 \mathrm{sec}$

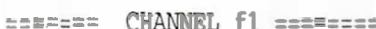
NOC1

P1 9.35 usec PL1 $-4.00 \mathrm{~dB}$

SEO1 400.1320007 MHz

F2 - Processing parameters

SI 32768

SF $\quad 400.1300000 \mathrm{MF}:$

WDW EM

$\begin{array}{ll}\text { SSB } & 0.30 \mathrm{~Hz}\end{array}$

$\begin{array}{lr}\mathrm{LB} & 0.30 \\ \mathrm{~GB} & 0\end{array}$

PC

1.00

1D NMR plot pa ameters

$\begin{array}{ll}\mathrm{CY} & 5.00 \mathrm{~cm}\end{array}$

F1P $\quad 11.000 \mathrm{ppm}$

F1 $4401.43 \mathrm{~Hz}$

$2 \mathrm{P}-1.000 \mathrm{ppm}$

$72-400.13 \mathrm{~Hz}$

PPMCM $\quad 0.60000 \mathrm{ppm} / \mathrm{cm}$

HZCM $240.07800 \mathrm{~Hz} / \mathrm{cm}$ 

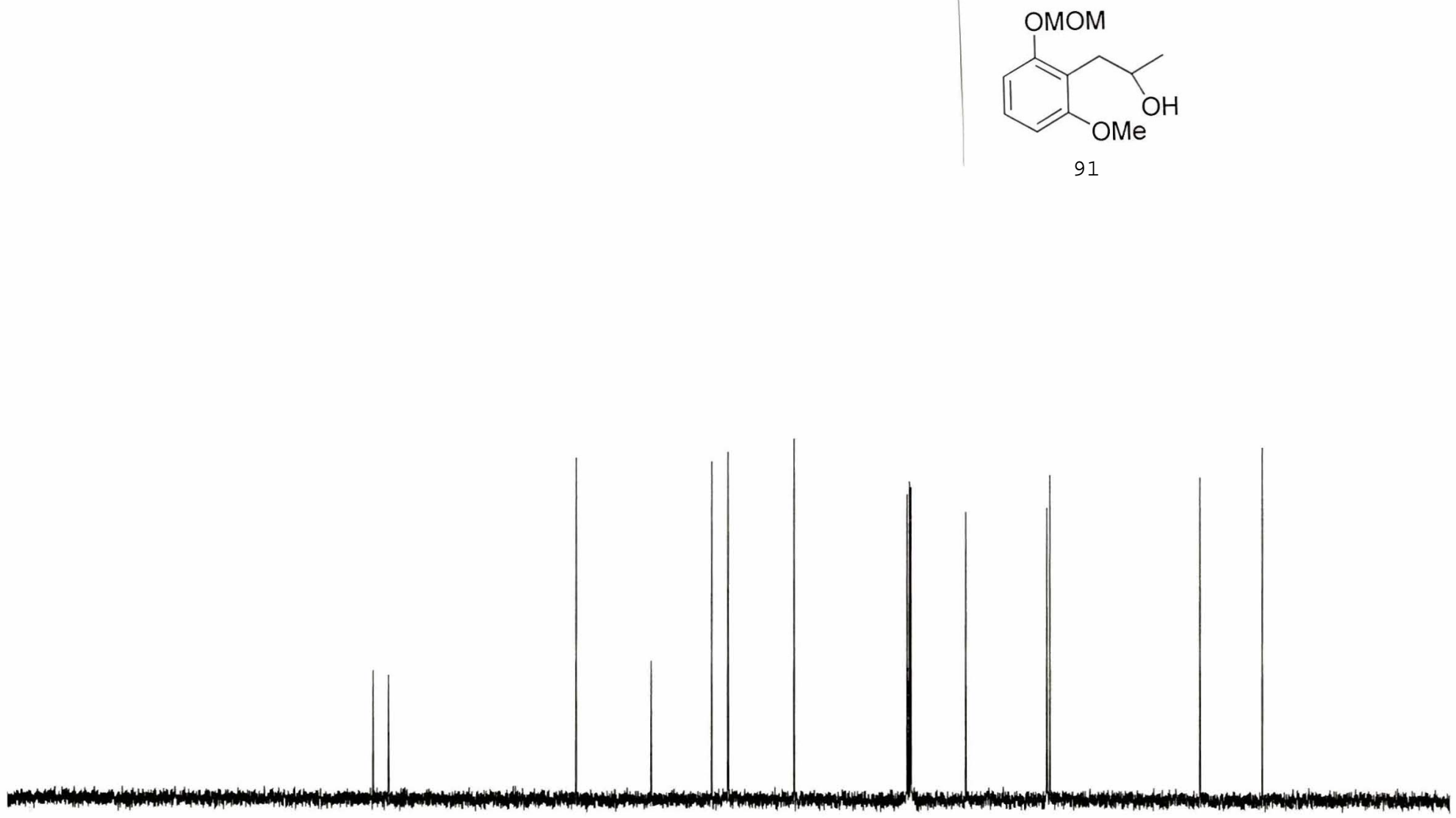

F2 - Acquisition Parameters

Date_ 20050920

Time $\quad 18.44$

INSTRUM $\quad \operatorname{dr} \times 400$

PROBHD 5 mm QNP $1 \mathrm{H} / 13$

PULPROG zgpg30

TD $\quad 6553$

$\begin{array}{lr}\text { SOLVENT } & \mathrm{CDCl3} \\ \text { NS } & 92\end{array}$

$\begin{array}{lr}\text { DS } & 4 \\ \text { SWH } & 23148.148 \mathrm{~Hz}\end{array}$

FIDRES $\quad 0.353213 \mathrm{~Hz}$

AQ $\quad 1.4156276 \mathrm{sec}$

$\begin{array}{lr}\text { RG } & 32768 \\ D W & 21.600 \text { use }\end{array}$

DE $\quad 5.50$ usec

TE $\quad 295.2 \mathrm{~K}$

D1 $\quad 0.15000001 \mathrm{sec}$

d11 $\quad 0.03000000 \mathrm{sec}$

DELTA $\quad 0.05000000 \mathrm{sec}$

MCREST $\quad 0.00000000 \mathrm{sec}$

MCWRK $\quad 0.01500000 \mathrm{sec}$

$\begin{array}{lr}==z====\text { CHANNEL } \mathrm{f} 1 \text { = }====== \\ \text { NUC1 } & 13 \mathrm{C} \\ \text { P1 } & 11.25 \mathrm{usec} \\ \text { PL1 } & 2.00 \mathrm{~dB} \\ \text { SF01 } & 100.6232933 \mathrm{MHz}\end{array}$

$\begin{array}{lc}====z== & \text { CHANNEL f2 }======= \\ \text { CPDPRG2 } & \text { waltz16 } \\ \text { NUC2 } & 1 \mathrm{H} \\ \text { PCPD2 } & 100.00 \text { usec } \\ \text { PL2 } & -4.00 \mathrm{~dB} \\ \text { PL12 } & 16.58 \mathrm{~dB} \\ \text { PL13 } & 17.00 \mathrm{~dB} \\ \text { SF02 } & 400.1316005 \mathrm{MHz}\end{array}$

F2 - Processing parameters

SI 65536

$\begin{array}{ll}\text { SF } & 100.6127290 \mathrm{MHz}\end{array}$

WDW
SSB

LB $\quad 1.00 \mathrm{~Hz}$

PC $\quad 1.40$

1D NMR plot parameters

$\begin{array}{lr}\text { CX } & 20.00 \mathrm{~cm} \\ \text { CY } & 5.00 \mathrm{~cm} \\ \text { F1P } & 215.000 \mathrm{ppm} \\ \text { F1 } & 21631.74 \mathrm{~Hz} \\ \text { F2P } & -5.000 \mathrm{ppm} \\ \text { F2 } & -503.06 \mathrm{~Hz} \\ \text { PPMCM } & 11.00000 \mathrm{ppm} ; \mathrm{cm} \\ \text { HZCM } & 1106.73999 \mathrm{~Hz} ; \mathrm{Cm}\end{array}$




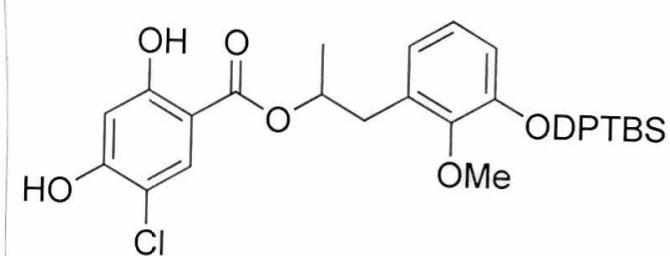

93

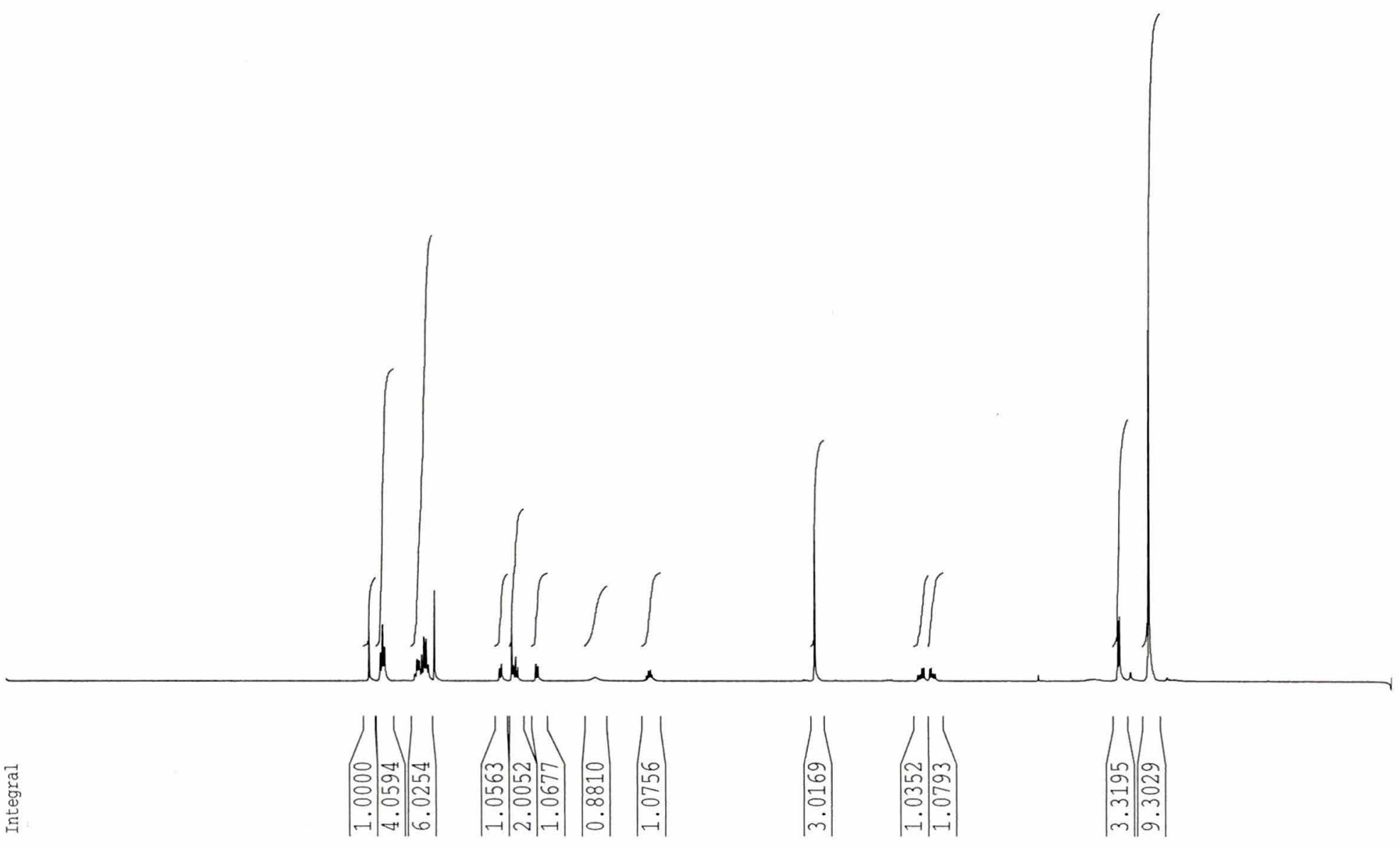

Current Data Parameters

NAME

EXPNO

PROCNO

F2 - Accuisition Parameters

Date

Time 20051116

INSTRUM drx400

PROBHD $5 \mathrm{~mm}$ ONP $1 \mathrm{H} / 13$

PUT,PROG

TD 32768

SOLVENT $\quad \mathrm{CDCl}$

SWH

FIDRES

4789.272

AQ $\quad 3.4210291 \mathrm{sec}$

RG 322.5

Dit $\quad 104.400$ usec

DE $\quad 5.50$ usec

TE $294.2 \mathrm{~K}$

D1 $\quad 1.00000000 \mathrm{sec}$

MCREST $\quad 0.00000000 \mathrm{sec}$

MCWRK $\quad 0.01500000 \mathrm{sec}$

=ニ=ニ=ニ== CHANNEL $\mathrm{fl}== \pm== \pm==$

NUC1 $1 \mathrm{H}$

P1 9.35 usec

PL1 $\quad-4.00 \mathrm{~dB}$

SF01 $\quad 400.1320007 \mathrm{MHz}$

F2 - Processing parameters

SI $\quad 32768$

SF $\quad 400.1300091 \mathrm{MHz}$

$\begin{array}{lr}\text { WDW } & \text { BM } \\ \text { SSB } & 0\end{array}$

SSB

LB

GB

$0.30 \mathrm{~Hz}$

1.00

1D MMR plot parameters

$\begin{array}{lr}\text { CX } & 20.00 \mathrm{~cm} \\ \text { CY } & 5.00 \mathrm{~cm}\end{array}$

F1P $\quad 11.000 \mathrm{ppm}$

$4401.43 \mathrm{~Hz}$

F2P

2P

PPMCM $-400.13 \mathrm{~Hz}$

$0.60000 \mathrm{ppm} / \mathrm{cm}$

HZCM

$240.07800 \mathrm{~Hz} / \mathrm{cm}$ 


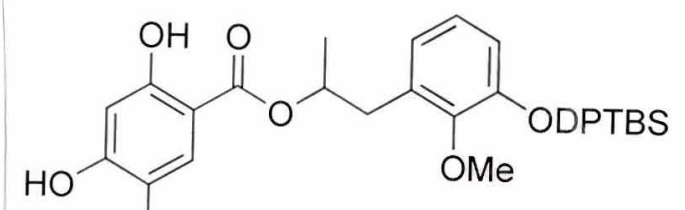

Current Data Parameters NAME

EXPNO

sg495

PROCNO

F2 - Acquisition Parameters

Date__ 20051116

Time 15.36

TROBHD

PULP

$5 \mathrm{~mm}$ CPDUL $13 \mathrm{C}$

PUILPOG

$\mathrm{TD}$

SOLVENT

NS

DS

SWH

FIDRES

AQ

$\mathrm{RG}$

DW

$\mathrm{DE}$

TE

d11

DELTA

DELTA
TDO

zgpg 30

65536

1024

$30030.029 \mathrm{~Hz}$

$0.458222 \mathrm{~Hz}$

$1.0912410 \mathrm{sec}$

13004

16.650 usec

6.00 usec $300.0 \mathrm{~K}$

$0.15000001 \mathrm{sec}$

$0.03000000 \mathrm{sec}$

$0.05000000 \mathrm{sec}$

$=====$

NUC

P1

PL1
SFO1

CHANNEL

1

CPDPRG2 waltz16

NUC2

PCPD2

PL2

PL12

PL13

$\mathrm{SFO} 2$

9.95 usec

$3.80 \mathrm{~dB}$

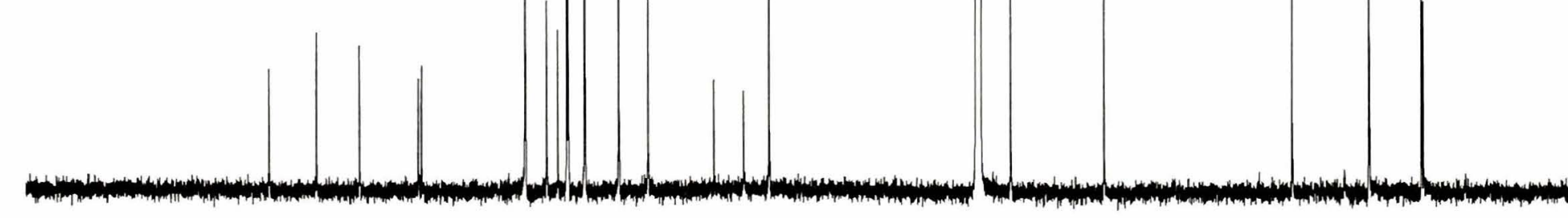

2 - Processing parameters

SI 32768

32768
$125.7577890 \mathrm{MHz}$

EM

0
$1.00 \mathrm{~Hz}$

0 


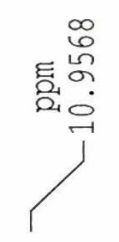

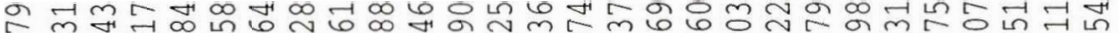

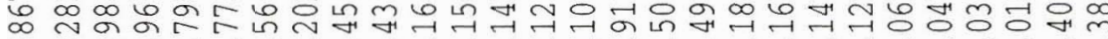
- م
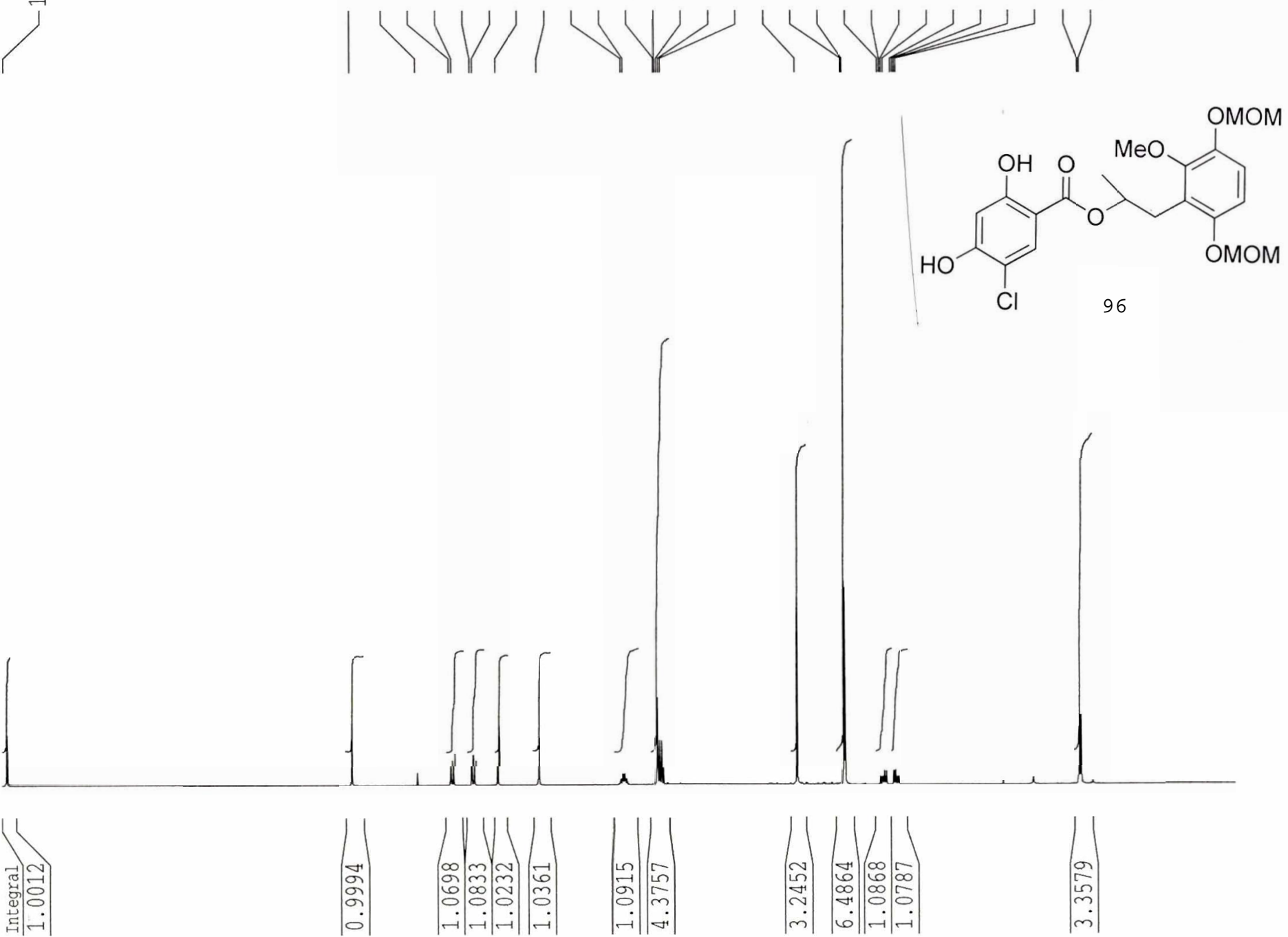

Current Data Parameters

F2 - Acquisition Parameters

Date_ 20050913

Time $\quad 17.46$

INSTRUM drx400

PROBHD 5 mat QNP $1 \mathrm{H} / 13$
PULPROG

32768

SOLVENT

NS

DS

SWH

FIDRES

$\mathrm{AQ}$

$\mathrm{RG}$

DW

$\mathrm{DE}$

TE

D1

MCREST

MCWRK

CDC13

2

$5592.841 \mathrm{~Hz}$

$0.170680 \mathrm{~Hz}$ $2.9295092 \mathrm{sec}$ 101.6

89. 400 usec 5.50 usec $296.2 \mathrm{~K}$

$1.00000000 \mathrm{sec}$ $0.00000000 \mathrm{sec}$ $0.01500000 \mathrm{sec}$

$=====\pi=+$ CHANNEL $\mathrm{fl}== \pm== \pm= \pm$ NUC1

P1

PL1 .35 usec $-4.00 \mathrm{~dB}$

SFO

$400.1328009 \mathrm{MHz}$

F2 - Processing parameters

$400.1300000 \mathrm{MHz}$

WDW EM

SSB

LB

GB

$$
\begin{gathered}
\text { EM } \\
0 \\
0.30 \mathrm{~Hz} \\
0
\end{gathered}
$$

1D NMR plot parameters

$\begin{array}{lr}\text { CX } & 20.00 \mathrm{~cm} \\ \text { CY } & 3.00 \mathrm{~cm} \\ \text { F1P } & 11.000 \mathrm{ppm} \\ \text { F1 } & 4401.43 \mathrm{~Hz} \\ \text { F2P } & -1.000 \mathrm{ppm} \\ \text { F2 } & -400.13 \mathrm{~Hz} \\ \text { PPMCM } & 0.60000 \mathrm{ppm} / \mathrm{cm} \\ \text { HZCM } & 240.07800 \mathrm{~Hz} / \mathrm{cm}\end{array}$


F2 - Accuisition Parameters

$\begin{array}{lr}\text { Date_ } & 20050913 \\ \text { Time } & 17.48\end{array}$

$\begin{array}{lr}\text { Time } & 17.48 \\ \text { INSTRUM } & \text { drx400 }\end{array}$

PROBHD $5 \mathrm{~mm}$ QNP $1 \mathrm{H} / 13$

PULPROG 2gpg30

TD

SOLVENT $\quad \mathrm{CDCl}$
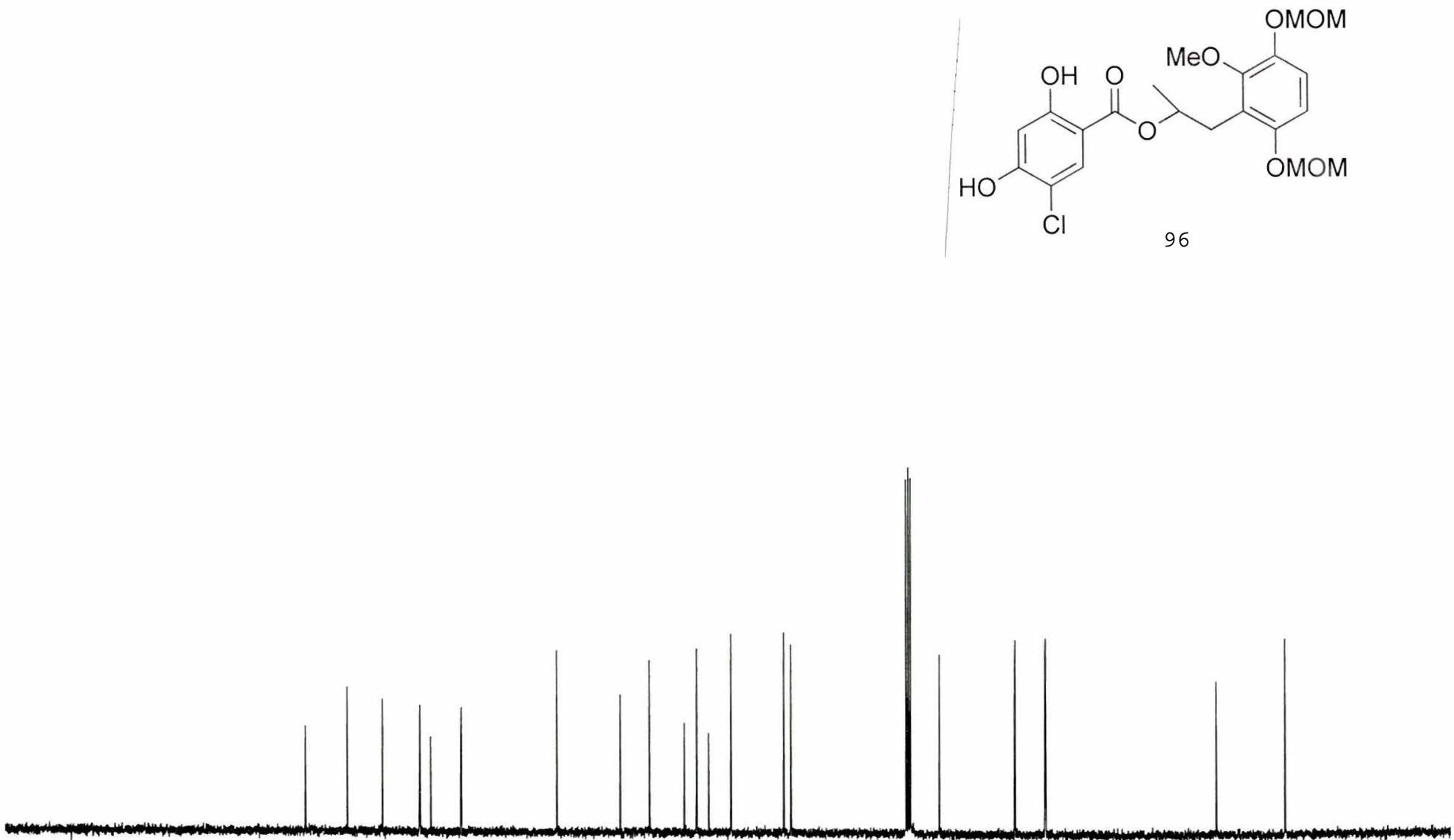

$$
\begin{gathered}
23148.148 \mathrm{~Hz} \\
0.353213 \mathrm{~Hz} \\
1.4156276 \mathrm{sec} \\
32768 \mathrm{H} \\
21.600 \mathrm{usec} \\
5.50 \mathrm{usec} \\
296.2 \mathrm{~K} \\
0.15000001 \mathrm{sec} \\
0.03000000 \mathrm{sec} \\
0.05000000 \mathrm{sec} \\
0.00000000 \mathrm{sec} \\
0.01500000 \mathrm{sec}
\end{gathered}
$$

$\begin{array}{lc}==z= \pm== & \text { CHANNEL } \mathrm{f} 2=z=z= \pm== \\ \text { CPDPRG2 } & \text { waltz16 } \\ \text { NUC2 } & 1 \mathrm{H} \\ \text { PCPD2 } & 100.00 \mathrm{usec} \\ \text { PL2 } & -4.00 \mathrm{~dB} \\ \text { PL12 } & 16.58 \mathrm{~dB} \\ \text { PL13 } & 17.00 \mathrm{~dB} \\ \text { SFO2 } & 400.1316005 \mathrm{MHz}\end{array}$



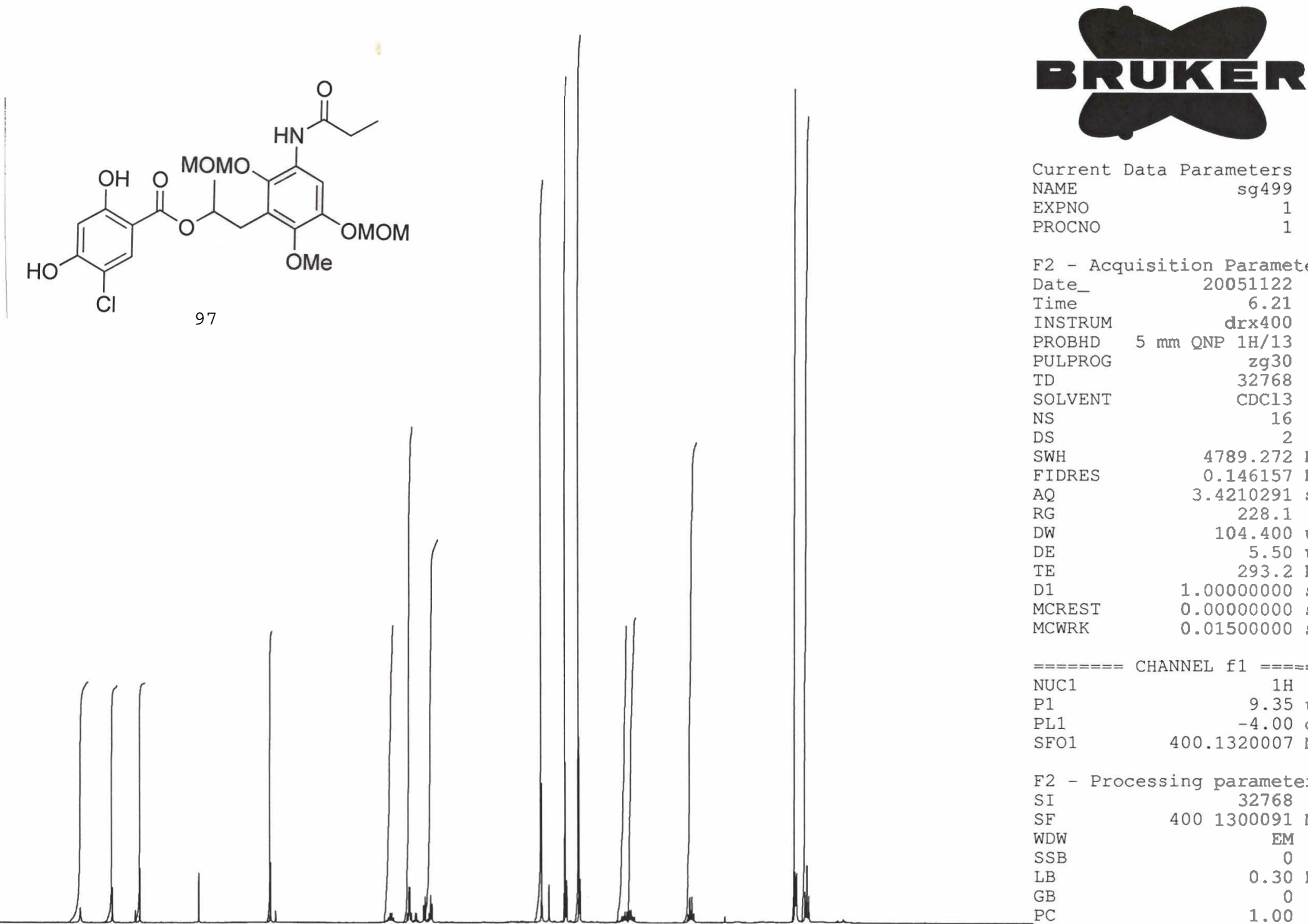

PROCNO

F2 - Acquisition Parameters

Date_ 20051122

Time

20051122
6.21

INSTRUM

PROBHD

PULPROG

arx400

TD

$5 \mathrm{~mm}$ QNP 1H/13

SOLVENT

NS

DS

SWH

IDRES

$A Q$

RG

DW

DE

MCREST

MCWRK

zg30
32768

CDC13

16

$4789.272 \mathrm{~Hz}$

$0.146157 \mathrm{~Hz}$

$3.4210291 \mathrm{sec}$ 228.1

104.400 usec 5.50 usec $293.2 \mathrm{~K}$

$1.00000000 \mathrm{sec}$

$0.00000000 \mathrm{sec}$ $0.01500000 \mathrm{sec}$

$\begin{array}{lr}=======\text { CHANNEL } \mathrm{fl}========1 & 1 \mathrm{H} \\ \text { NUC1 } & 9.35 \mathrm{usec} \\ \text { P1 } & -4.00 \mathrm{~dB} \\ \text { PL1 } & -4.007\end{array}$

SFO1

$400.1320007 \mathrm{MHz}$

F2 - Processing parameters

SI 32768

SF $4001300091 \mathrm{MHz}$

WDW

SSB

LB

EM

0
0.30
$H z$

0.30
0

1.00

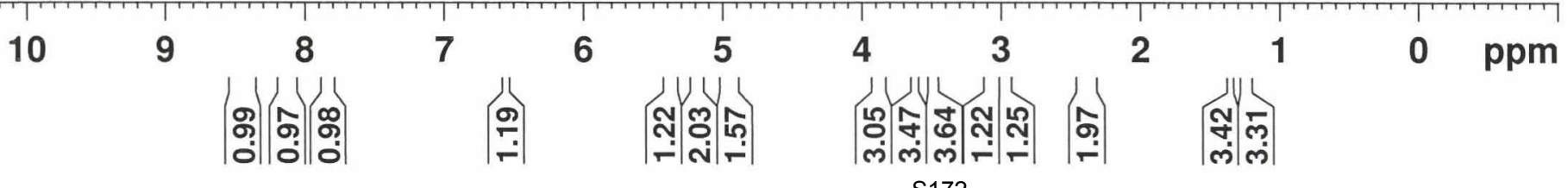



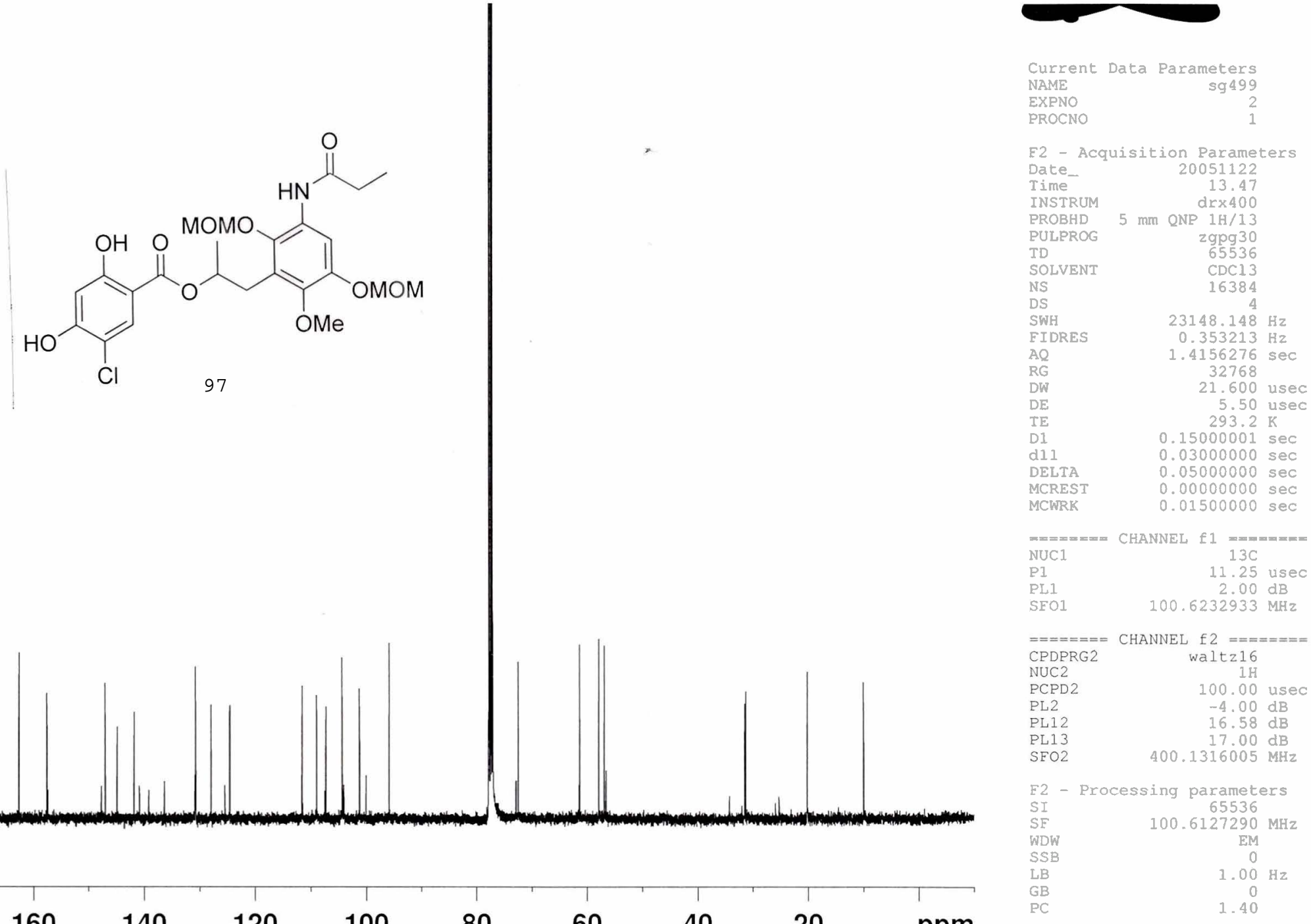

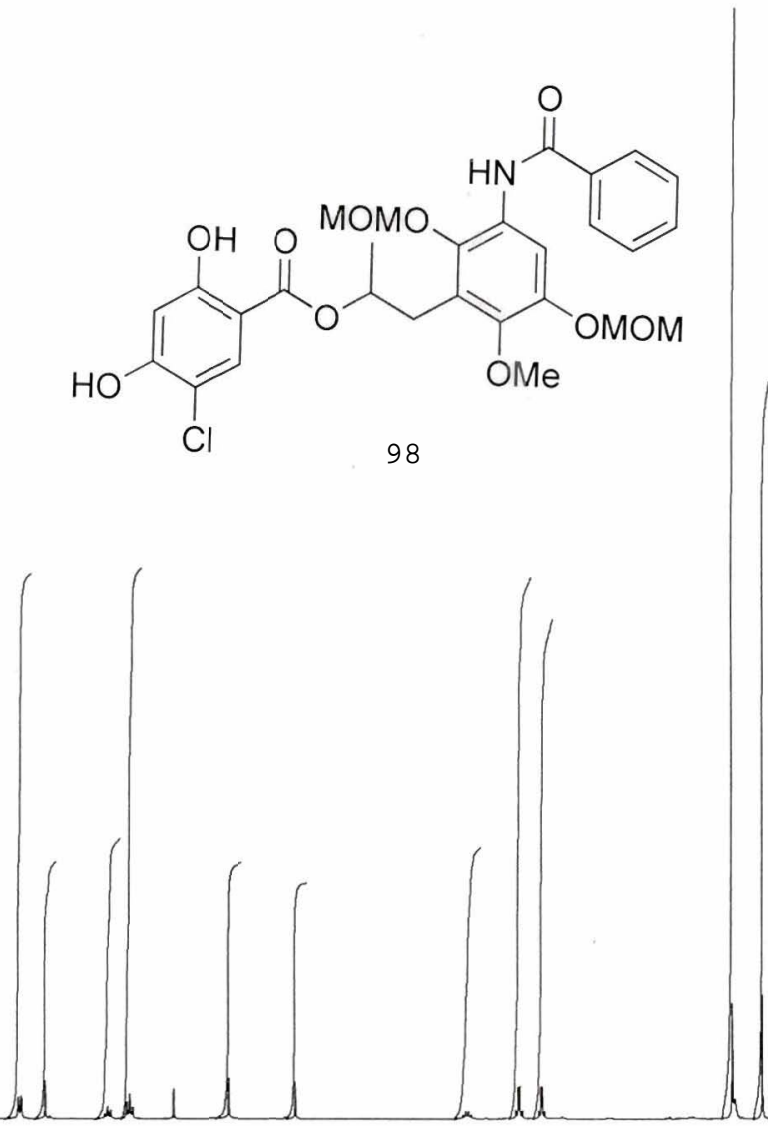

moumbur

(2)

Current Data Parameter

NAME

EXPNO

PROCNO

F2 - Acquisition Parameters

$\begin{array}{lr}\text { Date_ } & 20051121 \\ \text { Time } & 5.10\end{array}$

INSTRUM

PROBHD

PULPROG

TD

SOLVENT

NS

DS

SWH

FIDRES

$\mathrm{AQ}$

RG

DW

DE

TE

MCREST

MCWRK

5.10
dr. 400

$5 \mathrm{~mm}$ QNP 1H/13

2930
32768

CDC13

4789.272

$0.146157 \mathrm{~Hz}$

$3.4210291 \mathrm{sec}$

203.2

104.400 usec

5.50 usec

$293.2 \mathrm{~K}$

1.00000000 sed

$0.00000000 \mathrm{sec}$

0.01500000 sec

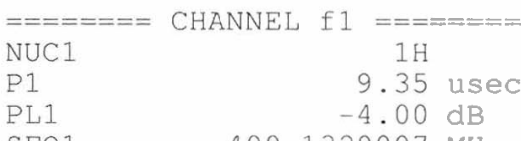

SFO1

400.1320007

F2 - Processing parameters SI $\quad 32768$

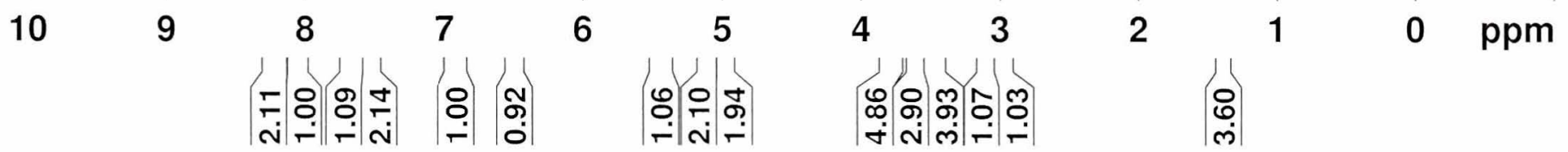




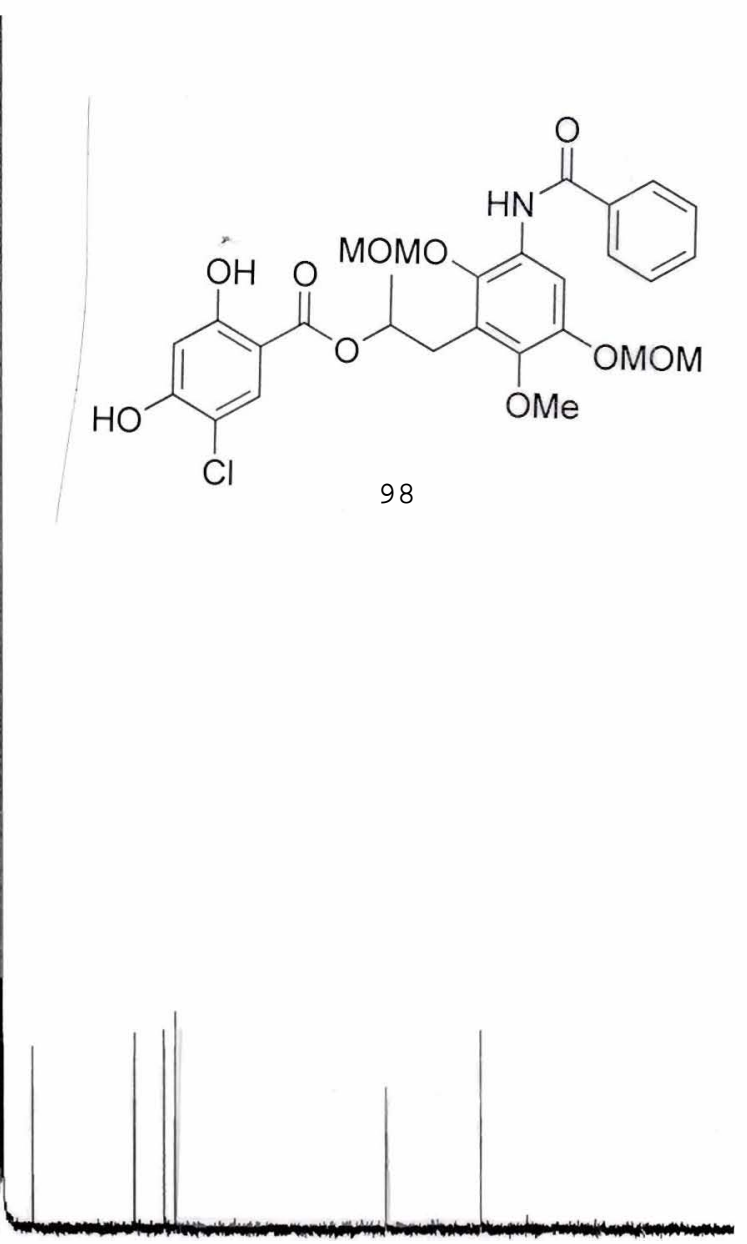

Current Data Parameters NAME PROCNO sg 498

F2 - Acquisition Parameters Date_ 20051121 $\begin{array}{lr}\text { Time } & 12.09\end{array}$ INSTRUM drX400 PROBHD 5 mU QNP $1 \mathrm{H} / 13$ PULPROG 2gpg 30 TD 65536 SOLVENT CDC13

NS

DS

SWH

FIDRES

AQ
RG

$\mathrm{RG}$
$\mathrm{DW}$
$\mathrm{DE}$
$\mathrm{TE}$

TE

d11

DELTA

DELTA

MCWRK

15360

$23148.148 \mathrm{~Hz}$

$0.353213 \mathrm{~Hz}$

$1.4156276 \mathrm{sec}$ 32768

21.600 usec

5.50 usec

$15000001 \mathrm{sec}$

$0.15000001 \mathrm{sec}$

$0.0500000 \mathrm{sec}$

0.0500000 sec

0.0000000 sec

NUC1

$\mathrm{P} 1$

PL1

SFO1

CHANNEL

$1=======$
$13 \mathrm{C}$
11.25 usec
$2.00 \mathrm{~dB}$
$32933 \mathrm{MHz}$

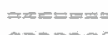

$100.6232933 \mathrm{MHz}$

NUC2 Waltz16

PCPD2

PL2

PL12

PL13

$\mathrm{SFO} 2$

100.00 use

$-4.00 \mathrm{~dB}$

$16.58 \mathrm{~dB}$

$400.1316005 \mathrm{MHz}$

SI

65536

$100.6127290 \mathrm{MHz}$

LB

$\mathrm{EM}$
0

1. $00 \mathrm{~Hz}$

1. 40 

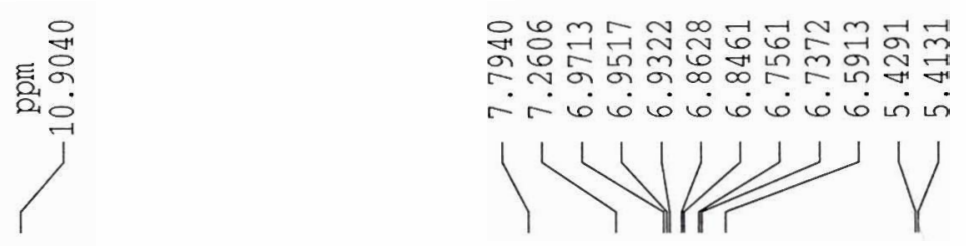

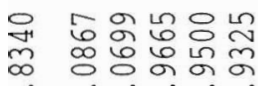

m mं $\dot{0}$ i

ํำ

111

मㄷ<smiles>COc1c(O)cccc1CC(C)OC(=O)c1cc(Cl)c(O)cc1O</smiles>

95

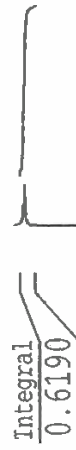

ppm
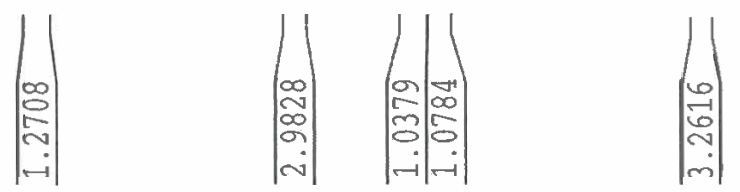

Current Data Parameters

F2 - Acquisition Paramete s

Date_ 20051122

Time $\quad 13.05$

INSTRUM drx400

PROBHD 5 mm ONP $1 \mathrm{H} / 13$

$\begin{array}{lr}\text { PROBHD } 5 \text { man ONP } & 1 \mathrm{H} / 13 \\ \text { PULPROG } & \mathrm{zg} 30\end{array}$

TD 32768

SOLVENT $\quad \mathrm{CDCl} 3$

NS

DS

SWH

FIDRES

$A Q$
RG

$\mathrm{DW}$

DW

TE

D1

MCREST

16
2

$4789.272 \mathrm{~Hz}$ $0.146157 \mathrm{~Hz}$

$3.4210291 \mathrm{sec}$ 406.4

104.400 usec 5.50 usec $293.2 \mathrm{~K}$

$1.00000000 \mathrm{sec}$

$0.00000000 \mathrm{sec}$

MCWRK $\quad 0.01500000 \mathrm{sec}$

$========$ CHANNEL f1 ==:==z==" NUC1

9.35 usec

PL1 $-4.00 \mathrm{~dB}$

SFO1 $400.1320007 \mathrm{MHz}$

F2 - Processing parameters

SI 32768

SF $\quad 400.1300091 \mathrm{MHz}$

$\begin{array}{lr}\text { SF } & 400.1300091 \\ \text { WDW } & \text { EM }\end{array}$

$\begin{array}{lc}S S B & 0 \\ L B & 0.30 \mathrm{~Hz}\end{array}$

GB $\quad 0$

PC

1D NMR plot parameters

CX $\quad 20.00 \mathrm{~cm}$

CY $\quad 5.00 \mathrm{~cm}$

F1P $\quad 11.000 \mathrm{pom}$

F1 $4401.43 \mathrm{~Hz}$

F2P $\quad-1.000 \mathrm{ppm}$

E2

PPNCY

$-400.13 \mathrm{~Hz}$

$0.60000 \mathrm{ppm} / \mathrm{cm}$

HZCM

$240.07800 \mathrm{~Hz} / \mathrm{cm}$ 
<smiles>COc1c(O)cccc1CC(C)OC(=O)c1cc(Cl)c(O)cc1O</smiles>

Current Data Parameters NAME sg500

EXPNO

PROCNO

F2 - Acquisition Parameters

Date_ 20051123

Time $\quad 2.45$

INSTRUM spect

PROBHD $5 \mathrm{~mm}$ CPDUL 13C

PULPROG zgpg 30

TD 65536

SOLVENT CDC13

NS

DS

SWH

FIDRES

AQ

RG

DW

TE

DI

d11

DELTA

TDO

2000

$30030.029 \mathrm{~Hz}$

$0.458222 \mathrm{~Hz}$

$1.0912410 \mathrm{sec}$ 13004

16.650 usec 6.00 usec $298.0 \mathrm{~K}$

$0.15000001 \mathrm{sec}$

0.03000000 sec

0.05000000 sec

$=======$ CHANNEL $\mathrm{f}$ NUC1

P1

$13 \mathrm{C}$

PL1

9.95 usec

SFO1

$125.7703640 \mathrm{MHz}$

$======$ CHANNEL $\mathrm{f}$

CPDPRG2 waltz16

NUC2

$1 \mathrm{H}$

80.00 usec

$2.20 \mathrm{~dB}$

$16.80 \mathrm{~dB}$

PL12

PL13

$10.00 \mathrm{~dB}$

$500.1320000 \mathrm{MHz}$

F2 - Processing parameters

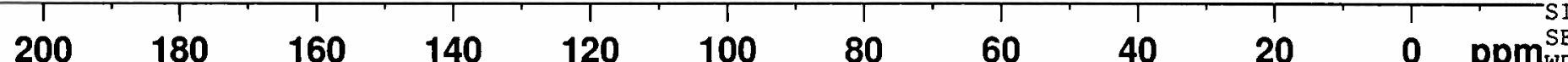

SE

parameter

WDW
SSB
LB
GB
PC

$125.7577890 \mathrm{MHz}$

EM
0
$1.00 \mathrm{~Hz}$
0
4




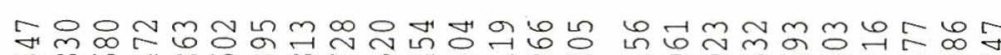

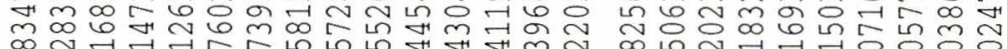
मिं

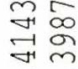
10112
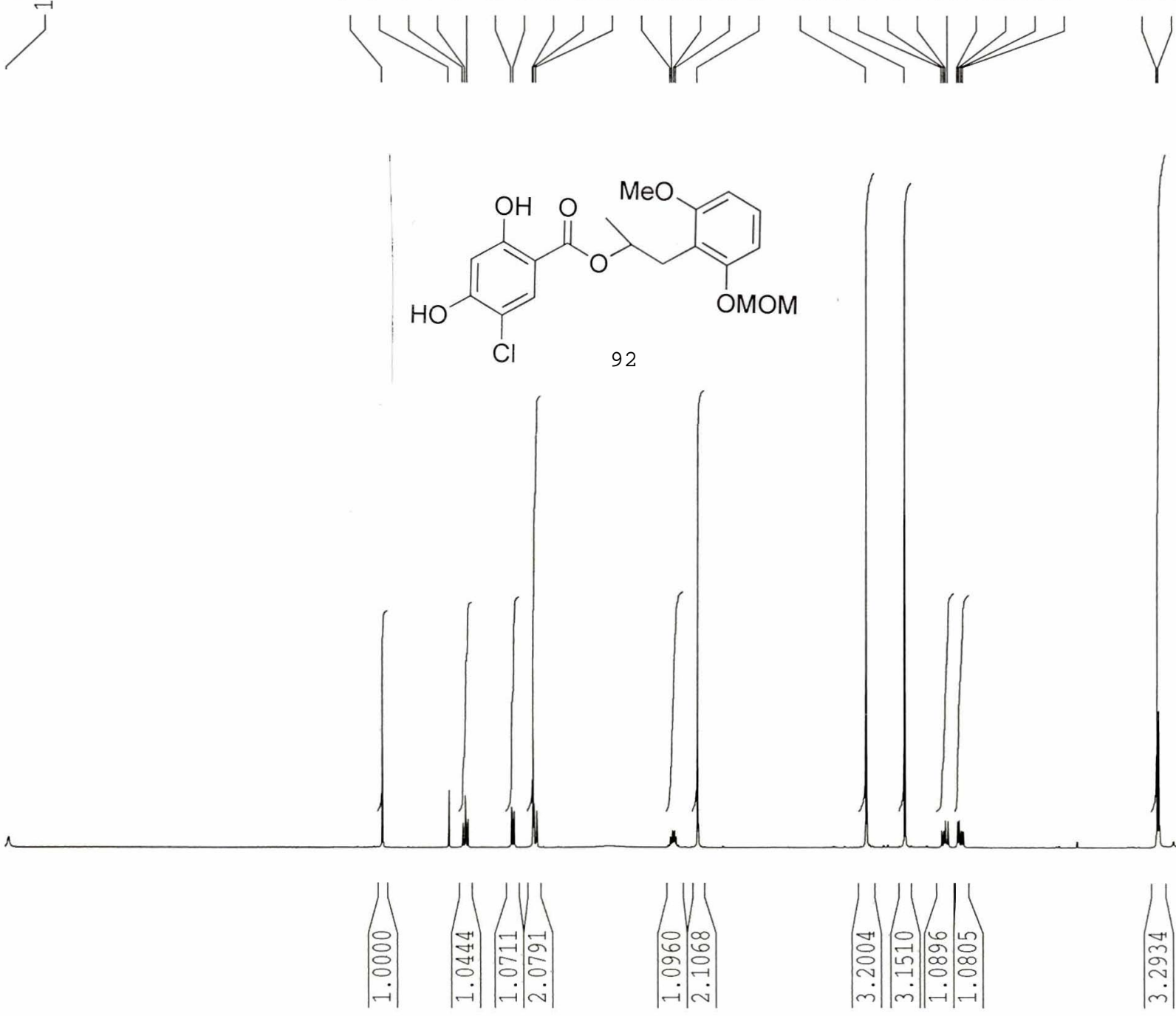

8
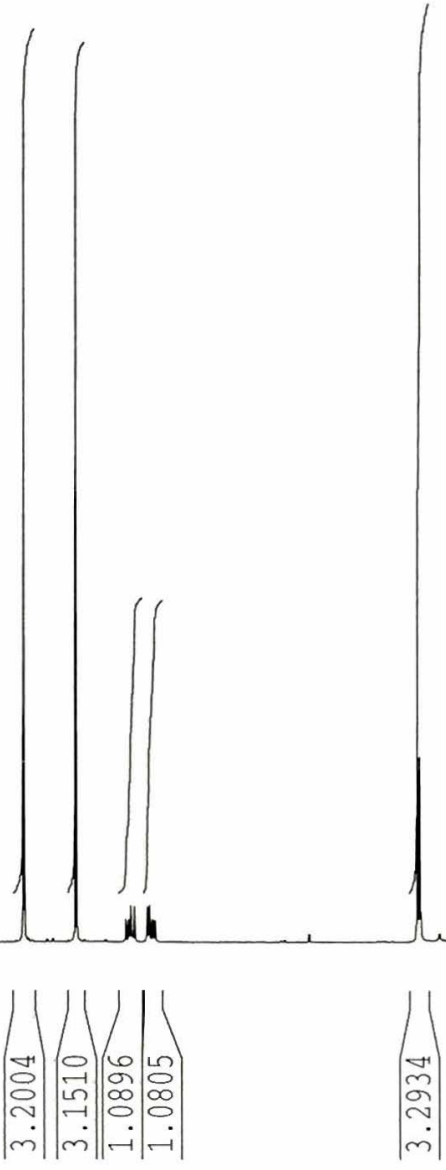

Current Data Parameters

EXPNO

PROCNO

$\operatorname{sg} 482$

F2 - Acquisition parameters

Date_ 20050929

Time $\quad 10.11$

INSTRUM drX400

PROBHD 5 man ONP 1H/13

PULPROG $\quad \mathrm{zg} 30$

TD 32768

SOLVENT $\quad \mathrm{CDCl3}$

NS

DS

SWH $\quad 4789.272$

FIDRES $\quad 0.146157$

$\mathrm{Al} \quad 3.4210291$

RG 228.1

DW $\quad 104.400$ usec

DE $\quad 5.50$ usec

TE $293.2 \mathrm{~K}$

D1 $1.00000000 \mathrm{sec}$

MCREST $\quad 0.00000000 \mathrm{sec}$

MCWRK $\quad 0.01500000 \mathrm{sec}$

$===\pi== \pm=$ CHANNBL $\mathrm{f}$

NUC1

P1

$-4.00 \mathrm{~dB}$

F2 - Processing parameters

SI $\quad 32768$

SF $\quad 400.1300000 \mathrm{MHz}$

$\begin{array}{lr}\text { WDW } & \text { EM } \\ \text { SSB } & 0\end{array}$

LB $\quad 0.30 \mathrm{~Hz}$

$\begin{array}{ll}P C & 1.00\end{array}$

1D NMR plot parameters

CX $\quad 20.00 \mathrm{~cm}$

CY $\quad 5.00 \mathrm{~cm}$

81P $\quad 11.000 \mathrm{ppm}$

F1 $\quad 4401.43 \mathrm{~Hz}$

F2P $\quad-1.000 \mathrm{ppm}$

F2

PPHCM

PPNCM

$-400.13 \mathrm{~Hz}$

$0.60000 \mathrm{ppm} / \mathrm{cm}$ $240.07800 \mathrm{~Hz} / \mathrm{cm}$ 
윰구 ह로용

กิ้

棺

$\stackrel{m}{\Sigma} \underset{m}{\Sigma}$

오 오
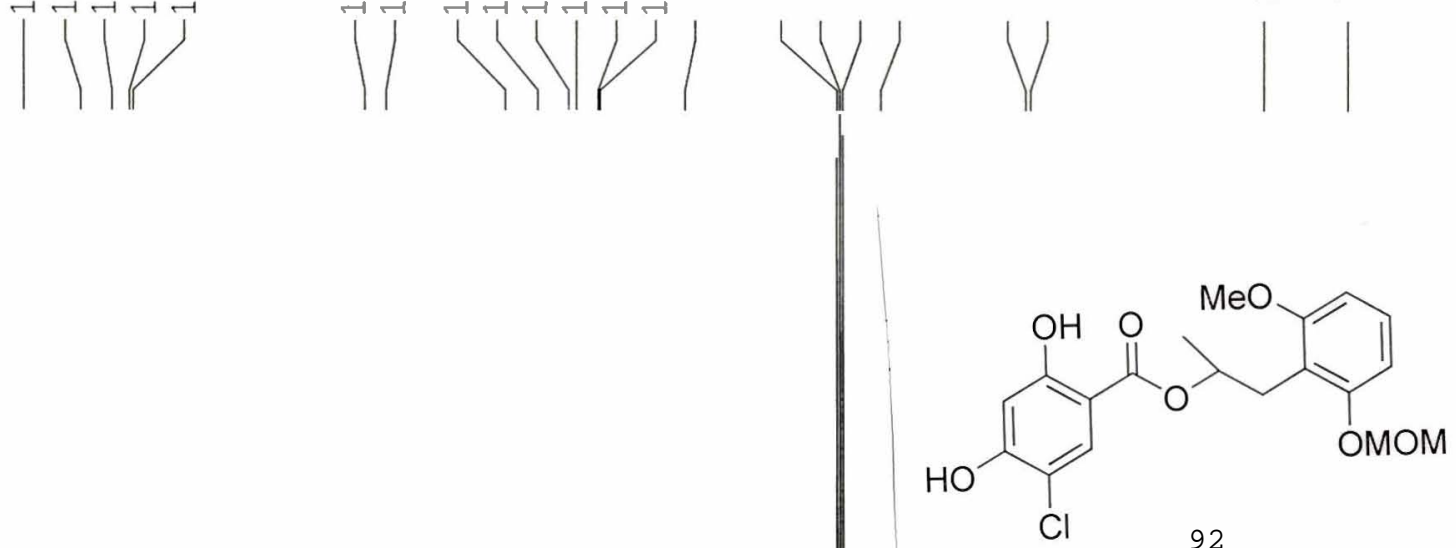

92

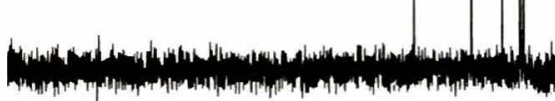

F2 - Acquisition Parameters

Date 20050929

Time- $\quad 10.15$

INSTRUIM drX400

TD

SOLVEN

NS

DS

FIDRES

AQ

RG

DW

TE

D1

d11

DELTA

KCRES

2gpg30
65536

$\mathrm{CDCl3}$

$$
23148.148 \mathrm{~Hz}
$$

$0.353213 \mathrm{~Hz}$

$1.4156276 \mathrm{sec}$

32768

21.600 usec 5.50 usec

$0.15000001 \mathrm{sec}$

$0.03000000 \mathrm{sec}$

$0.05000000 \mathrm{sec}$

$0.0500000 \mathrm{sec}$

$=-=-==$ CHANNEL $f 1$ =="====

$\begin{array}{lr}\text { NUC1 } & 13 \mathrm{C} \\ \text { P1 } & 11.25 \mathrm{useC} \\ \text { PL1 } & 2.00 \mathrm{~dB} \\ \text { SE01 } & 100.6232933 \mathrm{MHz}\end{array}$

$===== \pm=$ CHANDLL f2 ==:====" CPDPRG2 waltz16

NUC2

waltzi6
$1 \mathrm{H}$

100.00 usec

$-4.00 \mathrm{~dB}$

$16.58 \mathrm{~dB}$

PL2
PL12

PL12
PL13

$17.00 \mathrm{~dB}$
$\mathrm{SPO} 2$
$400.1316005 \mathrm{MHz}$

F2 - Processing parameters

SI $\quad 65536$

SP $\quad 100.6127290 \mathrm{kHz}$

WSW

LB $\quad 1.00 \mathrm{~Hz}$

$\mathrm{GB}$

15 MR plot parameters

CX Nat plot paraters

$\begin{array}{ll}\mathrm{CX} & 20.00 \mathrm{~cm} \\ \mathrm{CY} & 12.50 \mathrm{~cm}\end{array}$

$\begin{array}{lr}\text { CY } & 12.50 \mathrm{~cm} \\ \text { F1P } & 215.000 \mathrm{ppm}\end{array}$

F1 $21631.74 \mathrm{~Hz}$

$\begin{array}{ll}\text { F2P } & -5.000 \mathrm{ppm} \\ \text { F2 } & -503.06 \mathrm{~Hz}\end{array}$

PEMCM $\quad 11.00000 \mathrm{ppen} / \mathrm{Cm}$

$\mathrm{HZCM}$

$1106.73999 \mathrm{~Hz} / \mathrm{ctg}$ 
F2 - Acquisition Parameters

Date 20051004

Time $\quad 23.31$

INSTRUM $\quad \operatorname{drx} 400$

PROBHD 5 ant QNP 1H/13<smiles>COc1cccc(O)c1CC(C)OC(=O)c1cc(Cl)c(O)cc1O</smiles>

PULPROG

$\mathrm{zg} 30$

TD 32768

SOLVENT $\quad \mathrm{CDC13}$

NS

SWH $\quad 4789.272 \mathrm{~Hz}$

FIDRES $\quad 0.146157 \mathrm{~Hz}$

$\mathrm{AQ} \quad 3.4210291 \mathrm{sec}$

RG

DW

TE

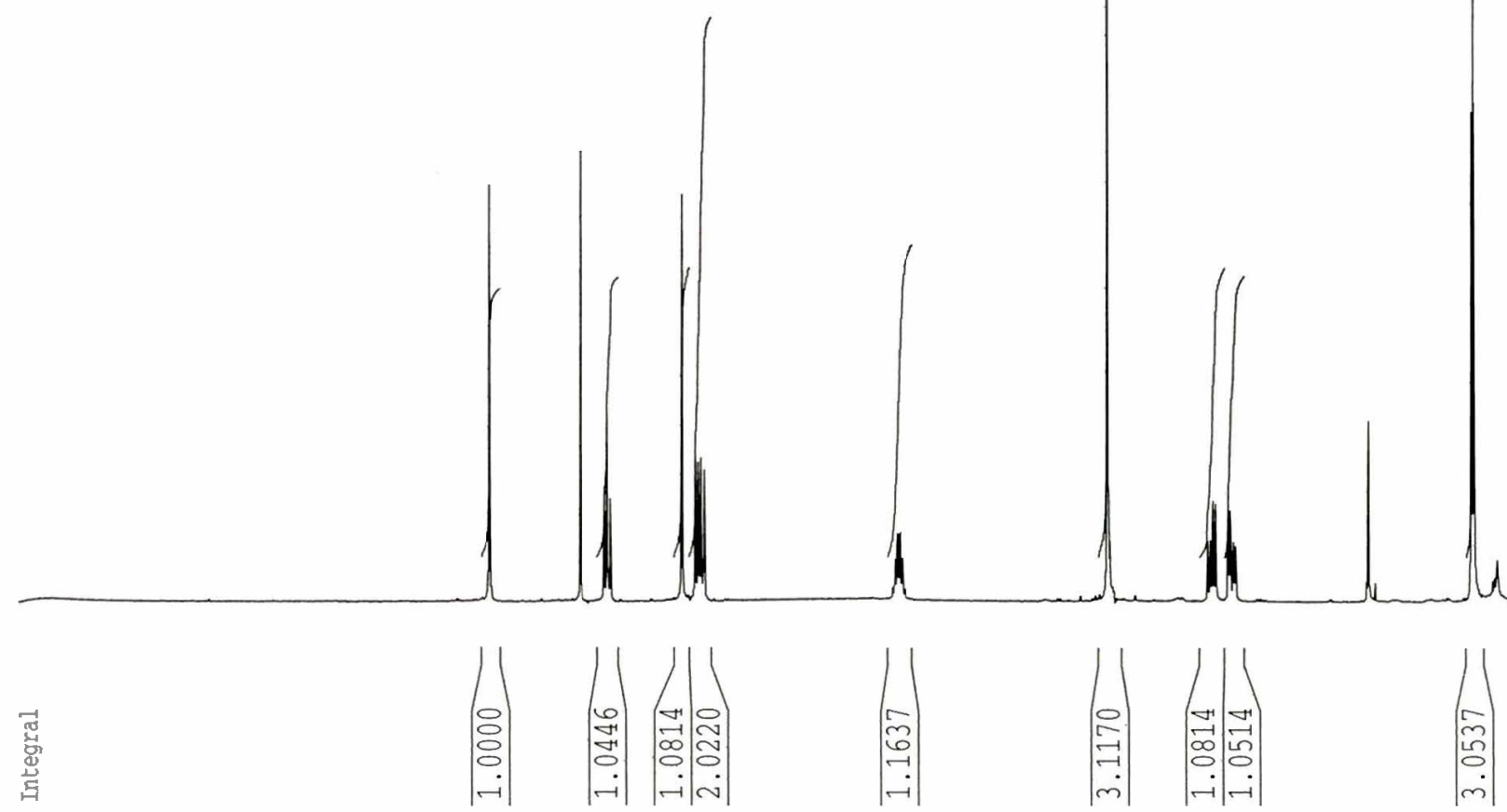

104.400 usec 5.50 usec $294.2 \mathrm{~K}$

D1 $\quad 1.00000000 \mathrm{sec}$

MCREST $\quad 0.00000000 \mathrm{sec}$

MCWRK $\quad 0.01500000 \mathrm{sec}$

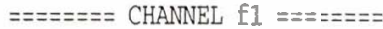
$\begin{array}{lr}\text { NUC1 } & 1 \mathrm{H} \\ \mathrm{P} 1 & 9.35 \mathrm{usec}\end{array}$ $\begin{array}{lr}\text { P1 } & 9.35 \text { usec } \\ \text { PL1 } & -4.00 \mathrm{~dB}\end{array}$

SF01 $\quad 400.1320007 \mathrm{MHz}$

F2 - Processing parameters

SI 32768

SF $\quad 400.1300000 \mathrm{MHz}$

WDW EM

LB $\quad 0.30 \mathrm{~Hz}$

GB

PC

0
1.00

1D NMR plot parameters

$\begin{array}{lc}\text { CX } & 20.00 \mathrm{~cm} \\ \text { CY } & 12.50 \mathrm{~cm} \\ \text { F1P } & 11.000 \mathrm{ppm} \\ \text { F1 } & 4401.43 \mathrm{~Hz} \\ \text { F2P } & -1.000 \mathrm{ppm} \\ \text { F2 } & -400.13 \mathrm{~Hz} \\ \text { PPMCM } & 0.60000 \mathrm{ppm} / \mathrm{cm} \\ \text { HZCM } & 240.07800 \mathrm{~Hz} / \mathrm{cm}\end{array}$




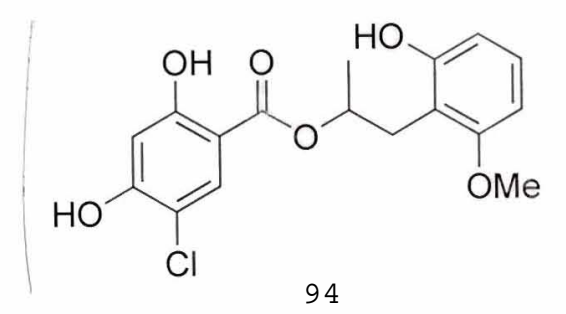

F2 - hoquisition Parameters

Date_ 20051005

Time $\quad 0.55$

INSTAUMA dr. $\times 400$

PROBHD 5 mIM ONP $1 H / 13$

PULPROG $\begin{array}{r}\text { 2gpg } 30 \\ \text { PR5 }\end{array}$

SOLVENT CEC13

NS

SWH $\quad 23148.148 \mathrm{~Hz}$

FIDAES $\quad 0.353213 \mathrm{~Hz}$

AQ $\quad 1.4156276 \mathrm{sec}$

$\begin{array}{ll}\text { RG } & 32768 \\ \text { DW } & 21.600 \text { ysec }\end{array}$

$\begin{array}{rr}\text { DHN } & 21.600 \text { usec } \\ \text { DEE } & 5.50 \text { use }\end{array}$ $294.2 \mathrm{~K}$

$0.15000001 \mathrm{sec}$

$0.03000000 \mathrm{sec}$

$\begin{array}{ll}\text { DII } & 0.03000000 \mathrm{sec} \\ \text { DELTA } & 0.05000000 \mathrm{sec}\end{array}$

MCREST $\quad 0.00000000 \mathrm{sec}$

MCHARK $\quad 0.01500000 \mathrm{sec}$

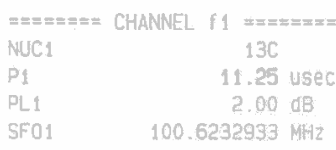

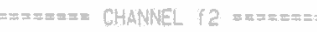

CPDPRG? CHANEL

NUC2 1 1H

PCPD2 100.00 use

$\begin{array}{ll}\text { PL2 } & -4.00 \mathrm{~dB} \\ \text { OL } 12 & 16.5 \mathrm{~d} \mathrm{~dB}\end{array}$

$\begin{array}{ll}\text { PL12 } & 46.58 \mathrm{~dB} \\ 0.13 & 17.00 \mathrm{~dB}\end{array}$

SF02 400 $1316005 \mathrm{MHZ}$

2 - Processing paraneters

51 processing par ativeters

SF $\quad 100.6127290 \mathrm{NHZ}$

WDW 태

$\triangle B \quad 1.00 \mathrm{~Hz}$

69

D NMP plot parameters

\begin{tabular}{|c|c|}
\hline $2 x$ & 20.00 \\
\hline$c y$ & 12.50 \\
\hline$=1 \mathrm{P}$ & 215.000 \\
\hline$=1$ & 21336.74 \\
\hline$=2 P$ & -5.000 \\
\hline$F_{2}$ & -503.06 \\
\hline DPMCM & 14.00000 \\
\hline
\end{tabular}



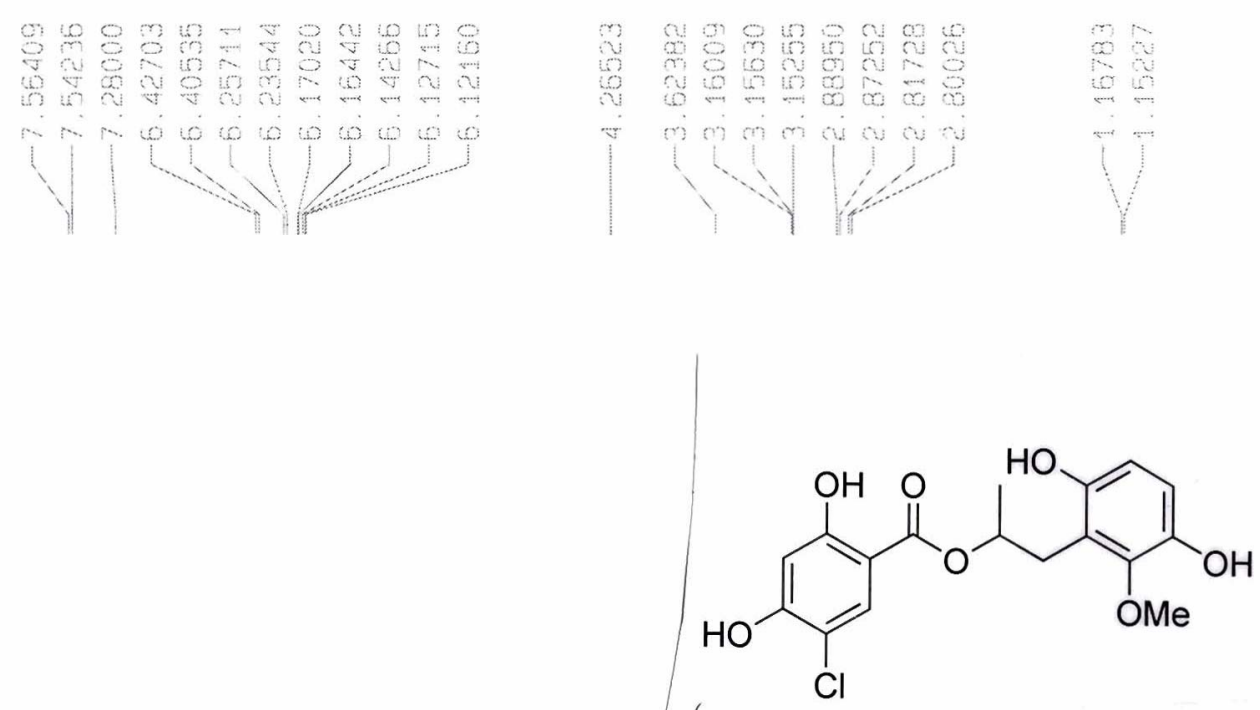

99
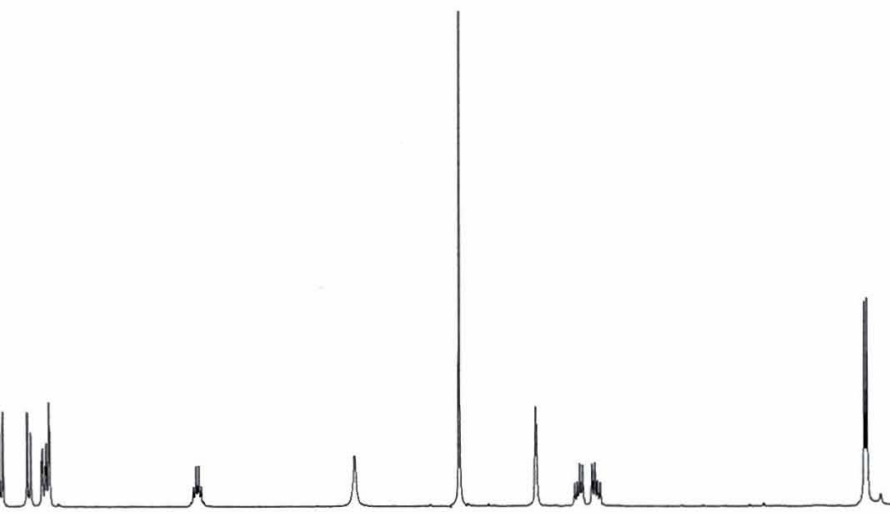

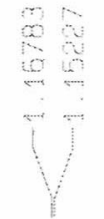

Curr

EXPNO

PROCNO

F2 - Acquisition Parameters

Date

Time

20051127
21.46

INSTAUM

dr $\times 400$

PROBHD $5 \mathrm{~mm}$ GNP $1 \mathrm{H} / 13$

PULPAOG

SOLVENT

NS

os

FIDRES

FIDAES

RG

DW

DE

TE

MCREST

MCWRK

2930

32768

$\mathrm{OH}$

16
2

$4789.272 \mathrm{~Hz}$

$0.146157 \mathrm{~Hz}$

$3.4210291 \mathrm{sec}$ 362

104.400 usec

5.50 usec

$293.2 \mathrm{~K}$

$1.00000000 \mathrm{sec}$

$0.00000000 \mathrm{sec}$

$0.01500000 \mathrm{sec}$

$==\Sigma== \pm= \pm$ CHANMEL

NUC 1

P1

PLI

$1 \mathrm{H}$

9.35 usec

100.1320007

F2 - Processing parameters

SI 65536

SF $\quad 400.1301132 \mathrm{MHz}$

WDW EM

$\begin{array}{lc}\text { SSB } & 0 \\ \text { LB } & 1.00 \mathrm{~Hz}\end{array}$

GB $\quad 0$

10 NMR plot parameters

CX $\quad 20.00 \mathrm{~cm}$

CY $5.00 \mathrm{~cm}$

F1P $\quad 10.811 \mathrm{ppm}$ $4325.99 \mathrm{~Hz}$ $4325.99 \mathrm{~Hz}$
$-1.158 \mathrm{ppm}$ $-463.28 \mathrm{~Hz}$

$0.59846 \mathrm{ppm} / \mathrm{cm}$ F2

$\mathrm{PPMCM}$

$239.46362 \mathrm{~Hz} / \mathrm{cm}$ 
<smiles>COc1c(O)ccc(O)c1CC(C)OC(=O)c1cc(Cl)c(O)cc1O</smiles>

99

$$
\begin{aligned}
& \text { F2 - Acquisition Parameters } \\
& \text { Date__ 20051127 } \\
& \text { Time } 21.49 \\
& \text { INSTRUM dr } \times 400 \\
& \text { PROBHD } 5 \mathrm{~mm} \text { ONP } 4 \mathrm{H} / 13 \\
& \text { PULPROG Zgpg30 } \\
& \text { TD } 65536 \\
& \text { NS 18432 } \\
& \text { SWH 23148.148 Hz } \\
& \text { SIDRES } \\
& \text { AQ } \quad 1.4156276 \mathrm{sec} \\
& \text { RG } \quad 32768 \\
& \begin{array}{rr}
\text { DW } & 21.600 \text { usec } \\
D E & 5.50 \text { usec }
\end{array} \\
& \begin{array}{l}
5.50 \text { usec } \\
294.2 \mathrm{~K}
\end{array} \\
& 0.15000001 \mathrm{sec} \\
& 0.03000000 \mathrm{sec} \\
& \begin{array}{ll}
\text { DELLTA } & 0.05000000 \mathrm{sec} \\
\text { MCREST } & 0.00000000 \mathrm{sec}
\end{array} \\
& \text { MCWRK } \quad 0.01500000 \mathrm{sec}
\end{aligned}
$$

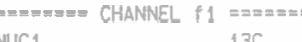

$$
\begin{aligned}
& \begin{array}{ll}
\text { NuC1 } & 13 \mathrm{C} \\
\text { P1 } & 11.25 \mathrm{usec}
\end{array} \\
& \begin{array}{l}
\text { P1 } \\
\text { PL1 } \\
\text { PLO }
\end{array} \\
& \text { SFO1 } \quad 100.6232933 \mathrm{MHz}
\end{aligned}
$$

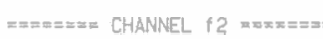

$$
\begin{aligned}
& \text { CPOPRGS waltz16 } \\
& \begin{array}{ll}
\text { NuC2 } & 1 \mathrm{H} \\
\text { PCPO2 } & 100.00 \text { usec }
\end{array} \\
& \begin{array}{lr}
\text { PCPD2 } & 100.00 \text { usec } \\
\text { PL2 } & -4.00 \mathrm{~dB}
\end{array} \\
& \text { PLI2 } 16.58 \mathrm{~dB} \\
& \text { PL13 } 17.00 \mathrm{~dB} \\
& \text { SF02 } 400.1316005 \mathrm{MHz} \\
& \text { F2 - Processing parameters } \\
& \begin{array}{lc}
\text { SI } & 65536 \\
\text { SF } & 100.6127290
\end{array} \\
& \text { SF } \\
& \text { SSB } \\
& \begin{array}{l}
\text { LB } \\
G B
\end{array} \\
& \text { PC } \\
& 10 \text { NMR plot parameters } \\
& \text { cx } \quad 20.00 \mathrm{~cm} \\
& 50.00 \mathrm{~cm} \\
& 215.000 \mathrm{ppm} \\
& 21631.74 \mathrm{~Hz} \\
& -5.000 \mathrm{ppm} \\
& -503.06 \mathrm{~Hz} \\
& \begin{array}{r}
-503.06 \mathrm{~Hz} \\
11.00000 \mathrm{pgm} / \mathrm{cm}
\end{array}
\end{aligned}
$$



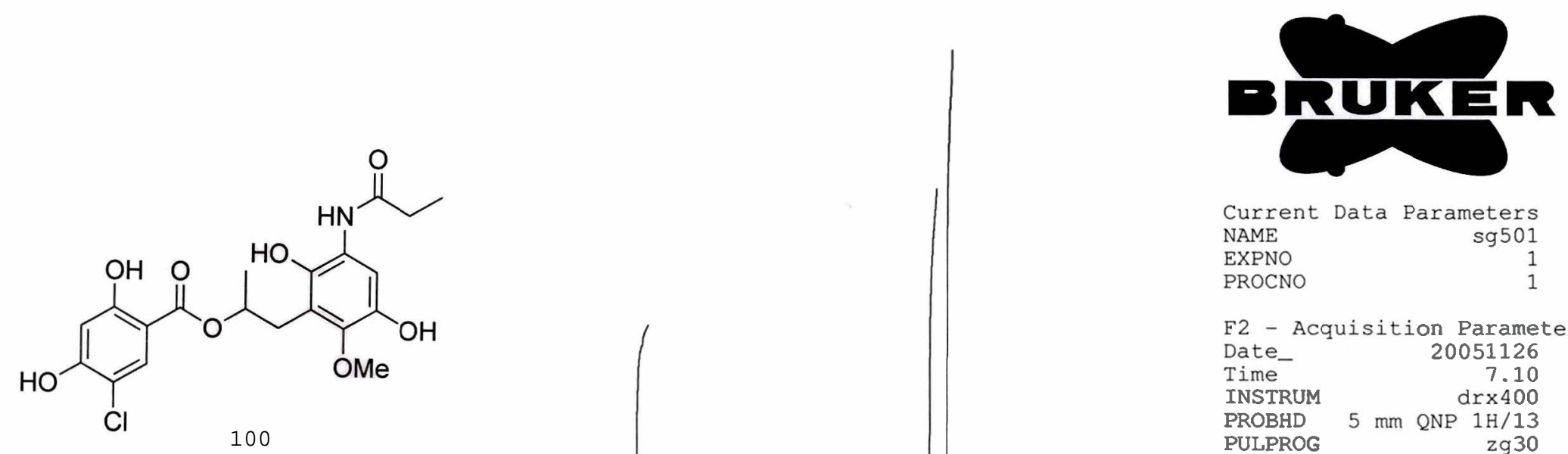

Current Data Parameters

NAME

EXPNO

PROCNO

$\mathrm{sg} 501$

F2 - Acquisition Parameters

Date_ 20051126

Time $\quad 7.10$

INSTRUM drx400

PROBHD $5 \mathrm{~mm}$ QNP $1 \mathrm{H} / 13$

PULPROG

$\mathrm{H} / 13$
$\mathrm{zg} 30$

SOLVENT

NS

DS

SWH

FID S

$A Q$

RG

DW

DE

DI

MCREST

MCWRK

CDC13

16

$4789.272 \mathrm{~Hz}$

$0.146157 \mathrm{~Hz}$

$3.4210291 \mathrm{sec}$ 287.4

104.400 usec 5.50 usec $293.2 \mathrm{~K}$

$1.00000000 \mathrm{sec}$

$0.00000000 \mathrm{sec}$

$0.01500000 \mathrm{sec}$

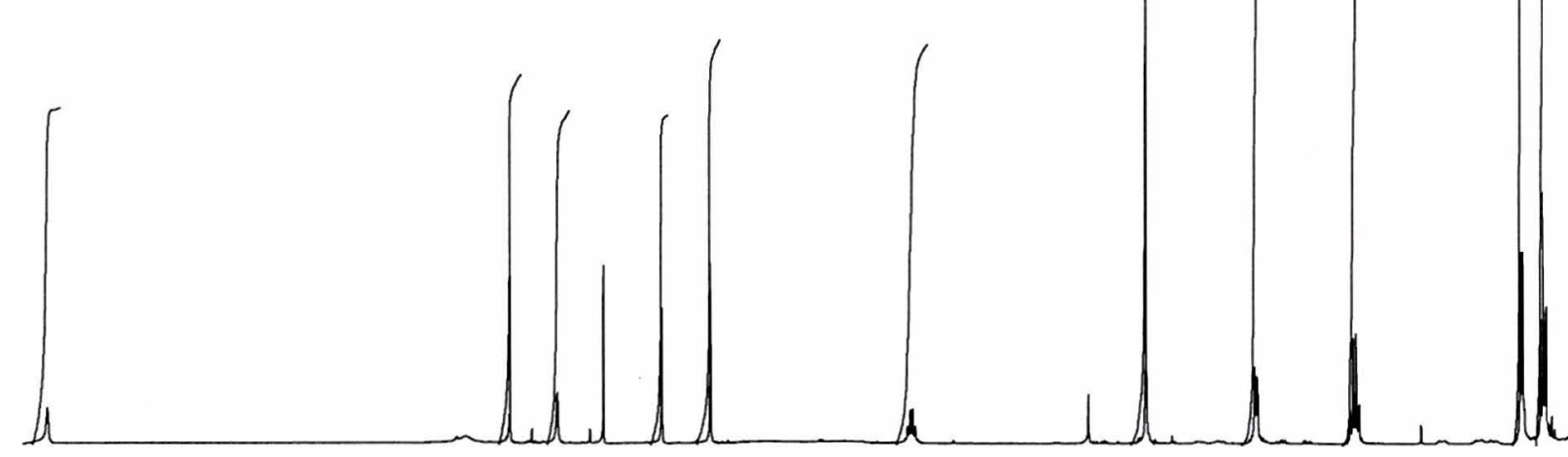
$=======$ CHANNEL $f$
NUC
P1
PL1

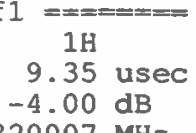

SFO1

$400.1320007 \mathrm{MHz}$

F2 - Processing parameters

SI 32768

SF $400.1300091 \mathrm{MH}$

WDW

SSB

GB

EM

0
0.30
0

0
1.00

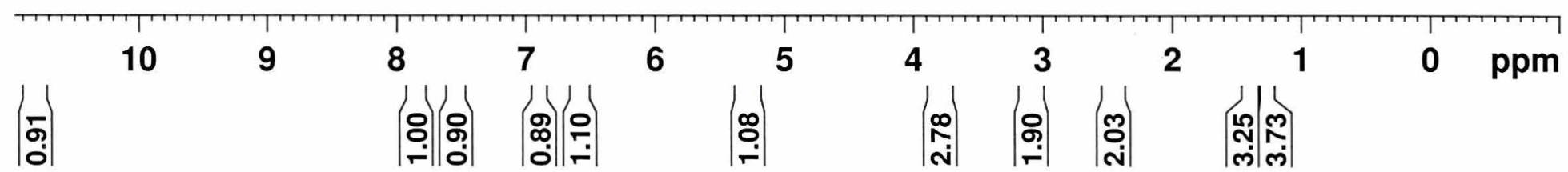




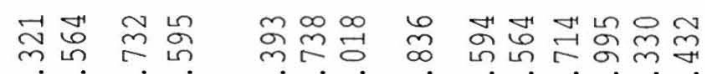

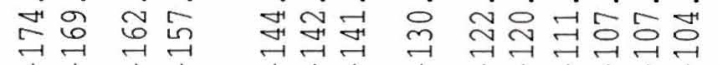

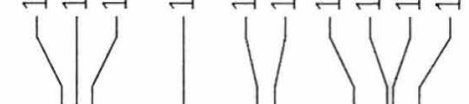<smiles>CCC(=O)Nc1cc(O)c(OC)c(CC(C)OC(=O)c2cc(Cl)c(O)cc2O)c1O</smiles>

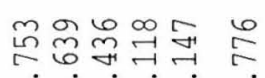

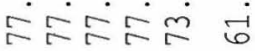

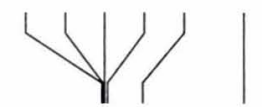

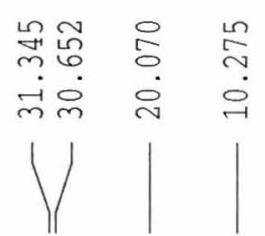

Current Data Parameters

F2 - Acquisition Parameters

Date_ 20051126

PROBHD 5 mm QNF $1 \mathrm{H} / 13$

PULPROG zgpc 30

SOLVEN

NS

DS
SWH
FIDRES

$\mathrm{AQ}$

RG

DW

$\mathrm{TE}$

$$
\begin{aligned}
& \text { D1 } \\
& \text { d11 }
\end{aligned}
$$

d11

\section{MCRE}

MCWRK

zgpg 30
65536

CDC13

43148.148

$23148.148 \mathrm{~Hz}$
$0.353213 \mathrm{~Hz}$ $0.353213 \mathrm{~Hz}$
$1.4156276 \mathrm{sec}$ 32768

21.600 usec 5.50 usec $293.2 \mathrm{~K}$

$0.15000001 \mathrm{sec}$ $0.03000000 \mathrm{sec}$ $0.05000000 \mathrm{sec}$ $0.00000000 \mathrm{sec}$

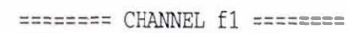
NUC1 P1 $\quad 11.25$ usec PL1 $2.00 \mathrm{~dB}$ 100.6232933 MH:

$\begin{array}{lc}====== & \text { CHANNEL } \mathrm{f} 2 \text { ======z } \\ \text { CPDPRG2 } & \text { waltz } 16 \\ \text { NUC2 } & 1 \mathrm{H} \\ \text { PCPD2 } & 100.00 \mathrm{usec} \\ \text { PL2 } & -4.00 \mathrm{~dB} \\ \text { PL12 } & 16.58 \mathrm{~dB} \\ \text { PL13 } & 17.00 \mathrm{~dB} \\ \text { SPO2 } & 400.1316005 \mathrm{mHz}\end{array}$

Processing parameters SI Processing parameters

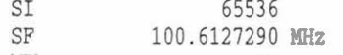
$\begin{array}{lr}\text { SF } & 100.6127290 \\ \text { WDW } & \text { EM }\end{array}$

SSB

$\mathrm{LB}$
$\mathrm{GB}$ $\mathrm{EM}$
0 $1.00 \mathrm{~Hz}$ 1.00
1.40

ID NMR pl t parameters CX CY $\quad 20.00 \mathrm{~cm}$ F1p $\quad \begin{array}{ll}12.50 \mathrm{~cm} \\ \text { F1 }\end{array}$ F1 $21531.74 \mathrm{~Hz}$ 


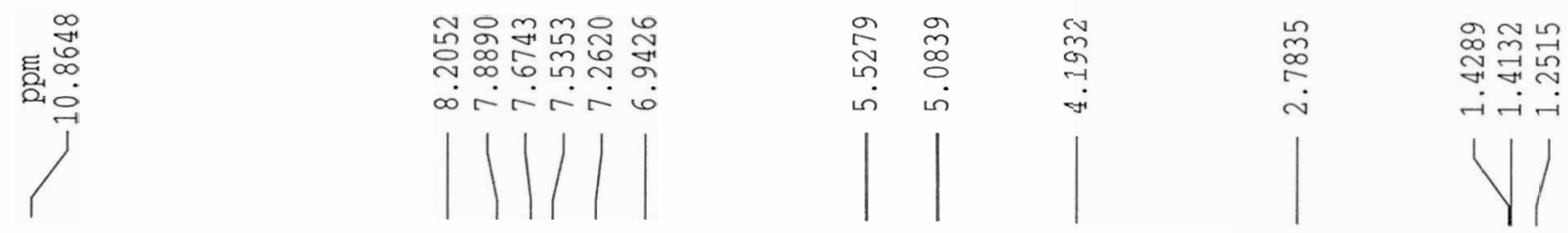
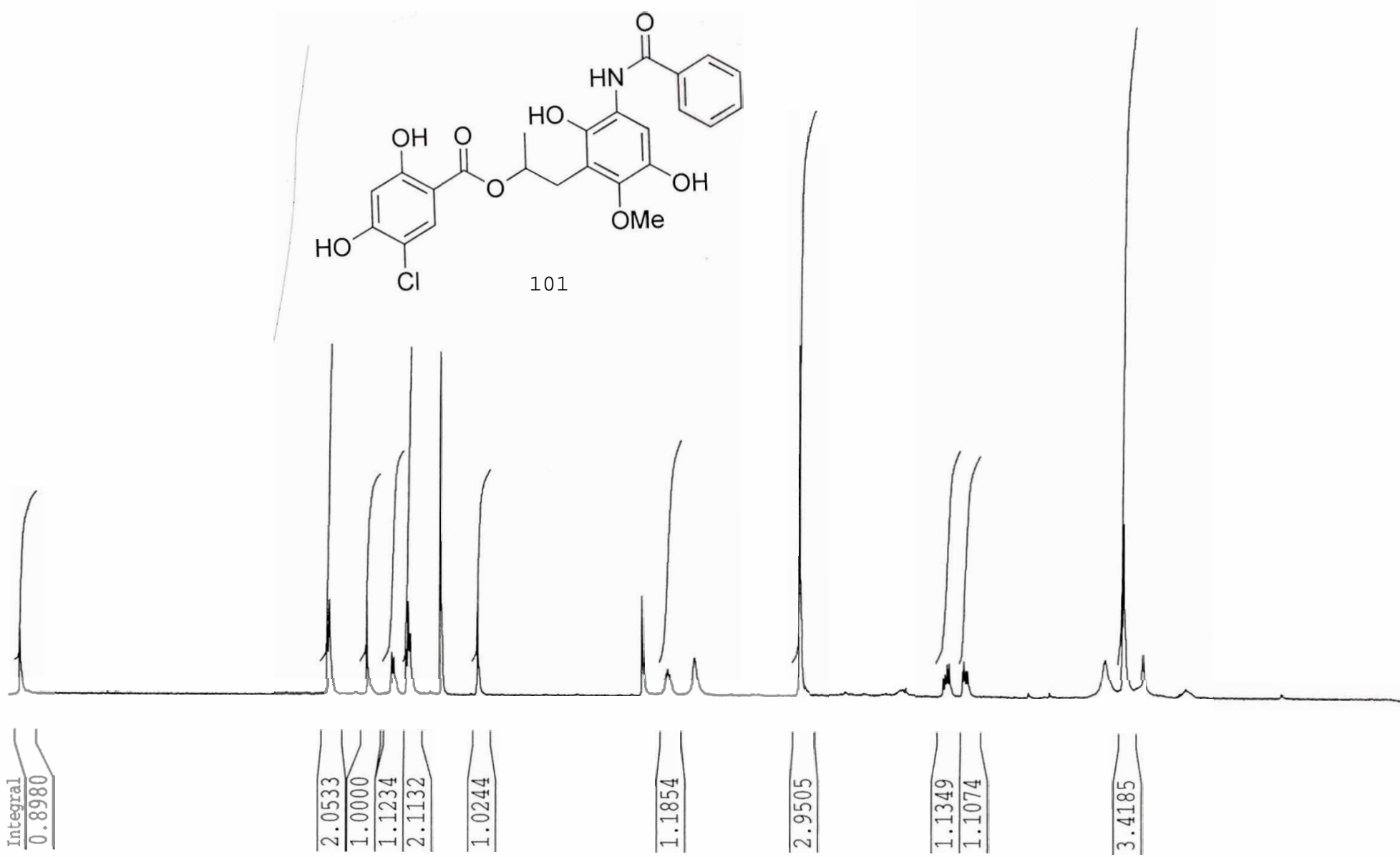

0043
8
Current Data Parameters

F2 - Acquisition Parameters

Date_ 20051127

Time 12.15

INSTRUM drX400

PROBHD $5 \mathrm{~mm}$ QNP 1H/13

PULPROG

$\mathrm{zg} 30$

32768

NS

SWH

FIDRES

$\mathrm{AQ}$

RG

DW

DE

D1

MCREST

MCWRK

2

$4789.272 \mathrm{~Hz}$

$0.146157 \mathrm{~Hz}$

$3.4210291 \mathrm{sec}$ 456.1

104.400 usec 5.50 usec $293.2 \mathbb{R}$

$1.00000000 \mathrm{sec}$

$0.00000000 \mathrm{sec}$

$0.01500000 \mathrm{sec}$

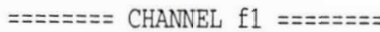

$\begin{array}{lr}\text { NUC1 } & 1 \mathrm{H} \\ \text { P1 } & 9.35 \text { usec } \\ \text { PL1 } & -4.00 \mathrm{~dB}\end{array}$

$\begin{array}{lr}\text { PL1 } & -4.00 \mathrm{~dB} \\ \text { SF01 } & 400.1320007 \mathrm{MHz}\end{array}$

F2 - Processing parameters

SI $\quad 32768$

$\begin{array}{cc}\text { SI } & 32768 \\ \text { SF } & 400.1300091 \mathrm{MHz}\end{array}$

WDW EM

$\begin{array}{ll}\text { SSB } & 0.30 \mathrm{~Hz}\end{array}$

$\begin{array}{lr}\text { GB } & 0 \\ \text { PC } & 1.00\end{array}$

1D NMR plot parameters

$\begin{array}{lr}\text { CX NMR plot parauteters } \\ \text { CX } & 20.00 \mathrm{~cm} \\ \text { CY } & 5.00 \mathrm{~cm} \\ \text { F1P } & 11.000 \mathrm{ppm} \\ \text { F1 } & 4401.43 \mathrm{~Hz} \\ \text { F2P } & -1.000 \mathrm{ppm} \\ \text { F2 } & -400.13 \mathrm{~Hz} \\ \text { PPMCM } & 0.60000 \mathrm{ppm} / \mathrm{cm} \\ \text { HZCM } & 240.07800 \mathrm{~Hz} / \mathrm{cm}\end{array}$



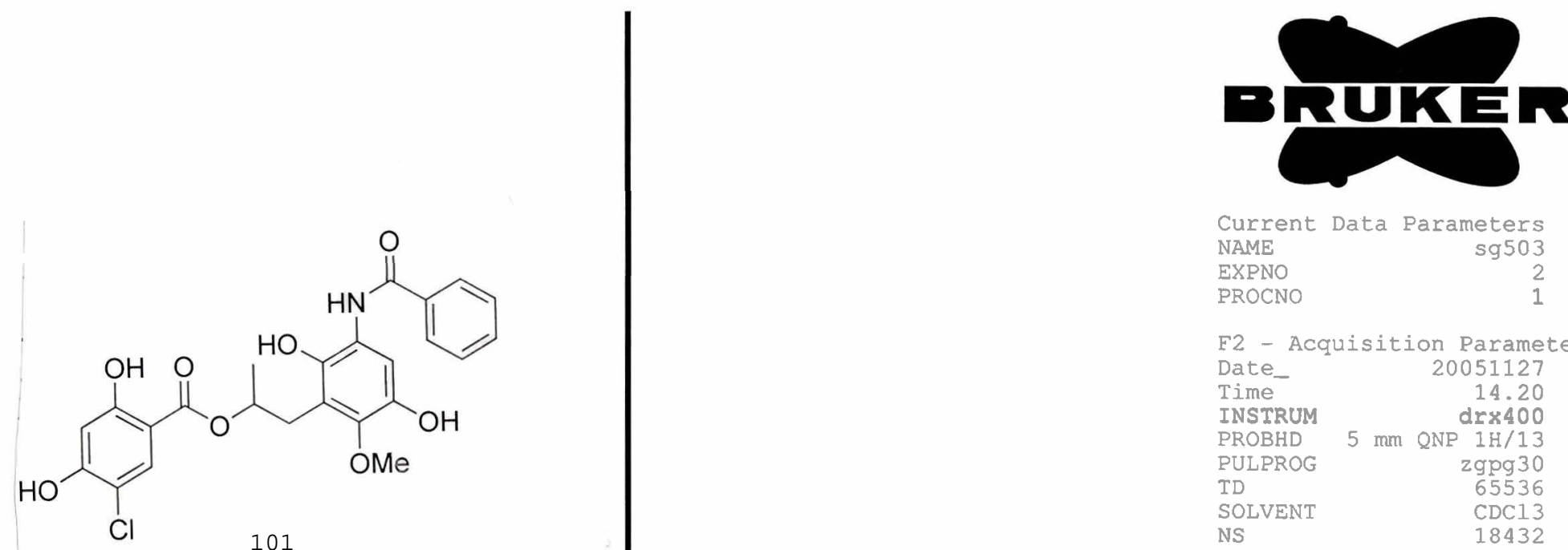

101

Current Data Parameters

NAME

PXPNO

sg503

F2 - Acquisition Parameters

Date_ 20051127

Time 14.20

INSTRUM dr $\times 400$

PROBHD

$5 \mathrm{~mm}$ ONP $1 \mathrm{H} / 13$

PUIPROG

TD

SOLVENT

NS

SWH

EIDRES

AQ

AQ

DW

$\mathrm{DE}$

D1

DELTA

MCRESI

MCWRK

P $1 \mathrm{H} / 13$

zgpg 30
65536

18432

$===$
NUC1

$\mathrm{PI}$

PL1

SFO1

$23148.148 \mathrm{~Hz}$

$0.353213 \mathrm{~Hz}$

$1.4156276 \mathrm{sec}$

32768

21.600 usec

5.50 usec

$294.2 \mathrm{~K}$

$0.15000001 \mathrm{sec}$

0.03000000 sec

$0.05000000 \mathrm{sec}$

$0.00000000 \mathrm{sec}$

$0.01500000 \mathrm{sec}$
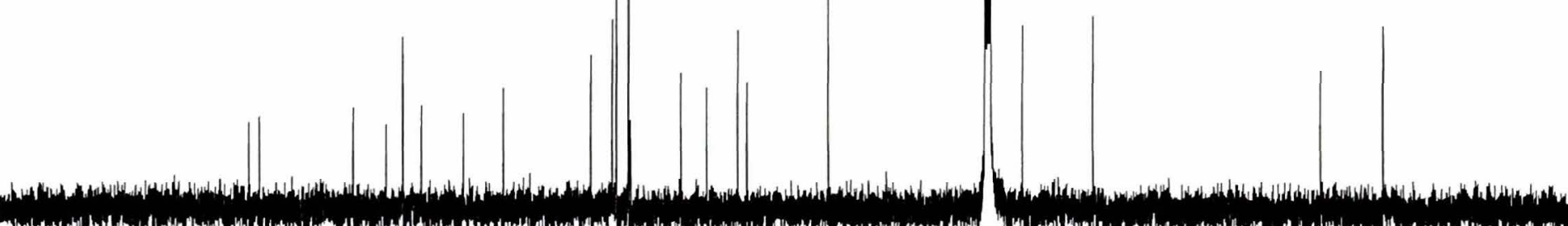

$=======$

CPDPRG2

NUC2

PCPD

Non

$100.6232933 \mathrm{MHz}$

2 - Processing parameters 

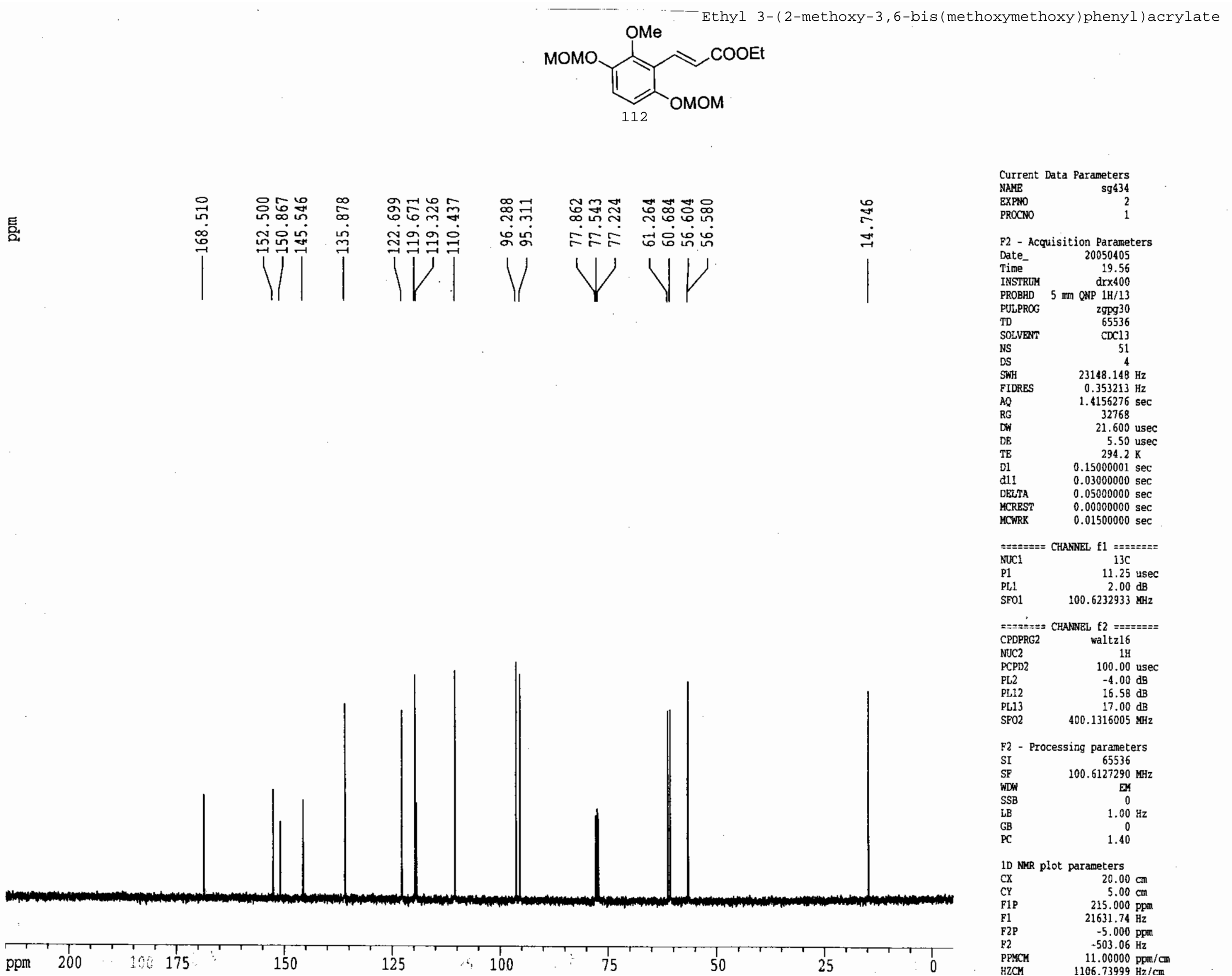

125

$\therefore \quad 100$

75

50

25

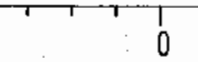

Current Data Parameters

SAME

EXPFO

OCNO

$\mathrm{sg} 434$

F2 - Acquisition Parameters

$\begin{array}{lr}\text { Date__ } & 20050405 \\ \text { Time } & 19.55\end{array}$

$\begin{array}{lr}\text { Time } & 19.56 \\ \text { INSTRIM } & \text { drx400 }\end{array}$

PROBHD $5 \mathrm{~mm}$ QNP $1 \mathrm{H} / 13$

PULPROG zGPG30

TD $\quad 65536$

$\begin{array}{lr}\text { SOLVENT } & \mathrm{CDCl3} \\ \text { NS } & 51\end{array}$

SWH $23148.148 \mathrm{~Hz}$

FIDRES $\quad 0.353213 \mathrm{~Hz}$

AQ $\quad 1.4156276 \mathrm{sec}$

RG 32768

$\begin{array}{lr}\text { DW } & 21.600 \text { usec } \\ D E & 5.50 \text { usec }\end{array}$

TE $294.2 \mathrm{~K}$

D1 $\quad 0.15000001 \mathrm{sec}$

dil $\quad 0.03000000 \mathrm{sec}$

DELTA $\quad 0.05000000 \mathrm{sec}$

MCREST $\quad 0.00000000 \mathrm{sec}$

MCWRK $\quad 0.01500000 \mathrm{sec}$

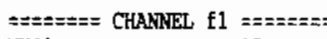
NUC1 $13 \mathrm{C}$

$\begin{array}{lc}\text { P1 } & 11.25 \text { usec } \\ \text { PL1 } & 2.00 \mathrm{~dB}\end{array}$

SFO1 $\quad 100.6232933 \mathrm{MHz}$

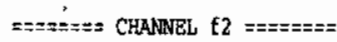
CPDPRG2 CHAnat f2

$\begin{array}{lc}\text { NUC2 } & 1 \mathrm{H} \\ \text { PCPD2 } & 100.00 \mathrm{usec} \\ \text { PL2 } & -4.00 \mathrm{~dB} \\ \text { PL12 } & 16.58 \mathrm{~dB} \\ \text { PL13 } & 17.00 \mathrm{~dB}\end{array}$

PL12 $16.58 \mathrm{~dB}$

$\begin{array}{ll}\text { PL13 } & 17.00 \mathrm{~dB} \\ \text { SP02 } & 400.1316005 \mathrm{MHz}\end{array}$

F2 - Processing parameters

SI 65536

SF $\quad 100.6127290 \mathrm{MHz}$

WDW

LB $\quad 1.00 \mathrm{~Hz}$

$\begin{array}{lr}\mathrm{GB} & 0 \\ \mathrm{PC} & 1.40\end{array}$

1D NMR plot parameters

$\begin{array}{lr}C X & 20.00 \mathrm{~cm} \\ \mathrm{CY} & 5.00 \mathrm{~cm}\end{array}$

F1P $215.000 \mathrm{ppm}$

F1 $21631.74 \mathrm{~Hz}$

F2P $\quad-5.000 \mathrm{ppm}$

$\begin{array}{lr}\text { P2 } & -503.06 \mathrm{~Hz} \\ \text { PPMCM } & 11.00000 \mathrm{ppm} / \mathrm{C}\end{array}$

$\begin{array}{lr}\text { HZCM } & 11.00000 \mathrm{ppm} / \mathrm{cm}\end{array}$ 
Ethyl 3-(2-methoxy-3,6-bis (methoxymethoxy) phenyl) acrylate

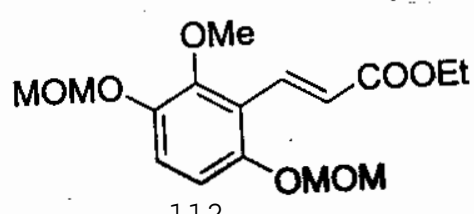

咅

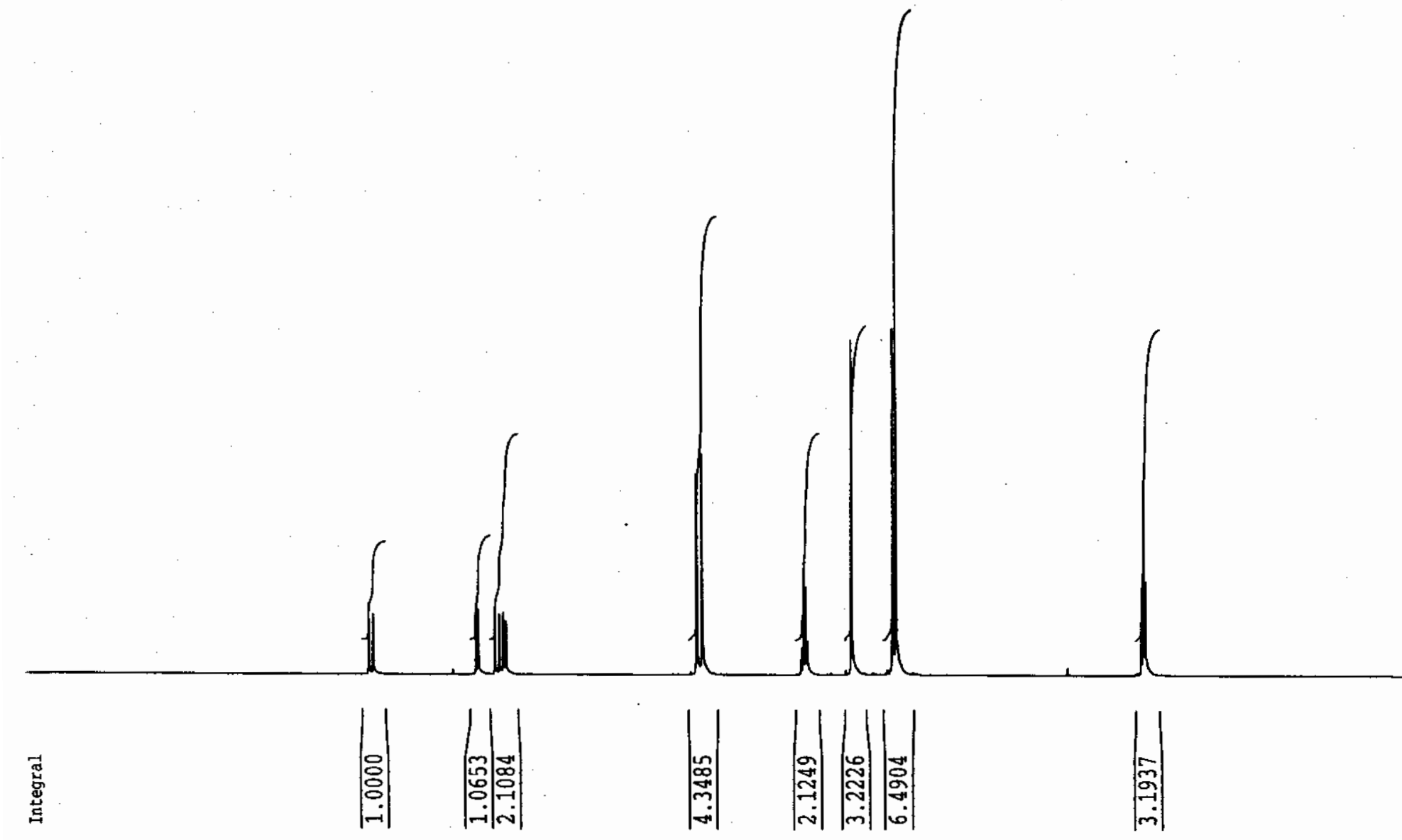

$\begin{array}{lr}\text { Current Data Parameters } \\ \text { NANE } & \text { sg434 } \\ \text { EXPNO } & 1 \\ \text { PROCNO } & 1\end{array}$

F2 - Acquisition Parameters Date. $\quad 20050405$ $\begin{array}{lr}\text { Time } & 19.53\end{array}$ INSTRUM drx400 PROBHD 5 and QNP $1 \mathrm{H} / 13$ PULPROG $\quad$ zg30 $\begin{array}{lr}\text { PULPROG } & \mathrm{zg} 30 \\ \text { TD } & 32768\end{array}$ SOLVENT $\quad \mathrm{CDCl3}$ NS

DS DS 2 $\begin{array}{ll}\text { SWH } & 4789.272 \mathrm{~Hz} \\ \text { FIDRES } & 0.146157 \mathrm{~Hz}\end{array}$ $\begin{array}{lc}\text { AQ } & 3.4210291 . \mathrm{sec} \\ \text { RG } & 64\end{array}$ DW 5.50 usec TE $294.2 \mathrm{~K}$ D1 $\quad 1.00000000 \mathrm{sec}$ $\begin{array}{ll}\text { MCREST } & 0.00000000 \mathrm{sec} \\ \text { MCARK } & 0.01500000 \mathrm{sec}\end{array}$ CHAMnEL NUC1 NUC1

P1 9.35 usec SF01 $\quad 400.1320007 \mathrm{kHz}$

F2 - Processing parameters

SI 32768 SF $\quad 400.1300000 \mathrm{MHz}$ WDW LB $\quad 0.30 \mathrm{~Hz}$

$\begin{array}{lr}\text { GB } & 0.30 \\ \text { PC } & 0 \\ & 1.00\end{array}$

1.00

1D NMR plot parameters $\begin{array}{lr}\text { CX } & 20.00 \mathrm{~cm} \\ \mathrm{CY} & 5.00 \mathrm{~cm}\end{array}$

F1P $\quad 11.000 \mathrm{ppm}$

F1 $\quad 4401.43 \mathrm{~Hz}$

F2P $\quad-1.000 \mathrm{ppm}$
PPMCM
$-400.13 \mathrm{~Hz}$

$\mathrm{HZCH}$ $240.07800 \mathrm{~Hz} / \mathrm{cm}$ 
2,3,6-trimethoxy-5-nitrophenethyl-2-(but-3-enyl)-3-chloro-4,6-dihydroxybenzoate

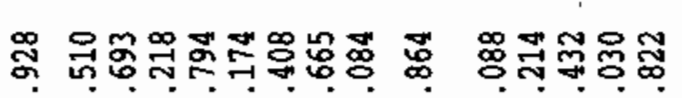

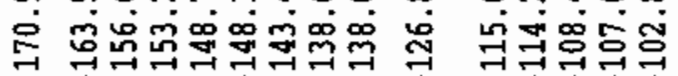

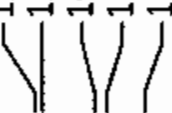

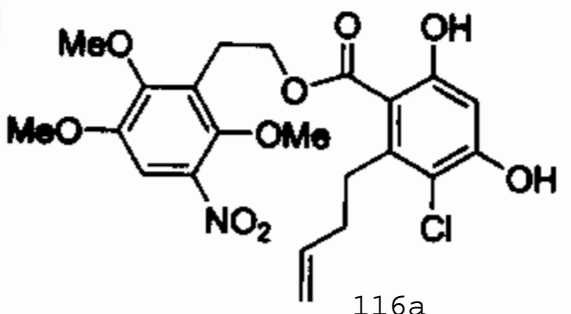

$116 a$

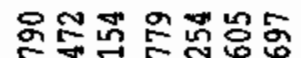

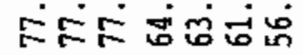

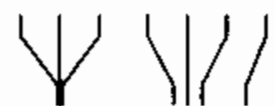

종윯 홍 लंख্ল

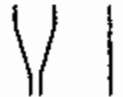

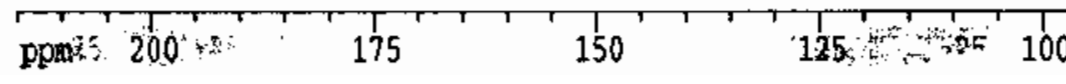

75

$50 \cdots$ 

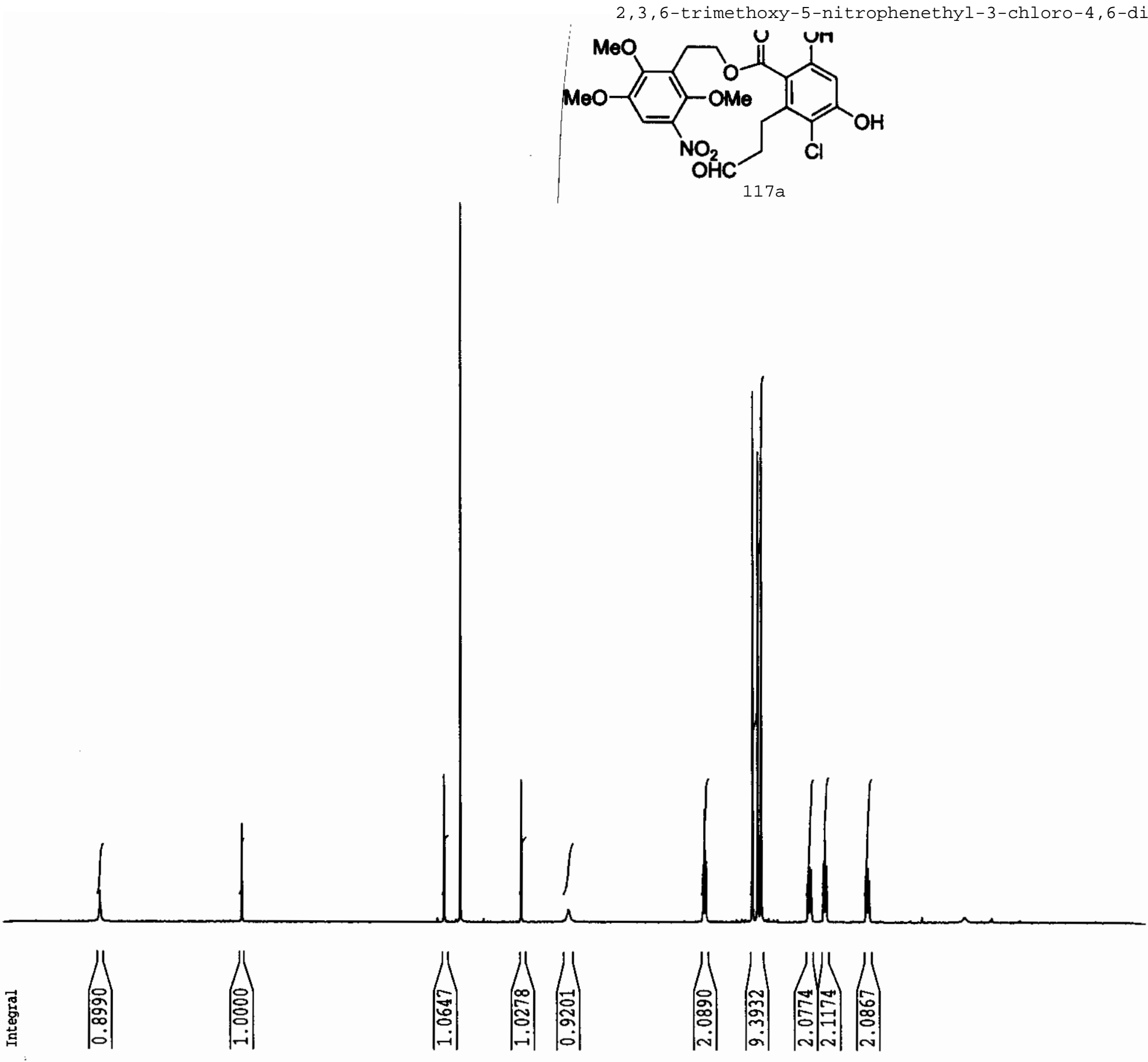

$\begin{array}{lr}\text { Current Data } & \text { Parameters } \\ \text { NAYE } & \text { wImw12271 } \\ \text { EXPNO } & 1 \\ \text { PROCNO } & 1\end{array}$

F2 - Acquisition Parameters Date_ 20041227

Time $\quad 17.14$

INSTRINA drx400 PROBHD $5 \mathrm{~mm} \mathrm{QNP} \mathrm{1H/13}$ PULPROG $\quad \mathrm{zg} 30$ TD 32768

SOLVENT $\quad \mathrm{CDCl3}$

NS

DS

SWH FIDRES

$A Q$

RG

DN

DE

D1

HCREST

MCWRK

16

$5175.983 \mathrm{~Hz}$ $0.157958 \mathrm{~Hz}$

$3.1654389 \mathrm{sec}$ 512

96.600 usec 5.50 usec $293.2 \mathrm{~K}$

$1.00000000 \mathrm{sec}$

$0.00000000 \mathrm{sec}$

$0.01500000 \mathrm{sec}$

="=z=s== CHANNEL f1 =:=z=z=s NUC1 $1 \mathrm{H}$

P1 9.35 usec $\begin{array}{lr}\text { PL1 } & -4.00 \mathrm{~dB} \\ \text { SFO1 } & 400.1324008 \mathrm{hHz}\end{array}$

F2 - Processing parameters SI 32768 SF $\quad 400.1300000 \mathrm{MHz}$ WDW ENI

LB $\quad 0.30 \mathrm{~Hz}$

$\begin{array}{lr}\text { GB } & 0 \\ \text { PC } & 1.00\end{array}$

1.00

1D NMR plot parameters

CX $20.00 \mathrm{~cm}$ CY $\quad 12.50 \mathrm{~cm}$ F1P $\quad 12.468 \mathrm{ppm}$ F1 $4988.77 \mathrm{~Hz}$ F2P $\quad-0.468 \mathrm{ppm}$ F2 $-187.21 \mathrm{~Hz}$ PPMCM $\quad 0.64679 \mathrm{ppm} / \mathrm{cm}$ HZCY $258.79919 \mathrm{~Hz} / \mathrm{cm}$ 
3-(2-chloro-3,5-dihydroxy-6-((2,3,6-trimethoxy-5-nitrophenethoxy) carbonyl)phenyl)propanoic acid
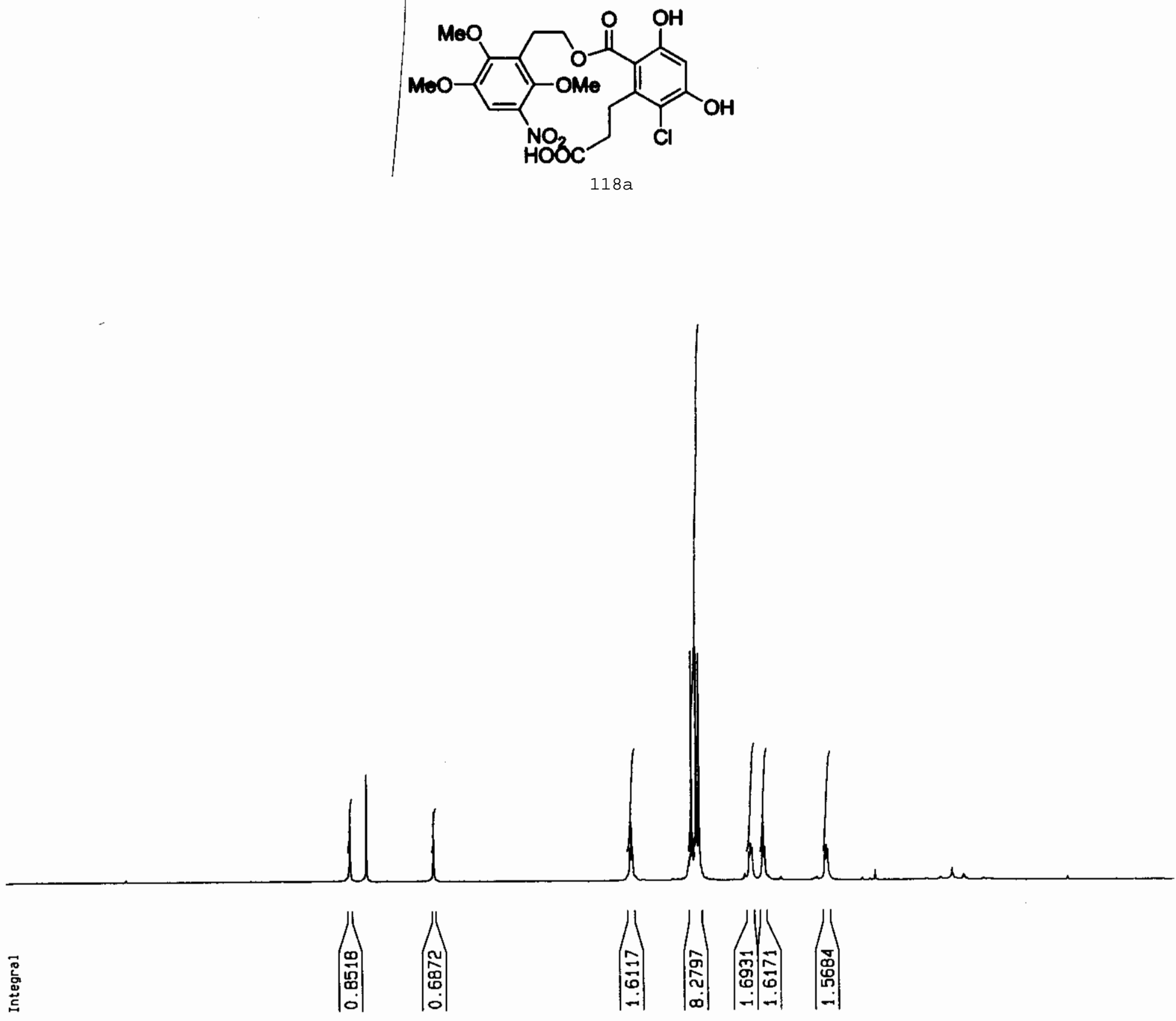

Current Data Parameters
NANE
EXPWO
PROCNO

F2 - Acquisition Paraneters Date_ 20041229 Time $\quad 9.45$ INSTRUM 5 PPOBHO 5 spect PAOBHO 5 B8O B8-1H
PUAPROG TO 32768 $\begin{array}{lr}\text { SOL VENT } & \operatorname{Coc} 13 \\ \text { N5 } & 16\end{array}$ $\begin{array}{lc}\text { DS } & 2 \\ \text { Sint } & 6009.615 \mathrm{~Hz}\end{array}$ $\begin{array}{ll}\text { SIWH } & 6009.615 \mathrm{~Hz} \\ \text { FIDRES } & 0.189399 \mathrm{~Hz}\end{array}$ AQ $2.7264309 \mathrm{sec}$ PG 362 $\begin{array}{lr}\text { DW } & 83.200 \text { usec } \\ \text { DE } & 6.00 \text { usec }\end{array}$ TE $0.0 \mathrm{~K}$ MCDEST $\quad 0.03000000 \mathrm{sec}$ MCREST $0.00000000 \mathrm{sec}$

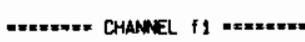
Mxi Ps PL1 $\quad 500.43000$

F1 - Acquisition parameters

$\mathrm{NOO}$

TC 2

$5 F 01 \quad 500.1325 \mathrm{MHz}$

FIORES $\quad 23.475060 \mathrm{~Hz}$

SH $\quad 12.016$ pop

undef ined

Fa - Processing paraneters

SI Processing paras

SP $\quad 500.1300000$

SCN 500.1300000

LB

GB

F1 - Processing parameters

SI 1024 $\begin{array}{lc}\text { HC2 } & \text { of } \\ \text { SF } & 500.1300000\end{array}$ NSB $\begin{array}{cc}58 & 0.30 \mathrm{~Hz}\end{array}$

10 NWP plot parameters

CX $20.00 \mathrm{ca}$

cr $4.00 \mathrm{cs}$

F1P $11.000 \mathrm{ppm}$

F1 $5501.43 \mathrm{~Hz}$

FrP $\quad-1.000$ pom

PPNCM $\quad-500.13 \mathrm{~Hz}$

HZZM $\quad \begin{array}{r}0.60000 \mathrm{ppa} / \mathrm{Cm} \\ 300.07800 \mathrm{~Hz} / \mathrm{cm}\end{array}$ 
3-(2-chloro-3,5-dihydroxy-6-((2,3,6-trimethoxy-5-nitrophenethoxy) carbonyl) phenyl pronoic acid

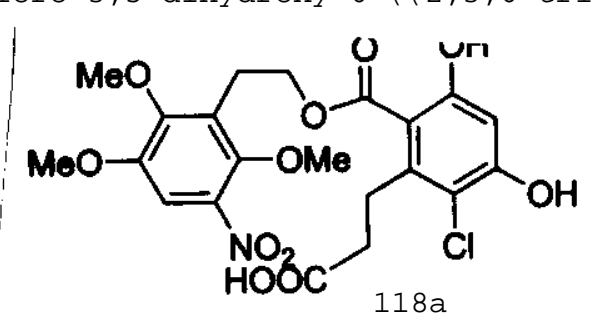

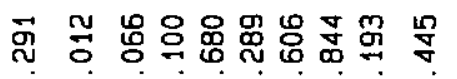

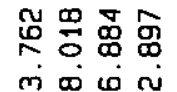

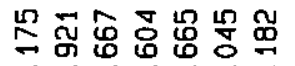

通哭哭

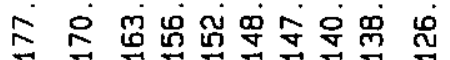

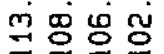

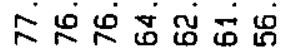

กั่ ลิ
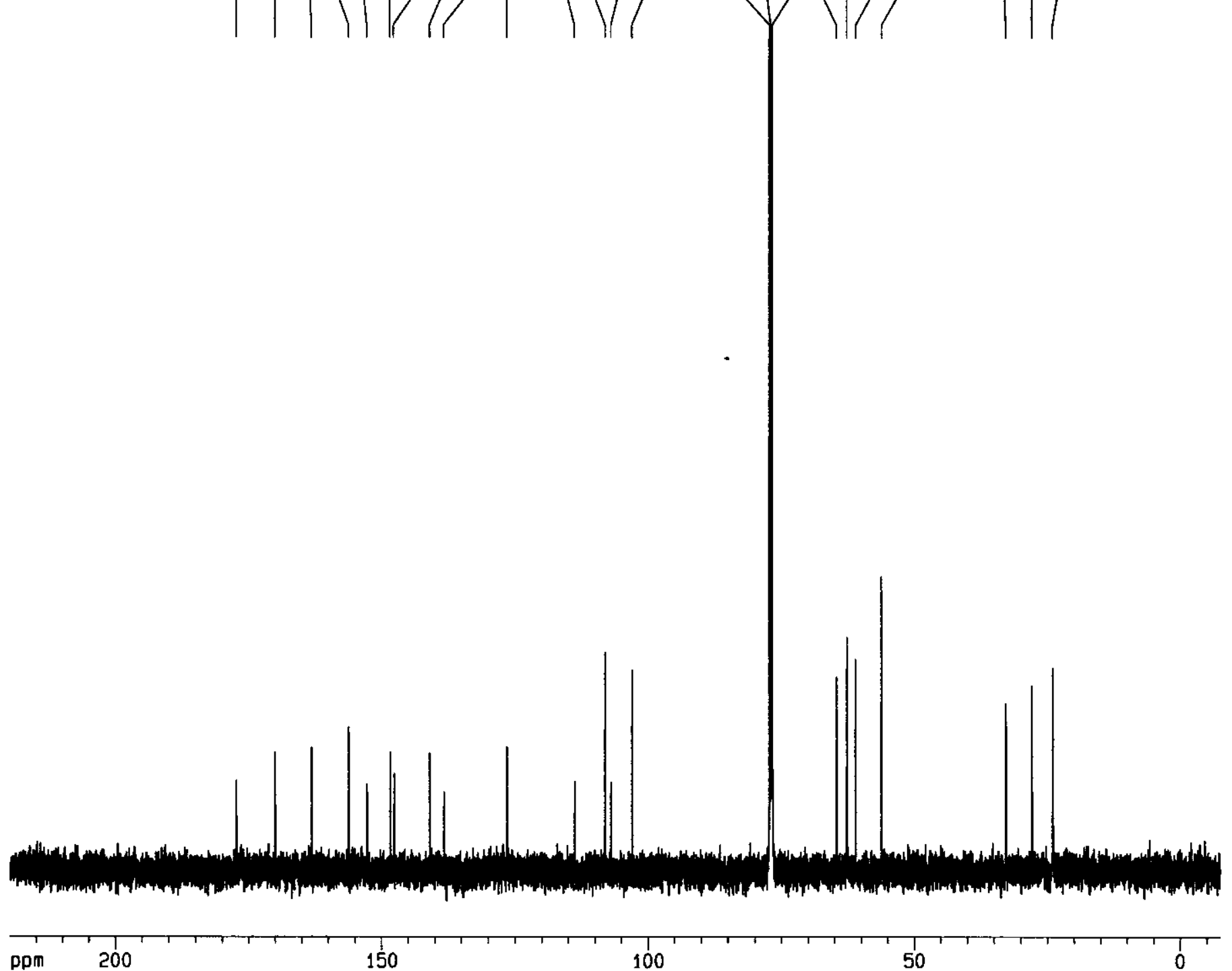

150

100

50

1 
3-(2-((3-amino-2,5,6-trimethoxyphenethoxy) carbonyl)-6-chloro-3,5-dihydroxyphenyl) propanoic acid 


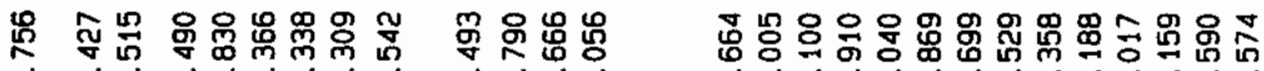

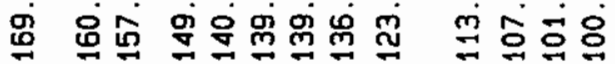
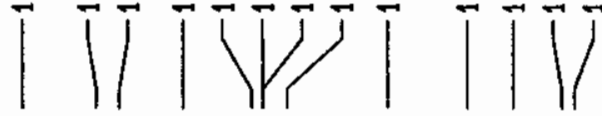

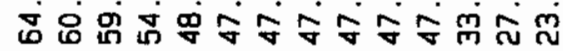

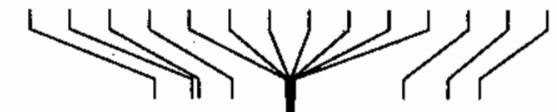




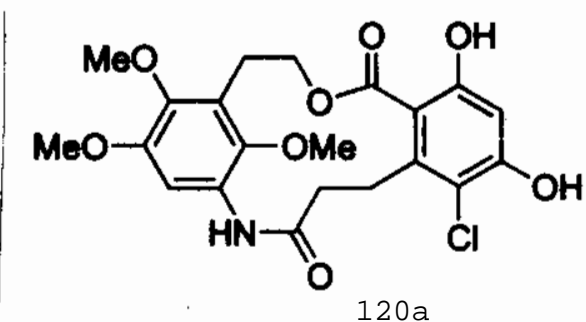

Current Data Parameters

NAME

Whw52091

EXPNO

PADCNO

F2 - Acquisition Paraneters

Date_ 20050209

14.11

Time

dr $\times 400$

INSTRUMM

$14 / 13$

PULPRO

2930

TD

SOLVENT

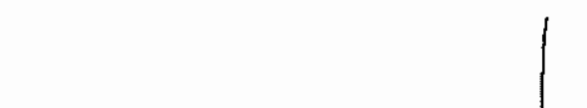

NS

DS

5NH

FIDAES

AQ

AG

DW

DE

TE

01

MCAEST

$\mathrm{COC} 13$

16
2

$5175.983 \mathrm{~Hz}$

$0.157958 \mathrm{~Hz}$

$3.1654389 \mathrm{sec}$

512

96.600 usec

5.50 usec

$292.2 \mathrm{~K}$

$1.00000000 \mathrm{sec}$

$0.00000000 \mathrm{sec}$

MCWFK 0.01500000 set

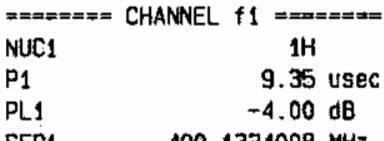

SFO1 $400.1324008 \mathrm{dHz}$

F2 - Processing parameters

SI 32768

SF $\quad 400.1300000 \mathrm{MHz}$

WON

SSB

LB

GB

EM

$.20 \mathrm{~Hz}$

0

10 NMR plot paraneters

CX NMR plot paraneters

$\mathrm{Cr} \quad 15.00 \mathrm{~cm}$

F士P $\quad 12.468 \mathrm{ppm}$

F1 $\quad 4989.77 \mathrm{~Hz}$

F2P $\quad-0.468 \mathrm{ppn}$

F2

PPNCI

$-0.468 \mathrm{ppm}$
$-187.21 \mathrm{~Hz}$ 


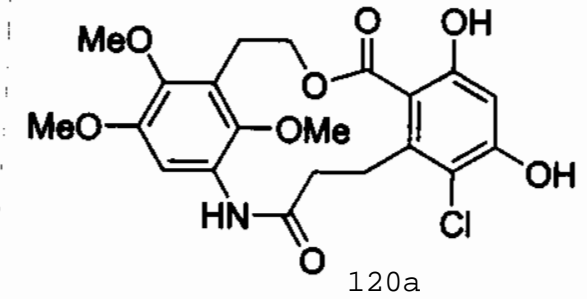

Desmethyltrimethoxy Radanamycin

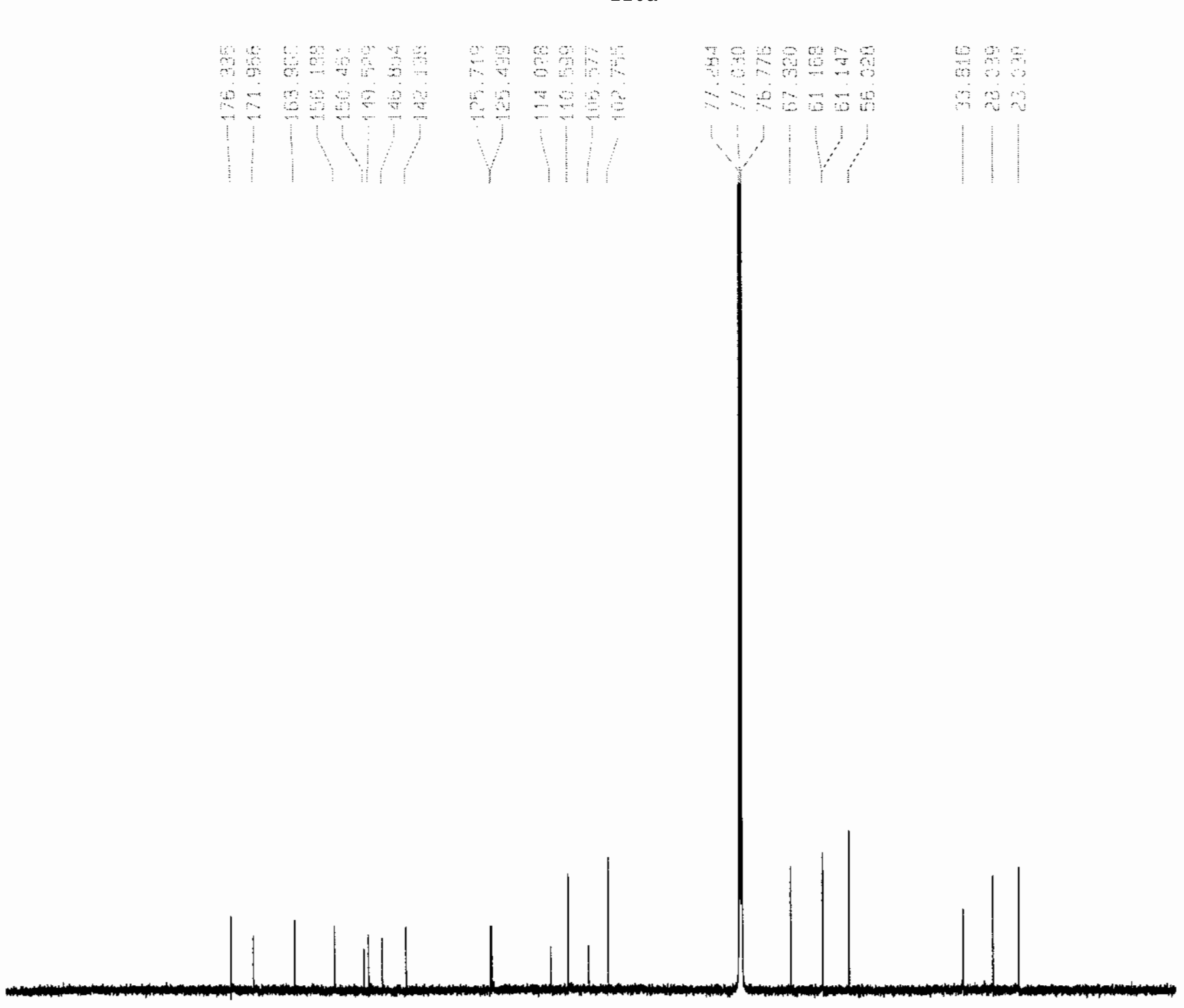

\begin{tabular}{|c|c|}
\hline Current & Data Parameters \\
\hline NAME & WMW_Me0_2 \\
\hline EXPNO & 2 \\
\hline PAOCNO & 1 \\
\hline$=2-A C C$ & quisition Рагаmeters \\
\hline Date_ & 20050210 \\
\hline Time & 21.38 \\
\hline INSTRUM & spect \\
\hline РROEHO & $5 \mathrm{~mm}$ CPDUL $13 \mathrm{C}$ \\
\hline PULPROG & $2 g \lg 30$ \\
\hline TD & 65536 \\
\hline SOLVENT & $\mathrm{CDCl} 3$ \\
\hline NS & 3072 \\
\hline DS & 4 \\
\hline SWH & $31446.541 \mathrm{~Hz}$ \\
\hline FIDRES & $0.479836 \mathrm{~Hz}$ \\
\hline$A Q$ & $1.0420724 \mathrm{sec}$ \\
\hline RG & 8192 \\
\hline DW & 15.900 usec \\
\hline DE & 6.00 usec \\
\hline TE & $298.0 \mathrm{~K}$ \\
\hline$D 1$ & $0.15000001 \mathrm{sec}$ \\
\hline d11 & $0.03000000 \mathrm{sec}$ \\
\hline DELTA & $0.05000000 \mathrm{sec}$ \\
\hline MCREST & $0.00000000 \mathrm{sec}$ \\
\hline MCWPK & $0.01500000 \mathrm{sec}$ \\
\hline$=== \pm=s$ & CHANNEL $f 1=======x$ \\
\hline NUC1 & $13 \mathrm{C}$ \\
\hline P1 & 10.00 usec \\
\hline PL1 & $-4.90 \mathrm{~dB}$ \\
\hline SF01 & $125.7697360 \mathrm{MHz}$ \\
\hline
\end{tabular}

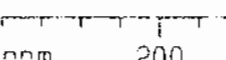

200

150

100

50

0

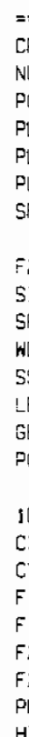

$== \pm===$ CHANNEL if CPDPRG2 waltz16 NUC2 $1 \mathrm{H}$ PCPO2 80.00 usec PL2 $\quad-5.00 \mathrm{~dB}$

$9.54 \mathrm{~dB}$
$\mathrm{PL} 12$
SF02
$500.1325000 \mathrm{MHz}$

F2 - Processing parameters

SI 65536

SF $\quad 125.7577890 \mathrm{MHz}$

WOW EM

$\begin{array}{lc}\text { SSB } & 0 \\ \text { LB } & 1.00 \mathrm{~Hz}\end{array}$

$\begin{array}{lr}68 & 0 \\ 9 C & 1.40\end{array}$

10 NMR plot parameters

$\begin{array}{lr}\text { CX } & 20.00 \mathrm{~cm} \\ \text { CY } & 100.00 \mathrm{~cm} \\ \text { FIP } & 242.517 \mathrm{ppm} \\ \text { F1 } & 30498.43 \mathrm{~Hz} \\ \text { F2P } & -7.539 \mathrm{ppm} \\ \text { F2 } & -948.11 \mathrm{HZ} \\ \text { PPMCM } & 12.50282 \mathrm{pgm} / \mathrm{cm} \\ \text { HZCM } & 1572.32690 \mathrm{~Hz} / \mathrm{cm}\end{array}$



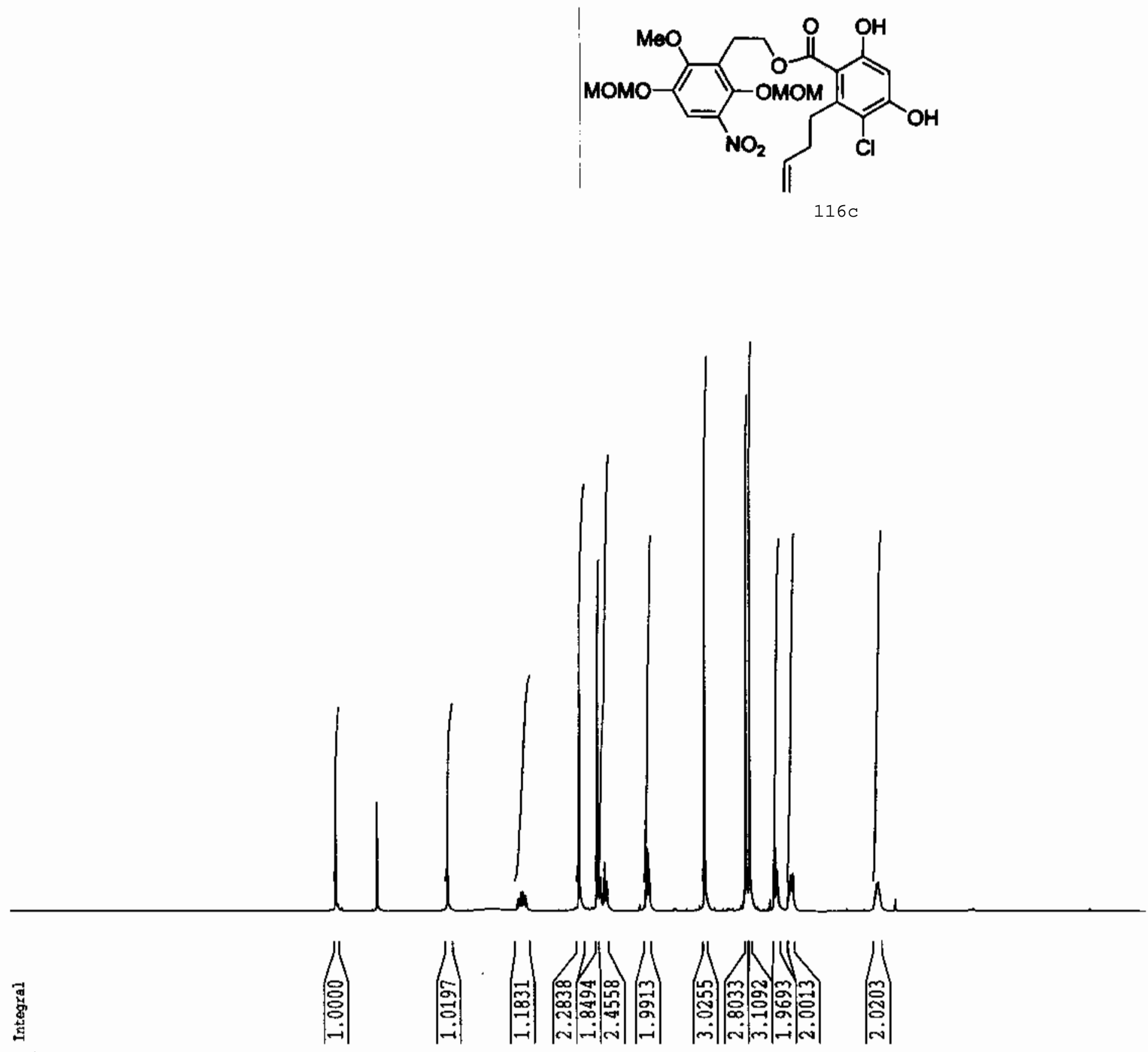

$\begin{array}{lr}\text { Current Data Parameters } \\ \text { NALE } & \text { wmw501291 } \\ \text { EXPYIO } & 2 \\ \text { PROCNO } & 1\end{array}$

F2 - Acquisition Parameters

Date_ 20050129

$\begin{array}{lr}\text { Time } & 15.03\end{array}$

INSTRUN $d r \times 400$

PROBFD 5 mाँ QNP 1H/13

PULPROG $\quad \mathbf{z g} 30$

TD 32768

SOLVEN $\quad \mathrm{CDCl} 3$

NS

DS

DS

FIDRES

AQ

$R G$

DN

TE

D1

HCREST

16

$5175.983 \mathrm{~Hz}$

$0.157958 \mathrm{~Hz}$

$3.1654389 \mathrm{sec}$

256

96.600 usec

5.50 usec

$293.2 \mathrm{~K}$

$1.00000000 \mathrm{sec}$

$0.00000000 \mathrm{sec}$

KCWRR

$0.01500000 \mathrm{sec}$

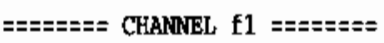
NUC1 $1 \mathrm{H}$

P1 9.35 usec

$-4.00 \mathrm{~dB}$

SFO1 $400.1324008 \mathrm{MHZ}$

F2 - Processing parameters

SI 32768

SF $\quad 400.1300000 \mathrm{rHz}$

WDW BN

SSB $\quad 0.30$

LB $\quad 0.30$

PC

1D MLR plot parameters

$\begin{array}{lr}\text { CX } & 20.00 \mathrm{~cm} \\ \text { CY } & 7.00 \mathrm{~cm} \\ \text { F1P } & 11.000 \mathrm{ppm} \\ \text { F1 } & 4401.43 \mathrm{~Hz} \\ \text { F2P } & -1.000 \mathrm{ppm} \\ \text { F2 } & -400.13 \mathrm{~Hz} \\ \text { PPKCX } & 0.60000 \mathrm{ppm} / \mathrm{cm} \\ \text { HZCY } & 240.07800 \mathrm{~Hz} / \mathrm{cm}\end{array}$




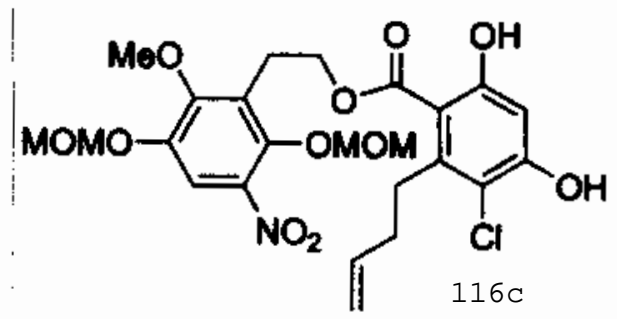

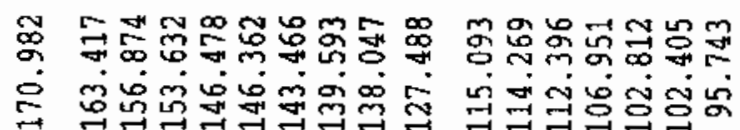

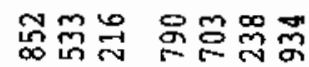

恕庶

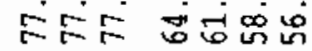

mi
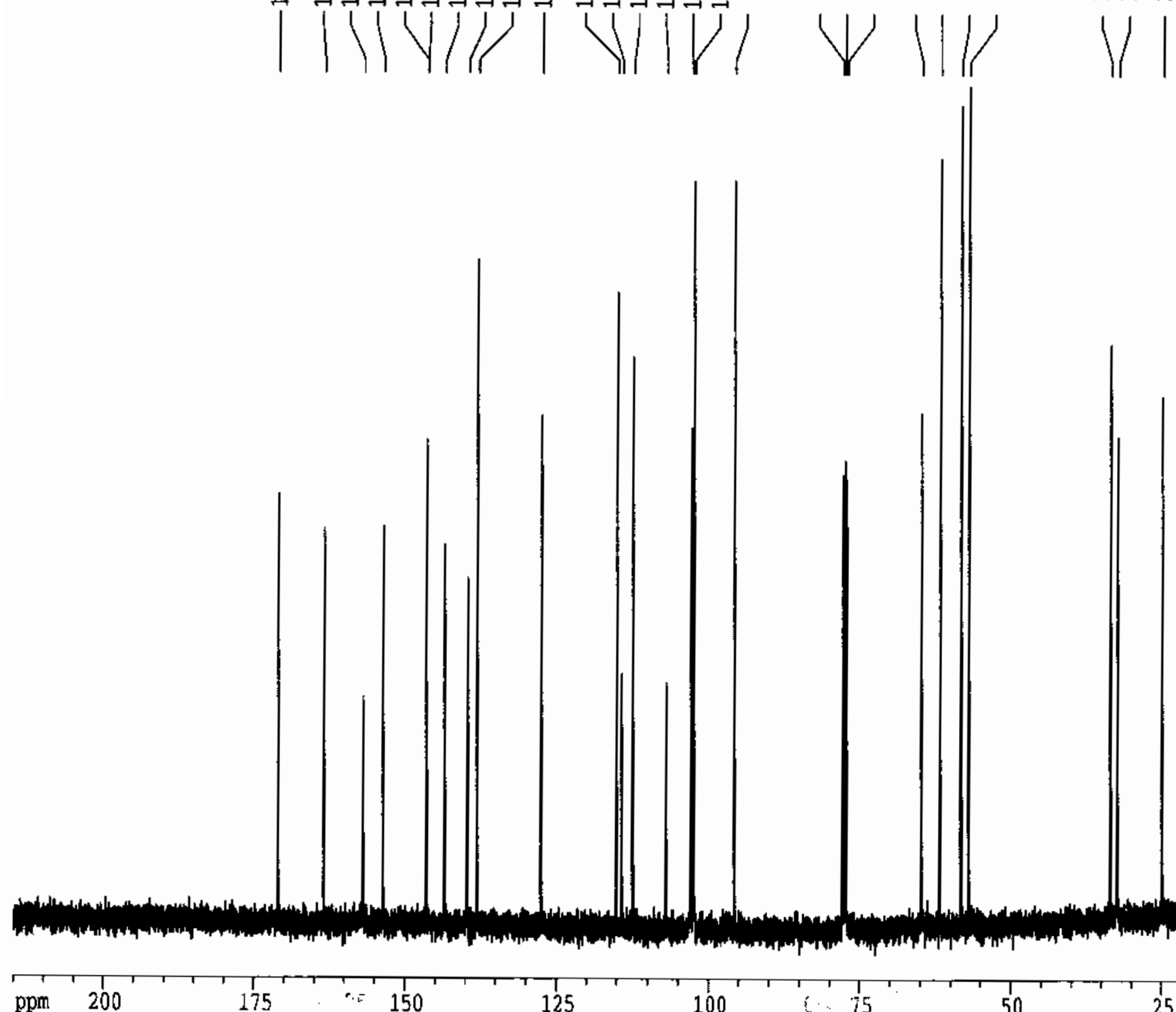

Current Datâ Parameters NAME

Axpio

PROCNO

F2 - Accuisition Parameters

Date_20050129

$\begin{array}{lr}\text { Time } & 2005018 \\ & 15.18\end{array}$

$\operatorname{drx} \times 190$

PROBHD 5 תIA ONP 1H/13

PJUPROG zgpg 30

TD 65536

SOLVENT

DS

DS

PIDRES $\quad 0.353213 \mathrm{~Hz}$

AQ $\quad 1.4156276 \mathrm{sec}$

$R G$

DE

TE

D1

6502

21.600 usec

5.50 usec 293.28

$0.15000001 \mathrm{sec}$

$0.03000000 \mathrm{sec}$

DELTA $\quad 0.05000000 \mathrm{sec}$

MCFEST $\quad 0.00000000 \mathrm{sec}$

$0.01500000 \mathrm{sec}$

$==\pi== \pm=$ CHARNEL $\mathrm{f} 1$

$\begin{array}{lr}\text { HOCl } & 13 \mathrm{C} \\ \text { P1 } & 11.25 \mathrm{usec} \\ \text { PL1 } & 2.00 \mathrm{~dB} \\ \text { SRO1 } & 100.6232933 \mathrm{LFH}\end{array}$

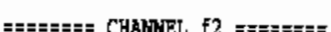

CPDPRG2 waltz16

MC2 $1 \mathrm{H}$

PCPD2 100.00 usec

PL12

PL13

$-4.00 \mathrm{~dB}$

16.58 di

$17.00 \mathrm{~dB}$

82 - Processing parameters

$\begin{array}{lc}\text { SI } & 65536 \\ \text { SF } & 100.6127290 \mathrm{MIIz}\end{array}$

WDN

LSB

$\mathrm{LB}$
$\mathrm{GB}$
$\mathrm{PC}$

EN
0

$1.00 \mathrm{~Hz}$

0
1.40

10 MR plot paraneters

$\begin{array}{lc}\mathrm{CX} & 20.00 \mathrm{~cm} \\ \mathrm{CY} & 12.50 \mathrm{~cm} \\ \mathrm{F1P} & 215.000 \mathrm{ppm} \\ \text { F1 } & 21631.74 \mathrm{~Hz} \\ \text { F2P } & -5.000 \mathrm{ppm} \\ \text { F2 } & -503.06 \mathrm{~Hz} \\ \text { PPHCH } & 11.00000 \mathrm{pPm} / \mathrm{cm} \\ \text { HZOH } & 1106.73999 \mathrm{~Hz} / \mathrm{cm}\end{array}$


3-(2-chloro-3,5-dihydroxy-6-((2-methoxy-3,6-bis (methoxymethoxy)-5-nitrophenethoxy) carbonyl) phenyl)propanoic acid

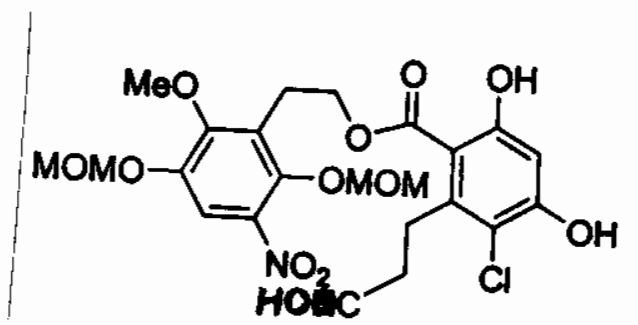

$118 \mathrm{C}$

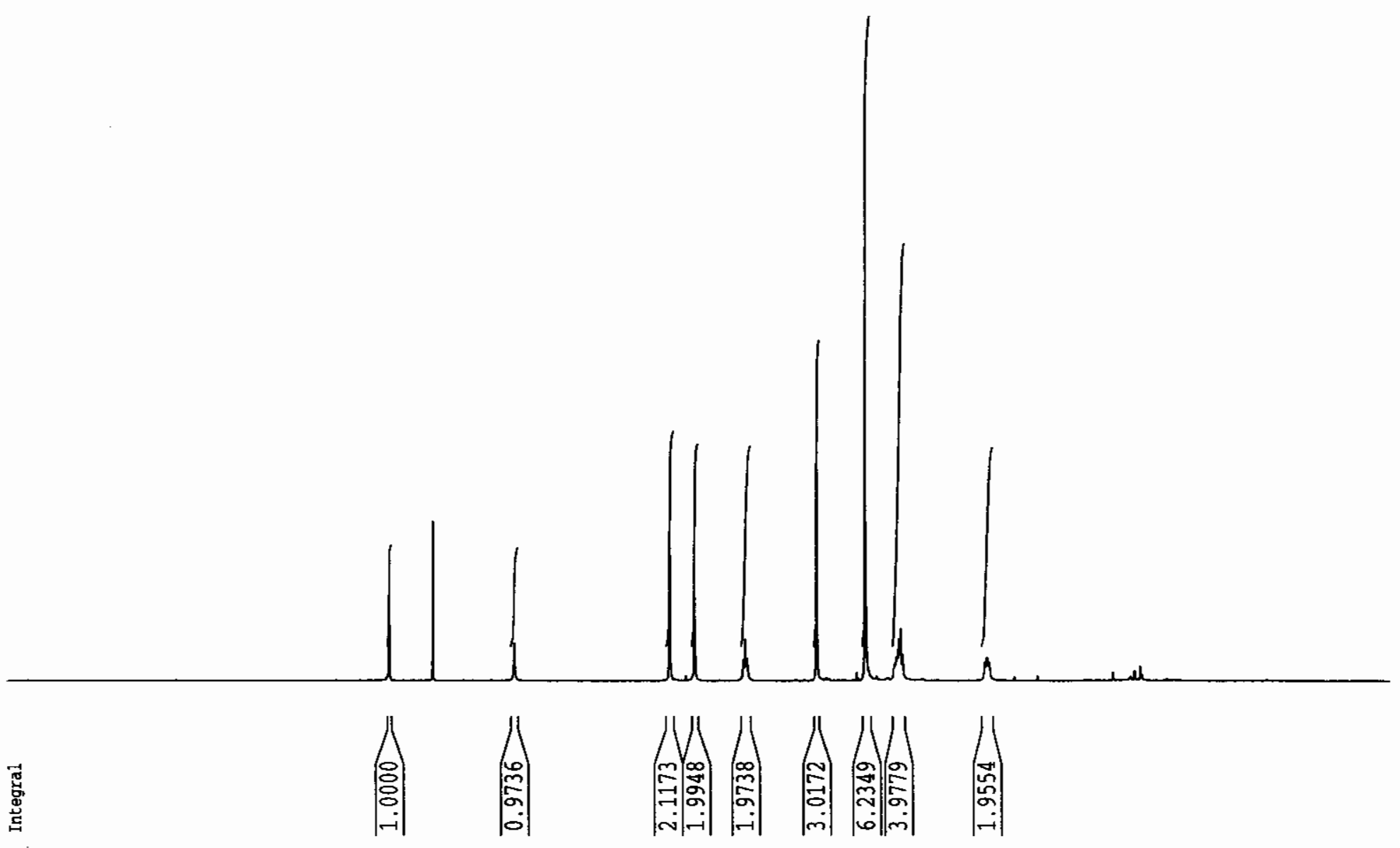

$\begin{array}{lr}\text { Current } & \text { Data } \\ \text { Narameters } \\ \text { EXPNO } & \text { wiw501314 } \\ \text { PROCNO } & 1 \\ & 1\end{array}$

F2 - Acquisition Parameters

Date_ 20050131

Time 20.57

INSTRUM drx400

PROBHD 5 QNP $1 \mathrm{H} / 13$

PULPROG $\quad \mathrm{zg} 30$

TD 32768

$\mathrm{CDC} 13$

NS

DS

$4789.272 \mathrm{~Hz}$

$\quad 0.146157 \mathrm{~Hz}$

$\mathrm{AQ} \quad 3.4210291 \mathrm{sec}$

RG $\quad 322.5$

$\begin{array}{lr}\text { DW } & 104.400 \text { usec } \\ \text { DE } & 5.50 \text { usec }\end{array}$

TE $292.2 \mathrm{~K}$

D1 $\quad 1.00000000 \mathrm{sec}$

MCREST $\quad 0.00000000 \mathrm{sec}$

MCWRK $\quad 0.01500000 \mathrm{sec}$

$=======$ CHANNEL f1 $=z===z==$

NUCl $1 \mathrm{H}$

P1 9.35 usec

PL1 $-4.00 \mathrm{~dB}$

SFO1 $400.1320007 \mathrm{MHz}$

F2 - Processing parameters

SI $\quad 32768$

SF $\quad 400.1300000 \mathrm{MHz}$

WDW

SSB

LB $\quad 0.30 \mathrm{~Hz}$

GB

EM

0
1.00

1D MMR plot parameters

$\begin{array}{lr}\text { CX } & 20.00 \mathrm{~cm} \\ \text { CY } & 4.00 \mathrm{~cm} \\ \text { F1P } & 11.000 \mathrm{ppm} \\ \text { F1 } & 4401.43 \mathrm{~Hz} \\ \text { F2P } & -1.000 \mathrm{ppm} \\ \text { F2 } & -400.13 \mathrm{~Hz} \\ \text { PPMCM } & 0.60000 \mathrm{ppm} / \mathrm{cm} \\ \text { HZCM } & 240.07800 \mathrm{~Hz} / \mathrm{cm}\end{array}$




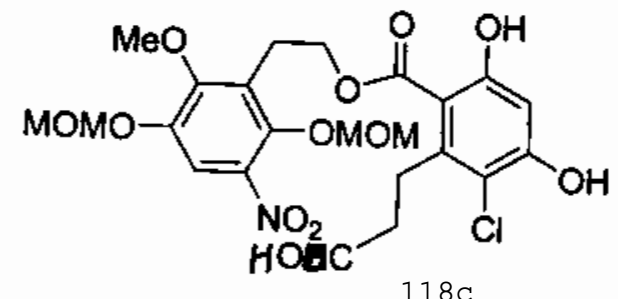

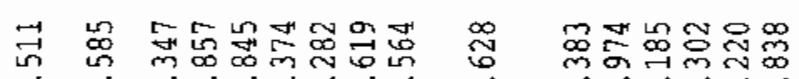

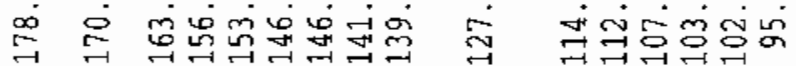

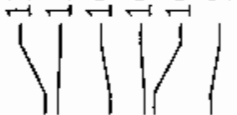

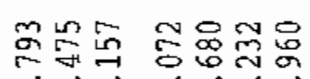

min sion

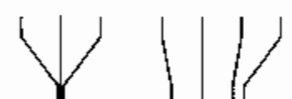

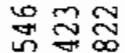

लिख

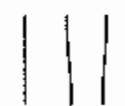

Current Data Paraneters

NAKE

EXPNO

PROCNO

F2 - Acquisition Paraneters

Date_ 20050131

INSTRUM drx4OD

PROBKD 5 IIII QNP $1 \mathrm{H} / 13$

PULPROG 2gpg30

TD 65536

SOLVEN

NS

DS

FIDR

so

RG

$\mathrm{IW}$
$\mathrm{DE}$
$\mathrm{TB}$

TB

D1 11

\section{DELTA}

MCRES

$\begin{array}{lr}=======\text { CHANNEL, } & \mathrm{f} 1 \mathrm{l}====== \\ \text { NUC1 } & 13 \mathrm{C} \\ \text { P1 } & 11.25 \text { usec } \\ \text { PL1 } & 2.00 \mathrm{~dB} \\ \text { SF01 } & 100.6232933 \mathrm{MHz}\end{array}$

$\mathrm{sro1}$

$100.6232933 \mathrm{mHz}$

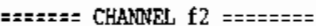

NuC2

PCPD

PL2

PL12

PL13

waltz16

100.00 usec

$-4.00 \mathrm{dg}$

$-4.00 \mathrm{~dB}$

$16.58 \mathrm{~dB}$

$400.1316005 \mathrm{MHz}$

F2 - Processing parameters

SI 65536

$\mathrm{SF} \quad 100.6127290 \mathrm{MHz}$

WDA

SSB

GB

PC

ID NMR plot parameters

$\begin{array}{ll}\mathrm{CX} & 20.00 \mathrm{~cm} \\ \mathrm{CY} & 12.50 \mathrm{~cm}\end{array}$

FIP $215.000 \mathrm{cpm}$

F1 $\quad 21631.74 \mathrm{~Hz}$

F2P

F2

PPYCN

$-5.000 \mathrm{pgm}$

HZCM 

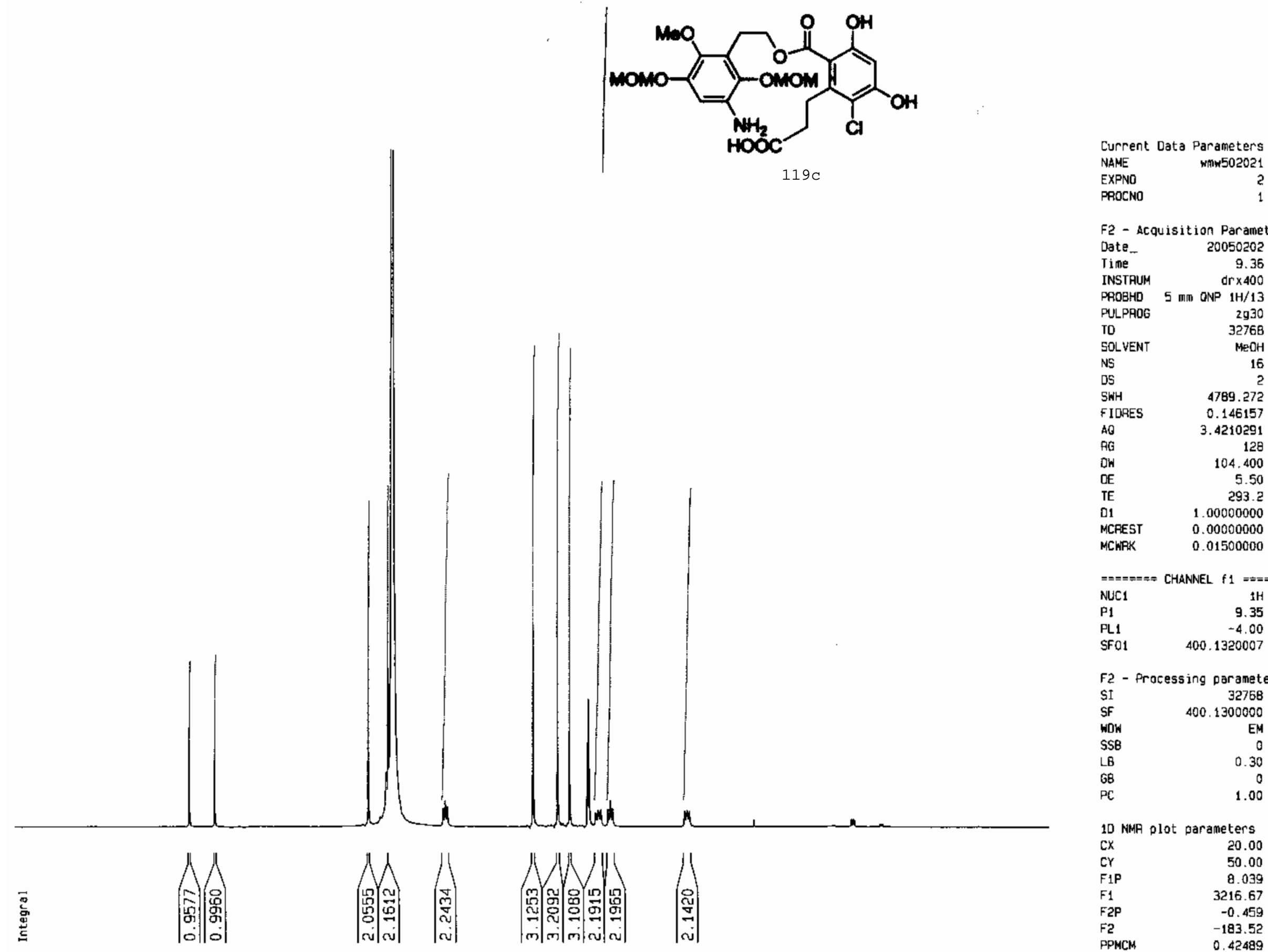

F2 - Acquisition Parameters

Date. 20050202

Time

20050202
9.36

dex 400

PULPPOG 2930

TO 32768

SOLVEN

NS

DS

SWH

F IDRES

AQ

DW

DE

TE

ธ1

MCFEST

$\mathrm{MEOH}$

MCHFKK

$4789.272 \mathrm{~Hz}$

$0.146157 \mathrm{~Hz}$

$3.4210291 \mathrm{sec}$

128

104.400 usec

5.50 usec $293.2 \mathrm{~K}$

$1.00000000 \mathrm{sec}$

0.00000000 sec

$====== \pm$ CHANNEL \&

NUC1

P1

PL1 $01500000 \mathrm{sec}$

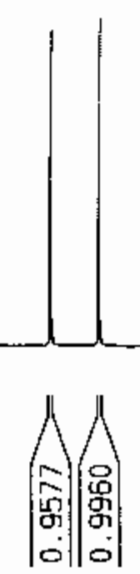

SFO1 iH 35 usec

$-4.00 \mathrm{~dB}$

F2 - Pracessing parameters

SI 32768 SF $\quad 400.1300000 \mathrm{MHz}$ WOW EM

SSB

LB
68

$\mathrm{EM}$
$0.30 \mathrm{~Hz}$
0

$\mathrm{PC}$

1.00

10 MMA plot parameters

$\begin{array}{lr}\text { CX } & 20.00 \mathrm{~cm} \\ \text { CY } & 50.00 \mathrm{~cm} \\ \text { F1P } & 8.039 \mathrm{ppm} \\ \text { F1 } & 3216.67 \mathrm{~Hz} \\ \text { F2P } & -0.459 \mathrm{ppm} \\ \text { F2 } & -183.52 \mathrm{~Hz} \\ \text { PPMCM } & 0.42489 \mathrm{ppm} / \mathrm{cm} \\ \mathrm{HZCM} & 170.00981 \mathrm{~Hz} / \mathrm{cm}\end{array}$


3-(2-((3-amino-6-methoxy-2,5-bis (methoxymethoxy) phenethoxy) carbonyl)-6-chloro-3,5-dihydroxyphenyl) propanoic acid

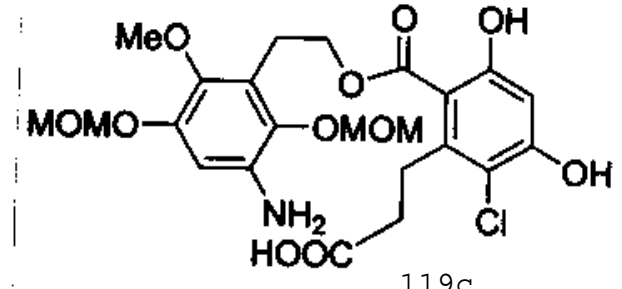

$119 \mathrm{c}$
38

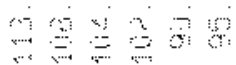

s a

89
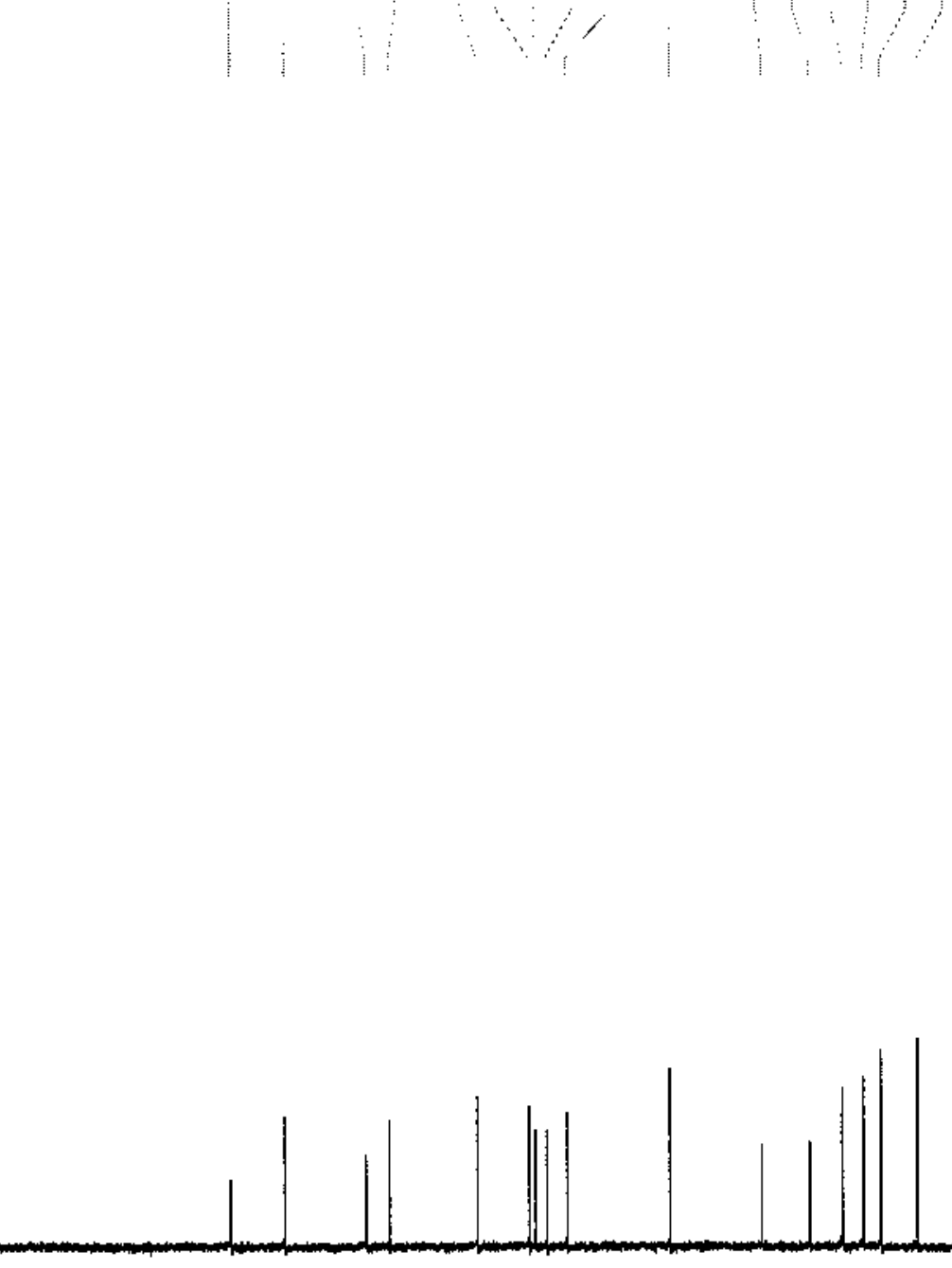

$$
\text { ppm }
$$


MOM-protected Desmethyl Radanamycin
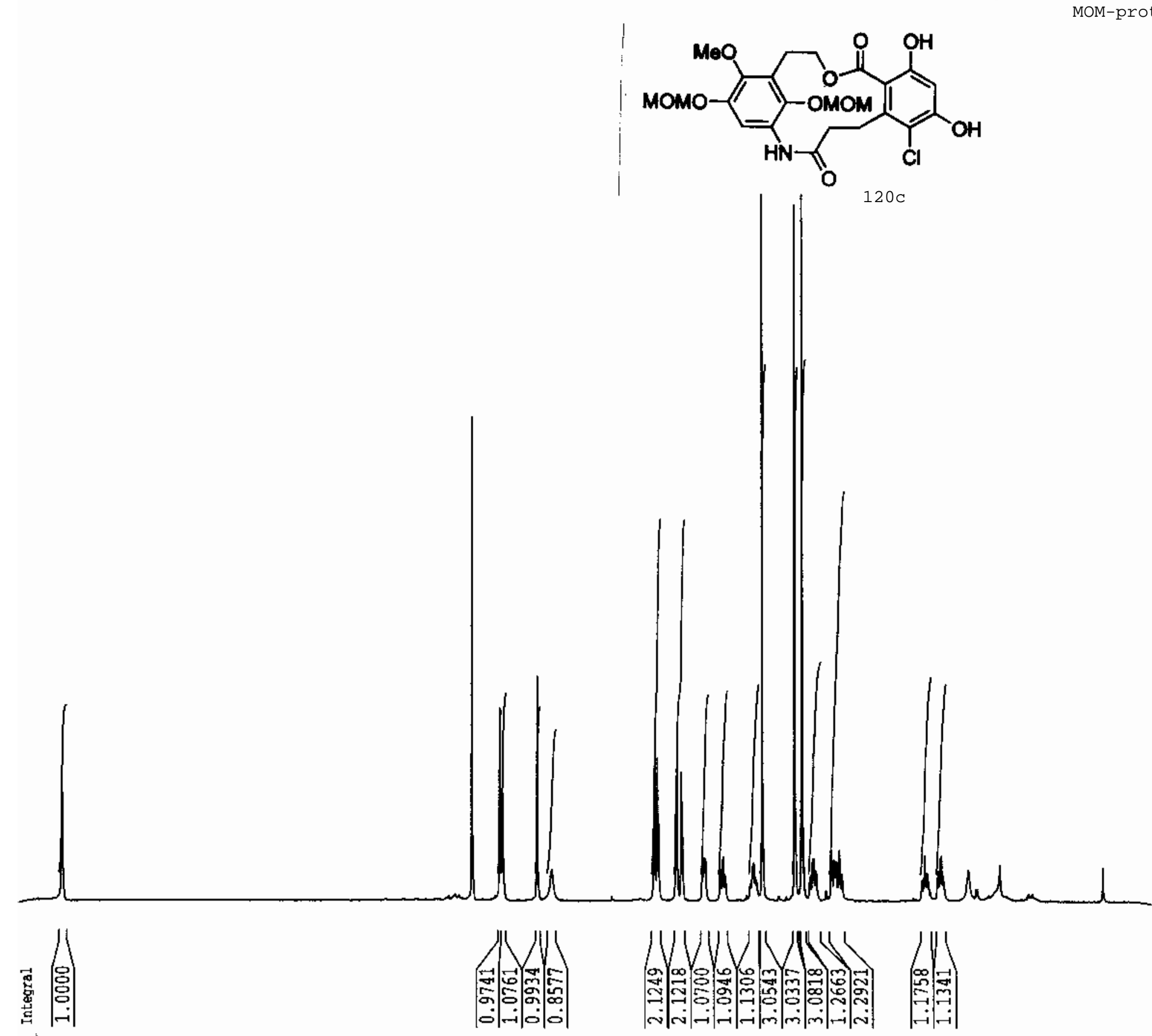
NAME
EXPNO
PROCNO
wow 2141

Current Data Parameters

F2 - Acquisition Paraneters

Date_ 20050215

$\begin{array}{lr}\text { Time } & 14.36 \\ \text { INSTRUM } & \mathrm{dr} \times 400\end{array}$

PROBHD 5 man ONP 1H/13

PULPROG

zg30

TD

SOLvEly

NS

DS

$\mathrm{CDCl} 3$

SWH

FIDRES

$\mathrm{AQ}$

RG

DW

DE

$\mathrm{TE}$

D1

MCREST

2

$5175.983 \mathrm{Bz}$

$0.157958 \mathrm{~Hz}$

$3.1654389 \mathrm{sec}$ 362

96.600 usec

5.50 usec $292.2 \mathrm{~K}$

$0.00000000 \mathrm{sec}$

$0.01500000 \mathrm{sec}$

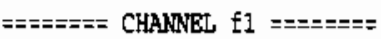
(I) 1

P1

9.35 usec

$4001324008 \mathrm{MHz}$

F2 - Processing parameters

SI. 32768

SF $\quad 400.1300000 \mathrm{MHz}$

WDW

SSB

LB $\quad 0.30 \mathrm{~Hz}$

GB

0

1D NMR plot parameters

$\begin{array}{lc}\text { CX } & 20.00 \mathrm{~cm} \\ \text { CY } & 12.50 \mathrm{~cm} \\ \text { F1P } & 12.468 \mathrm{ppm} \\ \text { FI } & 4988.77 \mathrm{~Hz} \\ \text { F2P } & -0.468 \mathrm{ppm} \\ \text { F2 } & -187.21 \mathrm{~Hz} \\ \text { PPHCM } & 0.64679 \mathrm{ppm} / \mathrm{Cm} \\ \text { HZCM } & 258.79916 \mathrm{~Hz} / \mathrm{cm}\end{array}$

10.

8

6

.) 4

2 


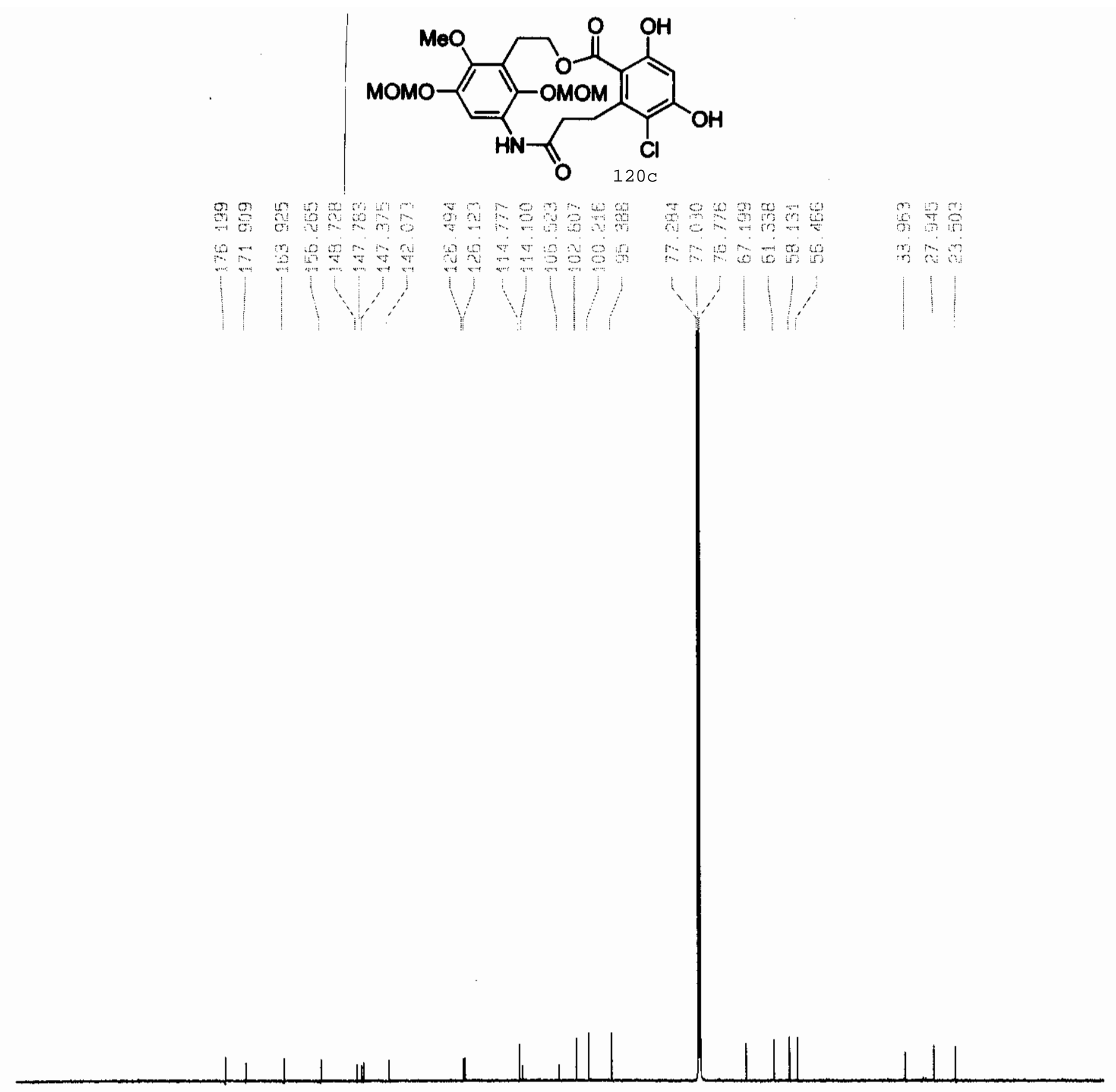
Current Data Parameters
NAME WMW MOM EXPNO PAOCNO

F2 - Acquisition Parameters Oate_ 20050210 TimeINSTRUM spect PROQHD $5 \mathrm{~mm}$ CPDUL 13C PULPROG $\begin{array}{r}\text { 2gpg } 30 \\ \text { POS }\end{array}$ $\begin{array}{lr}\text { TD } & 65536\end{array}$ SOLVENT $\quad \operatorname{CDCl3}$ NS DS $\quad 31446.541 \mathrm{~Hz}$ FIDRES $\quad 0.479836 \mathrm{~Hz}$ AQ $\quad 1.0420724 \mathrm{sec}$ PG 8192 $\begin{array}{lr}\text { DW } & 15.900 \text { usec } \\ \text { DE } & 6.00 \text { usec }\end{array}$ TE $298.0 \mathrm{~K}$

$01 \quad 0.15000001 \mathrm{sec}$

d11 $0.03000000 \mathrm{sec}$

DELTA $\quad 0.05000000 \mathrm{sec}$ MCAEST $\quad 0.00000000 \mathrm{sec}$ MCWRK $\quad 0.01500000 \mathrm{sec}$

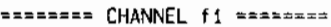
NUC1 $19 \mathrm{C}$ $P 1 \quad 10.00$ usec PL1 $\quad-4.90 \mathrm{OB}$ $125.7697360 \mathrm{MHz}$ $======$ CHANNEL $f 2=======$ CPOPAG2 waltz15 NUC2 $\quad 1 \mathrm{H}$ 80.00 use PL2 $\quad-5.00 \mathrm{~dB}$ $9.54 \mathrm{~dB}$ $\begin{array}{lr}\text { PL13 } & 10.00 \mathrm{~dB} \\ \text { SF02 } & 500.1325000 \mathrm{MHz}\end{array}$

F2 - Processing parameters $F_{2}$ - Processing Darameters
SI $\begin{array}{lr}\text { SI } & 65536 \\ \text { SF } & 125.7577890 \mathrm{MHZ}\end{array}$ WDW EM $\begin{array}{lc}\text { SSB } & 0 \\ \text { LO } & 1.00 \mathrm{~Hz}\end{array}$ $\begin{array}{rr}G B & 0 \\ P C & 1.40\end{array}$

i0 NMP plat parameters cx $\quad 20.00 \mathrm{~cm}$ $\begin{array}{lr}\mathrm{CY} & 0.00 \mathrm{~cm}\end{array}$ $\begin{array}{lr}\text { FIP } & 242.517 \mathrm{ppm} \\ \mathrm{FI} & 30498.43 \mathrm{~Hz}\end{array}$ $\begin{array}{ll}F 1 & 30498.43 \mathrm{~Hz} \\ F 2 \mathrm{P} & -7.539 \mathrm{pP}\end{array}$ $\begin{array}{ll} & -7.539 \mathrm{pp} \\ \mathrm{F2} & -948.11 \mathrm{~Hz}\end{array}$ PPMCM $12.50282 \mathrm{ppm} / \mathrm{Cm}$ $\mathrm{HZCM} \quad 1572.32690 \mathrm{~Hz} / \mathrm{cm}$ 

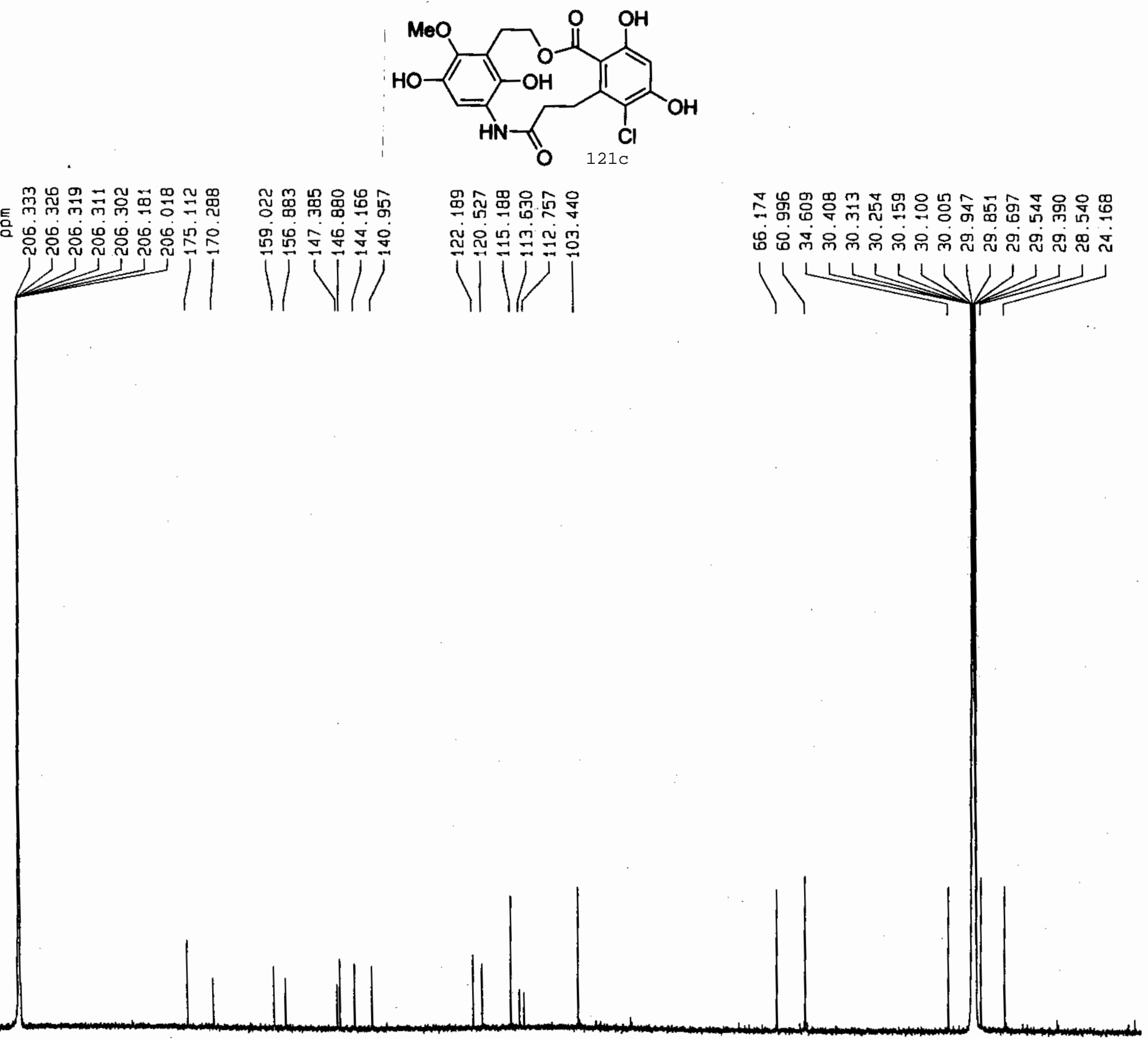

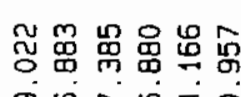

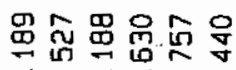

ญํำ

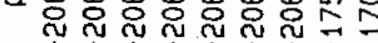

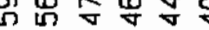

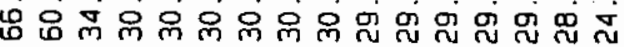

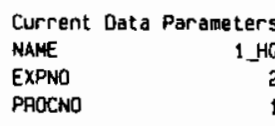

Fa - Acquisition Parameters

Oate__ 20050221

Time 10.34

PFDOHO Spect

PIIPROG 5 and CPOL $13 \mathrm{C}$

TO 290930

SOLVENT

NS $\quad 3050$

SWh

3060

FIDPES $\quad 31446.541 \mathrm{~Hz}$

AO $\quad 0.479836 \mathrm{~Hz}$

$1.0420883 \mathrm{sec}$

8192

$\begin{array}{lr}\text { DW } & 15.900 \text { usec } \\ \text { DE } & 6.00 \text { usec }\end{array}$

TE $298.0 \mathrm{~K}$

$01 \quad 0.15000001 \mathrm{sec}$

d11 $\quad 0.03000000 \mathrm{sec}$

DELTA $\quad 0.05000000 \mathrm{sec}$

HCPEST $\quad 0.00000000 \mathrm{sec}$

MCWFK $\quad 0.01500000 \mathrm{sec}$

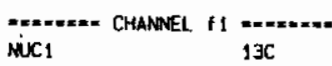

P1 $\quad 10.00$ usec

P.1 $-4.90 \mathrm{do}$

SF01 $125.7697360 \mathrm{MHz}$

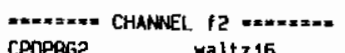

CPDPRG2 maltz16

NUC2 $\quad 5 H$

PCPD2 80.00 use

$\begin{array}{lr}\text { PL2 } & -5.00 \mathrm{~dB} \\ \mathrm{PL} 12 & 9.54 \mathrm{~dB}\end{array}$

PL.13 $10.00 \mathrm{dg}$

$5 F 02 \quad 500.1325000 \mathrm{MHz}$

F2 - Pracessing parameters

SI Processing praneters

SF $\quad 125.7576758 \mathrm{mHz}$

WOW $\quad 125.7576758$

EM

$\begin{array}{cc}\mathrm{LB} & 1.00 \mathrm{~Hz} \\ \mathrm{GO} & 0\end{array}$

GQ

10 NHP plok parameters

cX $20.00 \mathrm{~cm}$

cr $\quad 0.00 \mathrm{~cm}$

F1 $\quad 210.000 \mathrm{ppm}$

Fiv $26409.11 \mathrm{~Hz}$

$\begin{array}{ll}F 2 P & -1.000 \mathrm{ppm} \\ F 2 & -125.76 \mathrm{~Hz}\end{array}$

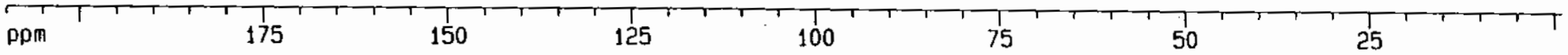

PPMCH 
Desmethyl Radanamycin Quinone

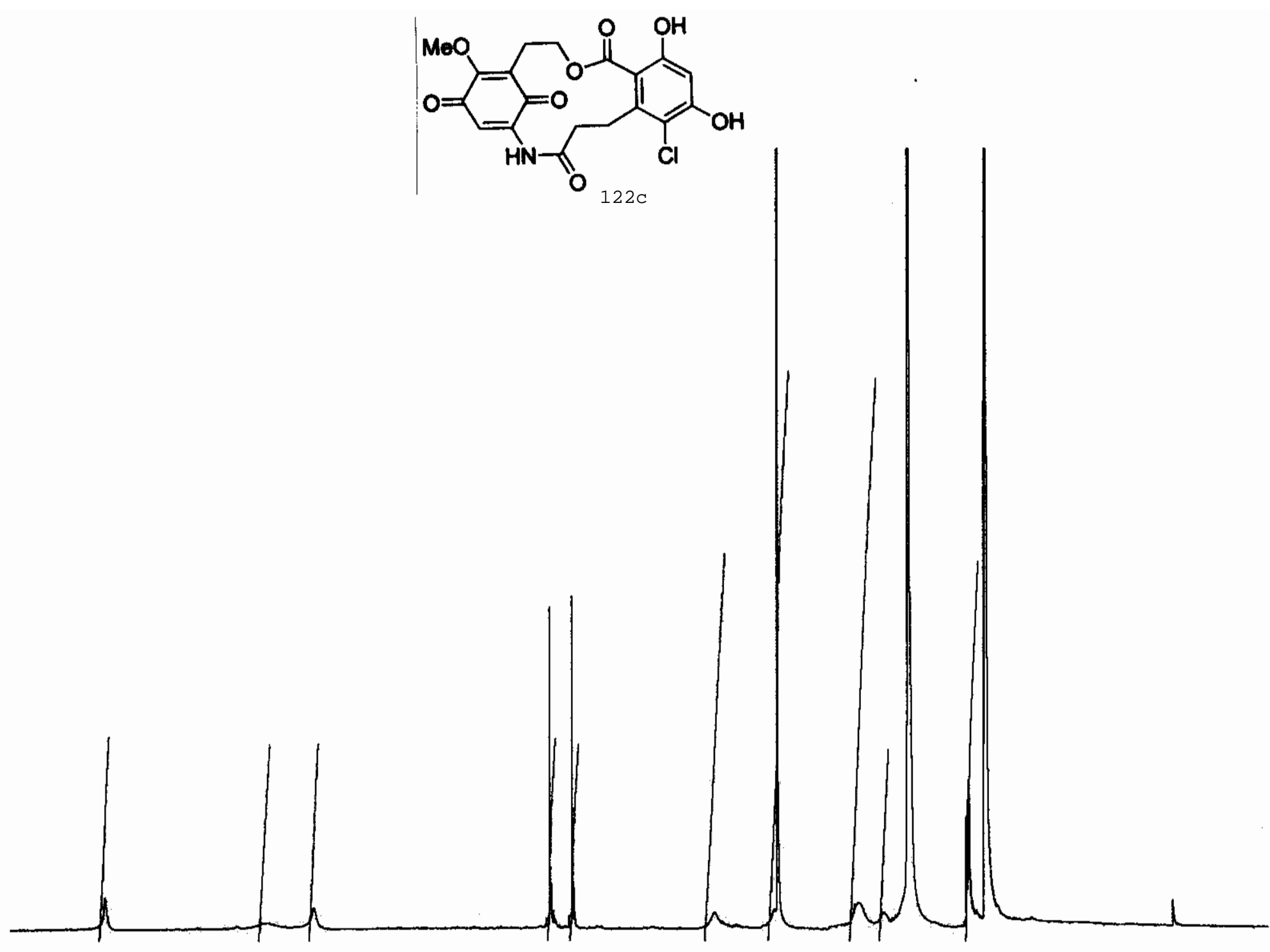

Current Doto Poraneters
NAVE
EXPNo
PROCNO

F2 - ACquisition Parameters

Date_ 20050291

$\quad 10.40$

PROBNIG 5 min cPove $43 \mathrm{C}$

PUPPROG

To $\quad 65536$

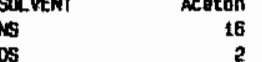

SWH $\quad 10330.578 \mathrm{~Hz}$

FIDPES $\quad 0.157852 \mathrm{~Hz}$

A日 $\quad 3.1719923 \mathrm{sec}$

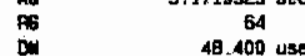

De $\quad 48.400$ usec

TE $298.0 \mathrm{~K}$

01 1.00000000 sec

$\begin{array}{ll}\text { MGREST } & 0.00000000 \text { SEc } \\ \text { VEWPK } & 0.01500000 \text { sec }\end{array}$

MUC1 CHANEL 11 -

$\begin{array}{ll}\text { NuC1 } & 1 \mathrm{H} \\ \text { P1 } & 15.00 \text { usec }\end{array}$

$\begin{array}{lr}\text { PL1 } & -5.00 \mathrm{~dB} \\ \text { SFO1 } & 500.1330895 \mathrm{MHt2}\end{array}$

Fi - ACquisftion parameters

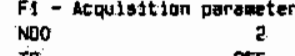

NDOO

TU

$5 F 01 \quad 500.1325 \mathrm{WHZ}$

23.47500

12.016

F2 - Processing parameters

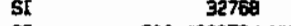

SF $\quad 500.1300734 \mathrm{Wtz}$

$\sin$

Le

PC

EN

$0.30 . \mathrm{Hz}$

F1 - processing paraneters

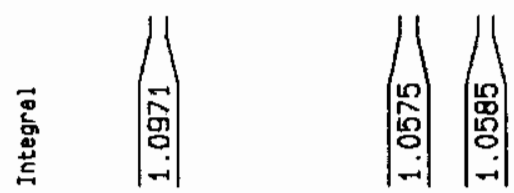

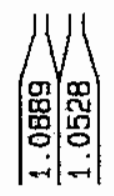

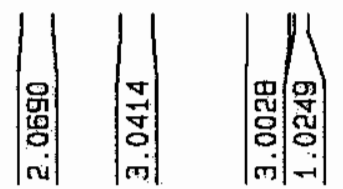

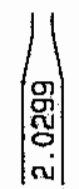

300.1300000 osine

550

Li

10 NwF plot porameters

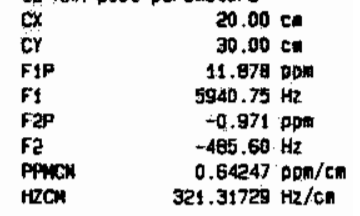



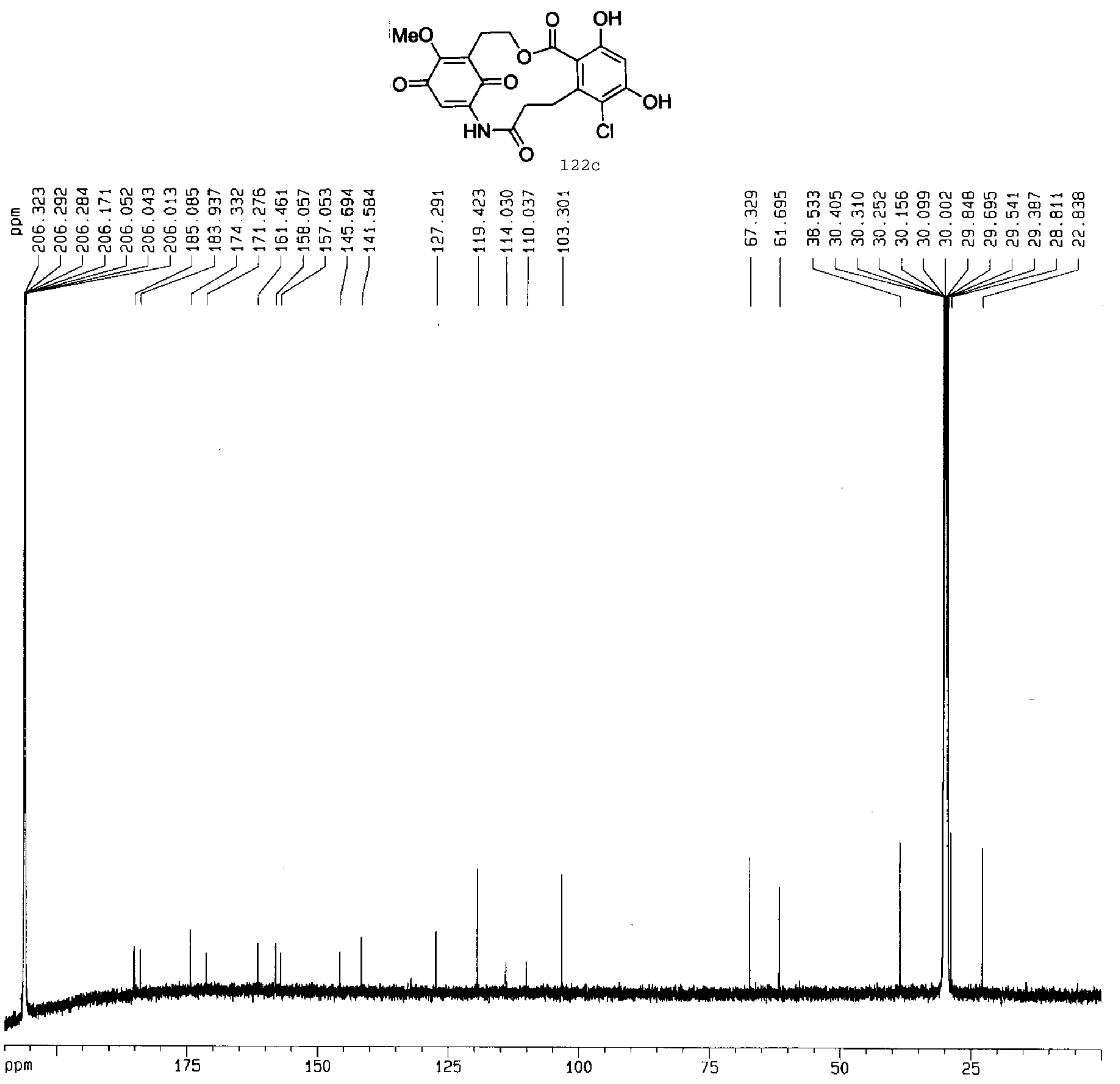

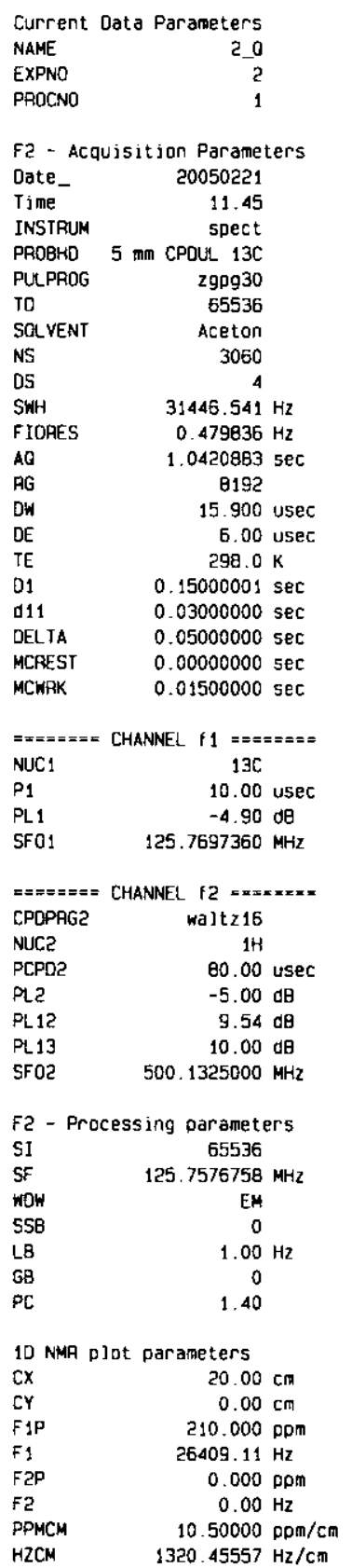


Methyl 4,6-bis (tert-butyldimethylsilyloxy)-3-chloro-2-(pent-4-enyl) benzoate

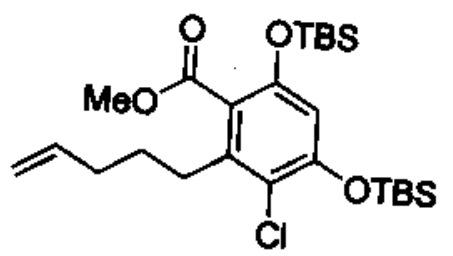

$110 a$

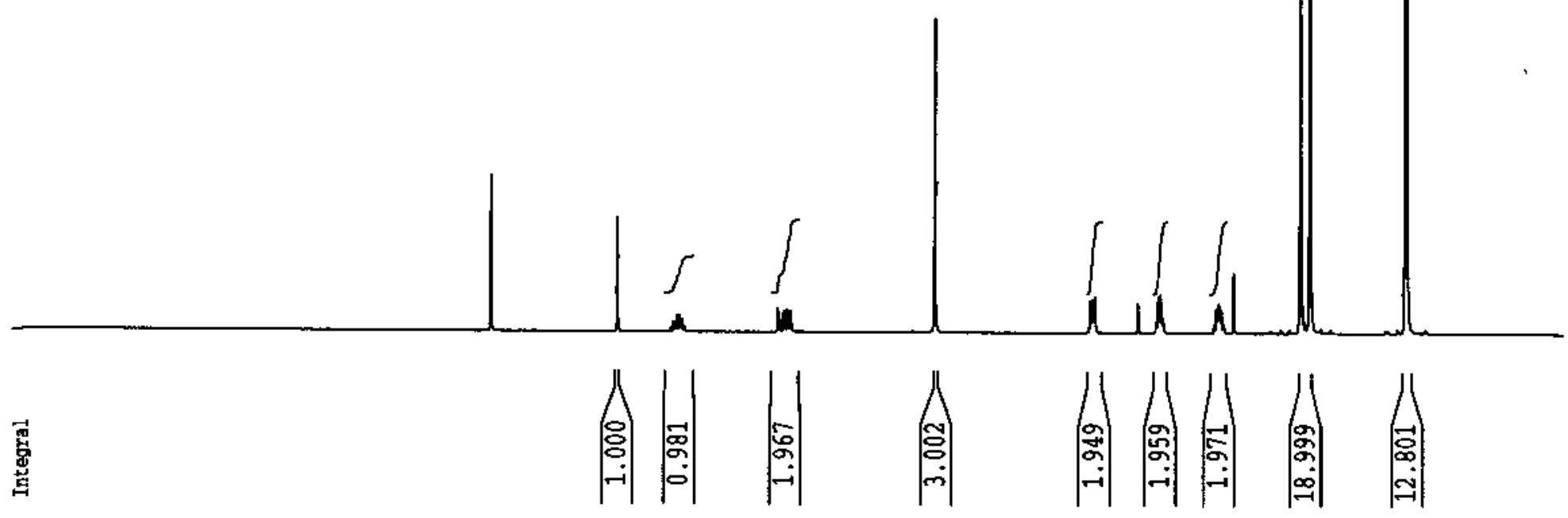

Current Data Parameters

$$
\text { EXPNO }
$$

PROCNO

01
1
1

E2 - Acquisition Parameters

Date_ 20050811

$\begin{array}{lr}\text { Time } & 20.55\end{array}$

INSTRUNI drx400

PROBHD 5 wII ONP $1 \mathrm{H} / 13$

PULPROG

$\begin{array}{lr}\text { PULPROG } & z g 30 \\ \text { TD } & 32768\end{array}$

SOLVANI CDCl3

NS

DS

DS

FIDRES

16

$\mathrm{AQ}$

RG

DW

DE

$\mathrm{TE}$

D1

$4789.272 \mathrm{~Hz}$

$0.146157 \mathrm{~Hz}$

$3.4210291 \mathrm{sec}$

256

104.400 usec

5.50 usec

$297.2 \mathrm{~K}$

$1.00000000 \mathrm{sec}$

$0.00000000 \mathrm{sec}$

MCWRK $\quad 0.01500000 \mathrm{sec}$

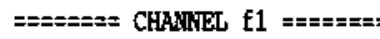
NUC1

P1

PL1 $1 \mathrm{H}$

SPO1

9.35 usec

$-4.00 \mathrm{~dB}$

F2 - Processing parameters

$\begin{array}{lc}\text { SI } & 32768 \\ \text { SF } & 400.1300000 \mathrm{MHzz} \\ \text { WIW } & \text { BN } \\ \text { SSB } & 0 \\ \text { LA } & 0.30 \mathrm{~Hz} \\ \text { GB } & 0 \\ \text { PC } & 1.00\end{array}$

1D NLR plot parameters

CX $20.00 \mathrm{~cm}$

CY $\quad 12.50 \mathrm{cr}$

F1P $\quad 11.000$ ppp

F1 $4401.43 \mathrm{~Hz}$

F2P $\quad-1.000 \mathrm{ppm}$

F2 $\quad-400.13 \mathrm{~Hz}$

PPHCY $\quad 0.60000 \mathrm{ppm} / \mathrm{cm}$

BzOI

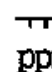

ppI
10
8
6
4
2 

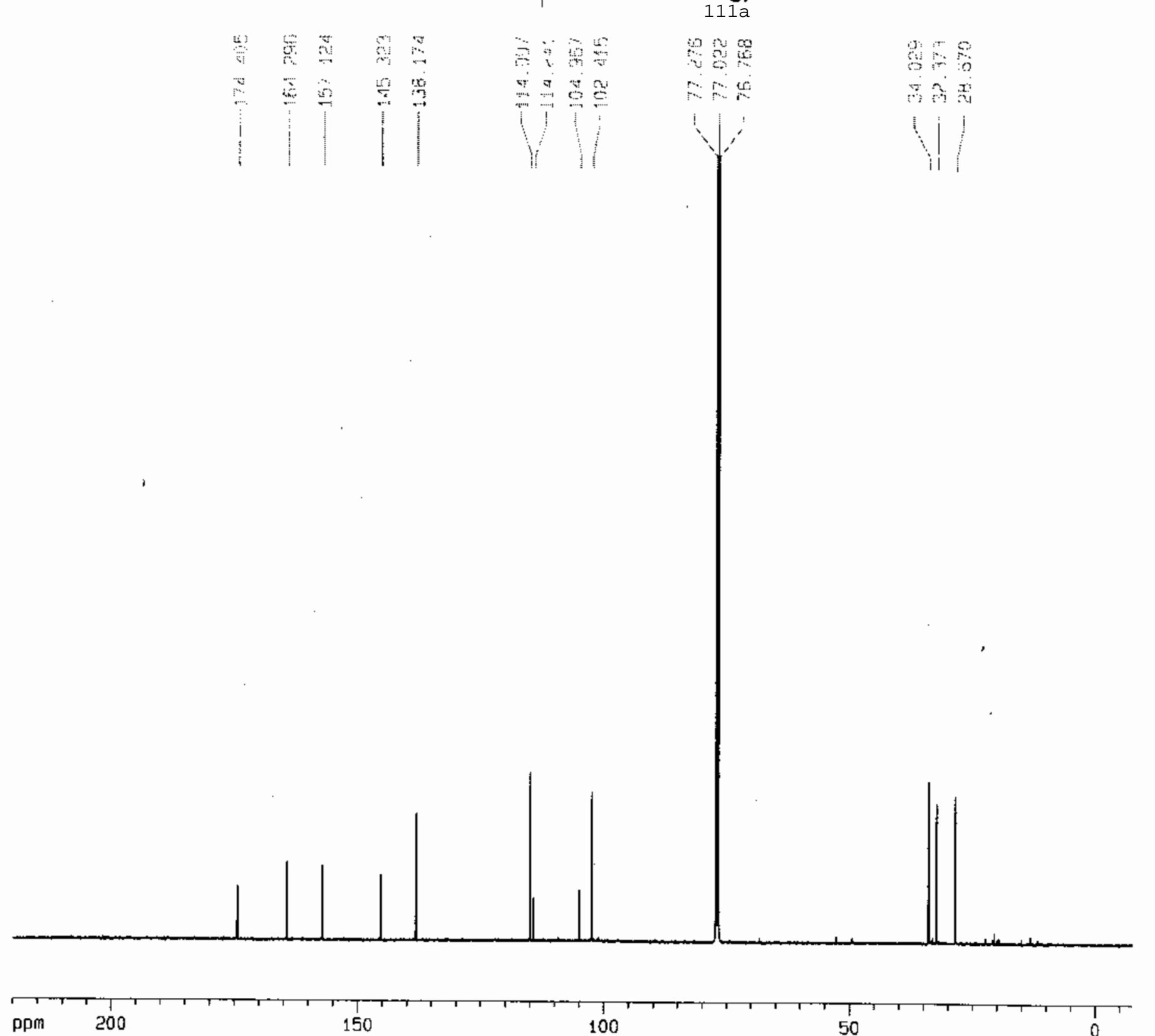

150

100

50

11

\begin{tabular}{|c|c|}
\hline \multicolumn{2}{|c|}{ Current Data Parameters } \\
\hline NAME & d2 \\
\hline EXPNO & 2 \\
\hline PROCNO & 1 \\
\hline \multicolumn{2}{|c|}{ F2 - Acquisitian Paraneters } \\
\hline Date & 20050809 \\
\hline Time & 10.42 \\
\hline INSTAUMN & spect \\
\hline PFOBH TD & 5 mem CPOLL $13 C$ \\
\hline PULPFOG & $29 \mathrm{pg} 30$ \\
\hline & 65536 \\
\hline SOL VENT & $\operatorname{coc} 13$ \\
\hline NS & 1024 \\
\hline os & 4 \\
\hline SWH & $31446.541 \mathrm{~Hz}$ \\
\hline FIDRES & $0.479836 \mathrm{~Hz}$ \\
\hline$A B$ & $1.0420724 \mathrm{sec}$ \\
\hline AG & 8192 \\
\hline DM & 15.900 usec \\
\hline DE & 6.00 usec \\
\hline & $300.0 \mathrm{~K}$ \\
\hline D1 & $0.15000001 \mathrm{sec}$ \\
\hline d11. & $0.03000000 \mathrm{sec}$ \\
\hline DELTA & $0.05000000 \mathrm{sec}$ \\
\hline MCREST & $0.00000000 \mathrm{sec}$ \\
\hline MCNFA & $0.01500000 \mathrm{sec}$ \\
\hline 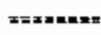 & CHANNEL IS $======$ \\
\hline NuC1 & 136 \\
\hline P1 & 10.00 usec \\
\hline PLt & $-4.90 \mathrm{~dB}$ \\
\hline SFO1 & $125.7697360 \mathrm{MHz}$ \\
\hline \multirow{2}{*}{\multicolumn{2}{|c|}{ 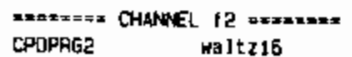 }} \\
\hline & \\
\hline Muca & IH \\
\hline PCPD2 & 80.00 usec \\
\hline PL.2 & $-5.00 \mathrm{~dB}$ \\
\hline PL12 & $9.54 \mathrm{~dB}$ \\
\hline P.13 & $10.00 \mathrm{~dB}$ \\
\hline $\mathrm{SF} 02$ & $500.1325000 \mathrm{MHz}$ \\
\hline \multicolumn{2}{|c|}{ F2 - Processing paraneters } \\
\hline sI & 65536 \\
\hline $\mathbf{S F}$ & $125.7577890 \mathrm{MHZ}$ \\
\hline NOW & EM \\
\hline SSB & 0 \\
\hline LQ & $1.00 \mathrm{~Hz}$ \\
\hline GB & 0 \\
\hline PC & 1.40 \\
\hline \multicolumn{2}{|c|}{ to plot paraneters } \\
\hline $\mathrm{cx}$ & $20.00 \mathrm{~cm}$ \\
\hline $\mathrm{cr}$ & $15.00 \mathrm{cn}$ \\
\hline FIP & $242.517 \mathrm{ppm}$ \\
\hline$f_{1}$ & $30498.43 \mathrm{H} 2$ \\
\hline F2P & $-7.539 \mathrm{ppm}$ \\
\hline F2 & $-948.11 \mathrm{~Hz}$ \\
\hline PFMCM & $12.50282 \mathrm{ppm} / \mathrm{cm}$ \\
\hline $\mathrm{HZCM}$ & $1572.32590 \mathrm{~Hz} / \mathrm{cm}$ \\
\hline
\end{tabular}




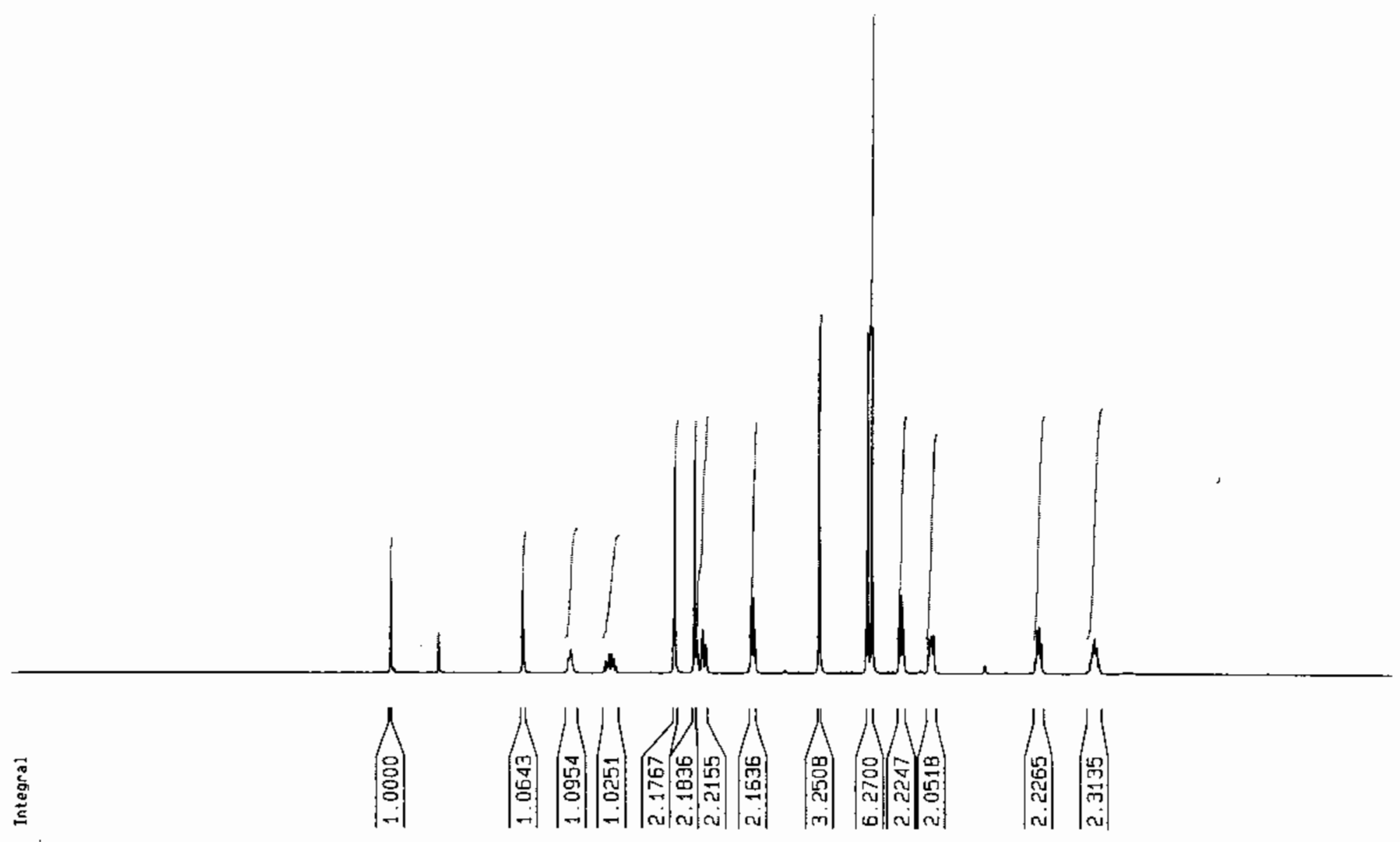

10

9

8

76

6

54

3

2

1

0

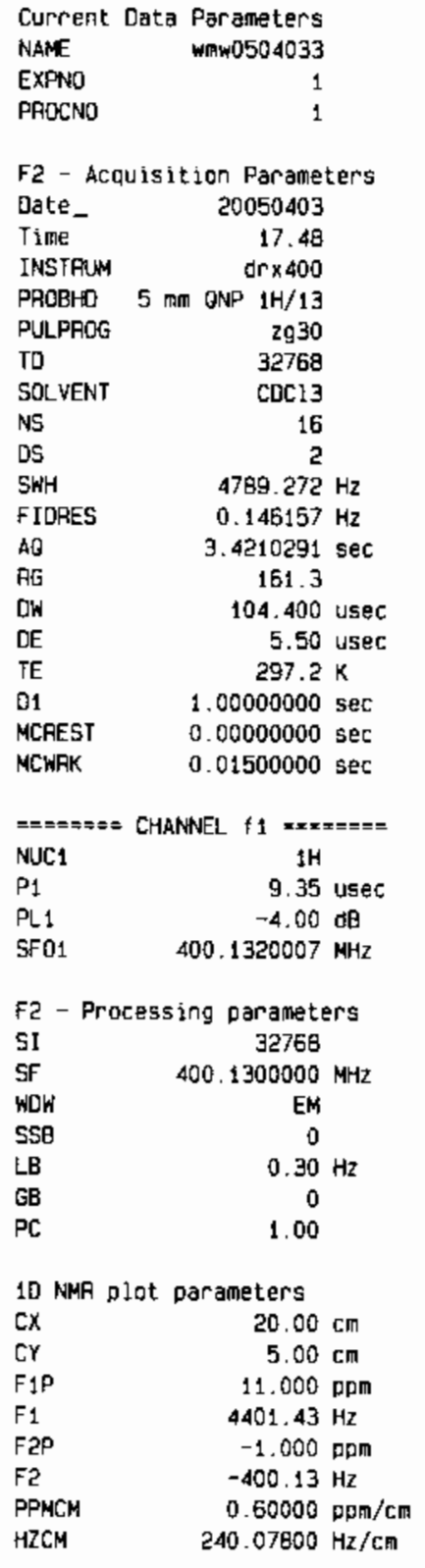



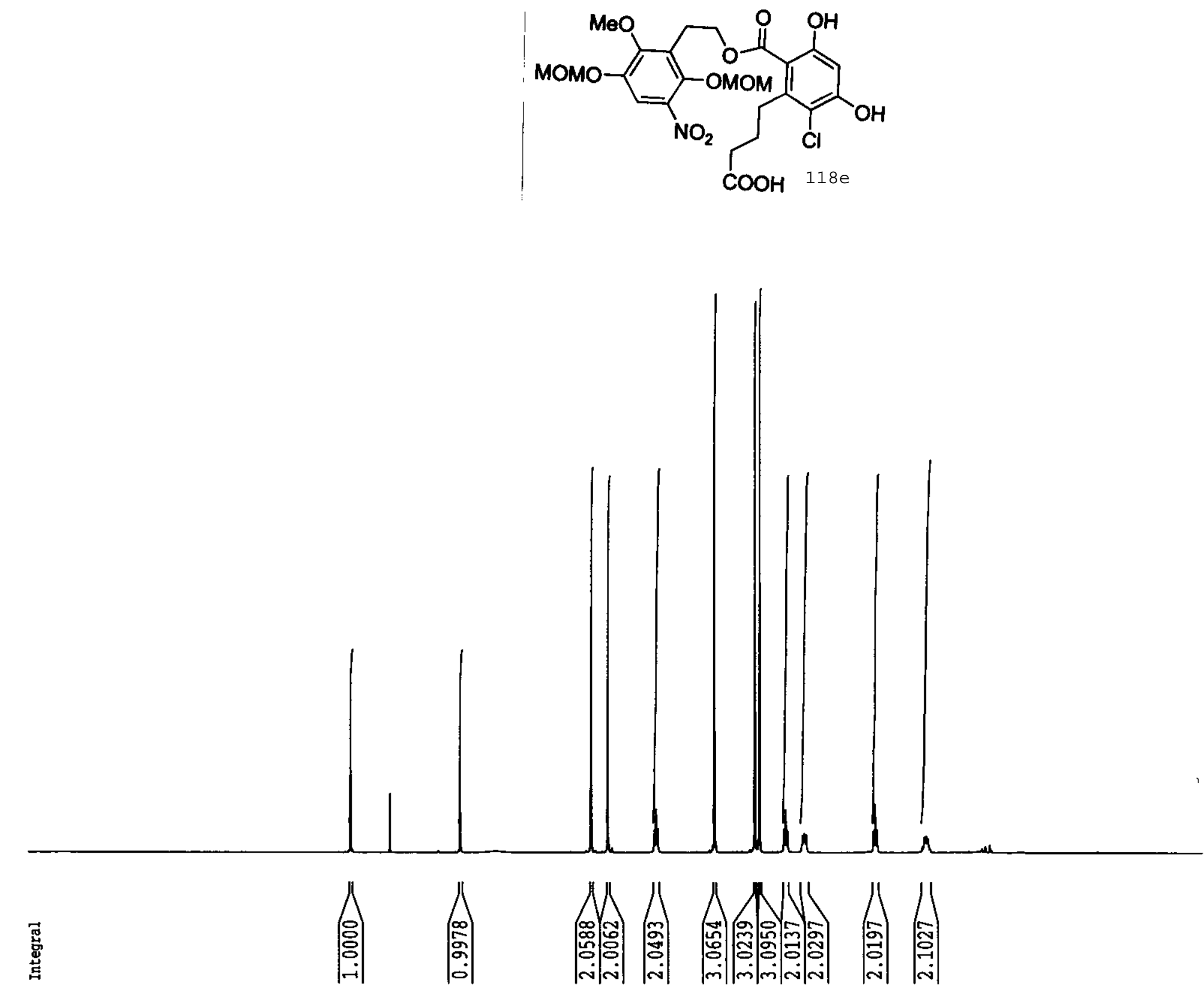

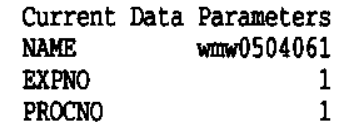

F2 - Acquisition Parameters Date 20050406 Time $\quad 21.12$ INSTRUM drx400 PROBHD 5 am ONP 1H/13 PULPROG TD SOLVENT NS DS DS 2 $\quad 4789.272 \mathrm{~Hz}$ $0.146157 \mathrm{~Hz}$ $\mathrm{AQ} \quad 3.4210291 \mathrm{sec}$ RG DW $\mathrm{DE}$ DI D1 104.400 usec 5.50 usec $293.2 \mathrm{~K}$ $1.00000000 \mathrm{sec}$ $0.00000000 \mathrm{sec}$ MCWRK $\quad 0.01500000 \mathrm{sec}$

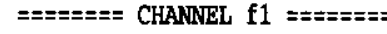
$\begin{array}{lr}\text { NUC1 } & \text { 1H } \\ \text { P1 } & 9.35 \text { usec } \\ \text { PL1 } & -4.00 \mathrm{~dB} \\ \text { SFO1 } & 400.1320007 \mathrm{MHz}\end{array}$

F2 - Processing parameters SI 32768 SF $\quad 400.1300000 \mathrm{MHz}$ WDW
SSB LB $\quad 0.30 \mathrm{~Hz}$ $\begin{array}{lr}\mathrm{GB} & 0 \\ \mathrm{PC} & 1.00\end{array}$

1D MR plot parameters CX $20.00 \mathrm{~cm}$ CY $\quad 5.00 \mathrm{~cm}$ F1P $\quad 11.000 \mathrm{ppm}$ F1 $\quad 4401.43 \mathrm{~Hz}$ F2P $\quad-1.000 \mathrm{ppm}$ F2 $\quad-400.13 \mathrm{~Hz}$ PPMCY $\quad 0.60000 \mathrm{ppm} / \mathrm{cm}$ HZCY $240.07800 \mathrm{~Hz} / \mathrm{cm}$ 
4-(2-chloro-3,5-dihydroxy-6-((2-methoxy-3, 6-bis (methoxymethoxy)-5-nitrophenethoxy) carbonyl) phenyl) butanoic acid
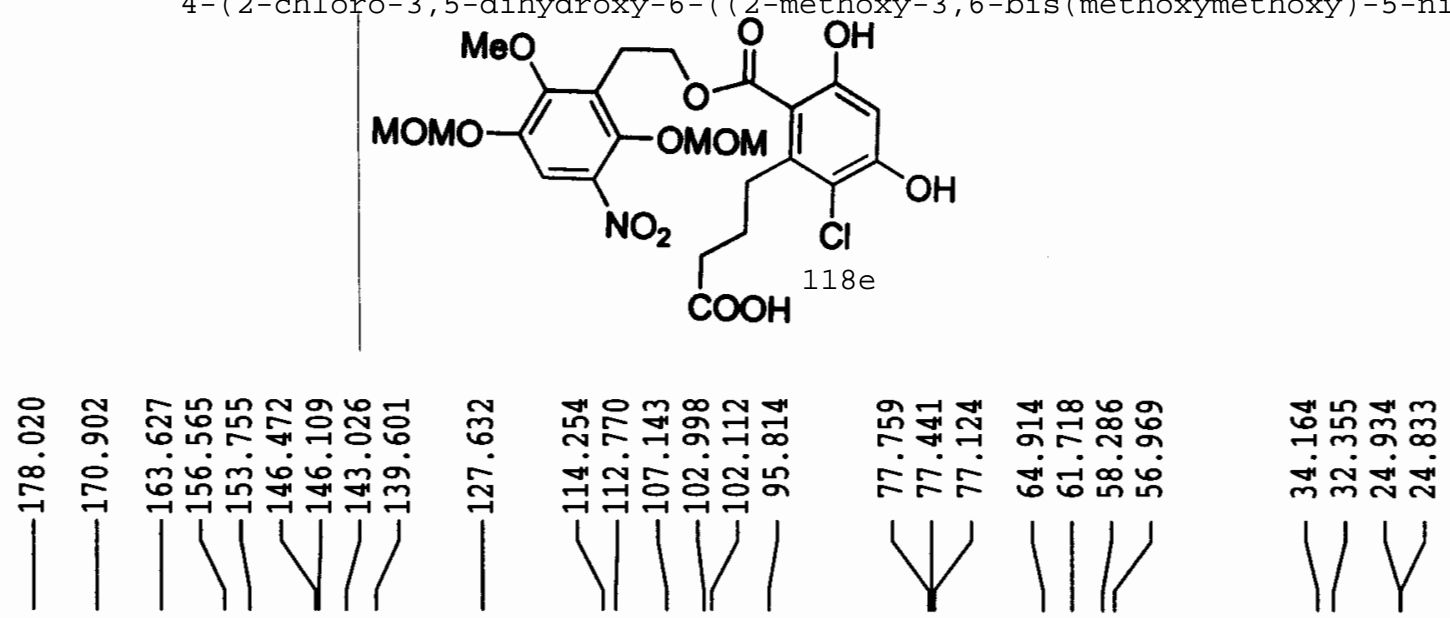

Current Data Parameters

NAME mato504061

PROCro

F2 - Acquisition Parameters

Date_ 20050406

Time $\quad 21.32$

$\begin{array}{lr}\text { INSTRUN } & \operatorname{drx} 400 \\ \text { PROBHD } 5 \mathrm{~mm} \text { OAP } 1 \mathrm{H} / 13\end{array}$

PULPROG 2gog30

$\begin{array}{ll}\text { TD } & 65536 \\ \text { SOLVENT } & \mathrm{CDCl3}\end{array}$

HS

DS

SWH

$\begin{array}{lr}\text { SWH } & 23148.148 \mathrm{~Hz} \\ \text { FIDRES } & 0.353213 \mathrm{~Hz}\end{array}$

505

$\mathrm{AQ} \quad 1.4156276 \mathrm{sec}$

RG $\quad 4597.6$

DN 21.600 usec

DR $\quad 5.50$ usec

TE $\quad 294.2 \mathrm{~K}$

$\quad 0.15000001 \mathrm{sec}$

di1 $\quad 0.0300000 \mathrm{sec}$

DELTA $\quad 0.05000000 \mathrm{sec}$

MCREST $\quad 0.0000000 \mathrm{sec}$

$========$ CHAMNEL $\mathrm{fl}$ =z==:=z=

$\begin{array}{lr}\text { NUC1 } & 13 \mathrm{C} \\ \text { P1 } & 11.25 \text { usec } \\ \text { PL1 } & 2.00 \mathrm{~dB} \\ \text { SF01 } & 100.6232933 \mathrm{dHz}\end{array}$

$======$ CHANVLL $\mathrm{f} 2$ ==:=:== CPDPRG2 waltz16 NUC2 100.00 use $\begin{array}{ll}\text { PCPD2 } & 100.00 \text { use } \\ \text { PL2 } & -4.00 \mathrm{~dB}\end{array}$

PL12

$\mathrm{PL} 13$

$16.58 \mathrm{~d}$

$17.00 \mathrm{~dB}$

SFO2 $\quad 400.1316005$

F2 - Processing parameters

SI 65536

SI $\quad 100.6127290 \mathrm{2HHz}$

$\begin{array}{lr}\text { SP } & 100.6127290 \\ \text { WDW } & \text { ER }\end{array}$

SSB

$\begin{array}{lr}\text { GB } & 1.00 \\ \text { GB } & 0\end{array}$
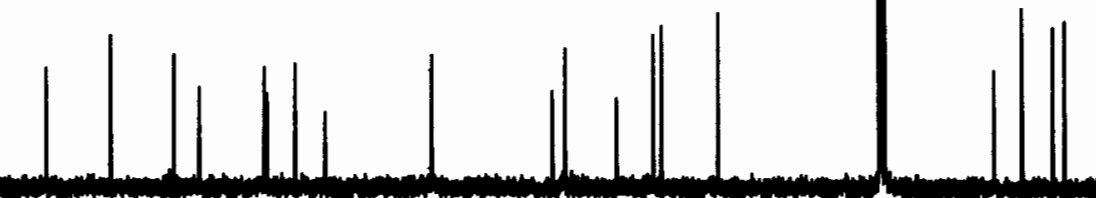

1D NRR plot parameters

$\begin{array}{lr}\mathrm{CX} & 20.00 \mathrm{~cm} \\ \mathrm{CY} & 8.00 \mathrm{~cm}\end{array}$

F1P $215.000 \mathrm{ppm}$

P1 $21631.74 \mathrm{~Hz}$

F2P $\quad-5.000 \mathrm{ppon}$

F2 $\quad-503.06 \mathrm{~Hz}$

$-503.06 \mathrm{~Hz}$

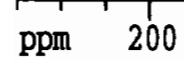

$175 \quad 75,150$

125

100

50

25

Hzor

$1106.73999 \mathrm{~Hz} / \mathrm{cm}$ 


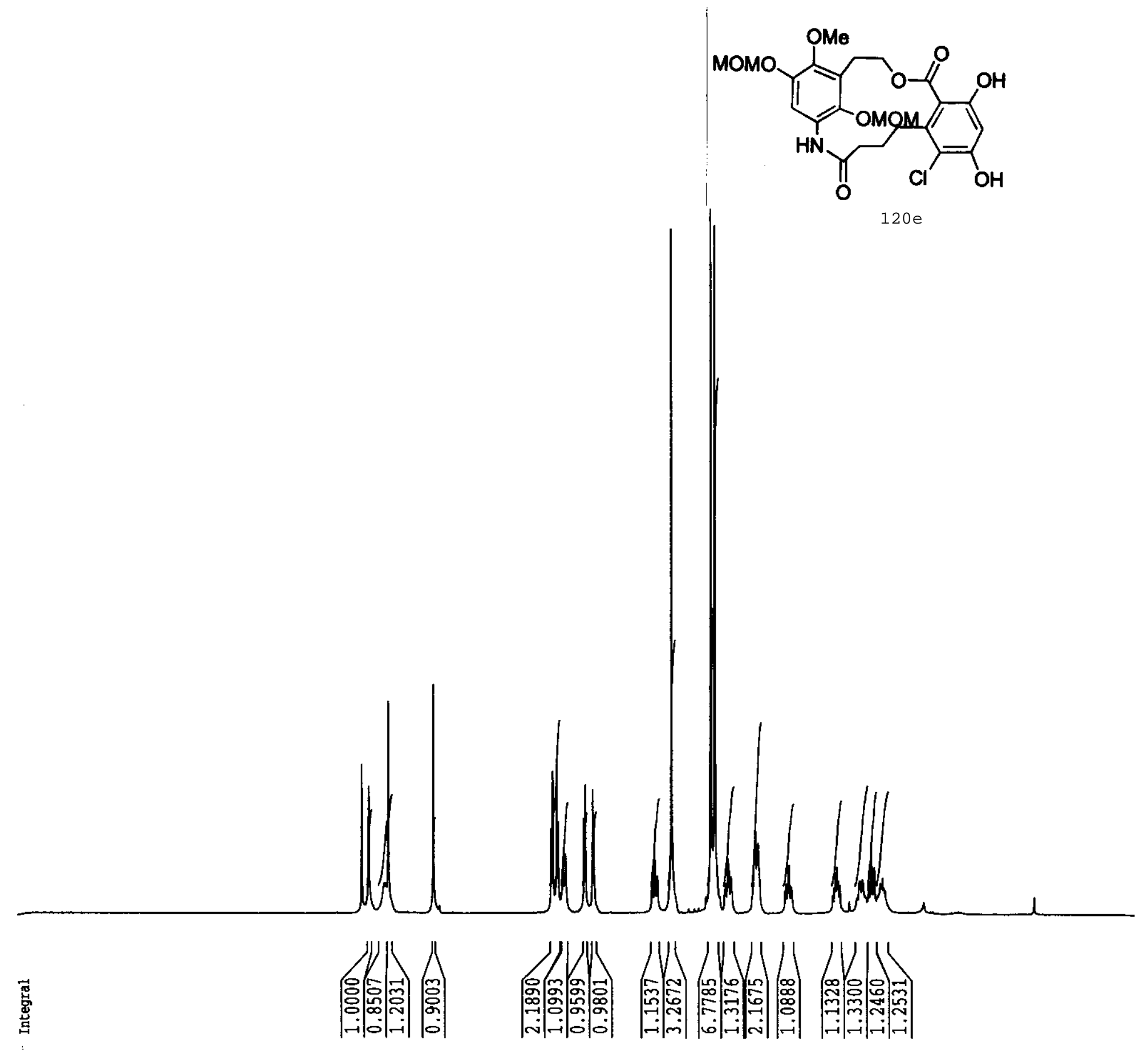

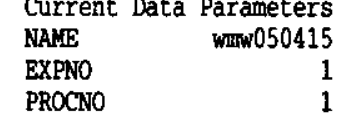

F2 - Acquisition Parameters Date 20050415

Time $\quad 14.54$ INSTRUM drx400 PROBHD 5 QN $1 \mathrm{H} / 13$ PULPROG $\quad$ zg30 TD 32768 SOLVENT $\quad \mathrm{CDCl3}$ NS DS SWH $\quad 4789.272 \mathrm{~Hz}$ FIDRES $\quad 0.146157 \mathrm{~Hz}$ $\mathrm{AQ} \quad 3.4210291 \mathrm{sec}$ RG

$$
203.2
$$

104.400 usec 5.50 usec $293.2 \mathrm{~K}$ $1.00000000 \mathrm{sec}$ $0.00000000 \mathrm{sec}$ $0.01500000 \mathrm{sec}$ $=== \pm=s==$ CHANNEL f1 ======s= $\begin{array}{ll}\text { NUC1 } & 1 \mathrm{H} \\ \text { P1 } & 9.35 \mathrm{usec}\end{array}$ SFO1 $\quad 400.1320007 \mathrm{MHz}$

F2 - Processing parameters SI $\quad 32768$ SF $\quad 400.1300000 \mathrm{MHz}$ SSB EY $\begin{array}{lc}\text { LB } & 0.30 \mathrm{~Hz} \\ \text { GB } & 0\end{array}$ GB 1.00

1D NMR plot parameters

$\begin{array}{lr}\text { CX } & 20.00 \mathrm{~cm} \\ \text { CY } & 12.50 \mathrm{~cm} \\ \text { F1P } & 11.000 \mathrm{ppm} \\ \text { F1 } & 4401.43 \mathrm{~Hz} \\ \text { F2P } & -1.000 \mathrm{ppm} \\ \text { F2 } & -400.13 \mathrm{~Hz} \\ \text { PPHCM } & 0.60000 \mathrm{ppm} / \mathrm{cm} \\ \text { HZCM } & 240.07800 \mathrm{~Hz} / \mathrm{cm}\end{array}$




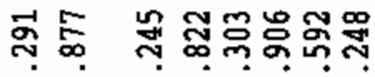

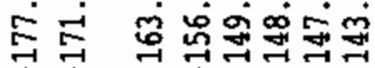

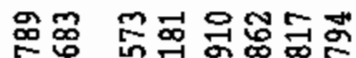

ஸ் $\dot{0} \dot{0} \dot{0}$ $\rightarrow \rightarrow-7$

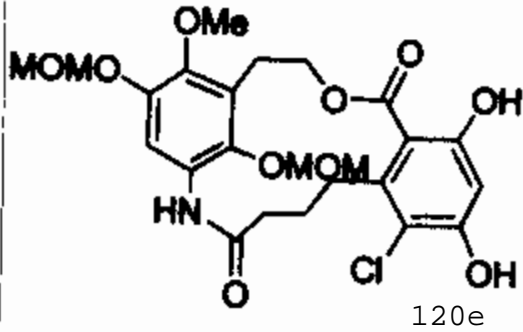

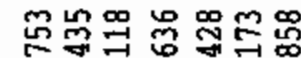

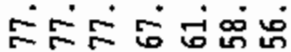

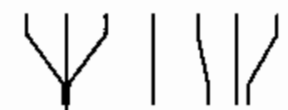

육공궈

ตี่

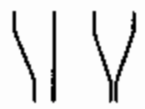
IIIV/

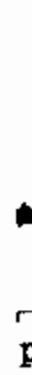

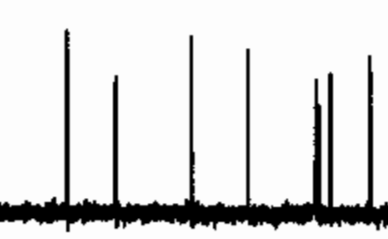

10.? 275

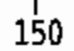

150
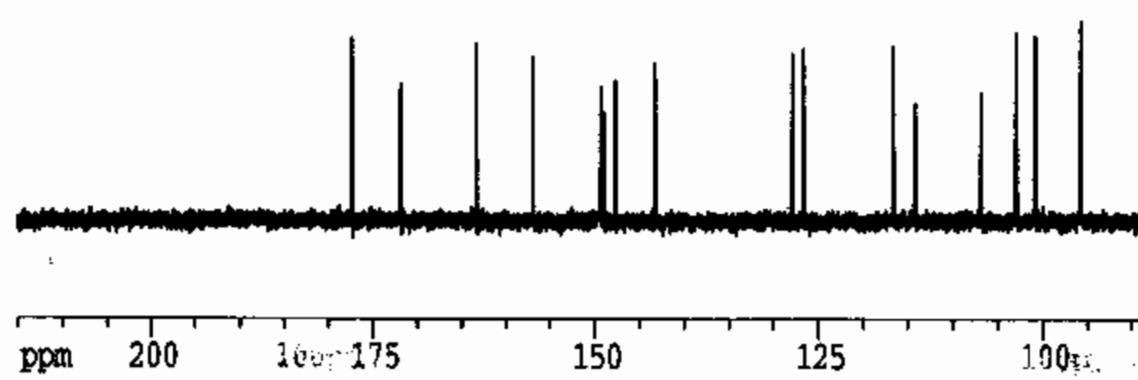

200

Current Data Parameters

PRiocio

$\operatorname{mos} 050415$

P2 - Acquisition Parameters

Date.

Tine

IISTERU

PITP 1H/13

TD

SOLVE:

us

SS

SWIT

PIDRES

RG

DE

IE

TE

a11

DELTA

DELTA

MCWRK

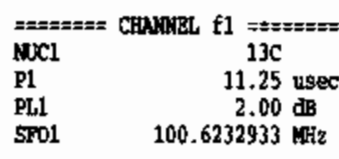

SFO1 $100.6232933 \mathrm{kHz}$

======= CHAYLTा f2 ======" CPDPRG2 waltz16

NOC2 $1 \mathrm{H}$

PCPD2 100.00 usec

$\mathrm{PL2} 2 \quad-4.00 \mathrm{~dB}$

PLI12 $16.58 \mathrm{~dB}$

Pill
SFO2

R2 - Processing paxameters

SI 65536

SP $\quad 100.6227290 \mathrm{vHz}$

in:

SSB

$\stackrel{\mathrm{LB}}{\mathrm{B}}$

EY

$1.00 \mathrm{~Hz}$

0
1.40

10 FiR plot paraneters

co $20.00 \mathrm{co}$

ct $\quad 20.50 \mathrm{~cm}$

F1P $215.000 \mathrm{ppow}$

$7121631.74 \mathrm{~Hz}$

72P $\quad-5.000 \mathrm{ppo}$

PPrCH $\quad \begin{array}{ll}-503.06 \mathrm{~Hz} \\ 11.00000 \mathrm{ppm} / \mathrm{cm}\end{array}$

$\begin{array}{lr}\text { PPACH } & 11.00000 \mathrm{ppm} / \mathrm{cm} \\ \text { HZOI } & 1106.73999 \mathrm{~Hz} / \mathrm{cr}\end{array}$ 

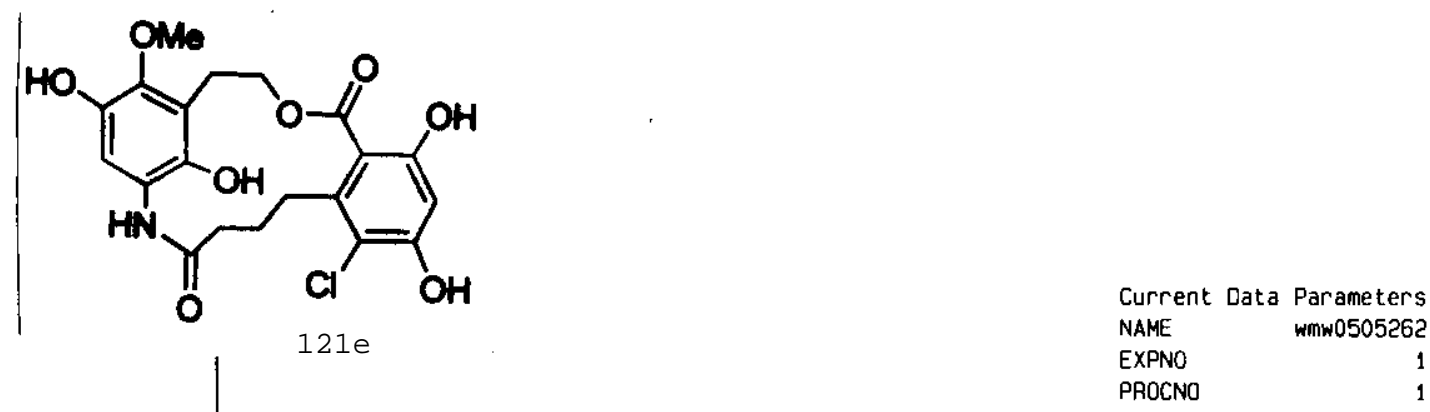

F2 - Acquisition Parameters

Date_ 20050526

Time 16.06

INSTRUM $\mathrm{d} \Gamma \times 400$

PROEHD $5 \mathrm{~mm}$ ONP $1 \mathrm{H} / 13$

PULPROG $\quad 2930$

32768

SOLVEN acefore ECTS

16
2

SWH $\quad 4789.272 \mathrm{~Hz}$

FIDRES $\quad 0.146157 \mathrm{~Hz}$

AQ $\quad 3.4210291 \mathrm{sec}$

AG 287.4

DW $\quad 104.400$ usec

IE

D1 $\quad 1.00000000 \mathrm{sec}$

MCREST $\quad 0.00000000 \mathrm{sec}$

MCWFiK $\quad 0.01500000 \mathrm{sec}$

$\begin{array}{lr}=======\text { CHANNEL } f 1 \quad======= \\ \text { NUC1 } & 1 \mathrm{H} \\ \text { P1 } & 9.35 \mathrm{useC} \\ \text { PL1 } & -4.00 \mathrm{cB} \\ \text { SF01 } & 400.1320007 \mathrm{MHz}\end{array}$

F2 - Processing parameters

$\begin{array}{lc}\text { SI } & 32768 \\ \text { SF } & 400.1300000 \mathrm{MHz}\end{array}$

WOW EM

$\begin{array}{lc}\text { SSB } & 0 \\ \text { LB } & 0.30 \mathrm{~Hz}\end{array}$

$\begin{array}{lr}\text { LB } & 0.30 \\ \text { GB } & 0\end{array}$

PC

1.00

1D NMP plot parameters

CX $20.00 \mathrm{~cm}$

CY $\quad 12.50 \mathrm{~cm}$

F1P $\quad 11.000 \mathrm{ppm}$

F1 $4401.43 \mathrm{~Hz}$

F2P $-1.000 \mathrm{PPm}$

F2 $\quad-400.13 \mathrm{~Hz}$

PPMCM $\quad 0.60000 \mathrm{ppm} / \mathrm{cm}$

$\mathrm{HZCM} \quad 240.07800 \mathrm{~Hz} / \mathrm{cm}$ 

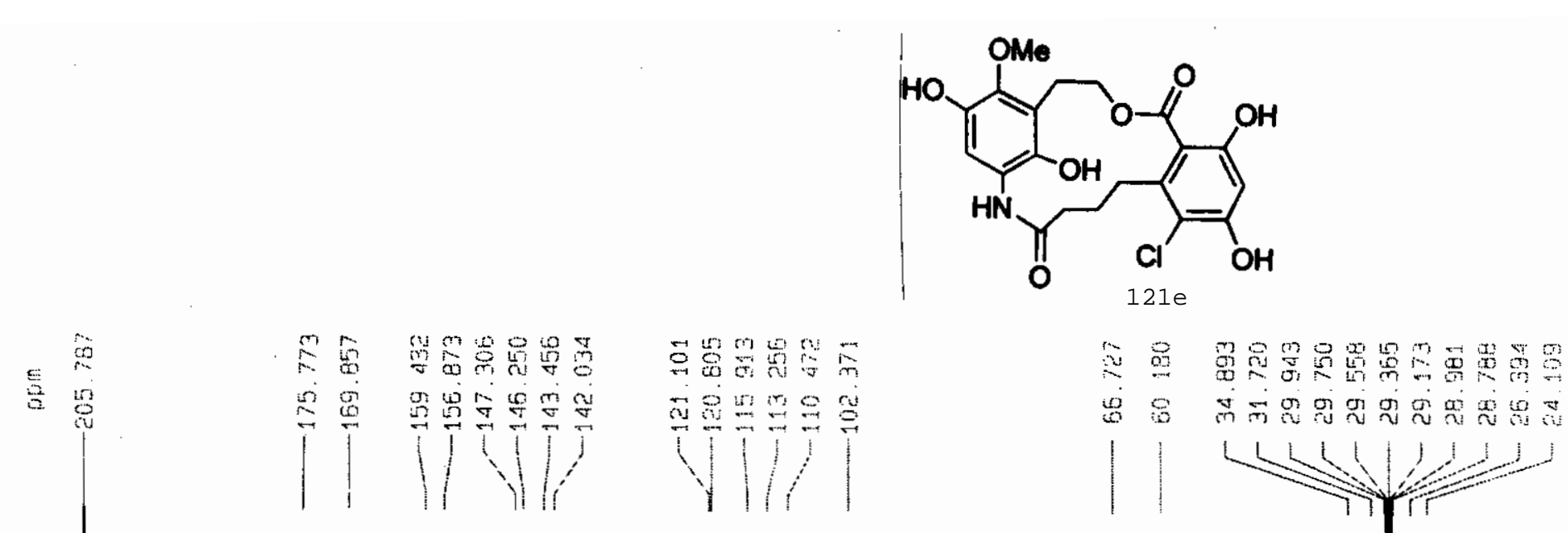

Current Data Parameters

NAME

EXPNO

Pan 05053

F2 - Acquisition Parameters

Date_ 20050526

Time

PROBHO 5 ma anp $1 \mathrm{H} / 13$

PUPAOG 2 ing ong

SOL VENI ace towe

NS

DS

SWH

日73

$23148.148 \mathrm{~Hz}$

FIOAES $0.353213 \mathrm{~Hz}$

$1.4156276 \mathrm{sec}$

5792.6

OW 21.600 usec

TE $\quad 5.50$ us

$01 \quad 0.15000001 \mathrm{sec}$

$011 \quad 0.03000000 \mathrm{sec}$

DELTA $0.05000000 \mathrm{seC}$

MCAEST $0.00000000 \mathrm{sec}$

MCHPa 0.01500000 sec

$== \pm= \pm x=$ CHANNEL. $f 1==x a x=x=$
NUC1 1 I3C

$\begin{array}{ll}\text { NuC1 } & 13 \mathrm{C} \\ \mathrm{Pl} & 11.25 \text { usec }\end{array}$

PL1 $\quad 2.00 \mathrm{~dB}$

$x== \pm=m=$ CHANNEL f $2 x=3=x=$

CPDPRG2 maltz16

NalC2 $1 \mathrm{H}$

$\begin{array}{lr}\mathrm{PCPO2} & 100.00 \text { usec } \\ \mathrm{PL2} & -4.00 \mathrm{dg}\end{array}$

$16.58 \mathrm{dg}$

PL13 $17.00 \mathrm{~dB}$

SF02 400.1356005

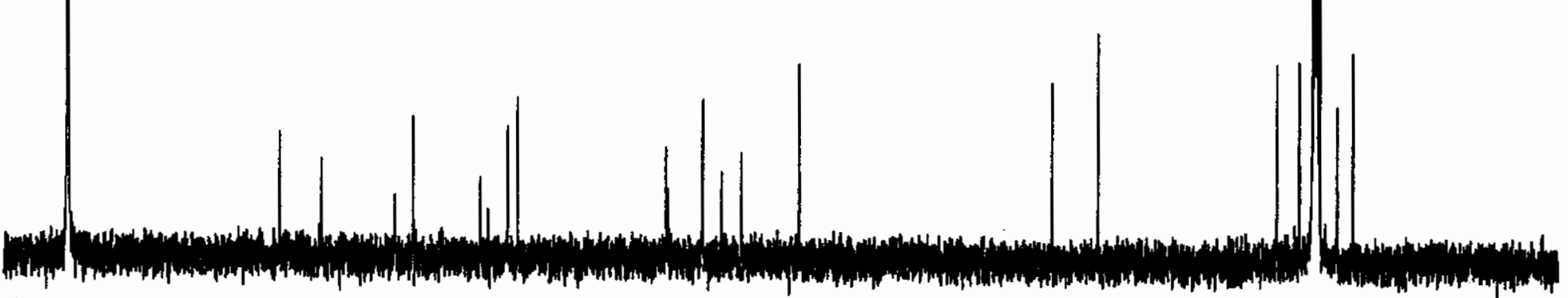

F2 - Processing parameters

St 65536

SF $\quad 100.6127290 \mathrm{WHZ}$

NOW EM

LB $\quad 1.00 \mathrm{~Hz}$

PC

$1.00 \mathrm{~Hz}$
0
1.40

10 plot parameters

CX

CY $\quad 20.00 \mathrm{~cm}$

FIP $\quad 215.000 \mathrm{ppm}$

F1 $21631.74 \mathrm{~Hz}$

$\begin{array}{lr}\text { Fap } & 21631.74 \mathrm{K2} \\ \text { F2 } & -5.000 \mathrm{ppm}\end{array}$

$\begin{array}{ll}\text { F2 } & -5.000 \mathrm{ppm} \\ \text { F2 } & -503.06 \mathrm{~Hz}\end{array}$

PPACH $\quad \begin{aligned} & -503.06 \mathrm{~Hz} \\ & 11.00000 \mathrm{ppm} / \mathrm{cm}\end{aligned}$ 


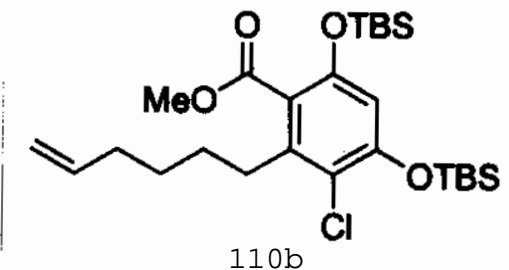

$\begin{array}{lr}\text { Current Data } & \text { Parameters } \\ \text { NANE } & \text { rCIV-93a } \\ \text { EXPNO } & 1 \\ \text { PROCNO } & 1\end{array}$

F2 - Acquisition Parameters

Date_ 20050521

$\begin{array}{lr}\text { Time } & 17.03\end{array}$

INSTRUM drx400

PROBHD 5 IIII QNP 1H/13

PULPROG zg30

$\begin{array}{ll}\text { TD } & 32768\end{array}$

SOLVENT $\quad \mathrm{CDCl3}$

NS

SWH

$\mathrm{AQ}$

RG

DW

TE

D1

MCREST

YCREST NUC1

P1

PL1

SFO1

4789.272

$4789.272 \mathrm{~Hz}$
$0.146157 \mathrm{~Hz}$

$3.4210291 \mathrm{sec}$ 64

104.400 usec 5.50 usec $293.2 \mathrm{~K}$

$1.00000000 \mathrm{sec}$

$0.00000000 \mathrm{sec}$

$0.01500000 \mathrm{sec}$

F2 - Processing paraneters

$\begin{array}{lc}\text { SI } & 32768 \\ \text { SF } & 400.1300000 \mathrm{MHz} \\ \text { WDW } & \text { EM } \\ \text { SSB } & 0 \\ \text { LB } & 0.30 \mathrm{~Hz} \\ \text { GB } & 0 \\ \text { PC } & 1.00\end{array}$

1D MNR plot parameters

CX $20.00 \mathrm{~cm}$

CY $20.00 \mathrm{~cm}$

F1P $\quad 10.985 \mathrm{ppm}$

F1 $\quad 4395.28 \mathrm{~Hz}$

F2P $\quad-0.985$ ppm

F2 $\quad-393.99 \mathrm{~Hz}$

PPMCM $\quad 0.59846 \mathrm{ppm} / \mathrm{c}$

HZCM $\quad 239.46359 \mathrm{~Hz} / \mathrm{cm}$ 


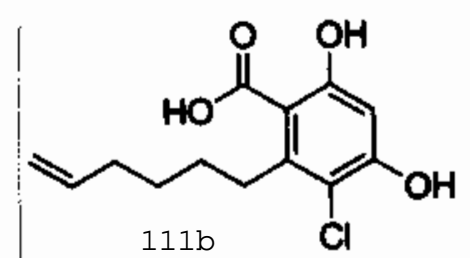

3-Chloro-2- (5-hexenyl)-4,6-dihydroxybenzoic acid

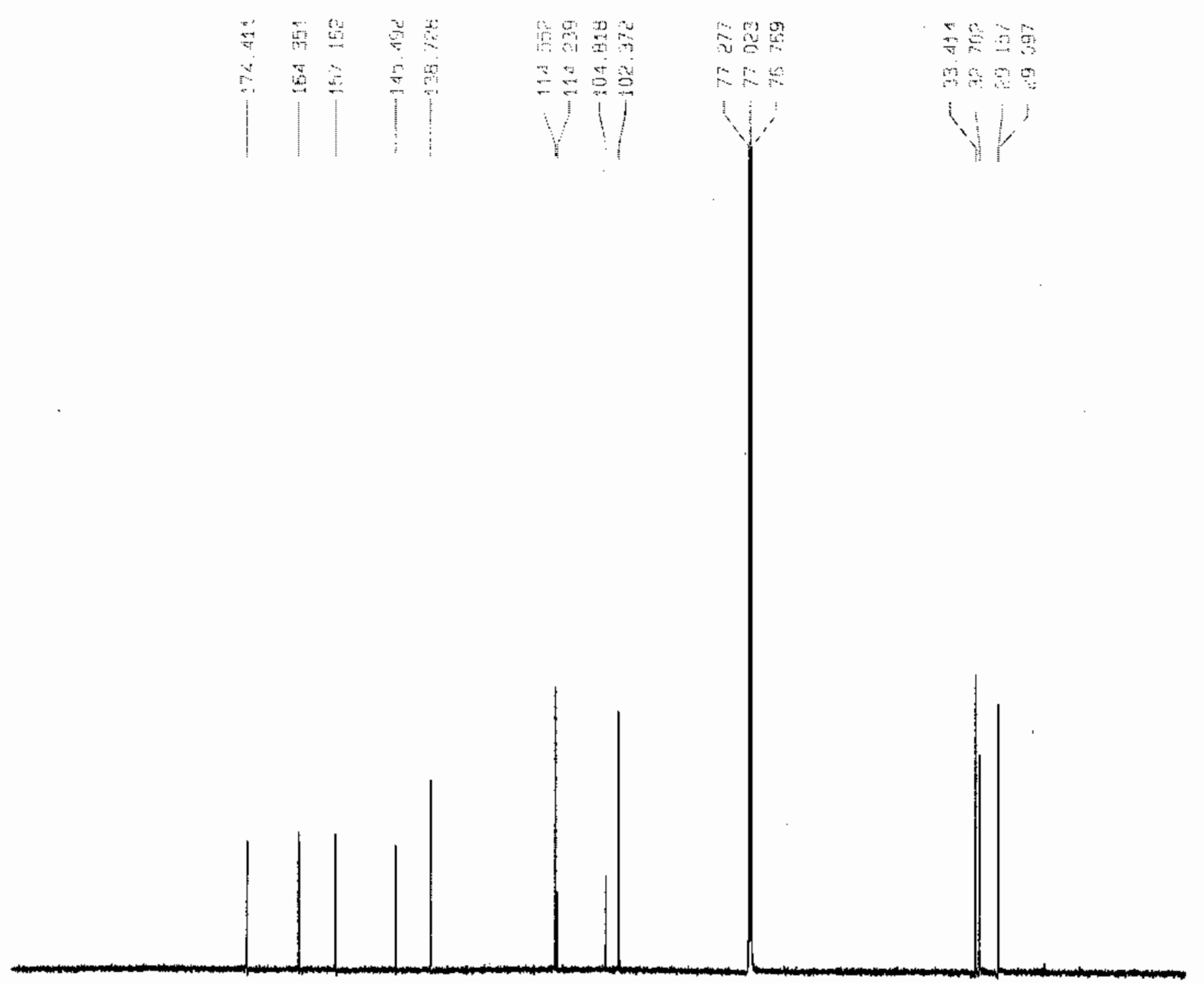

Current Data Parameters

NAME

EXPNO

F2 - Acquisitian Parameters

Oate_ 20050809

Iime $\quad 10.13$

PPOBHD 5 界 CPOA 130

PULPAOG 2 gag 30

ID 2 gpg 30

SOL VENT T

$\begin{array}{lr}\text { NS } & \text { COC } 13 \\ & 579\end{array}$

SWH $\quad 31446.541 \mathrm{~Hz}$

$\begin{array}{lr}\text { SWH } & 31446.541 \mathrm{~Hz} \\ \text { FIDRES } & 0.479836 \mathrm{~Hz}\end{array}$

AQ $\quad 1.0420724 \mathrm{sec}$

PG $\quad$ B192

OW $\$ 5.900$ บ5e

OE $\quad 5.00$ use

TE $\quad 0.1500000 \%$

0.1 $\quad 0.15000001 \mathrm{sec}$

d11 $0.03000000 \mathrm{sec}$

DELTA $\quad 0.05000000 \mathrm{sec}$

MCWFK $\quad 0.01500000 \mathrm{sec}$

$=====x=$ CHANNEL

$\begin{array}{ll}\text { NuC1 } & 13 \mathrm{C} \\ \rho_{1} & 10.00 \text { usec }\end{array}$

PL1 1 -4.90

SFO1 $125.7697360 \mathrm{HHz}$

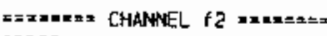

CPDPAG

NuC2

PCPD?

PL2

PL12

PL13

maitzi6

$1 \mathrm{H}$

Bo. 00 usec

-5.00 de

9.54 de

$500.1325000 \mathrm{WHZ}$

2 - Processing parameters

SI 65536

SF $\quad 125.7577290$ H.12

NSW

L8 $1.00 \mathrm{~Hz}$

GS

10 NMF olot parameters

$\begin{array}{ll}\mathrm{CX} & 20.00 \mathrm{~cm} \\ \mathrm{CY} & 30.00 \mathrm{~cm}\end{array}$

F1P 242.517 Dom

F1 30498.43 Hz

$\begin{array}{ll}\mathrm{FZP} & -7.539 \mathrm{DPM} \\ \mathrm{F} 2 & -948.11 \mathrm{~Hz}\end{array}$

PPMCM $12.50282 \mathrm{pgm} / \mathrm{cm}$

$\begin{array}{lr}12.50282 \mathrm{p \rho m} / \mathrm{cm} \\ \mathrm{HZCM} & 1572.32590 \mathrm{~Hz} / \mathrm{cm}\end{array}$ 


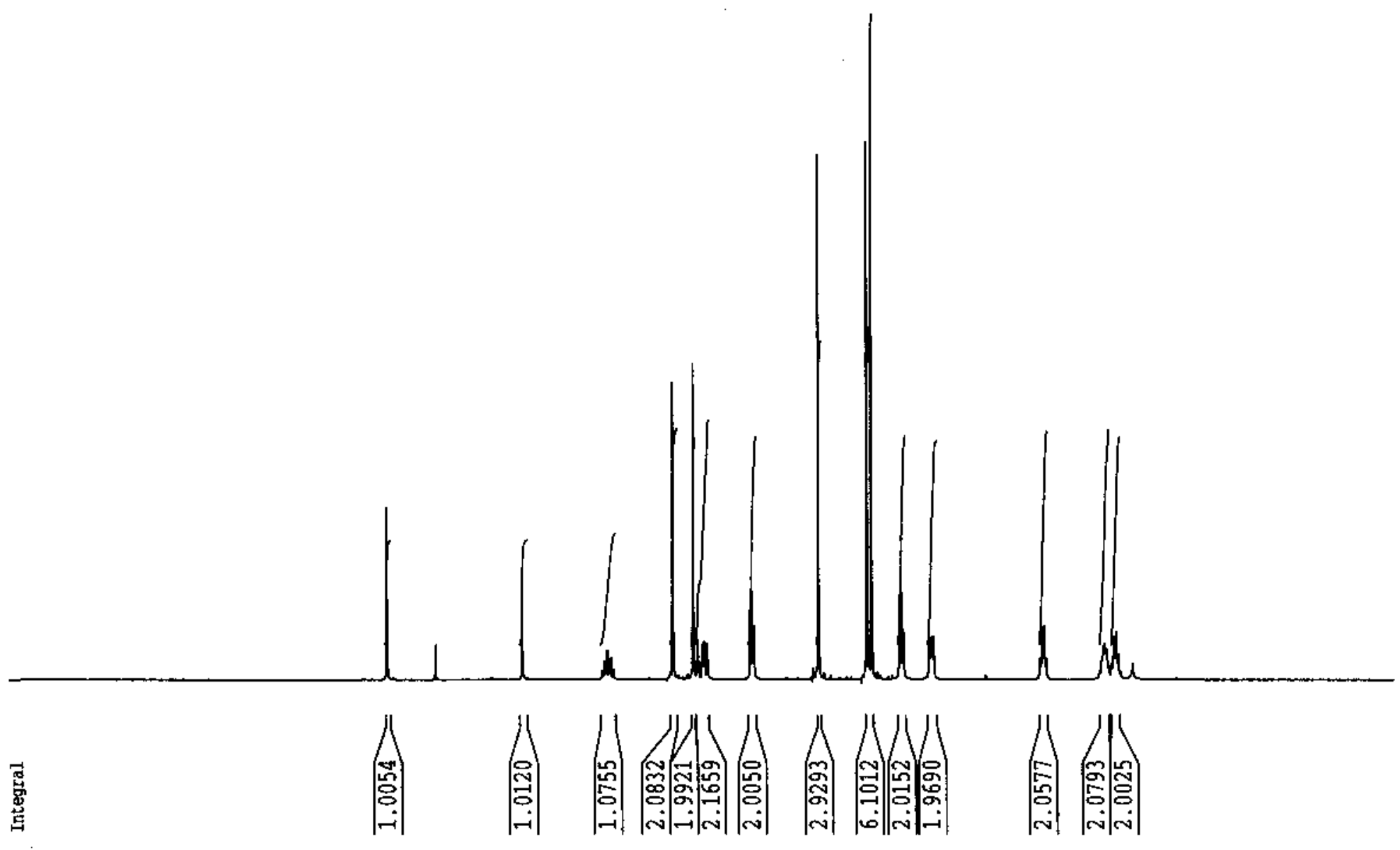

\section{Current Data Parameters NAKE \\ EXPNo waw0505041 \\ PROCNO}

F2 - Acquisition Parameters Date. 20050504

Time $\quad 14.46$

IFSTRTM drx400

PROBHD $5 \mathrm{~mm}$ QNP $1 \mathrm{H} / 13$

PULPROG $\quad \mathrm{gg} 30$

$\begin{array}{ll}\text { TD } & 32768 \\ \text { SOLVENT } & \text { CDC13 }\end{array}$

NS 16

DS

FIDRES

$\mathrm{AQ}$

$4789.272 \mathrm{~Hz}$

$0.146157 \mathrm{~Hz}$

$3.4210291 \mathrm{sec}$ 64

104.400 usec

$\begin{array}{lr}\mathrm{DE} & 5.50 \mathrm{usec} \\ \mathrm{TE} & 293.2 \mathrm{~K} \\ \mathrm{D} 1 & 1.00000000 \mathrm{sec}\end{array}$

$\begin{array}{lr}\mathrm{DE} & 5.50 \mathrm{usec} \\ \mathrm{TE} & 293.2 \mathrm{~K} \\ \mathrm{D} 1 & 1.00000000 \mathrm{sec}\end{array}$

D1 $\quad 0.00000000 \mathrm{sec}$

$\begin{array}{ll}\text { MCRRR } & 0.01500000 \mathrm{sec}\end{array}$

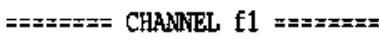
NUC1 $1 \mathrm{H}$

9.35 usec $\begin{array}{lr}\text { PLI } & -4.00 \mathrm{~dB} \\ \text { SF01 } & 400.1320007 \mathrm{MHz}\end{array}$

F2 - Processing parameters

$\begin{array}{lc}\text { SI } & 32768 \\ \text { SF } & 400.1300000 \mathrm{kHz} \\ \text { WDW } & \text { EM } \\ \text { SSB } & 0 \\ \text { LB } & 0.30 \mathrm{~Hz} \\ \text { GB } & 0 \\ \text { PC } & 1.00\end{array}$

1D MAR plot parameters

$\begin{array}{lc}\text { CX } & 20.00 \mathrm{~cm} \\ \text { CY } & 8.00 \mathrm{~cm} \\ \text { F1P } & 11.000 \mathrm{ppm} \\ \text { F1 } & 4401.43 \mathrm{bz} \\ \text { F2P } & -1.000 \mathrm{ppm} \\ \text { F2 } & -400.13 \mathrm{~Hz} \\ \text { PPHCM } & 0.60000 \mathrm{ppm} / \mathrm{cm} \\ \text { HZCH } & 240.07800 \mathrm{~Hz} / \mathrm{cm}\end{array}$



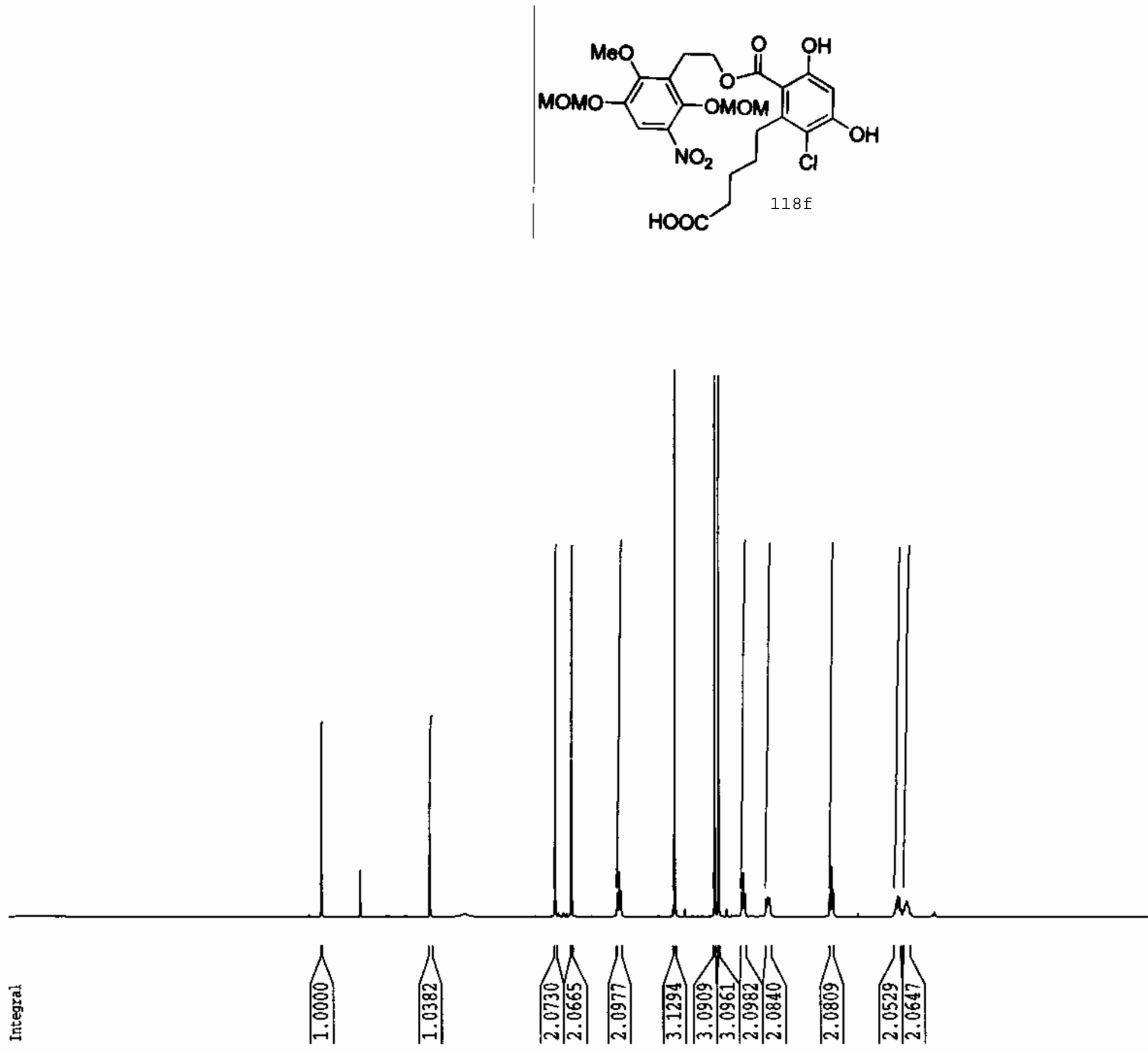

$$
\text { ppo }
$$

10

10

8

6

4

$2 \quad . . \because$

$\begin{array}{lr}\text { Current Data } & \text { Parameters } \\ \text { NAME } & \text { wmw0505093 } \\ \text { EXPNO } & 1 \\ \text { PROCNO } & 1\end{array}$

Date Acquisition Parameters

Time $\quad 19.07$

INSTRUM drx 400

PROBHD $5 \mathrm{~mm}$ QNP $1 \mathrm{H} / 13$

PULPROG 2930

TD 32768

SOLVENT $\quad$ CDCl3

NS

DS

Sint

FIDRES

16
2

$4789.272 \mathrm{~Hz}$

$0.146157 \mathrm{~Hz}$

$3.4210291 \mathrm{sec}$

$\begin{array}{lc}R G & 181 \\ D W & 104.400 \mathrm{usec}\end{array}$

DE $\quad 5.50$ usec

TE $\quad 294.2 \mathrm{~K}$

D1 $\quad 1.00000000 \mathrm{sec}$

VCRBST $\quad 0.00000000 \mathrm{sec}$

MCWRK $\quad 0.01500000 \mathrm{sec}$

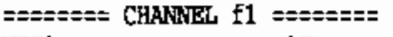

NUC1

P1

1H

9.35 usec

SFO1

$400.1320007 \mathrm{MHz}$

F2 - Processing parameters

SI $\quad 32768$

SF $\quad 400.1300000 \mathrm{MHz}$

$\begin{array}{ll}\text { WDW } & \text { EN } \\ \text { SSB } & 0\end{array}$

LB $\quad 0.30 \mathrm{~Hz}$

PC $\quad 1.00$

1D MR plot parameters

$\begin{array}{lc}\text { CX } & 20.00 \mathrm{~cm} \\ \text { CY } & 6.00 \mathrm{~cm} \\ \text { F1P } & 11.000 \mathrm{ppm} \\ \text { F1 } & 4401.43 \mathrm{~Hz} \\ \text { F2P } & -1.000 \mathrm{ppm} \\ \text { F2 } & -400.13 \mathrm{~Hz} \\ \text { PFCK } & 0.60000 \mathrm{ppm} / \mathrm{c} \\ \text { HZCY } & 240.07800 \mathrm{~Hz} / \mathrm{cm}\end{array}$


5-(2-chloro-3,5-dihydroxy-6-((2-methpxy-3,6-bis (methoxymethoxy)-5-nitrophenethoxy) carbonyl) phenyl) pentanoic acid
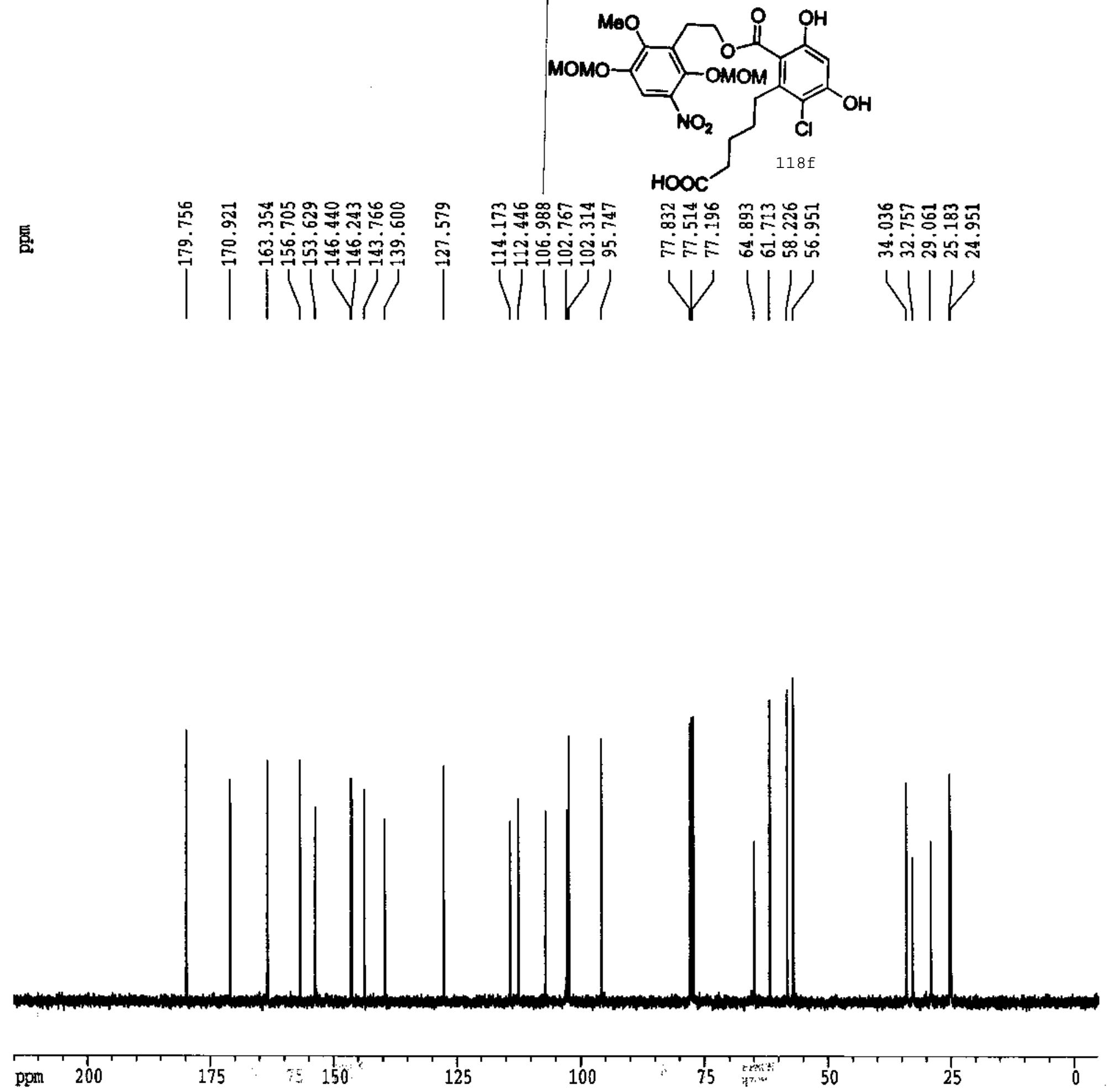


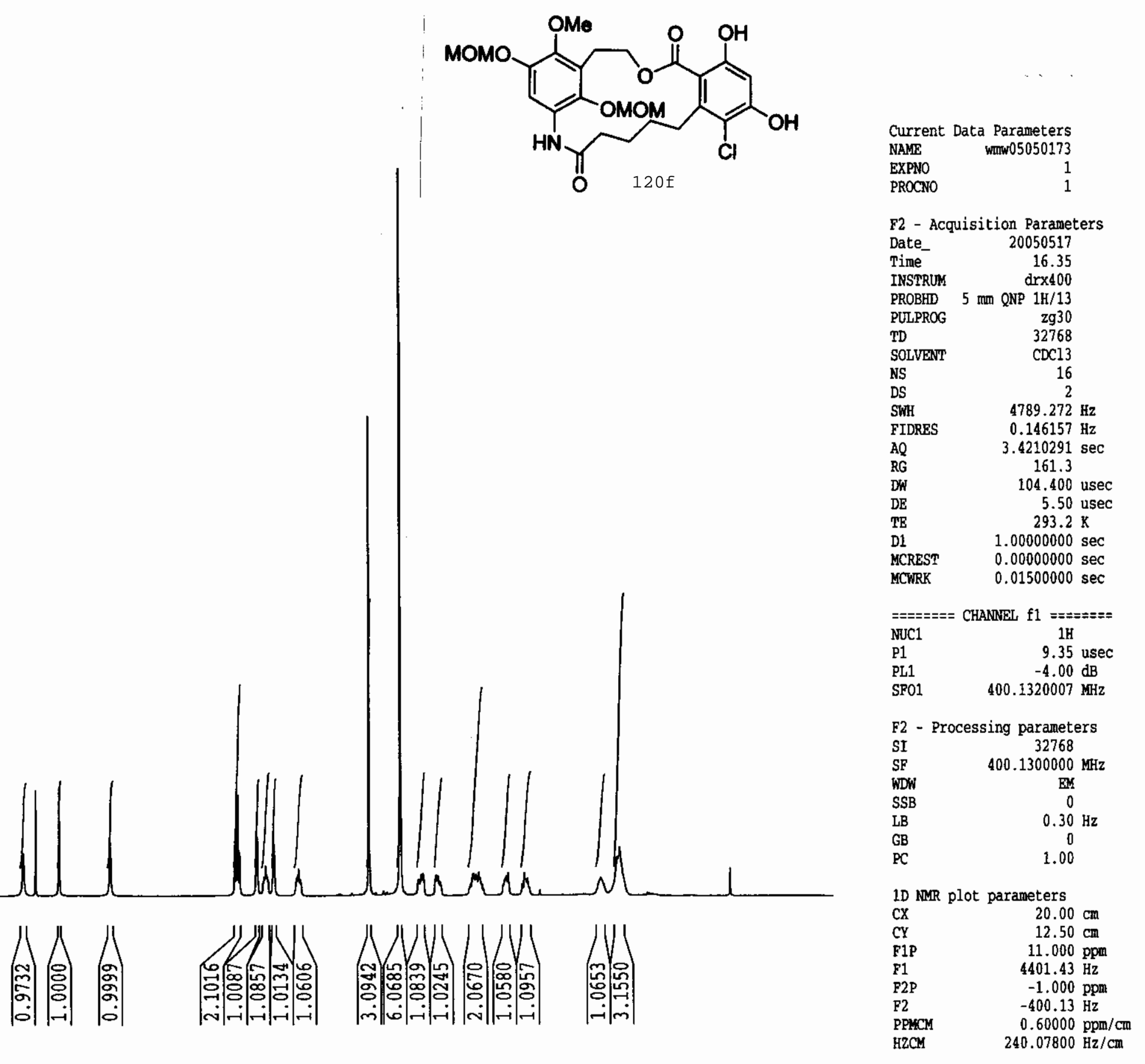

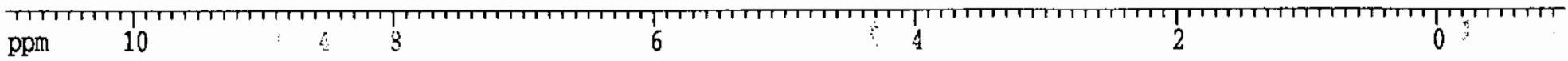




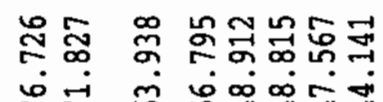

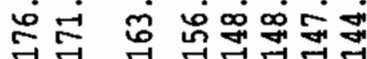

స్ㅎㅁㅇ 웜역

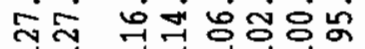

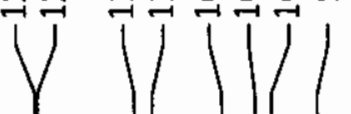

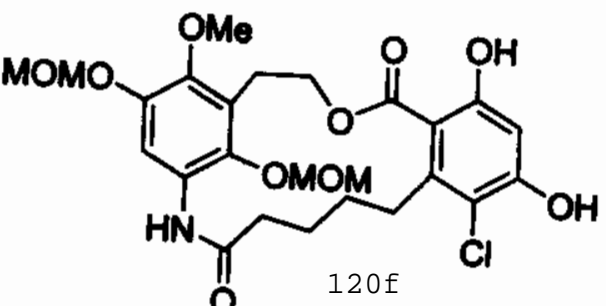

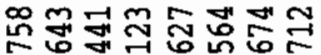
सहE⿱

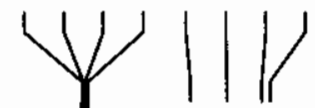

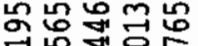

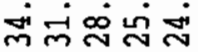

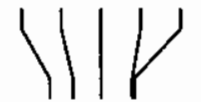
$\mathrm{pp}$

Current Data Parameters Narte

EXPNO

PROCAO

- Acquisition Paramete

Date_

Time

INSTRA

PULPROG

TD

SOLVEart

NS

DS

STH

FIDR

AQ

RG

$\mathrm{DW}$
$\mathrm{DR}$
$\mathrm{TE}$

D1

D1

DEITA

DELITA

MCWRK

$==$

NuC

P1

PL1

SFO1

$========$ CHANNELL

CPDPRG2

NUC2

PCPD2

PL12

PL13

$\mathrm{SF}_{\mathrm{O}} \mathrm{2}$

$1316005 \mathrm{BBz}$

P2 - Processing parameters

SI 65536

SF $\quad 100.6127290 \mathrm{MHz}$

WDW

SSB

LB

GB

5y

$1.00 \mathrm{~Hz}$

1.40

1D MyR plot parameters

CX

CY
F1P
F1

F1

$\mathrm{P} 2$

PPACIY

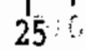

HZOY
$20.00 \mathrm{~cm}$ $25.00 \mathrm{~cm}$ $215.000 \mathrm{ppm}$ $21631.74 \mathrm{~Hz}$ $-5.000 \mathrm{ppm}$
$-503.06 \mathrm{~Hz}$ $11.00000 \mathrm{ppm} / \mathrm{cm}$ $1106.73999 \mathrm{~Hz} / \mathrm{cm}$ 
Desmethyl pentenyl Radanamycin quinone

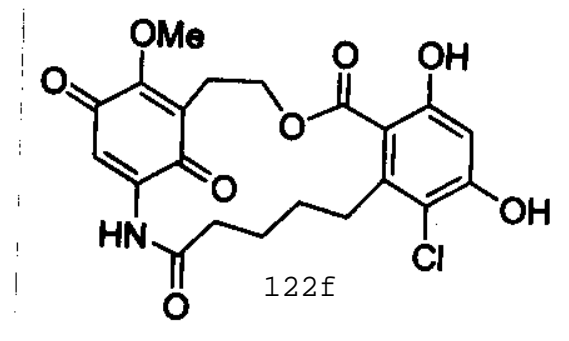

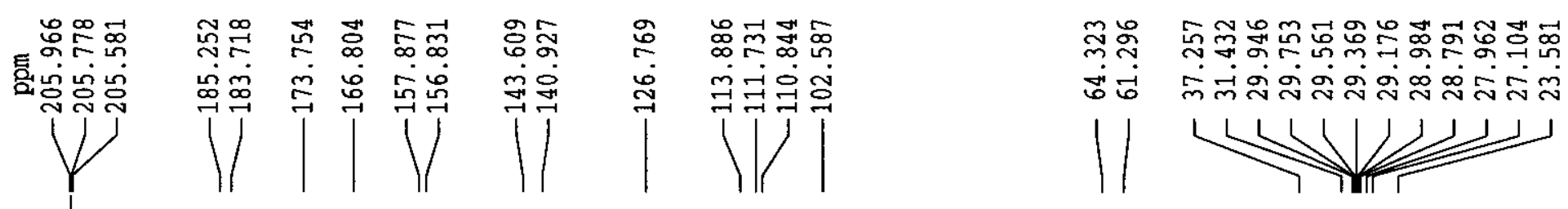

Current Data Parameters

EXPNO

PROCNO

F2 - Acquisition Parameters

Date_ 20050602

$\begin{array}{lr}\text { Time } & 17.04 \\ \text { INSTRUM } & \text { drx400 }\end{array}$

PROBHD 5 InI ONP $1 \mathrm{H} / 13$

PULPROG zgpg30

SOLVENT'

NS

DS

Sin

FIDRE

AQ

DW

DE

TE

D1

DELTA

MCRES

MCWRK

f1 ==="====

P1

PL1

SF01

11.25 usec $2.00 \mathrm{~dB}$

$100.6232933 \mathrm{kHz}$

:=::=:=: CHANARL f2 =s:=:=:= CPDPRG 2

NUC2

PCPD2

PL?

PL12

PL13 waltz16

100.00 usec $-4.00 \mathrm{~dB}$ $16.58 \mathrm{~dB}$ $17.00 \mathrm{~dB}$

$400.1316005 \mathrm{MHz}$

F2 - processing parameters

SI $\quad 65536$

SF $\quad 100.6127290 \mathrm{kHz}$

WDW

LB

GB

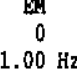

$1.00 \mathrm{~Hz}$

0
1.40

1D NMR plot parameters

CX

FIP

F1

F2P

F2

PPMC

$\mathrm{HZCM}$ $20.00 \mathrm{~cm}$
$12.50 \mathrm{~cm}$
$215.000 \mathrm{ppm}$
$21631.74 \mathrm{mz}$
$-5.000 \mathrm{ppm}$
$-503.06 \mathrm{~Hz}$
$11.00000 \mathrm{ppm} / \mathrm{c}$
$1106.73999 \mathrm{~Hz} / \mathrm{cm}$ 


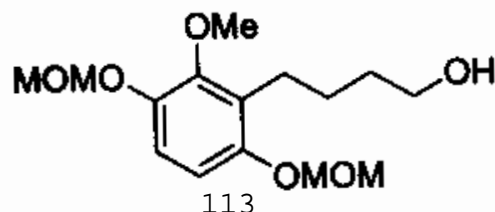

113

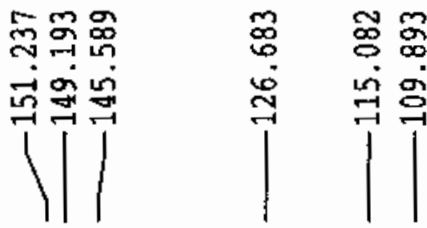
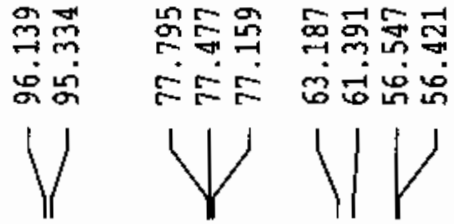

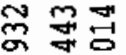

ஸ்
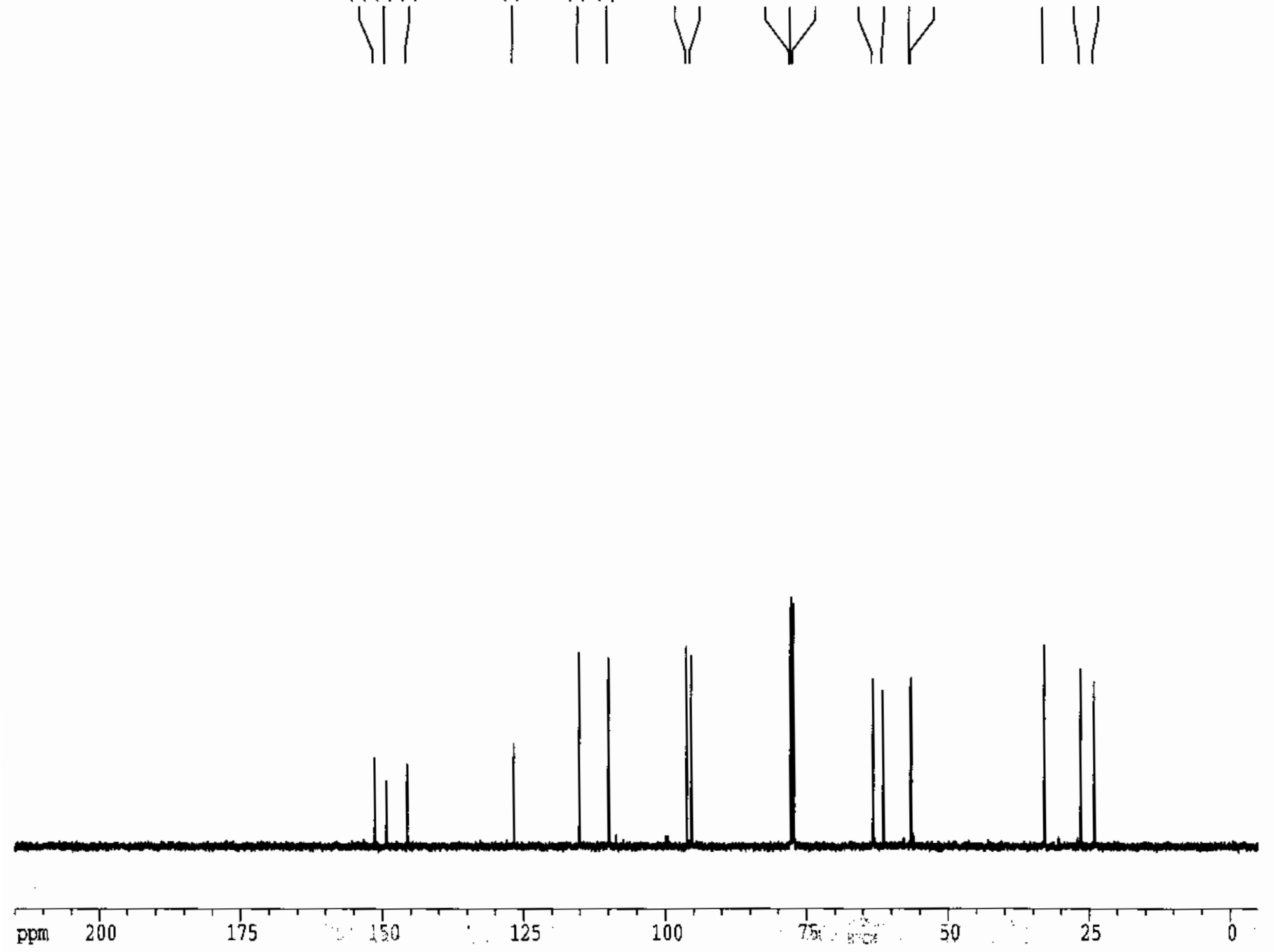

\section{pp}

175

150

125

50

Current Data Parameters

HWE

EXPNO

PROCNO mu1013

20051013

Time

18.33
$d r \times 100$

PROBHD 5 man QNP $1 H / 13$

PULPROC

2 gpg 30
65536

SOLVRT

NS

DS

SWI

PIDRES

$A Q$

$R G$

DE

TE

D1

d11

DRTA

MCREST

MTRK

=:=:="= CHANREL

NuC1

P1

PL1

CDC13

287

$23148.148 \mathrm{~Hz}$

$0.353213 \mathrm{~Hz}$

$.4156276 \mathrm{sec}$

4096

21.600 uses
5.50 use $294.2 \mathrm{R}$ $0.15000001 \mathrm{sec}$ $0.03000000 \mathrm{sec}$ $0.05000000 \mathrm{sec}$ 0.00000000 sec $0.01500000 \mathrm{sec}$

$$
13 \mathrm{C}
$$
$2.00 \mathrm{~dB}$ CPDPRe waltz16

NUC2

PCPO2

PL2

PL12

PL13

100.00 use

$-4.00 \mathrm{~dB}$
$16.58 \mathrm{~dB}$

$17.00 \mathrm{~dB}$

F2 - Processing parameters

SI 65536

SF $\quad 100.6127290 \mathrm{kHz}$

WDW

SSB

$\mathrm{LB}$

$\mathrm{GB}$

$$
\text { ax }
$$

$1.00 \mathrm{~Hz}$

1.

1D NIR plot parameters

CX $20.00 \mathrm{~cm}$

CX

FIP

B1

F2P

srax

Prex? $15.000 \mathrm{pps}$ $21631.74 \mathrm{~Hz}$ $-5.000 \mathrm{pgem}$
$-503.06 \mathrm{~Hz}$

$11.00009 \mathrm{ppon}$ (1) $6.73999 \mathrm{~Hz}$ 


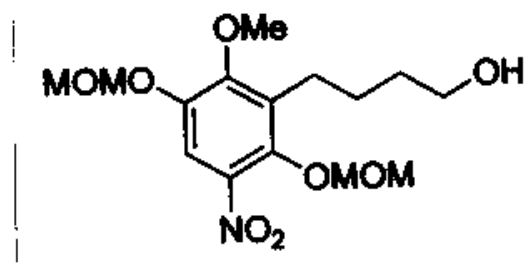

$\begin{array}{lr}\text { Current Data Parameters } \\ \text { NAKG } & \text { wmw12042 } \\ \text { EXPNO } & 1 \\ \text { PROCNO } & 1\end{array}$

114

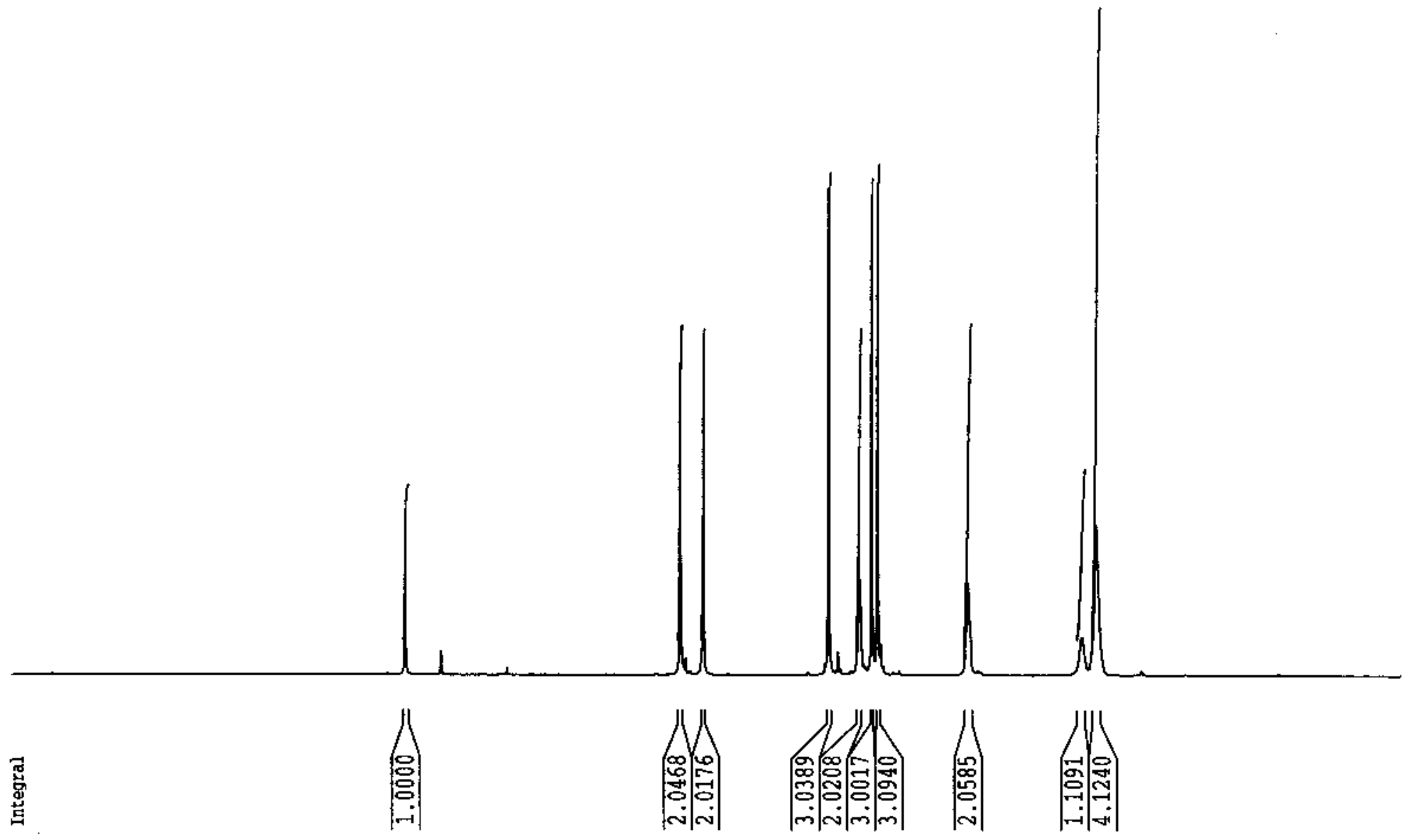




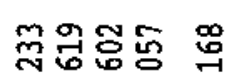
象象象离 |

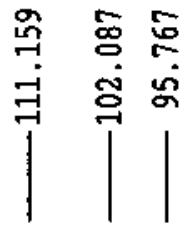<smiles>COc1cc([N+](=O)[O-])c(OC)c(CCCCCO)c1OC</smiles>

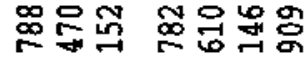 \\ 咅 总品

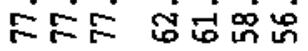 \\ mi் 똥

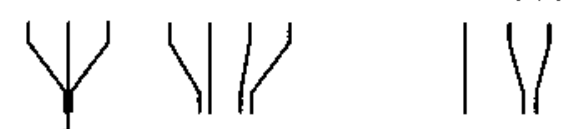

Current Data Parameters

PROCINO

F2 - Acquisition Paraneters $\begin{array}{lr}\text { Date_ } & 20051205 \\ \text { Time } & 11.08\end{array}$ TisTrior drx400 PROABD 5 ma OHP $1 \mathrm{H} / 13$ PULPROG 5 mu TD

SOLVRTI

ISS

DS

EIDR

X?

RG

DW

DE

TB

d11

DELTh

MCREST

ECWRR

65536
$\mathrm{CDC} 13$

415
43148.148

$23148.148 \mathrm{~Hz}$

$1.4156276 \mathrm{sec}$

3649.1

$21.600 \mathrm{usec}$ 5.50 usec $295.2 \mathrm{~K}$ $0.15000001 \mathrm{sec}$ $0.03000000 \mathrm{sec}$ $0.05000000 \mathrm{sec}$ $0.05000000 \mathrm{sec}$ $0.00000000 \mathrm{sec}$ $0.01500000 \mathrm{sec}$

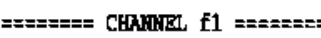

$\begin{array}{lr}\text { HWC1 } & 13 \mathrm{C} \\ \text { P1 } & 11.25 \mathrm{usec} \\ \text { PL1 } & 2.00 \mathrm{~dB} \\ \text { SFO1 } & 100.6232933 \mathrm{ut2}\end{array}$

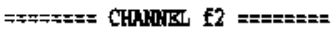
CPDPRG2 Waltz16

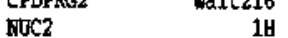

PCPD2 100.00 usec

PL2 $\quad-4.00$ di

$\begin{array}{ll}\text { PL2 } & -4.00 \mathrm{dP} \\ \text { PL12 } & 16.58 \mathrm{~dB}\end{array}$

PL12 $16.58 \mathrm{~dB}$

$\begin{array}{ll}\text { PL13 } & 17.00 \mathrm{~dB} \\ \text { SF02 } & 400.1316005 \mathrm{lPR}\end{array}$

F2 - Processing parameters

SI 65536

SF $\quad 100.5127290 \mathrm{WHz}$

NIN

LSB

GB

B:

$1.00 \mathrm{~Hz}$

0
1.40

1D MIR plot paranetera

1D MUR plot paraneters

\begin{tabular}{|c|c|}
\hline $\begin{array}{l}\text { X } \\
C Y \\
\text { P1P } \\
\text { F1 } \\
\text { P2P } \\
\text { P2 } \\
\text { PPACY } \\
\mathrm{HzO}\end{array}$ & $\begin{array}{c}20.00 \mathrm{~cm} \\
12.50 \mathrm{~cm} \\
215.000 \mathrm{pm} \\
21631.74 \mathrm{kz} \\
-5.000 \mathrm{ppm} \\
-503.06 \mathrm{~Hz} \\
13 \\
100000 \mathrm{~cm}\end{array}$ \\
\hline
\end{tabular}



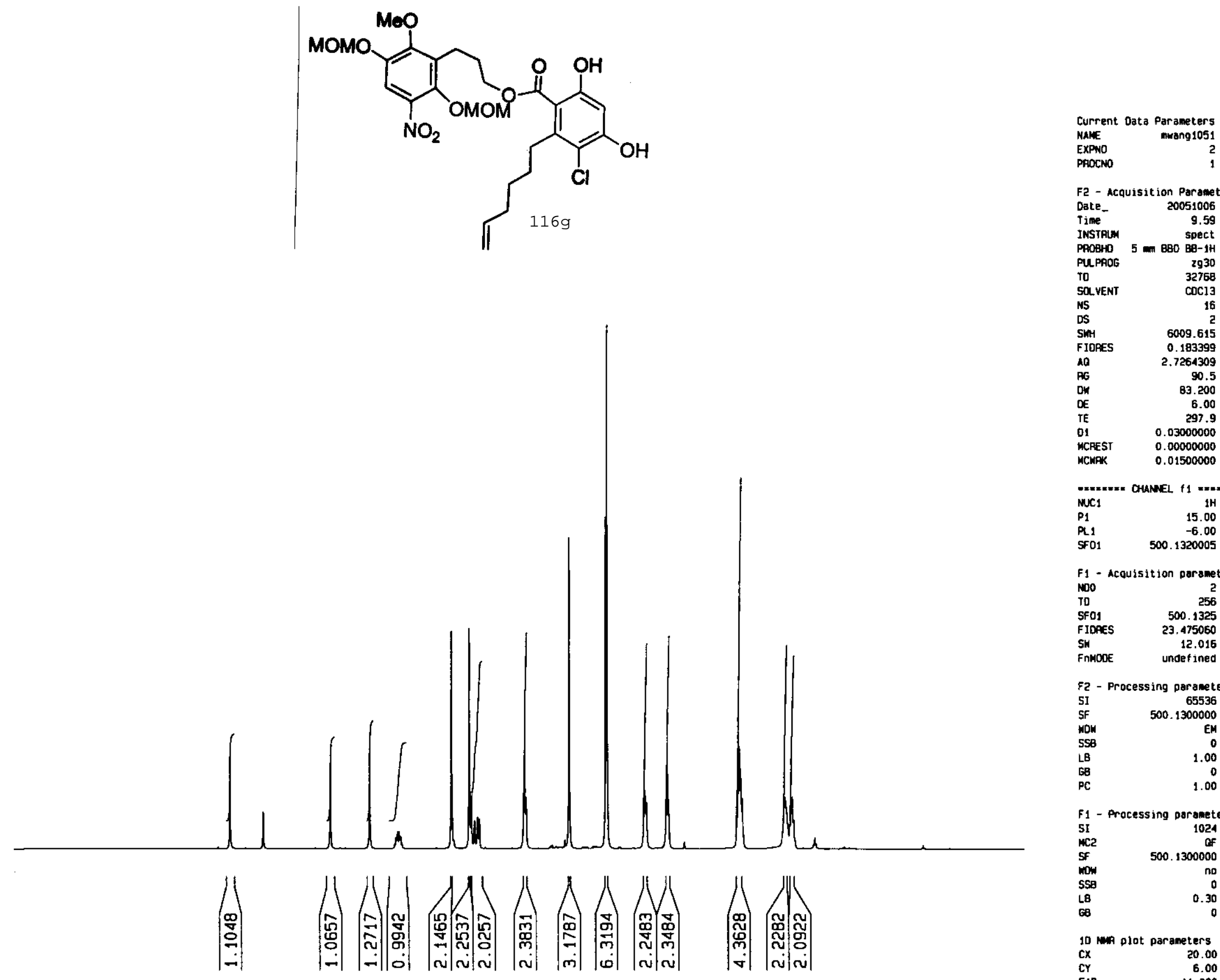

F2 - Acquisition Parameters

Date_ 20051006

Time

INSTRLM

PUPAOS 5 की

To

SO.vE

NS

DS

Sint

AO

DE

OE

OI

MCNFK

32768

$\operatorname{coc} 13$

2009

(1)

$0.19339 \mathrm{~Hz}$

90.5

83.200 usec

6.00 usec

$0.03000000 \mathrm{sec}$

$0.00000000 \mathrm{sec}$

0.01500000 sec

NuC1 $1 \mathrm{H}$

$\begin{array}{ll}P_{1} & 15.00 \text { usec } \\ P_{1} & -6.00 \mathrm{de}\end{array}$

SF01 $500.1320005 \mathrm{MHz}$

F1 - Acouisition parameters

NoO 25

SF01 $\quad 500.1325 \mathrm{mHz}$

FIDRES $\quad 23.475060 \mathrm{~Hz}$

SH 12.015 ppe

SN

$12.016 \mathrm{ppa}$
undef ined

62 - Processing paraneters

SI 65536

SF $\quad 500.1300000 \mathrm{MHz}$

NOW

SSB

LB

EM

$1.00 \mathrm{~Hz}$

F1 - Processing parameters

SI 1024

$\begin{array}{ll}\text { MC2 } & \text { Of } \\ \text { SF } & 500.1300000 \mathrm{MHz}\end{array}$

How

SSB

68

0.30

Dx plot paraneters

CX $20.00 \mathrm{~cm}$

FIP

F1

FaP

PPMCM

$6.00 \mathrm{~cm}$

$5501.43 \mathrm{~Hz}$

$-1.000 \mathrm{pom}$

$-500.13 \mathrm{~Hz}$

$0.60000 \mathrm{ppm} /$

$\mathrm{HZCM} \quad 300.07800 \mathrm{~Hz} / \mathrm{c}$ 


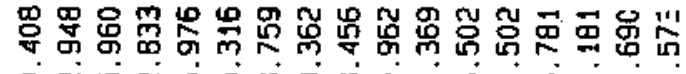

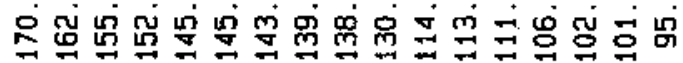
ओ

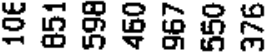

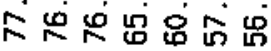

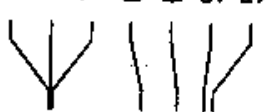

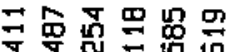

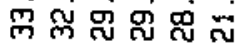

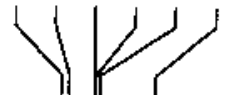

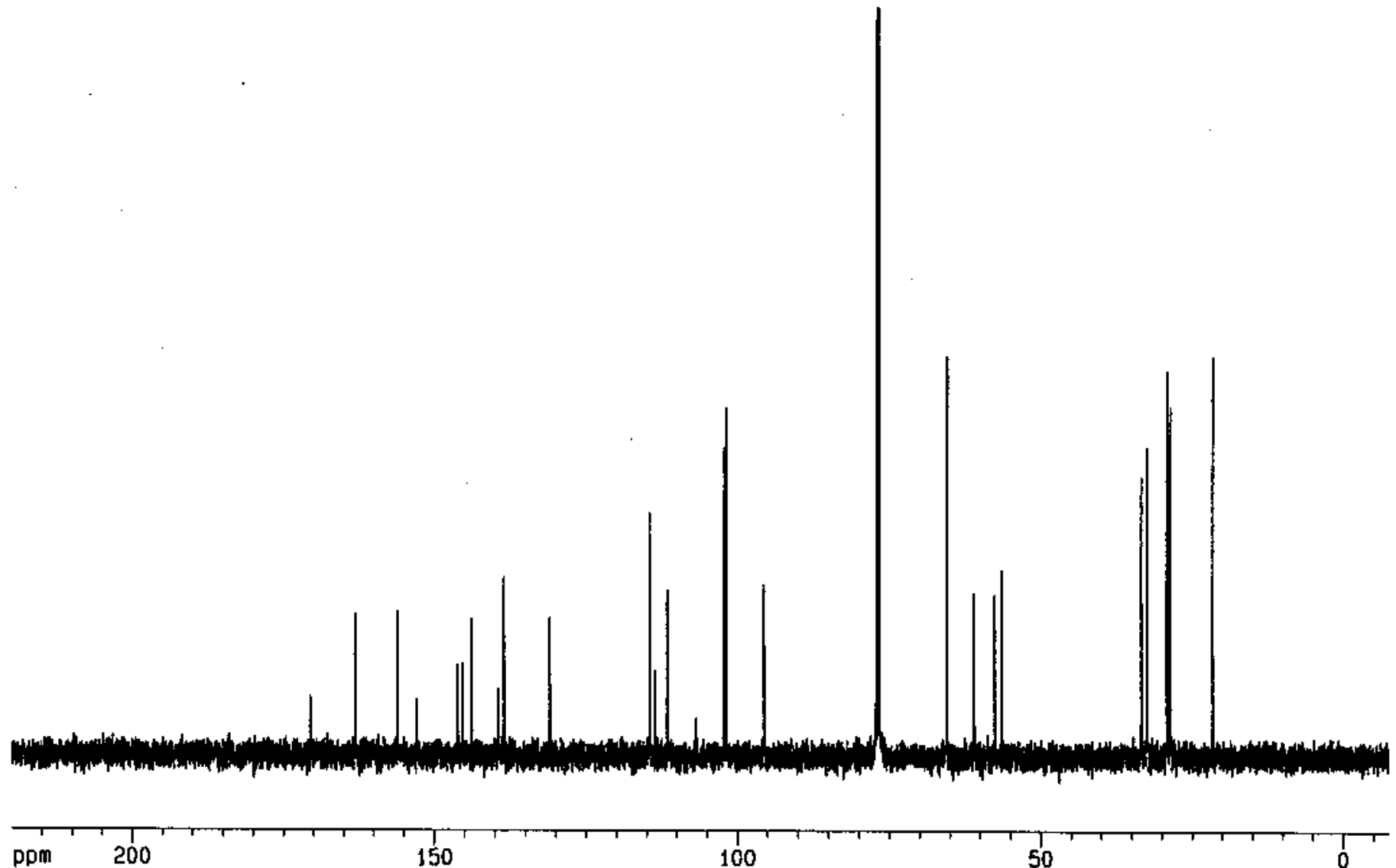




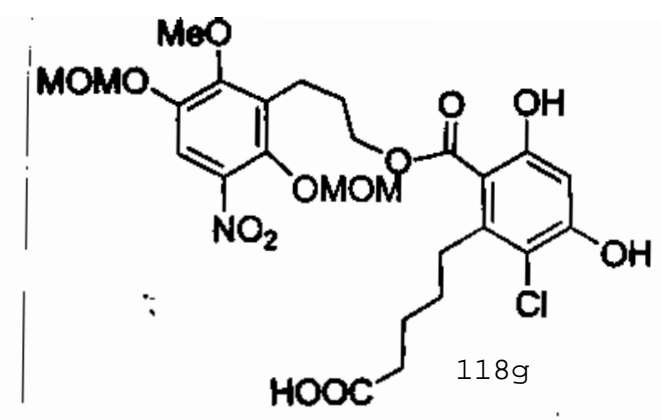

$\begin{array}{lr}\text { Current Data Parameters } \\ \text { NAME } & \text { Wmw109-4 } \\ \text { EXPNO } & 1 \\ \text { PROCNO } & 1\end{array}$

F2 - Acquisition Parameters

Date 20051010

Pime- $\quad 21.10$

INSTRTM drx400

PROBHD 5 IBI QNF $1 \mathrm{H} / 13$

PULPROG $\quad \mathbf{2 g} 30$

$\begin{array}{lr}\text { PDLPRG } & 2930 \\ \text { TD } & 32768\end{array}$

SOLVENT CDC13

NS 16

SWH

FIDRES

AQ

$4789.272 \mathrm{~Hz}$

$0.146157 \mathrm{~Hz}$

$3.4210291 \mathrm{sec}$

$$
203.2
$$

104.400 usec 5.50 usec $298.2 \mathrm{~K}$

$1.00000000 \mathrm{sec}$ $\begin{array}{ll}\text { HCREST } & 0.00000000 \mathrm{sec} \\ \text { HCWRR } & 0.01500000 \mathrm{sec}\end{array}$ $\begin{array}{ll}\text { HCREST } & 0.00000000 \mathrm{sec} \\ \text { HCWRR } & 0.01500000 \mathrm{sec}\end{array}$ $==:===-=$ CHANNEL $\mathrm{f} 1$ NUC

P1

PL1

SFO1 $1 \mathrm{H}$

9.35 usec $400.1320007 \mathrm{MHz}$

F2 - Processing parameters

SI 32758

SF $\quad 400.1300000 \mathrm{MHz}$

WDW

SSB

$\mathrm{LB}$

$\mathrm{GB}$

䍕

$.30 \mathrm{~Hz}$

$0.30 \mathrm{~Hz}$

1.00

1D MUR plot parameters

$\begin{array}{lr}\text { 1D MMR plot parameters } \\ \text { CX } & 20.00 \mathrm{~cm} \\ \text { CY } & 6.00 \mathrm{~cm} \\ \text { F1P } & 11.000 \mathrm{ppm} \\ \text { F1 } & 4401.43 \mathrm{gz} \\ \text { F2P } & -1.000 \mathrm{ppm} \\ \text { F2 } & -400.13 \mathrm{~Hz} \\ \text { PFYCM } & 0.60000 \mathrm{pPn} / \mathrm{c} \\ \text { HZCM } & 240.07800 \mathrm{~Hz} / \mathrm{Cm}\end{array}$



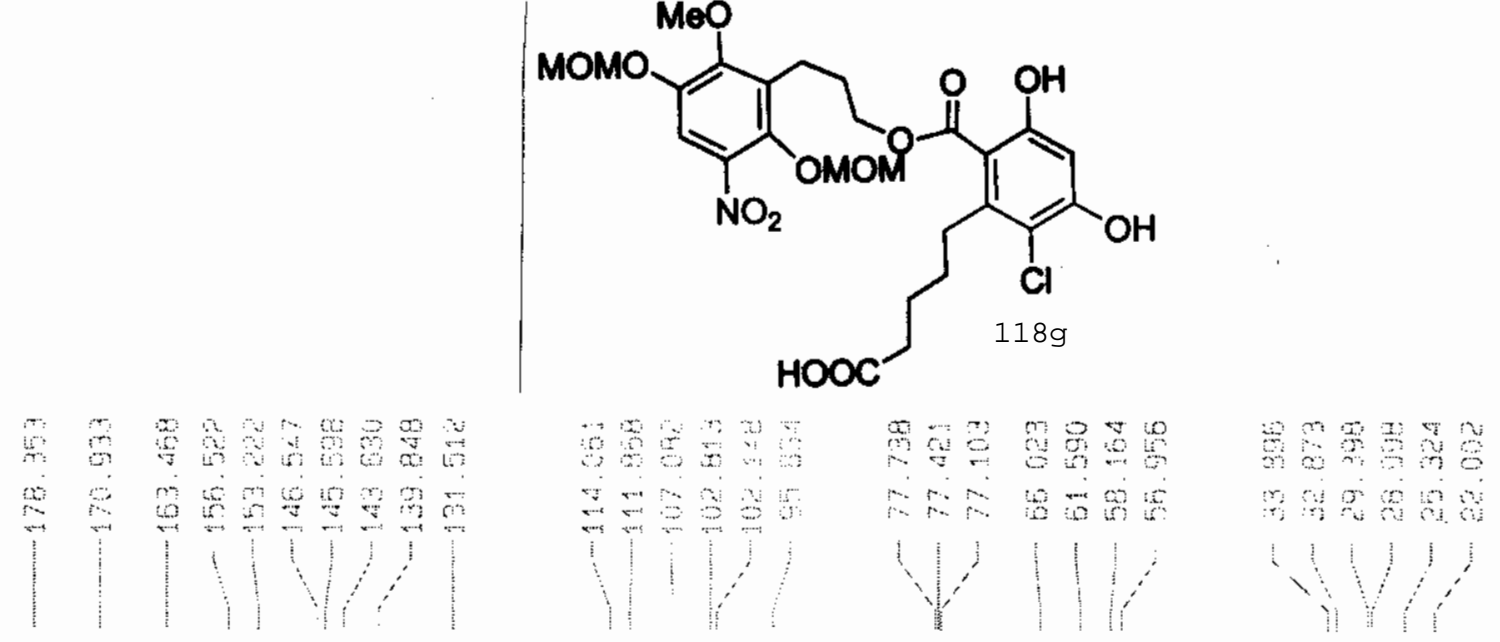

बक्ष

क्ष

Compound

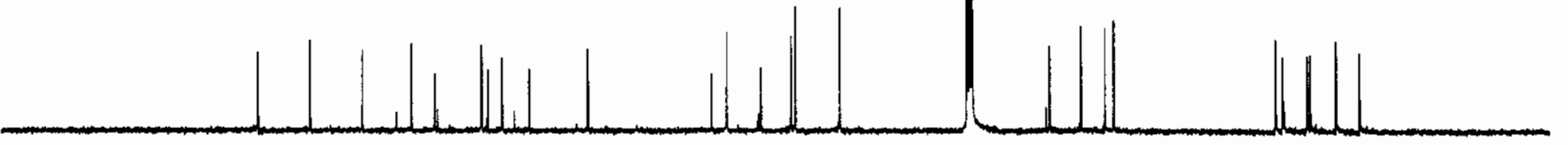

5


M_W34Pg 11 F: mwang

General

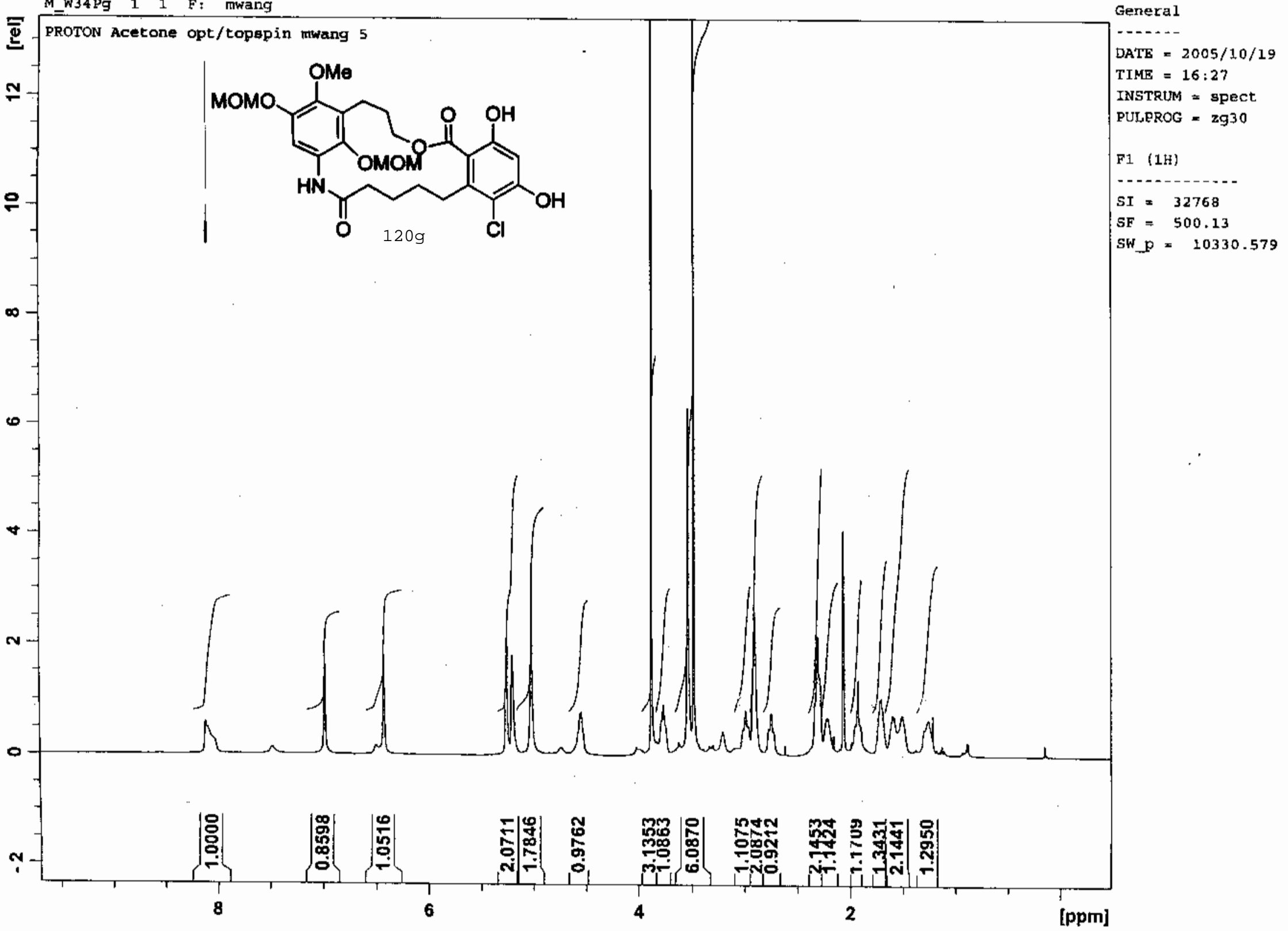




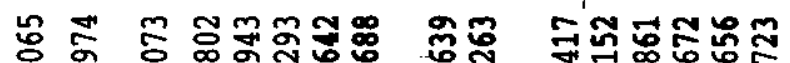
둥

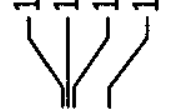

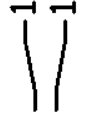

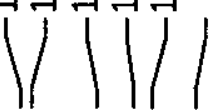

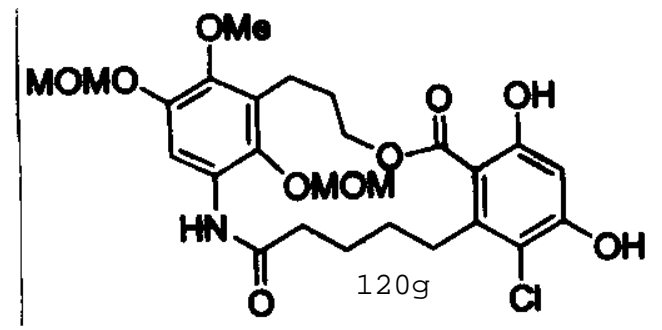

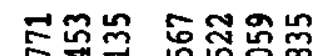

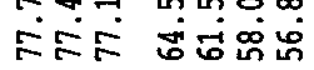

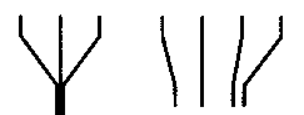

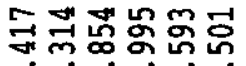

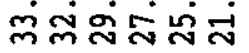

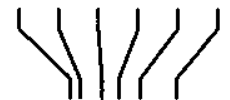

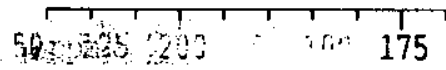

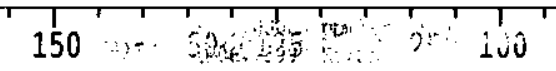

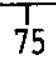

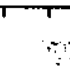

(1)

I)

(175 $\begin{array}{lr}\text { Current Data Parameters } \\ \text { FRMT } & 291029-4 \\ \text { EXPNO } & 2 \\ \text { PROCTO } & 1\end{array}$

F2 - Acquisition Paraneters

Date_ 20051030

Time $\quad 16.35$

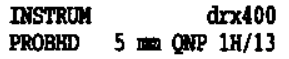

PULPROG 290930

TD $\quad 65536$

NS

DS

Sint

PIDRR

AQ

$23148.148 \mathrm{~Hz}$

$0.353213 \mathrm{~Hz}$

$1.4156276 \mathrm{sec}$

32768

21.600 usec
5.50 usec 5.50 usec
$293.2 \mathrm{~K}$ $0.15000001 \mathrm{sec}$

$0.03000000 \mathrm{sec}$

$0.05000000 \mathrm{sec}$

$0.05000000 \mathrm{sec}$
$0.00000000 \mathrm{sec}$ $0.00000000 \mathrm{sec}$
$0.01500000 \mathrm{sec}$

MCTRR

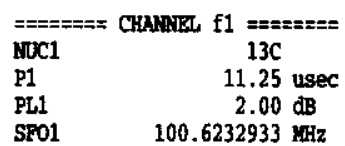

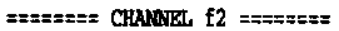

CPDPRG walt 21

PCPD2

PL2

PL2

PL13
SPO2

100.00 usec

$-4.00 \mathrm{~dB}$

$16.58 \mathrm{~dB}$

$17.00 \mathrm{~dB}$

72 - Processing parameters SI

SI $\quad 65536$

SP $\quad 100.6127290 \mathrm{MHz}$

WDN

SSB

LB

E.

0
$1.00 \mathrm{~Hz}$

$1.00 \mathrm{~Hz}$

10 M.R plot paraweters

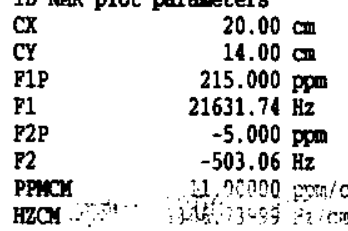


<smiles>COc1cc(O)c(NC(=O)CCCCc2c(O)c(Cl)cc(O)c2CCCOC(=O)c2c(O)cc(O)c(Cl)c2O)cc1O</smiles>

焉
总
点

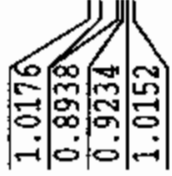

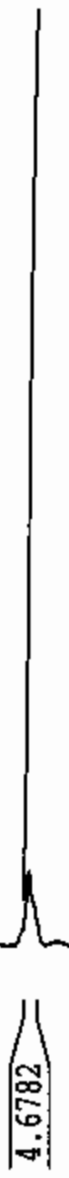

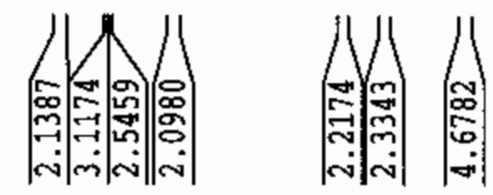

Current Data Parameters

NAKE

2-พnaw342

PROCNO

F2 - Acquisition Parameters

Date 20051109

$\begin{array}{lr}\text { Time } & 20051109 \\ \text { Tinstron } & 15.32\end{array}$

INSTRUL d d $\times 400$

PROBHD $5 \mathrm{~mm}$ QNF $1 \mathrm{H} / 13$

PULPROG $\quad z g 30$

TD 32768

SOLVENT CDCl3

NS

DS

SWH

FIDRES

AQ

RG

RG

DE

TE

D1

MCREST

MCWRK

NuC1

p1

PL1

SF01

4789.272

$0.146157 \mathrm{~Hz}$

$3.4210291 \mathrm{sec}$

645.1

104.400 usec 5.50 usec

$$
293.2 \mathrm{~K}
$$

$1.00000000 \mathrm{sec}$

0.00000000 sec

$0.01500000 \mathrm{sec}$

F2 - Processing parameters

SI 32768

SF $\quad 400.1300091 \mathrm{AHz}$

WDi

SSB

$\mathrm{LB}$

PC

EM

$.30 \mathrm{~Hz}$

1.40

1D NGR plot parameters

$\begin{array}{lr}\mathrm{CY} & 20.00 \mathrm{~cm} \\ \mathrm{CY} & 0.00 \mathrm{~cm}\end{array}$

F1P $\quad 11.000 \mathrm{ppm}$

F1 $\quad 4401.43 \mathrm{zz}$

E2P $\quad-1.000 \mathrm{ppm}$

F2

PPMCM

$\mathrm{HZCM}$
$-400.13 \mathrm{~Hz}$

$0.60000 \mathrm{ppm} / \mathrm{c}$ $240.07800 \mathrm{~Hz} / \mathrm{cm}$ rmato

8
4

$2: i$

0 


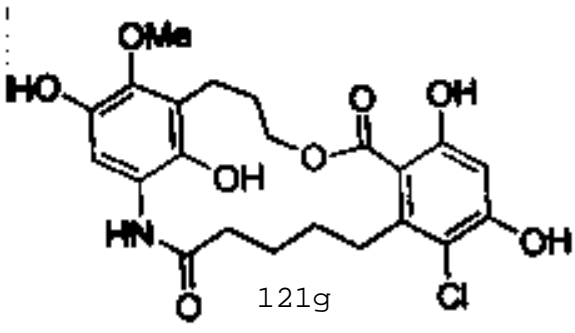

Currebt Data Paraneteré

$$
\text { EXPNO }
$$

2. Fint 34

PROCANO

P2 - Accuisition Parametera

Tate-

Time-

INsTru

PUTPROG

TD

SOrtwent

NS

Ds

SHH

$\mathrm{AQ}$

RG

DN

TE

T1

dil

DELTA

TDO

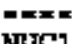

No1

BFOI

CPDPRG

NUC2

PCPD2

PL2

PL12

PI,13

SEOZ

Fi

SI

SF

SSB

LSB

GB
20051109

19.56

opect

$5 \mathrm{~mm}$ CPDU $13 \mathrm{C}$

xgpg 30

65536

Acetone

3072

$30030.029 \mathrm{~Hz}$

$0.458222 \mathrm{~Hz}$

1.0912410 eec 5792.6

16.650 uaes 6. DO vaer $298.0 \mathrm{~K}$

0.15000001 gec $0.03000000 \mathrm{gec}$ 0.05000000 sec

CHANNEL

1 xхx

10.00 uaec $-4.30 \mathrm{~dB}$ 125.7703640 MHz

\section{CHABNEL $f$}

waltz16

$00.00 \mathrm{ugec}$

$-5.50 \mathrm{~dB}$

$8.94 \mathrm{~dB}$ 10.00 dB

$500.1320000 \mathrm{MHz}$

Procesaing parametera 32769

125.7577890 Matz

EMI

$1.00 \mathrm{~Hz}$

1.0 

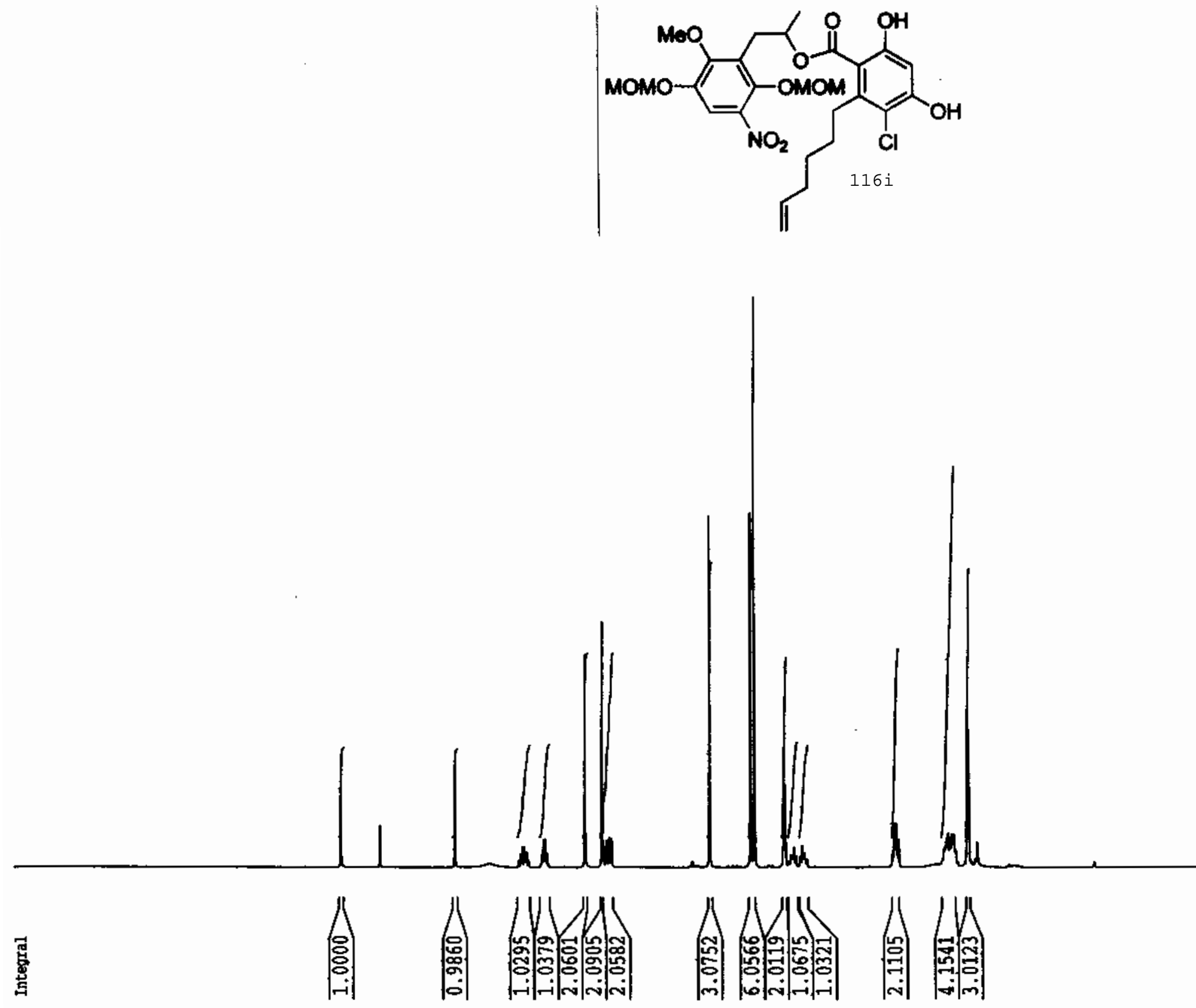

$$
\text { Tp }
$$

6
4

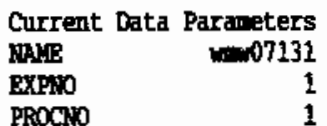

F2 - Acquisition Paraneters

Date 20050713

Tive 20.34

Disstion a drx400

PROB:D 5 In oup $1 \mathrm{H} / 13$

PULPROG

$\mathrm{zg} 30$

To

SOLVET

NS

DS

Sill

FIDRES

AQ

$\mathrm{RG}$

D.

DR

TE

MCREST

WCAREST

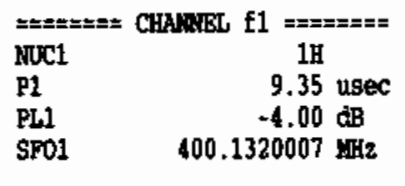

F2 - Processing paraneters

SI 32768

SF $\quad 400.1300000 \mathrm{LHZ}$

พDI

SSB

LB

PC

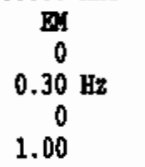

1D kisR plot parameters

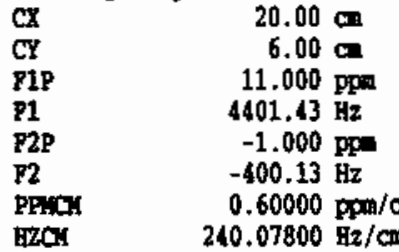




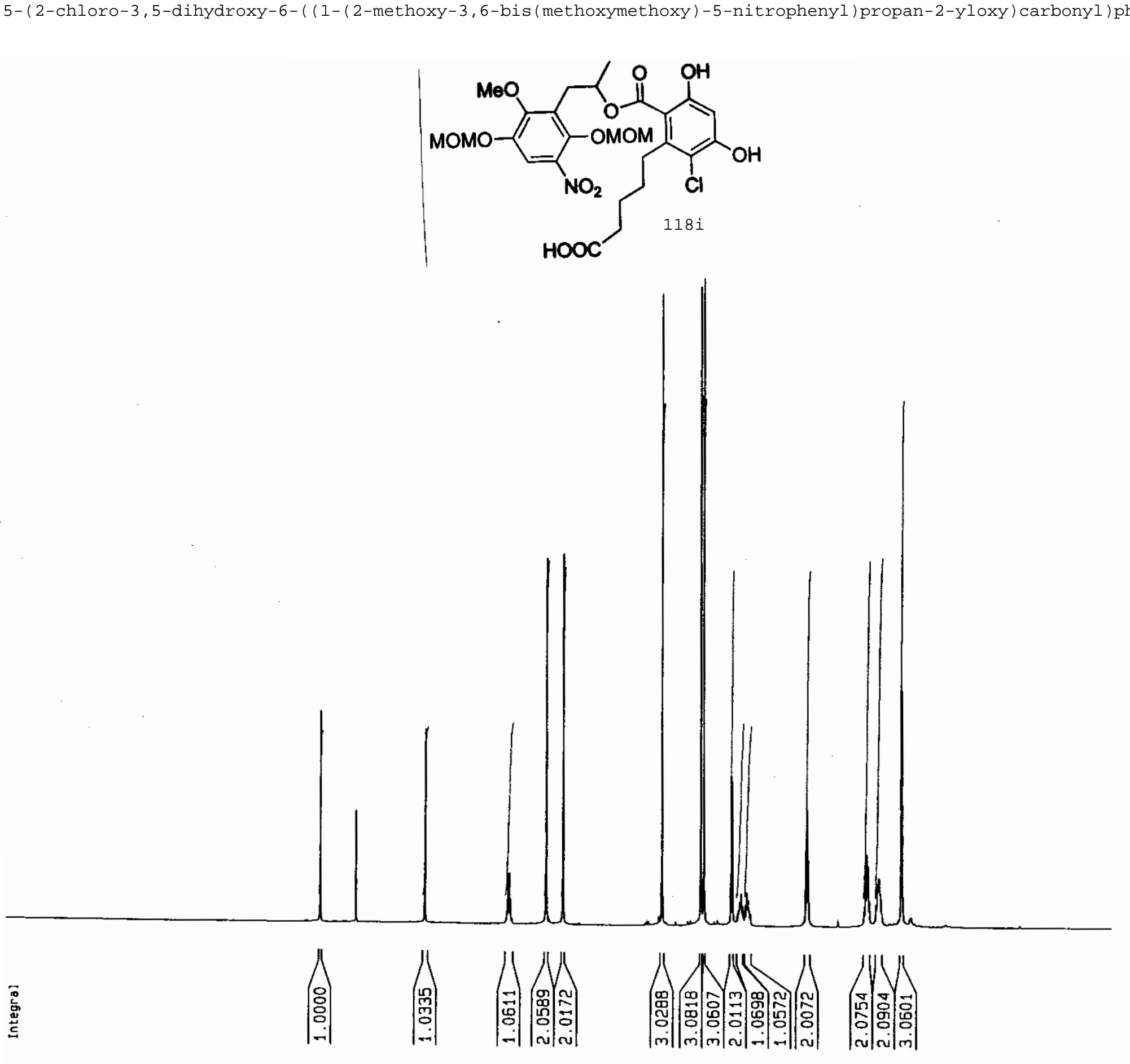

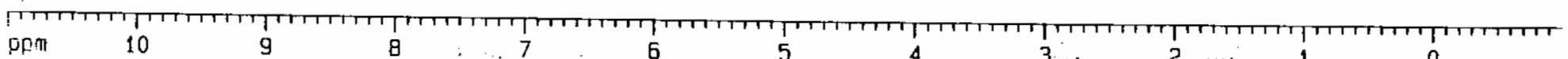




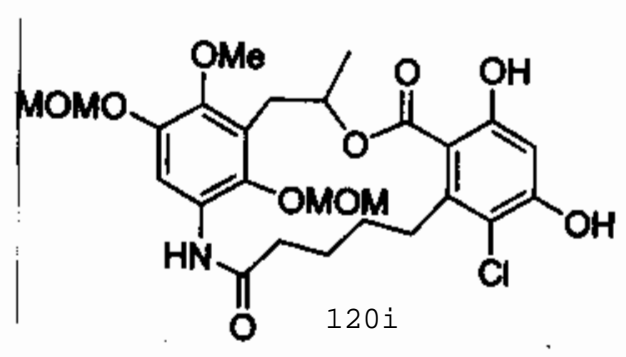

要

点

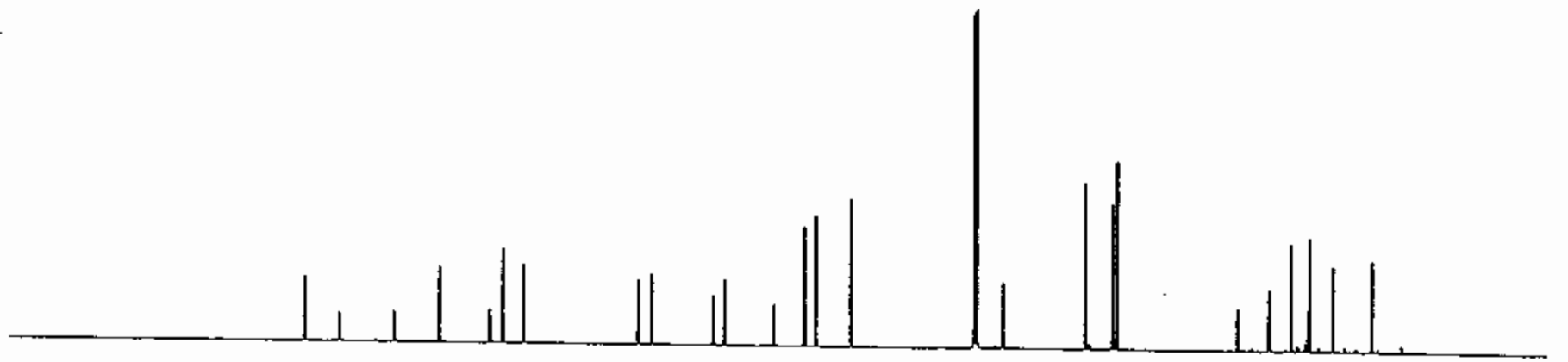

ppm 200 


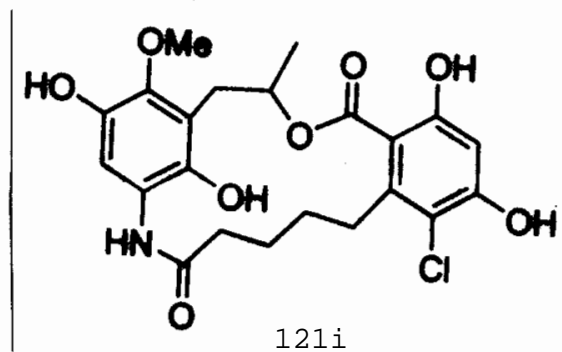

$\begin{array}{lr}\text { Current } & \text { Data } \text { Parameters } \\ \text { NAME } & \text { WMHHO24i } \\ \text { EXPNO } & 11 \\ \text { PROCNO } & 1\end{array}$

F2 - Acquisition Parameters Date 20050802

Time $\quad 15.26$

INSTRUM spect

PROBHD $5 \mathrm{~mm}$ CPDUL $13 \mathrm{C}$

2930
65536

SOLVENT

Aceton

NS

OS

SHH 2

$\begin{array}{lr}\text { SHH } & 10330.578 \mathrm{~Hz} \\ \text { FIDAES } & 0.157632 \mathrm{~Hz}\end{array}$

AQ $\quad 3.1719923 \mathrm{sec}$

RG

OW

DE

64
46.400 usec

6.00 usec

$300.0 \mathrm{~K}$

$01 \quad 1.00000000 \mathrm{sec}$

$0.00000000 \mathrm{sec}$

$0.01500000 \mathrm{sec}$
$= \pm x===$ CHANNEL $f 1=======$
$1 \mathrm{H}$
$\begin{array}{ll}\text { NUC1 } & 1 \mathrm{H} \\ \mathrm{P} 1 & 15.00 \text { us }\end{array}$
$-5.00 d \theta$

PL1

SF01 $500.1330805 \mathrm{MHZ}$

F2 - Processing parameters

SI 32768

SF $\quad 500.1300007 \mathrm{MHZ}$

$\begin{array}{lr}\text { WDW } & \text { EM } \\ \text { SSB } & 0\end{array}$

LB

PC

$E M$
0
$0.30 \mathrm{~Hz}$
0

1.00

F1 - Processing parameters

SI 1024

MC2

SF $\quad 500.1300000 \mathrm{MHZ}$

WDW OSINE

SSB

$0.30 \mathrm{~Hz}$

0

1D NMR plot parameters

CX $20.00 \mathrm{~cm}$

$\begin{array}{ll}\mathrm{CY} & 6.00 \mathrm{~cm}\end{array}$

F1P $\quad 10.979 \mathrm{ppm}$

$\mathrm{F}_{1} \quad 5490.98 \mathrm{~Hz}$

$\begin{array}{ll}\mathrm{F}_{2} \mathrm{P} & -0.7 \mathrm{B1} \mathrm{ppm}\end{array}$

$\begin{array}{ll}F 2 & -0.790 .59 \mathrm{~Hz}\end{array}$

$\begin{array}{lr}\mathrm{PPMCM} & 0.58000 \mathrm{pDm} / \mathrm{Cm} \\ \mathrm{HZCM} & 294.07846 \mathrm{~Hz} / \mathrm{Cm}\end{array}$ 

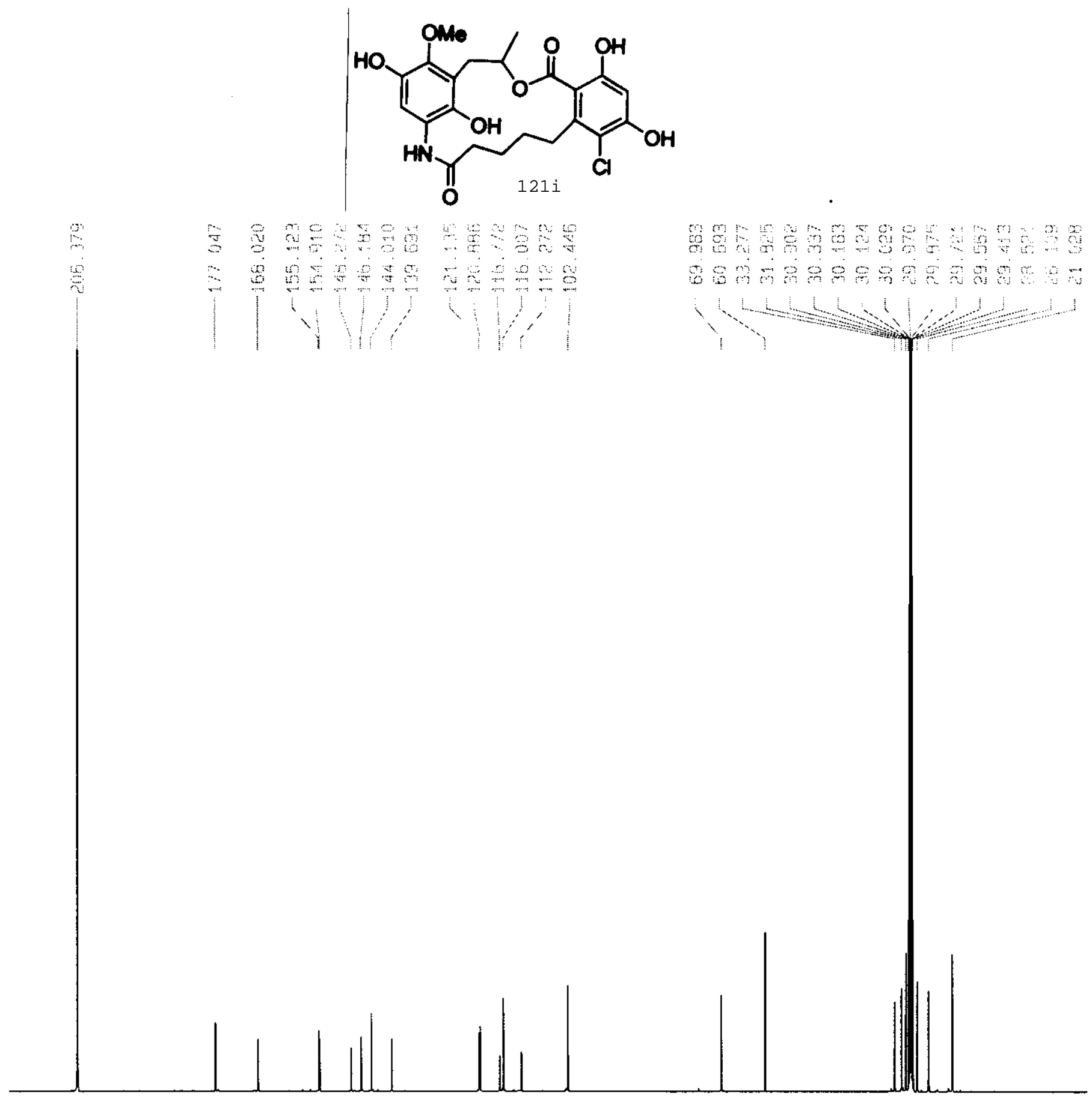

Current Data Parameters NAME

EXPNO
PROCNO

WMWHO24i Date_ 20050802 $\begin{array}{ll}\text { Time } & 15.59 \\ \text { INSTRUM } & \text { spect }\end{array}$ PROBHD 5 man CPOUL 13C PULPROG 2 ggog30 TD 65536 SOLVENT Aceton DS SWH FIDRES AO

AG

DW

DE

01

d11

DELTA

MCREST

MCWAK

31446.541 Hz

$0.479836 \mathrm{~Hz}$ $1.0420724 \mathrm{sec}$ B192 15.900 usec 6.00 usec $300.0 \mathrm{~K}$ $0.15000001 \mathrm{sec}$ (50000 $0.03000000 \mathrm{sec}$ $0.05000000 \mathrm{sec}$ $0.00000000 \mathrm{sec}$ ==xxmx= CHANNEL, NUC1

P1

PL1 $13 \mathrm{C}$

SF01

10.00 usec -4.90 ab $=======$ CHANHEL CPOPRG NUC2 PCPO2

PL2

PL12

PL13

fel $f 2===\pi===x$ walt 216

80.00 usec $-5.00 \mathrm{~dB}$ $9.54 \mathrm{~dB}$ 10.00 相 $500.1325000 \mathrm{MHz}$

F2 - Processing parameters SI $\quad 65536$ $\begin{array}{lr}\text { SF } & 125.7576758 \\ \text { WOW } & \text { EM }\end{array}$ $5 S B \quad 0$ LB $\quad 1.00 \mathrm{~Hz}$ GC 0
.40

10 NMR plot paraneters CX $\quad 20.00$ $\mathrm{CY} \quad 0.00 \mathrm{~cm}$ F1P $242.517 \mathrm{ppm}$ $\begin{array}{lr}F_{1} 1 & 30498.40 \mathrm{~Hz} \\ F_{20} & -7.539 \mathrm{ppm}\end{array}$ F2 PPMCM $-948.11 \mathrm{~Hz}$ $12.50282 \mathrm{ppm} / \mathrm{c}$ $\mathrm{HZCM}$ $1572.32544 \mathrm{~Hz} / \mathrm{Cr}$ 


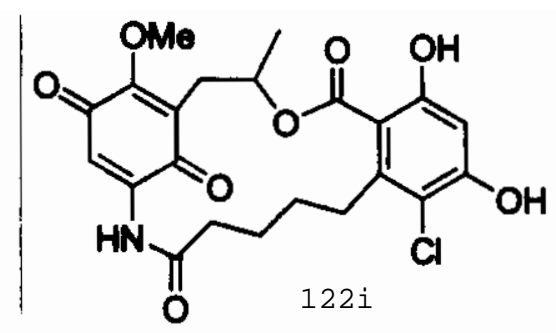

Pentenyl Radanamycin quinone
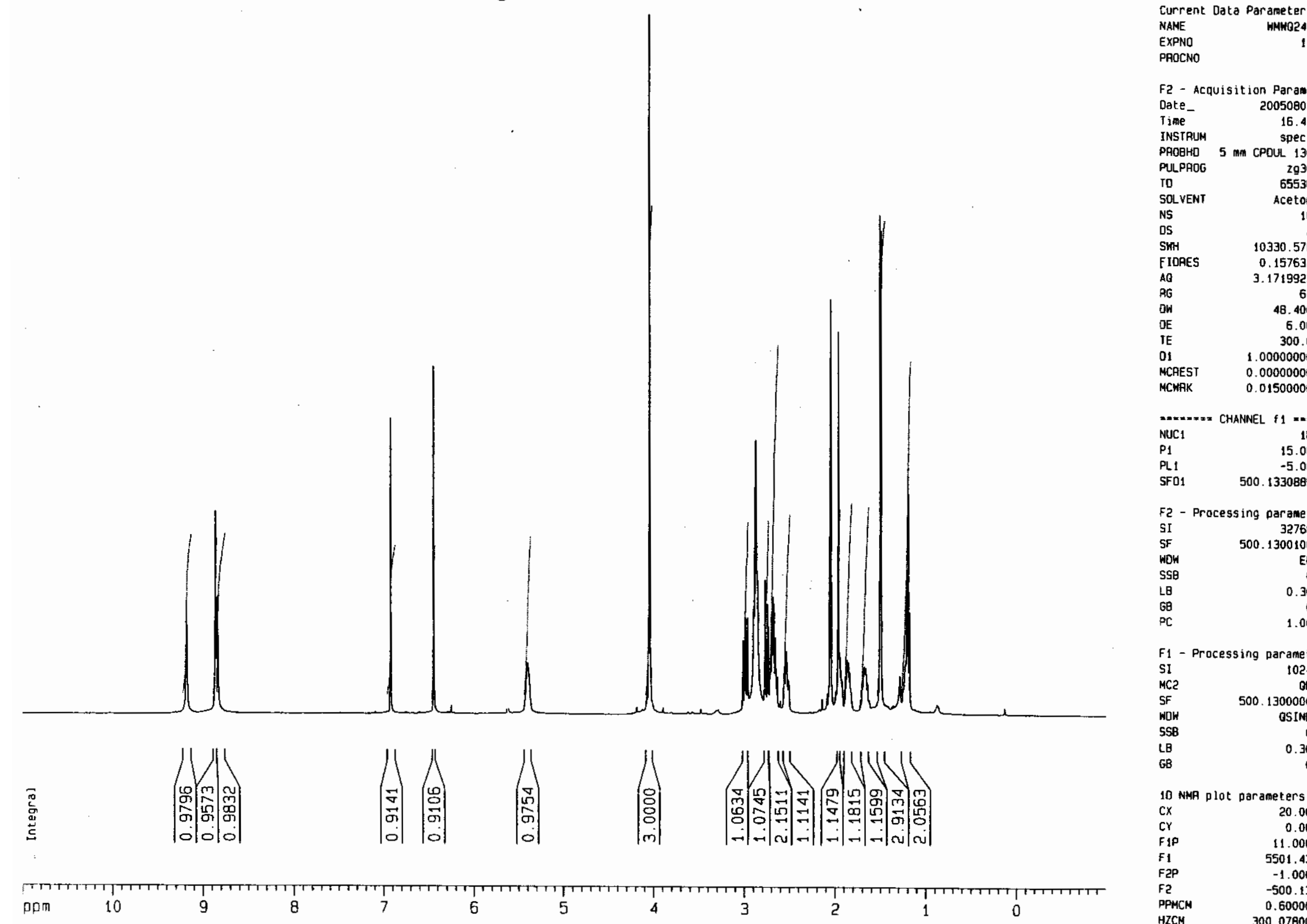

F2 - Acquisition Parameters

20050802

Time $\quad 16.47$

INSTRUM spect

PROBHD 5 m CPDUL 13C

PULPHOG 2930

$\begin{array}{lr}\text { ID } & 65536 \\ \text { SOLVENT } & \text { Aceton }\end{array}$

NS

Sin

FIDAES

$A Q$

RG

OW

IE

01

MCHAK

16

$10330.578 \mathrm{~Hz}$

$0.157632 \mathrm{~Hz}$

$0.157632 \mathrm{~Hz}$
$3.1719923 \mathrm{sec}$

64

48. 400 usec 6.00 usec $300.0 \mathrm{~K}$

$.00000000 \mathrm{sec}$

000000 $0.01500000 \mathrm{sec}$

$=\ldots=m=$ CHANNEL I

NUC

PLI

$f 1=\ldots$

SF01 500.1330885 MHZ

F2 - Processing parameters

SI 32768

SF $\quad 500.1300106 \mathrm{MHz}$

WOW

LQ $\quad 0.30 \mathrm{~Hz}$

68 o

F1 - Processing parameters

$\begin{array}{lr}\text { SI } & 1024 \\ \text { MC2 } & \text { BF }\end{array}$

SF

500. $1300000 \mathrm{MHZ}$

OSINE

0
$0.30 \mathrm{~Hz}$

$\begin{array}{lr}\text { LB } & 0.30 \\ \text { GB } & 0\end{array}$

10 NMA plot parameters

$\mathrm{CX} \quad 20.00 \mathrm{~cm}$

$0.00 \mathrm{~cm}$

Fip $\quad 11.000 \mathrm{ppm}$

F1 $5501.43 \mathrm{~Hz}$

$\begin{array}{ll}\text { F2P } & -1.000 \mathrm{ppm} \\ \mathrm{F2} & -500.13 \mathrm{~Hz}\end{array}$

PPMCM $0.60000 \mathrm{ppm} /$

$\mathrm{HZCM} \quad 300.07800 \mathrm{~Hz} / \mathrm{c}$ 


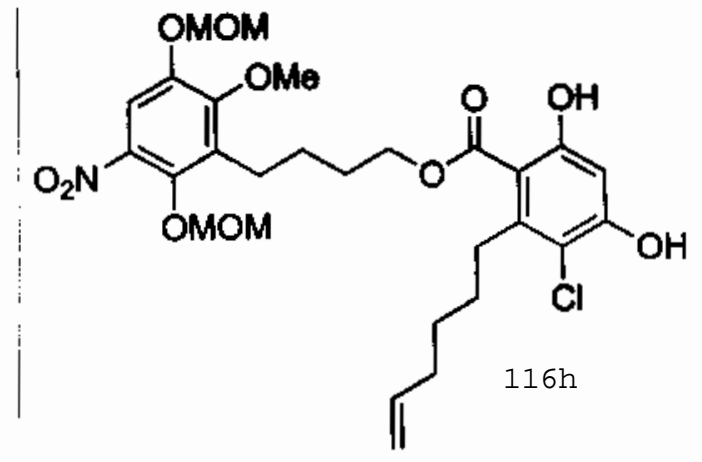

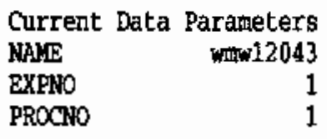

F2 - Accuisition Parameters Date 20051205

Time $\quad 15.52$

$\begin{array}{lr}\text { Time } & 15.52 \\ \text { INSTRON } & \mathrm{drx} \times 400\end{array}$

PROBHD 5 man QNP 1H/13

PULPROG zg30

TD 32758

SOLWANT $\quad$ CDC13

NS

DS

SWH

FIDRES

$\mathrm{AQ}$
$\mathrm{RG}$

DN

$\mathrm{DE}$

$\mathrm{DE}$

MCREST

MCWRR

जUए।

P1

PL1

16
2

$4789.272 \mathrm{~Hz}$

$0.146157 \mathrm{~Hz}$

$3.4210291 \mathrm{sec}$

$$
203.2
$$

104.400 usec

5.50 usec $293.2 \mathrm{~K}$

$1.00000000 \mathrm{sec}$

$0.00000000 \mathrm{sec}$

$0.01500000 \mathrm{sec}$

SFOI
SI $\quad 32768$
SP $\quad 400.1300091 \mathrm{MHz}$
WDN BNI
SSB
LB
GB

EII
0
$0.30 \mathrm{~Hz}$
0
1.00

1D KMR plot parameters

$\begin{array}{lr}\text { CX } & 20.00 \mathrm{~cm} \\ \text { CY } & 5.00 \mathrm{~cm} \\ \text { F1P } & 11.000 \mathrm{ppal} \\ \text { F1 } & 4401.43 \mathrm{~Hz} \\ \text { F2P } & -1.000 \mathrm{ppm} \\ \text { F2 } & -400.13 \mathrm{~Hz} \\ \text { PPNCY } & 0.60000 \mathrm{ppm} / \mathrm{cr} \\ \text { HZCY } & 240.07800 \mathrm{~Hz} / \mathrm{cm}\end{array}$
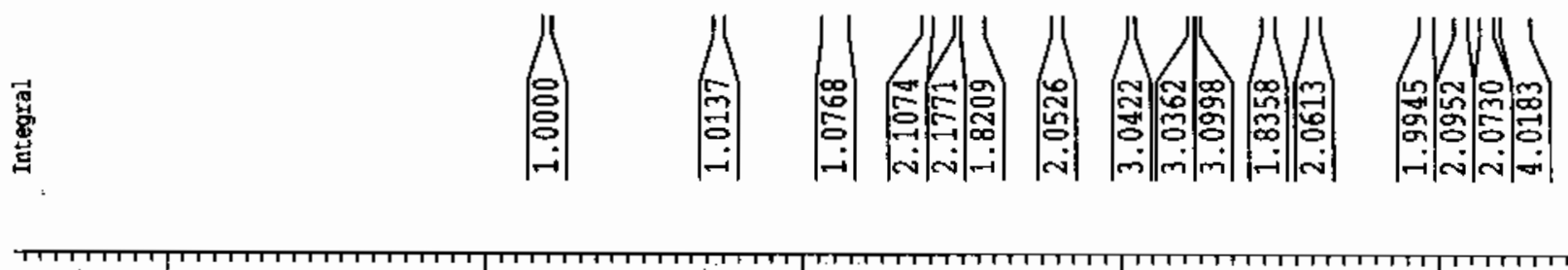
<smiles>C=CCCCCc1c(Cl)c(O)cc(O)c1C(=O)OCCCCc1c(OC)c([18O])cc([N+](=O)[O-])c1OC</smiles>

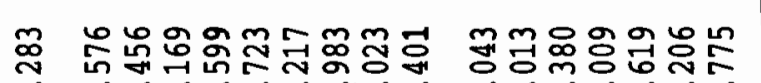
至

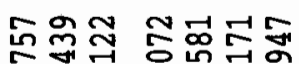
둥ํำ
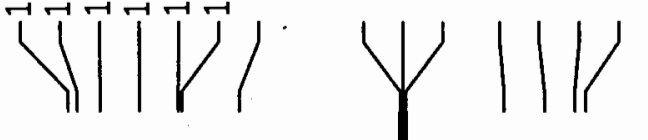

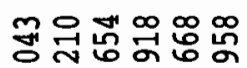

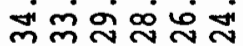

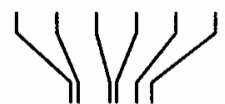

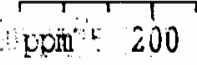

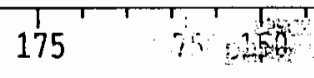

酐

50: 125

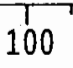

5 an

25 


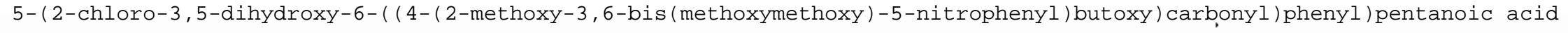

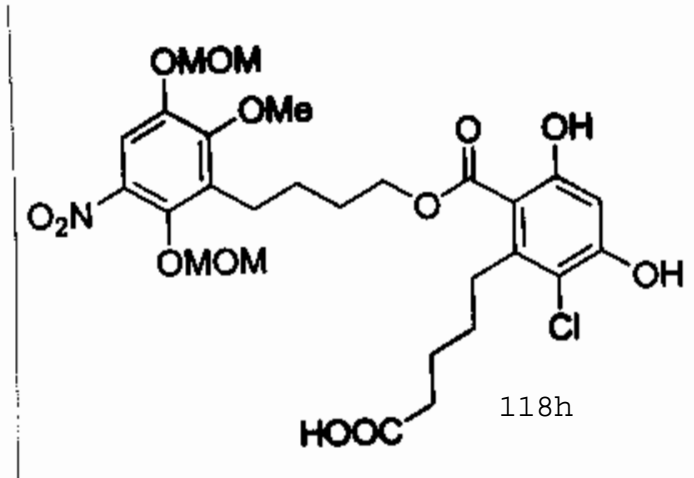

$\begin{array}{lr}\text { Current Data } & \text { Parameters } \\ \text { NAME } & \text { waw12132 } \\ \text { EXPNO } & 1 \\ \text { PROCNO } & 1\end{array}$

F2 - Acquisition Parameters

Date 20051216

$\begin{array}{lr}\text { Tine } & 9.32 \\ & \text { arx } 400\end{array}$

INSTRUM

PROBRD 5 mm ONP $1 \mathrm{H} / 13$

PULPROG $\quad \mathrm{zg} 30$

$\mathrm{PD} \quad 32768$

SOLVENT CDC13

NSS

DS

SWH

FIDRES

$\mathrm{AQ}$

RG

DN

DE

TE

D1

MCWRK

16

$4789.272 \mathrm{~Hz}$

$0.146157 \mathrm{~Hz}$

$3.4210291 \mathrm{sec}$ 256

104.400 usec 5.50 usec $293.2 \mathrm{~K}$

$1,00000000 \mathrm{sec}$
$0.00000000 \mathrm{sec}$

$0.01500000 \mathrm{sec}$

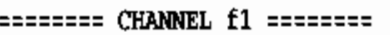
NoC

PL1

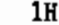

.35 usec

$-4.00 \mathrm{~dB}$

SF01 $400.1320007 \mathrm{MHz}$

F2 - Processing parameter

$\begin{array}{lc}\text { SI } & 32768 \\ \text { SF } & 400.1300091 \mathrm{kHz}\end{array}$

WDN $\mathrm{EN}$

$\begin{array}{ll}\text { SSB } & 0 \\ \text { LB } & 0.30 \mathrm{~Hz}\end{array}$

$\begin{array}{lr}\mathrm{LB} & 0.30 \\ \mathrm{~GB} & 0 \\ \mathrm{PC} & 1.00\end{array}$

1D

$c x$

CY

B1P

F1

F2

PPNCY
$20.00 \mathrm{~cm}$
$000 \mathrm{~cm}$ $4401.43 \mathrm{~Hz}$
$-1.000 \mathrm{ppm}$ $-400.13 \mathrm{~Hz}$
$0.60000 \mathrm{ppm} / \mathrm{c}$
$240.07800 \mathrm{~Hz} / \mathrm{cm}$

HZCM 


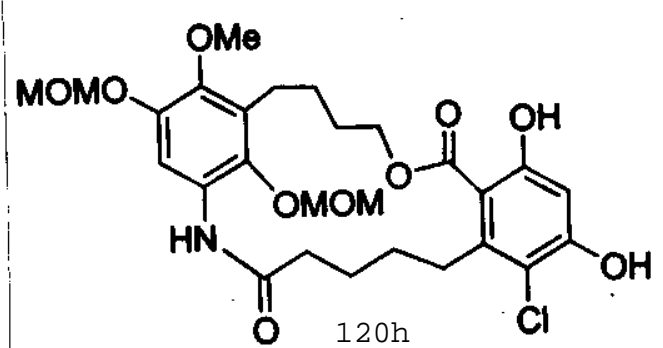

Current Data Parameters

EXPNO

PROCNO

F2 - Acquisition Parameters

20051221

Time

20.13

INSTRUN $d r \times 400$

PROBHD 5 mn QNP 1H/13

$\mathrm{zg} 30$

TD

SOLVENT

32768

NS

DS

SWH

FIDRES

$A Q$

RG

DW

DW

TE

IE

MCREST

MCWRK $\quad 0.01500000 \mathrm{sec}$

CDCl3

2

$0.146157 \mathrm{~Hz}$

$3.4210291 \mathrm{sec}$ 456.1

104.400 usec 5.50 usec $293.2 \mathrm{~K}$

$1.00000000 \mathrm{sec}$

$0.00000000 \mathrm{sec}$

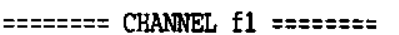
NUC1

1H

P1

9.35 usec

PL1
SF01

$-4.00 \mathrm{~dB}$

SFO1 $400.1320007 \mathrm{MHz}$

F2 - Processing parameters

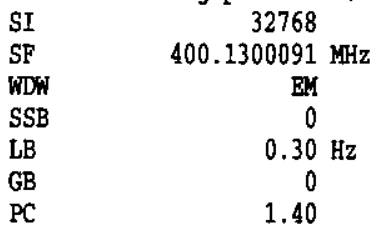

1D MMR plot parameters

$\begin{array}{lr}\text { CX } & 20.00 \mathrm{~cm} \\ \text { CY } & 0.00 \mathrm{~cm} \\ \text { F1P } & 11.000 \mathrm{ppm} \\ \text { F1 } & 4401.43 \mathrm{~Hz} \\ \text { F2P } & -1.000 \mathrm{ppm} \\ \text { F2 } & -400.13 \mathrm{~Hz} \\ \text { PPMCM } & 0.60000 \mathrm{ppm} / \mathrm{cm} \\ \text { HZCM } & 240.07800 \mathrm{~Hz} / \mathrm{cm}\end{array}$




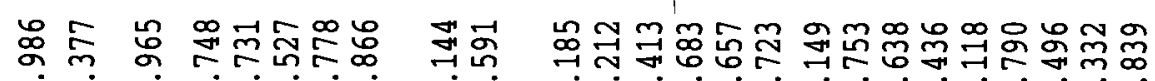

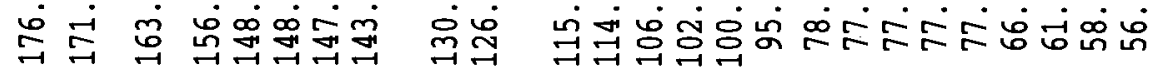

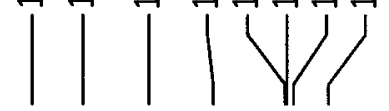

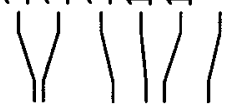

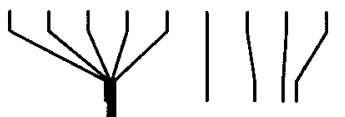

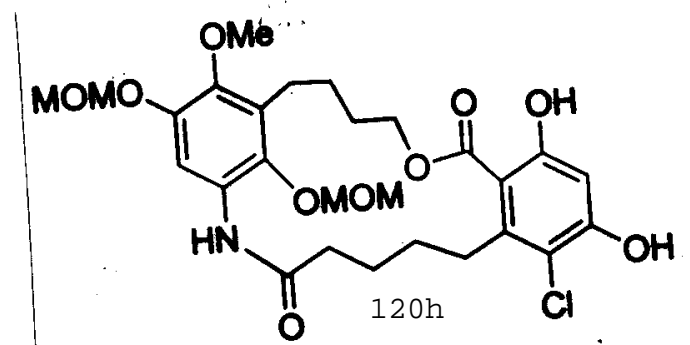

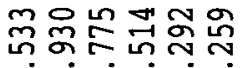

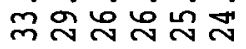

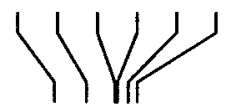

Macrocycle 


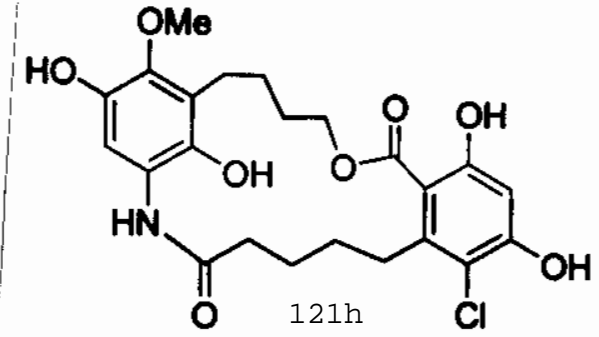

Current Data Parameters

NAME

wmw601042

PROCNO

F2 - Acquisition Parameters

Date 20060104

Time $\quad 15.10$

INSTRUM - drx400

PROBHD $5 \mathrm{~mm}$ ONP 1H/13

PULPROG $\quad \mathrm{zg} 30$

$\mathrm{zg} 30$
32768

SOLVENT

NS

DS

SWH

FIDRES

AQ

RG

DN

DE

TE

D1

MCWRK

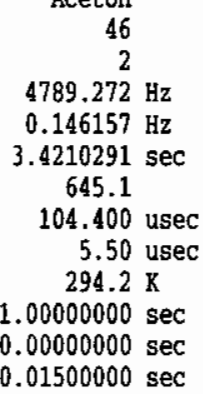

$=======$ CHANAEL NUC1

PL1

9.35 usec

SFO1 $400.1320007 \mathrm{MHz}$

F2 - Processing parameters SI $\quad 32768$ $\begin{array}{lc}\text { SI } & 32768 \\ \text { SF } & 400.1300091 \mathrm{kHz}\end{array}$ WDW EM

SSB

LB

GB

PC
EN
0
$0.30 \mathrm{~Hz}$ 1.00

1D NRR plot parameters

CX

CY

F1

F2P

F2

PPMCI

HZCY

pp

8

$7 \quad 6$

5

$4 t$

$\because 3$

2 


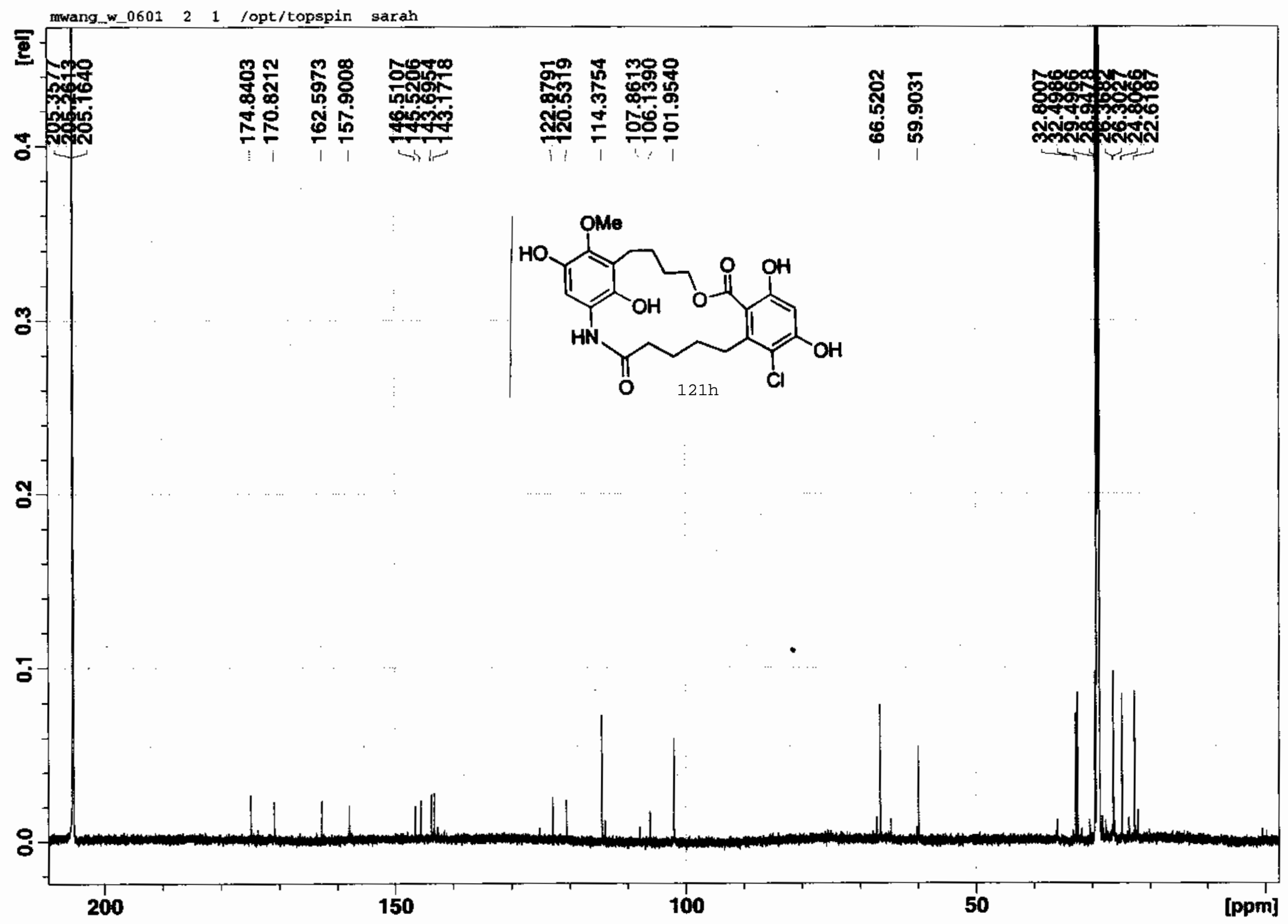

